Claude Tomaszewski

\title{
Bewertung strategischer Flexibilität beim Unternehmenserwerb
}

Der Wertbeitrag von Realoptionen 


\section{Claude Tomaszewski}

\section{Bewertung strategischer Flexibilität beim Unternehmenserwerb}

Bei Unternehmensakquisitionen werden häufig zusätzliche Investitionsoptionen geschaffen, über die erst in Zukunft entschieden wird. Die hierdurch entstehende unternehmerische Flexibilität besitzt einen ökonomischen Wert, der mit traditionellen Ertragswert- bzw. DCF-Verfahren nur schwer vollständig zu erfassen ist. Vor diesem Hintergrund werden zu deren Ergänzung Optionsbewertungskalküle vorgeschlagen. Der Autor erörtert sowohl theoretische Anwendungsvoraussetzungen als auch Gestaltungsalternativen einer praktischen Implementierung. Die im Zusammenhang mit solchen Investitionsoptionen diskutierten Aspekte werden anhand eines durchgängigen Zahlenbeispiels präsentiert. Die vorgestellte Bewertungsmethodik ist zudem nicht nur bei strategischen Akquisitionen einsetzbar, sondern kann auch beim Aufbau neuer Geschäftsfelder eine geeignete Beurteilungsgrundlage darstellen.

Claude Tomaszewski, 1969 in Luxemburg geboren, studierte von 1989 bis 1994 Betriebswirtschaftslehre an der Universität Münster. Seit 1995 arbeitete er als wissenschaftlicher Mitarbeiter am Lehrstuhl für Internationale Unternehmensrechnung von Prof. Dr. Bernhard Pellens an den Universitäten Münster und Bochum. Die Promotion erfolgte im Juni 1999. 
Bewertung strategischer Flexibilität

beim Unternehmenserwerb 


\section{BOCHUMER BEITRÄGE ZUR UNTERNEHMUNGSFÜHRUNG UND UNTERNEHMENSFORSCHUNG}

Herausgegeben von Prof. Dr. Dr. h.c. mult. Walther Busse von Colbe, Prof. Dr. Dr. h.c. Werner H. Engelhardt, Prof. Dr. Roland Gabriel, Prof. Dr. Arno Jaeger, Prof. Dr. Gert Laßmann, Prof. Dr. Wolfgang Maßberg, Prof. Dr. Bernhard Pellens, Prof. Dr. Marion Steven, Prof. Dr. Rolf Wartmann, Prof. Dr. Brigitte Werners, Prof. Dr. Daniel Zimmer

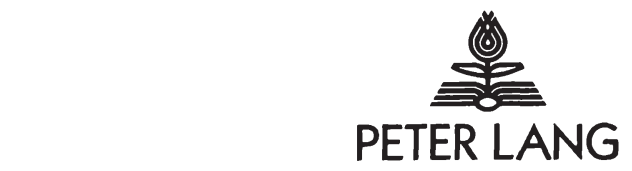

Frankfurt am Main - Berlin - Bern - Bruxelles - New York · Oxford - Wien 


\section{Bewertung strategischer Flexibilität beim Unternehmenserwerb}

Der Wertbeitrag von Realoptionen

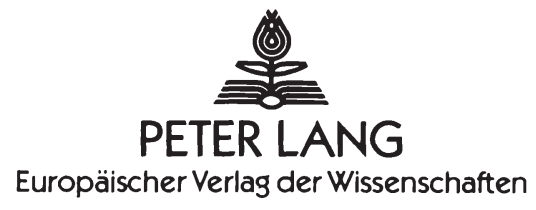


Die Deutsche Bibliothek - CIP-Einheitsaufnahme

Tomaszewski, Claude:

Bewertung strategischer Flexibilität beim Unternehmenserwerb : der Wertbeitrag von Realoptionen / Claude Tomaszewski. - Frankfurt am Main ; Berlin ; Bern ; Bruxelles ; New York ; Oxford ; Wien : Lang, 2000

(Bochumer Beiträge zur Unternehmungsführung und

Unternehmensforschung ; Bd. 57)

Zugl.: Bochum, Univ., Diss., 1999

ISBN 3-631-36188-2

Open Access: The online version of this publication is published on www.peterlang. com and www.econstor.eu under the international Creative Commons License CC-BY 4.0. Learn more on how you can use and share this work: http://creativecommons.org/ licenses/by/4.0.

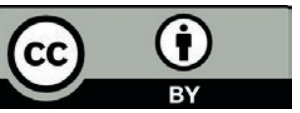

This book is available Open Access thanks to the kind support of ZBW - Leibniz-Informationszentrum Wirtschaft.

Die Veröffentlichung erfolgte mit freundlicher Unterstützung der Arthur Andersen Managementberatung.

Gedruckt auf alterungsbeständigem, säurefreiem Papier.

D 294

ISSN 0175-7105

ISBN 3-631-36188-2

ISBN 978-3-631-75492-4 (eBook)

(C) Peter Lang $\mathrm{GmbH}$

Europäischer Verlag der Wissenschaften

Frankfurt am Main 2000

Alle Rechte vorbehalten.

Das Werk einschließlich aller seiner Teile ist urheberrechtlich geschützt. Jede Verwertung außerhalb der engen Grenzen des

Urheberrechtsgesetzes ist ohne Zustimmung des Verlages unzulässig und strafbar. Das gilt insbesondere für

Vervielfältigungen, Übersetzungen, Mikroverfilmungen und die Einspeicherung und Verarbeitung in elektronischen Systemen.

Printed in Germany 123467 


\section{Geleitwort}

Im Vorfeld eines teilweisen bzw. vollständigen Unternehmenserwerbs ermittelt der potentielle Käufer i.d.R. denjenigen Preis, den er maximal für die gewünschten Anteile zu zahlen bereit ist. $\mathrm{Zu}$ dessen Bestimmung bietet die Lehre zur finanziellen Unternehmensbewertung schon seit längerem investitionsrechnerische Bewertungsverfahren an, deren Anwendungsmöglichkeiten durch die Forderung nach einer wertorientierten Unternehmensführung neu und intensiv diskutiert worden sind. Häufig wird jedoch beklagt, daß bei strategischen Akquisitionen tatsächlich gezahlte Kaufpreise oberhalb der hiermit ermittelten Barwerte liegen.

Der Wert einer strategisch motivierten Akquisition besteht zu einem großen Teil aus einem neu eingegangen Erfolgspotential, für dessen Ausschöpfung noch wichtige (Des-) Investitionsentscheidungen in der Zukunft zu treffen sind. Somit könnte eine mögliche Erklärung für die geschilderte Diskrepanz darin bestehen, daß sich mit den traditionellen Bewertungsverfahren der ökonomische Wert solcher Investitionsoptionen (häufig auch Realoptionen genannt) nicht bzw. nur schwer abbilden läßt. Dies steht im Einklang mit der z.T. vorzufindenden praktischen Vorgehensweise, den noch „fehlenden“ strategischen Wert über einen pauschalen Zuschlag abzugelten, der einen vorab ermittelten finanziellen (Basis-) Wert erhöht.

An dieser Stelle setzt die Arbeit von Herrn Tomaszewski an. Der Erwerb zusätzlicher Investitionsmöglichkeiten bietet neben der Ausschöpfung des neuen Erfolgspotentials bei günstiger Umweltentwicklung auch den Vorteil, bei ungünstiger Umweltentwicklung künftige Investitionsauszahlungen erst gar nicht zu tätigen. Bevor die endgültige Entscheidung getroffen wird, kann der bis dahin aufgelaufene Informationsstand abgewartet werden. Hierdurch entsteht ein ökonomischer Wert, der über den traditionell ermittelten Barwert hinausgeht. Unter der Maxime, daß sich auch bzw. insbesondere strategische Akquisitionen rechnen müssen und eine möglichst große Transparenz über die Entscheidungssituation herzustellen ist, präsentiert der Autor ein kapitalmarktorientiertes Bewertungskonzept, das den Wert solcher unternehmerischer Flexibilität durch Anwendung der Optionspreistheorie explizit und risikoadäquat berücksichtigt.

Herr Tomaszewski trägt mit dieser Arbeit zu einer aktuellen und breiten Diskussion bei, wie auch das kürzlich nahezu explosionsartige Erscheinen deutscher Beiträge und Stellungnahmen beweist. Die Arbeit leistet eine im Hinblick auf die Vollständigkeit der Darstellung sehr gute Zusammenfassung der bisherigen Erkenntnisse zum Thema Realoptionen. Zudem ist sie sauber strukturiert 
und gut lesbar formuliert, wodurch der Leser einen schnellen Überblick über die im Rahmen dieser Problemstellung diskutierten unterschiedlichen Aspekte erhält.

Bernhard Pellens 


\section{Vorwort}

Der vorliegenden Untersuchung liegt meine im Juni 1999 von der wirtschaftswissenschaftlichen Fakultät der Ruhr-Universität Bochum angenommene Dissertation zugrunde. Gegenüber der Fakultätsfassung unterscheidet sich diese Version insbesondere durch den aktuelleren Literaturstand. An der Arbeit, die während meiner wissenschaftlichen Tätigkeit am Lehrstuhl für Internationale Unternehmensrechnung an den Universitäten Münster und Bochum entstand, haben viele mitgewirkt, bei denen ich mich an dieser Stelle herzlichst bedanken möchte.

Meinem Doktorvater und akademischen Lehrer, Herrn Prof. Dr. Bernhard Pellens, kommt der besondere Verdienst zu, die Idee zu dieser Fragestellung entwickelt und mir einen großzügigen Spielraum bei dessen Bearbeitung gewährt zu haben. Ihm gebührt ebenfalls der Dank, mich in seiner ureigenen persönlichen Art für das wissenschaftliche Arbeiten motiviert und begeistert zu haben. Ein Dankeschön geht ebenfalls an Herrn Prof. Dr. Hans Dirrigl, der das Zweitgutachten übernahm und mit großem Engagement bei der endgültigen Titelformulierung geholfen hat.

Aus dem Kreise der Lehrstuhlmitarbeiter hat Nils Crasselt nicht nur in frühester Projektzeit unter vollem „Einsatz“ umfangreiche Literaturbeschaffungen durchgeführt, sondern im weiteren Verlauf auch unzählige wertvolle Anmerkungen geliefert. Mit ihm verbinden mich zudem zahlreiche gemeinsame Arbeiten und winterliche Unternehmungen. Ich würde mich freuen, wenn solche Kooperationen des „Real Options-Team“ auch in Zukunft möglich sind. Daneben hat sich Andreas Bonse als Rechnungslegungsexperte akribisch durch die Wirren des Realoptionsansatzes durchgekämpft. Ihm verdanke ich viele Verbesserungen zur Verständlichkeit und Lesefreundlichkeit. Auch Arndt Kempen möchte ich an dieser Stelle nicht vergessen, der in der zu kurzen gemeinsamen Zeit am Lehrstuhl stets ein begeisterungsfähiger Diskussionspartner war. Schließlich sei allen Kollegen gedankt, die gemeinsam mit mir die Entwicklung vom „freundlichen Lehrstuhl unterm Dach“ begleitet haben. Durch das dynamisch-produktive Klima konnte ich das Ziel der Fertigstellung nicht aus den Augen verlieren.

Auch außerhalb des Lehrstuhls ist mir Hilfe zuteil geworden. Dem Direktorium des Instituts für Unternehmungsführung und Unternehmensforschung und dem Peter Lang Verlag danke ich für die Aufnahme der Arbeit in diese Schriftenreihe, und der Arthur Andersen Managementberatung bin ich für die freundliche Unterstützung im Rahmen der Veröffentlichung zu Dank verpflichtet. 
Zuletzt und doch an erster Stelle gebührt jenen Menschen ein herzliches Dankeschön, die im privaten Umfeld - zum Teil unwissentlich - eine sehr große Hilfe darstellten: Freunden, bei denen ich trotz der vielen getrennten Jahre nie in Vergessenheit geraten bin; meiner Familie und insbesondere meinen Eltern, die bei den leider viel zu selten stattgefundenen gemeinsamen Unternehmungen stets ihrer bedingungslosen Liebe Ausdruck verliehen haben und schließlich Alexandra, die nicht nur unerbittlich dem Teufel der Rechtschreib- und Tippfehler hinterherjagte, sondern während der gesamten Zeit eine wertvolle Stütze war. Leider haben zwei von mir geliebte Menschen das fertige Produkt nicht mehr erleben dürfen. Ihnen sei die Arbeit im speziellen gewidmet.

Claude Tomaszewski 


\section{Inhaltsverzeichnis}

Abkürzungsverzeichnis

Symbolverzeichnis

XVII

Abbildungsverzeichnis

XXI

Tabellenverzeichnis

$\mathrm{XXV}$

\section{Kapitel I}

Einführung

1 Problemstellung und Ziel der Untersuchung

2 Gang der Untersuchung

\section{Kapitel II}

Begriff und Grundlagen der strategischen Unternehmensbewertung 6

1 Grundlagen der Unternehmensbewertung 6

1.1 Anlaß und Zweck der Bewertung 6

1.2 Traditionelle Bewertungsverfahren 9

1.2.1 Überblick 9

1.2.2 Einzelbewertungsverfahren $\quad 11$

$\begin{array}{ll}\text { 1.2.3 Preisfindungsverfahren } & 12\end{array}$

1.2.4 Investitionsrechnerische Verfahren $\quad 13$

1.2.4.1 Grundkonzeptionen 13

1.2.4.2 Ertragswert- und DCF-Methode 18

1.3 Eignung der Verfahren bei wertorientierter Unternehmensführung 21

1.3.1 Anforderungen bei wertorientierter Unternehmensführung 21

1.3.2 Eignung der einzelnen Bewertungsverfahren 22

2 Begriff der strategischen Unternehmensbewertung 24

2.1 Begriffsinterpretationen und Aufgabengebiete 24

2.2 Strategische Aspekte in der Unternehmensbewertung 26

2.2.1 Überblick 26

2.2.2 Restrukturierungspotentiale 28

2.2.3 Synergiepotentiale $\quad 29$

2.2.4 Beitrag zur geplanten Strategieumsetzung 33

2.3 Strategische und finanzielle Zielsetzungen in der

Unternehmensbewertung 
3 Ansätze strategischer Unternehmensbewertung 37

3.1 Bisherige Konzepte 37

3.1.1 Konzept der Mehrfachzielsetzung $\quad 37$

3.1.2 Shareholder Value-Analyse $\quad 40$

3.1.3 Konzept des strategischen Zuschlags 44

3.2 Theoretische Einteilung 46

4 Schlußfolgerungen für das weitere Vorgehen 49

4.1 Grundhaltungen zur Bewertung strategischer Aspekte 49

4.2 Strategische Aspekte mit Flexibilitätscharakter 50

4.3 Realoptionsorientierter Ansatz der strategischen

Unternehmensbewertung

\section{Kapitel III}

Berücksichtigung von Unsicherheit in investitionsrechnerischen Unternehmensbewertungsverfahren

1 Überblick

2 Korrekturverfahren $\quad 59$

2.1 Einperiodige Bewertung $\quad 59$

2.1.1 Sicherheitsäquivalent versus Risikozuschlag 59

2.1.2 Theoretisch fundierte Quantifizierung des

Sicherheitsäquivalents und des Risikozuschlags 61

2.1.2.1 Kapitalmarkttheoretische Vorgehensweise 61

2.1.2.2 Entscheidungstheoretische Vorgehensweise 64

$\begin{array}{ll}2.2 \text { Mehrperiodige Bewertung } & 67\end{array}$

2.2.1 Retrograde Vorgehensweise 67

2.2.2 Implikationen eines einheitlichen risikoadjustierten Zinsfußes $\quad 71$

3 Techniken zur Transparenz des Risikogehalts 75

$\begin{array}{ll}3.1 \text { Sensitivitätsanalyse } & 75\end{array}$

$\begin{array}{ll}3.2 \text { Risikoanalyse } & 76\end{array}$

4 Traditionelle Bewertungsverfahren und unternehmerische Flexibilität $\quad 78$

4.1 Traditionelle Bar- und Kapitalwertmethoden 78

4.2 Asymmetrische Risikostruktur als Folge unternehmerischer Flexibilität $\quad 80$

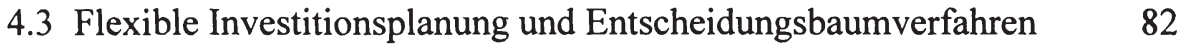




\section{Kapitel IV}

\section{Bewertung von Investitionsmöglichkeiten auf Basis finanz-} wirtschaftlicher Optionspreismodelle

1 Grundlagen der finanz- und realwirtschaftlichen Optionspreistheorie $\quad 85$

1.1 Grundbegriffe und Funktionsweise von Finanzoptionen 85

1.2 Systematisierung finanzwirtschaftlicher Optionspreismodelle $\quad 86$

1.3 Arbitragefreier Kapitalmarkt als zentrale Annahme gleichgewichtsorientierter Optionspreismodelle

1.4 Gegenüberstellung von Aktien- und Realoptionen 91

1.4.1 Interpretation von unternehmerischen

Handlungsspielräumen als Realoptionen

1.4.2 Systematisierung von Realoptionen

1.4.3 Einschränkung der Vergleichbarkeit von Real- mit Aktienoptionen

2 Binomialmodell als zeitdiskreter Bewertungsansatz

2.1 Einperiodiges Modell

2.1.1 Bewertung von Aktienoptionen $\quad 99$

2.1.1.1 Duplikationsansatz

2.1.1.2 Hegde- und Arbitrageansatz als zusätzliche Varianten

2.1.1.3 Risikoneutralisierte Bewertungsmethode $\quad 104$

2.1.2 Bewertung von Realoptionen

2.1.2.1 Ausgangssituation einer strategischen

Akquisition

2.1.2.2 Bestimmung des „Marktpreises“ des Bezugsguts

2.1.2.3 Optionspreistheoretische Bewertung der zusätzlichen Investitionsmöglichkeit

2.1.3 Zur Unterscheidung zwischen traditionellem Kapital-,

Realoptions- und Flexibilitätswert

2.2 Mehrperiodiges Modell

2.3 Vergleich zwischen Optionspreistheorie und flexibler Investitionsplanung

2.4 Berücksichtigung zwischenzeitlicher Rückflüsse

3 Black/Scholes-Modell als zeitstetiger Bewertungsansatz

3.2 Grundmodell der Black/Scholes-Bewertung 127

3.3 Berücksichtigung zwischenzeitlicher Rückflüsse 
3.4 Approximation des Black/Scholes-Ansatzes durch das

Binomialmodell

4 Sonstige Optionspreismodelle und Schlußfolgerungen für das weitere Vorgehen

\section{Kapitel V}

Berücksichtigung des Konkurrenzverhaltens bei der Bewertung von Investitionsmöglichkeiten

1 Ausgangsüberlegungen

1.1 Zwischenzeitlicher Konkurrenzeffekt

1.2 Investitionsverhalten bei konkurrenzbedingten Wertverlusten

1.3 Beeinflußbarkeit des Konkurrenzverhaltens

1.4 Unsicherheit des Konkurrenzverhaltens

1.5 Schlußfolgerungen für den weiteren Aufbau des Kapitels

2 Deterministischer Wettbewerbseffekt bei beeinflußbarem

Konkurrenzverhalten

2.1 Konkurrenzverhalten als exogener Modellparameter 155

2.1.1 Sinkende Marktanteile und Absatzpreise 155

2.1.2 Steigende Markteintrittsbarrieren

2.2 Endogenisierung des Konkurrenzverhaltens im

Bewertungsmodell

2.2.1 Begriffe und Grundlagen der Spieltheorie

2.2.2 Spieltheoretische Interpretation von Investitionsmöglichkeiten

2.2.3 Spieltheoretische Darstellung von Investitionsmöglichkeiten bei statischer Produktmarktstruktur

2.2.3.1 Ausgangssituation

2.2.3.2 Duopolbeispiel ohne dominante Marktstellung eines Wettbewerbers

2.2.3.3 Duopolbeispiel mit dominanter Marktstellung eines Wettbewerbers

2.2.3.4 Spieltheoretische Darstellung der Modellansätze mit ursprünglich exogenem Konkurrenzverhalten

2.2.4 Spieltheoretische Darstellung von Investitionsmöglichkeiten bei dynamischer Produktmarktstruktur 
3 Stochastischer Wettbewerbseffekt $\quad 177$

3.1 Unsicherheit des Zeitpunkts der Konkurrenzhandlung 177

3.2 Unsicherheit der Höhe des konkurrenzbedingten Wertverlusts $\quad 180$

3.2.1 Stochastische Markteintrittsbarrieren $\quad 180$

3.2.2 Stochastische Marktanteilsverluste und Preissenkungen $\quad 183$

\section{Kapitel VI}

Umsetzungsmöglichkeiten und -grenzen des Realoptionskonzepts bei der Bewertung von Investitionsmöglichkeiten

1 Überblick

2 Ausführliche Diskussion der Kapitalmarktannahmen

2.1 Arbitragefreiheit

2.2 Vollständigkeit

2.3 Handelbarkeit des Bezugsguts

2.4 Zusammenfassung der Diskussion

3 Verbundene Realoptionen

3.1 Art und Richtung der gegenseitigen Wertbeeinflussung 198

3.2 Ausmaß der gegenseitigen Wertbeeinflussung 201

3.3 Beispiel für sich kompensierende Realoptionen 204

3.4 Beispiel für komplementäre Realoptionen 206

4 Überlegungen zur praktischen Umsetzung der Realoptionsbewertung 208

4.1 Einsatz von Sensitivitätsanalysen 208

4.1.1 Isolierte Variation der Bewertungsparameter 208

4.1.2 Parallele Variation der Bewertungsparameter 213

4.2 Nutzen der Risikosimulation 216

4.3 Integration strategischer Analyse- und Planungsinstrumente 219

4.4 Einbettung des Realoptionskonzepts in die wertorientierte Unternehmensführung

5 Empirische Studien 225

5.1 Erklärungsgehalt von Realoptionsbewertungsmodellen 225

5.1.1 Untersuchung von Paddock/Siegel/Smith 225

5.1.2 Untersuchung von Quigg 227

5.2 Existenz von Realoptionswerten: Untersuchung von Kester 229 
5.3 Zum Verständnis und Einsatz der Realoptionstheorie in der Unternehmenspraxis

5.3.1 Untersuchung von Busby/Pitts

5.3.2 Untersuchung von Howell/Jägle

5.3.3 Eigene Untersuchung im Rahmen von Akquisitionen

5.3.4 Untersuchung von Laamanen

\section{Kapitel VII}

Zusammenfassung der Ergebnisse und Ausblick

Literaturverzeichnis 


\section{Abkürzungsverzeichnis}

\begin{tabular}{|c|c|}
\hline Abb. & Abbildung \\
\hline APT & Arbitrage Pricing Theory \\
\hline APV & Adjusted Present Value \\
\hline Aufl. & Auflage \\
\hline bzgl. & bezüglich \\
\hline bzw. & beziehungsweise \\
\hline CAPM & Capital Asset Pricing Model \\
\hline CFROI & Cash Flow Return on Investment \\
\hline c.p. & ceteris paribus \\
\hline CVA & Cash Value Added \\
\hline DAX & Deutscher Aktienindex \\
\hline DCF & Discounted Cash Flow \\
\hline d.h. & das heißt \\
\hline Div. & Dividende \\
\hline$€$ & Euro \\
\hline Ed. & Editor \\
\hline ed. & edited / edition \\
\hline $\mathrm{EP}$ & Economic Profit \\
\hline et al. & et alii \\
\hline EVA & Economic Value Added \\
\hline ex. & exklusiv \\
\hline f./ff. & folgende / fortfolgende \\
\hline Fn. & Fußnote \\
\hline FTE & Flow to Equity \\
\hline FT-SE & Financial Times Stock Exchange \\
\hline GARCH & Generalized Autoregressive Conditional Heteroskedastic \\
\hline ggf. & gegebenenfalls \\
\hline $\mathrm{GuV}$ & Gewinn und Verlust \\
\hline HFA & Hauptfachausschuß \\
\hline hrsg. & herausgegeben \\
\hline Hrsg. & Herausgeber \\
\hline i.d.R. & in der Regel \\
\hline
\end{tabular}


inkl. inklusive

insb. insbesondere

i.V.m. in Verbindung mit

IDW Institut der Wirtschaftsprüfer

Jg. Jahrgang

M\&A Mergers and Acquisitions

m.E. meines Erachtens

Mio. Millionen

OPT Optionspreistheorie

p.a. per annum

PIMS Profit Impact of Market Strategy

S. Seite

SHVA Shareholder Value Added

SG

Schmalenbach-Gesellschaft für Betriebswirtschaft, e.V.

Sp. Spalte

Tab. Tabelle

TCF Total Cash Flow

TSPM Time State Preference Model

u. und

u.a. unter anderem / und andere

USA United States of America

usw. und so weiter

u.U. unter Umständen

vgl. vergleiche

Vol. Volume

WACC Weighted Average Cost of Capital

WP Wirtschaftsprüfer

z.B. zum Beispiel

z.T. zum Teil

zzgl. zuzüglich 


\section{Symbolverzeichnis}

a

b

B

BG

Bin

C

$\operatorname{Cov}($.

CF

d

$\mathrm{d}_{1}$

$\mathrm{d}_{2}$

D

DI

Div

E (.)

$\mathrm{E}^{*}($.

$\mathrm{g}_{\mathrm{M}}$

GN

GW

$\mathrm{H}$

i

$\mathrm{i}_{\mathrm{A}}$

$i_{U}$

I

$I_{\text {Erw. }}$

j

k

K

KAP
Trendkomponente bzw. erwartete momentane Wachstumsrate eines generellen Wiener Prozesses oder eines Itô-Prozesses

Diffusionskomponente bzw. erwartete momentane Abweichungsrate eines generellen Wiener Prozesses oder eines Itô-Prozesses

Basispreis

Betriebsgewinn

Binomialprozeß

Wert einer Kaufoption

Kovarianz

Rückfluß, (operativer) Cash Flow

Senkungsfaktor

standardnormalverteilte Zufallsvariable

standardnormalverteilte Zufallsvariable

Wert der Desinvestitionsmöglichkeit

Desinvestitionseinzahlung

Dividendenzahlung

Erwartungswert

risikoneutralisierter Erwartungswert

Gewinnmarge

Gesamtnutzen

Gesamtwert von verbundenen Realoptionen

Hedgeportfolio

Summenindex

umsatzbezogene Investitionsrate des Anlagevermögens

umsatzbezogene Investitionsrate des Umlaufvermögens

Investitionsauszahlung

Erweiterungsinvestitionen

Summenindex

Ausmaß eines Sprungs im Rahmen eines Poisson-Sprung-

Prozesses

Aktienkurs

Kapitalbedarf 
kor

KT

KW

$\mathrm{kv}$

KV

1

$\mathrm{m}$

M

$\mathrm{n}$

$\mathrm{N}$

$\mathrm{N}($.

$\mathrm{p}$

$\mathrm{P}$

$\mathrm{P}_{\mathrm{M}}$

PF

$\mathrm{u}$

$\mathrm{r}_{\mathrm{A}}$

$\mathrm{r}_{\mathrm{CF}}$

$\mathrm{r}_{\mathrm{D}}$

$r_{\mathrm{EK}}$

$\mathrm{r}_{\mathrm{EK}}{ }^{*}$

$r_{f}$

$r_{\mathrm{FK}}$

$r_{\mathrm{GK}}$

$r_{M}$

$r_{p}$

$\mathrm{R}_{\mathrm{p}}$

$\mathrm{r}_{\tilde{\mathrm{t}}}$

$\mathrm{SÄ}$

$\mathrm{s}$

Korrelationskoeffizient

Kredittilgungs- bzw. -aufnahmezahlung

Kapitalwert

prozentualer konkurrenzbedingter Werteffekt

absoluter konkurrenzbedingter Werteffekt

Differenz zwischen Gleichgewichtsrendite und erwarteter momentaner Wachstumsrate

zustandsabhängiger Risikoparameter

risikolose Geldanlage bzw. Kreditaufnahme

Planungshorizont, Anzahl der Teilperioden im Binomialmodell

Natur, Umweltentwicklung

Wert der Standardnormalverteilungsfunktion

Pseudo- bzw. risikoneutralisierte Wahrscheinlichkeit

Wert einer Verkaufsoption

Marktpreis einer gehandelten Finanzposition

Portfolio

Steigungsfaktor

risikoadjustierter Zinsfuß

Rendite des zu bewertenden Rückflusses

Dividenden- bzw. zwischenzeitliche Wertverlustrate

Renditeforderung der Eigenkapitalgeber, Eigenkapitalkostensatz

Eigenkapitalkostensatz bei vollständiger Eigenfinanzierung risikoloser Zinsfuß

Renditeforderung der Fremdkapitalgeber, Fremdkapitalkostensatz

gewogener Gesamtkapitalkostensatz ohne Tax Shield-

Korrektur

Marktrendite

Risikozuschlag

Risikoabschlag

periodenspezifischer Zinsfuß

Sicherheitsäquivalent

Umweltzustand 


\begin{tabular}{|c|c|}
\hline st & durchschnittlicher Steuersatz \\
\hline SW & Substanzwert \\
\hline $\mathrm{t}$ & Perioden- bzw. Zeitindex \\
\hline $\mathrm{T}$ & Ende der Optionslaufzeit \\
\hline $\mathrm{TN}$ & Teilnutzen \\
\hline TS & (Bar-) Wert des Tax Shield \\
\hline $\mathrm{U}$ & Umsatz \\
\hline $\mathrm{U}()$. & Nutzenfunktion \\
\hline UW & Unternehmenswert \\
\hline $\mathrm{q}$ & Eintrittswahrscheinlichkeit \\
\hline $\mathrm{v}_{\mathrm{F}}$ & variantenspezifischer Faktor bei Mischverfahren \\
\hline $\mathrm{V}$ & Wert des Bezugsguts \\
\hline$V^{e x}$ & $\begin{array}{l}\text { Wert des Bezugsguts unter Abzug eines zwischenzeitlichen } \\
\text { Rückflusses bzw. eines konkurrenzbedingten Wertverlusts }\end{array}$ \\
\hline $\operatorname{Var}()$. & Varianz \\
\hline $\mathrm{V}_{\mathrm{EK}}$ & Wert des Eigenkapitals \\
\hline $\mathrm{V}_{\mathrm{EK}}^{*}$ & Wert des Eigenkapitals bei vollständiger Eigenfinanzierung \\
\hline $\mathrm{V}_{\mathrm{FK}}$ & Wert des Fremdkapitals \\
\hline $\mathrm{V}_{\mathrm{GK}}$ & Wert des Gesamtkapitals, Unternehmensgesamtwert \\
\hline $\mathrm{w}$ & Gewichtungsfaktor \\
\hline $\mathrm{w}_{\mathrm{U}}$ & Wachstumsrate des Umsatzes \\
\hline W & Wert der Investitionsmöglichkeit bzw. der Wachstumsoption \\
\hline wacc & $\begin{array}{l}\text { gewogener Gesamtkapitalkostensatz mit Tax Shield- } \\
\text { Korrektur }\end{array}$ \\
\hline $\mathrm{x}$ & Funktionsparameter einer Nutzenfunktion \\
\hline$\tilde{x}$ & Anteil eines Wertpapiers im Portfolio \\
\hline $\mathrm{x}_{\mathrm{MA}}$ & ursprünglicher Marktanteil \\
\hline $\mathrm{y}$ & Funktionsparameter einer Nutzenfunktion \\
\hline $\mathrm{X}$ & $\begin{array}{l}\text { Zufallsvariable eines generellen Wiener Prozesses bzw. eines } \\
\text { Itô-Prozesses }\end{array}$ \\
\hline$d \mathrm{z}$ & Inkrement eines generellen Wiener Prozesses \\
\hline $\mathrm{Z}$ & Fremdkapitalzinszahlung \\
\hline ZW & Zukunftserfolgswert \\
\hline
\end{tabular}


$\alpha$

$\alpha_{\mathrm{G}}$

$\beta$

$\hat{\beta}$

$\beta_{G}$

$\delta$

$\varepsilon$

$\gamma$

$\Gamma(. ;$.

$\eta$

$\varphi$

$\lambda$

$\lambda_{\mathrm{M}}$

$\mu$

$\pi$

$\sigma$

$\Omega$

Aktienanteil

Parameter der Gammaverteilungsfunktion

Betafaktor zur Bestimmung eines Risikozuschlags

„Betafaktor“ zur Bestimmung eines Risikoabschlags

Parameter der Gammaverteilungsfunktion

konkurrenzbedingter Wertverlust bei stochastischem

Konkurrenzverhalten

standardnormalverteilte Zufallsvariable

Verhältnis zwischen risikolosem und risikoadjustiertem

Zinsfuß

Wert der Gammaverteilung

Geschwindigkeitsvariable in einem Ornstein-UhlenbeckProzeß

poissonverteilte Zufallsvariable

Eintrittswahrscheinlichkeit eines Sprungs pro Zeiteinheit innerhalb eines Poisson-Sprung-Prozesses

Marktrisikoprämie

Mittelwert, erwartete momentane Wachstumsrate bzw. Aktienrendite

Arrow/Debreu-Preis, Preis eines reinen Wertpapiers

Standardabweichung, Volatilität, erwartete momentane Abweichungsrate

Verhältnis zwischen Sicherheitsäquivalent und

Erwartungswert 


\section{Abbildungsverzeichnis}

Abb. 1: Traditionelle Verfahren der Unternehmensbewertung 9

Abb. 2: Restrukturierungspotentiale 29

Abb. 3: Synergiepotentiale 32

Abb. 4: Zusammenhang zwischen wertorientierter Unternehmensführung und finanziellen sowie strategischen Zielen der Akquisition

Abb. 5: Shareholder Value-Netzwerk $\quad 42$

Abb. 6: Komponenten des strategischen Unternehmenswerts 44

Abb. 7: Ansätze strategischer Unternehmensbewertung 47

Abb. 8: $\quad$ Strategische Einflußfaktoren im erweiterten

Werttreibermodell 53

Abb. 9: Wertbestandteile und dazugehörige Ermittlungsmethoden 55

Abb. 10: Wertentwicklung einer unsicheren Zahlung bei konstantem Periodenrisiko

Abb. 11: Wertentwicklung einer unsicheren Zahlung bei spezifischem Periodenrisiko

Abb. 12: Asymmetrische Risikostruktur bei Existenz von unternehmerischen Handlungsspielräumen

Abb. 13: Entscheidungsbaum bei flexibler Investitionsplanung 83

Abb. 14: Überblick über gleichgewichtsorientierte Optionspreismodelle 87

Abb. 15: Systematisierung von Realoptionen 94

Abb. 16: Besonderheiten bei der Bewertung von Realoptionen 96

Abb. 17: Wertidentität von Aktienoption und Duplikationsportfolio 101

Abb. 18: Wertidentität von risikoloser Anlage und Hedgeportfolio 103

Abb. 19: Wertentwicklung des Arbitrageportfolios 104

Abb. 20: Markteintrittsentscheidung für neue Produktgruppe 108

Abb. 21: Bewertung einer Investitionsmöglichkeit im einperiodigen Binomialmodell

Abb. 22: Zusammensetzung des Werts einer Investitionsmöglichkeit

Abb. 23: Bewertung einer Investitionsmöglichkeit im dreiperiodigen Binomialmodell 
Abb. 24: Perioden- und zustandsabhängige Risikoprämien zur Bewertung der dreijährigen Investitionsmöglichkeit

Abb. 25: Bewertung einer Investitionsmöglichkeit mit zwischenzeitlich entgehenden Rückflüssen

Abb. 26: Zusammensetzung des Werts einer Investitionsmöglichkeit unter Berücksichtigung eines zwischenzeitlich entgehenden Rückflusses

Abb. 27: Genereller Wiener Prozeß

Abb. 28: Konvergenz zwischen dem Binomialmodell und dem Black/Scholes-Ansatz

Abb. 29: Weiterentwicklung der finanzwirtschaftlichen Optionspreismodelle nach dem Black/Scholes-Ansatz

Abb. 30: Investitionsverhalten bei konkurrenzbedingten Wertverlusten

Abb. 31: Entscheidung über eine frühzeitige Investition bei beeinflußbarem Konkurrenzverhalten

Abb. 32: Zusammensetzung des Werts einer Investitionsmöglichkeit unter Berücksichtigung des Konkurrenzeffekts

Abb. 33: Möglichkeiten zur Einbeziehung des Konkurrenzverhaltens im Realoptionsmodell

Abb. 34: Bewertung einer Investitionsmöglichkeit bei sinkendem Marktanteil bzw. Absatzpreis und zustandsunabhängiger Konkurrenzhandlung

Abb. 35: Bewertung einer Investitionsmöglichkeit bei sinkendem

Marktanteil bzw. Absatzpreis und zustandsabhängiger Konkurrenzhandlung

Abb. 36: Bewertung einer Investitionsmöglichkeit bei steigenden Markteintrittsbarrieren

Abb. 37: Matrixform des Spiels „Gefangenendilemma“

Abb. 38: Spielbaum des Spiels „Gefangenendilemma“

Abb. 39: Spieltheoretische Darstellung zur Bewertung einer exklusiven Investitionsmöglichkeit

Abb. 40: Investitionsspiel mit zwei Wettbewerbern

Abb. 41: Spieltheoretische Darstellung zur Bewertung einer Investitionsmöglichkeit im Duopol ohne dominante Marktstellung eines Wettbewerbers 
Abb. 42: Spieltheoretische Darstellung zur Bewertung einer Investitionsmöglichkeit im Duopol mit dominanter Marktstellung eines Wettbewerbers

Abb. 43: Spieltheoretische Darstellung zur Bewertung einer Investitionsmöglichkeit mit ursprünglich exogener Konkurrenzhandlung

Abb. 44: Verschiebung der Wahrscheinlichkeitsverteilung einer Investitionsmöglichkeit innerhalb der risikoneutralisierten Bewertungsmethode

Abb. 45: Ausmaß der gegenseitigen Wertbeeinflussung zwischen Realoptionen mit demselben Bezugsgut

Abb. 46: Vorhergehende Aufschubs- und nachfolgende

Erweiterungsoption als Beispiel für sich

kompensierende Realoptionen

Abb. 47: Vorhergehende und nachfolgende Erweiterungsoption als Beispiel für komplementäre Realoptionen

Abb. 48: Sensitivität des Werts der Investitionsmöglichkeit in Bezug auf den Barwert des Bezugsguts, die Investitionsauszahlung sowie die Standardabweichung des Bezugsguts

Abb. 49: Sensitivität des Werts der Investitionsmöglichkeit in Bezug auf den risikolosen Zinsfuß, die Optionslaufzeit und einen geringen oder einen hohen zwischenzeitlichen Rückfluß bzw. Wertverlust

Abb. 50: Entwicklung des traditionellen Kapital- sowie des Flexibilitätswerts bei paralleler Variation des Barwerts sowie der Standardabweichung des Bezugsguts

Abb. 51: Nutzen der Risikosimulation im Rahmen des Realoptionskonzepts

Abb. 52: Verbindung von strategischer Analyse bzw. Planung und optionspreistheoretischen Bewertungsansätzen

Abb. 53: Wertorientierte Steuerungsinstrumente in Abhängigkeit des Reifegrads eines Beurteilungsobjekts 
Claude Tomaszewski - 978-3-631-75492-4

Downloaded from PubFactory at 01/11/2019 04:15:58AM

via free access 


\section{Tabellenverzeichnis}

Tab. 1: Anlässe für Unternehmensbewertungen 7

Tab. 2: Strategische Aspekte bei Unternehmensakquisitionen 26

Tab. 3: Systematisierungskriterien für Synergiepotentiale 30

Tab. 4: Beispiele für Risikonutzenfunktionen 65

Tab. 5: Parallelen zwischen Aktienoptionen und Investitions- sowie Desinvestitionsmöglichkeiten

Tab. 6: Richtung der Wertbeeinflussung von nachfolgenden

Realoptionen auf den Wert von vorhergehenden

Realoptionen mit demselben Bezugsgut

Tab. 7: $\quad$ Richtung der Wertbeeinflussung von vorhergehenden

Realoptionen auf den Wert von nachfolgenden

Realoptionen mit demselben Bezugsgut

Tab. 8: Veränderung $\Delta$ des Werts der Investitionsmöglichkeit $W_{0}$ bei

Variation der Standardabweichung des Bezugsguts $\sigma$, des

Barwerts des Bezugsguts $V_{0}$ und der Laufzeit $t$

Tab. 9: Wertorientierte Steuerungsinstrumente im Vergleich

Tab. 10: Vergleich von Bewertungsmethoden und Unternehmensangeboten im Rahmen des Verkaufs von Rechten zur Erschließung und Förderung eines Petroleumfelds

Tab. 11: Flexibilitätsprämien bei Grundstückskäufen

Tab. 12: Bestimmtheitsmaß $R^{2}$ von Regressionsanalysen zur Überprüfung der Relevanz von Realoptionsbewertungsmodellen bei Grundstückskäufen (in \%)

Tab. 13: Vermuteter Anteil der Wachstumsoptionen am Unternehmenswert

Tab. 14: Existenz sowie explizite Beurteilung unternehmerischer Flexibilität im Rahmen von Investitionsprojekten (in \% der Befragten)

Tab. 15: Bewertungsparameter, theoretischer Optionswert sowie empirisches Ergebnis der einzelnen Fallstudien

Tab. 16: Berücksichtigung strategischer Faktoren bei Akquisitionen (Anzahl der Befragten) 
Tab. 17: Erklärungsgehalt hypothetischer Faktoren zum

Optionscharakter von Akquisitionen

Tab. 18: Erklärungsgehalt hypothetischer Faktoren zur Inanspruchnahme von Wachstumsoptionen bei Akquisitionen 


\section{Kapitel I}

\section{Einführung}

\section{Problemstellung und Ziel der Untersuchung}

Mit der Umsetzung des Shareholder Value-Ansatzes ${ }^{1}$ in der Unternehmenspra$\mathrm{xis}^{2}$ ist zugleich der Versuch unternommen worden, Elemente der strategischen Unternehmensführung zu quantifizieren, die zuvor einer eher qualitativen Betrachtung unterlagen. Über die Identifikation von Werttreibern soll eine Bewertung und Beurteilung von Geschäfts- und Investitionsstrategien sowie strategischen Geschäftsfeldern ermöglicht werden ${ }^{3}$. Dazu sind Finanzpläne aufzustellen und mit den Methoden der Investitionsrechnung zu Entscheidungswerten zu verdichten. Die hierbei vorgeschlagene Vorgehensweise zur Ermittlung von Bar- bzw. Kapitalwerten greift aber möglicherweise zu kurz.

Strategische Investitionsentscheidungen zeichnen sich im Hinblick auf die erwarteten Rückflüsse durch hohe Unsicherheit aus. Die Möglichkeit, auf unsichere Entwicklungen flexibel reagieren zu können, wird deshalb zu einem entscheidenden Erfolgsfaktor strategischer Unternehmensführung. Läßt sich eine Investition in die Zukunft verschieben und hierdurch der Informationsstand vor der Entscheidung verbessern, ist dieser Umstand in das Bewertungsmodell zu integrieren. Der Wert solcher unternehmerischen Handlungsspielräume - häufig auch als Realoptionen bezeichnet - läßt sich mit den traditionellen Ertragswertbzw. Discounted Cash Flow-Methoden nur schwer vollständig erfassen. Dies gilt insbesondere dann, wenn diese - wie allgemein üblich - entweder sofortige Investitionsentscheidungen und/oder einen im Zeitablauf konstanten Zinssatz unterstellen. Aus diesem Grund ist von einigen Autoren eine Erweiterung des investitionsrechnerischen Instrumentariums um Elemente der Optionspreistheorie vorgeschlagen worden ${ }^{4}$.

1 Vgl. allgemein den Überblick bei Hachmeister, Shareholder Value, 1997 und Lorson, Shareholder Value, 1999 sowie die dort zitierte Literatur.

2 Zur Umsetzung in Deutschland vgl. z.B. Pellens/Rockholtz/Stienemann, Konzerncontrolling in Deutschland, 1997, S. $1933 \mathrm{ff}$.

3 Vgl. Rappaport, Shareholder Value, 1998, Kapitel IV und V.

4 Vgl. stellvertretend Kester, Today’s Options, 1984; Myers, Financial Strategy, 1984; Trigeorgis/Mason, Managerial Flexibility, 1987. 
Im anglo-amerikanischen Raum ist die Anwendung der Optionspreistheorie bei realen Investitionsprojekten erstmals Mitte der achtziger Jahre intensiv erforscht worden'. Während in der Zwischenzeit in den USA neben einer Flut von Beiträgen auch Monographien ${ }^{2}$ erschienen sind sowie vielfältige Anwendungsfelder ${ }^{3}$ aufgezeigt wurden, ist dieser Themenkomplex in der deutschsprachigen Literatur bisher nur schleppend diskutiert worden. $\mathrm{Zu}$ Beginn dieser Untersuchung im Frühjahr 1995 waren nur wenige Stellungnahmen zu finden ${ }^{4}$. Die in letzter Zeit verstärkt zu verzeichnenden Bemühungen, den Realoptionsansatz ausführlicher darzustellen ${ }^{5}$, geben Anlaß zu der Vermutung, daß dieser nun auch hierzulande mehr beachtet wird und damit an Bedeutung gewinnt.

1 Vgl. Brennan/Schwartz, Evaluating, 1985; Mason/Merton, Contingent Claims Analysis, 1985; McDonald/Siegel, Investment and Option, 1985; Brennan/Schwartz, New Approach, 1986; Kester, Options Approach, 1986; McDonald/Siegel, Value of Waiting, 1986; Trigeorgis, Real Investment Opportunities, 1986; Majd/Pindyck, Time to Build, 1987; Paddock/Siegel/Smith, Option Pricing Models, 1987; Kensinger, Value of Active Management, 1987.

2 Zu Monographien vgl. Sick, Capital Budgeting With Real Options, 1989; Dixit/Pindyck, Investment Under Uncertainty, 1994; Trigeorgis, Real Options, 1996; Amram/Kulatilaka, Real Options, 1999. Zu Sammelbänden vgl. Trigeorgis (Ed.), Real Options, 1995; Pinches (Ed.), Real Options, 1998; Brennan/Trigeorgis (Ed.), Flexibility, 2000. Für einführende Darstellungen in Lehrbüchern vgl. Brealey/Myers, Principles, 1996, S. 589 ff; Damodaran, Corporate Finance, 1997, S. 743 ff.; Van Horne, Financial Management, 1998, S. $177 \mathrm{ff}$.

3 Hierzu gehören die Bewertung von natürlichen Ressourcen wie z.B. Öl, Gas, Kupfer usw., Grundstücken, Leasingverträgen, flexiblen Fertigungssystemen, Subventionen, Forschungsund Entwicklungsprojekten, Start Up-Unternehmen oder Auslandsinvestitionen. Vgl. den Überblick bei Cheung, Managerial Flexibility, 1993, S. 29 ff. und Trigeorgis, Overview, 1996, S. 24 ff. sowie die dort zitierte Literatur. Für typische industriespezifische Beispiele vgl. Copeland/Keenan, Real Options, 1998, S. 130.

4 Vgl. Herter, Berücksichtigung von Optionen, 1992; Eble/Völker, Optionen in der Investitionsrechnung, 1993; Fischer, Realoption beim Unternehmenskauf, 1993; Laux, Handlungsspielräume, 1993; Beißinger/Möller, Neue Investitionstheorie, 1994 sowie Nippel, Stellungnahme zu Eble und Völker, 1994.

5 Vgl. Kilka, Realoptionen, 1995; Spremann, Flexibilităt, 1995; Seppelfricke, Investitionstheorien unter Unsicherheit, 1996; Liebler, Strategische Optionen, 1996; Reinhardt, Kapitalmarktorientierte Bewertung, 1997; Stickel, Einsatz der Optionspreistheorie, 1997; Mostowfi, Flexibilităt, 1997; Meise, Realoptionen, 1998; Zimmermann, Fertigungssysteme, 1998 sowie jüngst Gintschel, Realoptionen, 1999; Breuer/Gürtler/Schuhmacher, Realoptionen, 1999; Koch, Unternehmensbewertung, 1999; Hommel/Pritsch, Realoptionsansatz, 1999; Hommel/Müller, Investitionsbewertung, 1999; Crasselt/Tomaszewski, Realoptionen, 1999; Fischer/Hahnenstein/Heitzer, Unternehmensbewertung, 1999; Lint/Pennings/Natter, Optionsmanagement, 1999; Rams, Unternehmensbewertung, 1999. Für eine Darstellung in einem Lehrbuch vgl. Gerke/Bank, Finanzierung, 1998, S. 215 ff. 
Ein mögliches Anwendungsfeld der Bewertung von Realoptionen liegt im Bereich der strategischen Unternehmensbewertung ${ }^{1}$. Die in dieser Arbeit vorgestellte und analysierte Bewertungsmethodik kann dabei sowohl zur Wertermittlung einer zusätzlichen Investitionsmöglichkeit innerhalb eines strategisch motivierten Unternehmenserwerbs als auch bei der laufenden Überprüfung des Geschäftsfeldportfolios zum Einsatz kommen. Während es beim Unternehmenserwerb um die Festlegung der Kaufpreisobergrenze vor dem speziellen Hintergrund einer durch die Akquisition, das Joint Venture oder die Minderheitsbeteiligung zusätzlich entstehenden Folgeinvestitionsmöglichkeit geht, unterstützt das Instrumentarium in der Unternehmensführung Entscheidungen zum Aufbau neuer Geschäftsfelder bzw. Geschäftsoptionen. Immer dann, wenn im Rahmen von Investitionen die Möglichkeit besteht, flexibel auf das zukünftige Unternehmensumfeld zu reagieren, stellt sich die Frage, ob die Realoptionsbewertung nicht zur Ergänzung traditioneller Verfahren der Unternehmensbewertung herangezogen werden kann.

Im Zentrum der vorliegenden Arbeit steht die ausführliche Präsentation der optionspreistheoretischen Bewertung unternehmerischer Flexibilität am Beispiel einer strategischen Akquisition. Das ökonomische Grundprinzip sowie Einsatzmöglichkeiten und -grenzen einer solchen Bewertung umfassend zu beleuchten, stellt das Hauptziel der Arbeit dar. Dabei werden folgende, bislang z.T. noch wenig diskutierte Schwerpunkte gesetzt:

- Erstens soll durch eine Auseinandersetzung mit den in der Literatur im Rahmen von Akquisitionen genannten strategischen Faktoren eine größere Transparenz bzgl. des in der vorliegenden Arbeit analysierten Aspekts der unternehmerischen Flexibilität entstehen.

- Zweitens wird den Unterschieden zwischen Finanz- und Realoptionen ein breiter Raum gewidmet, um zu vermeiden, daß die Übertragung finanzwirtschaftlicher Optionspreismodelle auf reale Investitionsentscheidungen $\mathrm{zu}$ Fehlbewertungen führt. Insbesondere die Existenz und das Verhalten von Wettbewerbern kann die eigenen Investitionsmöglichkeiten erheblich beeinträchtigen.

1 Vgl. auch Myers, Acquisition Target, 1986, S. 400 f.; Dirrigl, Konzepte, 1994, S. 423 ff.; Smith/Triantis, Strategic Acquisitions, 1995, S. 135 ff.; Crasselt/Tomaszewski, Akquisitionen, 1999, S. $517 \mathrm{ff}$. 
- Drittens soll eine intensive Auseinandersetzung mit den in den Realoptionsansätzen implizit unterstellten Kapitalmarktgegebenheiten dazu führen, daß der Blick für die Anwendbarkeit dieser Bewertungsmodelle geschärft wird.

- Schließlich ist es ein Anliegen der vorliegenden Arbeit, das Realoptionskonzept als ein wichtiges Element im „Instrumentenkasten“ der wertorientierten Unternehmensführung darzustellen.

\section{Gang der Untersuchung}

Die vorliegende Untersuchung beginnt im zweiten Kapitel mit einem Überblick grundlegender Erkenntnisse zur Bewertung von Unternehmen. Zunächst werden traditionelle Bewertungsverfahren dargestellt und in den Kontext der Shareholder Value-Diskussion bzw. wertorientierten Unternehmensführung eingeordnet. Nach einer Eingrenzung auf das in dieser Arbeit betrachtete Aufgabenfeld der strategischen Unternehmensbewertung werden die in der Akquisitionsliteratur unter dem Stichwort des „Strategischen“ aufgezählten Aspekte systematisiert und zu „finanziellen“ Faktoren abgegrenzt. Unter Heranziehung der bisher in der Literatur verbreiteten Ansätze zur strategischen Unternehmensbewertung werden theoretische Erweiterungsmöglichkeiten des finanziellen Basiskalküls aufgezeigt sowie der in der vorliegenden Arbeit analysierte strategische Aspekt der unternehmerischen Flexibilität präzisiert.

Die risikogerechte Berücksichtigung dieser Flexibilität steht im Zentrum der Ausführungen des dritten Kapitels. Zu diesem Zweck werden traditionelle Bewertungsverfahren und -techniken unter Unsicherheit präsentiert und verdeutlicht, daß sie zur Bewertung unternehmerischer Handlungsspielräume möglicherweise nicht geeignet sind. Hieraus resultiert der „Wunsch“ nach einer Bewertungsmethode, die in der Lage ist, die durch die Flexibilität hervorgerufene Besonderheit der vorliegenden Entscheidungssituation explizit zu erfassen.

Im vierten Kapitel werden zunächst Grundlagen der finanz- und realwirtschaftlichen Optionspreistheorie aufgeführt. Dabei wird auf Parallelen, aber auch auf Unterschiede zwischen Finanzoptionen - dem eigentlichen Bewertungsobjekt der Optionspreistheorie - und unternehmerischen Handlungsspielräumen eingegangen. Unter Verwendung der beiden am weitesten verbreiteten Optionspreismodelle wird anschließend die konkrete Bewertungstechnik sowohl von Aktienoptionen als auch von unternehmerischen Handlungsspielräumen ohne Konkurrenzeinfluß beschrieben. Abschließend wird kurz auf Weiterentwick- 
lungen des in der finanzwirtschaftlichen Optionspreistheorie als Standard geltenden Modells von Black und Scholes eingegangen.

Welche Auswirkungen das Konkurrenzverhalten auf die Beurteilung unternehmerischer Handlungsspielräume impliziert, wird im fünften Kapitel aufgezeigt. $\mathrm{Zu}$ diesem Zweck ist das Konkurrenzverhalten in verschiedene Kategorien einzuteilen. Folgende zwei Varianten der bis dahin vorgestellten Optionspreismodelle stehen im Mittelpunkt der Betrachtung. Einerseits lassen sich bereits vorhandene Bewertungsparameter in Konkurrenzeinflüsse uminterpretieren, andererseits kann das Bewertungskalkül mit Hilfe spieltheoretischer Elemente konzeptionell erweitert werden. Zur Verdeutlichung der verbalen Ausführungen wird ein bereits im dritten Kapitel gewähltes Zahlenbeispiel konsequent auf alle Bewertungsfälle angewandt und so lange wie möglich an der zugänglicheren zeitdiskreten Bewertung festgehalten.

Im sechsten Kapitel werden Aspekte diskutiert, die in direktem Zusammenhang mit einer praktischen Umsetzung der Realoptionsbewertung stehen. Zuerst werden die den einzelnen Bewertungsverfahren zugrunde liegenden Kapitalmarktannahmen besprochen, um hieraus Erkenntnisse zur Anwendbarkeit einer realoptionspreistheoretischen Bewertung zu ziehen. Danach wird auf die spezielle Bewertungsproblematik eingegangen, falls, wie in realistischen Entscheidungssituationen anzunehmen, mehrere unternehmerische Handlungsspielräume gleichzeitig bestehen. Abschließend werden Instrumente, die eine praktische Umsetzung der Realoptionsbewertung fördern, dargestellt und die bisher zum Themenkomplex „Realoptionen“ durchgeführten empirischen Studien präsentiert. Am Ende der Arbeit erfolgt im siebten Kapitel eine thesenförmige Zusammenfassung der wichtigsten Ergebnisse sowie ein kurzer Ausblick auf zukünftige Entwicklungen und den weiteren Forschungsbedarf. 


\section{Kapitel II}

\section{Begriff und Grundlagen der strategischen Unternehmensbewertung}

\section{Grundlagen der Unternehmensbewertung}

\subsection{Anlaß und Zweck der Bewertung}

Die Eignung eines Bewertungsverfahrens läßt sich nur beurteilen, wenn zuvor der Zweck der Unternehmensbewertung festgelegt ist ${ }^{1}$. Erste Anhaltspunkte zur Bestimmung dieses $Z$ wecks lassen sich eventuell über den Anlaß einer solchen Bewertung gewinnen ${ }^{2}$. Ein Blick in die Literatur zeigt, daß zu den verschiedenen Anlässen einer Unternehmensbewertung z.T. unterschiedliche Systematisierungen existieren ${ }^{3}$. Meist wird zwischen Situationen mit und ohne Wechsel des Eigentums an dem zu bewertenden Unternehmen differenziert ${ }^{4}$. Während der Unternehmenskauf zu der ersten Kategorie gehört, kann die Kreditwürdigkeitsprüfung als Beispiel für einen Bewertungsanlaß ohne Eigentumswechsel angeführt werden.

Anlässe mit Eigentumswechsel lassen sich zudem in dominierte und nicht dominierte Situationen aufteilen. Charakteristisches Merkmal nicht dominierter Anlässe ist die Tatsache, daß keine Partei die Eigentumsverhältnisse ohne das Einverständnis der anderen Partei ändern kann. Ein Eigentumswechsel kann nur über eine außergerichtliche Verhandlung und Einigung erfolgen. Wenn jedoch eine Partei in der Lage ist, die veränderten Eigentumsverhältnisse auch ohne den Willen der anderen Partei herbeizuführen, wird von dominierten Situationen gesprochen. Eventuell ist nun neben der Verhandlung ein gerichtliches Verfahren notwendig. Desweiteren wird zwischen dem Typ des Kaufs oder Verkaufs, bei dem die bisherigen Eigentümer ihre Unternehmensanteile gegen finanzielle oder sonstige Entschädigung aufgeben, und dem Typ der Fusion, bei dem sie Eigentum an einer neu entstehenden Einheit erlangen, differenziert.

I Vgl. stellvertretend Sieben/Schildbach, Stand der Entwicklung, 1979, S. 455; Moxter, Grundsätze, 1983, S. 6; Ballwieser/Leuthier, Grundprinzipien, 1986, S. 546.

2 Vgl. Dirrigl, Bewertung, 1988, S. 6.

3 Vgl. z.B. Jaensch, Wert und Preis, 1966, S. 4; Münstermann, Bewertung, 1970, S. 13 ff.; Engeleiter, Unternehmensbewertung, 1970, S. 10 ff.; Matschke, Entscheidungswert, 1975, S. 30 ff.; Künnemann, Unternehmensbewertung, 1985, S. 52 ff.

4 Vgl. zu folgendem Ballwieser/Leuthier, Grundprinzipien, 1986, S. 546; Sieben, Unternehmensbewertung, 1993, Sp. 4320 f.; Serfling/Pape, Grundlagen, 1995, S. 808. 
Im Rahmen dieser Arbeit wird ausschließlich auf den Kauf bzw. Verkauf eines Unternehmens (-teils) sowie auf die wertorientierte Unternehmensführung eingegangen. Zu letzterer zählen u.a. die am Wert des Unternehmens ausgerichtete Performancemessung von Unternehmensteilen sowie an den Unternehmenswert gekoppelte Entlohnungssysteme. Tab. 1 bietet einen Überblick zu den unterschiedlichen Bewertungsanlässen.

\begin{tabular}{|c|c|c|c|}
\hline & $\begin{array}{l}\text { mit Eigent } \\
\text { nicht dominiert }\end{array}$ & $\begin{array}{r}\text { umswechsel } \\
\text { dominiert }\end{array}$ & $\begin{array}{c}\text { ohne Eigentums- } \\
\text { wechsel }\end{array}$ \\
\hline $\begin{array}{l}\text { Typ Kauf oder } \\
\text { Verkauf }\end{array}$ & $\begin{array}{l}\text { - Kauf oder Verkauf } \\
\text { eines Unterneh- } \\
\text { mens oder Unter- } \\
\text { nehmensteils }\end{array}$ & $\begin{array}{l}\text { - Ausscheiden eines } \\
\text { Personengesell- } \\
\text { schafters } \\
\text { - Barabfindung von } \\
\text { Minderheitsgesell- } \\
\text { schaftern } \\
\text { - Enteignung } \\
\text { - Erbauseinander- } \\
\text { setzung }\end{array}$ & \multirow{2}{*}{$\begin{array}{l}\text { - Substanz- } \\
\text { besteuerung } \\
\text { - Kreditwürdigkeits- } \\
\text { prüfung } \\
\text { - Sanierung } \\
\text { - Wertorientierte } \\
\text { Unternehmens- } \\
\text { fuhrung } \\
\text { - Performance- } \\
\text { messung von } \\
\text { Unternehmens- } \\
\text { teilen } \\
\text { - Vergütung von } \\
\text { Managern } \\
\text { - Unternehmens- und } \\
\text { Finanzanalyse }\end{array}$} \\
\hline Typ Fusion & $\begin{array}{l}\text { - Unternehmens- } \\
\text { gründung mit Ein- } \\
\text { bringung } \\
\text { - Eintritt eines Ge- } \\
\text { sellschafters in be- } \\
\text { stehendes Unter- } \\
\text { nehmen } \\
\text { - Verschmelzung }\end{array}$ & $\begin{array}{l}\text { Abfindung von } \\
\text { Minderheitsgesell- } \\
\text { schaftern in } \\
\text { eigenen Aktien }\end{array}$ & \\
\hline
\end{tabular}

Tab. 1: Anlässe für Unternehmensbewertungen

Quelle: In Anlehnung an Künnemann, Unternehmensbewertung, 1985, S. 59.; Ballwieser/Leuthier, Grundprinzipien, 1986, S. 547; Sieben, Unternehmensbewertung, 1993, Sp. 4321.

Allein mit der Klärung des Bewertungsanlasses läßt sich jedoch der Zweck der Bewertung nicht hinreichend festlegen. Es könnte durchaus sein, daß in ein und demselben Anlaß verschiedene Bewertungszwecke verfolgt werden ${ }^{1}$. Aus diesem Grund unterscheidet die Kölner Funktionenlehre verschiedene Funktionen, die im Rahmen der Bewertung verfolgt werden können ${ }^{2}$. Neben diversen $\mathrm{Ne}$ -

1 Vgl. Künnemann, Unternehmensbewertung, 1985, S. 56 f.; Ballwieser/Leuthier, Grundprinzipien, 1986, S. 548; Serfling/Pape, Grundlagen, 1995, S. 811.

2 Vgl. stellvertretend Sieben, Funktionen, 1983, S. 539 ff. Zur Eignung der Kölner Funktionenlehre für Bewertungsanlässe außerhalb des nicht dominierten Eigentumswechsels vgl. kritisch Dirrigl, Bewertung, 1988, S. 10. 
benfunktionen, wie z.B. der Steuerbemessungsfunktion, sind folgende drei Hauptfunktionen zu nennen ${ }^{1}$. In der Beratungs- bzw. Entscheidungsfunktion versucht der Bewerter, die Grenze der Konzessionsbereitschaft auszumachen. Es wird derjenige Grenzwert ermittelt, bei dem er sich unter Berücksichtigung individueller Ziele und Handlungsalternativen ökonomisch gerade nicht verschlechtert. Der zu bestimmende Unternehmenswert ist dann ein Entscheidungswert. Demgegenüber geht es in der Vermittlungs- bzw. Schiedsfunktion darum, zwischen unterschiedlichen Interessenparteien zu vermitteln. Der Unternehmenswert ist dann ein Schieds- bzw. Arbitriumwert, der für beide Parteien akzeptabel ist. Schließlich werden in der Argumentationsfunktion Unternehmenswerte bestimmt, auf Basis derer ein bestimmtes Verhandlungsergebnis erreicht werden soll.

Wie bereits angeklungen, besteht zwischen Bewertungsanlässen und -zwecken nur eine lose Verbindung. Beispielsweise ist es beim Unternehmenskauf vorstellbar, daß zur Transparenz der individuellen Ausgangssituation ein Entscheidungswert und als Verhandlungsgrundlage parallel ein Argumentationswert bestimmt wird. Aus diesem Grund ist es dringend erforderlich, vor dem Bewertungsvorgang neben dem Bewertungsanlaß auch den Bewertungszweck festzulegen. In der vorliegenden Arbeit geht es ausschließlich um die Bestimmung von Entscheidungswerten.

Zudem ist an dieser Stelle auf den Unterschied zwischen Wert und Preis eines Unternehmens hinzuweisen. Beim Unternehmenskauf z.B. liegt der ausgehandelte Preis zwischen den Entscheidungswerten (Preisober- und -untergrenze) des Käufers und Verkäufers, vorausgesetzt die beiden Parteien handeln ratio$\mathrm{nal}^{2}$. Nur so kann eine Einigung im Hinblick auf die Übertragung des Eigentums erzielt werden.

I Vgl. zu folgendem Ballwieser/Leuthier, Grundprinzipien, 1986, S. 546 ff.; Sieben, Unternehmensbewertung, 1993, Sp. $4316 \mathrm{ff}$.

2 Vgl. Arbeitskreis „Unternehmensbewertung im Rahmen der unternehmerischen Zielsetzung”, Unternehmensbewertung, 1981, S. 2; Helbling, Wert und Preis, 1990, S. 14 f.; Ruhnke, Unternehmensbewertung, 1995, S. 6 f. 


\subsection{Traditionelle Bewertungsverfahren}

\subsection{1 Überblick}

Traditionelle Bewertungsverfahren lassen sich grob in Einzelbewertungs-, Gesamtbewertungs- sowie Mischverfahren einteilen (vgl. Abb. 1) ${ }^{1}$. Charakteristisches Merkmal von Einzelbewertungsverfahren ist es, den Unternehmenswert über die Summe der Werte der einzelnen Vermögensgegenstände zu bestimmen. Ausgangspunkt der Bewertung ist i.d.R. das Inventar und die Bilanz. Werden für die einzelnen Vermögensgegenstände Wiederbeschaffungswerte angesetzt, handelt es sich um das Substanzwertverfahren. Das Liquidationswertverfahren setzt hingegen die jeweiligen Liquidationserlöse an.

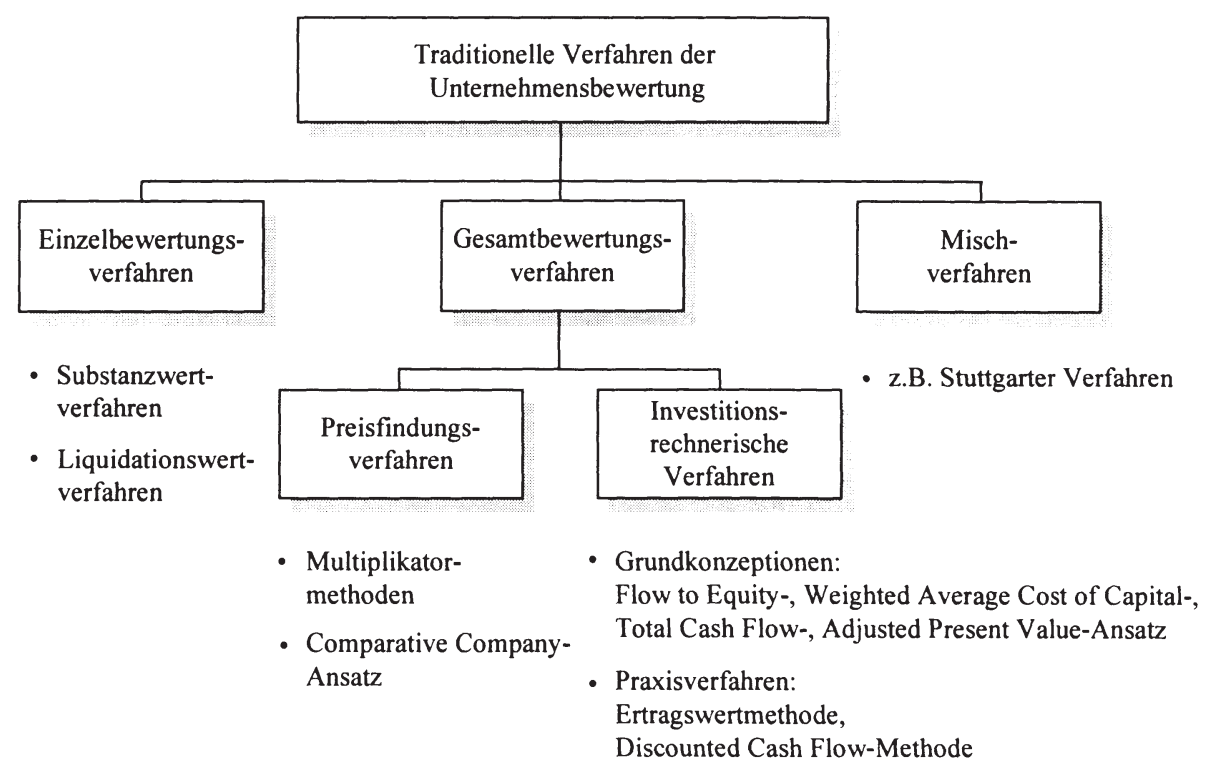

Abb. 1: Traditionelle Verfahren der Unternehmensbewertung

Bei der Ermittlung des Unternehmenswerts mit Gesamtbewertungsverfahren werden die durch die Gesamtheit der Vermögensgegenstände aus dem Unternehmen zu erzielenden Rückflüsse bewertet. Hierbei sind zwei Unterkategorien zu unterscheiden. Zum einen handelt es sich um kennzahlenorientierte Bewertungsverfahren, die durch eine äußerst hohe Praktikabilität und Wirtschaftlich-

1 Vgl. zu folgendem Ballwieser, Methoden, 1993, S. 152 ff.; Mandl/Rabel, Unternehmensbewertung, 1997, S. $28 \mathrm{ff}$. 
keit gekennzeichnet sind. Solche Methoden werden als Preisfindungsverfahren bezeichnet. Zum anderen existieren Bewertungsverfahren, die auf der traditionellen Investitionsrechnung aufbauen.

Im Rahmen von Preisfindungsverfahren wird mit Hilfe von Vergleichsunternehmen, deren Unternehmenswerten und ausgewählten Kennzahlen auf den Wert des zu bewertenden Unternehmens geschlossen. Hierbei kann danach unterschieden werden, ob die Vergleichsunternehmen explizit ausfindig gemacht werden oder nur implizit in die Bewertung eingehen. Der Comparative Company-Ansatz zählt zu der ersten Methode, während Multiplikatoren i.d.R. Durchschnittswerte einer Branche verwenden.

Demgegenüber wird in den investitionsrechnerischen Verfahren der Unternehmenswert durch Diskontierung der aus dem Unternehmen zu erwartenden Zahlungsströme an die Kapitalgeber gebildet. Hierin ist der Gedanke enthalten, daß sich der Unternehmenswert aus den zukünftigen Erfolgen zusammensetzt, die der Bewerter mit dem Unternehmen gemäß seiner individuellen Zielsetzung verbindet (Zukunftserfolgswert ${ }^{1}$ ). Je nach Ausgestaltung der Zahlungsströme und des Zinsfußes lassen sich verschiedene Varianten unterscheiden. Zum einen kann in verschiedene Grundkonzeptionen ${ }^{2}$ (Flow to Equity-, Weighted Average Cost of Capital-, Total Cash Flow-, Adjusted Present Value-Ansatz) unterteilt werden. Zum anderen konkurrieren in der Praxis Verfahren, die konkrete Ausgestaltungen dieser Grundkonzeptionen darstellen. Die bekanntesten sind das Ertragswertverfahren nach der HFA-Stellungnahme des Instituts der deutschen Wirtschaftsprüfer sowie die Discounted Cash Flow-Methode nach Copeland/Koller/Murrin ${ }^{3}$.

Im Rahmen von Mischverfahren wird der Unternehmenswert $U W$ aus einer Kombination von Einzel- und Gesamtbewertung ermittelt. In Abhängigkeit der Verknüpfung zwischen Substanz- $S W$ und Zukunftserfolgswert $Z W$ läßt sich eine Vielzahl von Methoden unterscheiden ${ }^{4}$. Eine der bekanntesten ist das steuerrechtliche „Stuttgarter Verfahren”. Allgemein gehorchen Mischverfahren der

1 Zum Begriff vgl. Busse von Colbe, Zukunftserfolg, 1957, S. $11 \mathrm{ff}$.

2 Vgl. Hachmeister, Unternehmenswert, 1996, S. 357 ff.; Ballwieser, Discounted Cash FlowVerfahren, 1998, S. 81 sowie die dort angegebene Literatur.

3 Vgl. IDW, Stellungnahme, 1983, S. 468 ff.; Dörner, Unternehmensbewertung, 1992, S. $1 \mathrm{ff}$; Copeland/Koller/Murrin, Valuation, 1994, S. $131 \mathrm{ff}$.

4 Vgl. Ballwieser, Methoden, 1993, S. $170 \mathrm{f}$. 
folgenden Beziehung, so daß sich für jede individuelle Mischmethode ein variantenspezifischer Faktor $v_{F}$ angeben läßt.

$$
U W=S W+v_{F} \cdot(Z W-S W)
$$

Da Mischverfahren lediglich eine Kombination anderer Verfahren darstellen, werden sie im folgenden nicht im Detail erläutert.

\subsubsection{Einzelbewertungsverfahren}

Als Einzelbewertungsverfahren gelten Methoden zur Ermittlung eines Substanz- bzw. Liquidationswerts. Der Substanzwert stellt den Betrag dar, der aufzuwenden wäre, um ein tatsächliches Unternehmen zu einem bestimmten Zeitpunkt zu rekonstruieren. Dabei werden die einzelnen Vermögensgegenstände bzw. Schulden zu ihren Einzelwerten bewertet und aufaddiert. Abhängig vom Umfang der berücksichtigten Wertgegenstände und deren Wertansätzen können abweichende Konzeptionen unterschieden werden ${ }^{1}$. Der Bruttorekonstruktionswert setzt sich aus den Wiederbeschaffungskosten der betriebsnotwendigen Vermögensgegenstände und den Veräußerungserlösen der nicht betriebsnotwendigen Vermögensgegenstände zusammen. Werden davon die Schulden abgezogen, entsteht der Nettorekonstruktionswert. Der Teilrekonstruktionswert umfaßt materielle und verkehrsfähige immaterielle Vermögensgegenstände, während der Vollrekonstruktionswert auch die nicht verkehrsfähigen immateriellen Vermögensgüter einbezieht.

Verfahrensimmante Probleme der Substanzwertverfahren liegen in der Bestimmung von Art und Kosten der wiederzubeschaffenden Vermögensgegenstände, insbesondere in der Berücksichtigung des technischen Fortschritts bei der Bewertung von Sachanlagen ${ }^{2}$. Desweiteren ist die Bewertung nicht verkehrsfähiger immaterieller Vermögensgegenstände nur über den Vergleich von Teilrekonstruktionswert und investitionsrechnerischem Verfahren theoretisch exakt durchführbar ${ }^{3}$.

Eine besondere Substanzwertkonzeption stellt der Substanzwert im Sinne ersparter Ausgaben dar, der als Differenz zwischen dem Barwert der einzelnen Auszahlungen des zu bewertenden Unternehmens und dem Barwert der einzel-

1 Vgl. Sieben, Unternehmensbewertung, 1993, Sp. 4327.

2 Vgl. Busse von Colbe, Gesamtwert, 1970, Sp. 573.

3 Vgl. Ballwieser, Methoden, 1993, S. 170. 
nen Auszahlungen im Falle der Neuerrichtung bestimmt wird ${ }^{1}$. Er stellt insofern den Barwert ersparter Auszahlungen dar. Unter dem Begriff Auszahlungen sind bei dieser Konzeption neben den zum Bewertungszeitpunkt anfallenden Auszahlungen sowohl die zukünftig einmalig auftretenden als auch die laufenden Zahlungsabflüsse zu verstehen ${ }^{2}$. Das Verfahren unterstellt implizit, daß die Neuerrichtung und das zu bewertende Unternehmen gleich hohe Einzahlungen erwarten lassen.

Im Gegensatz zu allen anderen Bewertungsmethoden geht das Liquidationswertverfahren nicht von der Fortführung des Unternehmens aus, sondern von dessen Zerschlagung. Durch Addition der Veräußerungserlöse der einzelnen Vermögensgegenstände und Subtraktion der Schulden sowie der bei Liquidation anfallenden Auflösungskosten erhält man den Liquidationswert. Verfahrensimmanente Probleme ergeben sich insofern, als daß der Liquidationswert von der zu schätzenden Zerschlagungsintensität sowie -geschwindigkeit abhängt ${ }^{3}$. Daneben muß der Bewerter eine Vorstellung über die mit der Liquidation verbundenen „Durchführungskosten” wie z.B. Abbruch- oder Sozialplankosten haben.

\subsubsection{Preisfindungsverfahren}

$\mathrm{Zu}$ den Preisfindungsverfahren zählen Multiplikatoren sowie der vorwiegend in den USA praktizierte Comparative Company-Ansatz ${ }^{4}$. In den Multiplikatormethoden wird der Wert des Unternehmens als Vielfaches einer Bezugsgröße bestimmt, für die beispielsweise der nachhaltige Gewinn, der operative Cash Flow, der Umsatz oder der Buchwert des Eigenkapitals herangezogen wird. Die Multiplikatoren werden dabei auf Basis von in der Vergangenheit zu beobachtenden Relationen ermittelt und in Abhängigkeit der Branche und der Bezugsgröße variiert. Fraglich ist hierbei, ob die in der Vergangenheit gefundenen Multiplikatoren auch für die Zukunft gelten. Es wird auf die bisherige Ange-

1 Zur Grundidee dieser Konzeption vgl. Sieben, Substanzwert, 1963, S. 79 ff.

2 Vgl. Busse von Colbe, Gesamtwert, 1970, Sp. 573 f.; Sieben, Unternehmensbewertung, 1993, Sp. 4328.

3 Die Zerschlagungsintensität bezeichnet den Grad der Zusammenfassung von Vermögensgegenständen. Die Zerschlagungsgeschwindigkeit bemißt den Zeitraum, innerhalb dessen die Veräußerung der Vermögensgegenstände abgeschlossen ist. Vgl. Ballwieser, Methoden, 1993, S. 169.

4 Vgl. zu folgendem Sanfleber-Decher, Unternehmensbewertung, 1992, S. 598 ff.; Ruhnke, Unternehmensbewertung, 1995, S. 12. 
bots- und Nachfragestruktur nach Unternehmen dieser Branche sowie auf das aktuelle Verhalten der Marktteilnehmer vertraut ${ }^{1}$. Während man in den USA für einige Branchen „relativ stramme Korrelationen hinsichtlich Kaufpreis, Umsatz, Price/Earnings-Ratio"2 festgestellt haben will, scheint dieser Zusammenhang für den deutschen Markt kaum belegt. Desweiteren ist nicht bekannt, welche individuellen Überlegungen und/oder welches Verhandlungsgeschick in der Vergangenheit zu einem bestimmten Unternehmenspreis geführt haben ${ }^{3}$.

Beim Comparative Company-Ansatz werden vergangene oder aktuelle Marktpreise konkreter Vergleichsunternehmen zur Bestimmung des zu ermittelnden Unternehmenswerts herangezogen. Die Marktpreise können dabei aus Börsennotierungen von Publikumsgesellschaften, aus abgeschlossenen Transaktionen im M\&A-Bereich oder aus erstmaligen Börsenplazierungen stammen ${ }^{4}$. Das Verfahren bedingt, daß in einem ersten Schritt anhand bestimmter Kriterien wie z.B. Branchenzugehörigkeit, Größe des Unternehmens, vergangene Gewinne und Umsätze, Gewinn- und Umsatzwachstum, Verschuldungsgrad, Absatzwege - Vergleichsunternehmen ausgewählt werden. In einem zweiten Schritt werden jahresabschlußorientierte Kennzahlen gebildet und zu den Unternehmenswerten der Vergleichsunternehmen in Beziehung gesetzt. Bei den resultierenden relativen Kennzahlen wie z.B. dem Kurs/Gewinn- oder dem Kurs/Buchwert-Verhältnis wird davon ausgegangen, daß sie die Performance des Unternehmens abbilden. Mit Hilfe der ermittelten Relation zwischen Kennzahl und Wert des Vergleichsunternehmens sowie der spezifischen Höhe dieser Kennzahl beim zu bewertenden Unternehmen läßt sich ein Anhaltspunkt für dessen Unternehmenswert gewinnen ${ }^{5}$.

\subsubsection{Investitionsrechnerische Verfahren}

\subsubsection{Grundkonzeptionen}

$\mathrm{Zu}$ den Varianten investitionsrechnerischer Bewertungsverfahren zählen das Flow to Equity-, das Weighted Average Cost of Capital-, das Total Cash Flow-

\footnotetext{
Vgl. Barthel, Theoretische Fundierung, 1996, S. 1704.

Jaensch, USA, 1989, S. 334.

Vgl. Ballwieser, Multiplikatoren, 1991, S. $58 \mathrm{ff}$.

Vgl. Böcking/Nowak, Vergleichsverfahren, 1999, S. 170 ff.

Vgl. Ruhnke, Unternehmensbewertung, 1995, S. 13.
} 
und das Adjusted Present Value-Konzept ${ }^{1}$. Allen gemeinsam ist die Diskontierung prognostizierter Rückflüsse an die Kapitalgeber. Um die Unterschiede aufzuzeigen, wird in der folgenden Darstellung der Cash Flow vor Abzug von Fremdkapitalzinsen (Brutto- bzw. operativer Cash Flow) als Ausgangspunkt der Argumentation verwendet. Der Cash Flow nach Abzug der Fremdkapitalzinsen (Netto-Cash Flow) gilt hier zur Vereinfachung als Steuerbemessungsgrundlage. Es wird ein einziger und von der Höhe des Netto-Cash Flows unabhängiger Steuersatz unterstellt. Desweiteren wird grundsätzlich von periodenunabhängigen Zinsfüßen ausgegangen.

Der Flow To Equity-Ansatz verwendet einen Rückfluß nach Zinsen und nach Steuern. Vom operativen Cash Flow der Periode $C F_{t}$ wird zuerst die Zinszahlung $Z_{t}$ auf das vorhandene Fremdkapital der Vorperiode abgezogen. Auf den verbleibenden Betrag werden anhand des durchschnittlichen Steuersatzes st Steuern berechnet und in Abzug gebracht. Die um geplante Kredittilgungszahlungen $K T_{t}$ geschmälerten ${ }^{2}$ Periodenergebnisse werden mit dem Eigenkapitalkostensatz nach Steuern $r_{E K}$ abgezinst. Die Addition der abgezinsten Periodenbeträge ergibt den Unternehmenswert $V_{E K}$ (Wert des Eigenkapitals) ${ }^{3}$.

$$
V_{E K}=\sum_{t=1}^{n} \frac{\left(C F_{t}-Z_{t}\right) \cdot(1-s t)-K T_{t}}{\left(1+r_{E K}\right)^{t}}
$$

Der Weighted Average Cost of Capital-Ansatz verwendet als Diskontierungssatz hingegen einen gemäß der Finanzierungsstruktur des Unternehmens gewogenen Kapitalkostensatz. Letzterer spiegelt sowohl die Renditeforderung der Eigen- als auch der Fremdkapitalgeber $r_{F K}$ wider. Folglich ist ein Rückfluß vor Zinsen und nach Steuern zu bestimmen. Auf den operativen Cash Flow werden fiktive Steuern unter der Annahme vollständiger Eigenfinanzierung berechnet. Die Steuererparnis, die durch die Abzugsfähigkeit der Fremdkapitalzinsen bei der Steuerbemessungsgrundlage entsteht (Tax Shield), wird durch Minderung

1 Zu einem Überblick und den folgenden Ausführungen vgl. Volpert, Kapitalwert, 1989, S. 90 ff.; Drukarczyk, Anmerkungen, 1995, S. 329 ff.; Hachmeister, Unternehmenswert, 1996, S. 357 ff.; Ross/Westerfield/Jaffe, Corporate Finance, 1996, S. 455 ff.; Ballwieser, Discounted Cash Flow-Verfahren, 1998, S. 81 ff.; Steiner/Wallmeier, Discounted Cash Flow-Methoden, 1999, S. 3 ff.

2 Im Fall der Kreditaufnahme ist der Rückfluß nach Zinsen und nach Steuern zu erhöhen.

3 Bei der Prognose der Rückflüsse wird i.d.R. für einen überschaubaren Zeitraum ( 5 bis 10 Jahre) periodenindividuell geplant. Ab diesem Detailplanungshorizont wird von einem konstanten nachhaltigen Rückfluß für darauf folgende Perioden ausgegangen. Dies zieht die Bildung einer ewigen Rente nach sich, von welcher an dieser Stelle abstrahiert wird. 
des Fremdkapitalkostensatzes im Nenner berücksichtigt. Durch Abzinsung der Cash Flows vor Zinsen mit dem gewogenen Kapitalkostensatz wacc resultiert in einem ersten Schritt der Unternehmensgesamtwert $V_{G K}$ (Wert des Gesamtkapitals).

$$
\begin{gathered}
V_{G K}=\sum_{t=1}^{n} \frac{C F_{t} \cdot(1-s t)}{(1+w a c c)^{t}} \\
w a c c=r_{E K} \cdot \frac{V_{E K}}{V_{G K}}+r_{F K} \cdot(1-s t) \cdot \frac{V_{F K}}{V_{G K}}
\end{gathered}
$$

Um zum Wert des Eigenkapitals zu gelangen, muß in einem zweiten Schritt vom Unternehmensgesamtwert der Wert des Fremdkapitals $V_{F K}$ abgezogen werden. Dieser kann durch Diskontierung der Zins- sowie Kredittilgungszahlungen $K T_{t}$ mit dem Fremdkapitalkostensatz gebildet werden.

$$
\begin{gathered}
V_{F K}=\sum_{t=1}^{n} \frac{Z_{t}+K T_{t}}{\left(1+r_{F K}\right)^{t}} \\
V_{E K}=V_{G K}-V_{F K}
\end{gathered}
$$

Beim WACC-Ansatz besteht ein Interdependenzproblem zwischen dem Wert des Eigenkapitals und den gewogenen Kapitalkosten ${ }^{1}$. Um letztere zu bilden, wird der Wert des Eigenkapitals vorausgesetzt, der eigentlich erst das Ergebnis der Berechnung sein soll. Theoretisch exakt lassen sich die beiden Gleichungen nur durch Iteration lösen. Um den Rechenaufwand gering zu halten, wird in der Literatur teilweise vorgeschlagen, von einer Zielkapitalstruktur auszugehen ${ }^{2}$. Im Vorfeld der Berechnung ist dann eine Eigen- und Fremdkapitalquote festzulegen. Diese Vorgehensweise bedingt, daß nach der Bewertung der Unternehmensgesamtwert in dem angenommenen proportionalen Verhältnis auf die Werte des Eigen- und Fremdkapitals aufgeteilt werden muß (Unternehmensgesamtwertorientierte Fremdfinanzierung). Wird hingegen aus pragmatischen oder anderen Gründen von einem fixen Fremdkapitalbestand ausgegangen, sollte nach der Bewertung die zuvor festgesetzte Kapitalstruktur mit dem Ergebnis überprüft werden. Die im Ergebnis enthaltene Finanzierungsstruktur sollte nicht gravierend von der angenommenen abweichen, da ansonsten ein

\footnotetext{
1 Vgl. Hachmeister, Abbildung der Finanzierung, 1996, S. 256.

2 Vgl. stellvertretend Copeland/Koller/Murrin, Valuation, 1994, S. $241 \mathrm{f}$.
} 
Unternehmenswert unter einer falschen Finanzierungsprämisse ermittelt wird und der Kapitalkostensatz neu bestimmt werden müßte.

Der Total Cash Flow-Ansatz arbeitet wie das WACC-Konzept mit einem gewogenen Gesamtkapitalkostensatz. Jedoch erfaßt das TCF-Konzept das Tax Shield nicht im Nenner durch Minderung des Fremdkapitalkostensatzes, sondern im Zähler durch Addition der durch Fremdfinanzierung zu erzielenden periodenabhängigen absoluten Steuerersparnis. Der gewogene Gesamtkapitalkostensatz $r_{G K}$ wird also ohne Tax Shield-Korrektur gebildet. Durch Abzug des Werts des Fremdkapitals vom Wert des Gesamtkapitals ergibt sich auch hier der Wert des Eigenkapitals.

$$
\begin{gathered}
V_{G K}=\sum_{t=1}^{n} \frac{C F_{t} \cdot(1-s t)+s t \cdot Z_{t}}{\left(1+r_{G K}\right)^{t}} \\
r_{G K}=r_{E K} \cdot \frac{V_{E K}}{V_{G K}}+r_{F K} \cdot \frac{V_{F K}}{V_{G K}} \\
V_{E K}=V_{G K}-V_{F K}
\end{gathered}
$$

Mit Ausnahme der unterschiedlichen Tax Shield-Erfassung stimmt der TCFAnsatz von der methodischen Konzeption her mit dem WACC-Ansatz überein. Im Vergleich zum WACC-Ansatz beinhaltet er neben dem dort beschriebenen ersten Zirkularitätsproblem eine zweite Interdependenz, die eine praktische Umsetzung erschwert. Zur Bestimmung der absoluten periodenindividuellen Steuerersparnis müßte die Zinszahlung der einzelnen Periode bzw. - was das gleiche bedeutet - der Fremdkapitalbestand der Vorperiode bekannt sein. Dieser ist aber bei theoretisch exakter Anwendung erst nach Berechnung des Unternehmensgesamtwerts und dessen proportionaler Aufteilung auf die Werte des Eigen- sowie Fremdkapitals ermittelbar.

Wie beim WACC- wird auch beim Adjusted Present Value-Ansatz der operative Cash Flow um fiktive Steuern gemindert. Anschließend erfolgt die Diskontierung dieses Rückflusses mit einem Eigenkapitalkostensatz nach Steuern $r_{E K}{ }^{*}$, der sich ohne Fremdfinanzierung ergeben würde. Dadurch wird im APVAnsatz in einem ersten Schritt von der Fiktion eines vollständig eigenfinanzierten Unternehmens ausgegangen, wodurch ein sogenannter Basiswert $V_{E K}{ }^{*}$ resultiert.

$$
V_{E K} *=\sum_{t=1}^{n} \frac{C F_{t} \cdot(1-s t)}{\left(1+r_{E K} *\right)^{t}}
$$


Den Unternehmensgesamtwert erhält man, indem zum Basiswert der Barwert des durch Fremdfinanzierung entstehenden Steuerspareffekts (Tax Shield) TS addiert wird.

$$
V_{G K}=V_{E K}^{*}+T S
$$

Um zum Wert des Eigenkapitals zu gelangen, muß auch beim APV-Ansatz vom Unternehmensgesamtwert der Wert des Fremdkapitals abgezogen werden.

$$
V_{E K}=V_{G K}-V_{F K}
$$

Wird von einem fixen Fremdkapitalbestand im Zeitablauf ausgegangen, so läßt sich der Barwert der periodischen Steuerersparnisse verhältnismäßig einfach ermitteln. Der Wert des Fremdkapitals ist mit dem Steuersatz zu multiplizieren, wobei der Fremdkapitalwert durch Division der periodisch konstanten Zinszahlung $Z$ mit dem Fremdkapitalkostensatz bestimmt werden kann.

$$
T S=s t \cdot V_{F K}=s t \cdot \frac{Z}{r_{F K}}
$$

Bei zeitlich schwankenden und zudem unsicheren Fremdkapitalbeträgen ist die Vorgehensweise nicht ganz so trivial. Aus Sicht der Periode $t$ ist die Steuerersparnis in $t+1$ als sicher anzusehen, da die Zinszahlung in $t+1$ durch die Kenntnis des Fremdkapitalbestands in $t$ eindeutig festgelegt ist. Die Steuerersparnis in $t+2$ gilt jedoch aus heutiger Sicht als unsicher, da der Fremdkapitalbestand in $t+1$ noch nicht bekannt ist. Folglich muß diese Steuergutschrift einmal mit dem risikolosen Zinsfuß und einmal mit einem, den Schwankungen des Fremdkapitalbestands entsprechenden, risikobehafteten Zinsfuß diskontiert werden ${ }^{1}$. Zur Abzinsung der einzelnen periodischen Steuergutschriften bedarf es folglich periodenspezifischer Zinsfüße $r_{\tilde{\imath}}$.

\footnotetext{
Wird eine konstante Kapitalstruktur im Zeitablauf unterstellt, sind die Schwankungen des Fremdkapitals auf die Schwankungen des Unternehmensgesamtwerts und jene auf die Schwankungen des operativen Cash Flows zurückzuführen. Der hier geforderte Zinsfuß stimmt dann mit dem Eigenkapitalkostensatz bei fiktiver vollständiger Eigenfinanzierung $r_{E K}{ }^{*}$ überein. Bei einer solchen Berechnung ergibt sich jedoch ein Zirkelproblem. Zur Bestimmung der periodenabhängigen Steuerersparnis in $t+l$ wird die Zinszahlung der gleichen Periode bzw. der Fremdkapitalbestand in $t$ benötigt. Letzterer kann jedoch erst nach Bestimmung des Unternehmensgesamtwerts in $t$, der das Ergebnis der Berechnung sein soll, ermittelt werden. Diese Interdependenz kann nur durch ein Roll Back-Verfahren gelöst werden. Vgl. Hachmeister, Discounted Cash Flow, 1998, S. 114 ff.
} 


$$
T S=\sum_{\substack { t=1 \\
\begin{subarray}{c}{t=1 \\
t{ t = 1 \\
\begin{subarray} { c } { t = 1 \\
t } }\end{subarray}}^{n} 1+r_{\tilde{t}}
$$

Bei Verwendung von periodenunabhängigen Kapitalkostensätzen $r_{E K}, r_{F K}$ bzw. $r_{E K}{ }^{*}$ führen die einzelnen Varianten zum gleichen Ergebnis, wenn erstens eine am Unternehmensgesamtwert orientierte Fremdfinanzierung und zweitens eine Abhängigkeit der Eigenkapitalkosten vom Verschuldungsgrad unterstellt wird ${ }^{1}$. Erstere Bedingung zielt auf die Konstanz der Kapitalstruktur im Zeitablauf ab. Diese ist nur sichergestellt, wenn in jeder Periode der Fremdkapitalbestand an die Veränderung des Unternehmensgesamtwerts - in dem angenommenen proportionalen Verhältnis - angepaßt wird. Die zweite Bedingung stellt sicher, daß sich die Eigenkapitalkosten bei fiktiver vollständiger Eigenfinanzierung $r_{E K}{ }^{*}$ von denjenigen bei tatsächlicher Finanzierungsstruktur $r_{E K}$ unterscheiden ${ }^{2}$.

\subsubsection{Ertragswert- und DCF-Methode}

Im Rahmen der investitionsrechnerischen Unternehmensbewertung ist in Literatur und Praxis oft die Rede von der Ertragswert- bzw. der Discounted Cash Flow (DCF)-Methode ${ }^{3}$. Dies sind nicht etwa zwei weitere Varianten, die in der bisherigen Darstellung noch nicht berücksichtigt wurden, sondern praktische Ausgestaltungen der präsentierten Grundkonzeptionen.

1 Die Identität des FTE- mit dem WACC-Ergebnis ist bereits bei Einhaltung der ersten Bedingung gegeben.

2 Vgl. Hachmeister, Discounted Cash Flow, 1998, S. 109 ff. sowie speziell S. 122 f. Zur Identität bzw. Nichtidentität der Bewertungsergebnisse sowie zur Leistungsfähigkeit und Anwendungsempfehlungen der einzelnen Grundkonzeptionen vgl. auch Ballwieser, Aktuelle Aspekte, 1995; Jonas, Anwendung in Deutschland, 1995; Drukarczyk/Richter, Unternehmensgesamtwert, 1995; Sieben, Zwei Ansätze, 1995; Schmidt, Discounted Cash FlowMethode, 1995; Richter, Finanzierungsprämissen, 1996; Born, Überleitung, 1996; Hachmeister, Abbildung der Finanzierung, 1996; Kirsch/Krause, Kritische Überlegungen, 1996; Richter, Abweichungen, 1997; Drukarczyk/Hunold, Unternehmensbewertung, 1998; Kruschwitz/Löffler, Revisited, 1998; Richter, Verschuldungsgrad, 1998; Schwetzler/Darijtschuk, Zirkularitätsproblem, 1999.

3 Es sei an dieser Stelle erwähnt, daß es die DCF-Methode nicht gibt. Vielmehr kann jede Variante der Abzinsung zukünftiger Rückflüsse als DCF-Verfahren bezeichnet werden. Im folgenden wird die DCF-Methode von Copeland/Koller/Murrin vorgestellt. Vgl. Copeland/ Koller/Murrin, Valuation, 1994, S. 131 ff. Die gleiche Argumentation läßt sich auch für die Ertragswertmethode fuhren. 
Der Begriff der Ertragswertmethode ist stark durch die vom Institut der deutschen Wirtschaftsprüfer zur Unternehmensbewertung herausgegebenen Verlautbarungen sowie die Ausführungen im WP-Handbuch geprägt ${ }^{1}$. Nach Auffassung des IDW ist der Unternehmenswert als Barwert der mit dem Eigentum an dem Unternehmen verbundenen Nettozuflüsse an die Unternehmenseigner definiert. Letztere sind beim Ertragswertverfahren auf Basis einer, auf Unternehmensebene durchzuführenden, handelsrechtlichen Ertragsüberschußrechnung zu bestimmen. Ergänzend ist eine Finanzbedarfsrechnung erforderlich, welche die finanziellen Auswirkungen der geplanten Ausschüttung erfaßt und den zukünftigen Mittelbedarf- bzw. -überschuß feststellt ${ }^{2}$. Auf Basis einer solchen Finanzplanung, in die auch die zukünftige Investitionsplanung eingeht, sind die zukünftigen Zinszahlungen festzulegen und Auswirkungen einer möglichen Veränderung der Kapitalstruktur auf den Diskontierungs- bzw. Fremdkapitalkostensatz abzuschätzen. Bei der Ermittlung der relevanten Nettozuflüsse werden zudem die Ertragsteuern des Unternehmens und des Unternehmenseigners abgezogen, soweit sie Definitiv-Charakter besitzen ${ }^{3}$.

Im Rahmen der Ermittlung eines Entscheidungswerts richtet sich der Diskontierungssatz nach den individuellen Verhältnissen des jeweiligen Eigentümers. Hierbei kommt sowohl die Rendite einer Alternativinvestition, der Zinssatz von Krediten, die zur Tilgung vorgesehen sind, oder ein gemäß der Zinszuschlagsmethode ermittelter Zinsfuß in Frage. Für letztere wird zuerst ein Basiszinssatz (z.B. langfristig erzielbare Rendite öffentlicher Anleihen) bestimmt, der dann um einen Risikozuschlag ${ }^{4}$ erhöht wird. Für einen solchen Risikozuschlag können am Kapitalmarkt beobachtbare Risikoprämien (z.B. auf Basis

1 Zu den Ausführungen im WP-Handbuch vgl. Dörner, Unternehmensbewertung, 1992, S. $1 \mathrm{ff}$. Zu den Verlautbarungen des IDW vgl. IDW, HFA-Stellungnahme, 1983, S. $468 \mathrm{ff}$. sowie IDW, Standard, 1999, S. 200 ff., wobei die HFA-Stellungnahme von 1983 durch den bis dato lediglich im Entwurf vorliegenden Standard von 1999 ersetzt werden soll. Die weiteren Ausfuhrungen beziehen sich deshalb auf den Entwurf von 1999 und hierbei nur auf die Ertragswertmethode. Alternativ ist nach dem neuen Entwurf eine Unternehmenswertermittlung nach der Grundkonzeption des WACC- bzw. APV-Ansatzes möglich.

2 Alternativ kann auch eine Einnahmenüberschußrechnung zugrundegelegt werden, die parallel um eine handelsrechtliche GUV-Rechnung ergänzt wird, um die Ausschüttungsfähigkeit der geplanten Ausschüttungen zu überprüfen. Vgl. IDW, Standard, 1999, S. 203.

3 Die Körperschaftsteuer ist aufgrund ihres Charakters als Vorauszahlung auf die persönliche Einkommensteuerschuld grundsătzlich nicht abzuziehen. Hiervon ausgenommen ist jedoch die auf nicht abzugsfähige Aufwendungen entfallende Körperschaftsteuer. Vgl. IDW, Standard, 1999, S. 204.

4 Alternativ wird auch die Berücksichtigung des sogenannten Unternehmerrisikos im Zähler der Diskontierungsformel erwähnt. Vgl. IDW, Standard, 1999, S. 208. 
des CAPM) herangezogen werden, die ggf. um die Besonderheiten des Bewertungsfalls anzupassen sind. Der i.d.R. vor Steuern ermittelte Zinsfuß ist um den persönlichen Ertragssteuersatz des Unternehmenseigners zu kürzen. Da die Ertragswertmethode von Rückflüssen nach Fremdkapitalzinsen und einem Eigenkapitalkostensatz als Kalkulationszinsfuß ausgeht, ist sie konzeptionell dem FTE-Ansatz zuzuordnen.

Bei der DCF-Methode von Copeland/Koller/Murrin werden sogenannte Free Cash Flows als zu diskontierende Zahlungsströme ermittelt ${ }^{1}$. Vom jahresabschlußorientierten operativen Ergebnis vor Steuern werden fiktive Ertragsteuern abgezogen und die verbleibende Größe um die Veränderung der Steuerrückstellungen korrigiert. Dieses operative Ergebnis nach Steuern wird um Abund Zuschreibungen bereinigt, um zum Cash Flow vor Investitionen zu gelangen $^{2}$. Durch Abzug der Investitionen in das Anlage- und Umlaufvermögen und Addition des nicht operativen Cash Flows erhält man den gewünschten Free Cash Flow, der den Eigen- und Fremdkapitalgebern gemeinsam zur Ausschüttung zur Verfügung steht ${ }^{3}$.

Bei der Kapitalkostenbestimmung wird auf einen gewogenen Zinssatz zurückgegriffen, der sich aus den mit der Zielkapitalstruktur gewichteten Eigen- und Fremdkapitalkosten ermittelt. Zur Bestimmung der Eigenkapitalkosten werden kapitalmarkttheoretische Modelle, wie z.B. das CAPM oder die APT, vorgeschlagen. Die Fremdkapitalkosten sind unter Berücksichtigung der steuerlichen Abzugsfähigkeit der Fremdkapitalzinsen zu schätzen ${ }^{4}$. Innerhalb der DCFMethode von Copeland/Koller/Murrin werden Rückflüsse vor Fremdkapitalzinsen angesetzt sowie zur Diskontierung ein gewogener Kapitalkostensatz herangezogen. Konzeptionell entspricht das Bewertungsverfahren damit dem WACC-Ansatz.

1 Vgl. zu folgendem Copeland/Koller/Murrin, Valuation, 1994, S. $131 \mathrm{ff}$.

2 Bei der hier vorgenommenen indirekten Ermittlung des Cash Flows vor Investitionen vermißt man die in Deutschland übliche Korrektur zur Veränderung der Pensionsrückstellungen. Dies ist auf die geringere Bedeutung der Pensionsrückstellungen in US-amerikanischen Jahresabschlüssen zurückzufuhren. Die Pensionsansprüche werden in den USA i.d.R. an Pensionskassen ausgezahlt.

3 Streng genommen steht den Kapitalgebern ein größerer Betrag zur Verfügung, da bei der Bestimmung des Free Cash Flows methodenbedingt eine zu hohe Steuerzahlung angesetzt wird.

4 Vgl. Copeland/Koller/Murrin, Valuation, 1994, S. $239 \mathrm{ff}$. 


\subsection{Eignung der Verfahren bei wertorientierter Unternehmensführung}

\subsubsection{Anforderungen bei wertorientierter Unternehmensführung}

Der Wert eines Unternehmens wird grundsätzlich durch den Nutzen bestimmt, den ein einzelnes Wirtschaftssubjekt durch seine Beteiligung an diesem Unternehmen erzielt. Für Eigenkapitalgeber - von denen im folgenden ausgegangen wird - wird angenommen, daß ihr Nutzen sich durch jene Rückflüsse konkretisiert, die ihnen aufgrund der Inhaberschaft der Unternehmensanteile zufließen. Dies steht im Einklang mit dem in jüngster Zeit in Literatur und Praxis zunehmend geforderten Ruf nach einer wertorientierten Unternehmensführung ${ }^{1}$. Darunter wird allgemein ein unternehmerisches Handeln verstanden, das alle Ziele, Strategien und operative Maßnahmen an der Maximierung des Marktwerts des Eigenkapitals ausrichtet. Dieser wird dabei als Summe der den Eigenkapitalgebern in Zukunft zur Verfügung stehenden, auf den heutigen Zeitpunkt diskontierten Zahlungsströme definiert.

Eine ganze Reihe von Autoren beschäftigen sich mit (Markt-) Bedingungen, die eine derartige Ausrichtung der Unternehmensführung begünstigen bzw. nahe legen ${ }^{2}$. Darauf wird an dieser Stelle nicht näher eingegangen. Hier wird lediglich die Frage gestellt, welche Anforderungen Bewertungsverfahren erfüllen müssen, um eine gewünschte Umsetzung der wertorientierten Unternehmensführung zu unterstützen.

Wenn der Nutzen eines Wirtschaftssubjekts durch Rückflüsse konkretisiert wird, liegt es nahe, zahlungsorientierte Überschußgrößen zur Bestimmung des Unternehmenswerts zu verwenden. Desweiteren werden die zukünftigen Rückflüsse durch den kombinierten Einsatz aller Vermögensgüter im Unternehmen erzielt. Dies schließt grundsätzlich eine Unternehmensbewertung über den Weg der Bewertung einzelner Vermögensgegenstände aus, so daß - abgesehen von Sonderfällen - lediglich eine Gesamtbewertung in Frage kommt. Letztlich ist der Kapitalgeber an jenen Rückflüssen interessiert, die er in Zukunft gemäß

\footnotetext{
Vgl. stellvertretend Hachmeister, Shareholder Value, 1997 sowie die dort zitierte Literatur.

2 Vgl. stellvertretend Wilhelm, Marktwertmaximierung, 1983, S. 516 ff.; Bischoff, Shareholder Value-Konzept, 1994, S. 168 ff.; Hachmeister, Discounted Cash Flow, 1998, S. 11 ff.; Breuer, Marktwertmaximierung, 1997, S. 222 ff. sowie kritisch Schneider, Klumpfuß, 1998, S. $1473 \mathrm{ff}$.
} 
seiner individuellen Ziele erreichen kann. Hierdurch ist eine konsequente $Z u$ kunftsbezogenheit sowie Subjektivität des Bewertungsverfahrens zu fordern ${ }^{1}$.

\subsubsection{Eignung der einzelnen Bewertungsverfahren}

Traditionelle Substanzwertverfahren verstoßen gegen sämtliche Bedingungen, die im vorigen Abschnitt zur Unterstützung der wertorientierten Unternehmensführung genannt worden sind. Für den Bewerter steht nicht die Errichtung eines strukturgleichen Unternehmens, sondern die Abbildung des erwartenden Erfolgs aus dem Unternehmen im Mittelpunkt der Betrachtung ${ }^{2}$. Mischverfahren sind, da sie sich Substanzwertkonzeptionen bedienen, ebenso wenig geeignet, die Bedingungen zu erfüllen wie die Substanzwertverfahren selbst.

Demgegenüber verstößt das Substanzwertverfahren im Sinne ersparter Ausgaben nicht grundsätzlich gegen die aufgestellten Bedingungen. Es wird davon ausgegangen, daß eine potentielle Neuerrichtung gleich hohe Einzahlungen erwarten läßt wie das zu bewertende Unternehmen. In dieser Annahme ist ansatzweise der Gedanke der Alternativrendite enthalten. Insofern könnte diese Konzeption eher als eine spezielle Form des Zukunftserfolgswerts mit Konzentration auf Auszahlungsgrößen interpretiert werden ${ }^{3}$.

Das Liquidationswertverfahren geht mit den Bedingungen für eine wertorientierte Unternehmensführung konform, falls die Zerschlagung des Unternehmens als Alternative zur Fortführung in Betracht gezogen wird ${ }^{4}$. Folgerichtig ist der Liquidationswert für den Bewerter von Belang, der an einer solchen Zerschlagung interessiert ist. Unter diesen Voraussetzungen kann der Liquidationswert als spezieller Zukunftserfolgswert aufgefaßt werden.

Der Anwendung von Branchenmultiplikatoren liegt die Überlegung zugrunde, daß Kapitalkosten sehr stark von Sektoren abhängen, in denen Unternehmen tätig sind. Stimmt die Bezugsgröße des Multiplikators inhaltlich mit der in der Investitionsrechnung im Rentenmodell verwendeten Überschußgröße überein,

1 Vgl. Ballwieser/Leuthier, Grundprinzipien, 1986, S. 548 f.; Serfling/Pape, Grundlagen, 1995, S. 812 f.

2 Vgl. Ballwieser, Methoden, 1993, S. 169; Sieben, Unternehmensbewertung, 1993, Sp. 4327; Serfling/Pape, Grundlagen, 1995, S. 816 f.

3 Vgl. Sieben, Unternehmensbewertung, 1993, Sp. 4328.

4 Vgl. Ballwieser/Leuthier, Grundprinzipien, 1986, S. 548. 
entspricht der Multiplikator dem Kehrwert des Kalkulationszinsfußes ${ }^{1}$. Er ist dann Ausdruck der in der jeweiligen Branche durchschnittlich zu erzielenden Alternativrendite.

In dieser Interpretation stellen Branchenmultiplikatoren eine im Durchschnitt vom Markt vorgenommene Gesamtbewertung dar. Jedoch werden die Besonderheiten des zu bewertenden Unternehmens, z.B. die mit dem Unternehmen konkret verfolgten Ziele des Bewerters, nicht erfaßt. Dazu müßten die Multiplikatoren für den individuellen Bewertungsfall angepaßt werden ${ }^{2}$. Für eine zukunftsbezogene Gesamtbewertung müßten prognostizierte Multiplikatoren verwendet werden. Diese sind aber nicht ohne umfangreiche Umwelt- und Unternehmensanalysen zu gewinnen, womit der Vorteil der Praktikabilität und Wirtschaftlichkeit von Multiplikatoren nicht mehr gegeben ist. Zudem weist Ballwieser darauf hin, daß Kapitalkosten sich nicht nur über die Branchenzugehörigkeit erklären lassen und sogar die Zuordnung zu einer Branche für diversifizierte Unternehmen nicht gelingt ${ }^{3}$.

Dem Comparative Company-Ansatz haften die gleichen theoretischen Mängel wie den Branchenmultiplikatoren an. Zur Erfassung der individuellen und zukünftigen Gegebenheiten wären umfassende Umwelt- und Unternehmensanalysen sowohl der Vergleichsunternehmen als auch des zu bewertenden Unternehmens notwendig ${ }^{4}$. Preisfindungsverfahren sind infolgedessen lediglich in der Lage, erste Anhaltspunkte für die Wertbestimmung zu liefern. Aufgrund der mangelnden Ausrichtung auf das individuelle Entscheidungsfeld und des Rückgriffs auf vergangene „Relationen” stellen sie jedoch keine geeignete Grundlage für eine an den Zielen des Bewerters orientierte, zukunftsbezogene Unternehmensbewertung dar 5 .

Durch Diskontierung von erwarteten Rückflüssen gehen investitionsrechnerische Bewertungsverfahren implizit vom Nutzen aus, den die Kapitalgeber durch die Gesamtheit der Vermögensgegenstände in Zukunft erzielen können. Die investitionsrechnerische Unternehmensbewertung stellt folglich per se eine

1 Vgl. Ballwieser, Multiplikatoren, 1991, S. 55.

2 Für Vorschläge dieser Art vgl. Bretzke, Risiken, 1988, S. 820; Sanfleber-Decher, Unternehmensbewertung, 1992, S. 602 f.; Barthel, Varianten des Umsatzverfahrens, 1996, S. $1462 \mathrm{ff}$.

3 Vgl. Ballwieser, Multiplikatoren, 1991, S. 58 f.

4 Vgl. dazu auch Ballwieser, Neue Lehre, 1997, S. 186 f.

s Vgl. Englert, Wertfindungsmethoden, 1997, S. 147. 
zukunftsbezogene zahlungsorientierte Gesamtbewertung dar $^{1}$. Werden die erwarteten Rückflüsse unter Berücksichtigung der individuellen Ziele des Bewerters prognostiziert, ist zudem das Kriterium der Subjektivität erfüllt. Investitionsrechnerische Verfahren entsprechen somit allen zuvor erwähnten Bedingungen, die die Unterstützung einer wertorientierten Unternehmensführung gewährleisten ${ }^{2}$. Im folgenden wird ausschließlich von diesen Bewertungsverfahren ausgegangen.

\section{Begriff der strategischen Unternehmensbewertung}

\subsection{Begriffsinterpretationen und Aufgabengebiete}

Der Einstieg in die im Rahmen dieser Arbeit behandelte Problemstellung wird durch die uneinheitliche Verwendung bzw. mehrfache Interpretation des Begriffs „Strategische Unternehmensbewertung“ erschwert. Unter diesem Stichwort können unterschiedliche betriebswirtschaftliche Aufgaben verstanden werden. Einigkeit scheint bei den Autoren lediglich darüber zu bestehen, daß es sich hierbei um die explizite Berücksichtigung bzw. Betrachtung strategischer Aspekte handelt. Die Literatur kann grob in drei Aufgabengebiete der strategischen Unternehmensbewertung eingeteilt werden ${ }^{3}$.

Als erstes sind Beiträge zu nennen, in denen sich die Autoren mit den Methoden und Instrumenten der strategischen Unternehmensplanung und -analyse auseinandersetzen. $\mathrm{Zu}$ den Konzepten dieser Art zählen beispielsweise das Stärken/Schwächen-Profil, die Wertschöpfungskette oder Portfoliomatrizen ${ }^{4}$. Hierbei findet jedoch keine Bewertung des Unternehmens im Sinne einer quantitativen Wertermittlung statt, sondern eine qualitative Beurteilung. Aufbauend auf der systematischen Suche nach günstigen und ungünstigen Umfeldentwicklungen sowie Stärken und Schwächen des Unternehmens wird die

1 Vgl. ähnlich Dirrigl, Bewertung, 1988, S. 137 f.

2 Für empirische Belege zur Anwendung investitionsrechnerischer Verfahren als zentrale Unternehmensbewertungsmodelle vgl. Peemöller/Bömelburg/Denkmann, Unternehmensbewertung, 1994, S. 742; Prietze/Walker, Unternehmensbewertung, 1995, S. 205; Beck, Unternehmensbewertung, 1995, S. 187; Pellens/Rockholtz/Stienemann, Konzerncontrolling in Deutschland, 1997, S. 1935.

3 Vgl. zu folgendem ausführlich Dirrigl, Konzepte, 1994, S. 412 f. Vgl. auch Serfling/Pape, Strategische Unternehmensbewertung, 1996, S. 57.

4 Für einen Überblick zu Methoden und Instrumenten der strategischen Unternehmensplanung und -analyse vgl. Aeberhard, Strategische Analyse, 1996, S. 115 ff.; Kreikebaum, Strategische Unternehmensplanung, 1997, S. 97 ff. 
Strategie des Unternehmens im Sinne einer Marschrichtung festgelegt. Die Erhaltung alter bzw. der rechtzeitige Aufbau neuer Erfolgspotentiale bildet den Hauptzweck dieses Aufgabenfelds. Die strategische Planung bzw. Analyse kann somit eher als Aufgabe vor der eigentlichen Wertermittlung angesehen werden.

Zweitens sind Ansätze der Unternehmenssteuerung vor dem Hintergrund der Zielsetzung „Steigere den Wert des Eigenkapitals!“ zu erwähnen. Meist wird hier auf traditionelle investitionsrechnerische Bewertungsverfahren zurückgegriffen. Mit solchen Konzepten können zum einen einzelne Geschäftseinheiten, zum anderen verschiedene Investitionsalternativen evaluiert werden. Die Tatsache, daß Unternehmenssteuerung bzw. -führung notwendigerweise auch strategische Aspekte beinhaltet, verleitet dazu, dieses Aufgabengebiet als strategische Unternehmensbewertung zu bezeichnen. Vielfach wird auch alternativ von der Bewertung strategischer Geschäftseinheiten bzw. von Strategiealternativen gesprochen.

Eine dritte Begriffswelt stellt die Ermittlung eines Unternehmenswerts bei strategisch motivierten Akquisitionen in den Mittelpunkt der Betrachtung. Der Unternehmenserwerb soll über die Realisation von Restrukturierungs- bzw. Synergiepotentialen oder über seinen Beitrag zur Umsetzung der geplanten Unternehmensstrategie neue Erfolgspositionen sichern. Aufgrund der mit der Akquisition insbesondere verfolgten strategischen Zielsetzung werden Ansätze diskutiert, die mehr oder minder von den traditionellen Methoden der „finanziellen" Unternehmensbewertungslehre abweichen. Im Vorfeld der anstehenden Kaufverhandlung und Preisfestlegung gilt es, den Entscheidungswert des Käufers unter besonderer Berücksichtigung dieser strategischen Aspekte zu bestimmen.

Die Ausführungen in dieser Arbeit sind vordergründig auf die zuletzt genannte Begriffsinterpretation zugeschnitten. Falls im Rahmen der wertorientierten Unternehmensführung der Barwert eines neu aufzubauenden Geschäftsbereichs bzw. einer Investitionsalternative ermittelt werden soll, lassen sich eine Vielzahl der Anmerkungen auch für diese (zweite) Aufgabenstellung verwenden. Die erste Begriffsinterpretation fließt nur dann in die Überlegungen ein, wenn kurz die Verknüpfung zwischen quantitativen Bewertungsverfahren und qualitativen Analyse- und Planungsmethoden diskutiert wird ${ }^{1}$.

1 Vgl. hierzu Abschnitt 4.3 in Kapitel VI. 


\subsection{Strategische Aspekte in der Unternehmensbewertung}

\subsection{1 Überblick}

Obwohl in der Unternehmenspraxis bei Akquisitionen vielfach von strategisch bedingten Unternehmenskäufen die Rede ist, scheint bis dato nicht vollständig geklärt, was eigentlich genau unter strategischen Aspekten bzw. Faktoren in der Unternehmensbewertung zu verstehen ist. Eine Analyse der relevanten Literatur $^{1}$ wird durch die Tatsache erschwert, daß die zur Erklärung herangezogenen Begriffe nicht einheitlich verwendet werden. Tab. 2 gibt einen Überblick über die verschiedenen Beschreibungen.

\begin{tabular}{|c|c|}
\hline Coenenberg/Sautter (1988), Sautter (1989) & Sieben (1988), Sieben/Diedrich (1990) \\
\hline $\begin{array}{l}\text { - Restrukturierungspotentiale } \\
\text { - leistungs- und finanzwirtschaftliche } \\
\text { Synergiepotentiale } \\
\text { - Verbesserung der Position im Multi-Markt- } \\
\text { Wettbewerb }\end{array}$ & $\begin{array}{l}\text { - Beitrag zur langfristig angelegten Unter- } \\
\text { nehmensstrategie (Verwirklichung eines } \\
\text { geplanten Unternehmensprofils) }\end{array}$ \\
\hline Schneider (1988), (1991) & Ruhnke (1991) \\
\hline $\begin{array}{l}\text { - Synergiepotentiale } \\
\text { - realisierbare Wettbewerbsvorteile }\end{array}$ & $\begin{array}{l}\text { nicht-finanzielle (jedoch ökonomische) } \\
\text { Ziele wie z.B. Synergieeffekte, Beitrag } \\
\text { zum Übergang auf das (Unternehmens-) } \\
\text { Zielportfolio, Marktanteil }\end{array}$ \\
\hline Valcarel (1992) & Dirrigl (1994) \\
\hline $\begin{array}{l}\text { zusătzlich offenstehende, interne oder } \\
\text { externe, Nutzenpotentiale und Multiplika- } \\
\text { tionsmöglichkeiten ihrer Ausnutzung }\end{array}$ & $\begin{array}{ll}\text { - } & \text { Synergieeffekte } \\
\text { (inkl. Restrukturierungsmaßnahmen) } \\
\text { - Aussicht auf zukünftige Akquisitionen } \\
\text { - } \quad \text { strategische Flexibilität }\end{array}$ \\
\hline Helling (1994) & Petersen (1994) \\
\hline $\begin{array}{l}\text { - Synergien } \\
\text { - Beitrag zur geplanten Strategieumsetzung }\end{array}$ & $\begin{array}{ll}\text { - } & \text { synergetische Ziele } \\
\text { - } & \text { nicht-synergetische Ziele wie z.B. Risiko- } \\
\text { minderung, Marktmacht, Restrukturierung }\end{array}$ \\
\hline Barthel (1996) & Schmidt (1996) \\
\hline $\begin{array}{l}\text { - } \text { künftige Wettbewerbsvorteile } \\
\text { Meränderungen strategischer Ziele wie z.B. } \\
\text { Marktanteil, Wachstum, Erlangung der } \\
\text { Preisführerschaft, Schaffung bzw. Abbau } \\
\text { von Marktzutrittshürden }\end{array}$ & $\begin{array}{l}\text { - interne Erfolgsfaktoren } \\
\text { - personell } \\
\text { - technologisch } \\
\text { - organisatorisch } \\
\text { - externe Erfolgsfaktoren }\end{array}$ \\
\hline
\end{tabular}

Tab. 2: Strategische Aspekte bei Unternehmensakquisitionen

1 Vgl. Coenenberg/Sautter, Strategische und finanzielle Bewertung, 1988; Schneider, Ermittlung, 1988; Sieben, Unternehmensstrategien, 1988; Sautter, Strategische Analyse, 1989; Sieben/Diedrich, Aspekte, 1990; Ruhnke, Akquisitionen, 1991; Schneider, Strategische Unternehmensbewertung, 1991; Valcarel, Brücke, 1992; Dirrigl, Konzepte, 1994; Helling, Strategieorientierte Unternehmensbewertung, 1994; Petersen, Unternehmungskăufe, 1994; Barthel, Unternehmenswert, 1996; Schmidt, Unternehmensbewertung, 1996. 
Die genannten strategischen Aspekte lassen sich grob in drei Klassen bzw. Komponenten einordnen. Als erstes sind Restrukturierungspotentiale zu nennen, die sich dadurch auszeichnen, daß sie theoretisch auch unabhängig von einem Unternehmenserwerb realisierbar sind. Restrukturierungsmaßnahmen zielen beispielsweise auf ein effizienteres Management der vorhandenen Vermögensgegenstände und Schulden ab oder stellen die betriebliche Notwendigkeit derselben in Frage. Eine zweite Gruppe der Beschreibungen nennt die Realisation von Synergiepotentialen als strategischen Faktor der Akquisition. Synergiepotentiale sind nicht ohne den Zusammenschluß der beiden Unternehmen realisierbar. Die hiermit verbundenen Vorteile werden beispielsweise durch die gemeinsame Ausübung von Aktivitäten oder die Übertragung von Know How geschaffen.

Ausführungen zur geplanten langfristigen Unternehmensstrategie und -entwicklung bilden den dritten Bestandteil der angeführten strategischen Aspekte ${ }^{1}$. Das Ausmaß, mit dem die Akquisition zur Erreichung des geplanten Unternehmensprofils beiträgt, wird als strategischer Faktor der Akquisition gesehen. Der Unternehmenserwerb stellt in einem solchen Fall eine Anpassungsmaßnahme dar, die als Reaktion auf geänderte Umfeldbedingungen oder unternehmensspezifische Gegebenheiten zur Sicherung des langfristigen Erfolgs notwendig erscheint ${ }^{2}$. Ein viel zitiertes Beispiel ist der durch die Akquisition ermöglichte Eintritt in einen neuen Markt ${ }^{3}$.

Ein inhaltlicher Vergleich der einzelnen Literaturbeiträge zeigt, daß einige Autoren sämtliche hier gebildeten Komponenten als strategische Faktoren einer Akquisition einordnen. Realisierbare Restrukturierungs- und Synergiepotentiale zählen dann zu den strategischen Aspekten einer Unternehmensbewertung. Andere wiederum legen den Schwerpunkt ihrer Ausführungen nur auf den Beitrag der Akquisition im Hinblick auf das geplante Unternehmensprofil.

1 Bei einer strengen theoretischen Einteilung läßt sich der Beitrag zur geplanten Strategieumsetzung nicht vom Synergiepotential unterscheiden. Entweder es handelt sich bei den angefürten strategischen Aspekten um Werterhöhungen, die auch ohne Zusammenschluß durchführbar sind, oder um solche, die nicht ohne Zusammenschluß realisierbar sind. Da bei der Beschreibung von Synergiepotentialen i.d.R. die klassische Sichtweise (z.B. Zusammenlegung von Aktivitäten, Know How-Transfer) dominiert, wurde in Anlehnung an diese Auffassung der Beitrag zur geplanten Strategieumsetzung separat aufgefuhrt.

2 Vgl. Sieben/Diedrich, Aspekte, 1990, S. 797.

3 Vgl. stellvertretend Porter, Wettbewerbsstrategie, 1995, S. 436 ff. 
Unabhängig davon, welche Faktoren nun eher strategischer oder eher nicht strategischer Natur sind, müssen alle Komponenten bei der Bestimmung des Unternehmenswerts berücksichtigt werden. Eine Einteilung im Sinne einer starren Trennung ist für die Bewertungstechnik nur insofern relevant, daß Klarheit über das Akquisitionsobjekt bzw. die Bewertungssituation gewonnen werden kann. Folgerichtig müßte präziser von einer Unternehmensbewertung, die notwendigerweise strategische Aspekte berücksichtigt, und nicht von einer strategischen Unternehmensbewertung gesprochen werden. Im folgenden werden die in diesem Abschnitt erwähnten Komponenten näher betrachtet.

\subsubsection{Restrukturierungspotentiale}

$\mathrm{Zu}$ Restrukturierungspotentialen sind in der Literatur vergleichsweise wenige Ausführungen zu finden. Meist wird diese Komponente zusammen mit der Erörterung von Synergiepotentialen abgehandelt, ohne dies ausdrücklich kenntlich zu machen. Helbling geht explizit auf die Möglichkeit der Unternehmenswertoptimierung durch Restrukturierungsmaßnahmen und durch Minimierung der betriebsnotwendigen Substanz ein. Zu seinen Maßnahmen zählen z.B. die Ausgliederung und rechtliche Verselbständigung bzw. die Veräußerung von Teilbereichen. Auch durch Verkauf nicht betriebsnotwendiger Vermögensteile werden Wertsteigerungsmöglichkeiten gesehen'.

Ein umfassender Ansatz zur Systematisierung von Restrukturierungspotentialen stammt von Coenenberg/Sautter (vgl. auch Abb. 2). Als erstes wird hier auf die Möglichkeit zu einem effizienteren Management der vorhandenen Vermögensgegenstände und Schulden eingegangen. Dazu sind zum einen Maßnahmen denkbar, die kostensenkend wirken wie z.B. ein erhöhter Effizienzgrad in den operativen Tätigkeiten oder die Einstellung unrentierlicher Produkte. Zum anderen wird erwogen, ob nicht der Umsatz durch ein verändertes Marketing-Mix gesteigert werden kann. Im Rahmen der zweiten Maßnahmengruppe wird der vorhandene Vermögens- und Schuldenbestand auf betriebliche Notwendigkeit überprüft. Hierbei handelt es sich um Überlegungen, die eine Reallokation der Aktiva und Passiva zum Inhalt haben. Bei den Aktiva sind Veräußerungen von Teilbereichen oder einzelnen Vermögensgütern denkbar. Das Eingehen von Leasingverträgen oder allgemeine Umfinanzierungen zählen zu den Handlungen, die eine effizientere Gestaltung der Passiva ermöglichen ${ }^{2}$.

1 Vgl. Helbling, Restrukturierungsmaßnahmen, 1989, S. $178 \mathrm{ff}$.

2 Vgl. Coenenberg/Sautter, Strategische und finanzielle Bewertung, 1988, S. 699. 
Aufgrund der in letzter Zeit zunehmenden Diskussion um die Bedeutung von Anreizsystemen im Rahmen einer wertorientierten Unternehmensführung ${ }^{1}$, scheint es geboten, diesbezügliche „Verbesserungen“ explizit als dritte Maßnahmengruppe auf der ersten Ebene des hier vorgestellten Ansatzes einzubeziehen. Die Veränderung der Anreizstruktur soll die Unternehmensleitung insgesamt zu effizienterer Leistung veranlassen.

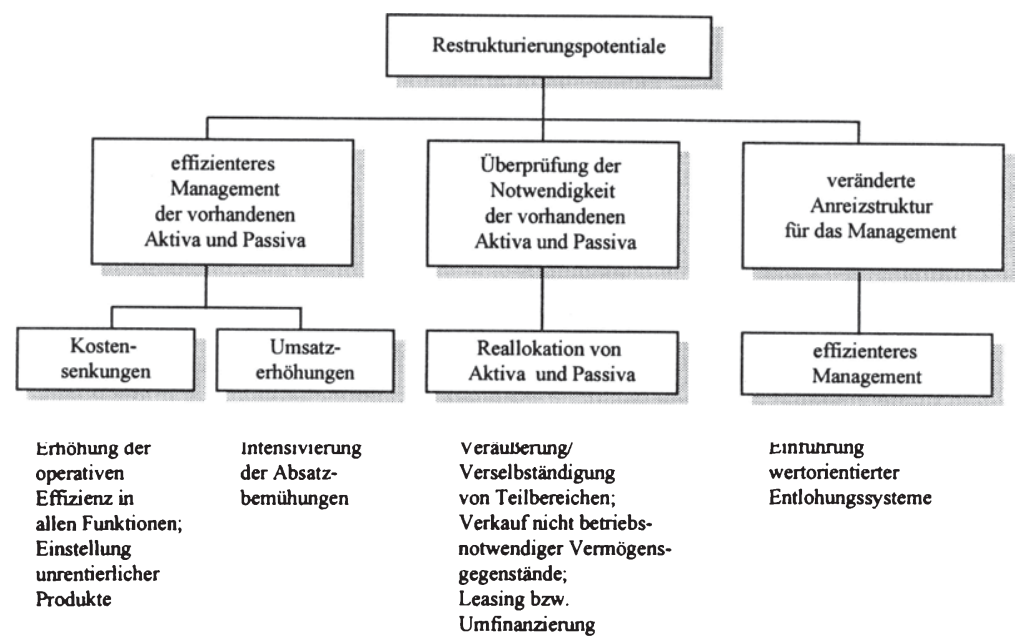

Abb. 2: Restrukturierungspotentiale

Quelle: In Anlehnung an Coenenberg/Sautter, Strategische und finanzielle Bewertung, 1988, S. 699.

\subsubsection{Synergiepotentiale}

Die bekanntesten Ansätze zur Systematisierung von Synergiepotentialen stammen von Ansoff und Porter ${ }^{2}$. Ersterer unterscheidet bei seinen Ausführungen in einmalige und permanente Synergien sowie in Verbundeffekte in Abhängigkeit einzelner Funktionsbereiche. Als Beispiel für letztere nennt Ansoff den Verkauf, die Produktion, die Investition und das Management. Porter entwickelte Mitte der achtziger Jahre den Ansatz der Wertkette, entlang derer die einzelnen Wertschöpfungsaktivitäten des Unternehmens dargestellt sind. Im Rahmen

1 Vgl. z.B. Pellens (Hrsg.), Unternehmenswertorientierte Entlohnungssysteme, 1998; Schnabel, Vergütung, 1998.

2 Vgl. Ansoff, Corporate Strategy, 1965, S. 79 ff.; Porter, Wettbewerbsvorteile, 1986, S. $59 \mathrm{ff}$. und S. $416 \mathrm{ff}$. 
seines Synergiekonzepts unterscheidet er in Synergien durch Zusammenlegung von Aktivitäten und solchen, die durch Know How-Transfer zustande kommen.

\begin{tabular}{|c|c|}
\hline Quelle & Systematisierungskriterien \\
\hline Küting (1981) & $\begin{array}{l}\text { monetäre und nicht monetäre, sofort oder später realisierbare, } \\
\text { Aufwands- oder Ertrags-, einmalige und permanente, positive und } \\
\text { negative Synergien; Synergien beim Akquisitionsobjekt oder beim } \\
\text { Erwerber, nach Funktionsbereichen, nach Entstehungsursachen }\end{array}$ \\
\hline Bühner/Spindler (1986) & $\begin{array}{l}\text { Finanzkraftvorteile; Verbundvorteile in Funktionsbereichen; Risi- } \\
\text { kovorteile }\end{array}$ \\
\hline Coenenberg/Sautter (1988) & $\begin{array}{l}\text { güter- und finanzwirtschaftiche, Gründungs- und Betriebssynergi- } \\
\text { en; Synergien in Verwaltung, Forschung und Entwicklung, Mar- } \\
\text { keting, Produktion }\end{array}$ \\
\hline Gälweiler (1989) & $\begin{array}{l}\text { absatzfördernde, technologische, investitionssparende, sonstige } \\
\text { stückkostenmindernde und Führungs-Synergien; einmalige und } \\
\text { permanente Synergien }\end{array}$ \\
\hline Ropella (1989) & Synergien in Abhängigkeit von Produktionsfaktoren \\
\hline Sandler (1991) & Scale Economy, Scope Economy, Machtsynergie \\
\hline Weber (1991) & Markt-, Kosten- und steuerliche Synergien \\
\hline $\begin{array}{l}\text { Abeitskreis „Die Unter- } \\
\text { nehmung im Markt““ } \\
\text { (1992) }\end{array}$ & $\begin{array}{l}\text { Synergien in einzelnen Funktionsbereichen; kosten-, ertrags- und } \\
\text { risikobedingte Synergien }\end{array}$ \\
\hline Kogeler (1992) & $\begin{array}{l}\text { finanzielle und funktionale Synergien; materielle und immaterielle } \\
\text { Synergien; positive und negative Synergien }\end{array}$ \\
\hline Reißner (1992) & $\begin{array}{l}\text { Synergien aus Zentralisation, Integration/Restrukturierung, Trans- } \\
\text { fer, Ergänzung/Zugang und Ausgleich }\end{array}$ \\
\hline Ehrensberger (1993) & $\begin{array}{l}\text { Verknüpfungen von materiellen, immateriellen sowie humanen } \\
\text { Aktiva }\end{array}$ \\
\hline Petersen (1994) & $\begin{array}{l}\text { leistungswirtschaftliche, finanzwirtschaftliche Synergieeffekte und } \\
\text { Synergieeffekte aus Management und Administration }\end{array}$ \\
\hline Paprottka (1996) & $\begin{array}{l}\text { Synergien in einzelnen Funktionsbereichen; Synergien durch } \\
\text { Größeneffekte, Know How-Transfer, Spill Over-Effekte, Quali- } \\
\text { tätsverbesserungen, Ausbau der Machtposition, Risikoverände- } \\
\text { rung, Transaktionskosteneinsparungen }\end{array}$ \\
\hline Perin (1996) & Finanz-, Markt- und Leistungssynergien \\
\hline Scharlemann (1996) & $\begin{array}{l}\text { finanzwirtschaftliche Synergiepotentiale durch risikomindernde } \\
\text { Senkung der Kapitalkosten sowie Größenvorteile }\end{array}$ \\
\hline Ziegler (1997) & $\begin{array}{l}\text { leistungs- und finanzwirtschaftliche Synergien; kosten- und lei- } \\
\text { stungsorientierte Synergien; Synergien nach Funktionsbereichen }\end{array}$ \\
\hline Ebert (1998) & $\begin{array}{l}\text { Synergien durch economies of scale, economies of scope, akqui- } \\
\text { sitionsinduzierte Marktpositionierung, Optimierung des Integrati- } \\
\text { onsgrades; Input-, Prozeß- und Output-Synergien }\end{array}$ \\
\hline Steidl (1999) & $\begin{array}{l}\text { finanzielle und materielle Ressourcen, immaterielle „Assets“, } \\
\text { Fähigkeiten }\end{array}$ \\
\hline
\end{tabular}

Tab. 3: Systematisierungskriterien für Synergiepotentiale 
Im Laufe der Zeit sind die Ansätze von Ansoff und Porter vielfach weiterentwickelt worden und neue Klassifizierungsansätze hinzugekommen. Tab. 3 vermittelt einen groben Überblick der deutschsprachigen Literatur. Den meisten Ansätzen ${ }^{1}$ liegt explizit oder implizit eine funktionsbereichs- oder wertschöpfungsorientierte Vorgehensweise zugrunde ${ }^{2}$.

Stellvertretend für andere wird an dieser Stelle ein Systematisierungsansatz aufgezeigt, der sich an die Ausführungen von Coenenberg/Sautter anlehnt (vgl. auch Abb. 3) ${ }^{3}$. Auf der ersten Ebene wird zwischen güter- und finanzwirtschaftlichen Synergien unterteilt. Die güterwirtschaftlichen Synergien sind entsprechend den Grundgedanken von Porter in Synergien, die durch funktionale Integration, und solchen, die durch Transfer von Know How entstehen, eingeteilt. Erstere zielen auf die gemeinsame Wahrnehmung bestimmter Funktionen bzw. Aktivitäten. Letztere sind durch die Weitergabe von organisatorischen oder allgemeinen Managementfähigkeiten bzw. technischem Wissen bedingt.

Die Höhe und die Art der güterwirtschaftlichen Synergien sind von der Art des Zusammenschlusses abhängig ${ }^{4}$. Bei einem horizontalen Zusammenschluß sind die Märkte und Produkte der beiden Unternehmen zumindest verwandt. Hier ist zu vermuten, daß Synergiepotentiale insbesondere in der Zusammenlegung von Aktivitäten zu finden sind. Ein vertikaler Unternehmenszusammenschluß liegt vor, wenn das zu akquirierende Unternehmen ein Lieferant oder Abnehmer des Erwerbers ist. Folglich ist die unternehmensspezifische Bedeutung der gehandelten Input bzw. Outputfaktoren für die Höhe der Synergien von Belang. Bei konglomeraten Unternehmenszusammenschlüssen, bei denen keinerlei Marktoder Produktbeziehungen zwischen Erwerber und Akquisitionsobjekt bestehen, sind die meisten Synergiepotentiale durch Transfer von allgemeinem Management- sowie organisatorischem Know How erzielbar.

1 Vgl. Küting, Analyse von Verbundeffekten, 1981; Bühner/Spindler, Synergieerwartungen, 1986; Coenenberg/Sautter, Strategische und finanzielle Bewertung, 1988; Gälweiler, Synergiepotentiale, 1989; Ropella, Synergie, 1989; Sandler, Synergie, 1991; Weber, Unternehmensbewertung, 1991; Arbeitskreis „Die Unternehmung im Markt", Synergie, 1992; Kogeler, Synergiemanagement, 1992; Reißner, Synergiemanagement, 1992; Ehrensberger, Unternehmensintegration, 1993; Petersen, Unternehmungskäufe, 1994; Paprottka, Unternehmenszusammenschlüsse, 1996; Perin, Synergien, 1996; Scharlemann, Finanzwirtschaftliche Synergiepotentiale, 1996; Ziegler, Synergieeffekte, 1997; Ebert, Synergien, 1998; Steidl, Synergiemanagement, 1999.

2 Vgl. Ossadnik, Aufteilung, 1995, S. 9.

3 Vgl. Coenenberg/Sautter, Strategische und finanzielle Bewertung, 1988, S. 699.

4 Vgl. Paprottka, Unternehmenszusammenschlüsse, 1996, S. $120 \mathrm{ff}$. 
Auf der finanzwirtschaftlichen Seite werden die Synergiepotentiale Risikodiversifikation, steuerliche Vorteile und Reduktion der Kapitalbeschaffungskosten unterschieden. Wenn das Akquisitionsobjekt in einem dem Erwerber fremden Produktfeld tätig ist, lassen sich durch die Akquisition Unternehmensrisiken diversifizieren'. Steuerliche Synergien hingegen zielen beispielsweise auf die Nutzung von steuerlichen Verlustvorträgen oder die Abzugsfähigkeit von Fremdkapitalzinsen ab. Eine Reduktion der Kapitalbeschaffungskosten ist auf vielfältige Art und Weise denkbar. Zum einen sind Größendegressionseffekte oder eine unter dem Marktdurchschnitt liegende Risikoprämie ${ }^{2}$ bei der Emission von Unternehmenstiteln in Betracht zu ziehen. Zuletzt ist auf die Möglichkeit hinzuweisen, daß Dritte (z.B. der Staat) ein besonderes Interesse am Akquisitionsobjekt hegen und aus diesem Grund zinsgünstige Darlehen anbieten.

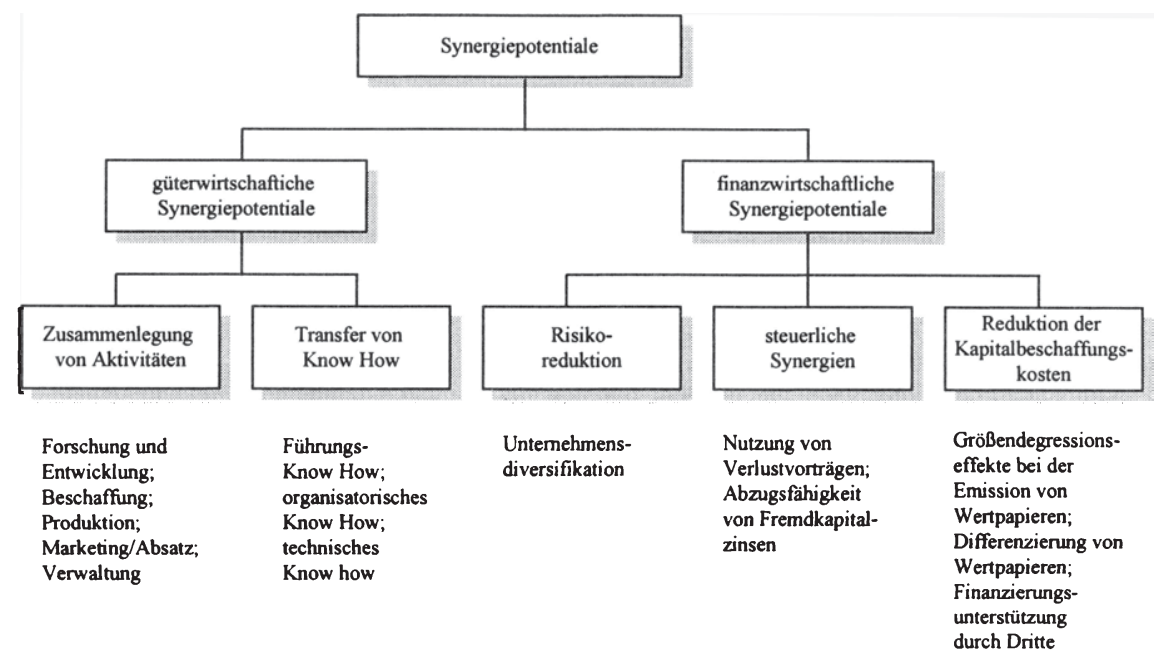

Abb. 3: Synergiepotentiale

Quelle: In Anlehnung an Coenenberg/Sautter, Strategische und finanzielle Bewertung, 1988, S. 699.

1 Aus Sicht der Eigenkapitalgeber ist eine solche Unternehmensdiversifikation nur von Vorteil, wenn sie die Risikoreduktion nicht selbst durch Kauf entsprechender Wertpapiere herstellen können.

2 Wenn ein Wertpapier in seinen Zahlungs- und Risikostrukturen auf ein ganz bestimmtes Klientel zugeschnitten wird, ist dieses eventuell bereit, auf einen Teil der Risikoprämie zu verzichten. 
Die eben beschriebenen Synergiepotentiale entstehen i.d.R. nicht ohne aktives Handeln der entsprechenden Geschäftsbereiche oder Abteilungen. Deswegen muß dafür Sorge getragen werden, daß die geplanten Synergieeffekte sich später auch realisieren lassen. Vor der tatsächlichen Akquisition sind nur potentielle Synergieeffekte vorhanden, die in der Integrationsphase noch umgesetzt werden müssen. Im Vorfeld der anstehenden Preisverhandlung ist zu fordern, daß nur solche Synergiepotentiale Eingang in die Bewertung finden, die auch eine Chance auf Verwirklichung besitzen. Ähnliches gilt auch für Restrukturierungspotentiale.

\subsubsection{Beitrag zur geplanten Strategieumsetzung}

Viele Autoren merken an, daß bei der Mehrzahl der Unternehmensakquisitionen Ziele verfolgt werden, deren Ursprung in der strategischen Leitlinie des akquirierenden Unternehmens zu finden ist ${ }^{1}$. Der Versuch, die mit solchen Unternehmenskäufen verfolgten strategischen Vorstellungen $\mathrm{zu}$ systematisieren, muß im Anblick der Fülle an Möglichkeiten zur Strategieformulierung und darin enthaltenen Schwerpunkten scheitern. Ungeachtet dessen wird an dieser Stelle auf einige besonders häufig zitierte Beispiele eingegangen, um zumindest eine Vorstellung von dem zu vermitteln, was unter der Komponente „Beitrag zur geplanten Strategieumsetzung" verstanden werden kann.

Laut der PIMS-Studie kommt dem Marktanteil eine besondere Bedeutung bei der Profitabilität von Geschäftsbereichen bzw. Unternehmen $\mathrm{zu}^{2}$. Es kann folglich nicht verwundern, daß viele Unternehmen nach einem hohen Marktanteil streben. Durch eine entsprechende Marktpräsenz kann Marktmacht aufgebaut werden, die sich in zukünftigen Lieferanten- oder Abnehmerverhandlungen positiv nutzen läßt. Desweiteren ist es denkbar, daß sich Investitionen erst ab einer bestimmten Beschäftigung rechnen. Durch Akquisitionen in gleiche bzw. verwandte Produktfelder läßt sich der Marktanteil ad hoc erhöhen.

Spezielle Produktionsprozesse, Geschäftsideen oder Markennamen sind i.d.R. durch Patente bzw. Urheberrechte vor Imitationen geschützt. Wenn der Weg über eine Lizenzvereinbarung mit dem Inhaber der Rechte nicht beschritten

1 Vgl. zu folgendem Sautter, Strategische Analyse, 1989, S. 263 ff.; Sieben/Diedrich, Aspekte, 1990, S. 797 ff.; Ruhnke, Akquisitionen, 1991, S. 1891; Arbeitskreis „Die Unternehmung im Markt", Synergie, 1992, S. 972; Helling, Strategieorientierte Unternehmensbewertung, 1994, S. 55 ff.

2 Vgl. Buzzell/Gale, PIMS, 1989, S. 82. 
werden kann, bleibt die Möglichkeit des Aufkaufs des entsprechenden Unternehmens inklusive der Rechte. Ähnliches gilt für das Umgehen nationaler gesetzlicher Vorschriften. Durch eine ausländische Akquisition ist es eventuell möglich, an Technologien zu partizipieren, die im Heimatland nicht genehmigt sind. Deutsche Unternehmenskäufe bzw. Beteiligungen im Ausland mit der Absicht, (partielle) Verbote im Bereich der Bio- und Gentechnologie zu umgehen, sind hierfür ein Beispiel.

Vielfach wird hervorgehoben, daß die Akquisition eines bestimmten Unternehmens als Voraussetzung für die Bearbeitung eines neuen Marktes gilt. Durch den Unternehmenskauf werden ein bestimmtes Vertriebsnetz, spezielle Marktkenntnisse bzw. technisches Know How erworben. Ohne den Erwerb dieses Unternehmens ließen sich die erforderlichen Startbedingungen sehr viel langsamer oder gar nicht realisieren. In diesem Kontext sind auch eine ganze Reihe von Joint Ventures zu sehen, bei denen über den Partner fehlende Erfolgsvoraussetzungen ausgeglichen werden. Durch die Unternehmensakquisition oder das Eingehen eines Joint Ventures werden allgemeine Markteintrittsbarrieren überwunden.

Auf erfolgte Wettbewerbsveränderungen reagieren Unternehmen oftmals mit Akquisitionen, um ihre als gefährdet eingestufte Erfolgsposition zu sichern. In diese Kategorie fallen z.B. das Aufkaufen eines Konkurrenten, um einen als zu stark empfundenen Wettbewerb zu vermeiden, oder der Unternehmenserwerb eines Lieferanten, um die Rohstoffbasis bzw. die gelieferten Teilleistungen zu sichern. Ähnliches gilt auch für anstehende Wettbewerbsmodifikationen. Hier geht es darum, sich eine günstige strategische Ausgangsposition für die $\mathrm{Zu}-$ kunft zu verschaffen. Ein offensichtliches Beispiel hierfür sind Unternehmenskäufe in der Telekommunikations- und Informationstechnologie, die vor dem Hintergrund des Zusammenwachsens der Produktmärkte getätigt werden.

\subsection{Strategische und finanzielle Zielsetzungen in der Unternehmens- bewertung}

Sowohl in der Unternehmenspraxis als auch in der Literatur wird bei der Bewertung von Akquisitionen oft zwischen "strategischen" und "finanziellen“ Zielen unterschieden ${ }^{1}$. Da sich ,selbstverständlich auch oder gerade eine stra-

1 Vgl. u.a. Coenenberg/Sautter, Strategische und finanzielle Bewertung, 1988, S. 691 ff.; Ruhnke, Akquisitionen, 1991, S. 1889. 
tegisch motivierte Akquisition rechnen muß ${ }^{\text {“1 }}$, stellt sich erstens die Frage, ob sich beide Zielsetzungen grundsätzlich widersprechen, und zweitens, wie sie gegebenenfalls auf einer höheren Ebene zusammengeführt werden können.

Die Beantwortung dieser Fragen wird durch die Tatsache erschwert, daß die betriebswirtschaftlichen Sachverhalte, die unter einer Strategie bzw. strategischen Zielen verstanden werden, nicht einheitlich festgelegt sind. Vielfach wird das Strategische dazu benutzt, um die Bedeutung einer Problemstellung oder die von einer Maßnahme ausgehende langfristige Wirkung auf den Unternehmenserfolg zu unterstreichen. Strategisches Verhalten läßt sich jedoch präziser abgrenzen, indem dessen Einflußnahme auf Wettbewerbspositionen in den Vordergrund der Betrachtung gerückt wird. Streng genommen verdienen nur solche Maßnahmen das Prädikat „strategisch“, die geeignet erscheinen, die eigene Wettbewerbsposition zu verändern ${ }^{2}$.

Übt eine Investitionsentscheidung einen Einfluß auf das Entscheidungsfeld eines anderen Investitionsprojekts aus, gilt diese Investitionsentscheidung folglich als strategisch. Dabei sind zwei Fälle denkbar ${ }^{3}$. Einerseits kann eine derzeitige Investition die Vorteilhaftigkeit bzw. die Verfügbarkeit zukünftiger Investitionsalternativen desselben Investors beeinflussen. Durch die aktuelle Investitionsentscheidung werden Bedingungen festgelegt, unter denen über das spätere Investitionsprojekt zu entscheiden ist. Andererseits kann eine Investitionsentscheidung die Vorteilhaftigkeit bzw. die Verfügbarkeit von Investitionsalternativen eines anderen Investors berühren. Hat die Investitionsentscheidung dieses anderen Investors einen Einfluß auf das eigene Investitionsvorhaben, ist dieser Umstand im eigenen Investitionskalkül zu berücksichtigen. In beiden Fällen wird die eigene Wettbewerbsposition berührt.

Entsprechend der eben festgelegten Begriffsbestimmung beeinflussen strategische Ziele bzw. Entscheidungen nicht nur unmittelbar, sondern auch und insbesondere mittelbar den eigenen Erfolg, indem von ihnen ein Einfluß auf zukünftige Erfolgspotentiale ausgeht. Dies steht im Einklang mit einem Großteil der Literatur zur strategischen Unternehmensführung, die das Erschließen von zu-

1 Sieben/Diedrich, Aspekte, 1990, S. 798.

2 Vgl. Neuss/Nippel, Verhalten, 1996, S. 423 ff. Die Autoren unterstreichen zudem, daß strategisches Verhalten eine Reaktionsverbundenheit zwischen den Wettbewerbern in der Art voraussetzt, daß die strategische Entscheidung des einen Handlungen des anderen beeinflußt mit der Absicht, das eigene Ziel besser verfolgen zu können.

3 Vgl. Nippel, Strategische Investitionsplanung, 1997, S. 2 f. 
künftigen sowie - in abgeschwächtem Maße - die Sicherung von gegenwärtigen Nutzenpotentialen, Wettbewerbsvorteilen bzw. Erfolgspositionen als Aufgabe der strategischen Planung sieht ${ }^{1}$. Demgegenüber ist das optimale Wirtschaften in einem gegebenen Bedingungsrahmen, z.B. bei aktueller Wettbewerbsposition, Gegenstand der operativen Planung.

Übertragen auf die in Literatur und Praxis zu beobachtende Trennung in strategische und finanzielle Zielsetzungen einer Akquisition, können folgende Aussagen getroffen werden. Mit der Formulierung strategischer Ziele wird die Schaffung neuer bzw. die Sicherung gegenwärtiger Erfolgspositionen verfolgt. Dies gilt unabhängig davon, ob diese Erfolgspositionen allein beim Akquisitionsobjekt oder aus Gesamtsicht des neu entstehenden Unternehmens gesehen werden. Unter finanziellen Zielen kann das Ausschöpfen bestehender Erfolgspositionen beim Akquisitionsobjekt verstanden werden. Vor dem Hintergrund aktuell gegebener Situations- und Kontextfaktoren gilt es, die besetzten Erfolgspositionen optimal zu nutzen ${ }^{2}$.

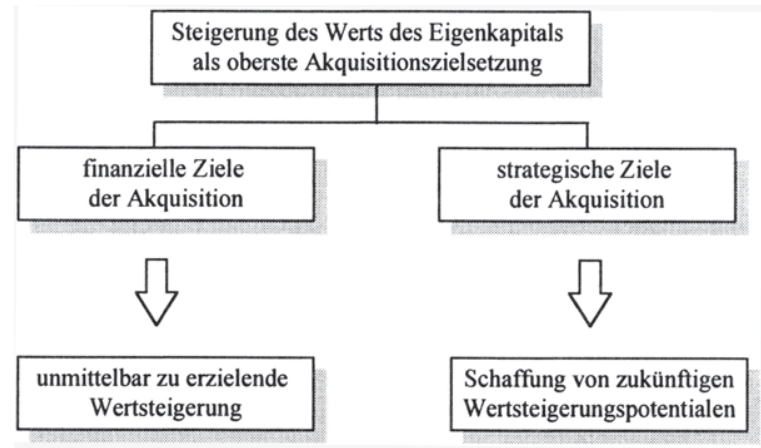

Abb. 4: Zusammenhang zwischen wertorientierter Unternehmensführung und finanziellen sowie strategischen Zielen der Akquisition

Wird die in Abschnitt 1 dieses Kapitels bereits vorgestellte wertorientierte Unternehmensführung unterstellt, müssen beide Zielsetzungen der Akquisition,

1 Vgl. stellvertretend Welge/Al-Laham, Planung, 1992, S. 4 f.; Aeberhard, Strategische Analyse, 1996, S. 11; Hinterhuber, Strategische Unternehmensführung, 1996, S. 18; Kreikebaum, Strategische Unternehmensplanung, 1997, S. 19.

2 Vgl. auch Pümpin, Strategische Unternehmensbewertung, 1990, S. 553 f. und Valcarel, Brücke, 1992, S. 591, die von besetzten Erfolgspositionen sowie erschlossenen Nutzenpotentialen im Gegensatz zu noch nicht besetzten Erfolgspositionen sowie offenstehenden Nutzenpotentialen sprechen. 
sowohl die finanzielle als auch die strategische Sichtweise, im Einklang mit der dort angenommenen Zielsetzung des Unternehmens „Steigere den Wert des Eigenkapitals!“ stehen. Dies ist nur möglich, wenn diese Unternehmenszielsetzung auch als oberstes Akquisitionsziel akzeptiert wird. Finanzielle und strategische Zielsetzungen sind dann Unterziele ein und derselben Oberzielsetzung.

Wird vereinfachend davon ausgegangen, daß Wertsteigerungen sich zum Zeitpunkt des Investitionszeitpunkts realisieren, kann folgende Abgrenzung getroffen werden. Während strategische Ziele der Akquisition die Schaffung von zukünftigen Wertsteigerungspotentialen zum Gegenstand haben, richten finanzielle Ziele der Akquisition ihr Augenmerk auf die in dem aktuell existierenden Bedingungsrahmen maximal erzielbaren Rückflüsse. Letztere verkörpern die zum Zeitpunkt der Akquisition unmittelbar zu erzielende Wertsteigerung (vgl. auch Abb. 4).

\section{Ansätze strategischer Unternehmensbewertung}

In Literatur und Praxis besteht Einigkeit darüber, daß die strategischen Faktoren einer Akquisition besonders schwierig zu bewerten sind. Viele halten sogar eine Wertermittlung strategischer Aspekte für unmöglich, da sie gerade nicht quantifizierbare Aspekte seien. Diese Haltung hilft jedoch vor dem Hintergrund einer anstehenden Preisverhandlung und der damit einhergehenden Notwendigkeit der Bestimmung einer Wertobergrenze wenig. Um die strategischen Aspekte einer Bewertung zugänglich zu machen, werden in der Literatur unterschiedliche Konzepte vorgeschlagen ${ }^{1}$.

\subsection{Bisherige Konzepte}

\subsubsection{Konzept der Mehrfachzielsetzung}

Bei Verfahren der Mehrfachzielsetzung gehen die strategischen Aspekte nicht über ihre Auswirkungen auf finanzielle Zielgrößen, sondern unmittelbar als separater Zielbestandteil in das Bewertungsmodell ein ${ }^{2}$. Die Zielfunktion des

1 Für die gleiche Einteilung sowie eine umfassende kritische Würdigung der einzelnen Konzepte vgl. Dirrigl, Konzepte, 1994, S. 415 ff. Vgl. auch Peemöller/Keller/Rödl, Verfahren, 1996, S. $74 \mathrm{ff}$.

2 Vgl. hierzu grundlegend Sieben, Bewertung bei heterogenen Zielen, 1969, S. $71 \mathrm{ff}$. 
Entscheidungsmodells wird um nicht-finanzielle Ziele erweitert ${ }^{1}$. Zur parallelen Berücksichtigung und Vergleichbarkeit der unterschiedlichen Zieldimensionen müssen die Erfolgsbeiträge der Akquisition hinsichtlich der einzelnen (Teil-) Ziele in einer einheitlichen Größe gemessen werden ${ }^{2}$. Dies geschieht mit Hilfe einer Höhenpräferenzfunktion, die die jeweiligen Ausprägungen der Zielmerkmale $^{3}$ in Nutzengrößen überführt. Bei der Bestimmung der einzelnen Ziele ist darauf $\mathrm{zu}$ achten, daß diese redundanzfrei sind. Viele strategischen Zielgrößen (z.B. Marktanteil) lassen sich als Ersatzmerkmale für finanzielle Zielgrößen (z.B. Cash Flow) interpretieren. Nur wenn die Ziele $i$ überschneidungsfrei definiert sind, lassen sich Doppelzählungen vermeiden ${ }^{4}$.

Um die einzelnen Teilnutzenwerte $T N_{i}$ auf einer Gesamtnutzenebene zu aggregieren, muß festgestellt werden, welches Gewicht dem einzelnen Teilziel zukommt. Es wird der relative Anteil $w_{i}$ des Teilziels gesucht, der die Vorziehenswürdigkeit dieses Ziels gegenüber den anderen Teilzielen ausdrückt ${ }^{5}$. Ist dies bewerkstelligt, kann der Gesamtnutzen $G N$ der zu bewertenden Akquisition ermittelt werden.

$$
G N=\sum_{i=1}^{l} w_{i} \cdot T N_{i}
$$

Damit liegt jedoch erst der dimensionslose Nutzenwert vor und noch kein Unternehmenswert in monetären Geldeinheiten. Um einen solchen zu ermitteln, wird beim Erwerber ein Investitionsprogramm aufgestellt, das zum einen das Akquisitionsvorhaben nicht enthält (Basisprogramm) und zum anderen das Kapitalbudget des Erwerbers gerade ausschöpft. Für jede Investitionsalternative in diesem Basisprogramm wird auf die gleiche Weise wie beim Akquisitionsobjekt ein Nutzenwert ermittelt. Über die Addition der einzelnen Nutzenwerte der geplanten Investitionen resultiert der Nutzen des Basisprogramms.

1 Neben den strategischen Zielen können hier auch andere nicht-finanzielle Ziele, wie z.B. Umweltschutz oder Arbeitsplatzsicherung, erfaßt werden.

2 Vgl. Arbeitskreis „Unternehmensbewertung im Rahmen der unternehmerischen Zielsetzung“, Unternehmensbewertung, 1981, S. 21.

3 Jedem Teilziel ist ein Zielmerkmal zugeordnet (z.B. Zielmerkmal Marktanteil für Teilziel Marktmacht), anhand dessen gemessener Ausprägung der Erfolgsbeitrag zum jeweiligen Teilziel (Zielerreichungsgrad) beschrieben werden kann.

4 Vgl. Hafner, Beitrag, 1989, S. 99.

5 Vgl. zur Zielgewichtung ausführlicher Hafner, Unternehmensbewertung, 1988, S. 495 ff. 
Wird das Basisprogramm um das Akquisitionsvorhaben erweitert, erhöht sich dessen Nutzenwert. Jedoch wird jetzt das vorgegebene Kapitalbudget überschritten. Im Basisprogramm vorgesehene Investitionen können nun nicht realisiert werden. Als Auswahlkriterium wird der Nutzen pro Kapitaleinheit herangezogen. Das Investitionsprojekt mit dem geringsten Nutzen pro Kapitaleinheit wird als erstes gestrichen. Dem folgen weitere Projekte, bis der ursprünglich ermittelte Nutzen des Basisprogramms mit dem Nutzen des sogenannten Bewertungsprogramms, das aus den noch verbleibenden Investitionsprojekten zuzüglich dem Akquisitionsvorhaben besteht, identisch ist. Über die verdrängten Investitionsprojekte $j$ und deren Kapitalbedarf $K A P$ kann nun auf denjenigen Unternehmenswert $U W$ geschlossen werden, bei dem sich der Erwerber gegenüber der Situation ohne Unternehmenskauf ökonomisch gerade nicht verschlechtert. Der Unternehmenswert ergibt sich aus den ersparten Anschaffungsauszahlungen der besten nicht realisierten Investitionsalternativen, die bei Durchführung den gleichen Nutzen gebracht hätten wie die realisierte Akquisition'.

$$
U W=\sum_{j=1}^{J} K A P_{j}
$$

Das bisher vorgestellte Grundmodell hat von dem Fall eines mehrperiodigen Bewertungskalküls unter Unsicherheit abstrahiert. Ein mehrperiodiges Kalkül setzt voraus, daß die periodenindividuellen Ergebnisse über einen bestimmten Mechanismus zusammengefaßt werden können. Bei finanziellen Cash FlowGrößen läßt sich dies über Diskontierung erreichen. Bei strategischen Zielen, wie z.B. Marktanteilen muß durch explizite Formulierung einer hierfür geltenden Zeitpräferenzfunktion ein anderer Weg beschritten werden ${ }^{2}$. Sind die in das Bewertungsverfahren eingehenden Ausprägungen der einzelnen Zielgrößen unsicher, muß diesem Umstand Rechnung getragen werden. Auf eine Formulierung von Risikopräferenzfunktionen kann dann nicht verzichtet werden ${ }^{3}$.

In einem mehrperiodigen Bewertungskalkül unter Unsicherheit gehen also neben der bereits im Grundmodell berücksichtigten Höhen- und Zielartenpräferenz zusätzlich Zeit- und Risikopräferenzen ein. Die vorige Darstellung einer

1 Vgl. Hafner, Unternehmensbewertung, 1988, S. 488 f. Diese Vorgehensweise wird auch tabellarische Methode genannt. Vgl. Sieben, Unternehmensstrategien, 1988, S. 90.

2 Vgl. Sieben/Schildbach, Entscheidungstheorie, 1994, S. 164.

3 Vgl. Hafner, Unternehmensbewertung, 1988, S. 496. 
additiven und multiplikativen Verknüpfung von Teilnutzen setzt voraus, daß eine wechselseitige Unabhängigkeit zwischen den Präferenzen besteht. Nur dann können z.B. die Gewichte der Teilziele ohne Berücksichtigung der Ausprägungshöhe der Zielgrößen festgelegt werden ${ }^{1}$. Die Berücksichtigung von Abhängigkeiten zwischen den Präferenzen führt zu einem sogenannten Präferenzsystem höherer Ordnung. Die Verfahren zu dessen Lösung ${ }^{2}$ sind so komplex, daß kaum eine Chance auf eine unmittelbare Anwendung in der Praxis besteht.

\subsubsection{Shareholder Value-Analyse}

Die von Rappaport entwickelte Shareholder Value-Analyse versucht, strategische Aspekte über ihre Auswirkungen auf die finanzielle Zielgröße „Cash Flow" ins Bewertungsverfahren zu integrieren ${ }^{3}$. Der für die einzelne Periode zu prognostizierende betriebliche Cash Flow wird dabei wie folgt ermittelt. Ausgehend vom aktuellen Umsatz $U_{0}$ wird mit Hilfe der geplanten Wachstumsrate des Umsatzes $w_{U}$ der Umsatz der ersten Periode festgelegt. Der prognostizierte Umsatz wird mit der betrieblichen Gewinnmarge $g_{M}$, die das Verhältnis zwischen dem Betriebsgewinn vor Zinsen und Steuern und dem Umsatz ausdrückt, multipliziert, um zum geschätzten Betriebsgewinn zu gelangen.

Beim Betriebsgewinn werden neben den Herstellungskosten der verkauften Produkte und den Verwaltungs- und Vertriebskosten auch Abschreibungen in Abzug gebracht. Dies unterstellt, daß Ersatzinvestitionen in Höhe der Abschreibungen getätigt werden. Durch Multiplikation des Betriebsgewinns vor Steuern mit dem Steuersatz st resultiert der Betriebsgewinn nach Steuern $B G_{\text {n. St. }}$.

$$
B G_{n . S t .}=U_{0} \cdot\left(1+w_{U}\right) \cdot g_{M} \cdot(1-s t)
$$

Vom Betriebsgewinn nach Steuern sind die Erweiterungsinvestitionen ins Anlage- und Umlaufvermögen abzuziehen, die aufgrund der Erhöhung des Umsatzes notwendig werden. Die Multiplikation des Umsatzwachstums mit den umsatzbezogenen Investitionsraten $i_{A}$ und $i_{U}$ ergibt den Investitionsbedarf zum Zweck der Geschäftserweiterung $I_{E w w}$. Bei der Ermittlung der Investitionsraten

1 Vgl. Ballwieser, Methoden, 1993, S. 173.

2 Vgl. Hafner, Beitrag, 1989, S. 151 ff.

3 Vgl. zu folgendem Rappaport, Shareholder Value, 1998, S. 33 ff. 
ist darauf zu achten, daß keine Ersatzinvestitionen (Gegenwert der Abschreibungen) berücksichtigt werden.

$$
I_{E r w .}=w_{U} \cdot U_{0} \cdot\left(i_{A}+i_{U}\right)
$$

Durch Abzug des Investitionsbedarfs vom Betriebsgewinn nach Steuern und anschließender Diskontierung mit dem Kapitalkostensatz erhält man den Barwert des Cash Flows der ersten Periode $V_{0}\left(C F_{l}\right)$. Der Kapitalkostensatz wacc wird dabei als gewogener Durchschnitt der Fremd- und Eigenkapitalkosten gebildet, wobei der Eigenkapitalkostensatz auf Basis eines kapitalmarkttheoretischen Modells (insb. CAPM) und der Fremdkapitalkostensatz unter Berücksichtigung des durch Fremdfinanzierung entstehenden Tax Shields ermittelt wird ${ }^{1}$.

$$
V_{0}\left(C F_{1}\right)=\frac{B G_{n . S t}-I_{E n w .}}{1+w a c c}
$$

Die eben dargestellte Vorgehensweise gilt für alle Cash Flows, die innerhalb des Detailplanungshorizonts $n$ anfallen. Für alle Cash Flows nach dem Planungshorizont wird von einem konstanten Rückfluß ausgegangen. Der sogenannte Residualwert (Wert der außerhalb des Detailplanungshorizonts anfallenden Cash Flows zum Zeitpunkt des Detailplanungshorizonts) kann durch Bildung einer ewigen Rente errechnet werden. Es wird angenommen, daß ab dem Planungshorizont keine Erweiterungsinvestitionen mehr getätigt werden bzw. diese bei Realisierung einen Kapitalwert von null erwirtschaften und insofern nicht berücksichtigt werden brauchen.

Die Addition der Barwerte der periodenindividuellen Cash Flows sowie des Residualwerts führt zum Unternehmensgesamtwert $V_{G K}$. Von diesem wird der Wert des Fremdkapitals $V_{F K}$ abgezogen, um zum Wert des Eigenkapitals $V_{E K}$ (Shareholder Value) zu gelangen.

$$
V_{E K}=V_{G K}-V_{F K}
$$

Bei der Shareholder Value-Analyse wird davon ausgegangen, daß der Unternehmensgesamt- bzw. Eigenkapitalwert neben dem aktuellen Umsatz von sieben sogenannten Werttreibern bestimmt wird. Zu diesen zählen

1 Vgl. Rappaport, Shareholder Value, 1998, S. 37 ff. sowie die Ausführungen zum WACCAnsatz und zur DCF-Methode nach Copeland/Koller/Murrin in Abschnitt 1.2.4 dieses Kapitels. 
- die betriebliche Gewinnmarge,

- die Wachstumsrate des Umsatzes,

- der auf die Cash Flows bezogene Gewinnsteuersatz,

- die Rate für Erweiterungsinvestitionen ins Anlagevermögen,

- die Rate für Erweiterungsinvestitionen ins Umlaufvermögen,

- der gewogene Kapitalkostensatz und

- der Detailplanungshorizont (Dauer der geplanten Wertsteigerung) ${ }^{1}$.

Zielsetzung des

Unternehmens

Bewertungs-

komponenten

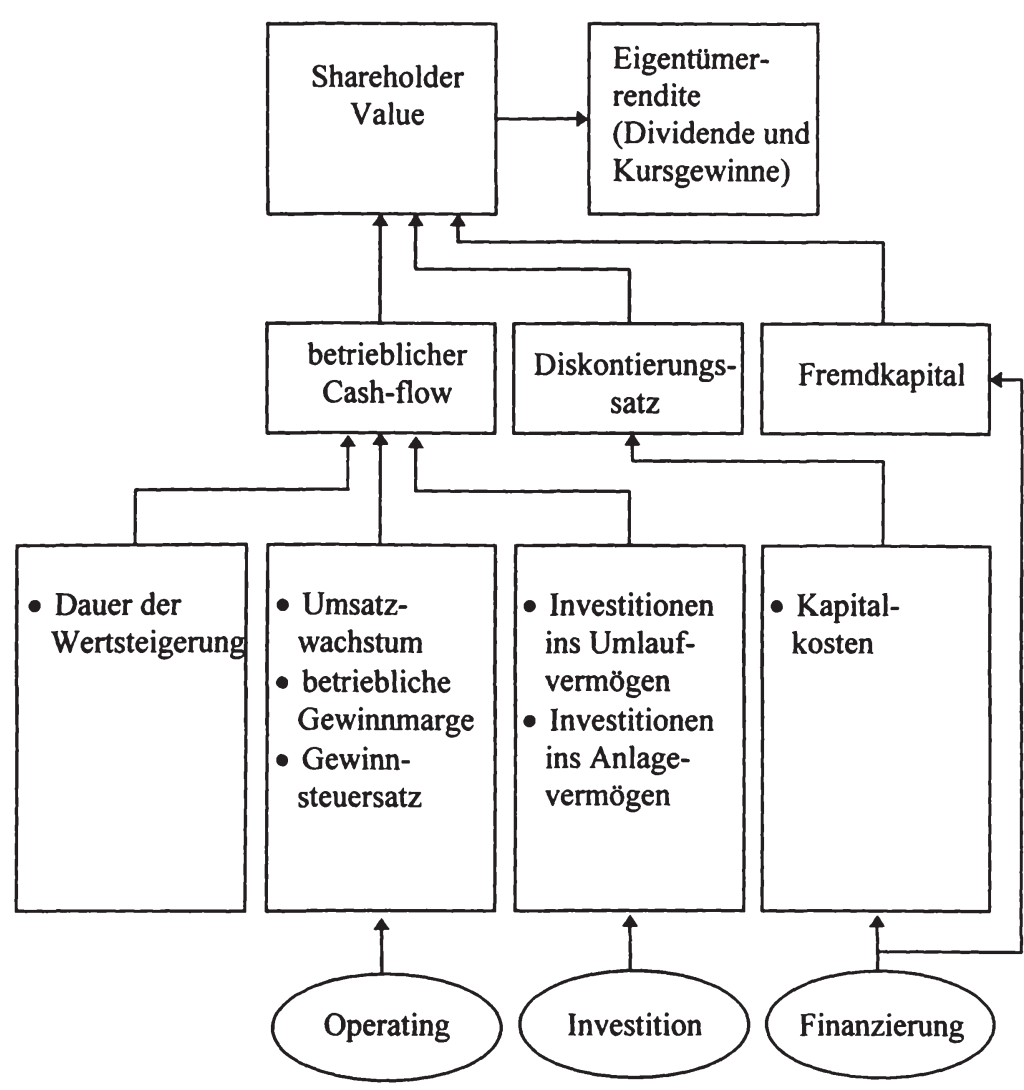

Führungs-

entscheidungen

Abb. 5: Shareholder Value-Netzwerk

Quelle: Rappaport, Shareholder Value, 1998, S. 56.

I Vgl. Rappaport, Shareholder Value, 1998, S. 55 f. 
Mit Ausnahme des Detailplanungshorizonts können diese Werttreiber den drei Hauptbereichen betrieblicher Führungsentscheidungen zugeordnet werden (vgl. Abb. 5). Die ersten drei der oben genannten Werttreiber werden maßgeblich durch operative Entscheidungen, wie z.B. Produkt- und Leistungsprogramm, Preis- und Kommunikationspolitik, beeinflußt. Entscheidungen über mögliche Erweiterungsinvestitionen gehören dem Investitionsbereich an, während der Kapitalkostensatz die Finanzierungshandlungen des Unternehmens widerspiegelt. Die Verbindung zwischen den hier in den Vordergrund gestellten Werttreibern und dem konkreten Bewertungsverfahren soll die Umsetzung der Zielsetzung „wertorientierte Unternehmensführung“ fördern.

Die bislang geschilderte Vorgehensweise läßt nur schwer erkennen, wieso die Shareholder Value-Analyse zu den Ansätzen der strategischen Unternehmensbewertung gezählt werden kann. Erst durch die von Rappaport im zweiten Teil seines Werks propagierte Verknüpfung zwischen den Instrumenten der strategischen Unternehmensplanung und -analyse auf der einen und den Werttreibern der Wertsteigerungsanalyse auf der anderen Seite rechtfertigt jene Bezeichnung. Ausgehend von konkreten Strategien für ganze Unternehmen oder einzelne Geschäftsbereiche wird über die Formulierung von Eckdaten in der strategischen Planung auf die Höhe der Werttreiber geschlossen ${ }^{1}$.

Rappaport greift bei seinen Ausführungen vorwiegend auf Porter'sche Analyseinstrumente zurück, wie z.B. das Industriestrukturmodell oder die Wertschöpfungskette ${ }^{2}$. So zeigt er beispielsweise mögliche Verbindungen zwischen den einzelnen Aktivitäten der Wertkette und den von ihm vorgeschlagenen Werttreibern auf. Betriebskosten, Abschreibungen sowie Zunahme des Umlaufbzw. Anlagevermögens werden in Abhängigkeit der wertschöpfungsorientierten Aktivitäten geschätzt ${ }^{3}$. Für die Strategie der Kostenführerschaft bzw. Differenzierung erläutert der Autor wertsteigernde Maßnahmen mit Einfluß auf die einzelnen Werttreiber und zwingt auf diese Weise, die „richtigen“ Fragen für die beabsichtigte Strategie zu stellen ${ }^{4}$.

1 Vgl. Rappaport, Wertsteigerung, 1995, S. 83 ff.

2 Zu den Analyseinstrumenten vgl. ausfuhrlich Porter, Wettbewerbsvorteile, 1996, S. 22 ff. und S. $59 \mathrm{ff}$.

3 Vgl. Rappaport, Wertsteigerung, 1995, S. $88 \mathrm{ff}$.

4 Vgl. Rappaport, Shareholder Value, 1998, S. 66 ff. 


\subsubsection{Konzept des strategischen Zuschlags}

Im Konzept des strategischen Zuschlags wird der Unternehmenswert in einem zweistufigen Vorgehen bestimmt. In einem ersten Bewertungsschritt wird in einem sogenannten Basiskalkül der Stand Alone-Wert ermittelt. Der Stand Alone ist der Unternehmenswert, der sich unter der Annahme einer unveränderten Fortführung des zu akquirierenden Unternehmens ergibt. Hiervon ist insbesondere dann auszugehen, wenn die bisherige Unternehmenspolitik beibehalten wird und die Unternehmensleitung nicht wechselt. Zum Stand Alone wird in einem zweiten Bewertungsschritt ein strategischer Zuschlag addiert, der den Wert der strategischen Aspekte verkörpert. In Anlehnung an die weiter oben vorgenommene Einteilung der strategischen Faktoren in Restrukturierungs-, Synergiepotentiale und Beitrag zur geplanten Strategieumsetzung ließe sich für jede Komponente ein strategischer Zuschlag ermitteln. Der strategische Unternehmenswert könnte somit als Summe der vier Komponenten Stand Alone, Restrukturierungspotentiale, Synergiepotentiale sowie Beitrag zur Umsetzung der geplanten Unternehmensstrategie gesehen werden (vgl. Abb. 6).

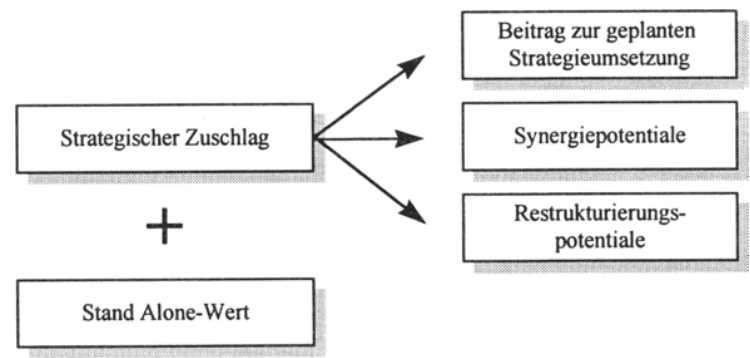

Abb. 6: Komponenten des strategischen Unternehmenswerts

Das zweistufige Vorgehen bei der Ermittlung eines strategischen Unternehmenswerts wird beispielsweise von Coenenberg/Sautter vorgeschlagen ${ }^{1}$. Neben dem Stand Alone-Wert wird eine Werterhöhung durch Restrukturierungsmaßnahmen sowie eine Werterhöhung durch Realisierung von Synergiepotentialen berücksichtigt. Die Autoren stellen einen Systematisierungsansatz ${ }^{2}$ sowie eine ganze Reihe von Instrumenten vor, die die Identifikation der strategischen Faktoren zum Ziel haben. Jedoch sind kaum Hinweise auf eine konkrete Wertermittlung im Sinne einer Quantifizierung vorhanden. Es wird lediglich allge-

1 Vgl. Coenenberg/Sautter, Strategische und finanzielle Bewertung, 1988, S. $693 \mathrm{ff}$.

2 Vgl. die Ausführungen in Abschnitt 2.2.2 und 2.2.3 dieses Kapitels. 
mein auf die Ertragswertmethode mit der Besonderheit eingegangen, den Risikozuschlag auf Basis eines Kapitalmarktmodells zu ermitteln.

Ähnliches läßt sich auch für den Vorschlag von Valcarel feststellen ${ }^{1}$. Die Autorin fordert, daß neben dem „Ertragswert auf der Grundlage der Cash Flows“ die dem Unternehmen noch zusätzlich offenstehenden Nutzenpotentiale und die Multiplikationsmöglichkeiten ihrer Ausnutzung zu berücksichtigen sind. Durch den „Ertragswert auf der Grundlage der Cash Flows“ sind für sie lediglich die aktuelle Investitionspolitik des Unternehmens, die Ergiebigkeit von erschlossenen Nutzenpotentialen sowie die vom Unternehmen besetzten strategischen Erfolgspositionen in die Bewertung eingeflossen. Valcarel fügt ihren Ausführungen eine Übersicht über mögliche externe und interne Nutzenpotentiale hinzu, ohne jedoch auf eine konkrete Wertermittlung einzugehen. Die Autorin selbst gibt sogar zu bedenken, daß ,im einzelnen nicht klar ist, wie sie erfolgen soll, bedenkt man zumal, daß es sich eben nicht um Gewinngrößen, sondern nur um Potentiale handelt, deren tatsächliche Ausschöpfung nicht gesichert ist $^{\star 2}$.

Neben den soeben erläuterten strategischen Zuschlägen wird bei Akquisitionen ein besonderer Wertzuschlag gesehen, wenn innerhalb einer Minderheitsbeteiligung ein Recht auf Aufstockung der Anteile vorgesehen ist ${ }^{3}$. Bereits zum Zeitpunkt des Erwerbs der Minderheitsbeteiligung wird vertraglich festgehalten, daß ein Recht zum Kauf weiterer Anteile innerhalb eines bestimmten Zeitraums zu einem vorab festgelegten Preis besteht. Damit besitzt der Käufer der Minderheitsbeteiligung die Möglichkeit, einseitig ein größeres Kapitalengagement an dem betreffenden Unternehmen zu bewirken. Er ist jedoch hierzu nicht verpflichtet. Die durch das Aufstockungsrecht bedingte unternehmerische Flexibilität stellt eine Wertkomponente dar, die es bei der Bewertung der Minderheitsbeteiligung zu berücksichtigen gilt ${ }^{4}$.

1 Vgl. Valcarel, Brucke, 1992, S. 590 ff.

2 Valcarel, Brücke, 1992, S. 595.

3 Vgl. Fischer, Realoption beim Unternehmenskauf, 1993, S. 110 ff.; Dirrigl, Konzepte, 1994, S. $423 \mathrm{f}$.

4 Vgl. Fischer, Realoption beim Unternehmenskauf, 1993, S. 115 f. Zu vertraglich fixierten Optionen, in denen der Käufer bzw. Verkäufer eine nachträgliche Korrektur des Kaufpreises fur den Fall verlangen kann, daß die Unternehmensentwicklung sich außerhalb einer bestimmten Bandbreite bewegt, vgl. Delcker, Besserungsoption, 1992, S. 2453 f. 
Dieser Flexibilitätswert existiert jedoch nicht nur bei vertraglich fixierten Handlungsspielräumen. Er ist ebenfalls in Betracht zu ziehen, wenn durch eine Akquisition erst die Voraussetzungen geschaffen werden, um zukünftige Folgeinvestitionen $\mathrm{zu}$ ermöglichen. Ist dabei die zukünftige Investitionspolitik noch nicht klar vorgegeben, so daß erst in der Zukunft in Abhängigkeit des dann vorzufindenden Informationsstands über Folgeinvestitionen entschieden wird, beinhaltet eine solche Ausgangssituation ebenfalls eine werterhöhende Flexibilitätskomponente ${ }^{1}$.

Diese liegt beispielsweise vor, wenn mit der Akquisition die strategische Intention verfolgt wird, einen „Brückenkopf“ in einem neuen Markt aufzubauen. Das strategische Vorhaben läßt sich dann gedanklich in zwei zeitlich voneinander getrennte Projekte zerlegen. Als erstes ist über die Akquisition bzw. den Markteintritt zu befinden. Erst später wird über Investitionen zur Marktdurchdringung entschieden. Gleichwohl stellt die Möglichkeit der späteren Marktdurchdringung bei der Bewertung des Akquisitionsobjekts einen nicht zu übersehenden Wertbestandteil dar ${ }^{2}$. Techniken zur Bewertung einer solchen Flexibilitätskomponente stehen ab Kapitel IV im Mittelpunkt dieser Untersuchung.

\subsection{Theoretische Einteilung}

Vergleicht man die drei bisher vorgestellten Konzepte miteinander, stellt man fest, daß das „Strategische“ auf völlig unterschiedliche Art und Weise in die Bewertung einfließt. Ausgehend von einem finanziellen aus der Investitionsrechnung stammenden Basiskalkül wird versucht, Erkenntnisse aus anderen betriebswissenschaftlichen Teildisziplinen in ein Konzept der strategischen Unternehmensbewertung zu integrieren. Neben der konzeptionellen Heterogenität kommt für eine theoretische Einteilung erschwerend hinzu, daß die einzelnen Ansätze zum Teil spezielle und damit unterschiedliche strategische Aspekte in den Vordergrund ihrer Betrachtung rücken ${ }^{3}$. Im folgenden wird deswegen, wie aus Abb. 7 ersichtlich, der Schwerpunkt auf Erweiterungsmöglichkeiten des Basiskalküls gelegt, die unabhängig von einem speziellen - zuvor beschriebenen - Konzept gelten.

1 Für frühe Empfehlungen, diese Flexibilitătskomponente mit Hilfe der Optionspreistheorie zu bewerten, vgl. Myers, Financial Strategy, 1984, S. 135 f.; Myers, Acquisition Target, 1986, S. $400 \mathrm{f}$.

2 Vgl. Sautter, Strategische Analyse, 1989, S. 342 ff.

3 Vgl. hierzu sowie zu folgendem Dirrigl, Konzepte, 1994, S. 428 f. 
Erste Versuche zur Anreicherung des investitionsrechnerischen Kalküls mit strategischen Elementen stammen aus der strategischen Unternehmensplanung. Die Unternehmensbewertung beginnt hier mit einer detaillierten Analyse zum Umfeld des zu bewertenden Unternehmens. Aufbauend auf den Erkenntnissen dieser Analyse lassen sich unternehmensspezifische strategische Planungen durchführen, die das Grundgerüst für die anschließend zu erfolgende Prognose der Cash Flows darstellen'. Dieser Weg wird z.B. von der Shareholder ValueAnalyse beschritten, in der strategische Planungs- und Analyseinstrumente mit finanziellen Werttreibern verknüpft werden. Die strategischen Faktoren werden ungleich welcher Art über ihre Auswirkungen auf monetäre Größen in die Bewertung einbezogen.

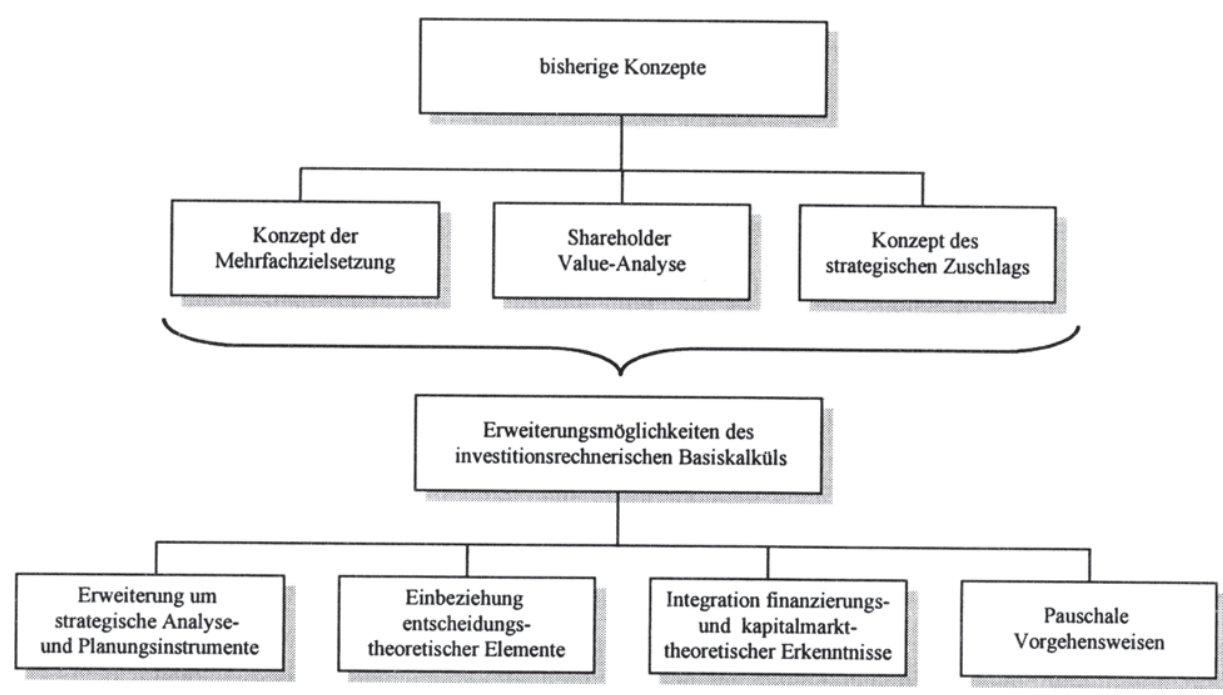

Abb. 7: Ansätze strategischer Unternehmensbewertung

Eine zweite Möglichkeit zur Einbeziehung strategischer Faktoren beruht auf entscheidungstheoretischen Erkenntnissen. Auf die Aufgabenstellung der Unternehmensbewertung wird die Verfahrensweise bei multikriteriellen Entscheidungsproblemen angewandt. Neben einem finanziellen Ziel werden ein oder mehrere strategische Ziele in das Zielsystem des Entscheidungsträgers einbezogen. Dabei lassen sich unabhängig von der Art alle strategischen Aspekte durch explizite (Ziel-) Formulierung berücksichtigen. Auf der anderen Seite ist es ebenso denkbar, daß nur die strategischen Faktoren, bei denen von einem

1 Vgl. auch Ballwieser, Komplexitätsreduktion, 1990, S. 75 ff. 
quantifizierbaren Einfluß auf die monetären Zielgrößen abgesehen wird, auf die entscheidungstheoretische Nutzenebene gehoben werden. Die anderen strategischen Aspekte sind dann durch ihre Auswirkungen auf Cash Flows im „Gewinnziel" zu berücksichtigen.

Eine dritte Weiterentwicklung der investitionsrechnerischen Unternehmensbewertung wird durch die Einbeziehung finanzierungs- oder kapitalmarkttheoretischer Zusammenhänge erreicht. Besonders die aus den USA stammenden DCF-Verfahren im Sinne des WACC-Ansatzes propagieren die explizite Festlegung der Finanzierungsstruktur des zu bewertenden Unternehmens. Unter realistischen Marktbedingungen ist davon auszugehen, daß der Verschuldungsgrad einen Einfluß auf den Unternehmenswert ausübt, so daß die Möglichkeit zur Veränderung der Kapitalstruktur als strategischer Spielraum interpretiert werden kann ${ }^{1}$. Die finanzierungstheoretische Erweiterung konzentriert sich somit ausschließlich auf den Aspekt der Kapitalstruktur. Ein anderer Weg wird durch die Integration optionspreistheoretischer Erkenntnisse beschritten. Mit Hilfe von Optionspreismodellen läßt sich die in einem Investitionsvorhaben eventuell vorhandene strategische Flexibilität quantifizieren ${ }^{2}$. Hier wird als strategischer Faktor die Möglichkeit zur Entscheidungsrevidierung bzw. zum Entscheidungsaufschub gesehen. Eine solche Flexibilität kann grundsätzlich in allen zuvor gebildeten strategischen Komponenten enthalten sein. In konkreten Bewertungssituationen sind jedoch nur jene strategischen Faktoren betroffen, in denen der zu bewertende Flexibiltätsscharakter auch wirklich vorhanden ist.

Die vierte Möglichkeit zur Erweiterung des Investitionskalküls um strategische Aspekte ist keiner betriebswirtschaftlichen Theorie zuzuordnen. Vielmehr handelt es sich hier um mehr oder minder pauschale Verfahren zur Bestimmung eines strategischen Zuschlags. Auf Basis von Durchschnitts- und/oder Erfahrungswerten werden die spezifisch hervorgehobenen strategischen Aspekte in einer prozentualen Zuschlagsprämie abgegolten. Dieser Zuschlagssatz wird dabei auf den ohne Betrachtung dieser strategischen Aspekte ermittelten Unternehmenswert bezogen. Welche strategischen Faktoren nun im prozentualen Zuschlag berücksichtigt sind und welche in die Basisrechnung einfließen, ist vom spezifischen Bewertungsfall abhängig.

1 Demgegenüber sehen Serfling/Pape die strategische Einbeziehung in der Möglichkeit, den Einfluß strategischer Entscheidungen auf den Eigen- und den Fremdkapitalkostensatz explizit sichtbar zu machen. Vgl. Serfling/Pape, Strategische Unternehmensbewertung, 1996, S. 63.

2 Vgl. hierzu auch die Ausführungen zum besonderen Wertzuschlag im vorigen Abschnitt. 


\section{Schlußfolgerungen für das weitere Vorgehen}

\subsection{Grundhaltungen zur Bewertung strategischer Aspekte}

Die im vorigen Abschnitt dargestellten Konzepte sowie theoretischen Weiterentwicklungen des investitionsrechnerischen Basiskalküls lassen den Schluß $\mathrm{zu}, \mathrm{da} ß$ zur Einbeziehung strategischer Aspekte in die Unternehmensbewertung zwei Grundhaltungen existieren. Die erste Vorstellung geht davon aus, da $\beta$ es nicht möglich ist, die Auswirkungen der strategischen Aspekte auf monetäre Größen aufzuzeigen. Für die im Vorfeld des Unternehmenserwerbs jedoch unerläßliche Quantifizierung sind dadurch zwei Vorgehensweisen denkbar. Entweder es werden Informationen zu Durchschnitts- oder Erfahrungswerten gesammelt, um den strategischen Faktor einer Akquisition pauschal „einzufangen" oder man geht den Umweg über einen Nutzenvergleich, um den spezifisch verfolgten strategischen Zielen gerecht zu werden. Erstere Variante ist kaum in der Lage, eine der spezifischen Akquisition angemessene individuelle Bewertung zu gewährleisten. Wie soll etwa über einen Durchschnittswert das Besondere am strategischen Vorhaben erfaßt werden? Die zweite Variante ist mit den Problemen der Formulierung von Nutzenfunktionen sowie der Berücksichtigung gegenseitiger Abhängigkeit bei den einzubeziehenden Präferenzen konfrontiert. Dies macht die damit einhergehenden Bewertungsverfahren einer Praxisanwendung nur schwer zugänglich.

Die zweite Grundhaltung gegenüber strategischen Aspekten ist durch Bemühungen charakterisiert, ihren Einfluß auf finanzielle Zielgrößen explizit darzustellen. Auch wenn direkte Auswirkungen in vielen Fällen nur schwer und mit hohem Aufwand herzustellen sind, ist dies ein gangbarer Weg einer individuellen Bewertung, wenn mathematische Gleichungssysteme höherer Ordnung wie z.B. im Konzept der Mehrfachzielsetzung - abgelehnt werden. Zur Bewertung der einzelnen strategischen Faktoren stehen grundsätzlich die gleichen Bewertungsverfahren $^{1}$ zur Verfügung wie zur Bewertung des Stand Alone. Dies gilt unabhängig, ob es sich bei der zu bewertenden strategischen Komponente um Restrukturierungs-, Synergiepotentiale oder dem Beitrag zur Umsetzung der geplanten Unternehmensstrategie handelt. $\mathrm{Zu}$ klären ist lediglich, ob die durch den strategischen Aspekt hervorgerufenen Rückflüsse in einer separaten Rechnung oder zusammen mit den aus der unveränderten Fortführung des

1 Vgl. die in Abschnitt 1.2.4 dieses Kapitels dargestellten Varianten der investitionsrechnerischen Unternehmensbewertung. 
Unternehmens erwarteten Rückflüssen in einer Gesamtrechnung erfaßt werden sollen.

In der Literatur finden sich speziell zu Synergiepotentialen einige Stellungnahmen ${ }^{1}$, die sich problemlos auf die anderen strategischen Aspekte übertragen lassen. Hier wird dem Vorschlag von Weber gefolgt, der empfiehlt, den Wert von Synergiepotentialen gesondert zu ermitteln. Dies geschieht vor allem, weil die Bewertungsgrundlagen für die Stand Alone-Rückflüsse und die Cash Flows aufgrund von Synergiepotentialen eine unterschiedliche Qualität aufweisen. Für letztere liegen z.B. keine Vergangenheitsergebnisse vor, die als Ausgangspunkt der Prognose dienen können ${ }^{2}$. Zudem besitzen die beiden Cash Flow-Reihen i.d.R. unterschiedliche Realisierungs- und Risikograde ${ }^{3}$.

\subsection{Strategische Aspekte mit Flexibilitätscharakter}

Strategisch motivierte Akquisitionen beinhalten vergleichsweise hohe Risiken bezüglich der Erreichung der mit dem Unternehmenserwerb gesteckten Zielsetzung. Um so wichtiger ist es für Unternehmen, bestimmte mit dem Akquisitionsvorhaben in Zusammenhang stehende Investitionsentscheidungen später teilweise revidieren zu können, um damit das Verlustpotential nach unten zu begrenzen. Genau so förderlich ist es, wenn sich mit der Akquisition verbundene Investitionsentscheidungen aufschieben lassen, um verläßlichere Daten über die Erfolgswahrscheinlichkeit des investierten Kapitals zu gewinnen. Zu fragen ist dann, wie die hiermit einhergehende strategische Flexibilität zum Zeitpunkt der Akquisitionsentscheidung bewertet werden kann. Läßt sich diese auch auf Basis der in Abschnitt 1 dargestellten Varianten der investitionsrechnerischen Unternehmensbewertung quantifizieren?

Zur Beantwortung der soeben gestellten Frage ${ }^{4}$ ist es unerheblich, ob die strategische Flexibilität durch Ausschöpfen von Restrukturierungs-, Synergiepotentialen oder des Beitrags zur geplanten Strategieumsetzung entsteht. Es ist le-

1 Vgl. stellvertretend Arbeitskreis „Unternehmensbewertung im Rahmen der unternehmerischen Zielsetzung“, Unternehmensbewertung, 1981, S. 44 ff.; Weber, Unternehmensbewertung, 1991, S. 100 f.; Paprottka, Unternehmenszusammenschlüsse, 1996, S. 67 f.

2 Vgl. Weber, Unternehmensbewertung, 1991, S. $100 \mathrm{f}$.

3 Werden für zwei Cash Flow-Reihen unterschiedliche Risikograde angenommen, bedingt dies gemäß dem fundamentalen finanzierungstheoretischen Rendite/Risiko-Zusammenhang die Verwendung unterschiedlicher Diskontierungssätze.

4 Die konkrete Beantwortung erfolgt im nächsten Abschnitt. 
diglich entscheidend, daß der strategische Aspekt mit dem oben beschriebenen Flexibilitätscharakter ausgestattet ist. Entweder läßt sich durch zukünftige Entscheidungen das Verlustpotential begrenzen oder das Gewinnpotential erhöhen. In diesem Zusammenhang scheint es an dieser Stelle wichtig, auf die beiden von Sieben/Diedrich konstruierten Fälle einer Akquisition mit Beitrag zur Umsetzung der geplanten Unternehmensstrategie einzugehen ${ }^{1}$.

Im ersten Fall wird eine Akquisition beschrieben, die den ersten Schachzug einer langfristig angelegten, schon heute relativ klar umrissenen Unternehmensstrategie darstellt. Die weiteren Schachzüge, insbesondere die Akquisition eines zweiten Unternehmens, sind bereits vorgegeben. Auch wenn die aktuell zur Disposition stehende Akquisition eine unabdingbare Voraussetzung für den Erwerb des zweiten Kaufobjekts darstellt, mangelt es in diesem Beispiel am Flexibilitätscharakter des strategischen Aspekts ${ }^{2}$. Die Bewertung des ersten Akquisitionsobjekts kann grundsätzlich mit den traditionellen investitionsrechnerischen Verfahren erfolgen. Dabei sind die erfolgsmäßigen Konsequenzen der weiteren Schachzüge zu berücksichtigen, damit sämtliche dem Investitionsprojekt zurechenbaren Rückflüsse erfaßt werden. In der Lehre zur entscheidungsorientierten Unternehmensbewertung spricht man von einer konsequenten Auslegung des Prinzips der Bewertungseinheit ${ }^{3}$. Nur wenn die durch das Anfangsprojekt „verursachten“ Folgeprojekte einen Kapitalwert von null aufweisen, ist eine isolierte Bewertung des Anfangsprojekts gerechtfertigt.

Der zweite Fall ist ebenfalls durch eine Akquisition gekennzeichnet, die den ersten Schritt in einer langfristigen Unternehmensstrategie verkörpert. Im Gegensatz zur ersten Situation ist jedoch nicht völlig geklärt, wie die weitere Investitionspolitik aussehen soll. Über die auf die Erstakquisition folgenden Investitionsprojekte ist noch nicht endgültig entschieden. Es bestehen vielmehr mehrere mögliche Pfade für die zukünftige Entwicklung. Hier besteht nun der weiter oben beschriebene Flexibilitätscharakter, da noch ein unternehmerischer Handlungsspielraum besteht, einen der vorgezeichneten Pfade zu verfolgen bzw. die ursprüngliche Planung völlig aufzugeben. Die aktuell zur Disposition stehende Akquisition schafft ein (neues) Erfolgspotential, über dessen Ausschöpfung erst in der Zukunft entschieden wird. Sieben/Diedrich lösen das hiermit verbundene Bewertungsproblem, in dem sie fragen, was es alternativ,

1 Vgl. zum folgenden Sieben/Diedrich, Aspekte, 1990, S. $798 \mathrm{ff}$.

2 Gleiches gilt furr ein von Helling angeführtes Beispiel. Vgl. Helling, Strategieorientierte Unternehmensbewertung, 1994, S. 55 ff.

3 Vgl. Sieben/Diedrich, Aspekte, 1990, S. 800. 
z.B. durch die Gründung eines eigenen Betriebs, kosten würde, ähnliche Voraussetzungen für die Umsetzung der geplanten Unternehmensstrategie zu schaffen ${ }^{1}$. Damit wird jedoch strenggenommen ein Vergleichsverfahren angewandt ${ }^{2}$. Eine eigentliche investitionsrechnerische Wertermittlung findet nicht statt bzw. wird auf die Vergleichsalternative geschoben.

\subsection{Realoptionsorientierter Ansatz der strategischen Unternehmens- bewertung}

Im Rahmen der wissenschaftlichen Auseinandersetzung mit dem Thema "Strategische Unternehmensbewertung“ stellt sich die Frage, was ausgehend von den bisherigen Ausführungen zu dessen Weiterentwicklung beigetragen werden kann. Da in dieser Arbeit der Vorstellung gefolgt wird, daß im Hinblick auf eine wertorientierte Unternehmensführung die Auswirkungen strategischer Aspekte auf monetäre Größen grundsätzlich quantifiziert werden sollten, ergeben sich zwei Betätigungsfelder. Zum einen lassen sich in der Tradition des Rappaport'schen Ansatzes weitere Verknüpfungen zwischen den Instrumenten der strategischen Unternehmensplanung und -analyse und den ins Diskontierungsmodell eingehenden Größen aufzeigen. In diese Richtung geht z.B. die Arbeit von Peschke ${ }^{3}$, der das Werttreibermodell von Rappaport unter expliziter Einbeziehung strategischer Einflußfaktoren erweitert (vgl. auch Abb. 8).

Zum anderen kann auf die durch die strategische Flexibilität hervorgerufene spezifische Bewertungssituation näher eingegangen werden. Ein solcher Weg wird in der vorliegenden Arbeit verfolgt. Dazu ist es nützlich, einen zur Shareholder Value-Analyse und zum Konzept des strategischen Zuschlags leicht veränderten Ansatz der strategischen Unternehmensbewertung zu wählen. Rappaport berücksichtigt zwar zukünftige, fest eingeplante Investitionen ins Anlage- und Umlaufvermögen ${ }^{4}$, jedoch keine Investitionen, über die erst in Zukunft in Abhängigkeit des dann vorzufindenden Informationsstandes entschieden wird. Insofern kann es sich bei dem von ihm ermittelten strategischen Unternehmenswert nur um einen Wert ohne Berücksichtigung eines betreffenden Flexibilitätscharakters handeln.

Vgl. Sieben/Diedrich, Aspekte, 1990, S. 806.

2 Vgl. Valcarel, Brücke, 1992, S. 593.

3 Vgl. Peschke, Wertorientierte Strategiebewertung, 1997.

4 Vgl. Abschnitt 3.1.2 dieses Kapitels. 


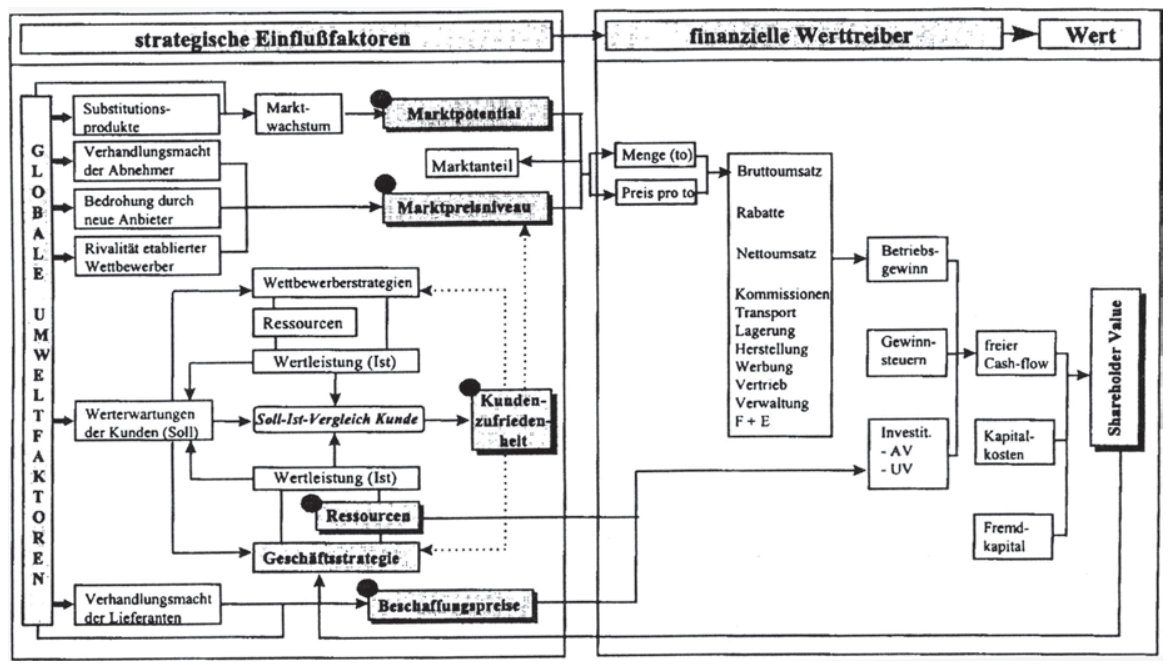

Abb. 8: Strategische Einflußfaktoren im erweiterten Werttreibermodell

Quelle: Peschke, Wertorientierte Strategiebewertung, 1997, S. 146.

Das Konzept des strategischen Zuschlags ist für die weitere Untersuchung insofern irreführend, daß es für die zu analysierende Werterhöhung nicht notwendigerweise auf die spezielle Art des strategischen Zuschlags, sondern vielmehr auf das Vorhandensein der unternehmerischen Flexibilität ankommt. Selbst wenn man die Trennung der strategischen Aspekte in drei verschiedene $\mathrm{Zu}$ schläge (Restrukturierungs-, Synergiepotentiale und Beitrag zur geplanten Strategieumsetzung) aufgibt, und somit nur von einem strategischen Zuschlag spricht, ist das angesprochene Konzept wenig nützlich, um den in dieser Arbeit hervorgehobenen Sachverhalt $\mathrm{zu}$ unterstreichen. Flexibilität ist keine Frage strategischer, operativer oder finanzieller Betrachtung. Für die Existenz eines Flexibilitätswerts reicht es aus, daß im Rahmen von zukünftigen Entscheidungen der durch die Flexibilität bedingte unternehmerische Handlungsspielraum gegeben ist ${ }^{1}$. Die Tatsache, daß bei strategisch motivierten Akquisitionen Handlungsspielräume strategischer Art in den Vordergrund der Betrachtung gerückt werden, verleitet dazu, vorwiegend von einer strategischen Flexibilitätskomponente zu sprechen ${ }^{2}$.

1 Folglich sind auch Flexibilitätswerte bei unveränderter Fortführung des Unternehmens denkbar.

2 Vgl. auch die Ausführungen in Abschnitt 2.3 dieses Kapitels. 
Zudem kann das Konzept des strategischen Zuschlags leicht zu begrifflichen Mißverständnissen führen. Wird durch eine strategische Akquisition ein Folgeprojekt erst ermöglicht, ist man einerseits in einer Gesamtsicht geneigt, den Wert dieser Folgeinvestition als strategischen Zuschlag zu charakterisieren, der den für das Akquisitionsobjekt isoliert ermittelten Wert erhöht. Wie später gezeigt wird, setzt sich andererseits der Wert einer künftigen Investitionsmöglichkeit aus dem traditionellen Kapitalwert und einem Flexibilitätswert zusammen. Bei einer isolierten Betrachtung der durch die Akquisition erst gegebenen Folgeinvestitionsmöglichkeit läßt sich dieser Flexibilitätswert ebenfalls als strategischer Zuschlag (zum traditionellen Kapitalwert) interpretieren. Aus diesem Grund wird im folgenden der Begriff des strategischen Zuschlags vermieden und statt dessen zum einen vom Gesamtwert (bzw. einfach Wert) und zum anderen vom Flexibilitätswert der Folgeinvestitionsmöglichkeit gesprochen.

Zur Bestimmung des strategischen Unternehmenswerts wird in dieser Arbeit der Vorschlag von Myers aufgegriffen, der zwischen ,assets in place bzw. real assets" und „future investment opportunities bzw. real options“ unterscheidet ${ }^{1}$. Der Wert des Unternehmens bestimmt sich demnach durch den Wert der Vermögenspositionen, dessen erwartete Rückflüsse nicht durch zukünftige Investitionen beeinflußt sind, und dem Wert solcher Vermögenspositionen, dessen erwartete Rückflüsse sich erst in Abhängigkeit zukünftiger Investitionsauszahlungen bestimmen lassen.

Übertragen auf den Fall einer strategisch motivierten Akquisition lassen sich somit folgende Vermögenspositionen unterscheiden. Die beim Akquisitionsobjekt bzw. durch den Zusammenschluß auch ohne zukünftige Investitionen erwarteten Rückflüsse stellen ebenso wie Rückflüsse, die aufgrund von unmittelbar nach der Transaktion zu erfolgenden Investitionen prognostiziert werden, das Erfolgspotential aus den Vermögenspositionen der ersten Kategorie (assets in place) dar. Dabei ist es unabhängig, ob diese Rückflüsse beim Akquisitionsobjekt ober beim Erwerber anfallen ${ }^{2}$ (vgl. auch Abb. 9). Demgegenüber

1 Vgl. Myers, Corporate Borrowing, 1977, S. 150 und S. 163 sowie ähnlich Broyles/Cooper, Growth Opportunities, 1981, S. 108; Pindyck, Value of the Firm, 1988, S. 970; Chung/ Charoenwong, Investment Options, 1991, S. 21; Berk/Green/Naik, Growth Options, 1999, S. $1560 \mathrm{ff}$.

2 Für eine Hinzurechnung zum Entscheidungswert der Akquisition ist zu fordern, daß die beim Erwerber anfallenden Rückflüsse durch die Akquisition bedingt sind. 
verkörpern Rückflüsse, deren generelle ${ }^{1}$ Realisierung erst von zukünftigen Investitionsauszahlungen abhängt, das Erfolgspotential aus den Vermögenspositionen der zweiten Kategorie (future investment opportunities). Letztere werden im folgenden Realoptionen bzw. Investitionsmöglichkeiten genannt.

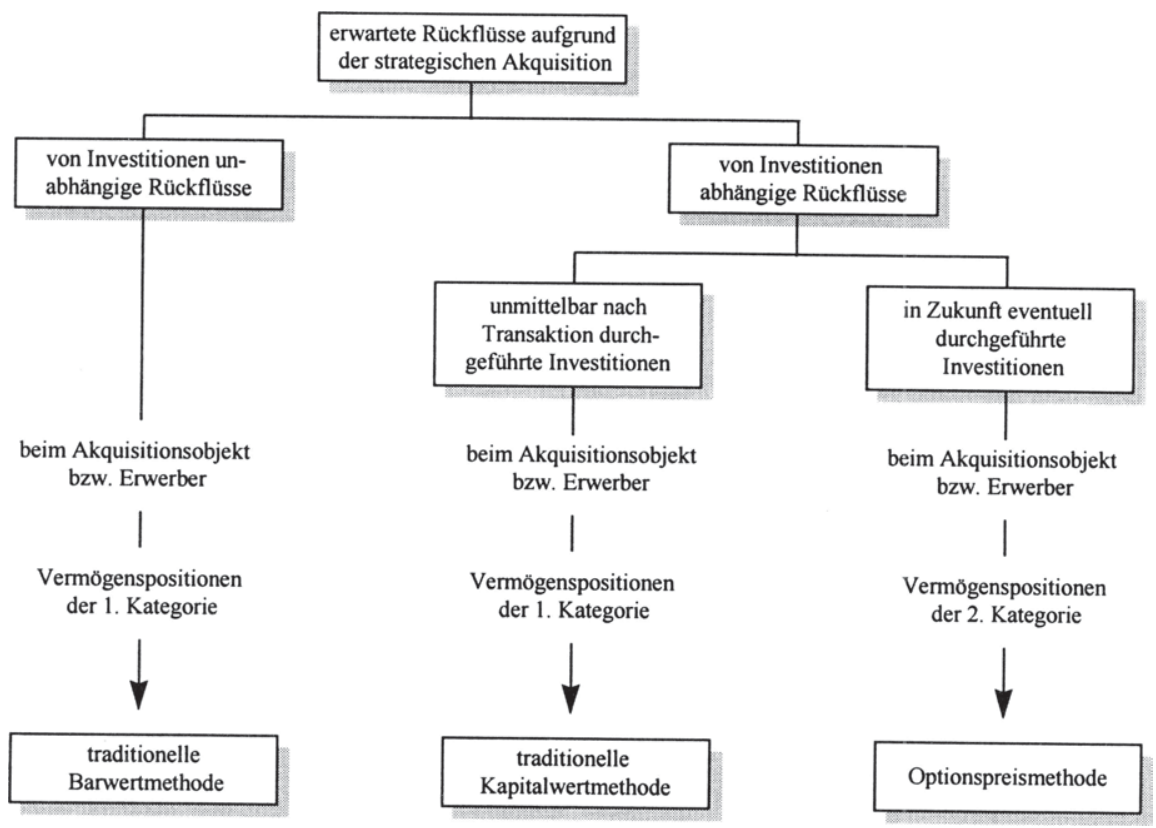

Abb. 9: Wertbestandteile und dazugehörige Ermittlungsmethoden

Der heutige Wert des Erfolgspotentials aus Vermögenspositionen der ersten Kategorie kann mit Hilfe der in Abschnitt 1 dargestellten investitionsrechnerischen Bewertungsverfahren erfolgen. Für die nach Akquisition unmittelbar durchgeführten Investitionen ergibt sich die Besonderheit, daß vom Barwert der erwarteten Rückflüsse die jeweils noch zu zahlende Anschaffungsauszahlung in Abzug zu bringen ist. Zur Bestimmung des heutigen Werts des Erfolgspotentials aus Vermögenspositionen der zweiten Kategorie werden hingegen op-

1 Wie bei der ersten Kategorie ist die tatsächliche Realisierung der Rückflüsse aufgrund von Umweltentwicklungen unsicher. Bei der zweiten Kategorie kommt zusätzlich hinzu, daß die zukünftige Investition eventuell unterlassen wird und somit auch die generelle Realisierung dieser Rückflüsse unsicher ist. 
tionspreistheoretische Modelle vorgeschlagen ${ }^{1}$. Die Anwendung dieser Modelle und damit die Bewertung der mit der Akquisition im Zusammenhang stehenden zusätzlichen Investitionsmöglichkeiten bilden den Schwerpunkt dieser Arbeit.

Mit dem hier vorgeschlagenen Ansatz zur Bestimmung eines strategischen Unternehmenswerts wird keine eigenständige - neue - Lehre der strategischen Unternehmensbewertung propagiert. Es wird vielmehr in strategischen Ausgangskonstellationen eine konsequente Einhaltung der entscheidungsorientierten Bewertungsgrundsätze verfolgt. Neben den geforderten Elementarprinzipien der Bewertungseinheit, der Subjektivität und der Zukunftsbezogenheit ${ }^{2}$ wird eine gemäß der Entscheidungssituation vorzufindende konsistente Risikoberücksichtigung gefordert ${ }^{3}$. Wie im Rahmen dieser Arbeit zu zeigen sein wird, kann diese „einfacher" durch Anwendung optionspreistheoretischer Bewertungsmodelle als über eine Modifikation traditioneller Barwert- bzw. Unternehmensbewertungsmethoden gewährleistet werden. Da die situationsadäquate Berücksichtigung des eingegangenen Risikos im Mittelpunkt der Betrachtung steht, wird im dritten Kapitel im Detail auf Verfahren zur Berücksichtigung von Unsicherheit eingegangen, bevor im vierten Kapitel die konkrete Ermittlung des Werts einer durch die Akquisition zusätzlich geschaffenen Investitionsmöglichkeit anhand eines optionspreistheoretischen Modells aufgezeigt wird.

1 Vgl. zu einer ähnlichen Einteilung inklusive des damit einhergehenden Bewertungsvorschlags Sautter, Strategische Analyse, 1989, S. 128 f.

2 Vgl. Ballwieser/Leuthier, Grundprinzipien, 1986, S. 548 f.; Sieben/Diedrich, Aspekte, 1990, S. 796 f.

3 Vgl. auch Serfling/Pape, Strategische Unternehmensbewertung, 1996, S. 64. 


\section{Kapitel III}

\section{Berücksichtigung von Unsicherheit in investitionsrechnerischen Unternehmensbewertungsverfahren}

\section{1 Überblick}

Der Barwert $V_{0}$ von in der Zukunft liegenden Rückflüssen wird durch deren Diskontierung auf den heutigen Zeitpunkt ermittelt. Ist ein zukünftiger Rückfluß $C F$ hinsichtlich seiner Höhe sicher, entspricht der risikolose Zinsfuß $r_{f}$ den Opportunitätskosten dafür, daß der entsprechende Geldbetrag erst eine Periode später zu Konsumzwecken zur Verfügung steht ${ }^{1}$.

$$
V_{0}=\frac{C F}{1+r_{f}}
$$

Beispielsweise beläuft sich der Barwert einer am Ende der Periode anfallenden Zahlung $C F$ in Höhe von $125 €$ bei einem risikolosen Zinsfuß von $5 \%$ auf $119 €^{2}$.

$$
V_{0}=\frac{125}{1,05}=119 €
$$

Ist der Rückfluß am Ende der Periode jedoch unsicher, bedarf es zusätzlicher Überlegungen. Unter Unsicherheit werden zum einen Situationen verstanden, bei denen für das unsichere Eintreten des zukünftigen Umweltzustands objektive oder subjektive Wahrscheinlichkeiten genannt werden können (Risiko) und zum anderen solche, bei denen dies nicht möglich ist (Ungewißheit) ${ }^{3}$. Im folgenden wird ausschließlich auf Risikosituationen eingegangen ${ }^{4}$.

$\mathrm{Zu}$ den traditionellen Ansätzen zur Berücksichtigung von Unsicherheit zählen Korrekturverfahren, die Sensitivitätsanalyse, die Risikoanalyse und das Ent-

1 Vgl. Schmidt/Terberger, Grundzüge, 1997, S. $111 \mathrm{ff}$.

2 Im weiteren Verlauf der Arbeit wird dieses Zahlenbeispiel kontinuierlich fortgeführt.

3 Vgl. Bamberg/Coenenberg, Entscheidungslehre, 1996, S. 66 und S. 105; Kruschwitz, Investitionsrechnung, 1998, S. $233 \mathrm{f}$.

4 Die Begriffe Unsicherheit und Risiko werden dabei synonym verwendet. 
scheidungsbaumverfahren bzw. die flexible Investitionsplanung ${ }^{1}$. Bei den Korrekturverfahren wird die aufgezeigte Diskontierungsformel entweder in der Zählergröße oder im Zinsfuß korrigiert. Risikoaverses Verhalten unterstellt, kann das Risiko einerseits durch Erhöhung des Zinsfußes oder andererseits durch Verringerung des zu diskontierenden Rückflusses berücksichtigt werden. Beides führt dazu, daß der Barwert eines unsicheren Rückflusses kleiner ist als der eines in der Höhe vergleichbaren sicheren Cash Flows. In Abschnitt 2 dieses Kapitels wird zunächst auf ein einperiodiges Modell zurückgegriffen, bevor anschließend die Bewertungstechnik auf mehrere Perioden ausgedehnt wird.

Im Gegensatz zu den Korrekturverfahren, bei denen ein einwertiges Rechenergebnis unter Unsicherheit resultiert, wird bei der Sensitivitäts- oder der Risikoanalyse die Mehrwertigkeit des Ergebnisses explizit untersucht. Sowohl die Sensitivitäts- als auch die Risikoanalyse stellen kein alternatives Bewertungsverfahren im engeren Sinne dar, sondern lediglich ergänzende Verfahrenstechniken im Umgang mit Unsicherheit. Die durch das einwertige Rechenergebnis gewonnene Information soll um die in diesen Verfahren durch die Mehrwertigkeit zusätzlich gewonnenen Einblicke in den Risikogehalt der zukünftigen Zahlung erweitert werden.

Ist die Höhe des Rückflusses nicht nur von der Unsicherheit des zukünftig eintretenden Umweltzustands abhängig, sondern zusätzlich von in der Zukunft in Abhängigkeit der Umweltentwicklung zu treffenden Entscheidungen, muß diesem Umstand im Bewertungskalkül Rechnung getragen werden. Dazu bietet sich das Gedankengut der flexiblen Investitionsplanung auf Basis eines Entscheidungsbaums an. Wie noch zu zeigen sein wird, scheint insbesondere bei asymmetrischen und sich kontinuierlich ändernden Risikostrukturen eine Diskontierung mit risikoadäquaten Zinsfüßen in solchen Entscheidungsbäumen nur schwer realisierbar. Daher wird am Ende des Kapitels kurz auf die Grundidee der Optionspreistheorie eingegangen.

1 Vgl. allgemein Busse von Colbe/Laßmann, Investitionstheorie, 1990, S. 158 ff.; Blohm/Lüder, Investition, 1995, S. 248 ff.; Kruschwitz, Investitionsrechnung, 1998, S. 254 ff. sowie speziell im Rahmen der Unternehmensbewertung Janschek, Risiko, 1989, S. 111; Siegel, Risikoprofil, 1991, S. 622 f. 


\section{Korrekturverfahren}

\subsection{Einperiodige Bewertung}

\subsubsection{Sicherheitsäquivalent versus Risikozuschlag}

Im folgenden wird unterstellt, daß am Ende der betrachteten Periode ein unsicherer Rückfluß erwartet wird. Dieser läßt sich in Form einer Wahrscheinlichkeitsverteilung beschreiben. Am Ende der betrachteten Periode ist einer von vielen möglichen Umweltzuständen eingetreten, dem dann ein eindeutiger Wert für den Rückfluß zugeordnet werden kann. Über die Eintrittswahrscheinlichkeiten der einzelnen Umweltzustände in Verbindung mit den jeweils möglichen Ausprägungen des Rückflusses läßt sich aus heutiger Sicht ein Erwartungswert für den unsicheren Cash Flow $E(C F)$ ermitteln.

Im weiteren Verlauf der Arbeit wird stets risikoaverses Verhalten vorausgesetzt. Eine Erfassung des Risikos im Zähler der Diskontierungsformel bedingt somit, daß der Erwartungswert des unsicheren Rückflusses um einen Risikoabschlag $R_{P}$ gemindert wird ${ }^{1}$. Dieser ist so zu wählen, daß ein gedanklich sicherer Rückfluß in Höhe der korrigierten Zählergröße den gleichen (Risiko-) Nutzen stiftet, wie der erwartete unsichere Cash Flow. Die korrigierte Zählergröße stellt ein Sicherheitsäquivalent $S \ddot{A}$ für den unsicheren Rückfluß dar.

$$
V_{0}=\frac{E(C F)-R_{p}}{1+r_{f}}=\frac{S \ddot{A}}{1+r_{f}}
$$

Wird beispielsweise ein erwarteter, jedoch unsicherer Rückfluß von $125 €$ um einen Risikoabschlag von $20 €$ gemindert, errechnet sich ein Barwert von $100 €$. Das Sicherheitsäquivalent in Höhe von $105 €$ wird mit dem risikolosen Zinsfuß von $5 \%$ diskontiert.

$$
V_{0}=\frac{125-20}{1,05}=100 €
$$

Bei der Bildung eines Sicherheitsäquivalents werden Zeit- und Risikopräferenz getrennt abgebildet. Die Zeitkomponente, die den Verzicht auf heutigen Kon-

1 Vgl. zum folgenden auch Busse von Colbe/Laßmann, Investitionstheorie, 1990, S. 158 ff; Trigeorgis, Real Options, 1996, S. 34 ff.; Kruschwitz, Finanzierung und Investition, 1999, S. 171 sowie speziell im Rahmen der Unternehmensbewertung Ballwieser, Methoden, 1993, S. 155 ff.; Drukarczyk, Unternehmensbewertung, 1998, S. 313; Schwetzler, Unternehmensbewertung, 1998, S. 2 f.; Steiner/Wallmeier, Discounted Cash Flow-Methoden, 1999, S. 2. 
sum verkörpert, wird im Nenner erfaßt, während die Risikokomponente, die die Unsicherheit über die Höhe des in einer Periode möglichen Konsums darstellt, im Zähler berücksichtigt wird.

Bei Erfassung des Risikos im Nenner wird der risikolose Zinsfuß um einen Risikozuschlag $r_{p}$ erhöht. Der Erwartungswert des unsicheren Rückflusses wird mit dem risikoadjustierten Zinsfuß $r_{A}$ abgezinst.

$$
V_{0}=\frac{E(C F)}{1+r_{f}+r_{p}}=\frac{E(C F)}{1+r_{A}}
$$

Bei einer erwarteten Zahlung von $125 €$, einem risikolosen Zinssatz von 5\% und einem Risikozuschlag von 20\% resultiert ein Barwert von $100 €$.

$$
V_{0}=\frac{125}{1,05+0,20}=100 €
$$

Die Bildung von Risikozuschlägen führt zu einer gemeinsamen Erfassung der Zeit- und Risikopräferenz im Zinsfuß. Der Zähler der Diskontierungsformel bleibt von den Präferenzen des Bewerters unberührt.

Wird ein spezifischer Bewerter mit einer bestimmten Risikopräferenz unterstellt, ist es bei konsistentem Vorgehen unerheblich, ob das Risiko über ein Sicherheitsäquivalent oder über einen Risikozuschlag in die Diskontierungsformel eingeht. Beide Verfahren führen zum gleichen Ergebnis, wenn folgende Beziehung sichergestellt ist ${ }^{1}$.

$$
1-\frac{R_{p}}{E(C F)}=\frac{1+r_{f}}{1+r_{f}+r_{p}}
$$

Der Term $R_{p} / E(C F)$ läßt sich als prozentualer Risikoabschlag interpretieren. Er ist notwendig, um vom Erwartungswert des unsicheren Rückflusses zum Sicherheitsäquivalent zu gelangen, falls ein Risikozuschlag in Höhe von $r_{p}$ angenommen wird.

Vgl. ähnlich Ballwieser, Risiko, 1981, S. 102 f. 


\subsubsection{Theoretisch fundierte Quantifizierung des Sicherheitsäquivalents und des Risikozuschlags}

\subsubsection{Kapitalmarkttheoretische Vorgehensweise}

Unter Rückgriff auf kapitalmarkttheoretische Erkenntnisse, insbesondere auf das Capital Asset Pricing Modell ${ }^{1}$, läßt sich der Risikoabschlag z.B. mit Hilfe von Kapitalmarktdaten ermitteln. Unter den Annahmen des CAPM wird für eine Einheit übernommenes systematisches Risiko ${ }^{2}$ vom Markt eine Prämie in Höhe von $\lambda_{M}$ entgolten. Zur Bestimmung dieser Marktrisikoprämie wird in einem ersten Schritt der risikolose Zinsfuß von der erwarteten Marktrendite $E\left(r_{M}\right)$ abgezogen, um anschließend die ermittelte Differenz durch die Varianz dieser Marktrendite $\operatorname{Var}\left(r_{M}\right)$ zu teilen. Zum Risikoabschlag gelangt man, wenn die Marktrisikoprämie mit der Kovarianz zwischen dem unsicheren Rückfluß und der Marktrendite $\operatorname{Cov}\left(C F, r_{M}\right)$ multipliziert wird ${ }^{3}$.

$$
\begin{gathered}
\lambda_{M}=\frac{E\left(r_{M}\right)-r_{f}}{\operatorname{Var}\left(r_{M}\right)} \\
V_{0}=\frac{E(C F)-\lambda_{M} \cdot \operatorname{Cov}\left(C F, r_{M}\right)}{1+r_{f}}
\end{gathered}
$$

Durch Umformulierung kann der Risikoabschlag auch in „Betaform“ dargestellt werden. Der hier verwendete Faktor $\hat{\beta}$ unterscheidet sich vom verbreiteteren Betafaktor zur Bildung von Risikozuschlägen, indem bzgl. des unsicheren Rückflusses keine Rendite-, sondern absolute Daten verwendet werden.

$$
\hat{\beta}=\frac{\operatorname{Cov}\left(C F, r_{M}\right)}{\operatorname{Var}\left(r_{M}\right)}
$$

I Zum CAPM vgl. allgemein Sharpe, Capital Asset Prices, 1964; Lintner, Risky Assets, 1965; Mossin, Capital Asset Market, 1966.

2 Unter dem systematischen Risiko wird jenes Risiko verstanden, das selbst bei risikoeffizienter Diversifikation über alle Wertpapiere des Marktes nicht eliminiert werden kann. Im CAPM wird von ,perfekt" diversifizierten Marktteilnehmern ausgegangen, weshalb nur das systematische Risiko bewertungsrelevant ist.

3 Vgl. zum folgenden Kruschwitz, Finanzierung und Investition, 1999, S. 171 ff. Für Darstellungen im Rahmen der Unternehmensbewertung Göppl, Unternehmensbewertung, 1980, S. 240; Schmidt, Discounted Cash Flow-Methode, 1995, S. 1103 ff.; Nippel, Zirkularitätsprobleme, 1999, S. $337 \mathrm{ff}$. 


$$
V_{0}=\frac{E(C F)-\left[E\left(r_{M}\right)-r_{f}\right] \cdot \hat{\beta}}{1+r_{f}}
$$

In Erweiterung des zuvor eingeführten Zahlenbeispiels sei angenommen, daß der unsichere Rückfluß am Ende der Periode mit Hilfe einer Binomialverteilung abgebildet werden kann. In einem ersten Umweltzustand fließen dem Bewerter 200 und einem zweiten Umweltzustand $50 €$ zu. Bei gleichwahrscheinlichem Eintreten der beiden Umweltzustände beläuft sich der Erwartungswert des unsicheren Rückflusses auf $125 €$. Desweiteren werden Marktrenditen von $28,75 \%$ bzw. $-8,75 \%$ für die beiden Umweltzustände unterstellt, so daß Erwartungswert und Varianz der Marktrendite $10 \%$ bzw. 3,52\% betragen. Die Marktrisikoprämie und die Kovarianz zwischen Zahlung und Marktrendite ergeben sich folglich zu 1,42 bzw. 14,06, so daß sich ein Risikoabschlag von $20 €$ ergibt.

$$
V_{0}=\frac{125-1,42 \cdot 14,06}{1,05}=100 €
$$

Das weitaus bekanntere auf dem CAPM basierende Diskontierungsmodell berücksichtigt das Risiko über einen Risikozuschlag im Zinsfuß. Dazu wird die Marktrisikoprämie mit der Kovarianz zwischen der Rendite des zu bewertenden Rückflusses und der Marktrendite $\operatorname{Cov}\left(r_{C F}, r_{M}\right)$ multipliziert.

$$
V_{0}=\frac{E(C F)}{1+r_{f}+\lambda_{M} \cdot \operatorname{Cov}\left(r_{C F}, r_{M}\right)}
$$

Durch Umformulierung kann die Darstellung des Risikozuschlags auf Basis des Betafaktors $\beta$ erfolgen. Der Betafaktor ergibt sich dabei durch Division der Kovarianz zwischen der Rendite der unsicheren Zahlung und der Marktrendite durch die Varianz der Marktrendite ${ }^{1}$.

1 Der Betafaktor kann auch auf Basis der Korrelation kor zwischen Rendite des unsicheren Rückflusses und Marktrendite sowie dem Verhältnis der beiden Standardabweichungen $\sigma$ dargestellt werden.

$$
\beta=k o r_{r_{C F}}, r_{M} \cdot \frac{\sigma_{r_{C F}}}{\sigma_{r_{M}}}
$$

Diese Schreibweise macht besonders deutlich, daß es sich hierbei um ein Risikomaß handelt, das den Gleichlauf zwischen dem Markt und des zu bewertenden Rückflusses berücksichtigt. 


$$
\begin{gathered}
\beta=\frac{\operatorname{Cov}\left(r_{C F}, r_{M}\right)}{\operatorname{Var}\left(r_{M}\right)} \\
V_{0}=\frac{E(C F)}{1+r_{f}+\left[E\left(r_{M}\right)-r_{f}\right] \cdot \beta}
\end{gathered}
$$

Bei Anwendung der kapitalmarkttheoretischen Risikozuschlagsmethode besteht ein Zirkularitätsproblem ${ }^{1}$. Die exakte Ermittlung der Cash Flow-Renditen ist nur bei Kenntnis des Barwerts des unsicheren Rückflusses möglich. Dieser ist jedoch gerade das Ziel der Bewertung. Zwei Möglichkeiten zur Umgehung dieser Zirkularität bieten sich an. Zum einen kann näherungsweise auf die zur Erlangung des Rückflusses notwendige Investitionsauszahlung zurückgegriffen werden ${ }^{2}$. Zum anderen wird in der Unternehmenspraxis i.d.R. historisches Datenmaterial verwendet ${ }^{3}$. Die zwischen den Renditen einer vergleichbaren unsicheren Zahlung und denen des Marktes gefundenen Beziehungen aus der Vergangenheit werden als „Proxy“ in die Bewertung des jetzt zur Disposition stehenden Rückflusses eingesetzt.

Vorausgesetzt der Barwert des unsicheren Rückflusses beträgt $100 €$, so kann die Rendite dieses Cash Flows mit $100 \%$ respektive $-50 \%$ angegeben werden. Bei einer Kovarianz zwischen Rendite des Cash Flows und Marktrendite von 0,1406 und einer Varianz der Marktrendite von 0,0352 ergibt sich für den zu bewertenden Rückfluß ein Betafaktor von 4. Dadurch stellt sich ein Risikozuschlag von $20 \%$ ein, womit der adäquate risikoadjustierte Zinsfuß $25 \%$ beträgt.

$$
V_{0}=\frac{125}{1,05+(0,10-0,05) \cdot 4}=100 €
$$

Auch bei kapitalmarkttheoretischer Vorgehensweise muß bei konsistentem Vorgehen unabhängig davon, ob die Sicherheitsäquivalents- oder die Risikozuschlagsmethode gewählt wird, der gleiche Barwert resultieren. Unter Zuhilfe-

Vgl. Nippel, Zirkularitätsprobleme, 1999, S. 337 ff., der zudem darauf hinweist, daß auch bei der kapitalmarkttheoretischen Risikoabschlagsmethode ein (weniger offensichtliches) Zirkularitätsproblem besteht.

2 Vgl. Kruschwitz, Finanzierung und Investition, 1999, S. 246.

3 Für eine kritische Darstellung dieser Vorgehensweise vgl. Baetge/Krause, Unternehmensbewertung, 1994, S. $440 \mathrm{ff}$. 
nahme der beiden folgenden Beziehungen läßt sich nach wenigen Umformungen schnell die Identität der Bewertungsformeln belegen ${ }^{1}$.

$$
\begin{gathered}
r_{C F}=\frac{C F}{V_{0}}-1 \\
\operatorname{Cov}\left(r_{C F}, r_{M}\right)=\frac{\operatorname{Cov}\left(C F, r_{M}\right)}{V_{0}}
\end{gathered}
$$

\subsubsection{Entscheidungstheoretische Vorgehensweise}

Das entscheidungstheoretische Vorgehen beruht auf dem Bernoulli-Prinzip und den darin enthaltenen Axiomen rationalen Entscheidungsverhaltens unter Unsicherheit ${ }^{2}$. Mit Hilfe von Risikonutzenfunktionen $U(C F)$ wird jeder für möglich gehaltenen Ausprägung des unsicheren Rückflusses ein Nutzenwert zugeordnet. Die einzelnen (Teil-) Nutzenwerte werden mit den für die jeweiligen Umweltzustände $s$ geltenden Eintrittswahrscheinlichkeiten $q$ multipliziert und zu einem erwarteten Gesamtnutzen $G N$ addiert $^{3}$. Wird letzterer auf Basis der Umkehrfunktion $U^{l}(G N)$ in ein Sicherheitsäquivalent des unsicheren Cash Flows transformiert, kann dieses zur Barwertermittlung mit dem risikolosen Zinsfuß abgezinst werden ${ }^{4}$.

$$
V_{0}=\frac{U^{-1}[G N]}{1+r_{f}}=\frac{U^{-1}\left[\sum_{s=1}^{S} q_{s} \cdot U\left(C F_{s}\right)\right]}{1+r_{f}}
$$

1 Vgl. Trigeorgis, Real Options, 1996, S. 47 f.

2 Zum Bernoulli-Prinzip vgl. ausführlich Bitz, Entscheidungstheorie, 1981, S. 153 ff.; Sieben/Schildbach, Entscheidungstheorie, 1994, S. 62 ff.; Bamberg/Coenenberg, Entscheidungslehre, 1996, S. $85 \mathrm{ff}$.

3 Vgl. Schmidt/Terberger, Grundzüge, 1997, S. 289; Kruschwitz, Investitionsrechnung, 1998, S. 242.

4 Im originären Ansatz des Bernoulli-Prinzips wird lediglich die Risikopräferenz des Entscheidungsträgers erfaßt. Um die Konvergenz zu den davor aufgezeigten Vorgehensweisen zu gewährleisten, wird hier mit Hilfe der risikolosen Diskontierung zusätzlich die Zeitpräferenz berücksichtigt. 
Zur Bestimmung der Risikonutzenfunktion werden in der Literatur verschiedene Funktionstypen vorgeschlagen ${ }^{1}$, von denen einige beispielhaft in Tab. 4 abgebildet sind. Der spezifische Funktionsparameter $x$ (bzw. $y$ ) ist dabei gemäß der individuellen Risikoeinstellung des Entscheidungsträgers auszugestalten. Dieser kann grundsätzlich risikoscheu, risikoneutral oder risikofreudig sein ${ }^{2}$. In Anlehnung an die vorigen Abschnitte wird auch im folgenden von einem risikoaversen Entscheidungsträger ausgegangen.

\begin{tabular}{|c|c|c|}
\hline Risikonutzenfunktion & \\
\hline$x \cdot C F+y \cdot C F^{2}$ & absolute Risikoaversion & relative Risikoaversion \\
\hline$-\mathrm{e}^{-x \cdot C F}$ & zunehmend & zunehmend \\
\hline$C F^{x}$ & konstant & zunehmend \\
\hline & abnehmend & konstant \\
\hline $\ln (C F+x)$ & abnehmend & $x>0$ : zunehmend \\
& & $x=0$ : konstant \\
& & $x<0$ : abnehmend \\
\hline
\end{tabular}

Tab. 4: Beispiele fur Risikonutzenfunktionen

Quelle: In Anlehnung an Kasanen/Trigeorgis, Merging, 1995, S. 55; Kruschwitz, Finanzierung und Investition, 1999, S. 112.

Das Ausmaß der in den einzelnen Nutzenfunktionen zum Ausdruck kommenden Risikoabneigung kann über die absolute und die relative Risikoaversion näher beschrieben werden. Baut der Entscheidungsträger bei wachsendem Vermögen keine (weniger bzw. mehr) zusätzliche(n) risikobehaftete(n) Vermögenspositionen auf, wird von konstanter (zunehmender bzw. abnehmender) absoluter Risikoaversion gesprochen. Je nachdem ob der Investor bei Vermögenszuwachs den Anteil des risikobehafteten Vermögens am Gesamtkapital erhöht, reduziert oder konstant läßt, wird dies als abnehmende, zunehmende oder konstante relative Risikoaversion bezeichnet.

Der Annahme einer zunehmenden absoluten Risikoaversion kann nur schwer zugestimmt werden. Mit größerem Vermögen dürfte der Entscheidungsträger

1 Vgl. beispielhaft die bei Kasanen/Trigeorgis, Merging, 1995 zitierte Literatur. Zur Konkretisierung einer Risikonutzenfunktion eines bestimmten Entscheidungsträgers vgl. Laux, Entscheidungstheorie, 1995, S. 170 ff.; Bamberg/Coenenberg, Entscheidungslehre, 1996, S. $76 \mathrm{ff}$.

2 Vgl. Schmidt/Terberger, Grundzüge, 1997, S. 288; Kruschwitz, Investitionsrechnung, 1998, S. $247 \mathrm{f}$.

3 Damit die quadratische Risikonutzenfunktion risikoaverses Verhalten ausdrückt muß gelten: $x>0$ und $y<0$. Für die exponentielle Nutzenfunktion ist hier $x>0$ und für die Potenzfunktion $0<x<1$ unterstellt. 
eher geneigt sein, zusätzliche absolute Risikopositionen einzugehen ${ }^{1}$. Quadratische Risikonutzenfunktionen stellen deswegen ein wenig überzeugendes Risikoverhalten dar. Am plausibelsten scheinen Nutzenfunktionen, die durch abnehmende absolute und konstante bzw. abnehmende relative Risikoaversion gekennzeichnet sind ${ }^{2}$.

Wenn z.B. angenommen wird, daß die mit Hilfe des individuellen Entscheidungsträgers bestimmte Risikonutzenfunktion folgende Gestalt annimmt

$$
U\left(C F_{S}\right)=-e^{-0,00747 \cdot C F_{S}},
$$

resultiert bei zustandsabhängigen und gleichwahrscheinlichen Rückflüssen von $200 €$ respektive $50 €$ ein Sicherheitsäquivalent von $105 €$. Die Abzinsung mit einem risikolosen Zinsfuß von $5 \%$ führt zu einem Barwert des unsicheren Rückflusses von $100 €^{3}$.

Um die kapitalmarkt- und die entscheidungstheoretische Vorgehensweise zu verbinden, kann das Konzept des Marktnutzens herangezogen werden ${ }^{4}$. Kern dieses Gedankenguts ist die Annahme, daß der Kapitalmarkt, begriffen als Aggregation aller Investoren, eine bestimmte Risikoeinstellung besitzt, die wie bei einem individuellen Entscheidungsträger durch eine Risikonutzenfunktion dargestellt werden kann. Konkret bedeutet dies, daß für einen bestimmten Funktionstyp der spezifische Parameter gefunden werden muß, der mit den Preisen am Kapitalmarkt übereinstimmt ${ }^{5}$. Mit Hilfe der gefundenen Marktnutzenfunktion ließen sich dann auch andere unsichere Rückflüsse bewerten.

Neben der Bewertung von risikobehafteten Vermögenspositionen kann das Bernoulli-Prinzip auch dazu herangezogen werden, das im CAPM vorausge-

1 Vgl. Drukarczyk, Theorie und Politik, 1993, S. 109.

2 Vgl. Kruschwitz, Finanzierung und Investition, 1999, S. $111 \mathrm{ff} . \mathrm{Zu}$ empirischen Ergebnissen vgl. Oehler, Risikoaversion, 1998, S. 230 ff. sowie die dort zitierte Literatur.

3 Das Beispiel ist bewußt so gewählt worden, daß das entscheidungstheoretische Ergebnis mit demjenigen aus der kapitalmarkttheoretischen Vorgehensweise übereinstimmt. Wird die Bestimmung der individuellen Nutzenfunktion ohne Rückgriff auf Kapitalmarktdaten durchgeführt, dürften in einer praktischen Anwendung die Ergebnisse nur zufällig übereinstimmen.

4 Vgl. Kasanen/Trigeorgis, Approach, 1994, S. 294 ff.; Kasanen/Trigeorgis, Merging, 1995, S. $47 \mathrm{ff}$.

5 Ob sich unter realen Gegebenheiten ein solcher Parameter überhaupt ermitteln läßt, kann angezweifelt werden. 
setzte $\mu$ - $\sigma$-Prinzip ${ }^{1}$ zu durchleuchten. Dieses Prinzip setzt voraus, daß unsichere Zahlungen allein anhand ihres Erwartungswerts und ihrer Standardabweichung bewertet werden. Dies steht nur dann im Einklang mit dem BernoulliPrinzip, wenn die Wahrscheinlichkeitsverteilung der unsicheren Zahlung vollständig durch diese beiden Parameter beschrieben werden kann, d.h. eine Normalverteilung vorliegt oder der Entscheidungsträger sich gemäß einer quadratischen Risikonutzenfunktion ${ }^{2}$ verhält ${ }^{3}$.

Quadratische Risikonutzenfunktionen können jedoch gegen das Dominanzaxiom verstoßen, das besagt, daß eine Investitionsalternative $\mathrm{zu}$ bevorzugen ist, wenn sie in einem Umweltzustand einen größeren und in allen anderen Umweltzuständen keinen geringeren Cash Flow als eine andere Alternative verspricht ${ }^{4}$. Zudem stellen sie kein sehr realistisches Risikoverhalten dar, so daß bei Anwendung des $\mu$ - $\sigma$-Prinzips im allgemeinen von einer Normalverteilung des unsicheren Rückflusses ausgegangen wird. Dies ist eine wichtige Schlußfolgerung für den im weiteren Verlauf dieses Kapitels noch nachzuweisenden Vorteil von Optionspreismodellen bei der Bewertung einer unsicheren Zahlung, die einer asymmetrischen und sich kontinuierlichen ändernden Risikostruktur folgt.

\subsection{Mehrperiodige Bewertung}

\subsubsection{Retrograde Vorgehensweise}

Im folgenden wird davon ausgegangen, da $§$ die unsichere Zahlung nicht wie bisher nach einer, sondern erst nach mehreren Perioden erfolgt. Die dadurch notwendige mehrperiodige Bewertung wird an dieser Stelle exemplarisch anhand der kapitalmarkttheoretischen Vorgehensweise aufgezeigt. Letztere eignet sich bestens für die Ableitung der im nächsten Abschnitt zu treffenden Aussa-

1 Zum $\mu-\sigma$-Prinzip vgl. ausführlich Schmidt/Terberger, Grundzüge, 1997, S. 294 ff.; Kruschwitz, Investitionsrechnung, 1998, S. 237 ff.

2 Bei einer quadratischen Nutzenfunktion von $x \cdot C F+y \cdot C F^{2}$ kann der erwartete Nutzen über $x \cdot \mu+y \cdot\left(\mu^{2}+\sigma^{2}\right)$ und damit neben den Funktionsparametern $x$ und $y$ vollständig über den Erwartungswert des Cash Flows $\mu$ und dessen Standardabweichung $\sigma$ ermittelt werden.

3 Vgl. Franke/Hax, Finanzwirtschaft, 1994, S. 300 ff.; Laux, Entscheidungstheorie, 1995, S. 208 ff.; Kruschwitz, Finanzierung und Investition, 1999, S. $121 \mathrm{ff}$.

4 Vgl. Schmidt/Terberger, Grundzüge, 1997, S. 297; Kruschwitz, Investitionsrechnung, 1998, S. 240. Zudem sind bei Risikoaversion nur Ergebnisgrößen bis zur Höhe von $C F=-x / 2 y$ mit $x>0$ und $y<0$ möglich. Vgl. Franke/Hax, Finanzwirtschaft, 1994, S. 303 f.; Kruschwitz, Finanzierung und Investition, 1999, S. 122. 
gen, in denen die impliziten Annahmen von Bewertungsverfahren erläutert werden, die einen - wie in der Unternehmenspraxis üblich ${ }^{1}$ - einheitlichen risikoadjustierten Zinsfuß verwenden.

Um den Barwert eines unsicheren Rückflusses, der nach mehreren Perioden fließt, zu ermitteln, wird retrograd vorgegangen. In Analogie zu der bei Einperiodigkeit entwickelten Bewertung werden die Werte zu Anfang einer Periode aus den Werten am Ende der Periode bestimmt ${ }^{2}$. Aufgrund der bereits geschilderten Zirkularität bei der Bildung von Risikozuschlägen wird zuerst auf Sicherheitsäquivalente zurückgegriffen. Der zu bestimmende Risikoabschlag wird durch die zu Beginn der Periode geltenden Markt- und Risikoparameter ${ }^{3}$ beeinflußt. Wie der erwartete Cash Flow sind diese vom bis dahin eingetretenen Umweltzustand $s$ abhängig ${ }^{4}$.

$$
V_{t-1}=\frac{E\left(C F_{t} / s\right)-\lambda_{s} \cdot \operatorname{Cov}\left(C F, r_{M} / s\right)}{1+r_{f, s}}
$$

Im Rahmen des oben angeführten Zahlenbeispiels werden nun zwei Perioden angenommen. Die Wertentwicklung des unsicheren Rückflusses folgt in beiden Perioden einer Binomialverteilung. Am Ende der ersten Periode sind zwei Umweltzustände und folglich zwei Werte für den unsicheren Rückfluß denkbar $\left(V_{l, l}\right.$ bzw. $\left.V_{l, 2}\right) . V_{t, s}$ bezeichnet dabei den Gegenwartswert dieses Rückflusses zum Zeitpunkt $t$ im Umweltzustand $s$. Nach einer Periode ist bekannt, welcher der beiden Werte realisiert wurde. Hiervon ausgehend entstehen wiederum jeweils zwei Umweltzustände (vgl. Abb. 10). Am Ende der zweiten Periode können somit vier Umweltzustände bzw. vier Werte des unsicheren Rückflusses abgebildet werden $\left(V_{2,1} ; V_{2,2} ; V_{2,3} \text { bzw. } V_{2,4}\right)^{5}$.

1 Vgl. die empirischen Ergebnisse bei Peemöller/Bömelburg/Denkmann, Unternehmensbewertung, 1994, S. 744 f.; Walker/Prietze, Unternehmensbewertung, 1995, S. 205 f.; Pellens/Rockholtz/Stienemann, Konzerncontrolling in Deutschland, 1997, S. $1935 \mathrm{f}$.

2 Zur Zurückführung eines mehrperiodigen auf mehrere einperiodige Bewertungsprobleme vgl. allgemein Schmidt/Terberger, Grundzüge, 1997, S. 307 ff.

3 Als Marktparameter gelten an dieser Stelle der risikolose Zinsfuß $r_{f}$ und der Erwartungswert der Marktrendite $E\left(r_{M}\right)$, während die Varianz der Marktrendite $\operatorname{Var}\left(r_{M}\right)$ und die Kovarianz zwischen zu bewertender Zahlung und Marktrendite $\operatorname{Cov}\left(C F, r_{M}\right)$ als Risikoparameter angesehen werden können.

4 Vgl. stellvertretend Copeland/Weston, Theory and Policy, 1988, S. 405.

5 Wird die unsichere Wertentwicklung mit einer Normalverteilung beschrieben, vergrößert sich der Bereich der möglichen Werte am Ende der jeweiligen Perioden. Der Unsicherheitszusammenhang zwischen den Perioden bleibt jedoch bestehen. 
Die Eintrittswahrscheinlichkeit eines Umweltzustands in der jeweiligen Binomialverteilung wird konstant mit $50 \%$ angenommen. Für die unsichere Zahlung wird am Planungshorizont von Beträgen von 400, 100, 100 respektive $25 €$ ausgegangen und vereinfachend unterstellt, daß in beiden Perioden die gleichen Markt- und Risikoparameter herrschen. Wie im einperiodigen Beispiel soll der risikolose Zinsfuß 5\%, der Erwartungswert der Marktrendite 10\% sowie die Varianz der Marktrendite 3,52\% betragen. Dies impliziert die bereits bekannte Marktrisikoprämie von 1,42.

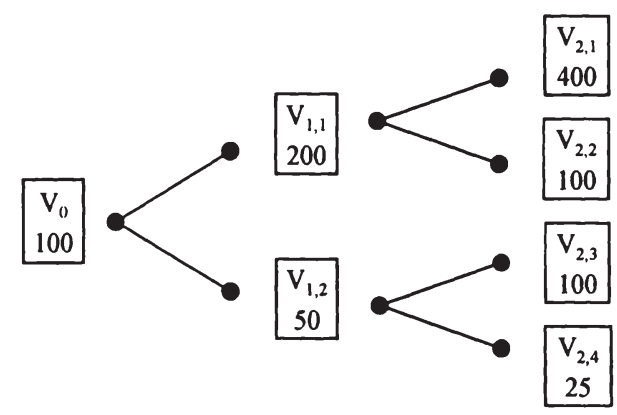

Abb. 10: Wertentwicklung einer unsicheren Zahlung bei konstantem Periodenrisiko

Tritt am Ende der ersten Periode Umweltzustand 1 ein, ist mit einem erwarteten Rückfluß am Ende der zweiten Periode von $250(0,5 \cdot 400+0,5 \cdot 100) € \mathrm{zu}$ rechnen. Bei einer Kovarianz zwischen Cash Flow und Marktrendite von 28,13 und damit einem Risikoabschlag in Höhe von $40(1,42 \cdot 28,13) €$ beläuft sich der Wert des unsicheren Rückflusses am Ende der ersten Periode auf $200 €$ $\left(V_{l, l}\right)$.

$$
V_{l, l}=\frac{250-1,42 \cdot 28,13}{1,05}=200 €
$$

Für den Fall, daß sich Umweltzustand 2 realisiert, resultiert ein erwarteter Rückfluß am Ende der zweiten Periode von $62,5(0,5 \cdot 100+0,5 \cdot 25) €$, eine Kovarianz von 7,03 und somit ein Risikoabschlag von $10(1,42 \cdot 7,03) €$. Der entsprechende Wert des unsicheren Rückflusses am Ende der ersten Periode beträgt somit $50 €\left(V_{l, 2}\right)$.

$$
V_{l, 2}=\frac{62,5-1,42 \cdot 7,03}{1,05}=50 €
$$


Damit hat sich das mehrperiodige Bewertungsproblem auf das bei Einperiodigkeit in Abschnitt 2.1.2.1 dieses Kapitels geschilderte Vorgehen reduziert. Für den Barwert des unsicheren Cash Flows, der nach zwei Perioden erfolgt, resultiert ein Ergebnis von $100 €$.

$$
V_{0}=\frac{125-1,42 \cdot 14,06}{1,05}=100 €
$$

Wie bei der Sicherheitsäquivalentmethode muß auch bei der Bildung von Risikozuschlägen auf die Abhängigkeit der Markt- und Risikoparameter vom Umweltzustand $s$ geachtet werden.

$$
V_{t-1}=\frac{E\left(C F_{t} / s\right)}{1+r_{f, s}+\lambda_{s} \cdot \operatorname{Cov}\left(r_{C F}, r_{M} / s\right)}
$$

Da über die Sicherheitsäquivalentmethode alle perioden- und zustandsabhängigen Werte des unsicheren Rückflusses bekannt sind, kann nun das (theoretisch korrekte) Vorgehen bei der Risikozuschlagsmethode aufgezeigt werden. Die Kovarianz zwischen Rendite des Rückflusses und Rendite des Marktes ist in diesem Beispiel für alle Perioden und Zustände identisch und beträgt 0,1406. Dadurch resultiert ein perioden- und zustandsunabhängiger Risikozuschlag von $20 \%$ und somit ein risikoadjustierter Zinsfuß von $25 \%$. Die Werte des unsicheren Cash Flows am Ende der ersten Periode von 200 bzw. $50 €$ werden über risikoadjustierte Abzinsung des jeweiligen Erwartungswerts am Ende der zweiten Periode $(250 / 1,25$ bzw. 62,5/1,25) gewonnen. Die Diskontierung des erwarteten unsicheren Rückflusses am Ende der ersten Periode $(125 / 1,25)$ führt zu einem Barwert von $100 €$.

Durch die Annahme perioden- und zustandsunabhängiger Markt- und Risikoparameter wurden im Zahlenbeispiel die einzelnen Rechenschritte stark vereinfacht. Das vorgestellte Bewertungskonzept ist gleichwohl universell einsetzbar, indem für jede Periode und jeden Umweltzustand die entsprechenden Daten ermittelt und zur Bewertung des Werts der Vorperiode genutzt werden ${ }^{1}$. Für solche Fälle ist jedoch zu fordern, daß die perioden- und zustandsabhängigen

1 Vgl. Drukarczyk, Theorie und Politik, 1993, S. 287. Zu einem Zahlenbeispiel vgl. Kruschwitz, Finanzierung und Investition, 1999, S. $257 \mathrm{ff}$. 
Markt- und Risikoparameter keiner Unsicherheit im Zeitablauf unterliegen'. Aus heutiger Sicht müssen sie deterministisch prognostizierbar $\operatorname{sein}^{2}$, so daß die zukünftigen - nach der ersten Periode geltenden - Markt- und Risikoparameter zwar aus heutiger Sicht unbekannt, jedoch in Abhängigkeit des Umweltzustands eindeutig festgelegt sind.

\subsubsection{Implikationen eines einheitlichen risikoadjustierten Zinsfußes}

Für den Fall, daß die Markt- und Risikoparameter wie im obigen Beispiel unabhängig von der Periode und dem eingetretenen Umweltzustand sind, ist eine rechentechnische Vereinfachung möglich. Der risikoadjustierte Zinsfuß ist für alle Perioden und Umweltzustände konstant. Der Barwert läßt sich deshalb direkt durch Diskontierung eines zum heutigen Zeitpunkt erwarteten Werts am Ende der zweiten Periode ermitteln. Letzterer wird durch die aus heutiger Sicht vorzufindenden Eintrittswahrscheinlichkeiten der vier Umweltzustände am Planungshorizont bestimmt. Bei einem Erwartungswert der unsicheren Zahlung von $156,25 \epsilon^{3}$ und einem risikoadjustierten Zinsfuß von $25 \%$ ergibt sich bei zweiperiodiger Abzinsung ein Barwert von $100 €$.

$$
V_{0}=\frac{156,25}{1,25^{2}}=100 €
$$

Das gewählte Zahlenbeispiel zeigt deutlich, welche Implikationen eine Diskontierung über mehrere Perioden mit einem einheitlichen risikoadjustierten Zinsfuß mit sich bringt. Es setzt voraus, daß sich die perioden- und zustandsabhängigen Markt- und Risikoparameter im Zeitablauf nicht verändern ${ }^{4}$. Nur dann kann folgende Diskontierungsformel verwendet werden.

1 Dies bedeutet, daß ,... schon im Zeitpunkt $t_{0}$ mit Sicherheit die zustandsabhängigen Möglichkeiten zur Geldanlage ... bekannt sind.“ Schmidt/Terberger, Grundzüge, 1997, S. 309.

2 Vgl. Fama, Discount Rates, 1977, S. 4; Kruschwitz/Schöbel, CAPM, 1987, S. 71. Constantinides zeigt, daß auch bei stochastischen Markt- und Risikoparametern eine mehrperiodige Bewertung in der CAPM-Welt möglich ist. Eine Anwendung dieser Vorgehensweise ist jedoch als ,computationally complex and of little practical use“ einzuschätzen. Vgl. Constantinides, Admissible Uncertainty, 1980, S. $71 \mathrm{ff}$.

$30,25 \cdot 400+0,25 \cdot 100+0,25 \cdot 100+0,25 \cdot 25$.

4 Vgl. Fama, Discount Rates, 1977, S. 20; Hachmeister, Diskontierung bei Unsicherheit, 1998, S. 26. 


$$
V_{0}=\sum_{t=1}^{n} \frac{E\left(C F_{t}\right)}{\left(1+r_{A}\right)^{t}}
$$

Dieses Ergebnis läßt sich unabhängig von der speziellen Methode zur Bestimmung eines risikoadjustierten Zinsfußes verallgemeinern. Ein einheitlicher, über alle Perioden und Zustände konstanter risikoadjustierter Zinsfuß impliziert, daß die pro Periode auftretende Unsicherheit über die Wertentwicklung der künftigen Zahlung (Periodenrisiko) konstant ist. Gleichzeitig bedeutet dies, daß sich das Gesamtrisiko im Zeitablauf gleichmäßig entsprechend diesem Periodenrisiko auflöst bzw. sich aus heutiger Sicht mit zunehmender zeitlicher Entfernung in einem bestimmten konstanten Verhältnis erhöht ${ }^{1}$.

Dies läßt sich z.B. veranschaulichen, indem das Sicherheitsäquivalent als proportionaler Bestandteil des erwarteten Rückflusses ausgedrückt wird ${ }^{2}$. Ist das pro Periode relevante Risiko konstant, erhöht sich dieser proportionale Bestandteil $\Omega$ im Zeitablauf gemäß der folgenden Beziehung ${ }^{3}$.

$$
\frac{S \ddot{A}_{t}}{E\left(C F_{t}\right)}=\Omega_{t} \equiv \gamma^{\prime}
$$

Für den Barwert des unsicheren Rückflusses gilt unter diesen Voraussetzungen folgende Diskontierungsformel.

$$
V_{0}=\sum_{t=1}^{n} \frac{\gamma^{t} \cdot E\left(C F_{t}\right)}{\left(1+r_{f}\right)^{t}}
$$

Über das Verhältnis zwischen dem risikolosen und dem innerhalb der Risikozuschlagsmethode verwendeten risikoadjustierten Zinsfuß läßt sich $\gamma$ direkt, d.h. ohne explizite Formulierung einer Nutzenfunktion, ermitteln ${ }^{4}$.

$1, \ldots$ the risk borne per period is constant, i.e. ... the total riskiness of future cash flows and hence the risk premium increases at a constant rate over time." Trigeorgis, Real Options, 1996, S. 35. Vgl. auch Schmidt/Terberger, Grundzüge, 1997, S. 368.

2 Vgl. ähnlich Ballwieser, Methoden, 1993, S. 158.

3 Vgl. Trigeorgis, Real Options, 1996, S. 35 f. Vgl. ähnlich bereits Robichek/Myers, Conceptual Problems, 1966, S. 727 ff., die jedoch auf Basis von individuellen Nutzenfunktionen argumentieren.

4 Vgl. Trigeorgis, Real Options, 1996, S. 37. Diese Beziehung ist materiell mit der bereits in Abschnitt 2.1.1 dieses Kapitels aufgezeigten Überleitung zwischen der Sicherheitsäquivalents- und der Risikozuschlagsmethode identisch. 


$$
\gamma=\frac{1+r_{f}}{1+r_{A}}
$$

Bei einem risikolosen bzw. risikoadjustierten Zinsfuß von $5 \%$ bzw. $25 \%$ beläuft sich $\gamma$ auf 0,84 . Der erwartete Cash Flow von $125 €$ nach einer Periode führt somit zu einem gültigen Sicherheitsäquivalent von $105 €$, dessen risikolose Diskontierung um eine Periode einen Barwert von $100 €$ hervorbringt. Nach zwei Perioden beträgt der Erwartungswert des unsicheren Rückflusses 156,25 $€$, wodurch bei einem proportionalen Bestandteil von $0,84^{2}(=0,71)$ ein relevantes Sicherheitsäquivalent von 110,25€ und aufgrund der risikolosen Abzinsung um zwei Perioden wiederum ein Barwert von $100 €$ resultiert.

Dieses proportionale Wachsen des Gesamtrisikos eines unsicheren Rückflusses mit zunehmender zeitlicher Entfernung des Anfalls läßt sich auch über die Standardabweichung $\sigma$ der zeitstetigen ${ }^{1}$ Rendite dieses Rückflusses zeigen. Nach einer Periode ergibt sich im Beispiel eine Standardabweichung von 0,69, wohingegen dieselbe nach zwei Perioden $0,98(=0,69 \cdot \sqrt{2})$ beträgt. Allgemein gilt zwischen dem Perioden- und dem Gesamtrisiko folgende Beziehung ${ }^{2}$.

$$
\sigma_{\text {Gesamt }}=\sigma_{\text {Periode }} \cdot \sqrt{t}
$$

Nur unter den eben aufgezeigten Prämissen, daß das Periodenrisiko konstant ist und sich das Gesamtrisiko mit zunehmender zeitlicher Entfernung in einem bestimmten konstanten Verhältnis erhöht, ist die mehrperiodige Diskontierung des Erwartungswerts eines unsicheren Rückflusses mit einem einheitlichen risikoadjustierten Zinsfuß gerechtfertigt.

Wäre beispielsweise die Unsicherheit über den Cash Flow am Ende der zweiten Periode ausschließlich durch den Verlauf der zweiten Periode begründet (vgl. auch Abb. 11), müßte der aus heutiger Sicht erwartete Betrag am Ende der zweiten Periode einmal mit dem risikoadjustierten und einmal mit dem risikolosen Zinsfuß abgezinst werden. Das Risiko in der ersten Periode ist gleich

1 Bei der zeitstetigen Rendite wird davon ausgegangen, daß Wertzuwächse nicht erst am Ende eines Zeitraums entstehen, sondern sich stetig im Laufe der Zeit bilden. Die zeitstetige Rendite $r_{\text {stetig }}$ wird wie folgt berechnet.

$$
r_{\text {stetig }}=\ln \frac{V_{t}}{V_{0}}
$$

2 Vgl. ăhnlich Sick, Real Options, 1995, S. 669. 
null, weshalb hier nur der risikolose Zinsfuß zur Diskontierung herangezogen werden darf ${ }^{l}$.

$$
V_{0}=\frac{156,25}{1,25 \cdot 1,05}=119 €
$$

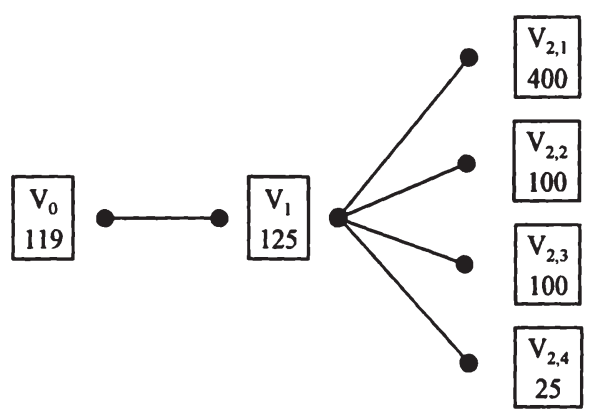

Abb. 11: Wertentwicklung einer unsicheren Zahlung bei spezifischem Periodenrisiko

Eine solche Vorgehensweise bedingt in der Diskontierungsformel allgemein eine multiplikative Verknüpfung der periodenspezifischen Zinssätze ${ }^{2}$.

$$
V_{0}=\sum_{\substack { t=1 \\
\begin{subarray}{c}{t \\
t=1{ t = 1 \\
\begin{subarray} { c } { t \\
t = 1 } }\end{subarray}} \frac{E\left(C F_{t}\right)}{A, \tilde{t}}
$$

Die für einen einzelnen unsicheren Rückfluß gemachten Aussagen gelten auch für ein mehrperiodiges Investitionsprojekt, das sich durch mehrere, zu unterschiedlichen Zeitpunkten erfolgende Rückflüsse auszeichnet. Bei traditionellen Bar- bzw. Kapitalwertmethoden werden die erwarteten periodenindividuellen Rückflüsse i.d.R. mit einem einheitlichen, perioden- und zustandsunabhängigen, risikoadjustierten Zinsfuß diskontiert. Dies unterstellt, daß sich das pro Periode angenommene Projektrisiko im Zeitablauf nicht verändert ${ }^{3}$.

1 Vgl. Copeland/Weston, Theory and Policy, 1988, S. 408; Schmidt, Discounted Cash FlowMethode, 1995, S. 1103; Drukarczyk, Unternehmensbewertung, 1998, S. 258 f.

2 Vgl. Ballwieser, Methoden, 1993, S. 158.

3 Vgl. u.a. Brealey/Myers, Principles, 1996, S. 228. Drukarczyk, Unternehmensbewertung, 1998, S. 315 und Schwetzler, Unternehmensbewertung, 1998, S. 9 sprechen von stochastischer Abhängigkeit der den einzelnen Perioden zugeordneten Überschußverteilungen. 


\section{Techniken zur Transparenz des Risikogehalts}

\subsection{Sensitivitätsanalyse}

Im vorigen Abschnitt ist ein einwertiges Ergebnis unter Unsicherheit ermittelt worden. Der Barwert einer unsicheren Zahlung wird unter der Annahme eines vorgegebenen Risikos errechnet. Mit Hilfe der im folgenden erläuterten Sensitivitäts- und Risikoanalysen wird hingegen versucht, durch mehrwertige Ergebnisse Einblicke über den Risikogehalt der zu bewertenden Rückflüsse bzw. des Investitionsprojekts zu gewinnen. Über die hierdurch zu erzielenden Informationen lassen sich einerseits kritische Grenzen aufzeigen und anderseits Planungsprämissen oder innerhalb der Korrekturverfahren verwendete Risikozuschläge überprüfen.

Im Rahmen der Sensitivitätsanalyse wird beispielsweise aufgezeigt, wie empfindlich eine Ergebnisgröße auf Veränderungen der im Rahmen eines Rechenverfahrens verwendeten Bewertungsparameter reagiert ${ }^{1}$. Wie verändert sich z.B. der Barwert, wenn die einzelnen Rückflüsse, der Kalkulationszinsfuß, der Planungshorizont oder die sie determinierenden Faktoren (Marktvolumen, Marktwachstum, Marktanteil, Absatzmengen, Preise, variable Stückkosten, Fixkosten usw.) modifiziert werden? Mit der Sensitivitätsanalyse sollen diejenigen kritischen Bewertungsparameter ausfindig gemacht werden, die den ursprünglich ermittelten Barwert besonders sensitiv gegenüber Veränderungen erscheinen lassen.

Innerhalb der Sensitivitätsanalyse lassen sich zudem mehrere Varianten unterscheiden ${ }^{2}$. Zum einen sind Aussagen zu gewinnen, die die prozentuale bzw. absolute ceteris paribus-Veränderung des Barwerts durch eine prozentuale bzw. absolute Erhöhung bzw. Senkung eines Bewertungsparameters darstellen. In einer erweiterten Fassung findet die gleichzeitige Modifikation mehrerer Inputgrößen statt. Zum anderen werden kritische Werte der Bewertungsparameter für den Fall gesucht, daß der Barwert eine bestimmte vorgegebene Abweichung überschreitet.

1 Vgl. allgemein Busse von Colbe/Laßmann, Investitionstheorie, 1990, S. 161 ff.; Kruschwitz, Investitionsrechnung, 1998, S. 257 ff. sowie im Rahmen der Unternehmensbewertung Janschek, Risiko, 1989, S. 112.

2 Vgl. stellvertretend Blohm/Lüder, Investition, 1995, S. 250 ff.; Kruschwitz, Investitionsrechnung, 1998, S. $258 \mathrm{ff}$. 


\subsection{Risikoanalyse}

Im Rahmen der Risikoanalyse wird eine vollständige Wahrscheinlichkeitsverteilung für die Ergebnisgröße aufgestellt ${ }^{1}$. Dazu müssen zuerst Verteilungen für die im Rahmen eines bestimmten Rechenverfahrens verwendeten Bewertungsparameter aufgestellt werden. Auf Basis dieser Verteilungen wird dann auf die Verteilung der Ergebnisgröße (z.B. Barwert) geschlossen ${ }^{2}$. Dies läßt sich über zwei verschiedene Wege bewerkstelligen.

Die analytische Variante der Risikoanalyse nutzt statistisch-theoretische Erkenntnisse, um aus den Einzelverteilungen der Bewertungsparameter eine Endverteilung des Barwerts zu erhalten ${ }^{3}$. Problematisch hieran ist die Berücksichtigung der Abhängigkeiten - insbesondere Korrelationen - zwischen den Bewertungsparametern. Zudem ist das zur Verfügung stehende statistische Instrumentarium vergleichsweise bescheiden, falls Funktionstypen angenommen werden, die nicht einer Normalverteilung entsprechen ${ }^{4}$.

Die in der Unternehmenspraxis eher angewandte simulative Variante umgeht das direkte Errechnen einer Endverteilung, indem durch eine Vielzahl von sogenannten Probeläufen eine Stichprobe für die Verteilung des Barwerts gebildet wird ${ }^{5}$. In jedem Probelauf werden auf Basis der angenommenen Verteilungen per Zufallsgenerator ganz bestimmte Ausprägungen der einzelnen Bewertungsparameter ausgewählt und anschließend jeweils ein Barwert errechnet. Bei einer genügend großen Anzahl an Probeläufen entsteht auf diesem Weg eine simulierte Wahrscheinlichkeitsverteilung des Barwerts.

Bei der innerhalb der Risikoanalyse zu erfolgenden Diskontierung stellt sich die Frage, welcher Zinsfuß hierfür verwendet werden soll. In der Literatur wird i.d.R. ein - sicherer - risikoloser Zinsfuß vorgeschlagen ${ }^{6}$. Dieses Vorgehen bedingt, daß die Endverteilung des Barwerts ohne Kenntnis der individuellen

1 Hierin unterscheidet sich grundsätzlich die Risiko- von der Sensitivitätsanalyse. Bei letzterer werden keine Informationen über die Wahrscheinlichkeiten der mehrwertigen Ergebnisse gewonnen.

2 Vgl. stellvertretend Blohm/Lüder, Investition, 1995, S. 263.

3 Vgl. Hillier, Probabilistic Information, 1963, S. 447 ff.

4 Vgl. ähnlich Coenenberg, Monte-Carlo-Simulation, 1970, S. 801.

5 Vgl. Hertz, Risk Analysis, 1964, S. 99 ff.

6 Vgl. Coenenberg, Monte-Carlo-Simulation, 1970, S. 800; Siegel, Risikoprofil, 1991, S. 630; Van Horne/Wachowicz, Fundamentals, 1992, S. 413; Brealey/Myers, Principles, 1996, S. 255. 
Risikoneigung des Entscheidungsträgers aufgestellt werden kann ${ }^{1}$. Wird der Erwartungswert dieser Endverteilung als Entscheidungswert angesehen, setzt dies risikoneutrales Verhalten voraus.

Zur Berücksichtigung eines risikoaversen Verhaltens sind weitere Überlegungen anzustellen. Aus der risikoneutralen Endverteilung ist derjenige Barwert auszusuchen, der hinsichtlich seines Risikonutzens einem sicheren Betrag gleichkommt ${ }^{2}$. Dies kann z.B. durch Minderung des Erwartungswerts um einen pauschalen Risikoabschlag oder mit Hilfe einer entscheidungstheoretisch fundierten Risikonutzenfunktion geschehen ${ }^{3}$. Andererseits ließen sich innerhalb der Risikoanalyse die Rückflüsse mit einem vorgegebenen - sicheren - risikoadjustierten Zinsfuß diskontieren. Dies würde zu einer risikobereinigten Verteilung des Barwerts führen, so daß der Erwartungswert dieser Endverteilung als Entscheidungswert gelten könnte.

Unter der Annahme eines sicheren Planungshorizonts bzw. einer sicheren Lebensdauer des Investitionsprojekts ${ }^{4}$ bedingt die Verwendung von sicheren Zinsfüßen ${ }^{5}$, daß streng genommen nicht der Barwert, sondern die Rückflüsse einer Risikoanalyse unterzogen werden. Unter diesen Bedingungen stimmt nämlich der Erwartungswert der Endverteilung mit dem Barwert überein, der sich ergibt, wenn die Erwartungswerte der Rückflüsse mit diesem sicheren, risikolosen oder risikoadjustierten, Zinsfuß diskontiert werden. Aus diesem Grund

1 Vgl. Siegel, Risikoprofil, 1991, S. 628.

2 Siegel schlägt vor, dieses Sicherheitsăquivalent durch graphische „Visualisierung“ (Anlegen einer Geraden an die kumulierte Wahrscheinlichkeitsverteilung) zu ermitteln. Vgl. Siegel, Risikoprofil, 1991, S. 630; Siegel, Unsicherheit, 1994, S. 469.

3 In diesem Zusammenhang ist darauf hinzuweisen, daß bei einer kapitalmarkttheoretischen Vorgehensweise nicht das Gesamtrisiko des Projekts, ausgedrückt durch die Standardabweichung der Projektrückflüsse, sondern lediglich dessen systematisches Risiko bewertungsrelevant ist. Vgl. Brealey/Myers, Principles, 1996, S. 255.

4 Bei unsicherem Planungshorizont liegt der Erwartungswert der Endverteilung unter dem Barwert der diskontierten Erwartungswerte der Rückflüsse, wobei der Unterschied jedoch marginal ist. Vgl. Lewellen/Long, Simulation, 1972, S. 23 ff.; Sautter, Strategische Analyse, 1989, S. 314 f.

5 Die Verwendung eines sicheren Zinsfußes ist konsistent mit dem im vorigen Abschnitt betrachteten Fall, in dem der Risikozuschlag lediglich für jenes Risiko entschädigt, das durch die Unsicherheit der Rückflüsse entsteht. Wird zusätzlich ein unsicherer risikoloser Zinsfuß angenommen, fallt eine Interpretation der auf diese Weise simulierten Barwertverteilung umso schwieriger. 
kann es nicht verwundern, daß umstritten ist, welche Informationen nun wirklich aus einer solchen Endverteilung zu ziehen sind ${ }^{1}$.

Für eine ökonomische Interpretation der Verteilung des Barwerts muß bei risikoloser Diskontierung der Rückflüsse entweder risikoneutrales Verhalten oder für den Fall der Risikoaversion ein Auflösen der Unsicherheit in der Sekunde nach Initiierung des Projekts unterstellt werden ${ }^{2}$. Wird zur Diskontierung der Rückflüsse innerhalb der Risikoanalyse hingegen ein risikoadjustierter Zinsfuß ausgewählt, der die Risikopräferenz des Entscheidungsträgers reflektiert, enthält die vollständige Endverteilung des Barwerts nicht mehr entscheidungsrelevante Informationen als der bloße Erwartungswert dieser Verteilung. Durch Betrachtung der gesamten Endverteilung muß der Entscheidungsträger zu der gleichen Entscheidung wie bei alleiniger Betrachtung des Erwartungswerts gelangen. Ist dies nicht der Fall, entspricht das Verhalten des Entscheidungsträgers nicht dem zuvor verwendeten risikoadjustierten Zinsfuß.

Für die Anwendung der Risikoanalyse ergeben sich hierdurch folgende Implikationen. Entweder werden die Rückflüsse in einem mehrwertigen Kalkül mit einem risikolosen Zinsfuß abgezinst, wodurch bei risikoaversem Verhalten das Problem entsteht, aus dieser Endverteilung ein Sicherheitsäquivalent $\mathrm{zu}$ bestimmen. Oder man verwendet die Risikoanalyse lediglich zur Ermittlung der erwarteten Rückflüsse, um diese dann in einem einwertigen Kalkül mit einem risikoadjustierten Zinsfuß zu diskontieren ${ }^{3}$. Ob sich die Ermittlung des Sicherheitsäquivalents auf Basis der Endverteilung des Barwerts oder die Bestimmung des risikoadjustierten Zinsfußes auf Basis der Verteilungen der Rückflüsse einfacher erweist, sei dahingestellt.

\section{Traditionelle Bewertungsverfahren und unternehmerische Flexibilität}

\subsection{Traditionelle Bar- und Kapitalwertmethoden}

In der traditionellen Bar- bzw. Kapitalwertmethode werden für die unsicheren Rückflüsse $E\left(C F_{t}\right)$ i.d.R. Erwartungswerte gebildet, die sich aus heutiger Sicht

1 Vgl. Van Horne/Wachowicz, Fundamentals, 1992, S. 415 f.; Schmidt/Terberger, Grundzüge, 1997, S. 303 f. Für Schmidt/Terberger kann es für den Bar- bzw. Kapitalwert „aufgrund seiner Funktion und Interpretation ... keine Wahrscheinlichkeitsverteilung" geben.

2 Vgl. Brealey/Myers, Principles, 1996, S. 255. Nach dieser Sekunde ist das betrachtete Unsicherheitsproblem nicht mehr existent, wodurch sich die Risikoanalyse erübrigt.

3 Vgl. so auch Brealey/Myers, Principles, 1996, S. 255. 
bei passivem Management ergeben. Die Fähigkeit, auf veränderte ökonomische Rahmenbedingungen in der Zukunft zu reagieren, fließt damit nicht in die Prognose ein. Die Erwartungswerte werden fortan mit einem risikoadjustierten Zinsfuß $r_{A}$ diskontiert, der dem Risiko des Investitionsprojekts entspricht ${ }^{1}$. Wird von diesem Barwert $V_{0}$ die heutige Investitionsauszahlung $I$ abgezogen, bleibt der Kapitalwert $K W_{0}$ des Projekts übrig.

$$
K W_{0}=V_{0}-I=\sum_{t=1}^{n} \frac{E\left(C F_{t}\right)}{\left(1+r_{A}\right)^{t}}-I
$$

Zur Verdeutlichung sei in einem einperiodigen Modell ein Unternehmen angenommen, daß vor der Entscheidung über die Einführung eines neuen Produkts steht. Für den Aufbau des notwendigen Vertriebsnetzes sind 90 Mio. $€$ zu veranschlagen. Am Ende der Periode wird von zwei gleichwahrscheinlichen Umweltzuständen ausgegangen. Bei hoher Produktnachfrage generiert das Basisprojekt einen Rückfluß von 200 Mio. $€$, während bei niedriger Produktnachfrage lediglich ein Rückfluß von 50 Mio. $€$ resultiert ${ }^{2}$. Der Erwartungswert des Rückflusses des Basisprojekts beträgt somit 125 Mio. $€$. Bei einem risikoadjustierten Zinsfuß von $25 \%$ beläuft sich der Barwert dieses unsicheren Rückflusses auf 100 Mio. $€$ und der traditionelle Kapitalwert auf 10 Mio. $€$.

$$
K W_{0}=\frac{0,5 \cdot 200+0,5 \cdot 50}{1,25}-90=10 \text { Mio. } €
$$

Besteht die Möglichkeit, den Start des Projekts zu verschieben, muß die Investitionssumme erst am Ende der Periode zur Verfügung stehen. Eine "naive“ Anwendung der Kapitalwertmethode reduziert lediglich die - hier aus Vereinfachungsgründen als konstant angenommene - Investitionsauszahlung. Bei einem risikolosen Zinsfuß von 5\% bedingt dies ein Zinsgewinn von 4,3 $(90 / 1,05)$ Mio. $€$, so daß ein naiver Kapitalwert von 14,3 Mio. $€$ resultiert.

Eine solche starre Investitionsplanung vernachlässigt jedoch die mit dem Entscheidungsaufschub verbundene Flexibilität. Sie stellt deshalb eine unsinnige Vorgehensweise dar. Das Unternehmen kann bis zum Ende der Periode warten, um dann in Abhängigkeit der Umweltentwicklung zu entscheiden. Bei niedri-

1 Die gleiche Vorgehensweise liegt den traditionellen Ertragswert- bzw. DCF-Methoden zugrunde. Vgl. hierzu Abschnitt 1.2.4 in Kapitel I.

2 An Stelle eines einzelnen Rückflusses lassen sich die angegebenen Beträge auch als Gegenwartswerte der ab diesem Zeitpunkt in den einzelnen Umweltzuständen erwarteten Rückflüsse verstehen. 
ger Produktnachfrage wird sich das Unternehmen gegen die Produkteinführung aussprechen, da der Rückfluß von 50 Mio. $€$ die Investitionsauszahlung von 90 Mio. $€$ unterschreitet. Wird ein aus heutiger Sicht bei passivem Management vorzufindender Erwartungswert gebildet, können im Bewertungskalkül Rückflüsse enthalten sein, die auf keinen Fall realisiert werden. Implizit wird dadurch von einer Jetzt oder Nie-Entscheidung ausgegangen.

Da Sensitivitäts- oder Risikoanalysen keine eigenständige, sondern ergänzende Verfahren im Umgang mit Unsicherheit darstellen, gelten die für eine besondere Bewertungsmethode getätigten Aussagen folglich auch für Sensitivitäts- oder Risikoanalysen, die auf dieser Methode aufbauen ${ }^{1}$. Werden beispielsweise für Sensitivitäts- oder Risikoanalysen in der Unternehmenspraxis traditionelle Barbzw. Kapitalwertmethoden herangezogen, sind erstere ebenfalls mit den eben erläuterten Schwächen behaftet.

\subsection{Asymmetrische Risikostruktur als Folge unternehmerischer Flexibilität}

Die Berücksichtigung eines unternehmerischen Handlungsspielraums im Bewertungsverfahren wird durch die Tatsache erschwert, daß durch seine Existenz für den zu diskontierenden Rückfluß ein anderer Verteilungstyp vorliegt, als dies ohne Handlungsspielraum der Fall wäre. Um dies zu verdeutlichen, sei in einem Einperiodenmodell zuerst auf eine Investition ohne Handlungsspielraum (= Basisprojekt) verwiesen ${ }^{2}$. Nimmt man z.B. an, der unsichere Rückfluß des Basisprojekts $C F$ am Ende der Periode gehorche einer Normalverteilung ${ }^{3}$, so folgt daraus, daß die möglichen Rückflüsse des Basisprojekts symmetrisch um den Erwartungswert $E(C F)$ gestreut sind. Dieser Erwartungswert charakterisiert den Rückfluß des Basisprojekts mit der höchsten Eintrittswahrscheinlichkeit (vgl. auch Abb. 12).

Besteht die Möglichkeit, die Investitionsentscheidung um eine Periode zu verzögern und in der Zwischenzeit Sicherheit über den „wahren“ Wert des Rückflusses des Basisprojekts zu erlangen, stellt sich folgende Situation ein. Falls der Rückfluß des Basisprojekts $C F$ die Investitionsauszahlung $I$ unterschreitet, wird nicht investiert. Der Rückfluß aus der Investitionsmöglichkeit (= Ba-

1 Vgl. auch Meise, Realoptionen, 1998, S. 43.

2 Zur folgenden Argumentation vgl. auch Trigeorgios, Real Options, 1996, S. $121 \mathrm{ff}$.

3 Die folgende Argumentation läßt sich auch für einen anderen Verteilungstyp durchführen, ohne das resultierende Ergebnis grundsätzlich zu verändern. 
sisprojekt + Handlungsspielraum) $C F-I$ ist für diese Fälle gleich null. Übersteigt der Rückfluß des Basisprojekts am Ende der Periode die Investitionsauszahlung, wird investiert. Für diese Fälle ist der Rückfluß aus der Investitionsmöglichkeit größer als null.
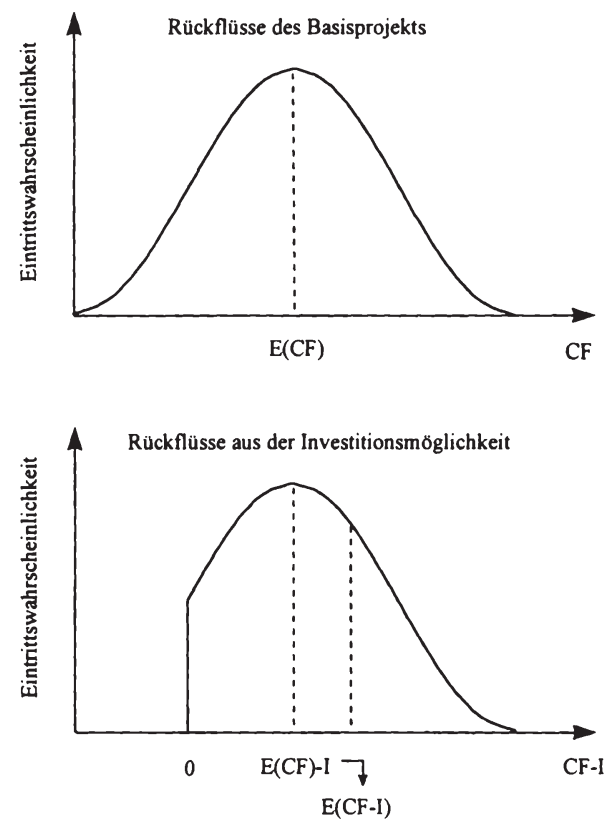

Abb. 12: Asymmetrische Risikostruktur bei Existenz von unternehmerischen Handlungsspielrăumen

Quelle: In Anlehnung an Trigeorgis, Options Framework, 1988, S. 147.

Die durch den Entscheidungsaufschub gewonnene Flexibilität führt dazu, daß die Verteilung des Rückflusses aus der Investitionsmöglichkeit an der Stelle $C F=I$ abrupt abbricht ${ }^{1}$. Fälle, in denen der Rückfluß des Basisprojekts die Investitionsauszahlung unterschreitet, kann keine Eintrittswahrscheinlichkeit mehr zugeordnet werden. Der Erwartungswert des Rückflusses aus der Investitionsmöglichkeit $E(C F-I)$ ist nicht der Betrag mit der höchsten Eintrittswahrscheinlichkeit. Auf der Abzisse befindet sich dieser Erwartungswert rechts von dem wahrscheinlichsten Wert, der mit $E(C F)$ - I gekennzeichnet werden kann.

1 In Abb. 12 ist vereinfachend von einem sicheren Investitionsbetrag ausgegangen worden. Die gleiche Argumentation ließe sich auch für eine unsichere Investitionsauszahlung führen. 
Die Existenz des unternehmerischen Handlungsspielraums bedingt, daß die möglichen Rückflüsse aus der Investitionsmöglichkeit nicht symmetrisch um ihren Erwartungswert gestreut sind. Im Vergleich zu den Rückflüssen des Basisprojekts gehorchen sie einer anderen Verteilung. Letztere ist insbesondere durch eine asymmetrische Risikostruktur gekennzeichnet. Dies ist eine wichtige Schlußfolgerung für die im nächsten Abschnitt diskutierte Anwendung des Entscheidungsbaumverfahrens.

\subsection{Flexible Investitionsplanung und Entscheidungsbaumverfahren}

Kann der Start eines Investitionsprojekts in die Zukunft verschoben werden, ist es nicht sinnvoll, bereits heute eine endgültige Entscheidung für oder gegen das geplante Vorhaben zu treffen. Über eine solche Entscheidung sollte erst dann nachgedacht werden, wenn der betreffende zukünftige Zeitpunkt eingetreten ist, damit alle bis dahin aufgelaufenen Informationen berücksichtigt werden kön$\mathrm{nen}^{1}$. Da in einer solchen Situation die aus der Investitionsmöglichkeit zu erwartenden Rückflüsse nicht nur von dem unsicheren Eintritt zukünftiger Umweltzustände, sondern auch von den zukünftigen Entscheidungen des Unternehmens abhängen, sind diese beiden Einflußfaktoren in einem geeigneten Bewertungsverfahren gemeinsam zu berücksichtigen ${ }^{2}$.

Einen solchen Ansatz stellt die flexible Investitionsplanung dar ${ }^{3}$. Dessen Gedankengut kann am besten durch einen sogenannten Entscheidungsbaum visualisiert werden. Beim Entscheidungsbaumverfahren werden künftige, von unsicheren Umweltentwicklungen abhängige, Entscheidungen explizit in die Bewertung unter Unsicherheit einbezogen. Dadurch wird der in Abschnitt 4.1 dieses Kapitels geschilderte Mangel der traditionellen Bar- bzw. Kapitalwertmethoden behoben und die Investition bei optimaler Ausübung der unternehmerischen Handlungsspielräume bewertet. Bei zukünftigen Entscheidungen wird jeweils die Alternative in die Bewertung einbezogen, bei der der höhere Rückfluß erwartet wird. Die jeweils dominierten Alternativen sind für die Bewertung zu eliminieren. Abb. 13 zeigt den vollständigen Entscheidungsbaum der zuvor in Abschnitt 4.1 beschriebenen Investitionsmöglichkeit.

Vgl. Laux, Unsicherheit, 1971, S. 526.

2 Vgl. ähnlich Bretzke, Unternehmungsbewertung, 1993, S. 40.

3 Vgl. allgemein zur flexiblen Investitionsplanung Franke/Hax, Finanzwirtschaft, 1994, S. 273 f.; Adam, Planung, 1996, S. 299 f.; Schmidt/Terberger, Grundzüge, 1997, S. 305 f.; Kruschwitz, Investitionsrechnung, 1998, S. 276 ff. 


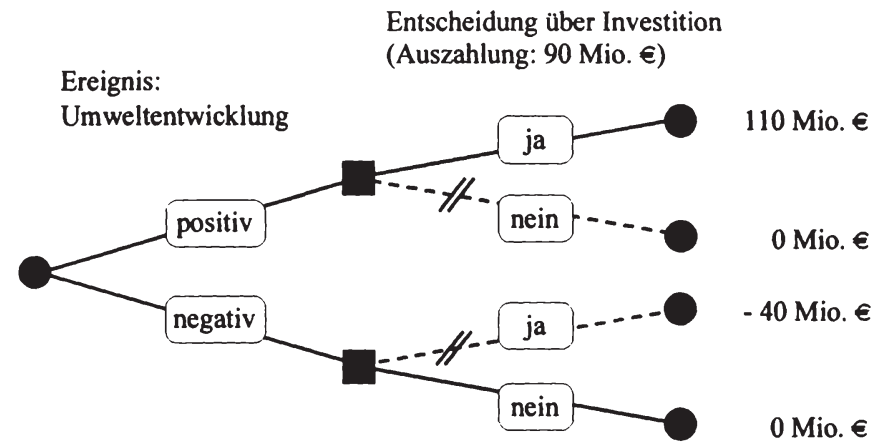

- Ereignisknoten antscheidungsknoten // dominierte Alternative

Abb. 13: Entscheidungsbaum bei flexibler Investitionsplanung

Der Wert der Investitionsmöglichkeit beträgt am Ende der Periode bei hoher Produktnachfrage 110 Mio. $€$ und bei niedriger Produktnachfrage 0 Mio. $€$. Um den Barwert dieser Rückflüsse zu ermitteln, kann der vorher verwendete risikoadjustierte Zinsfuß von $25 \%$ nicht mehr herangezogen werden, da die Rückflüsse der Investitionsmöglichkeit (110 bzw. 0 Mio. $€$ ) eine andere Risikostruktur aufweisen als jene des Basisprojekts (200 bzw. 50 Mio. $€$ ). Folglich muß ein ,neuer“ risikoadjustierter Zinsfuß bestimmt werden, der der durch den Handlungsspielraum bedingten asymmetrischen Risikostruktur gerecht wird.

Dies scheint für das angeführte Beispiel mit einer Periode und zwei Umweltzuständen nicht allzu schwer zu sein. Das Problemausmaß, das die Neubestimmung des Zinsfußes hervorruft, wird jedoch klar, wenn mehrere Perioden mit mehreren Umweltzuständen angenommen werden. Die Existenz von unternehmerischen Handlungsspielräumen kann unter Umständen ein kontinuierlich wechselndes Projektrisiko zur Folge haben mit der Notwendigkeit, für jede Periode und jeden Umweltzustand den hierfür entsprechenden Zinsfuß zu bestimmen. Aus Vereinfachungsgründen könnte ein für alle Perioden und Umweltzustände konstanter Zinsfuß verwendet werden, der das durchschnittliche Risiko des Projekts widerspiegelt. Zweifelsohne ist dies jedoch keine ökonomisch korrekte Bewertung des Projekts. Der Bewertungsfehler, der auf diese Weise begangen wird, ist insbesondere dann nicht trivial, wenn sich das Risiko des Projekts im Zeitablauf gravierend verändert.

In der deutschsprachigen Literatur findet sich auch der Vorschlag, auf zustandsund periodenabhängige Zinsfüße zu verzichten und statt dessen eine nichtrisikobereinigte Wahrscheinlichkeitsverteilung für das Vermögen am Ende des 
Planungshorizonts aufzustellen'. Das von Laux vorgeschlagene Modell läuft gedanklich auf einen Vergleich zwischen dem erwarteten Nutzen des Endwerts bei Investition und demjenigen bei Unterlassung der Investition hinaus.

Für eine Anwendung in der Praxis lastet diesem Ansatz jedoch die gleiche Schwäche wie einer Risikoanalyse an, die erwartete Rückflüsse unter expliziter Berücksichtigung der zukünftigen zustandsabhängigen Entscheidungen simuliert. Durch den Verzicht auf perioden- und zustandsabhängige Zinsfüße ${ }^{2}$ (bzw. Nutzenfunktionen ${ }^{3}$ ) wird das eigentliche Problem der ökonomisch korrekten Risikoberücksichtigung nur verlagert. Spätestens bei Betrachtung der erstellten Verteilung muß sich der Entscheidungsträger über seine Risikopräferenzen bewußt werden. $\mathrm{Ob}$ dies mit einer entscheidungstheoretisch fundierten Risikonutzenfunktion oder pauschal mit „einfachem“ Blick auf die Verteilung zu bewerkstelligen ist, kann angezweifelt werden ${ }^{4}$.

Eine im Vergleich zur flexiblen Investitionsplanung ähnliche Vorgehensweise findet sich in der Optionspreistheorie wieder. Deren Bewertungsmodelle umgehen das durch den unternehmerischen Handlungsspielraum hervorgerufene Problem der adäquaten Risikoberücksichtigung. Voraussetzung ist, daß der Barwert des Basisprojekts beobachtet bzw. abgeschätzt werden kann, wobei u.U. auf traditionelle Barwertmethoden zurückgegriffen werden muß. Die Ermittlung eines risikoadjustierten Zinsfußes zur Diskontierung der erwarteten Rückflüsse ist jedoch beim Basisprojekt verhältnismäßig einfacher als im Rahmen der Investitionsmöglichkeit, da das Basisprojekt definitionsgemäß keinen unternehmerischen Handlungsspielraum beinhaltet.

Bei Kenntnis des Barwerts des Basisprojekts läßt sich der Wert der Investitionsmöglichkeit ermitteln, ohne die jeweilige perioden- und zustandsabhängige Risikoprämie explizit bestimmen zu müssen. Es wird lediglich darauf geachtet, daß die Investitionsmöglichkeit relativ zum Basisprojekt ,richtig“ bewertet ist. Diese Lösung der Optionspreistheorie im Umgang mit asymmetrischer und sich im Zeitablauf kontinuierlich verändernder Risikostruktur steht im Vordergrund der Ausführungen des nächsten Kapitels.

1 Vgl. Laux, Unsicherheit, 1971, S. 528 ff.; Laux, Flexible Investitionsplanung, 1971, S. $45 \mathrm{ff}$.

2 Eine Ausnahme findet sich bei Krag, Ungewißheit, 1978, S. 442 ff., der bestimmten Bereichen im Entscheidungsbaum unterschiedliche (nicht näher erläuterte) Zinsfüße zuordnet.

3 Zum Einsatz zeit- und zustandsabhängiger Nutzenfunktionen vgl. Haumer, Investitionsplanung, 1983, S. 43 ff.; von Nitzsch, Investitionsbewertung, 1997, S. 85 ff.

4 Vgl. ähnlich kritisch Ballwieser, Möglichkeiten der Komplexitătsreduktion, 1980, S. 68 ff. 


\section{Kapitel IV}

\section{Bewertung von Investitionsmöglichkeiten auf Basis finanzwirtschaftlicher Optionspreismodelle}

\section{Grundlagen der finanz- und realwirtschaftlichen Optionspreistheorie}

\subsection{Grundbegriffe und Funktionsweise von Finanzoptionen}

Eine Finanzoption ist ein Titel, der dem Inhaber das Recht einräumt, einen am Kapitalmarkt gehandelten Vermögensgegenstand innerhalb einer bestimmten Frist (Optionsfrist) zu einem im voraus festgelegten Basispreis zu kaufen (Call, Kaufoption) oder zu verkaufen (Put, Verkaufsoption). Als zugrunde liegende Vermögensgegenstände (underlying assets, Bezugsgüter) von Finanzoptionen sind Aktien, Anleihen und Devisen üblich. Grundsätzlich kommt jeder Finanztitel bzw. jede Kombination von Finanztiteln (z.B. Indizes) in Frage, sofern hierfür auf einem Kapitalmarkt ein Preis gegeben ist ${ }^{1}$. Im folgenden werden ausschließlich Aktienoptionen betrachtet.

Nimmt der Optionsinhaber das ihm gewährte Recht wahr, erhält er bei einer Kaufoption die dem Optionsgeschäft zugrunde liegende Aktie und zahlt dafür den Basispreis. Bei einer Verkaufsoption liefert er bei Ausübung die entsprechende Aktie und erhält den Basispreis. Es wird zwischen sogenannten amerikanischen Optionen, die zu jedem Zeitpunkt innerhalb der Optionsfrist, und europäischen Optionen, die nur am Ende der Optionslaufzeit ausgeübt werden können, unterschieden ${ }^{2}$. Die Ausübung einer Aktienoption hängt von der Entwicklung des Aktienkurses ab. Der Optionsinhaber wird die Option nur dann ausüben, wenn er dadurch einen finanziellen Vorteil erzielen kann. Er ist so gegen eine ungünstige Entwicklung des Aktienkurses geschützt und sein Risiko ist dementsprechend asymmetrisch verteilt. So ist z.B. bei Kaufoptionen der maximale Verlust auf den gezahlten Optionspreis begrenzt, während die Gewinnmöglichkeit keiner Einschränkung nach oben unterliegt.

Der Wert einer Aktienoption setzt sich aus zwei Komponenten zusammen. Der innere Wert entspricht dem möglichen Gewinn bei sofortiger Ausübung. Bei einer Kaufoption ist es der Betrag, um den der aktuelle Aktienkurs den Basispreis übersteigt, bei einer Verkaufsoption wird er genau umgekehrt ermittelt.

\footnotetext{
Vgl. Kruschwitz, Finanzierung und Investition, 1999, S. 263 f.

2 Vgl. zu folgendem Brealey/Myers, Principles, 1996, S. 558 ff.; Steiner/Bruns, Wertpapiermanagement, 1998, S. $267 \mathrm{ff}$.
} 
Die zweite Komponente des Optionswertes ist der Zeitwert, der durch die Möglichkeit entsteht, die unsichere Entwicklung des Aktienkurses abzuwarten, bevor eine Kauf- oder Verkaufentscheidung im Hinblick auf die dem Optionsgeschäft zugrunde liegende Aktie getroffen wird. Am Ende der Optionsfrist ist der Wert einer Aktienoption eindeutig bestimmt. Er entspricht dann dem inneren Wert. Welchen Wert besitzt eine Aktienoption jedoch zu Beginn bzw. während der Optionslaufzeit? Dieses Problem wird in den Bewertungsmodellen der Optionspreistheorie ${ }^{1}$ behandelt.

\subsection{Systematisierung finanzwirtschaftlicher Optionspreismodelle}

In der Literatur sind eine ganze Reihe von Systematisierungen zu finden, die sich hinsichtlich Umfang der erfaßten Optionspreismodelle sowie Benennung der einzelnen Modellklassen unterscheiden ${ }^{2}$. Die Grundeinteilung ist jedoch ähnlich und geht auf Smith zurück. Im folgenden wird der Vorgehensweise von Terstege gefolgt, da sie die umfassendste Ausführung darstellt.

Auf der ersten Ebene wird zwischen Ad Hoc-Modellen und gleichgewichtsorientierten ${ }^{3}$ Bewertungsmodellen differenziert. Ad Hoc-Modelle zeichnen sich dadurch aus, daß sie auf Basis vergangener Daten einen Zusammenhang zwischen den beobachtbaren Optionspreisen und den vermuteten Einflußgrößen herstellen ${ }^{4}$. Dies geschieht mit Hilfe statistischer oder ökonometrischer Modelle. Die aufgrund von vergangenheitsorientierten Daten gefundenen Beziehungen werden dann in die Zukunft projeziert. Problematisch an diesen Mo-

1 Streng genommen handelt es sich um eine Optionsbewertungstheorie, da die im nächsten Abschnitt angefuhrten Bewertungsmodelle nicht den Preis einer Option an einem bestimmten Kapitalmarkt, sondern den Wert bzw. Wertgrenzen einer Option unter bestimmten, teilweise idealisierten, Marktbedingungen ermitteln. Der Preis einer am Kapitalmarkt gehandelten Option ergibt sich durch Angebot und Nachfrage. Vgl. hierzu auch die Ausfuhrungen bei Terstege, Optionsbewertung, 1995, S. 27 ff.

2 Vgl. stellvertretend Smith, Review, 1976, S. 3 ff.; Geske/Trautmann, Option Valuation, 1986, S. 81 ff.; Bös, Optionsbewertung, 1991, S. 33 ff.; Hauck, Optionspreise, 1991, S. 161 ff.; Schäfer, Optionsbewertung, 1994, S. 25 ff.; Terstege, Optionsbewertung, 1995, S. 30 ff., Beinert, Kurssprünge, 1997, S. 9 ff.; Perridon/Steiner, Finanzwirtschaft, 1997, S. 321 ff.; Andres, GARCH-Optionsbewertungsmodell, 1998, S. 125 ff.

3 Das Attribut gleichgewichtsorientiert macht deutlich, daß in diesen Modellen eine Gleichgewichtsrelation zwischen Bezugsgut und Option vor dem Hintergrund eines arbitragefreien Kapitalmarkts hergestellt wird. Ob neben diesem relativen auch ein absolutes Gleichgewicht besteht, in dem sich die beiden betrachteten Werttitel im Gleichgewicht zu anderen existierenden Vermögensgütern befinden, ist nicht Gegenstand dieser Optionspreismodelle.

4 Zu einem Überblick über Ad Hoc-Modelle vgl. z.B. Bös, Optionsbewertung, 1991, S. 34 ff. 
dellen ist sowohl die Auswahl der relevanten Einflußgrößen als auch die Bestimmung des zu beobachtenden Zeitraums, da davon auszugehen ist, daß der Zusammenhang zwischen den Variablen nicht zeitstabil ist ${ }^{1}$. Außerdem fehlt diesen Ansätzen eine theoretisch fundierte, deduktive Vorgehensweise ${ }^{2}$.

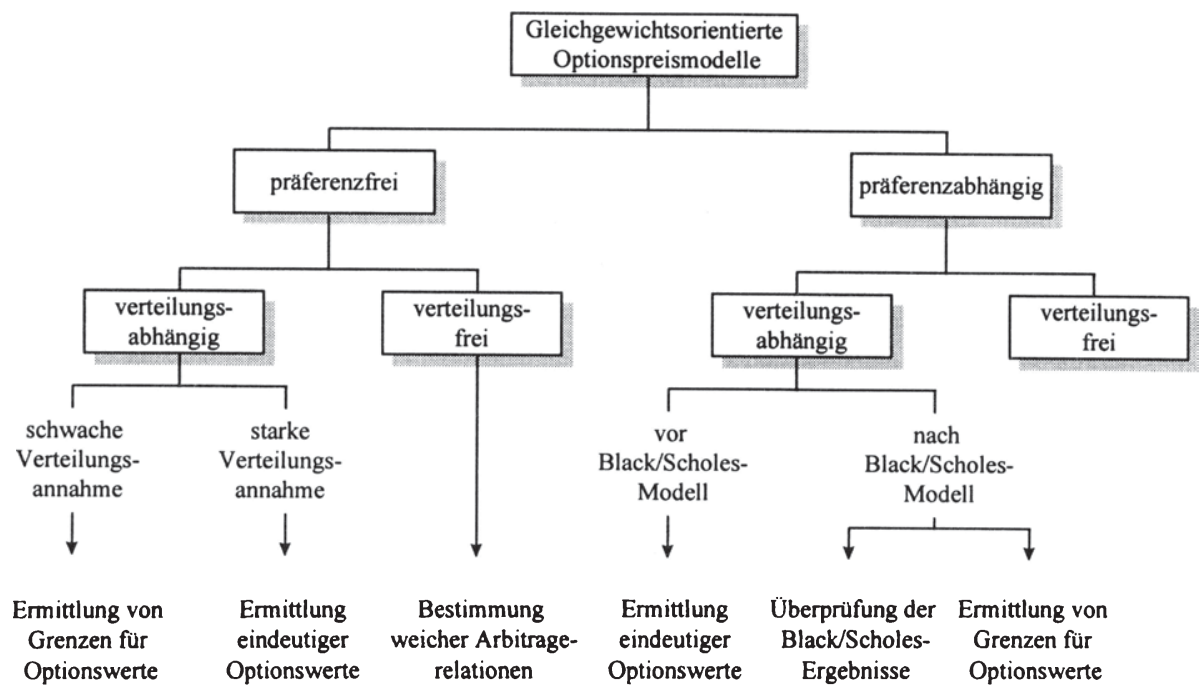

Abb. 14: Überblick über gleichgewichtsorientierte Optionspreismodelle

Die gleichgewichtsorientierten Bewertungsansätze können danach unterschieden werden, ob sie zum einen Annahmen über die Risikopräferenzen der Kapitalmarktteilnehmer unterstellen ${ }^{3}$ und zum anderen eine Wahrscheinlichkeitsverteilung des Aktienkurses voraussetzen (vgl. Abb. 14). Präferenzfreie Modelle, die eine „starke“ Verteilungsannahme voraussetzen, sind die bekanntesten und am weitesten verbreiteten Optionspreisansätze. So geht z.B. das Cox/ Ross/Rubinstein-Modell von einer Binomialverteilung und das Black/Scholes-

1 Vgl. Bös, Optionsbewertung, 1991, S. 36.

2 Aufgrund der vergangenheitsorientierten Vorgehensweise bezeichnet Terstege solche Ansătze als Optionsbeschreibungsmodelle. Vgl. Terstege, Optionsbewertung, 1995, S. 33.

3 Smith unterscheidet zwischen „incomplete“ und „general equilibrium models“, je nachdem ob Präferenzen der Kapitalmarktteilnehmer explizit einbezogen werden oder nicht. Perridon/Steiner bezeichnen dies als partielle und vollständige Gleichgewichtsmodelle und Bös trennt zwischen Gleichgewichtsmodellen mit und ohne Hedge-Ansatz. Vgl. Smith, Review, 1976, S. 13 ff.; Bös, Optionsbewertung, 1991, S. 39 ff.; Perridon/Steiner, Finanzwirtschaft, 1997, S. 322. 
Modell von einer logarithmischen Normalverteilung des Aktienkurses aus ${ }^{1}$. Anhand von Gleichgewichtsüberlegungen zwischen der Aktie und der Option wird auf einen eindeutigen Optionswert geschlossen. Bewertungsmodelle, die nur eine "schwache“ Verteilungsannahme voraussetzen, sind lediglich in der Lage, einen Bereich an möglichen Optionswerten anzugeben ${ }^{2}$. Präferenzfreie Ansätze, die zudem verteilungsfrei sind, bilden eine Minderheit unter den gleichgewichtsorientierten Bewertungsmodellen. Sie erlauben weder eine eindeutige Bestimmung des Optionswerts noch die Angabe von Wertgrenzen. Hier sind nur ,weiche“ Arbitragerelationen (zwischen Aktie und Option) ableitbar ${ }^{3}$.

Präferenzabhängige Ansätze mit Verteilungsannahme lassen sich danach einteilen, ob sie vor oder nach dem Black/Scholes-Modell entstanden sind. In den älteren Ansätzen wird aus einer angenommenen Verteilung des Aktienkurses für den Endzeitpunkt der Optionsfrist eine Verteilung für den Optionswert generiert ${ }^{4}$. Der Erwartungswert dieser Verteilung wird mit Hilfe eines Kalkulationszinsfußes auf den heutigen Zeitpunkt diskontiert. Die bei der Zinsfußbestimmung erfolgende Einbeziehung des speziellen, vom Aktienkursrisiko verschiedenen, Optionsrisikos stellt das eigentliche Problem dieser Bewertungsansätze dar. Häufig wird der risikolose Zinsfuß verwendet und damit risikoneutrales Verhalten der Marktteilnehmer unterstellt.

Ein Teil der nach der Arbeit von Black und Scholes entwickelten Ansätze beschäftigen sich mit der Überprüfung der Black/Scholes-Ergebnisse. Fragestellungen, $\mathrm{zu}$ welchen Bewertungsergebnissen man gelangt, wenn die im Black/Scholes-Modell angenommenen permanenten Hedge- und Duplikationsmöglichkeiten nicht vorhanden sind, stehen im Vordergrund. Einige Autoren zeigen, daß unter der Annahme bestimmter Präferenzen der Marktteilnehmer - so z.B. konstante proportionale Risikoaversion - die im Black/ScholesModell gewonnenen Bewertungsergebnisse auch für Marktsituationen ohne Hedge- und Duplikationsmöglichkeiten gelten ${ }^{5}$. Eine andere Gruppe von Ansätzen, die nach dem Black/Scholes-Modell entstanden sind und bestimmte

1 Vgl. die in Abschnitt 3 und 4 dieses Kapitels zitierte Literatur.

2 Im Ansatz von Levy und Levy wird beispielsweise eine Gleichverteilung für einen nach unten und oben begrenzten Wertebereich angenommen. Vgl. hierzu die Ausführungen bei Bös, Optionsbewertung, 1991, S. 74 ff. sowie Levy/Levy, Extension, 1991, S. 49 ff.

3 Vgl. hierzu Terstege, Optionsbewertung, 1995, S. 91 ff.

4 Für einen Überblick dieser Arbeiten vgl. Smith, Review, 1976, S. 15 ff. sowie Bös, Optionsbewertung, 1991, S. 39 ff.

5 Für einen Überblick vgl. Jurgeit, Bewertung von Optionen, 1989, S. 143 ff. 
Präferenzen der Marktteilnehmer unterstellen, zielt nicht darauf ab, eindeutige Optionswerte zu ermitteln. Vielmehr werden hier vor dem Hintergrund konkreter Marktunvollkommenheiten (wie z.B. diskrete Handelsmöglichkeiten) Wertunter- und -obergrenzen abgeleitet ${ }^{1}$.

Einige präferenzabhängige Modelle unterstellen keine Verteilung des Aktienkurses. Obwohl diese Ansätze in der Optionspreistheorie praktisch keine Rolle spielen, ist diese Unterteilung in der Übersicht in Abb. 14 der Vollständigkeit halber aufgeführt.

Im Rahmen dieser Arbeit werden ausschließlich präferenzfreie Optionspreismodelle mit starker Verteilungsannahme angewandt. Zum einen erlauben sie die Ermittlung eindeutiger Optionswerte, welche vor dem Hintergrund der vorliegenden Entscheidungssituation erstrebenswert erscheint, und zum anderen sind sie in der Lage, eine konsistente Einbeziehung des Optionsrisikos im Vergleich zum Risiko des Bezugsguts sicherzustellen.

\subsection{Arbitragefreier Kapitalmarkt als zentrale Annahme gleichgewichts- orientierter Optionspreismodelle}

Gleichgewichtsorientierte Optionspreismodelle gehen grundsätzlich von einem arbitragefreien Kapitalmarkt aus. Ein Kapitalmarkt ist arbitragefrei, wenn er keine sogenannten Free Lunch-Situationen erlaubt. Dies bedeutet, daß keine Vermögensposition existiert, die zu einem zukünftigen Zeitpunkt mit positiver Wahrscheinlichkeit eine Einzahlung verspricht (und mit Sicherheit keine Auszahlung verlangt), ohne heute eine Auszahlung zu fordern ${ }^{2}$. Diese Annahme setzt folglich voraus, daß bei kurzfristiger Existenz solcher Vermögenspositionen die hierdurch entstehende Möglichkeit zur Realisation risikoloser Gewinne durch reaktionsschnelle Marktteilnehmer ausgeräumt wird.

Auf einem arbitragefreien Kapitalmarkt weisen Vermögenspositionen, die in allen zukünftigen Umweltzuständen die gleichen Rückflüsse versprechen, den gleichen Preis auf ${ }^{3}$. Daraus folgt, daß durch bloße Zerlegung bzw. Zusammenführung von Vermögenspositionen kein risikoloser Gewinn erzielt werden kann. Der Preis eines Portfolios ist gleich der Summe der Preise der in ihm

1 Vgl. Terstege, Optionsbewertung, 1995, S. 43 f. sowie die dort zitierte Literatur.

$2 \mathrm{Zu}$ Arbitragegelegenheiten unter Unsicherheit vgl. Kruschwitz, Finanzierung und Investition, 1999, S. $141 \mathrm{ff}$.

3 Vgl. Nietert/Wilhelm, Arbitrage, 1998, S. 5. 
enthaltenen Werttitel (Wertadditivitätstheorem) ${ }^{1}$. Andererseits muß eine Vermögensposition, die in allen Umweltzuständen außer einem, die gleichen Rückflüsse wie eine andere Vermögensposition verspricht und in eben diesem Umweltzustand einen höheren Rückfluß garantiert, vergleichsweise einen höheren Preis aufweisen ${ }^{2}$.

Formal läßt sich die Arbitragefreiheit wie folgt definieren. In einer Modellwelt mit Unsicherheit über den Eintritt des zukünftigen Umweltzustands existiert kein Portfolio, das einerseits mit Sicherheit eine nicht negative Zahlung verspricht und andererseits einen negativen Preis hat. Ein Portfolio $P F$, das aus $i$ Wertpapieren mit jeweiligem Anteil $\tilde{x}_{i}$, erwartetem Rückfluß $C F_{i}$ sowie heutigem Preis $P_{M, i}$ besteht und mit Sicherheit $(q=1)$ einen nicht negativen Rückfluß garantiert, muß einen nicht negativen Preis $P_{M, P F}$ aufweisen ${ }^{3}$.

$$
q\left(\sum_{i=1}^{I} \tilde{x}_{i} \cdot C F_{i} \geq 0\right)=1 \Rightarrow \sum_{i=1}^{l} \widetilde{x}_{i} \cdot P_{M, i}=P_{M, P F} \geq 0
$$

Verspricht dieses Portfolio zudem mit positiver Wahrscheinlichkeit einen strikt positiven Rückfluß, besitzt es einen strikt positiven Preis ${ }^{4}$.

$$
q\left(\sum_{i=1}^{I} \widetilde{x}_{i} \cdot C F_{i} \geq 0\right)=1 \text { und } q\left(\sum_{i=1}^{I} \widetilde{x}_{i} \cdot C F_{i}>0\right)>0 \Rightarrow \sum_{i=1}^{I} \widetilde{x}_{i} \cdot P_{M, i}=P_{M, P F}>0
$$

Dieser Zusammenhang macht ebenfalls deutlich, daß wenn das betrachtete Portfolio mit Sicherheit einen Rückfluß von null verspricht, zur Erlangung dieses Portfolios ein Preis von null verlangt wird. In dieser Formulierung kommt die später aufgezeigte Optionsbewertung mit Hilfe eines Arbitrageportfolios zum Ausdruck, während die weiter oben vorgestellte Definition, daß identische Vermögenspositionen einen identischen Preis fordern, die Bewertung anhand eines Duplikationsportfolios verkörpert ${ }^{5}$. Bevor jedoch die Bewertung von Optionen anhand solcher Arbitrage- bzw. Duplikationsportfolios im Detail aufgezeigt wird, stehen Parallelen sowie fundamentale Unterschiede zwischen Akti-

1 Vgl. Kruschwitz, Finanzierung und Investition, 1999, S. 147.

2 Vgl. Terstege, Optionsbewertung, 1995, S. 36.

3 Vgl. Varian, Arbitrage, 1987, S. 59.

4 Vgl. Kruschwitz, Finanzierung und Investition, 1999, S. 146 f.

5 Vgl. Abschnitt 2.1.1 in diesem Kapitel. 
enoptionen und unternehmerischen Handlungsspielräumen im Mittelpunkt der Betrachtung.

\subsection{Gegenüberstellung von Aktien- und Realoptionen}

\subsubsection{Interpretation von unternehmerischen Handlungsspielräumen als Realoptionen}

Handlungsspielräume im Leistungsbereich eines Unternehmens weisen viele Parallelen zu Aktienoptionen auf ${ }^{1}$. Die Möglichkeit zu investieren, stellt beispielsweise eine Gelegenheit dar, gegen Entrichtung der Investitionsauszahlung eine Vermögensposition mit unsicherer Wertentwicklung zu erwerben. Dieses Vermögens- bzw. Bezugsgut ist durch den Gegenwartswert der aus der Investition resultierenden Rückflüsse gekennzeichnet. Die Investitionsauszahlung läßt sich hingegen als Basispreis interpretieren.

Kann die endgültige Investitionsentscheidung in die Zukunft verschoben werden, geht dies i.d.R. mit zusätzlichen Informationen über den tatsächlichen Wert des durch die Investition zu erzielenden Bezugsguts einher. Die Entscheidung für oder gegen die Investition läßt sich folglich auf Grundlage einer verbesserten Informationsbasis treffen. Der Zeitraum, bis zu dessen Ende mit der Investitionsauszahlung gewartet werden kann, stellt die Laufzeit einer solchen Realoption ${ }^{2}$ dar (vgl. auch Tab. 5). Da in den meisten Fällen Investitionsentscheidungen zu einem beliebigen Zeitpunkt getroffen werden können, entsprechen Investitionsmöglichkeiten in der Regel amerikanischen Optionen. Jedoch sind auch Beispiele denkbar, in denen erst nach Ablauf einer Frist - z.B. behördliche Genehmigungsfristen - eine bestimmte Investition möglich oder sinnvoll ist.

Im Rahmen eines in Betrieb befindlichen Investitionsprojekts läßt sich die Möglichkeit, einen Teil der bereits geleisteten Investitionsauszahlung wieder-

1 Auch im Finanzbereich eines Unternehmens lassen sich Handlungsspielräume ausfindig machen, die als Optionen interpretiert werden können. Diese werden jedoch im Rahmen dieser Arbeit nicht näher betrachtet.

2 Myers verwendete als erster den Begriff der Realoptionen, indem er darauf hinwies, daß der Wert des Unternehmens sich aus dem Wert der ,real assets“ und dem Wert der „real options" zusammensetzt. Vgl. Myers, Corporate borrowing, 1977, S. 163 sowie Abschnitt 4.3 in Kapitel II. Die Bezeichnung Realoptionen macht deutlich, daß es sich hierbei um Investitions- bzw. Desinvestitionsmöglichkeiten im Rahmen realer Investitionsprojekte im Gegensatz zu den auf einem organisierten Kapitalmarkt gehandelten Finanzoptionen handelt. 
zugewinnen, ebenfalls als Realoption interpretieren. Zum möglichen Desinvestitionszeitpunkt wird die Unternehmensleitung von dieser Desinvestitionsmöglichkeit Gebrauch machen, falls der Gegenwartswert der Rückflüsse unter den Desinvestitionserlös gefallen ist. Während auch hier der Gegenwartswert der Rückflüsse das Bezugsgut der Realoption verkörpert, stellt die Desinvestitionseinzahlung den Basispreis dar.

\begin{tabular}{|l|l|l|}
\hline $\begin{array}{c}\text { Kauf-/Verkaufsoption } \\
\text { auf Aktien }\end{array}$ & \multicolumn{1}{|c|}{ Investitionsmöglichkeit } & \multicolumn{1}{c|}{ Desinvestitionsmöglichkeit } \\
\hline $\begin{array}{l}\text { Aktienkurs } \\
\text { (= Bezugsgut) }\end{array}$ & $\begin{array}{l}\text { Gegenwartswert der erwarteten } \\
\text { Rückflüsse } \\
\text { (ohne Investitionsauszahlung) }\end{array}$ & $\begin{array}{l}\text { Gegenwartswert der erwarteten } \\
\text { Rückflüsse }\end{array}$ \\
\hline Basispreis & Investitionsauszahlung & Desinvestitionseinzahlung \\
\hline Optionslaufzeit & $\begin{array}{l}\text { Zeitraum, bis zu dessen Ende mit } \\
\text { der Investitionsentscheidung } \\
\text { gewartet werden kann }\end{array}$ & $\begin{array}{l}\text { Zeitraum, bis zu dessen Ende mit } \\
\text { der Desinvestitionsentscheidung } \\
\text { gewartet werden kann }\end{array}$ \\
\hline
\end{tabular}

Tab. 5: Parallelen zwischen Aktienoptionen und Investitions- sowie Desinvestitionsmöglichkeiten

\subsubsection{Systematisierung von Realoptionen}

Realoptionen lassen sich in Abhängigkeit der mit ihnen verbundenen Entscheidung klassifizieren. Zunächst kann - wie im vorigen Abschnitt praktiziert - eine grobe Unterscheidung danach erfolgen, ob durch eine Realoption das Gewinnpotential des Unternehmens erhöht oder das Verlustrisiko begrenzt wird. Erstere stellen Investitionsmöglichkeiten bzw. Optionen mit Kaufcharakter dar, während letztere Desinvestitionsmöglichkeiten bzw. Optionen mit Verkaufscharakter verkörpern. Die erste umfassende Arbeit zur Einordnung und Bewertung von Realoptionen stammt von Trigeorgis, in der sechs voneinander abweichende Kategorien unterschieden werden ${ }^{1}$.

\footnotetext{
Vgl. zum folgenden Trigeorgis, Real Investment Opportunities, 1986, S. 130 ff. Für frühe Versuche zur Bewertung von Aufgabeoptionen ohne Zuhilfenahme der Optionspreistheorie vgl. Robichek/Van Horne, Abandonment Value, 1967; Dyl/Long, Abandonment Value, 1969; Robichek/Van Horne, Reply, 1969; Bonini, Abandonment Options, 1977 sowie zur Beurteilung und Bewertung von Aufschubs- und Wachstumsoptionen Arrow/Fisher, Irreversibility, 1974; Henry, Irreversibility Effect, 1974; Cukierman, Effects of Uncertainty on Investment, 1980; Baldwin, Sequential Investment, 1982; Bernanke, Irreversibility, 1983. Für frühe Arbeiten zur optionspreistheoretischen Bewertung von Realoptionen vgl. Emery/ Parr/Mokkelbost/Gandhi/Saunders, Real Investment, 1978; Tourinho, Natural Resources, 1979.
} 
Bei einem noch nicht in Betrieb befindlichen Investitionsprojekt besteht eventuell die Möglichkeit, den Startzeitpunkt zu verschieben. Auf diese Weise lassen sich zusätzliche Informationen über mögliche Absatzpreise und sonstige Marktbedingungen generieren, bevor die erste Investitionsauszahlung getätigt wird. Auch nach der ersten Investitionsauszahlung existieren unter Umständen unternehmerische Handlungsspielräume, die bei der Bewertung der Investitionsmöglichkeit berücksichtigt werden sollten. Übersteigt beispielsweise das tatsächliche Marktvolumen die Erwartungen, ist es vorteilhaft, wenn sich die Kapazität gegen eine Folgeauszahlung unverzüglich erhöhen läßt. Umgekehrt läßt sich auf eine ungünstige Marktentwicklung flexibel reagieren, indem die Kapazität eingeschränkt wird oder die Produktion gar zeitweise eingestellt wird, falls die Absatzpreise unter die variablen Kosten fallen. Besteht auch auf längere Sicht keine Aussicht auf Gewinne, kann das Projekt vollständig aufgegeben und ein Liquidationserlös realisiert werden. Ein unternehmerischer Handlungsspielraum besteht ebenfalls, wenn in Abhängigkeit von Marktpreisen auf verschiedene Inputfaktoren zurückgegriffen werden kann bzw. unterschiedliche Produkte gefertigt werden können.

In einer späteren Veröffentlichung hat Trigeorgis seine Ausführungen um drei Kategorien erweitert ${ }^{1}$. In mehrstufigen Investitionsprojekten besteht gleich mehrmals die Gelegenheit, das Projekt abzubrechen. Jede Investitionsstufe stellt dabei die Erreichung eines Meilensteins in der Entwicklung des Projekts dar und kann als Option auf den Eintritt in die nächste Stufe angesehen werden. Demgegenüber besteht eine Wachstumsoption für den Fall, daß ein Anfangsprojekt als Vorreiter für ein mögliches zukünftiges Folgeprojekt gilt. Schließlich sind verbundene Realoptionen durch die Tatsache charakterisiert, daß innerhalb eines Projektes mehrere Handlungsspielräume gleichzeitig existieren und der Gesamtwert dieser Realoptionen nicht der Summe der Einzelwerte entspricht ${ }^{2}$. Einen zusammenfassenden Überblick gewährt Abb. 15.

Die von Trigeorgis vorgenomme Systematisierung von Realoptionen scheint aus praktischen Gesichtspunkten nützlich. Gleichzeitig fällt jedoch auf, daß sich einige der vorgestellten Realoptionsarten sehr ähneln bzw. die von ihm

1 Vgl. Trigeorgis, Overview, 1995, S. 3 ff. Zu jüngeren, hierzu z.T. leicht abweichenden Systematisierungen vgl. Amram/Kulatilaka, Decisions, 1999, S. 96 f.; Hommel/Pritsch, Realoptionsansatz, 1999, S. 13 ff.

2 Verbundene Realoptionen stellen eine Sonderklasse dar, da hier auf die anderen Kategorien Bezug genommen wird. 
vorgenommene Einteilung nicht trennscharf ist ${ }^{1}$. Dies ist einerseits auf die bereits erwähnte grundsätzliche Unterscheidung zwischen Investitions- und Desinvestitionsmöglichkeiten und andererseits auf begriffliche Abgrenzungen zurückzuführen. Beispielsweise ist die Unterscheidung zwischen einer Wachstums- und einer Erweiterungsoption nur auf den Umstand der unterschiedlichen Projektabgrenzung zurückzuführen ${ }^{2}$. Während bei letztgenannter die Möglichkeit zur Erweiterung innerhalb des gleichen Projektes besteht, beinhaltet bei einer Wachstumsoption das Anfangsprojekt eine Investitionsgelegenheit für ein eigenständiges Folgeprojekt.

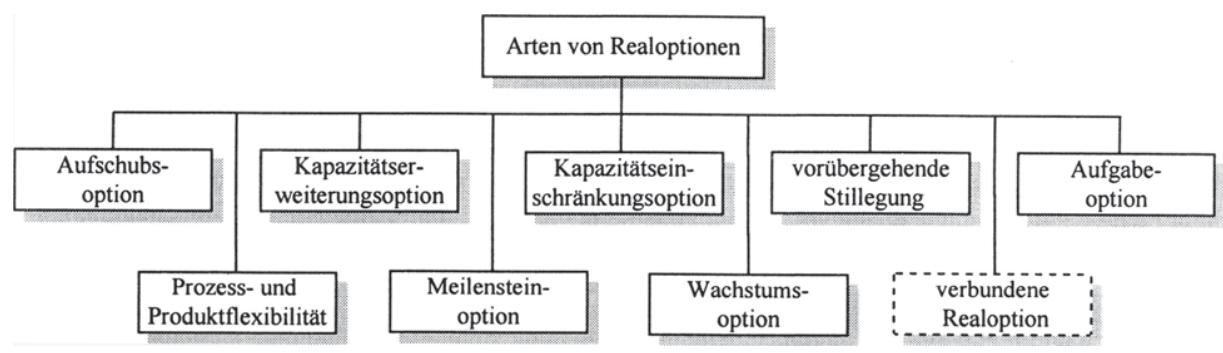

Abb. 15: Systematisierung von Realoptionen

In Abhängigkeit der konkreten Entscheidungssituation sowie der spezifischen Charakteristika des zu beurteilenden Investitionsprojekts kommt den einzelnen Realoptionsarten eine unterschiedliche Bedeutung zu. Während bei flexiblen Fertigungssystemen das Augenmerk auf einem möglichen technischen Wechsel liegen dürfte, sind bei Forschungs- und Entwicklungsprojekten insbesondere Meilensteinoptionen von Interesse. Im Rahmen einer strategischen Akquisition werden neben dem eigentlichen Erwerb des Unternehmens vor allem zusätzliche Investitionsmöglichkeiten gesehen.

Wird durch die Realisation des Anfangsprojekts (Akquisition des Unternehmens) die Möglichkeit geschaffen, in ein eigenständiges Folgeprojekt (z.B. die Möglichkeit zur Erschließung eines neuen Markts) zu investieren, handelt es sich hierbei um eine Wachstumsoption. Besteht die Möglichkeit das Anfangsprojekt zu vergrößern, kann von einer Erweiterungsoption gesprochen werden. Diese ist z.B. beim Erwerb einer Minderheitsbeteiligung bzw. eines Joint

1 Für eine theoretisch genauere Abgrenzung von Realoptionstypen vgl. Gintschel, Realoptionen, 1999, S. $70 \mathrm{ff}$.

2 Zur Projektabgrenzung vgl. auch Trigeorgis, Options Framework, 1988, S. 152 f. 
Ventures gegeben, wenn neben dem Kauf der aktuellen Beteiligungstranche zusätzlich die Möglichkeit eines zukünftigen Erwerbs weiterer Anteile zu einem bereits heute festgelegten Preis ausgehandelt wird ${ }^{1}$.

Daneben sind bei strategischen Akquisitionen auch Umstellungsoptionen ${ }^{2}$, die alternative Verwendungsmöglichkeiten eines bereits beim Akquisitionskandidaten befindlichen Vermögensguts (z.B. ausgebautes Vertriebsnetz) erlauben, oder Desinvestitionsmöglichkeiten, die eine vollständige oder teilweise Wiedergewinnung des ursprünglich gezahlten Kaufpreises ermöglichen, denkbar ${ }^{3}$. In den weiteren Ausführungen wird jedoch schwerpunktmäßig auf zusätzlich geschaffene Investitionsmöglichkeiten eingegangen. Dies scheint insofern gerechtfertigt, als diese zum einen das Hauptmotiv einer strategisch motivierten Akquisition darstellen dürften und zum anderen eine Übertragung der vorgestellten Bewertungstechniken auf Umstellungs- und Desinvestitionsoptionen leicht zu bewerkstelligen ist.

\subsubsection{Einschränkung der Vergleichbarkeit von Real- mit Aktienoptionen}

Obwohl zwischen Real- und Aktienoptionen viele Parallelen bestehen, darf nicht übersehen werden, daß keine vollständige Übereinstimmung existiert. Insbesondere sind folgende fünf Unterschiede zu beachten (vgl. Abb. 16) ${ }^{4}$. Während die der Aktienoption zugrunde liegende Aktie an einem organisierten Kapitalmarkt notiert ist, so daß für das Bezugsgut der Option laufend ein Marktpreis festgestellt werden kann, ist dies bei Realoptionen nicht der Fall. Folglich ist kein Marktpreis des Bezugsguts beobachtbar. Die hierdurch erfor-

1 Zu Erweiterungsoptionen im Rahmen von Minderheitsbeteiligungen vgl. Dirrigl, Konzepte, 1994, S. 424 f.; Crasselt/Tomaszewski, Akquisitionen, 1999, S. 517 ff. Zum Optionsgedanken bei Joint Ventures vgl. Kogut, Joint Ventures, 1991, S. 19 ff.; Kogut/Kulatilaka, Investing in Opportunity, 1994, S. 63 f.; Nödelke/Schmidt, Options to Own, 1998, S. 633 ff.

2 Bei entsprechender Interpretation des Bezugsguts und des Basispreises stellt jede Realoption eine Umstellungsoption dar. Mit einer Realoption ist immer die Möglichkeit verbunden, eine andere Art der Kapitalverwendung zu wählen. Vgl. Kulatilaka/Marcus, Corporate Real Options, 1988, S. 198; Ritchken/Rabinowitz, Contingent Claims Analysis, 1988, S. 141; Kulatilaka/Trigeorgis, Flexibility to Switch, 1994, S. 792. An dieser Stelle wird jedoch unter einer Umstellungsoption die engere Begriffsauffassung verstanden.

3 Für eine solche Einteilung von Realoptionen bei strategischen Akquisitionen vgl. Smith/ Triantis, Strategic Aquisitions, 1995, S. $137 \mathrm{ff}$.

4 Vgl. jüngst auch Hommel/Pritsch, Realoptionsansatz, 1999, S. 15 ff., die zusätzlich noch die Möglichkeit des Unternehmens zur Beeinflussung der Investitionsauszahlung und der projektbezogenen Unsicherheit sowie Anreizprobleme zwischen untergeordneten Entscheidungstrăgern und Unternehmensleitung erwähnen. 
derlichen Schätz- bzw. Bewertungsschritte zur Bestimmung eines „Marktpreises" des Bezugsguts werden bei der erstmaligen Bewertung einer Investitionsmöglichkeit in Abschnitt 2.1.2.2 dieses Kapitels erläutert.

Auch hinsichtlich der Ausübungszahlung unterscheiden sich Aktien- und Realoptionen. Bei Finanzoptionen ist der Basispreis vertraglich festgelegt und unterliegt i.d.R. keiner Bedingung. Die Höhe der zukünftig geplanten Investitionsauszahlungen ist bei realen Investitionsprojekten jedoch unsicher. Hierauf wird im Rahmen dieser Arbeit nur kurz eingegangen, da die erforderliche Korrektur lediglich eine mathematische Verfeinerung der vorgestellten Bewertungstechnik darstellt. Beispielhaft läßt sich die Vorgehensweise in Abschnitt 3.2.1 in Kapitel V anführen.

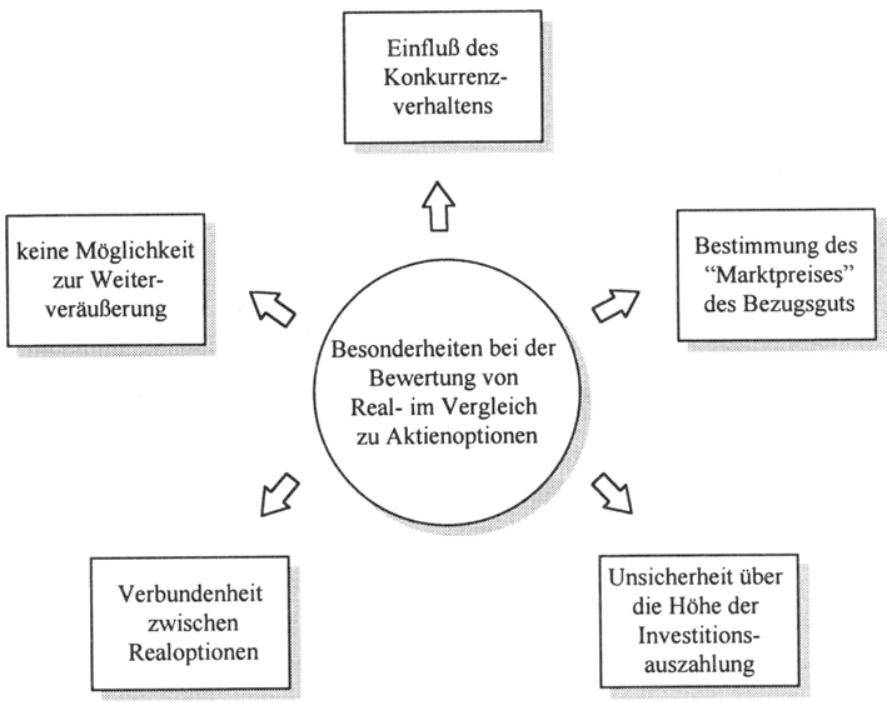

Abb. 16: Besonderheiten bei der Bewertung von Realoptionen

Greifen mehrere Handlungsspielräume auf das gleiche Bezugsgut zurück, handelt es sich um verbundene Realoptionen. Dies ist z.B. der Fall, wenn ein Investitionsprojekt zu einem bestimmten Zeitpunkt erweitert und zu einem anderen Zeitpunkt abgebrochen werden kann. Innerhalb des gleichen Investitionsprojekts existieren eine Erweiterungs- und eine Aufgabeoption gleichzeitig. Die 
Addition der einzelnen Realoptionswerte entspricht nur selten dem Gesamtoptionswert ${ }^{1}$. Diese Problematik wird in Abschnitt 3 in Kapitel VI behandelt.

Desweiteren sind Realoptionen zumeist nicht weiterveräußerbar, während für Aktienoptionen häufig aufnahmefähige Sekundärmärkte bestehen. Für die konkrete Bewertungstechnik spielt dieser Unterschied keine Rolle. Er hat aber eine Bedeutung im Rahmen der Ausübungsentscheidung und damit auch auf den Wert einer Realoption. Bei letzterer kann ein erwarteter Wertverlust lediglich durch frühzeitige Investition und nicht durch Verkauf auf einem organisierten Markt verhindert werden ${ }^{2}$.

Der Käufer einer Aktienoption erhält das Recht, die Finanzoption unabhängig von Handlungen anderer Marktteilnehmer auszuüben. Im Gegensatz dazu stehen Realoptionen dem Inhaber nicht immer exklusiv zur Verfügung. Vielmehr ist zwischen exklusiven Realoptionen und solchen, die mit Wettbewerbern geteilt werden, zu unterscheiden ${ }^{3}$. Wenn eine Realoption mit Wettbewerbern geteilt wird, besitzen mehrere Unternehmen eine Realoption mit demselben bzw. einem ähnlichen Bezugsgut, wodurch sich der Wert dieser Realoption aus Sicht des einzelnen Unternehmens im Vergleich zur Situation ohne Konkurrenz verändert.

Die Existenz von Wettbewerbern, die dem betrachteten Unternehmen die zu beurteilende Investitionsmöglichkeit streitig machen können, kann als der bedeutendste Unterschied im Vergleich zwischen Aktien- und Realoptionen angesehen werden. Bei der Übertragung finanzwirtschaftlicher Optionspreismodelle auf realwirtschaftliche Investitionen gilt es, diesem Umstand besondere Aufmerksamkeit zukommen zu lassen. Aus diesem Grund wird in Kapitel V ausschließlich der Einfluß des Konkurrenzverhaltens auf die zur Verfügung stehenden Realoptionen und deren Werte diskutiert.

In den nun folgenden Abschnitten wird die konkrete Bewertung von Aktienund Realoptionen dargestellt, indem auf die beiden am weitesten verbreiteten finanzwirtschaftlichen Optionspreisansätze zurückgegriffen wird. Als zeitdiskreter Bewertungsansatz wird das Binomialmodell von Cox, Ross und Rubinstein und als zeitstetiges Bewertungsmodell das Modell von Black und Scholes vorgestellt. Im Rahmen des Binomialmodells wird dabei ausführlich auf alle

\footnotetext{
1 Vgl. Trigeorgis, Options Interactions, 1993, S. 7 ff.

2 Vgl. Kester, Options Approach, 1986, S. 24.

3 Vgl. Kester, Growth Options, 1993, S. 193.
} 
Varianten der relativen Bewertungstechnik zwischen Option und Bezugsgut eingegangen und zudem mit der risikoneutralisierten Optionsbewertung die Brücke zu den in Kapitel III erläuterten Korrekturverfahren geschlagen. 


\section{Binomialmodell als zeitdiskreter Bewertungsansatz}

\subsection{Einperiodiges Modell}

\subsubsection{Bewertung von Aktienoptionen}

\subsubsection{Duplikationsansatz}

Die Bewertung von Aktienoptionen vor dem Ende der Optionslaufzeit beruht auf der Grundannahme eines arbitragefreien Kapitalmarkts ${ }^{1}$. Unter dieser Annahme müssen eine Option und ein Portfolio, das die möglichen Rückflüsse der Option genau dupliziert, den gleichen Wert haben. Ein solches Portfolio kann für eine Aktienoption durch den Kauf oder Verkauf von Aktien des Typs, auf den die Option ausgestellt ist, und eine gleichzeitige Kreditaufnahme bzw. Kapitalanlage gebildet werden ${ }^{2}$. Der Duplikationsansatz wird im folgenden anhand einer europäischen Kaufoption dargestellt. Neben einem arbitragefreien Kapitalmarkt wird angenommen ${ }^{3}$ :

- Es besteht ein friktionsloser und kompetitiver Kapitalmarkt. Insbesondere existieren keine Transaktionskosten oder Steuern. Leerverkäufe sind uneingeschränkt möglich. Die Kapitalmarktteilnehmer sind Mengenanpasser.

- Der risikolose Zinsfuß ist im Zeitablauf konstant. Zu diesem Zinsfuß können Kapitalmarktteilnehmer beliebig Kapital anlegen bzw. aufnehmen.

- Die Kapitalmarktteilnehmer haben homogene Erwartungen bezüglich der möglichen Aktienkurse am Verfalltag der Option.

Weiterhin werden zunächst folgende vereinfachenden Annahmen getroffen, die im weiteren Verlauf der Arbeit aufgehoben bzw. ersetzt werden:

- Während der Optionslaufzeit fallen keine Dividenden an.

- Der Aktienkurs folgt einem zeitdiskreten, einperiodigen und multiplikativen Binomialprozeß.

\footnotetext{
Vgl. die Ausführungen in Abschnitt 1.3 dieses Kapitels.

2 Vgl. Cox/Ross/Rubinstein, Option Pricing, 1979, S. $231 \mathrm{f}$.

3 Vgl. Kruschwitz, Finanzierung und Investition, 1999, S. 267.
} 
Aufgrund der Annahme eines einperiodigen Binomialprozesses können am Verfalltag der Option nur zwei Aktienkursausprägungen auftreten ${ }^{1}$. Ausgehend vom heutigen Aktienkurs $K_{0}$ wird der Aktienkurs bis zum Ende der Optionslaufzeit in $T$ entweder auf $u \cdot K_{0}$ steigen oder auf $d \cdot K_{0}$ fallen. Für den Fall, daß der Aktienkurs den Basispreis $B$ übersteigt, ist der Optionswert gleich der Differenz zwischen Aktienkurs und Basispreis, ansonsten ist die Option wertlos. Entsprechend der Aktienkursentwicklung nimmt die Option in $T$ in Abhängigkeit der Umweltzustände $s=1$ und $s=2$ zwei mögliche Werte an $\left(C_{u}\right.$ bzw. $\left.C_{d}\right)$.

$$
\begin{aligned}
& C_{u}=\max \left\{u \cdot K_{0}-B ; 0\right\} \\
& C_{d}=\max \left\{d \cdot K_{0}-B ; 0\right\}
\end{aligned}
$$

Wird nun ein Duplikationsportfolio aus $\alpha$ Anteilen der Aktie zu $K_{0}$ und einem Anlage- bzw. Kreditbetrag $M$ zum risikolosen Zinsfuß $r_{f}$ derart gebildet, daß die Rückflüsse des Portfolios in beiden Umweltzuständen in $T$ genau denen der Option entsprechen, muß der Barwert des Portfolios $\alpha \cdot K_{0}+M$ dem Barwert der Option $C_{0}$ entsprechen.

$$
\begin{aligned}
& C_{u}=\alpha \cdot u \cdot K_{0}+M \cdot\left(1+r_{f}\right) \\
& C_{d}=\alpha \cdot d \cdot K_{0}+M \cdot\left(1+r_{f}\right)
\end{aligned} \quad \Rightarrow C_{0}=\alpha \cdot K_{0}+M
$$

Aus den beiden linken Gleichungen lassen sich $\alpha$ und $M$ ermitteln. Der Wert der Aktienoption kann anschließend unter den angenommen Bedingungen durch Einsetzen in die dritte (rechte) Gleichung bestimmt werden.

Ein Beispiel soll die Bewertungstechnik verdeutlichen. Bei einem Aktienkurs von $30 €$, einer möglichen Steigung von $50 \%(u=1,5)$ und einer möglichen Senkung von $30 \%(d=0,7)$ sowie einer einjährigen Optionslaufzeit wird der Aktienkurs in einem Jahr entweder 45 oder $21 €$ betragen. Bei einem Basispreis von $30 €$ nimmt die Option am Verfalltag folgende zwei Werte an.

$$
\begin{aligned}
& C_{u}=\max \{45-30 ; 0\}=15 € \\
& C_{d}=\max \{21-30 ; 0\}=0 €
\end{aligned}
$$

Vgl. zu folgendem Cox/Ross/Rubinstein, Option Pricing, 1979, S. 232 ff.; Kruschwitz/ Schöbel, Optionspreistheorie, 1984, S. 69 ff. 
Für den Zeitraum von einem Jahr soll eine risikolose Verzinsung von 5\% gelten $^{1}$. Ein künstlich gebildetes Portfolio, das aus einem bestimmten (noch zu ermittelnden) Anteil $\alpha$ der Aktie zu $30 €$ und einem (noch zu ermittelnden) risikolosen Anlage- bzw. Kreditbetrag $M$ besteht, soll die Option nachbilden. Am Verfalltag muß der Wert dieses Portfolios für jeden Umweltzustand dem Wert der Option entsprechen.

$$
\begin{aligned}
& 15=\alpha \cdot 45+M \cdot 1,05 \\
& 0=\alpha \cdot 21+M \cdot 1,05
\end{aligned}
$$

Daraus ergibt sich $\alpha=0,625$ und $M=-12,5$. Der positive Anteil $\alpha$ und der negative Anteil $M$ bedeuten, daß 62,5\% der Aktie gekauft und ein Kreditbetrag in Höhe von 12,5€ aufgenommen werden. Der Anleger eines Portfolios aus 0,625 Anteilen der Aktie zu $30 €$ sowie einer Kreditaufnahme von 12,5 $€$ und der Inhaber einer Kaufoption auf diese Aktie gehen die gleiche Position hinsichtlich der erwarteten Rückflüsse ein (vgl. Abb. 17). Unter den angenommenen Bedingungen ist der Wert des gebildeten Portfolios und der Kaufoption identisch.

$$
C_{0}=0,625 \cdot 30-12,5=6,25 €
$$

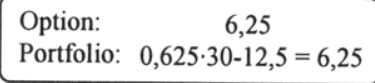

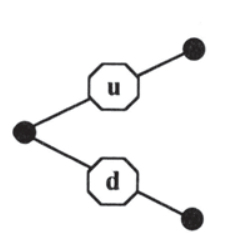

Option:

15

Portfolio: $0,625 \cdot 45-12,5 \cdot 1,05=15$

Option: $\quad 0$

Portfolio: $0,625 \cdot 21-12,5 \cdot 1,05=0$

Abb. 17: Wertidentităt von Aktienoption und Duplikationsportfolio

Auf analoge Art und Weise kann für eine Verkaufsoption mit einem Basispreis von $30 €$ aus

$$
\begin{aligned}
& 0=\alpha \cdot 45+M \cdot 1,05 \\
& 9=\alpha \cdot 21+M \cdot 1,05
\end{aligned}
$$

\footnotetext{
Aufrund der Annahme der Arbitragefreiheit muß gelten: $d<1+r_{f}<u$. Die risikobehaftete Rendite des Bezugsguts darf nicht mit Sicherheit die risikolose Rendite übertreffen und umgekehrt darf die risikolose Rendite nicht mit Sicherheit die risikobehaftete Rendite übersteigen.
} 
die duplikationsbestimmenden Parameter $\alpha=-0,375$ sowie $M=16,07$ und damit der Wert dieser Verkaufsoption $P_{0}$ errechnet werden.

$$
P_{0}=-0,375 \cdot 30+16,07=4,82 €
$$

\subsubsection{Hegde- und Arbitrageansatz als zusätzliche Varianten}

Während beim Duplikationsansatz das Portfolio aus $\alpha$ Anteilen der Aktie $K_{0}$ sowie dem risikolosen Anlage- bzw. Kreditbetrag $M$ besteht, wird beim Hedgeansatz ein Portfolio aus $\alpha$ Anteilen der Aktie $K_{0}$ und einer (leer) verkauften Option $C_{0}$ gebildet. Der Anteil $\alpha$ wird dabei so gewählt, daß die Rückflüsse des Hedgeportfolios in jedem Umweltzustand identisch und somit unabhängig von den Schwankungen der Aktie sind ${ }^{1}$. Das Gleichsetzen der Portfoliorückflüsse aus den beiden Umweltzuständen führt zu dem gesuchten Aktienanteil am Hedgeportfolio.

$$
\begin{gathered}
\alpha \cdot u \cdot K_{0}-C_{u}=\alpha \cdot d \cdot K_{0}-C_{d} \\
\Rightarrow \alpha=\frac{C_{u}-C_{d}}{u \cdot K_{0}-d \cdot K_{0}}
\end{gathered}
$$

Bei Kenntnis des Aktienanteils $\alpha$ ist auch gleichzeitig der Wert des Hedgeportfolios am Ende der Optionslaufzeit festgelegt. Da dieser Wert nicht von den Aktienkursschwankungen abhängt, verzinst sich ein solches Hedgeportfolio auch nur zum risikolosen Zinsfuß. Die risikolose Diskontierung des Portfoliowerts am Ende der Optionslaufzeit auf den heutigen Zeitpunkt führt zum Barwert des Hedgeportfolios, der hier - in Übereinstimmung mit dem oben eingeführten risikolosen Anlagebetrag - mit dem Symbol $M$ belegt wird. Aus diesem kann der aktuelle Wert der Option herausgerechnet werden.

$$
\begin{aligned}
& \alpha \cdot K_{0}-C_{0}=M \\
& \Rightarrow C_{0}=\alpha \cdot K_{0}-M
\end{aligned}
$$

Zur Verdeutlichung sei das Beispiel zur Kaufoption aus dem vorigen Abschnitt aufgegriffen. Über

$1 \mathrm{Zu}$ einer ähnlichen Vorgehensweise im Binomialmodell vgl. Rendleman/Barter, Option Pricing, 1979, S. 1093 f. sowie im Rahmen der zeitstetigen Bewertung Black/Scholes, Pricing of Options, 1973, S. $641 \mathrm{f}$. 


$$
\alpha=\frac{15-0}{1,5 \cdot 30-0,7 \cdot 30}=0,625
$$

läßt sich der Anteil der Aktie am Hedgeportfolio ermitteln. Am Verfalltag der Option beträgt der Portfoliowert unabhängig vom Umweltzustand 13,125 € (vgl. Abb. 18). Somit beträgt der Wert des Portfolios am Periodenanfang 12,5 $(13,125 / 1,05) €$. Über diesen Barwert, den aktuellen Aktienkurs und den Aktienanteil am Hedgeportfolio läßt sich der Optionswert ermitteln.

$$
\begin{gathered}
0,625 \cdot 30-C_{0}=12,5 \\
\Rightarrow C_{0}=6,25 €
\end{gathered}
$$

risikolose Anlage:

Portfolio:
12,5

$0,625 \cdot 30-6,25=12,5$

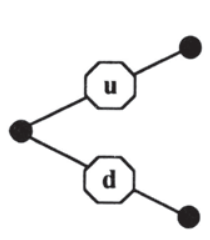

risikolose Anlage: $12,5 \cdot 1,05=13,125$

Portfolio: $0,625 \cdot 45-15=13,125$

risikolose Anlage: $12,5 \cdot 1,05=13,125$

Portfolio: $\quad 0,625 \cdot 21-0=13,125$

Abb. 18: Wertidentität von risikoloser Anlage und Hedgeportfolio

Im Rahmen des Arbitrageansatzes wird ein Portfolio aus allen drei bisher betrachteten Werttiteln gebildet ${ }^{1}$. Das Arbitrageportfolio besteht aus $\alpha$ Anteilen der Aktie $K_{0}$, einem risikolosen Anlage- bzw. Kreditbetrag $M$ und einer (leer) verkauften Option $C_{0}$. Der Anteil $\alpha$ sowie der risikolose Anlage- bzw. Kreditbetrag $M$ werden dabei so bestimmt, daß die Rückflüsse des Arbitrageportfolios in jedem Umweltzustand null betragen (vgl. auch Abb. 19). Dies führt zu zwei Gleichungen, die bereits im Rahmen des Duplikationsansatzes aufgestellt worden sind. Das Lösen dieser Gleichungen führt zu den bereits bekannten Parameterwerten von $\alpha=0,625$ und $M=12,5$. Da das Arbitrageportfolio mit Sicherheit einen Rückfluß von null garantiert, muß es auch einen aktuellen Wert von null besitzen. Aus

$$
\begin{aligned}
& \alpha \cdot K_{0}+M-C_{0}=0 \\
& \Rightarrow C_{0}=\alpha \cdot K_{0}+M
\end{aligned}
$$

$1 \mathrm{Zu}$ einer solchen Vorgehensweise, jedoch im Rahmen einer zeitstetigen Bewertung, vgl. Merton, Rational Option Pricing, 1973, S. 164 f. 
folgt

$$
\begin{gathered}
0,625 \cdot 30-12,5-C_{0}=0 \\
\Rightarrow C_{0}=6,25 € .
\end{gathered}
$$

Rückfluß:

Portfolio: $0,625 \cdot 30-12,5-6,25=0$,

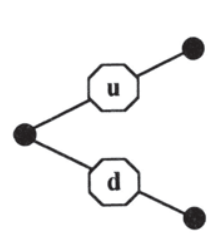

Rückfluß:

Portfolio: $0,625 \cdot 45-12,5 \cdot 1,05-15=0$

Rückfluß:

Portfolio: $0,625 \cdot 21-12,5 \cdot 1,05-0=0$

Abb. 19: Wertentwicklung des Arbitrageportfolios

Die Ausführungen dieses sowie des vorigen Abschnitts haben gezeigt, daß es sich beim Duplikations-, Hedge- und Arbitrageansatz nicht um unterschiedliche Bewertungstechniken, sondern lediglich um unterschiedliche Formulierungen bzw. Varianten der gleichen Bewertungstechnik handelt.

\subsubsection{Risikoneutralisierte Bewertungsmethode}

Die Bewertung von Aktienoptionen kann auch ohne Konstruktion eines Duplikations-, Hedge- oder Arbitrageportfolios erfolgen. Aus dem aktuellen Aktienkurs, der angenommenen Verteilung für den Aktienkurs und dem risikolosen Zinsfuß läßt sich ein Sicherheitsäquivalent für die erwarteten Rückflüsse aus der Aktienoption ableiten, daß mit dem risikolosen Zinsfuß diskontiert werden kann'. Um die Idee zu verdeutlichen, soll das Vorgehen zunächst bei der Bewertung der Aktie, die der Option zugrunde liegt, erläutert werden.

In Ergänzung zu den in den beiden vorangegangenen Abschnitten gewählten Beispielen zur Kauf- und Verkaufsoption möge für die Aktie gelten, daß die Eintrittswahrscheinlichkeiten $q$ und 1-q der beiden Umweltzustände $s=1$ und $s=2$ als gleich wahrscheinlich eingeschätzt werden und der aus dem Kapitalmarkt abgeleitete risikoadjustierte Zinsfuß $r_{A}$ für den einjährigen Zeitraum 10\% beträgt. Für einen risikoaversen Kapitalmarktteilnehmer wird der aktuelle Wert der Aktie bestimmt, indem der Erwartungswert der Rückflüsse mit dem risikoadjustierten Zinsfuß diskontiert wird.

1 Vgl. hierzu stellvertretend Brealey/Myers, Principles, 1996, S. 575 f. 


$$
K_{0}=\frac{q \cdot u \cdot K_{0}+(1-q) \cdot d \cdot K_{0}}{1+r_{A}}=\frac{0,5 \cdot 45+0,5 \cdot 21}{1,1}=30 €
$$

Eine Bewertung der Aktie auf Basis der Sicherheitsäquivalentmethode muß zum gleichen Ergebnis führen. Da der aktuelle Aktienkurs bekannt ist, läßt sich unter Zuhilfenahme der angenommenen Verteilung und des risikolosen Zinsfusses aus der folgenden Formel eine „Kennzahl“ $p$ ermitteln, durch die die Transformation des erwarteten (unsicheren) Rückflusses in das benötigte Sicherheitsäquivalent gelingt.

$$
\begin{gathered}
K_{0}=\frac{p \cdot u \cdot K_{0}+(1-p) \cdot d \cdot K_{0}}{1+r_{f}}=\frac{p \cdot 45+(1-p) \cdot 21}{1,05}=30 € \\
\Rightarrow p=0,4375
\end{gathered}
$$

Vereinfacht läßt sich $p$ auch über folgende Formel ermitteln ${ }^{1}$.

$$
p=\frac{1+r_{f}-d}{u-d}=\frac{1,05-0,7}{1,5-0,7}=0,4375
$$

Die „Kennzahlen“ $p$ bzw. 1- $p$ lassen sich als Wahrscheinlichkeiten interpretieren. Im Gegensatz zu den tatsächlich erwarteten Eintrittswahrscheinlichkeiten $q$ und $1-q$, von denen bei der Bewertung der Aktie unter der Annahme risikoaverser Marktteilnehmer ausgegangen wird, stellen sie Wahrscheinlichkeiten dar, die sich im aktuellen Aktienkurs widerspiegeln müßten, wenn von risikoneutralen Marktteilnehmern ausgegangen würde. Tatsächlich wird jedoch nicht von risikoneutralen Marktteilnehmern ausgegangen ${ }^{2}$. Die „Kennzahl“ $p$ wird deshalb auch als Pseudo- bzw. risikoneutralisierte Wahrscheinlichkeit bezeichnet ${ }^{3}$.

Da der Wert der Kaufoption von der gleichen Umweltentwicklung abhängt wie der Wert der Aktie, können auch die erwarteten Rückflüsse der Option mit Hilfe dieser Pseudowahrscheinlichkeiten in ein Sicherheitsäquivalent umgewandelt werden.

1 Vgl. Cox/Ross/Rubinstein, Option Pricing, 1979, S. 234 f.

2 Würde eine solche Annahme indes getroffen, ergäbe sich bei gegebener Datenkonstellation ein höherer Aktienkurs. Im Zahlenbeispiel wäre der Erwartungswert des unsicheren Rückflusses von $33(0,5 \cdot 45+0,5 \cdot 21) €$ mit dem risikolosen $Z i n s f u ß$ von $5 \%$ zu diskontieren. Der unter dieser geänderten Annahme ermittelte aktuelle Wert der Aktie beträgt $31,4 \in$.

3 Vgl. Kruschwitz/Schöbel, Optionspreistheorie, 1984, S. 72. 


$$
C_{0}=\frac{p \cdot C_{u}+(1-p) \cdot C_{d}}{1+r_{f}}=\frac{0,4375 \cdot 15+0,5625 \cdot 0}{1,05}=6,25 €
$$

Diese Vorgehensweise gilt auch für eine Verkaufsoption auf die betrachtete Aktie. Mit Hilfe der Pseudowahrscheinlichkeiten läßt sich ein Sicherheitsäquivalent für den unsicheren Rückfluß der Verkaufsoption bilden, der durch risikoloses Abzinsen in den aktuellen Wert der Verkaufsoption überführt werden kann.

$$
P_{0}=\frac{p \cdot P_{u}+(1-p) \cdot P_{d}}{1+r_{f}}=\frac{0,4375 \cdot 0+0,5625 \cdot 9}{1,05}=4,82 €
$$

Ist der Wert der Option erst einmal bekannt, läßt sich im Umkehrschluß auch ein risikoadjustierter Zinsfuß ermitteln, der unter Berücksichtigung der erwarteten Eintrittswahrscheinlichkeiten der Umweltzustände zum gleichen Ergebnis führt. Für die Kaufoption gilt ein risikoadjustierter Zinsfuß in Höhe von 20\%. Bemerkenswert ist die Tatsache, daß die hierin enthaltene Risikoprämie drei Mal so hoch ist wie diejenige zur Bewertung der Aktie. Das Risiko der Option unterscheidet sich gravierend vom Risiko des Bezugsguts.

$$
\begin{gathered}
C_{0}=\frac{q \cdot C_{u}+(1-q) \cdot C_{d}}{1+r_{A}}=\frac{0,5 \cdot 15+0,5 \cdot 0}{1+r_{A}}=6,25 € \\
\Rightarrow r_{A}=20 \%
\end{gathered}
$$

Für die Verkaufsoption gilt entsprechend ein risikoadjustierter Zinsfuß in Höhe von $-6,6 \%$.

$$
\begin{gathered}
P_{0}=\frac{q \cdot P_{u}+(1-q) \cdot P_{d}}{1+r_{A}}=\frac{0,5 \cdot 0+0,5 \cdot 9}{1+r_{A}}=4,82 € \\
\Rightarrow r_{A}=-6,6 \%
\end{gathered}
$$

An dieser Stelle offenbart sich ein zentrales Problem der Optionsbewertung. Wäre der risikoadjustierte Zinsfuß bekannt, könnte auf die vorgestellten Bewertungsmethoden (Bildung von Duplikations-, Hedge- bzw. Arbitrageportfolio oder risikoneutralisierte Bewertungsmethode) verzichtet werden. Die Ermittlung eines risikoadjustierten Zinsfußes für Optionen wird jedoch aufgrund der Abhängigkeit des Optionsrisikos von der Wertentwicklung des Bezugsguts 
abgelehnt ${ }^{1}$. Im einperiodigen Binomialmodell mit nur einer Veränderung des Bezugsguts während der Optionslaufzeit tritt die Zeit- und Zustandsabhängigkeit eines solchen risikoadjustierten Zinsfußes noch nicht deutlich zum Vorschein. Das Problem wird jedoch klar, wenn mehrere oder gar kontinuierliche Veränderungen angenommen werden. Mit jeder Änderung des Werts des Bezugsguts ändert sich das Optionsrisiko.

Im folgenden werden die Erkenntnisse zur Bewertung von Aktienoptionen auf die Beurteilung von Realoptionen übertragen. Die Darstellung erfolgt anhand des Beispiels einer strategischen Akquisition, bei der neben dem eigentlichen Erwerb des Unternehmens eine zusätzliche Investitionsmöglichkeit in Form einer Wachstumsoption gesehen wird.

\subsubsection{Bewertung von Realoptionen}

\subsubsection{Ausgangssituation einer strategischen Akquisition}

Ein Unternehmen A plant, ein anderes Unternehmen B zu 100\% zu erwerben. Durch die Akquisition erwirbt Unternehmen A einen Anspruch auf die künftig von Unternehmen B erwirtschafteten Rückflüsse. Die aus dem bestehenden Geschäft des Unternehmens B zu erwartenden Rückflüsse werden i.d.R. periodenindividuell erfaßt und mit einem einheitlichen risikoadjustierten Zinsfuß abgezinst (traditioneller Ertragswert bzw. Discounted Cash Flow). Bei der Prognose der Rückflüsse wird an dieser Stelle vereinfachend von einem konstanten unendlichen Rückfluß ab der dritten Periode ausgegangen. Bei prognostizierten Rückflüssen von $C F_{1}=25$ Mio. $€, C F_{2}=35$ Mio. $€, C F_{3}=42$ Mio. $€$ und einem risikoadjustierten Zinsfuß von $25 \%$ beläuft sich der traditionelle DCFWert auf 150 Mio. $€$.

$$
\mathrm{DCF}=\frac{25}{1,25}+\frac{35}{1,25^{2}}+\frac{42}{0,25 \cdot 1,25^{2}}=150 \text { Mio. } €
$$

Neben den aus dem bestehenden Geschäft realisierbaren Rückflüssen wird von Unternehmen A die Möglichkeit gesehen, mit Hilfe von Unternehmen B in Zukunft eine zweite - bisher noch nicht vertriebene - Produktgruppe auf den Markt zu bringen. Die Entscheidung über die Einführung dieser neuen Produktgruppe und die dafür notwendige Ausweitung des bestehenden Vertriebs-

1 Vgl. stellvertretend Brealey/Myers, Principles, 1996, S. 573 sowie speziell fur Realoptionen Sick, Capital Budgeting With Real Options, 1989, S. 39 f.; Lund, Option Values, 1991, S. 9; Ehrhardt, Search for Value, 1994, S. 212 ff.; Trigeorgis, Real Options, 1996, S. 23 ff. 
netzes soll nach eingehender Marktbeobachtung getroffen werden. Für diese sei zunächst ein Zeitraum von einer Periode angenommen. Die Ausweitung des Vertriebsnetzes hätte eine Investitionsauszahlung von 90 Mio. $€$ zur Folge.

Für den Zeitpunkt der Markteinführung der neuen Produktgruppe werden zwei gleichwahrscheinliche Szenarien erwartet. Bei Eintritt von Szenario 1 kann mit einer hohen Nachfrage und einem Gegenwartswert der Investitionsmöglichkeit von 200 Mio. $€$ gerechnet werden. Dieser ergibt sich z.B. wenn bei einem risikoadjustierten Zinsfuß von $25 \%$ von einem unendlichen periodenunabhängigen Rückfluß von 50 Mio. $€$ ausgegangen wird. Der erwartete Rückfluß soll erstmalig eine Periode nach Markteinführung fließen. Tritt Szenario 2 ein, ist von einer niedrigen Nachfrage und einem Gegenwartswert von 50 (z.B. 12,5/0,25) Mio. $€$ auszugehen. Unabhängig davon, ob die Entscheidung heute oder in einem Jahr getroffen wird, wird erst in zwei Jahren mit Rückflüssen aus dem Projekt gerechnet.

Unternehmen A ist nicht verpflichtet, die Markteinführung der neuen Produktgruppe vorzunehmen. Es wird die Möglichkeit wahrnehmen, falls zum Entscheidungszeitpunkt der Gegenwartswert der erwarteten Rückflüsse die notwendige Investitionsauszahlung übersteigt. Dies ist dann der Fall, wenn die einjährige Marktbeobachtung zum Ergebnis geführt hat, daß Szenario 1 eingetreten ist (vgl. Abb. 20).

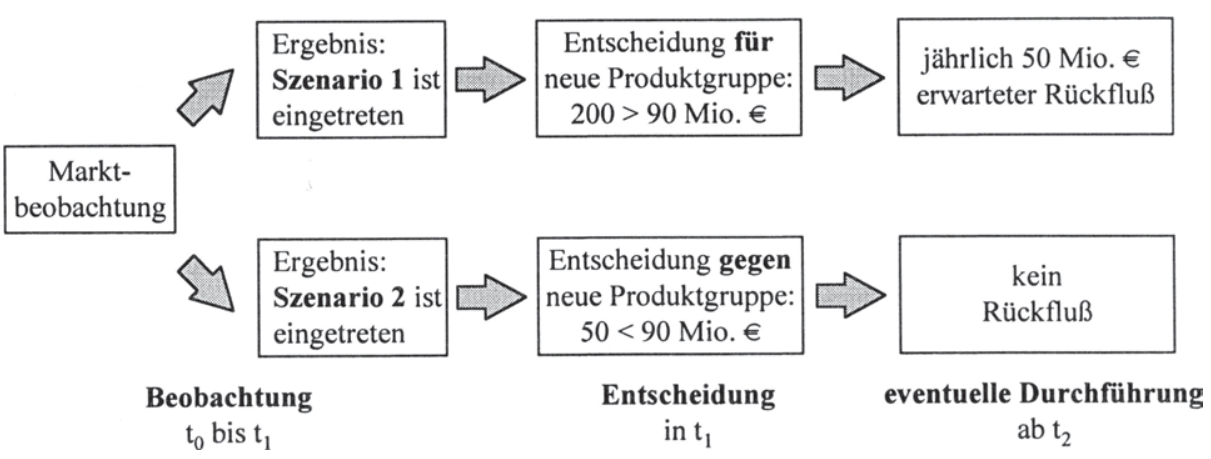

Abb. 20: Markteintrittsentscheidung für neue Produktgruppe

In der eben skizzierten Ausgangslage einer strategischen Akquisition ist die Akquisitionsentscheidung als Jetzt oder Nie-Beschluß und die zusätzliche Investitionsmöglichkeit als Wachstumsoption modelliert. Folglich läßt sich das Anfangsprojekt Akquisition mit der oben skizzierten Vorgehensweise des traditionellen DCF-Verfahrens beurteilen. Demgegenüber besitzt die Möglichkeit, die Entscheidung über die neue Produktgruppe in die Zukunft zu verschieben, 
einen ökonomischen Wert, der auf Basis des traditionellen DCF-Konzepts nur schwer erfaßt werden kann. Die Diskontierung der erwarteten Cash Flows mit einem periodenunabhängigen risikoadjustierten Zinsfuß impliziert ein konstantes Risiko des Investitionsprojekts im Zeitablauf. Dies ist jedoch definitiv nicht gegeben, wenn die endgültige Entscheidung zur Investition in die neue Produktgruppe erst in der Zukunft in Abhängigkeit der zwischenzeitlichen Umweltentwicklung getroffen wird ${ }^{1}$. Unbestritten besitzt die zusätzliche Investitionsmöglichkeit jedoch einen Wert, der im Rahmen der Preisobergrenze der strategischen Akquisition nicht vernachlässigt werden kann. Im folgenden wird dessen optionspreistheoretische Ermittlung vorgestellt.

\subsubsection{Bestimmung des „Marktpreises“ des Bezugsguts}

Bei der Anwendung von Optionspreismodellen auf reale Investitionsmöglichkeiten stellt sich das Problem, daß das Bezugsgut der Realoption - die erwarteten Rückflüsse - nicht auf einem organisierten Markt gehandelt wird. Es existiert deshalb kein Marktpreis, in dem sich die tatsächlich erwarteten Eintrittswahrscheinlichkeiten sowie die Kenntnis der Risikopräferenzen der Marktteilnehmer widerspiegeln. Während z.B. bei Aktienoptionen der Aktienkurs diesen Marktpreis repräsentiert, muß hier vor der eigentlichen Optionsbewertung der „Marktpreis“ des Bezugsguts ermittelt werden.

Zur Lösung dieses Problems bieten sich zwei Möglichkeiten an. Zum einen kann der „Marktpreis“ des Bezugsguts mit Hilfe eines marktgängigen, in der Risikostruktur stark korrelierenden Vermögenstitels abgeschätzt werden. So wird beispielsweise vorgeschlagen, die Preisentwicklung natürlicher Ressourcen (Gold, Kupfer, Öl) zur Bewertung von Gold- und Kupferminen bzw. Ölbohrrechten zu nutzen ${ }^{2}$. Zum anderen kann der „Marktpreis“ des Bezugsguts durch Diskontierung der erwarteten Projektrückflüsse mit einem über den Kapitalmarkt ermittelten risikoadjustierten Zinsfuß bestimmt werden. Dabei kann auf Standardbewertungsmethoden zur Ermittlung eines Barwerts, wie z.B. traditionelle DCF-Ansätze, zurückgegriffen werden. Deren Ziel ist ohnehin die Bestimmung eines Werts, zu dem ein Investitionsprojekt unter den in diesen Modellen angenommenen Bedingungen am Kapitalmarkt gehandelt würde $e^{3}$.

1 Vgl. die Ausfuhrungen in Abschnitt 2.2.2 sowie 4.1 in Kapitel III.

2 Vgl. Brennan/Schwartz, Natural Resource, 1985, S. 136 f.; Paddock/Siegel/Smith, Option Valuation, 1988, S. $486 \mathrm{f}$.

3 Vgl. Mason/Merton, Contingent Claims Analysis, 1985, S. 31 f. und 38 f.; Teisberg, Methods, 1995, S. $38 \mathrm{f}$. 
Zur Bestimmung eines solchen Barwerts werden tatsächlich erwartete Eintrittswahrscheinlichkeiten und Risikopräferenzen benötigt, was auf den ersten Blick als Rückschritt erscheinen mag. Die Anwendung von traditionellen Bewertungsmethoden erfordert jedoch die gleichen Informationen. Der hier vorgestellte Lösungsansatz bietet den Vorteil, diesen Schritt einmalig bei der Bewertung des Bezugsguts und nicht mehr im Rahmen der Bewertung der Investitionsmöglichkeit durchführen zu müssen ${ }^{1}$.

Für die Periode der Marktbeobachtung soll für das Bezugsgut ebenfalls ein risikoadjustierter Zinsfuß von $25 \%$ gelten. Der Barwert des Bezugsguts $V_{0}$ beträgt somit 100 Mio. $€$.

$$
V_{0}=\frac{0,5 \cdot 200+0,5 \cdot 50}{1,25}=100 \text { Mio. } €
$$

\subsubsection{Optionspreistheoretische Bewertung der zusätzlichen Investitions- möglichkeit}

Für die Bewertung der zusätzlichen Investitionsmöglichkeit liegen nun folgende Daten vor. Der Barwert des Bezugsguts (= geschätzter Marktpreis) beläuft sich auf 100 Mio. $€$. Der Markteintritt der neuen Produktgruppe macht eine Investitionsauszahlung zum Ausbau des Vetriebsnetzes von 90 Mio. $€$ erforderlich. Tritt nach einer Periode Szenario 1 ein, verdoppelt sich gemäß den in Abschnitt 2.1.2.1 diesen Kapitels gemachten Annahmen der Gegenwartswert des Bezugsguts. Dies entspricht einer Steigerung von $100 \%$ bzw. einem Steigungsfaktor von 2. Tritt Szenario 2 ein, halbiert sich der Gegenwartswert, was einem Senkungsfaktor von 0,5 entspricht. Die Laufzeit der Investitionsmöglichkeit beträgt eine Periode. Der risikolose Zinsfuß soll sich auf 5\% pro Periode belaufen.

$\begin{array}{ll}\text { Barwert des Bezugsguts: } & V_{0}=100 \text { Mio. } € \\ \text { Investitionsauszahlung: } & I=90 \text { Mio. } € \\ \text { Steigungsfaktor: } & u=2 \\ \text { Senkungsfaktor: } & d=0,5 \\ \text { Risikoloser Zinsfuß der Binomialperiode: } & r_{f}=5 \% \\ \text { Optionslaufzeit: } & T=1 \text { Periode }\end{array}$

1 Vgl. Kester, Options Approach, 1986, S. 30; Laux, Handlungsspielräume, 1993, S. 940 sowie Abschnitt 2.3 in Kapitel VI. 
Nach Ablauf der Periode beträgt der Wert der Investitionsmöglichkeit bei hoher Produktnachfrage (Szenario 1) 110 Mio. $€$ und bei niedriger Produktnachfrage (Szenario 2) 0 Mio. $€$.

$$
\begin{gathered}
W_{u}=\max \left\{u \cdot V_{0}-I ; 0\right\}=\max \{200-90 ; 0\}=110 \text { Mio. } € \\
W_{d}=\max \left\{d \cdot V_{0}-I ; 0\right\}=\max \{50-90 ; 0\}=0 \text { Mio. } €
\end{gathered}
$$

Aus dem Steigungs- und Senkungsfaktor sowie dem risikolosen Zinsfuß können Pseudowahrscheinlichkeiten von $36,7 \%$ bzw. 63,3\% berechnet werden, mit denen sich eine risikoneutralisierte Bewertung der Investitionsmöglichkeit vornehmen läßt.

$$
p=\frac{1+r_{f}-d}{u-d}=\frac{1,05-0,5}{2-0,5}=36,7 \% \text { bzw. } 1-p=63,3 \%
$$

Der aktuelle Wert der Investitionsmöglichkeit $W_{0}$ beträgt somit 38,4 Mio. $€$.

$$
W_{0}=\frac{p \cdot W_{u}+(1-p) \cdot W_{d}}{1+r_{f}}=\frac{0,367 \cdot 110+0,633 \cdot 0}{1,05}=38,4 \text { Mio. } €
$$

Abb. 21 zeigt den Binomialbaum für die betrachtete Investitionsmöglichkeit. $V_{t, s}$ beschreibt den Gegenwartswert des Bezugsguts zum Zeitpunkt $t$ im Umweltzustand $s, K W_{t, s}$ den entsprechenden Kapitalwert nach Abzug der Investitionsauszahlung und $W_{t, s}$ den Wert der Investitionsmöglichkeit.

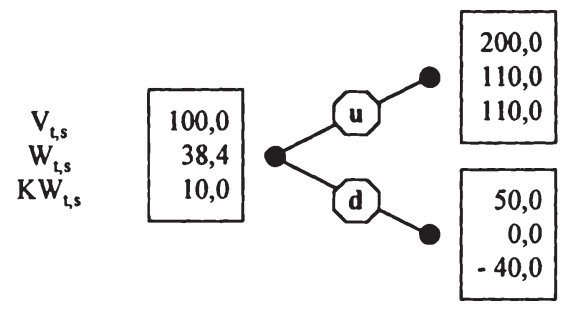

Abb. 21: Bewertung einer Investitionsmöglichkeit im einperiodigen Binomialmodell

Der Wert des Unternehmens B aus Sicht des Unternehmens A setzt sich aus dem in Abschnitt 2.1.2.1 ermittelten traditionellen Discounted Cash Flow von 150 Mio. $€$ und dem Wert der zusätzlichen Investitionsmöglichkeit in Höhe von rund 38 Mio. $€$ zusammen. Die Preisobergrenze beim Erwerb des Unternehmens B beläuft sich unter diesen Annahmen auf 188 Mio. $€$. 


\subsubsection{Zur Unterscheidung zwischen traditionellem Kapital-, Realoptions- und Flexibilitätswert}

Der soeben ermittelte Wert der Investitionsmöglichkeit von 38,4 Mio. $€$ ist um 28,4 Mio. $€$ höher als der zum Zeitpunkt $t_{0}$ geltende traditionelle Kapitalwert der Investition von 10 (100 - 90) Mio. €. Dieser zusätzliche Flexibilitätswert entsteht durch die Möglichkeit, die unsichere Umfeldentwicklung vor der endgültigen Entscheidungsfindung abzuwarten, die in der traditionellen Kapitalwertberechnung nicht berücksichtigt wird. Würde das Unternehmen die Investitionsentscheidung aufgrund des positiven Kapitalwerts sofort treffen, würde es auf den Flexibilitätswert verzichten.

Der traditionelle Kapitalwert der Investitionsmöglichkeit korrespondiert mit dem inneren Wert der Realoption. Der Kapitalwert ist der Wert, der sich bei sofortiger Investition, d.h. dem Ausüben der Realoption, ergibt. Der Flexibilitätswert stellt den Zeitwert der Realoption dar ${ }^{1}$. Wird eine im Zeitablauf konstante Investitionsauszahlung unterstellt, setzt sich der Zeitwert der Realoption aus zwei Komponenten zusammen ${ }^{2}$. Durch die Stundung der Investitionsauszahlung um eine Periode wird ein Zins- bzw. Opportunitätsertrag erwirtschaftet. Unter der Prämisse, daß der zum Ausbau des Vertriebsnetzes benötigte Betrag zum risikolosen Zinsfuß von 5\% angelegt wird, ergibt sich am Ende der Periode ein Zinsertrag von 4,5 $(90 \cdot 0,05)$ Mio. $€$. Der Wert dieses Zinsertrags beträgt zu Beginn der Periode 4,3 (4,5/1,05) Mio. €. Die Differenz zwischen dem Zinsertrag und dem Zeitwert der Realoption von 24,1 (28,4 - 4,3) Mio. $€$ kann als Schutzkomponente gegen eine ungünstige Entwicklung angesehen werden.

Wird die Prämisse einer konstanten Investitionsauszahlung im Zeitablauf aufgehoben, entspricht eine risikolose Investitionsauszahlung von 90 Mio. $€$ nach einer Periode einer heutigen Investitionsauszahlung von 85,7 (90/1,05) Mio. $€$. Der traditionelle Kapitalwert steigt dann um den Opportunitätsertrag von 4,3 auf 14,3 Mio. $€$ an, wodurch der Flexibilitätswert nur noch die Schutzkomponente von 24,1 Mio. $€$ verkörpert. Unabhängig davon, ob nun der Flexibilitätswert mit oder ohne Opportunitätsertrag definiert wird, setzt sich der auf Basis von Optionspreismodellen ermittelte Wert einer Investitionsmöglichkeit

1 Wird davon ausgegangen, daß der innere Wert - wie in der finanzwirtschaftlichen Optionspreistheorie üblich - nicht negativ werden kann, gilt die dargestellte Identität streng genommen nur bei einem positiven Kapitalwert. Die Aufspaltung des Werts der Investitionsmöglichkeit in zwei unterschiedliche Wertkomponenten bleibt jedoch hiervon unberührt.

2 Vgl. ähnlich Laux, Handlungsspielräume, 1993, S. 944. 
bzw. Realoptionswert aus dem traditionellen Kapitalwert und dem Flexibilitätswert zusammen (vgl. Abb. 22) ${ }^{1}$.

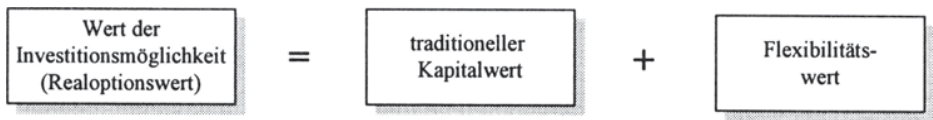

Abb. 22: Zusammensetzung des Werts einer Investitionsmöglichkeit

Der gleiche unternehmerische Handlungsspielraum wie bei der bisher beschriebenen Investitionsmöglichkeit besteht bei einem sofortigen Ausbau des Vertriebsnetzes, falls dieses nach einer Periode zu einem Preis von 90 Mio. $€$ wieder verkauft werden $\mathrm{kann}^{2}$. Bei einer derartigen Realoption handelt es sich dann jedoch um eine Desinvestitionsmöglichkeit bzw. Aufgabeoption. Bei Eintritt des Umweltzustands 1 wird die Möglichkeit des Verkaufs des eine Periode zuvor aufgebauten Vertriebsnetzes nicht wahrgenommen, da der Gegenwartswert der erwarteten Rückflüsse die Desinvestitionseinzahlung übersteigt. Der Wert der Desinvestitionsmöglichkeit ist in diesem Fall wertlos. In Umweltzustand 2 wird jedoch das Vertriebsnetz wieder veräußert. Der Wert der Desinvestitionsmöglichkeit beläuft sich auf $40(90$ - 50) Mio. $€$. Der aktuelle Wert dieser Realoption $D_{0}$ kann in Analogie zur Bewertung von Aktienoptionen mit Verkaufscharakter ermittelt werden.

$$
D_{0}=\frac{0,367 \cdot 0+0,633 \cdot 40}{1,05}=24,1 \text { Mio. } €
$$

Der auf Basis von Optionspreismodellen ermittelte Wert einer Desinvestitionsmöglichkeit bzw. Realoptionswert setzt sich nicht aus Kapital- und Flexibilitätswert zusammen. Da es sich nun um ein bereits in Betrieb befindliches Investitionsprojekt handelt, ist einerseits der Kapitalwert bereits realisiert und andererseits die Investitionsauszahlung geleistet worden, so daß kein Zinsertrag entstehen kann. Das Ergebnis spiegelt lediglich die Schutzkomponente der Desinvestitionsmöglichkeit von 24,1 Mio. € wider.

1 Vgl. hierzu stellvertretend Trigeorgis, Real Options, 1996, S. 124.

2 Dixit/Pindyck sprechen in diesem Zusammenhang von einem reversiblen im Gegensatz zu einem irreversiblen Projekt, bei dem die anfängliche Investitionsauszahlung bei ungünstiger Entwicklung nicht wiedergewonnen werden kann. Vgl. Dixit/Pindyck, Investment Under Uncertainty, 1994, S. 8 f. 
Es ist kein Zufall, daß sich die Schutzkomponenten der hier beschriebenen Investitions- und Desinvestitionsmöglichkeit entsprechen. $\mathrm{Da}$ in beiden Fällen der gleiche unternehmerische Handlungsspielraum dargestellt wird, sind Bezugsgut und Basispreis dieser Realoptionen identisch. Die Übereinstimmung der Schutzkomponenten ist somit das Ergebnis der aus der finanzwirtschaftlichen Optionspreistheorie bekannten Put/Call-Parität ${ }^{1}$.

$$
C_{0}=K_{0}-\frac{B}{1+r_{f}}+P_{0}
$$

\subsection{Mehrperiodiges Modell}

Zur Darstellung des mehrperiodigen Binomialmodells soll die Ausgangssituation leicht modifiziert werden. Bisher wurde unterstellt, daß das akquirierende Unternehmen A eine Periode nach der Akquisition endgültig über einen Ausbau des Vertriebsnetzes entscheiden muß. Nun sei angenommen, daß mit der Entscheidung über den Markteintritt der neuen Produktgruppe drei Perioden gewartet werden kann ${ }^{2}$. Das Binomialmodell wird dafür auf drei Binomialbzw. Teilperioden ausgeweitet. Jedem Umweltzustand zu Beginn einer Teilperiode können genau zwei Umweltzustände am Ende einer Teilperiode folgen. Aus Vereinfachungsgründen wird in allen Teilperioden von den gleichen Annahmen bezüglich der Wertentwicklung des Bezugsguts ausgegangen ${ }^{3}$.

Mit Ausnahme der Optionslaufzeit werden die im einperiodigen Binomialmodell verwendeten Ausgangsdaten übernommen. Der Barwert des Bezugsguts beträgt 100 Mio. $€$. Innerhalb einer Periode kann sich der Wert des Bezugsguts entweder verdoppeln oder halbieren. Bei drei Teilperioden mit gleichen Steigungs- und Senkungsfaktoren ergeben sich vier mögliche Umweltzustände am Ende der Optionslaufzeit ${ }^{4}$. Der Ausbau des Vetriebsnetzes koste unabhängig vom Investitionszeitpunkt 90 Mio. $€$. Der risikolose Zinsfuß betrage weiterhin $5 \%$ pro Teilperiode.

1 Zur Put/Call-Parităt vgl. Kruschwitz/Schöbel, Puts, 1984, S. 380.

2 Unabhängig vom Investitionszeitpunkt werden erst nach Ablauf dieser drei Perioden die ersten Rückflüsse erwartet.

3 Zum mehrperiodigen Binomialmodell vgl. Cox/Ross/Rubinstein, Option Pricing, 1979, S. 236 ff.; Kruschwitz/Schöbel, Optionspreistheorie, 1984, S. 116 ff.

4 Bei gleichwahrscheinlichem Eintreten der beiden Umweltzustände am Ende jeder Teilperiode folgen daraus Eintrittswahrscheinlichkeiten von $12,5 \%$ respektive $37,5 \%$ fur die vier Umweltzustănde am Ende der Optionslaufzeit. 
Die Ermittlung des aktuellen Werts der Investitionsmöglichkeit erfolgt rekursiv. Ausgehend von den Werten der Investitionsmöglichkeit in $t=3$ werden analog zur Vorgehensweise im einperiodigen Modell die Werte der Investitionsmöglichkeit in $t=2$ berechnet. Da sich Steigungs- und Senkungsfaktor sowie risikoloser Zinsfuß für eine Teilperiode im Vergleich zum vorherigen Abschnitt nicht verändert haben, können die dort ermittelten Pseudowahrscheinlichkeiten auch hier verwendet werden. Die Werte der Investitionsmöglichkeit in $t=1$ werden anschließend mit Hilfe der Werte in $t=2$, den Pseudowahrscheinlichkeiten und dem risikolosen Zinsfuß pro Teilperiode bestimmt. Sie bilden ihrerseits wiederum die Eingangsdaten für die Berechnung des Werts in $t=0$. Allgemein gilt ${ }^{1}$ :

$$
W_{t, s}=\frac{p \cdot W_{t+1, s_{u}}+(1-p) \cdot W_{t+1, s_{d}}}{1+r_{f}} .
$$

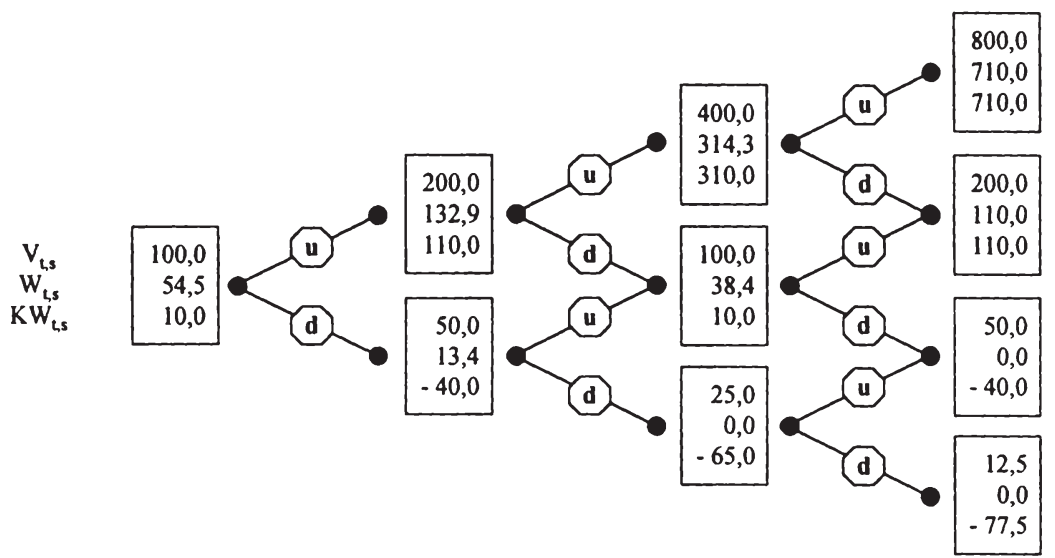

Abb. 23: Bewertung einer Investitionsmöglichkeit im dreiperiodigen Binomialmodell

Wie aus Abb. 23 ersichtlich beträgt der aktuelle Wert der dreiperiodigen Investitionsmöglichkeit 54,5 Mio. $€$. Gegenüber der einperiodigen Investitionsmöglichkeit ist der Wert gestiegen. Der Wertzuwachs von 16,1 Mio. $€$ ist auf die angenommene längere Optionslaufzeit zurückzuführen.

Durch die unterschiedlichen Annahmen hinsichtlich der Optionslaufzeit sind bislang zwei unterschiedliche Investitionsmöglichkeiten bewertet worden. Wird davon ausgegangen, daß eine Teilperiode einem Zeitraum von einem Jahr

Beispielsweise erhält man den Wert der Investitionsmöglichkeit in Teilperiode $t=2$ und Umweltzustand $s=1$ uber $W_{2,1}=(0,367 \cdot 710+0,633 \cdot 110) / 1,05=314,3$ Mio. $€$. 
entspricht, ist bislang im einperiodigen Modell eine einjährige und im dreiperiodigen Modell eine dreijährige Investitionsmöglichkeit bewertet worden. Soll mit dem einperiodigen Binomialmodell ein Wert für die dreijährige Investitionsmöglichkeit berechnet werden, darf die Teilperiode des Binomialmodells nicht mehr einem Jahr entsprechen. Um eine dreijährige Investitionsmöglichkeit in einem einperiodigen Binomialmodell abzubilden, muß das Bewertungsmodell modifiziert werden. Es ist eine Teilperiode mit einer Länge von drei Jahren anzunehmen. Damit jedoch unabhängig von der gewählten Anzahl an Teilperioden die gleiche Investitionsmöglichkeit bewertet wird, muß der Wert des Bezugsguts in beiden Kalkülen der gleichen jährlichen Standardabweichung unterliegen. Aus diesem Grund sind neue Steigungs- und Senkungsfaktoren $u$ und $d$ zu berechnen.

Gemäß der Parameterüberführung von Cox/Ross/Rubinstein ${ }^{1}$ ergeben sich $u$ und $d$ in Abhängigkeit der Optionslaufzeit in Jahren $T$, der Anzahl Binomialperioden $n$ sowie der jährlichen Standardabweichung des Bezugsguts $\sigma$ durch folgende Beziehung.

$$
u=e^{\sigma \cdot \sqrt{T / n}} \text { und } d=e^{-\sigma \cdot \sqrt{T / n}}=\frac{1}{u}
$$

Die Steigungs- und Senkungsfaktoren von $u=2$ bzw. $d=0,5$ entsprechen mit $T=3$ und $n=3$ bzw. mit $T=1$ und $n=1$ einer jährlichen Standardabweichung des Bezugsguts von $\sigma=69,31 \%$. Für ein einperiodiges Binomialmodell mit $T=3, n=1$ und $\sigma=69,31 \%$ gilt somit $u=3,32$ und $d=0,30$.

Daneben muß der risikolose Zinsfuß der Teilperiode angepaßt werden. Bei einer dreijährigen Optionslaufzeit und nur einer Teilperiode ergibt sich ein risikoloser Zinsfuß von $15,8 \%\left(1,05^{3}-1\right)$. Der Wert der dreijährigen Investitionsmöglichkeit beträgt im einperiodigen Binomialmodell 59,3 Mio. $€^{2}$ und unterscheidet sich damit um rund 5 Mio. $€$ vom Ergebnis aus dem dreiperiodigen Binomialmodell. Dies ist nun nicht mehr auf eine veränderte Optionslaufzeit zurückzuführen. Es wird die gleiche Investitionsmöglichkeit bewertet. Der Ergebnisunterschied ist durch die unterschiedliche Verteilung des Bezugsguts zu erklären.

1 Vgl. Cox/Ross/Rubinstein, Option Pricing, 1979, S. 246 ff.

2 Die Pseudowahrscheinlichkeiten betragen $p=(1,158-0,30) /(3,32-0,30)=28,4 \%$ und $1-p=71,6 \%$. Daraus ergibt sich $W_{0}=(0,284 \cdot 242+0,716 \cdot 0) / 1,158=59,3$ Mio. $€$. 
Sowohl das ein- als auch das dreiperiodige Binomialmodell liefern wegen ihrer vereinfachenden Annahmen zur Wertentwicklung des Bezugsguts nur eine grobe Approximation des Werts der Investitionsmöglichkeit. Eine Verbesserung der Bewertung läßt sich durch eine Verfeinerung der Verteilungsannahme erreichen. Dies bedeutet, daß die gleiche Optionslaufzeit in eine größere Anzahl an Teilperioden aufgeteilt wird ${ }^{1}$. Die Entscheidung über den Markteintritt der neuen Produktgruppe erfolgt gleichwohl nach dem zuvor festgelegten Zeitraum. Es wird lediglich die Wertentwicklung des Bezugsguts detaillierter dargestellt. Für eine konsistente Anwendung einer solchen Verfeinerung der Verteilungsannahme ist darauf $\mathrm{zu}$ achten, daß jeweils die Steigungs- und Senkungsfaktoren für die kürzeren Teilperioden neu bestimmt werden, damit die angenommene jährliche Standardabweichung des Bezugsguts nicht verändert wird. Zudem ist der risikolose Zinsfuß an die modifizierte Teilperiode anzupas$\operatorname{sen}^{2}$.

\subsection{Vergleich zwischen Optionspreistheorie und flexibler Investitions- planung}

Um den entscheidenden Vorteil von Optionspreismodellen zu unterstreichen, wird an dieser Stelle kurz auf Gemeinsamkeiten und Unterschiede im Vergleich zur traditionellen flexiblen Investitionsplanung eingegangen. Wie die Optionspreistheorie berücksichtigt die flexible Investitionsplanung den Umstand, daß in der Zukunft noch Entscheidungen in Abhängigkeit von zwischenzeitlichen unsicheren Umweltentwicklungen zu treffen sind. Auch in der flexiblen Investitionsplanung wird die Investition unter Berücksichtigung der optimalen Ausübung der über die Laufzeit des Investitionsprojekts vorhandenen Handlungsspielräume bewertet. Im Rahmen des i.d.R. verwendeten Entscheidungsbaumverfahrens wird jeweils die Alternative mit dem höheren (erwarteten) Rückfluß in die Bewertung einbezogen ${ }^{3}$.

Das Grundproblem des Modellansatzes der flexiblen Investitionsplanung besteht in der Berücksichtigung der für die jeweilige Periode sowie den jeweili-

1 Werden bei einer dreijährigen Investitionsmöglichkeit z.B. 36 Teilperioden zur Abbildung der Wertentwicklung des Bezugsguts angenommen, beträgt die Länge einer Teilperiode 0,083 Jahre bzw. 1 Monat.

2 Die Berechnung erfolgt allgemein anhand folgender Beziehung.

$$
1+r_{f, \text { Teilperiode }}=\left(1+r_{f, \text { p.a. }}\right)^{T n}
$$

3 Vgl. die Ausführungen in Abschnitt 4.2.2 in Kapitel III sowie die dort zitierte Literatur. 
gen Umweltzustand geltenden Risikosituation. Da sich das Risiko der Investitionsmöglichkeit laufend mit der Wertentwicklung des Bezugsguts verändert ${ }^{1}$, bedürfte es für eine risikogerechte Berechnung des Werts der Investitionsmöglichkeit mehrerer risikoadjustierter Zinsfüße. Genau genommen müßte für jede Periode und jeden Umweltzustand eine eigene Risikoprämie mit einem eigenen Zinsfuß ermittelt werden ${ }^{2}$. Eine Diskontierung der erwarteten Rückflüsse mit einem vom Informationsstand der jeweiligen Periode unabhängigen Zinsfuß ist aufgrund der vorliegenden Entscheidungs- und Risikosituation nicht gerechtfertigt $^{3}$.

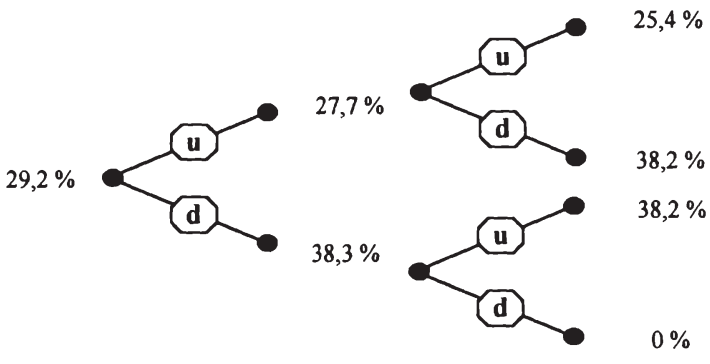

Abb. 24: Perioden- und zustandsabhängige Risikoprämien zur Bewertung der dreijährigen Investitionsmöglichkeit

Bei Kenntnis des optionspreistheoretisch ermittelten Ergebnisses lassen sich für jede Periode und jeden Umweltzustand die dazugehörigen Risikoprämien bestimmen. Im Beispiel der dreijährigen Investitionsmöglichkeit betragen sie

1 Im Rahmen des kapitalmarkttheoretischen Risikozuschlagmodells läßt sich der Betafaktor der Investitionsmöglichkeit $\beta_{W}$ in Abhängigkeit des Betafaktors des Bezugsguts $\beta_{V}$ wie folgt abbilden.

$$
\beta_{W}=\eta \cdot \beta_{V} \text { mit } \eta=\frac{\delta W / W}{\delta V / V}
$$

Diese Beziehung zeigt, daß mit jeder Veränderung des Bezugsguts der Betafaktor und damit der risikoadjustierte Zinsfuß der Investitionsmöglichkeit zu modifizieren ist, selbst wenn die Risikoprämie des Bezugsguts im Zeitablauf konstant ist. Vgl. hierzu im Kontext von Aktienoptionen Black/Scholes, Pricing of Options, 1973, S. 645; Jarrow/Rudd, Option Pricing, 1983, S. 107 ff.; Cox/Rubinstein, Options Markets, 1985, S. 185 ff. sowie für Realoptionen, Kulatilaka/Marcus, When Does DCF Fail?, 1992, S. 100.

2 Vgl. Ross, Simple Approach, 1978, S. 453 f.; Teisberg, Methods under Uncertainty, 1995, S. $33 \mathrm{f}$.

3 Vgl. Ritchken/Rabinowitz, Contingent Claims Analysis, 1988, S. 120 f.; Laux, Handlungsspielräume, 1993, S. 935 f.; Mostowfi, Flexibilität, 1997, S. 582. 
zwischen 0 und 38,3\% ${ }^{1}$. Mit Hilfe dieser Risikoprämien (vgl. auch Abb. 24) lassen sich perioden- und zustandsabhängige Zinsfüße bestimmen, die in dem Modellansatz der flexiblen Investitionsplanung zu dem gleichen Ergebnis führen wie der im Rahmen der Optionspreistheorie errechnete Wert ${ }^{2}$.

Wird innerhalb der flexiblen Investitionsplanung der Wert der Investitionsmöglichkeit mit Hilfe des einheitlichen risikolosen Zinsfußes von $5 \%$ ermittelt, resultiert ein Ergebnis von 112,3 Mio. $€$. Wird hingegen der einheitliche risikoadjustierte Zinsfuß des Bezugsguts von $25 \%$ und damit eine durchschnittliche Risikoprämie von $20 \%$ verwendet, beläuft sich der Wert der Investitionsmöglichkeit auf 66,6 Mio. $€$. Im Vergleich zum optionspreistheoretisch ermittelten Ergebnis von 54,5 Mio. $€$ stellt dies eine Abweichung von über $20 \%$ dar. Auf einem arbitragefreien Kapitalmarkt kann sich lediglich der zuletzt genannte Preis einstellen. Niemand ist bereit für eine Vermögensposition 112,3 bzw. 66,6 Mio. $€$ zu zahlen, wenn er die gleiche Vermögensposition auf anderem Wege für 54,5 Mio. $€$ erhalten kann.

Es läßt sich festhalten, daß sowohl die flexible Investitionsplanung als auch die Optionspreistheorie zukünftige bedingte Entscheidungen explizit in die Beurteilung der zu bewertenden Vermögensposition einbeziehen. Während jedoch die flexible Investitionsplanung keine Anhaltspunkte enthält, wie man der spezifischen und sich kontinuierlich ändernden Risikosituation der Investitionsmöglichkeit ökonomisch gerecht wird, bietet die Optionspreistheorie den Vorteil, diese über den indirekten Weg der risikoadjustierten Bewertung des Bezugsguts zu integrieren. Eine explizite Erfassung bzw. Quantifizierung des informationsstandabhängigen Risikos der Investitionsmöglichkeit ist dann nicht mehr notwendig. Bei Kenntnis dieses Sachverhalts kann es nicht überraschen, daß Trigeorgis/Mason die optionspreistheoretische Vorgehensweise als „Economically Corrected Version“ der „Decision Tree Analysis“ ${ }^{\text {“3 }}$ bezeichnen.

1 Beispielsweise ergibt sich in $t=1$ und $s=2$ ein risikoadjustierter Zinsfuß von $43,3 \%=$ $[(0,5 \cdot 38,4+0,5 \cdot 0) / 13,4]-1$. Bei einem risikolosen Zinsfuß von $5 \%$ resultiert eine Risikoprămie von $38,3 \%$.

2 Vgl. so auch Pindyck, Irreversibility and Investment Behaviour, 1991, S. 134.

3 Trigeorgis/Mason, Managerial Flexibility, 1987, S. 15. Vgl. ăhnlich Ritchken/Rabinowitz, Contingent Claims Analysis, 1988, S. 129; Kemna, Case Studies, 1993, S. 269; Copeland/ Koller/Murrin, Valuation, 1994, S. 453 f. 


\subsection{Berücksichtigung zwischenzeitlicher Rückflüsse}

Die Bewertung der Investitionsmöglichkeit erfolgte bislang unter der Annahme, daß die Rückflüsse aus dem Projekt unabhängig vom Investitionszeitpunkt erst nach Ablauf der „Wartezeit" anfallen. Dies ist gleichbedeutend mit der zur Aktienoptionsbewertung getroffenen Annahme, daß während der Optionslaufzeit keine Dividenden ausgeschüttet werden ${ }^{1}$. Ist jedoch davon auszugehen, daß während der Optionsfrist Dividendenzahlungen erfolgen bzw. durch eine frühzeitige Investition bereits Rückflüsse aus dem Projekt zu verdienen sind, bedarf es einer Modifikation des bisher vorgestellten Bewertungskalküls.

Im Fall der Aktienoptionsbewertung verringert sich durch die Dividendenzahlung c.p. der Aktienkurs, was aufgrund der direkten Abhängigkeit der Aktienoption vom Aktienkurs ebenfalls eine Wertveränderung der Option hervorruft. Während der Aktionär seinen Wertverlust durch den Erhalt der Dividende ausgleicht und somit die Aktie keiner wirklichen Neubewertung bedarf, partizipiert der Inhaber einer Kaufoption nicht an dem ausgeschütteten Betrag. Die zwischenzeitliche Dividendenzahlung führt somit definitiv zu einer Senkung seiner Vermögensposition ${ }^{2}$ mit der Notwendigkeit, die Bewertung der Aktienoption zu modifizieren ${ }^{3}$.

Im Fall der Realoptionsbewertung fließen durch einen Entscheidungsaufschub die zwischenzeitlich zu verdienenden Rückflüsse dem Unternehmen nicht zu. Wenn diese Rückflüsse durch die Startverzögerung definitiv verloren gehen, ist der hiervon ausgehende Effekt auf den Wert von Investitionsmöglichkeiten direkt mit demjenigen einer Dividendenzahlung bei Aktienoptionen vergleich$\mathrm{bar}^{4}$. Durch die zwischenzeitlich entgehenden Rückflüsse verringert sich der zukünftige (erwartete) Gegenwartswert des Bezugsguts $V_{t, s}^{e x}$ und somit auch gleichzeitig der Wert der betrachteten Investitionsmöglichkeit.

Im Binomialmodell können unterschiedliche Arten zwischenzeitlich entgehender Rückflüsse $C F_{t, s}$ erfaßt werden. Zum einen lassen sich die Bewertungssi-

1 Vgl. Abschnitt 2.1.1.1 in diesem Kapitel.

2 Bei Verkaufsoptionen stellt sich der entgegengesetzte Effekt ein. Die Aussagen gelten nur für nicht dividendengeschützte Optionen. Bei dividendengeschützten Optionen, bei denen nach Dividendenzahlung dem Optionsinhaber ein Recht auf Kauf bzw. Verkauf von einem um den Anteil der Dividendenausschüttung erhöhten Aktienbetrag zusteht, verändert sich der Optionswert nicht.

3 Vgl. allgemein Cox/Rubinstein, Options Markets, 1985, S. 236 ff.

4 Vgl. stellvertretend Pickles/Smith, Binomial Lattice Implementation, 1993, S. 3. 
tuationen danach einteilen, ob solche Projektverluste nur am Ende einer Teilperiode oder am jeweiligen Ende aller Teilperioden auftreten. Zum anderen kann zwischen entgehenden Rückflüssen, die nur in einem bestimmten Umweltzustand und solchen, die unabhängig vom Umweltzustand auftreten, unterschieden werden. Drittens stellt sich die Frage, ob die Höhe der zwischenzeitlich entgehenden Rückflüsse in einem proportionalen Verhältnis zum Wert des Bezugsguts stehen oder in einer bestimmten, vom Wert des Bezugsguts unabhängigen, absoluten Höhe auftreten ${ }^{1}$. Unabhängig von der spezifischen Art des unterstellten zwischenzeitlichen Projektrückflusses erfolgt dessen Integration in das bisherige Bewertungskalkül, indem der Wert des Bezugsguts am Ende der jeweiligen Teilperiode verringert wird ${ }^{2}$.

$$
\begin{aligned}
& V_{t, s_{u}}^{e x}=u \cdot V_{t-1, s}^{e x}-C F_{t, s_{u}} \\
& V_{t, s_{d}}^{e x}=d \cdot V_{t-1, s}^{e x}-C F_{t, s_{d}}
\end{aligned}
$$

Ohne zwischenzeitlich entgehende Rückflüsse ist es bei Investitionsmöglichkeiten immer vorteilhaft, bis zum spätest möglichen Investitionszeitpunkt zu warten. Bei Existenz zwischenzeitlicher Projektrückflüsse muß hingegen während der Optionslaufzeit überprüft werden, ob der erwartete Nutzen der Informationsverbesserung den Verlust der zwischenzeitlichen Rückflüsse rechtfertigt $^{3}$. Im Bewertungskalkül ist deshalb zu Anfang jeder Teilperiode der innere Wert der Realoption mit dem Realoptionswert zu vergleichen ${ }^{4}$.

Ist der zu diesem Zeitpunkt geltende Kapitalwert unter Berücksichtigung des realisierbaren Rückflusses der nächsten Periode höher als der Gegenwartswert der Investitionsmöglichkeit ohne diesen Rückfluß, sollte trotz Aufgabe des Flexibilitätswerts investiert werden.

1 Vgl. im Hinblick auf Aktienoptionen Schroder, Fixed Cash Payouts, 1988, S. 54 ff.; Loistl, Wertpapiermanagement, 1992, S. 354 ff.; Pflüger/Ulrich, Amerikanische Optionen, 1997, S. $65 \mathrm{ff}$.

2 Vgl. zu letzterem Cox/Ross/Rubinstein, Option Pricing, 1979, S. 255 ff.

3 Vgl. u.a. Teisberg, Methods under Uncertainty, 1995, S. 39.

4 Bei amerikanischen Verkaufsoptionen auf Aktien bzw. Desinvestitionsmöglichkeiten ist dieser Vergleich auch ohne Dividenden bzw. laufende Rückflüsse durchzufuhren. Zur vorzeitigen Ausübung von amerikanischen Aktienoptionen ohne Dividenden und den resultierenden Bewertungskonsequenzen vgl. Kruschwitz/Schöbel, Puts, 1984, S. 380 ff.; Pflüger/Ulrich, Amerikanische Optionen, 1997, S. 64. 


$$
K W_{t, s}>W_{t, s} \text { bzw. } V_{t, s}^{e x}-I>\frac{p \cdot W_{t+1, s_{u}}+(1-p) \cdot W_{t+1, s_{d}}}{1+r_{f}}
$$

Die in jeder Teilperiode stattfindende Überprüfung der Investitionsentscheidung bedingt, daß sich aus diesem Bewertungskalkül auch Hinweise für den optimalen Investitionszeitpunkt gewinnen lassen ${ }^{1}$.

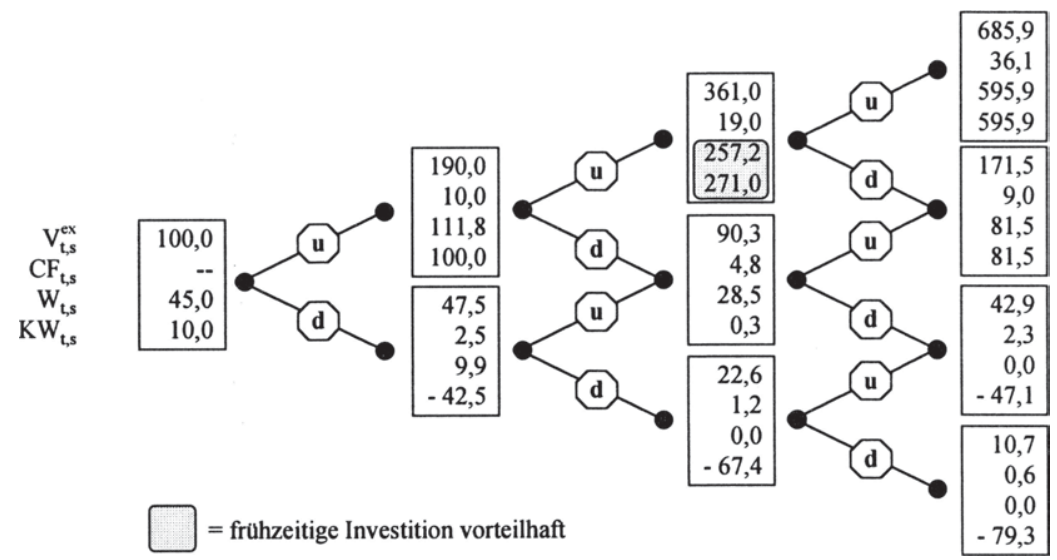

Abb. 25: Bewertung einer Investitionsmöglichkeit mit zwischenzeitlich entgehenden Rückflüssen

Im Beispiel der Investitionsmöglichkeit mit dreijähriger Laufzeit sei nun angenommen, daß durch die Verschiebung der Investitionsentscheidung der Gegenwartswert des Bezugsguts in jeder Teilperiode und in jedem Umweltzustand um 5\% sinkt. Nach einem Jahr werden also nicht mehr 200 Mio. $€$ bzw. 50 Mio. $€$ sondern nur noch 190 Mio. $€$ bzw. 47,5 Mio. $€$ als erwartete Gegenwartswerte des Bezugsguts unterstellt. Die Berechnung der Optionswerte in den einzelnen Teilperioden vollzieht sich gleichwohl nach dem Prinzip wie im Modell ohne zwischenzeitliche Rückflüsse ${ }^{2}$. Zu beachten ist jedoch, daß im Rahmen der rekursiven Vorgehensweise gegebenenfalls in bestimmten Teilperioden bzw. Umweltzuständen der Realoptionswert durch den höheren Kapitalwert für die weitere Berechnung ersetzt werden muß. Abb. 25 verdeutlicht diesen Bewertungsvorgang. Der Wert des Bezugsguts zum jeweiligen Zeitpunkt und Umweltzustand ist jetzt unter Abzug des zwischenzeitlichen Cash

1 Vgl. stellvertretend Lee, Optimal Timing, 1988, S. $155 \mathrm{ff}$.

2 Von Vorteil ist, daß sich die bereits bestimmten Pseudowahrscheinlichkeiten auch in diesem Fall verwenden lassen. Neben dem risikolosen Zinsfuß beruht ihre Ermittlung lediglich auf der (möglichen) Wertentwicklung des Bezugsguts bei frühzeitiger Investition. 
Flows dargestellt $\left(V_{t, s}^{e x}\right) . C F_{t, s}$ repräsentiert den zwischenzeitlichen Rückfluß zum Zeitpunkt $t$ im Umweltzustand $s$.

Unter den hier angenommenen Bedingungen beträgt der aktuelle Wert der dreijährigen Investitionsmöglichkeit 45 Mio. $€$. Dieser setzt sich aus dem traditionellen Kapitalwert von 10 Mio. $€$ und dem Flexibilitätswert von nun 35 Mio. $€$ zusammen. Gegenüber der Situation ohne zwischenzeitlich entgehende Rückflüsse beinhaltet der Flexibilitätswert nun nicht nur einen reinen, ohne zwischenzeitliche Effekte ermittelten, Wert des Abwartens von 44,5 Mio. $€$, sondern ebenfalls den durch den zwischenzeitlich entgehenden Rückfluß bedingten Wertverlust von 9,5 Mio. $€\left(\right.$ vgl. Abb. 26) ${ }^{1}$.

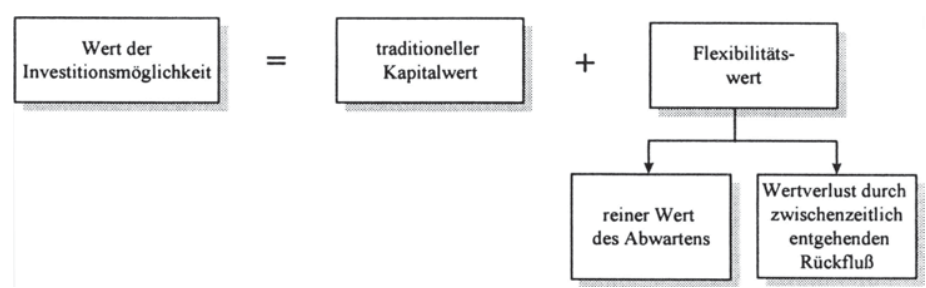

Abb. 26: Zusammensetzung des Werts einer Investitionsmöglichkeit unter Berücksichtigung eines zwischenzeitlich entgehenden Rückflusses

Der Wertverlust von 9,5 Mio. $€$ ist zunächst auf die in jeder Teilperiode und in jedem Umweltzustand geltenden niedrigeren Gegenwartswerte des Bezugsguts zurückzuführen. Andererseits ist in Teilperiode $t=2$ sowie Umweltzustand $s=$ 1 eine frühzeitige Investition gegenüber eines weiteren Investitionsaufschubs vorteilhaft. Der Gegenwartswert der Investitionsmöglichkeit von 257,2 Mio. $€$ liegt unter dem zu diesem Zeitpunkt geltenden Kapitalwert von 271,0 Mio. $€$. Gegenüber der Situation ohne zwischenzeitliche Rückflüsse wird dadurch der reine Wert des Abwartens für die noch verbleibende Optionslaufzeit aufgegeben.

Dieser Verzicht auf den reinen Wert des Abwartens ist jedoch vorteilhaft, da der ansonsten, durch den zwischenzeitlichen Rückfluß, eintretende Wertverlust noch größer ist. Die frühzeitige Investition führt deshalb zu einer Werterhö-

I Vgl. auch Beißinger/Möller, Neue Investitionstheorie, 1994, S. 271, die eine ähnliche Gleichung fur einen risikoneutralen Investor aufstellen. 
hung der Investitionsmöglichkeit ${ }^{1}$. Wird in Teilperiode $t=2$ trotz Umweltzustand $s=1$ nicht investiert, führt dies zu einem Wert der Investitionsmöglichkeit von 43,3 Mio. $€$. Die Differenz zum oben berechneten Wert der Investitionsmöglichkeit von 45,0 Mio. € erklärt sich durch den Umstand, daß durch die frühzeitige Investition $1,7\left[0,367^{2} \cdot(271,0-257,2) / 1,05^{2}\right]$ Mio. $€$ gegenüber dem Fall der Nichtinvestition gesichert werden können.

1 Ohne den zwischenzeitlichen Projektverlust in $t=3$ ergäbe sich ein Wert der Investitionsmöglichkeit in $t=2, s=1$ von 275,5 Mio. $€$. Der durch diesen zwischenzeitlichen Rückfluß bedingte Wertverlust von 18,3 (275,5 - 257,2) Mio. $€$ ist größer als der zu diesem Zeitpunkt aufgegebene reine Wert des Abwartens von 4,5 (275,5 - 271,0) Mio. $€$. 


\section{Black/Scholes-Modell als zeitstetiger Bewertungsansatz}

\subsection{Grundlagen zeitstetiger Zufallsprozesse}

Die Annahme der Wertentwicklung des Bezugsguts entlang eines zeitdiskreten mutiplikativen Binomialprozesses kann durch die Annahme anderer stochastischer Prozesse ersetzt werden ${ }^{1}$. Beispielsweise wird im Black/Scholes-Modell angenommen ${ }^{2}$, daß die Wertentwicklung des Bezugsguts einem zeitstetigen generellen Wiener Prozeß (geometrisch Brownsche Bewegung) folgt ${ }^{3}$.

Zeitstetige Prozesse zeichnen sich dadurch aus, daß Veränderungen der $\mathrm{Zu}$ fallsvariablen nicht nur zu bestimmten diskreten Zeitpunkten, sondern im Zeitablauf kontinuierlich möglich sind. In einem infinitesimal kleinen Zeitintervall $d t$ vollzieht die Zufallsvariable $X$ eine infinitesimal kleine Veränderung $d z$. Damit der Verlauf einer Zufallsvariablen als einfacher Wiener Prozeß bezeichnet werden kann, müssen folgende zwei Bedingungen erfüllt sein. Zum einen muß die Veränderung $d z$ vom Zeitintervall $d t$ abhängen in der Form

$$
d z=\varepsilon \cdot \sqrt{d t},
$$

wobei $\varepsilon$ einer Standardnormalverteilung gehorcht. Daraus folgt, daß $d z$ einer Normalverteilung mit Mittelwert $\mu(d z)=0$ und Standardabweichung $\sigma(d z)$ $=\sqrt{d t}$ gehorcht. Zum anderen müssen die einzelnen Veränderungen $d z$ der einzelnen Zeitintervalle $d t$ unabhängig voneinander sein. Diese zweite Bedingung sichert die Markov-Eigenschaft, die besagt, daß einzig der aktuelle Wert der Zufallsvariablen einen Einfluß auf zukünftige Werte hat. Welchen Weg die Zufallsvariable in der Vergangenheit hin zu diesem aktuellen Wert zurückgelegt hat, ist bedeutungslos. Der aktuelle Wert enthält bereits alle Informationen der Vergangenheit.

Der bisher betrachtete einfache Wiener Prozeß besitzt eine Wachstumsrate von null und eine Varianzrate von eins. Dies bedeutet, daß zum einen der Erwartungswert der Zufallsvariablen nach einem Zeitintervall $t$ dem aktuellen Wert entspricht und zum anderen das Ausmaß der Streuung der Zufallsvariablen

\footnotetext{
Vgl. auch die Ausführungen in Abschnitt 4 dieses Kapitels.

2 Vgl. Black/Scholes, Pricing of Options, 1973, S. 637 ff.

3 Zu Grundlagen zeitstetiger Prozesse und den folgenden Ausführungen vgl. Dixit/Pindyck, Investment Under Uncertainty, 1994, S. 63 ff.; Loistl, Kapitalmarkttheorie, 1994, S. 95 ff.; Hull, Options, 1997, S. 210 ff. Eine gute Einführung bietet auch Mann, Einführung, 1994, S. 674 ff. sowie Mann, Zeithorizonteffekte, 1995, S. 299 ff.
} 
nicht verändert werden kann. Beides schränkt die Anwendung dieses Prozesses zur Abbildung von Werteverläufen am Kapitalmarkt bzw. von realen Investitionsprojekten erheblich ein. Um diesen Mangel zu beheben, kann zu dem einfachen Wiener Prozeß eine pro Zeiteinheit $d t$ definierte Trendkomponente bzw. Wachstumsrate $a$ der Zufallsvariablen $X$ sowie eine allgemeine Diffusionskomponente bzw. Abweichungsrate $b$ hinzugefügt werden ${ }^{1}$. Der resultierende generelle Wiener Prozeß läßt sich durch folgende Form charakterisieren.

$$
d X=a \cdot d t+b \cdot d z
$$

In Abb. 27 ist ein solcher Prozeß graphisch dargestellt.

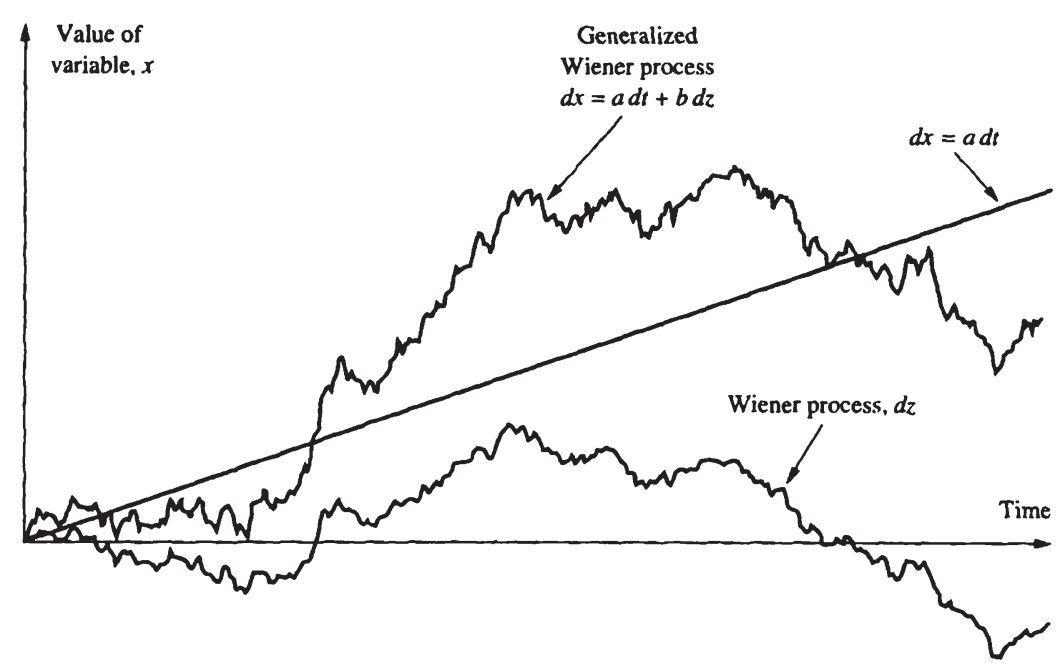

Abb. 27: Genereller Wiener Prozeß

Quelle: Hull, Options, 1997, S. 214.

Im folgenden wird das Grundmodell von Black und Scholes zunächst am Beispiel von Aktienoptionen illustriert. Anschließend erfolgt die Bewertung einer realen Investitionsmöglichkeit, indem die im Black/Scholes-Modell benötigten Bewertungsparameter uminterpretiert werden.

1 Hängen $a$ und $b$ von der Zufallsvariablen $X$ und der Zeit $t$ ab, wird von einem Itô-Prozeß gesprochen. Die Wachstumsrate $a$ und die Abweichungsrate $b$ unterliegen dann Veränderungen im Zeitablauf.

$$
d X=a(X, t) \cdot d t+b(X, t) \cdot d z
$$




\subsection{Grundmodell der Black/Scholes-Bewertung}

Bis auf die Aktienkursentwicklung stimmen die Annahmen, die Black und Scholes in ihrem Modell treffen, mit denen im Binomialmodell überein ${ }^{1}$. Der Aktienkurs (bzw. die Aktienkursveränderung $d K / K$ ) folgt nun nicht einem diskreten Binomialprozeß sondern einem zeitstetigen generellen Wiener Prozeß

$$
d K=\mu \cdot K \cdot d t+\sigma \cdot K \cdot d z
$$

wobei $\mu$ die erwartete momentane Aktienkursrendite und $\sigma$ die erwartete momentane Standardabweichungsrate darstellen ${ }^{2}$.

Durch Kauf eines bestimmten Anteils der Aktie und Verkauf einer Option auf diese Aktie ist es möglich, ein risikoloses Hedgeportfolio zu konstruieren ${ }^{3}$. Der Aktienanteil entspricht dabei der relativen Veränderung des Optionswerts in Bezug auf den Aktienkurs $(\delta C / \delta K)^{4}$. Der Wert dieses Portfolios vor einer Aktienkursänderung beträgt

$$
H=\frac{\delta C}{\delta K} \cdot K-C
$$

Erfolgt eine Aktienkursschwankung in Höhe von $d K$, verändert sich der Portfoliowert um

$$
d H=\frac{\delta C}{\delta K} \cdot d K-d C
$$

Die Veränderung des Aktienkurses hängt gemäß dem oben beschriebenen Prozeß ausschließlich vom Aktienkurs $K$ und der Zeit $t$ ab. Aufgrund des direkten Abhängigkeitsverhältnisses der Option von der zugrundeliegenden Aktie gilt

1 Es sei daran erinnert, daß entgegen der Gliederung dieser Arbeit Black und Scholes ihr zeitstetiges Bewertungsmodell vor dem zeitdiskreten Ansatz von Cox, Ross und Rubinstein aufgestellt haben.

2 Black und Scholes gehen bei der Formulierung ihrer Annahmen nicht explizit auf die erwartete Aktienkursrendite ein. Vgl. Black/Scholes, Pricing of Options, 1973, S. 640.

3 Zur originären Ableitung der Black/Scholes-Formel vgl. Black/Scholes, Pricing of Options, 1973, S. 641 ff. Für deutschsprachige Darstellungen vgl. Loistl, Wertpapiermanagement, 1992, S. 414 f.; Steiner/Bruns, Wertpapiermanagement, 1998, S. 298 f.

4 Das risikolose Hedgeportfolio kann auch über eine Aktie und eine bestimmte Anzahl Optionen auf diese Aktie gebildet werden. Der Optionsanteil beträgt dann $\delta K / \delta C$. 
dies auch für eine Veränderung des Optionswerts. Gemäß Itô's Lemma läßt sich die Veränderung des Optionswerts als Taylor-Series-Expansion schreiben.

$$
d C=\frac{\delta C}{\delta t} \cdot d t+\frac{\delta C}{\delta K} \cdot d K+\frac{1}{2} \cdot \frac{\delta^{2} C}{\delta K^{2}}(d K)^{2}
$$

Wenn der Aktienkurs $K$, wie oben angenommen, einem generellen Wiener Prozeß folgt, dann kann $(d K)^{2}$ durch $\sigma^{2} \cdot K^{2} \cdot d t$ ersetzt werden. Durch Substitution der Taylor-Series-Expansion in die Veränderung des Portfoliowerts $d H$ ergibt sich

$$
d H=\frac{\delta C}{\delta K} \cdot d K-\frac{\delta C}{\delta t} \cdot d t-\frac{\delta C}{\delta K} \cdot d K-\frac{1}{2} \cdot \frac{\delta^{2} C}{\delta K^{2}} \cdot \sigma^{2} \cdot K^{2} \cdot d t
$$

An dieser Stelle ist die Aktienkursschwankung durch geschickte Bildung eines Portfolios eliminiert worden. Diese Tatsache bewirkt, daß der Inhaber eines solchen risikolosen Hedgeportfolios für den betrachteten Zeitraum eine risikolose Rendite erhält. Durch Verzinsung des Portfoliowerts in Höhe des risikolosen Zinsfußes

$$
d H=r_{f} \cdot d t \cdot H=r_{f} \cdot d t \cdot\left(\frac{\delta C}{\delta K} \cdot K-C\right)
$$

und Gegenüberstellung mit der zuvor gebildeten Gleichung gelangt man zu folgender partiellen Differentialgleichung.

$$
\frac{\delta C}{\delta t}+\frac{1}{2} \cdot \frac{\delta^{2} C}{\delta K^{2}} \quad \sigma^{2} \cdot K^{2}+r_{f} \cdot \frac{\delta C}{\delta K} \cdot K-r_{f} \cdot C=0
$$

In Anlehnung an ein analytisches Lösungsverfahren, das insbesondere in der Physik angewandt wird, und unter den folgenden drei Nebenbedingungen,

- der Wert einer Kaufoption am Laufzeitende $T$ ist gleich dem inneren Wert, falls dieser positiv ist, ansonsten null

$$
C_{t=T}=\max (K-B ; 0),
$$

- für den Fall, daß die Aktie $K$ keinen Wert besitzt, ist auch die Kaufoption wertlos,

1 Zu Itô's Lemma vgl. Klump, Itô-Theorem, 1985, S. 183 f.; Dixit/Pindyck, Investment Under Uncertainty, 1994, S. 79 ff.; Hull, Options, 1997, S. 220 ff. 


$$
C_{K=0}=0,
$$

- bei unendlich hohen Aktienkursen sind Aktienkurs und Optionswert identisch

$$
C_{K=\infty}=K \text {, }
$$

haben Black und Scholes die partielle Differentialgleichung zu einer analytischen (eindeutigen geschlossenen) Lösung zur Bewertung von europäischen Kaufoptionen umgeformt ${ }^{1}$.

$$
C=K \cdot N\left(d_{1}\right)-B \cdot e^{-r_{f} \cdot t} \cdot N\left(d_{2}\right)
$$

mit $d_{l}=\frac{\ln \frac{K}{B}+\left(r_{f}+0,5 \cdot \sigma^{2}\right) \cdot t}{\sigma \cdot \sqrt{t}}$ sowie $d_{2}=d_{l}-\sigma \cdot \sqrt{t}$

$$
\begin{aligned}
\text { wobei } & N(.) \\
K & =\text { Wert der Standardnormalverteilung } \\
B & =\text { Bastueller Aktienkurs } \\
\sigma & =\text { jährliche Volatilität der Aktie } \\
r_{f} & =\text { risikoloser Zinsfuß p.a. } \\
t & =\text { Optionslaufzeit in Jahren }
\end{aligned}
$$

Bei der Anwendung dieser Bewertungsformel ist darauf zu achten, daß die jährliche Volatilität der Aktie auf Basis zeitstetiger Aktienkursrenditen gemessen wird, damit die Kompatibilität zu der hier erfolgenden zeitstetigen Bewertung und der im Black/Scholes-Modell angenommenen Verteilungsannahme

\footnotetext{
$\mathrm{Zu}$ einer alternativen, grundsätzlich jedoch ähnlichen Ableitung der Black/Scholes-Formel vgl. Merton, Rational Option Pricing, 1973, S. 162 ff. Merton konstruiert ein Arbitrageportfolio aus Aktie, risikolosem Wertpapier und Option, das eine erwartete Rendite von null aufweist. Desweiteren kann die Black/Scholes-Formel auch aufgrund von Grenzbetrachtungen des Binomialmodells, mit Hilfe einer risikoneutralisierten Erwartungswertberechnung bzw. innerhalb eines intertemporalen CAPM hergeleitet werden. Vgl. Sick, Capital Budgeting with Real Options, 1989, S. 19; Schäfer, Optionsbewertung, 1994, S. 45. Zur Darstellung der einzelnen Ansätze vgl. stellvertretend Black/Scholes, Pricing of Options, 1973, S. 645 f.; Kesting/ Schulte-Mattler, Herleitung, 1992, S. 170 f.; Galitz, Financial Engineering, 1994, S. $210 \mathrm{ff}$.
} 
gewährleistet ist ${ }^{1}$. Die möglichen Aktienkurse am Ende der Optionslaufzeit gehorchen einer logarithmischen Normalverteilung, d.h. die logarithmierten Aktienkurse sind normalverteilt. Damit unterliegen auch die logarithmierten Aktienkursrenditen einer Normalverteilung. Letztere ist durch die Parameter Erwartungswert $\mu \cdot d t$ und Standardabweichung $\sigma \cdot \sqrt{d t}$ gekennzeichnet ${ }^{2}$.

Die Bewertung europäischer Verkaufsoptionen erfolgt über eine ähnliche Vorgehensweise ${ }^{3}$. Für amerikanische Kaufoptionen ohne Dividenden kann die gleiche Bewertungsformel wie für europäische Kaufoptionen verwandt werden. Das Recht zur vorzeitigen Ausübung hat bei amerikanischen Kaufoptionen ohne Dividenden keinen Wert. Bei amerikanischen Verkaufsoptionen hingegen kann es sinnvoll sein, die Option vor dem Laufzeitende auszuüben ${ }^{4}$. Dies ist dann der Fall, wenn zu einem bestimmten Zeitpunkt der innere Wert den Optionswert übersteigt. Für solche Optionen stellt das Black/Scholes-Ergebnis lediglich eine Wertuntergenze dar, die das Recht zur vorzeitigen Ausübung nicht beinhaltet.

Durch Uminterpretation der Bewertungsparameter kann die Black/ScholesFormel auch zur Bewertung der dreijährigen Investitionsmöglichkeit herangezogen werden. Als aktueller Aktienkurs wird der Barwert des Bezugsguts $V_{0}$ von 100 und als Basispreis die Investitionsauszahlung $I$ von 90 Mio. $€$ eingesetzt. Bei einer jährlichen Standardabweichung $\sigma$ von $69,31 \%$, einem risikolosen Zinsfuß $r_{f}$ von 5\% p.a. sowie einer Optionslaufzeit $t$ von 3 Jahren ergibt sich ein Wert der Investitionsmöglichkeit von 52,1 Mio. €.

Dieses und die zuvor im Binomialmodell erzielten - hiervon abweichenden Ergebnisse zeigen, daß der Wert der dreijährigen Investitionsmöglichkeit wie bei allen Barwertkalkülen unter Unsicherheit von der unterstellten Verteilung

1 Die zeitstetige Rendite läßt sich über $\ln \left(K^{*} / K\right)$ ermitteln, wobei $K$ den aktuellen und $K^{*}$ den zukünftigen Aktienkurs darstellt. Durch die Logarithmierung wird unterstellt, daß Wertzuwächse stetig geschaffen werden. Außerdem können negative Aktienkurse ausgeschlossen werden. Drittens entsteht der Vorteil, daß logarithmierte Werte eher einer Normalverteilung gehorchen als nicht-logarithmierte Werte. Vgl. hierzu auch Cox/Rubinstein, Options Markets, 1985, S. 201 ff.; Loistl, Kapitalmarkttheorie, 1994, S. 118 ff.

2 Vgl. Cox/Rubinstein, Options Markets, 1985, S. 203.

$3 P=B \cdot e^{-r_{f} \cdot t} \cdot N\left(d_{2}\right)-K \cdot N\left(d_{l}\right) ; d_{l}=\frac{\ln \frac{B}{K}-\left(r_{f}+0,5 \cdot \sigma^{2}\right) \cdot t}{\sigma \cdot \sqrt{t}} ; d_{2}=d_{l}+\sigma \cdot \sqrt{t}$.

4 Zum Wert der vorzeitigen Ausübung bei amerikanischen Aktienoptionen vgl. Kruschwitz/ Schöbel, Puts, 1984, S. 380 ff. 
der Zufallsvariablen abhängt ${ }^{1}$. Von Interesse ist ebenfalls welche Veränderung das Ergebnis erfährt, wenn ein dem Optionspreismodell zugrundegelegter Eingangsparameter modifiziert wird. Während unter ceteris paribus-Bedingungen der Wert der Investitionsmöglichkeit steigt, falls sich die Laufzeit, der Barwert oder die Standardabweichung des Bezugsguts vergrößert, sinkt er bei einer erhöhten Investitionsauszahlung oder einem zunehmenden risikolosen Zinsfuß ${ }^{2}$.

\subsection{Berücksichtigung zwischenzeitlicher Rückflüsse}

Merton entwickelt das Grundmodell von Black und Scholes weiter zur Berücksichtigung von zwischenzeitlichen Zahlungen, indem er eine kontinuierliche, über die Optionslaufzeit konstante Dividendenrate in Höhe von $r_{D}$ annimmt ${ }^{3}$. Die „Basisformel“ wird dafür an zwei Stellen modifiziert. Durch zwischenzeitliche Ausschüttungen verringert sich der potentielle Aktienkurs am Ende der Optionslaufzeit, was durch Verringerung des aktuellen Aktienkurses um die unterstellte Dividendenrate berücksichtigt wird. Zum anderen erhält der Inhaber des Hedgeportfolios nur noch eine Rendite in Höhe des risikolosen Zinsfußes abzüglich der Dividendenrate $\left(r_{f}-r_{D}\right)^{4}$. Dies wird bei der Bestimmung des Terms $d_{l}$ berücksichtigt.

$$
\begin{gathered}
C=K \cdot e^{-r_{D} \cdot t} \cdot N\left(d_{l}\right)-B \cdot e^{-r_{f} \cdot t} \cdot N\left(d_{2}\right) \\
\text { mit } d_{l}=\frac{\ln \frac{K}{B}+\left(r_{f}-r_{D}+0,5 \cdot \sigma^{2}\right) \cdot t}{\sigma \cdot \sqrt{t}} \text { sowie } \quad d_{2}=d_{l}-\sigma \cdot \sqrt{t}
\end{gathered}
$$

Eine andere Erfassung von zwischenzeitlichen Zahlungen im Black/ScholesModell ist die Berücksichtigung von absoluten, bezüglich ihrer Höhe und ihres zeitlichen Anfalls sicheren, Dividendenzahlungen Div. Hierfür wird vom aktu-

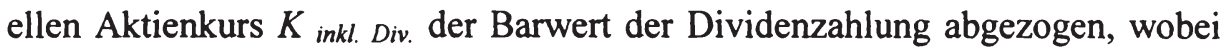
aufgrund der Annahme der Sicherheit eine risikolose Verzinsung unterstellt

1 Zur Konvergenz zwischen dem Binomial- und dem Black/Scholes-Modell vgl. Abschnitt 3.4 in diesem Kapitel.

2 Eine ausfuhrliche Erörterung dieser Ergebnisse erfolgt in Abschnitt 4.1 in Kapitel VI.

3 Vgl. Merton, Rational Option Pricing, 1973, S. 170.

4 Zur resultierenden Formel vgl. u.a. Smith, Review, 1976, S. 26. 
wird $^{1}$. Der bereits um die Dividendenzahlung geminderte Aktienkurs $K_{\text {ex. Div. }}$ wird anschließend in die ursprüngliche Bewertungsformel eingesetzt.

$$
K_{\text {ex. Div. }}=K_{\text {inkl. Div. }}-\text { Div } \cdot e^{-r_{f} \cdot t}
$$

wobei $D i v=$ Dividendenzahlung $i$ zum Zeitpunkt $t$

Bei amerikanischen Kauf- und Verkaufsoptionen mit Dividendenzahlungen entsteht eine ähnliche Problematik wie bereits bei der Bewertung amerikanischer Verkaufsoptionen ohne Dividendenzahlungen. Aufgrund von zwischenzeitlichen Zahlungen, die dem Optionsinhaber nicht zur Verfügung stehen, kann es sinnvoll sein, die Option vorzeitig auszuüben. Vor jeder Dividendenzahlung muß geprüft werden, ob der innere Wert der Option nicht den Optionswert unmittelbar nach Dividendenzahlung übersteigt. Dies führt dazu, daß in all diesen Fällen mit der Black/Scholes-Formel lediglich eine Wertuntergrenze ermittelbar ist ${ }^{2}$.

Ähnlich wie der Ansatz von Black und Scholes läßt sich auch das Modell von Merton zur Bewertung von realen Investitionsmöglichkeiten heranziehen, indem die notwendigen Bewertungsparameter uminterpretiert werden. Zwischenzeitlich entgehende Rückflüsse werden dabei durch den im Vergleich zum Black/Scholes-Modell zusätzlich benötigten Bewertungsparameter (Dividendenrate) abgebildet ${ }^{3}$. Im Beispiel der dreijährigen Investitionsmöglichkeit sei nun unter Beibehaltung der Daten des vorigen Abschnitts ein zwischenzeitlicher kontinuierlicher Rückfluß von 5\% angenommen. Dadurch verringert sich der Wert der Investitionsmöglichkeit von 52,1 auf nun 41,4 Mio. $€$.

1 Vgl. Steiner/Bruns, Wertpapiermanagement, 1998, S. 306 f.

2 Zu analytischen Lösungen für die Bewertung einer amerikanischen Kaufoption mit Dividendenzahlungen vgl. Roll, Known Dividends, 1977, S. 251 ff.; Geske, Known Dividends, 1979, S. 375 ff.; Whaley, Known Dividends, 1981, S. 207 ff.; Selby/Hodges, Compound Options, 1987, S. 351 ff. Für amerikanische Verkaufsoptionen mit und ohne Dividendenzahlungen Geske/Johnson, American Put, 1984, S. $1511 \mathrm{ff}$.

3 Vgl. Kemna, Case Studies, 1993, S. 261 f. Zu weiteren zeitstetigen Bewertungsansätzen bei realen Investitionsprojekten unter Berücksichtigung zwischenzeitlicher Rückflüsse vgl. Brennan/Schwartz, Natural Resource, 1985, S. 138 ff.; McDonald/Siegel, Value of Waiting, 1986, S. 709 ff.; Majd/Pindyck, Time to Build, 1987, S. 10 ff.; Paddock/Siegel/Smith, Option Valuation, 1988, S. 486 ff.; Lee, Optimal Timing, 1988, S. 157 ff.; Myers/Majd, Abandonment Value, 1990, S. 7 ff. 


\subsection{Approximation des Black/Scholes-Ansatzes durch das Binomial- modell}

Im einperiodigen Binomialmodell beträgt der Wert der dreijährigen Investitionsmöglichkeit ohne zwischenzeitlich entgehende Rückflüsse 59,3 Mio. $€$, während im dreiperiodigen Modell ein Ergebnis von 54,5 und im Ansatz von Black und Scholes ein Wert von 52,1 Mio. $€$ resultiert. Diese Unterschiede sind auf die in den einzelnen Bewertungen implizit unterstellten Verteilungen des Bezugsguts zurückzuführen. Im folgenden wird kurz dargestellt, unter welchen Bedingungen das Ergebnis des mehrperiodigen Binomialmodells gegen dasjenige der Black/Scholes-Bewertung konvergiert.

Im Binomialmodell folgt die Wertentwicklung des Bezugsguts einem zeitdiskreten und multiplikativen Binomialprozeß. Die Werte des Bezugsguts am Ende einer Binomialperiode sind binomialverteilt, so daß im mehrperiodigen Modell diese Werte am Ende der Optionslaufzeit einer Multinomialverteilung gehorchen. Die Black/Scholes-Bewertung geht von einer Wertentwicklung des Bezugsguts entsprechend einem zeitstetigen generellen Wiener Prozeß aus. Die möglichen Werte des Bezugsguts am Ende der Optionslaufzeit sind logarithmisch normalverteilt.

Da die Binomialverteilung bei entsprechend großer Anzahl von Binomialversuchen die Normalverteilung approximiert, liegt es nahe, daß das Ergebnis aus dem Binomialmodell bei wachsender Anzahl an Binomialperioden gegen das Ergebnis aus dem Black/Scholes-Modell konvergiert. Um an dieser Stelle die Konvergenzargumentation darzustellen, wird auf Erwartungswert und Varianz der beiden Zufallsprozesse näher eingegangen ${ }^{1}$. Als Zufallsvariable wird dabei die zeitstetige Rendite des Bezugsguts (hier am Beispiel des Aktienkurses) zugrunde gelegt ${ }^{2}$.

Erwartungswert $\mu_{B i n}$ und Varianz $\sigma_{B i n}^{2}$ eines diskreten einperiodigen Binomialprozesses lassen sich wie folgt ermitteln.

$$
\begin{gathered}
E\left[\ln \left(K^{*} / K\right)\right]=q \cdot \ln u+(1-q) \cdot \ln d \equiv \mu_{B i n} \\
\operatorname{Var}\left[\ln \left(K^{*} / K\right)\right]=q \cdot \ln ^{2} u+(1-q) \cdot \ln ^{2} d-[q \cdot \ln u+(1-q) \cdot \ln d]^{2} \equiv{\sigma_{B i n}}^{2}
\end{gathered}
$$

1 Vgl. zum folgenden Cox/Ross/Rubinstein, Option Pricing, 1979, S. 248 f.

2 Eine ähnliche Argumentation mit gleichem Ergebnis läßt sich auch über die Wertentwicklung des Bezugsguts führen. Vgl. Cox/Ross/Rubinstein, Option Pricing, 1979, S. 249. 
Bei $n$ Teilperioden betragen Erwartungswert und Varianz:

$$
E\left[\ln \left(K^{*} / K\right)\right]=\mu_{B i n} \cdot n, \quad \operatorname{Var}\left[\ln \left(K^{*} / K\right)\right]=\sigma_{B i n}{ }^{2} \cdot n .
$$

Ein normalverteilter Zufallsprozeß ist durch den Erwartungswert $\mu$ und die Varianz $\sigma^{2}$ pro Zeiteinheit $T$ charakterisiert. Beträgt $T$ genau ein Jahr, so handelt es sich bei Erwartungswert und Varianz um annualisierte Größen.

$$
E\left[\ln \left(K^{*} / K\right)\right]=\mu \cdot T, \operatorname{Var}\left[\ln \left(K^{*} / K\right)\right]=\sigma^{2} \cdot T
$$

Die Parameter $u, d$ und $q$ sind nun so zu wählen, daß der diskrete Binomialprozeß möglichst dem normalverteilten Zufallsprozeß entspricht. Cox/Ross/Rubinstein zeigen, daß unter den Bedingungen

$$
\begin{aligned}
& u=e^{\sigma \cdot \sqrt{T / n}} \text { und } d=e^{-\sigma \cdot \sqrt{T / n}} \\
& \text { sowie } q=0,5+0,5 \cdot \frac{\mu}{\sigma} \sqrt{T / n}
\end{aligned}
$$

für jedes $n$ folgende Beziehungen gelten:

$$
\begin{gathered}
\mu_{B i n} \cdot n=\mu \cdot T \\
{\sigma_{B i n}}^{2} \cdot n=\sigma^{2} \cdot T-\mu^{2} \cdot \frac{T^{2}}{n} .
\end{gathered}
$$

Damit Erwartungswert und Varianz der beiden Zufallsprozesse genau identisch sind, müssen im Binomialmodell unendlich viele Teilperioden angenommen werden $(n \rightarrow \infty)$. Wenn die zeitstetige Rendite im Black/Scholes-Modell keinen Trend aufweist $(\mu=0)$ entsprechen sich Erwartungswert und Varianz der beiden Zufallsprozesse sogar unabhängig von der gewählten Anzahl der Teilperioden ${ }^{1}$.

Für die oben genannten Bedingungen weisen Cox/Ross/Rubinstein nach, daß bei $n \rightarrow \infty$ die mehrperiodige Binomial- in die Black/Scholes-Formel übergeht $^{2}$. Das Black/Scholes-Modell stellt insofern einen Grenzfall des mehrperi-

Diese Annahme besagt zugleich, daß von identischen Eintrittswahrscheinlichkeiten für die beiden Umweltzustände in einer Teilperiode ausgegangen wird $(q=0,5)$.

2 Die ersten beiden Bedingungen sind bereits im Rahmen der Parameterüberführung in Abschnitt 2.2 sowie implizit in Abschnitt 3.2 dieses Kapitels verwendet worden. 
odigen Binomialmodells dar'. Anhand von Abb. 28 ist die Konvergenz der beiden Formeln leicht nachzuvollziehen ${ }^{2}$. Die Schwankungen des Ergebnisses aus dem Binomialmodell um den Black/Scholes-Wert verringern sich mit zunehmender Anzahl an unterstellten Teilperioden, bis sich beide schließlich entsprechen. Die Konvergenz erfolgt dabei um so schneller, je näher die unterstellte Eintrittswahrscheinlichkeit $q$ an dem für die Approximation idealen Wert 0,5 ist.

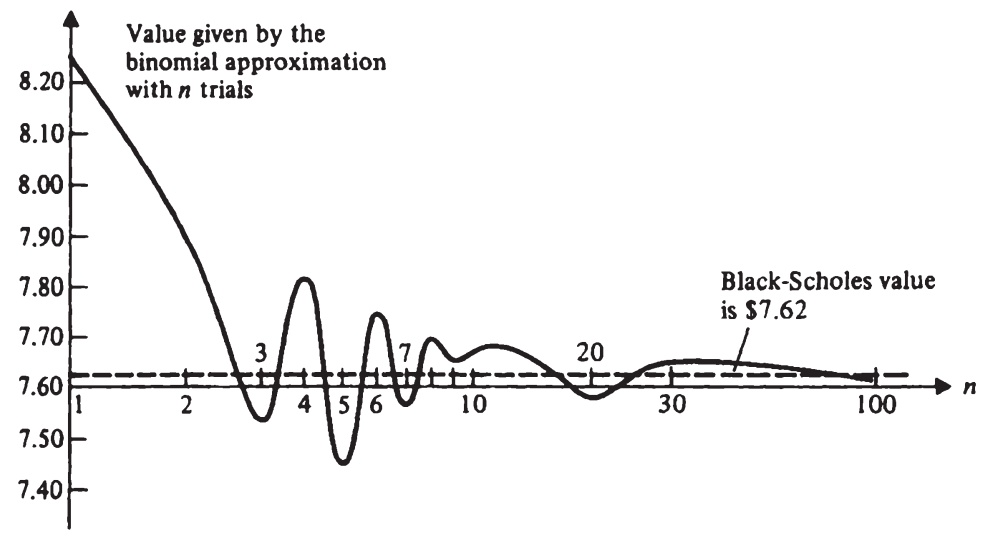

Abb. 28: Konvergenz zwischen dem Binomialmodell und dem Black/Scholes-Ansatz

Quelle: Copeland/Weston, Theory and Policy, 1988, S. 273.

Eine andere Parameterüberführung zwischen dem Binomialmodell und dem Black/Scholes-Ansatz nehmen Jarrow/Rudd vor $^{3}$. Sie schlagen vor, $u$ und $d$ durch folgende Transformationen zu ermitteln.

1 Vgl. Cox/Ross/Rubinstein, Option Pricing, 1979, S. 251 ff. Hsia argumentiert, daß die von Cox/Ross/Rubinstein aufgezeigte Konvergenz ein Spezialfall darstellt. Es sei lediglich zu fordern, daß $n \cdot p \rightarrow \infty$ strebt, wenn $n$ unendlich groß wird, wobei $p$ die Pseudowahrscheinlichkeit darstellt. Vgl. Hsia, Binomial Option Pricing, 1983, S. 43 f.

2 Der Abbildung liegt die Bewertung einer europäischen Kaufoption mit aktuellem Aktienkurs in Höhe von 50 \$, einem Basispreis von 45 \$, einem risikolosen Zinsfuß von 6\% p.a., einer Volatilităt von $20 \%$ sowie einer Laufzeit von 3 Monaten zugrunde. Vgl. ebenfalls die Zahlenbeispiele bei Cox/Ross/Rubinstein, Option Pricing, 1979, S. 259; Kruschwitz/Schöbel, Optionspreistheorie, 1984, S. 175; Kesting/Schulte-Mattler, Optionspreismodell, 1992, S. 215.

3 Vgl. Jarrow/Rudd, Option Pricing, 1983, S. 187 ff. Zu einer dritten Überfuhrung, die der von Jarrow und Rudd sehr nahe kommt vgl. Rendleman/Bartter, Option Pricing, 1979, S. 1098. 


$$
\begin{gathered}
\ln u=\left(r_{f}-0,5 \cdot \sigma^{2}\right) \cdot \frac{T}{n}+\sigma \cdot \sqrt{\frac{T}{n}} \\
\ln d=\left(r_{f}-0,5 \cdot \sigma^{2}\right) \cdot \frac{T}{n}-\sigma \cdot \sqrt{\frac{T}{n}} \\
\text { bei } q=0,5
\end{gathered}
$$

Jarrow/Rudd argumentieren, daß sich bei ihrer Parametersubstitution sowohl Erwartungswert als auch Varianz des binomialverteilten und des log-normalverteilten Zufallsprozesses entsprechen, wenn $n \rightarrow \infty$ strebt, während dies bei dem Vorschlag von Cox/Ross/Rubinstein für die Erwartungswerte gilt und die Varianzen nur im Grenzfall identisch sind. Dieses Ergebnis „erkaufen“ sie sich durch die Bedingung $q=0,5$. 


\section{Sonstige Optionspreismodelle und Schlußfolgerungen für das weitere Vorgehen}

Aufbauend auf dem Black/Scholes-Modell sind eine ganze Reihe weiterer Optionspreismodelle entwickelt worden. Die hier vorgenommene Aufzählung der Arbeiten erhebt keinen Anspruch auf Vollständigkeit, welche vor dem Hintergrund der Vielzahl an Varianten nur schwer möglich ist. Vielmehr soll überblicksmäßig aufgezeigt werden, in welche Richtungen sich die finanzwirtschaftliche Optionspreistheorie nach dem im Jahre 1973 veröffentlichten Ansatz von Black und Scholes entwickelt hat (vgl. Abb. 29).

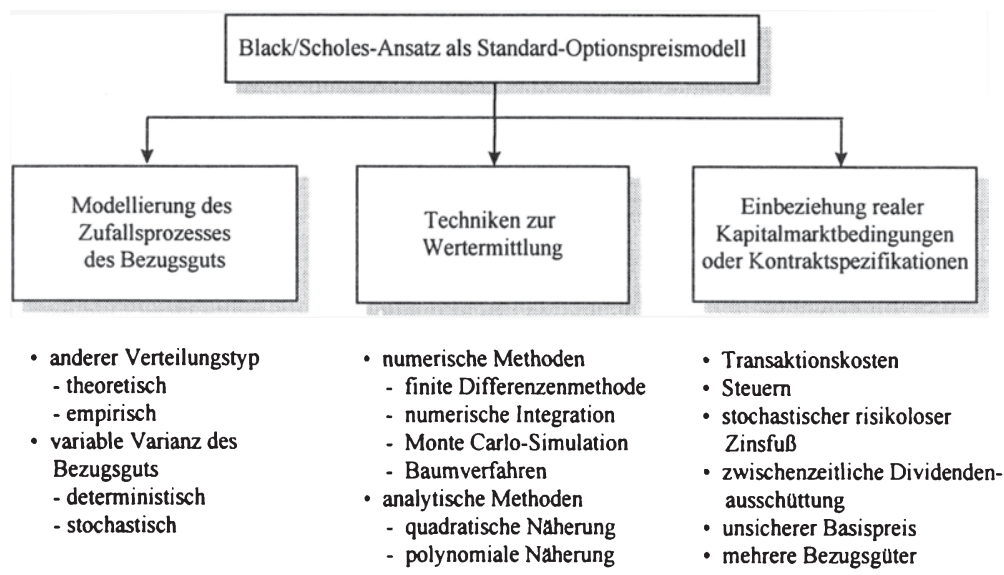

Abb. 29: Weiterentwicklung der finanzwirtschaftlichen Optionspreismodelle nach dem Black/Scholes-Ansatz

Eine erste Gruppe von Autoren nimmt zur Beschreibung der Aktienkursentwicklung andere Verteilungen als die logarithmische Normalverteilung des Aktienkurses $K$ an. Cox/Ross gehen z.B. von einem Sprungprozeß aus, der aus einer erwarteten momentanen Aktienkursrendite und einer Sprungkomponente besteht ${ }^{1}$.

$$
d K=[\mu-\lambda \cdot(k-1)] \cdot K \cdot d t+(k-1) \cdot K \cdot d \varphi
$$

wobei $\quad d \varphi=1$ mit Eintrittswahrscheinlichkeit $\lambda \cdot d t$

$d \varphi=0$ mit Eintrittswahrscheinlichkeit $I-\lambda \cdot d t$

1 Vgl. Cox/Ross, Alternative Stochastic Processes, 1976, S. 147. 
Abweichungen von der erwarteten momentanen Aktienrendite werden in diesem Modell nicht mehr durch die Streuung eines kontinuierlichen Wiener Prozesses, sondern durch diskontinuierliche (deterministische) Sprünge beschrieben. Letztere könnten beispielsweise durch neue Informationen auftreten. Der Term $d \varphi$ unterliegt einer Poissonverteilung, bei der mit einer Eintrittswahrscheinlichkeit von $\lambda \cdot d t$ erwartet wird, daß der Aktienkurs sich um $(k-1) \cdot K$ verändert. Der Parameter $\lambda$ drückt die Intensität des Sprungprozesses aus. Mit dem Parameter $k$ wird das Ausmaß eines Sprungs modelliert. Je näher $k$ bei eins liegt, desto geringer sind die für möglich gehaltenen Kurssprünge.

Merton führt den generellen Wiener Prozeß mit der Sprungkomponente zusammen. Abweichungen von der erwarteten Aktienrendite sind hier sowohl durch die Diffusions- als auch die Sprungkomponente möglich. Überdies ist nun auch die Sprunghöhe $k$ stochastisch ${ }^{1}$.

$$
d K=[\mu-\lambda \cdot E(k-1)] \cdot K \cdot d t+\sigma \cdot K \cdot d z+(k-1) \cdot K \cdot d \varphi
$$

wobei $E()=$. Erwartungswert

Wenn davon ausgegangen wird, daß der Wert des Bezugsguts um einen bestimmten „Normalwert" schwankt, kann diese Eigenschaft durch einen sogenannten Mean Reverting-Proze $\beta$ dargestellt werden. Als einfachste Form eines Mean Reverting-Prozesses gilt der Ornstein-Uhlenbeck-Proze $\beta^{2}$. Bei diesem schwankt der Aktienkurs um ein spezifisch angenommenes Normalniveau $\bar{K}$, auf das er sich bei Abweichung mit einer „Geschwindigkeit" $\eta$ zubewegt.

$$
d K=\eta \cdot(\bar{K}-K) \cdot d t+\sigma \cdot K \cdot d z
$$

Nach den Prinzipien von Black und Scholes fließt die erwartete Aktienrendite nur mittelbar über den aktuellen Preis der Aktie in die Optionsbewertung ein. Allerdings zeigen Lo/Wang $^{3}$, daß der Volatilitätsparameter für die Black/ Scholes-Formel mit einem Korrekturfaktor zu versehen ist, wenn das Bezugsgut nicht wie im Rahmen eines Wiener Prozesses einem Random Walk folgt,

1 Vgl. Merton, Discontinuous, 1976, S. 128 f. Im Modell von Merton wird das Risiko eines Aktiensprungs als diversifizierbar angesehen. Für eine Optionsbewertung bei systematischem Sprungrisiko des Aktienkurses vgl. z.B. Beinert, Kurssprünge, 1997, S. 95 ff.

2 Vgl. Dixit/Pindyck, Investment under Uncertainty, 1994, S. 74 f.

3 Vgl. Lo/Wang, Predictable, 1995. 
sondern die aufeinanderfolgenden Aktienkurse wie im Beispiel des Mean Reverting-Prozesses z.T. autokorreliert sind.

Anstelle von theoretischen gehen Gastineau/Madansky von empirischen Verteilungen des Aktienkurses aus, die sie aus statistischen bzw. ökonometrischen Untersuchungen gewonnen haben ${ }^{1}$. In neueren Ansätzen wird die Entwicklung einer gegebenen Wahrscheinlichkeitsverteilung der Aktienkurse durch „flexible" Binomialbäume modelliert. Im Unterschied zu den in Abschnitt 2 dieses Kapitels vorgestellten ,starren“ Binomialbäumen kann sich bei erstgenannten in jedem Zeitpunkt und Zustand die (lokale) Volatilität unterscheiden. Dabei wird die Wahrscheinlichkeitsverteilung am Ende der Optionslaufzeit oder sogar der gesamte Binomialbaum implizit aus den am Markt beobachtbaren Optionspreisen erschlossen ${ }^{2}$.

Bis auf die zuletzt genannten Beiträge sind bisher ausschließlich Abweichungen von der erwarteten momentanen Aktienrendite betrachtet worden, die einer konstanten Streuung um den Erwartungswert folgen. Während der Optionslaufzeit verändert sich die Volatilität des Aktienkurses nicht. Andere Modelle lassen eine variable Streuung im Zeitablauf zu. Dabei kann zwischen Ansätzen, die die Veränderung der Streuung in Abhängigkeit einer Veränderung anderer Parameter, wie z.B. dem Aktienkurs, deterministisch modellieren ${ }^{3}$, und solchen, die die Entwicklung der Streuung selbst als stochastischen Zufallsprozeß abbilden ${ }^{4}$, unterschieden werden. Zu den Weiterentwicklungen auf Basis eines anderen Verteilungstyps und/oder einer variablen Varianz sind auch Optionspreismodelle auf Basis von GARCH-Modellen zu zählen 5 .

1 Vgl. Gastineau, Options Manual, 1988, S. $202 \mathrm{ff}$.

2 Vgl. Rubinstein, Implied, 1994, S. 771 ff.; Derman/Kani, Riding, 1994, S. 32 ff. Einen Überblick gibt Neumann, Optionsbewertung, 1999.

3 Vgl. Cox/Ross, Alternative Stochastic Processes, 1976; Geske, Compound Options, 1979; Rubinstein, Displaced Diffusion, 1983. Vgl. auch Goldenberg, Diffusion Processes, 1991.

4 Vgl. beispielhaft Hull/White, Stochastic Volatilities, 1987 sowie die bei Finucane, Stochastic Volatilities, 1994, S. 115 zitierte Literatur.

5 Vgl. stellvertretend Andres, GARCH-Optionsbewertungsmodell, 1998, S. 175 ff. und Ritchken/Trevor, GARCH, 1999. Zu empirischen Untersuchungen zur Performance von in der Wahl des Zufallsprozesses des Bezugsguts sich unterscheidenden Optionspreismodellen vgl. u.a. Jackwerth/Rubinstein, Recovering Processes, 1996; Bakshi/Cao/Chen, Empirical Performance, 1997; Dumas/Fleming/Whaley, Empirical Tests, 1998. 
Eine zweite Gruppe von Autoren bietet alternative Bewertungstechniken an ${ }^{1}$. Für die Fälle, in denen im zeitstetigen Bewertungsansatz keine geschlossene analytische Lösung der partiellen Differentialgleichung ableitbar oder diese rechentechnisch nicht handhabbar ist, werden zur Bestimmung des Optionswerts numerische Verfahren herangezogen. Hierzu zählen die finite (explizite und implizite) Differenzenmethode, die numerische Integration, die Monte Carlo-Simulation sowie Baumverfahren wie z.B. das Binomialmodell ${ }^{2}$. Neben diesen numerischen Vorgehensweisen existieren verschiedene analytische, quadratische bzw. polynomiale, Näherungsmethoden. Die meisten dieser alternativen Bewertungstechniken sind ursprünglich zur Wertermittlung von amerikanischen Verkaufsoptionen entwickelt worden ${ }^{3}$.

Eine dritte Gruppe von Autoren versucht, die in den traditionellen Modellen angenommenen idealisierenden Kapitalmarktbedingungen aufzuheben ${ }^{4}$. So beschreibt Scholes den Effekt, den die Erhebung von Steuern auf Optionspreise ausübt ${ }^{5}$. Bereits Merton hebt die Prämisse eines während der Optionslaufzeit konstanten risikolosen Zinsfußes auf, indem er diesen als stochastischen $\mathrm{Zu}$ fallsprozeß abbildet ${ }^{6}$. Für die Bewertung von Zinsoptionen ist es zudem unverzichtbar, zusätzlich die Abhängigkeit des Bezugsguts von der Zinsentwicklung explizit zu modellieren. Unter Berücksichtigung der aktuellen Zinsstrukturkurve gelingt dies erstmals Ho/Lee. Diese Erkenntnisse nutzt Kishimoto zur Bewertung von Aktienoptionen, indem er die Abhängigkeit der Aktienkurse von der gegenwärtigen Zinsstrukturkurve in sein Modell integriert ${ }^{?}$.

1 Zu einem Überblick vgl. Jarrow/Rudd, Option Pricing, 1983, S. 202 ff.; Hull, Options, 1997, S. 343 ff. Speziell zur Monte Carlo-Bewertung vgl. Schäfer, Optionsbewertung, 1994, S. $91 \mathrm{ff}$.

$2 \mathrm{Zu}$ einem Anwendungsvergleich numerischer Methoden an konkreten Zahlenbeispielen vgl. Geske/Shastri, Approximation, 1985 und Broadie/Detemple, Comparison, 1996.

3 Vgl. Brennan/Schwartz, American Put, 1977; Parkinson, American Put, 1977; Boyle, Monte Carlo Approach, 1977; Johnson, American Put, 1983; Geske/Johnson, American Put, 1984; Blomeyer, American Put, 1986; MacMillan, American Put, 1986; BaroneAdesi/Whaley, Analytic Approximation, 1987.

4 Zur Optionsbewertung auf unvollkommenen Kapitalmärkten vgl. allgemein Figlewski, Imperfect Markets, 1989; Bell, Market Imperfections, 1993.

5 Vgl. Scholes, Taxes, 1976.

6 Vgl. Merton, Rational Option Pricing, 1973, S. 164 ff.

$7 \mathrm{Vgl}$. Ho/Lee, Interest Rate, 1986; Kishimoto, Interest Rate, 1989. Vgl. jüngst auch Ho/ Stapleton/Subrahmanyam, Stochastic Interest Rates, 1997 zur Bewertung von amerikanischen Optionen in dem hier skizzierten Kontext. 
Im zeitstetigen Bewertungsansatz wird aufgrund der laufenden Änderung des Aktienkurses eine kontinuierliche Anpassung des Hedgeportfolios notwendig, um das Prinzip der Arbitragebewertung zu gewährleisten. Dies ist unter Umständen gar nicht möglich bzw. verursacht erhebliche Transaktionskosten. Rubinstein und Brennan bestätigen die Ergebnisse der Black/Scholes-Formel jedoch auch für den Fall, daß von den Marktteilnehmern nur zeitdiskrete Anpassungen des besagten Portfolios vorgenommen werden. Dafür muß jedoch angenommen werden, daß die Marktteilnehmer eine konstante relative Risikoaversion aufweisen'. Die explizite Berücksichtigung von Transaktionskosten bei der Bestimmung von Optionspreisen haben als erste Gilster/Lee sowie Leland vorgenommen. Sie gingen von einer vollständigen Duplizierung eines bestimmten Zahlungsstroms aus. Ediringshe/Naik/Uppal entwickeln eine Optionsbewertungstechnik, in der der betrachtete Zahlungsstrom nicht exakt dupliziert wird, mit dem Ziel, Transaktionskosten zu sparen ${ }^{2}$. Desweiteren werden lineare Programmierungsansätze vorgeschlagen, in denen zur Minimierung der Transaktionskosten das Handeln in mehreren Werttiteln ermöglicht wird ${ }^{3}$.

Andere Autoren berücksichtigen in ihren Bewertungsmodellen spezifische reale Kontraktspezifikationen, die im Zusammenhang mit Optionsgeschäften auftreten können. Zum einen ist hier die in diesem Kapitel bereits behandelte Problematik der Dividendenausschüttung auf Aktien während der Optionslaufzeit bei nicht-dividendengeschützten Optionen zu nennen. Zum anderen gibt es Optionsverträge, in denen der Basispreis von zukünftigen Bedingungen abhängt. Der Ausübungskurs ist somit bei Abschluß des Optionsgeschäfts noch unsicher ${ }^{4}$. Zuletzt sind Optionsgeschäfte zu nennen, bei denen das Bezugsgut ex-ante nicht feststeht. Entscheidet sich beispielsweise der Käufer einer Kaufoption für die Ausübung, kann er zwischen mehreren Bezugsgütern auswäh-

1 Die explizite Formulierung einer dementsprechenden Risikonutzenfunktion ist dabei jedoch nicht notwendig. Vgl. Rubinstein, Uncertain Income Streams, 1976; Brennan, Discrete Time Models, 1979. Vgl. auch Egle/Trautmann, Preference-Dependent Pricing, 1981. Für eine Übertragung dieser Ergebnisse ins Binomialmodell vgl. Stapleton/Subrahmanyam, Binomial Process, 1984.

2 Vgl. Gilster/Lee, Transaction Costs, 1984; Leland, Transaction Costs, 1985; Ediringshe/ Naik/Uppal, Transaction Costs, 1993.

3 Vgl. Dennis/Rendleman, Transaction Costs, 1995 und Boyle/Lin, Transaction Costs, 1997. Zur Berucksichtigung von Transaktionskosten im Binomialmodell vgl. Boyle/Vorst, Transaction Costs, 1992. Vgl. auch Hoggard/Whalley/ Wilmott, Transaction Costs, 1994, die ein Bewertungsmodell für Optionsportfolios vorschlagen, und Reiß, Transaktionskosten, 1998.

$4 \mathrm{Zu}$ den wichtigsten resultierenden Bewertungsformeln vgl. Fischer, Exercice Price is Uncertain, 1978; Margrabe, Exchange, 1978. 
$\operatorname{len}^{1}$. Von hier aus öffnet sich das weite Feld der exotischen Optionen, die regelmäßig nur noch mit Hilfe numerischer Ermittlungstechniken bewertet werden können².

Das Ziel dieser Arbeit ist nicht, ein weiteres spezielles Optionspreismodell der ohnehin nicht mehr überschaubaren Anzahl an Bewertungsansätzen hinzuzufügen, sondern die Prinzipien der finanzwirtschaftlichen Optionsbewertung für die Beurteilung von realen Investitionsprojekten zu nutzen. Dabei stehen Übertragungsmöglichkeiten und -grenzen der finanzwirtschaftlichen Optionspreistheorie auf Realoptionen im Vordergrund. Aus diesem Grund wird im weiteren Verlauf der Arbeit grundsätzlich auf die beiden bisher ausführlich dargestellten Bewertungsansätze eingegangen.

In Kapitel V wird vorwiegend auf das Binomialmodell zurückgegriffen. Die Wahl des Binomialmodells zur Darstellung der im nächsten Kapitel anzutreffenden Problemstellungen kann begründet werden, indem auf die grundsätzliche Überlegenheit des Binomialmodells über das Black/Scholes-Modell hinsichtlich Anwendungsvielfalt und -flexibilität hingewiesen wird ${ }^{3}$. Zudem bietet das Binomialmodell den Vorteil, daß es im Vergleich zu zeitstetigen Bewertungsansätzen wesentlich leichter zu kommunizieren ist ${ }^{4}$. Dieser Vorteil kann im Hinblick auf eine Umsetzung in der Unternehmenspraxis als unverzichtbar angesehen werden. Nur in Ausnahmefällen, in denen die vorliegende Entscheidungssituation nicht oder nur noch schwer mit diesem Bewertungsansatz abgebildet werden kann, wird kurz auf eine - wenn mögliche - zeitstetige Bewertung hingewiesen.

1 Vgl. Stulz, Two Risky Assets, 1982. Für ein solches Bewertungsmodell ohne permanente Hedge- bzw. Duplikationsmöglichkeiten vgl. Stapelton/Subrahmanyam, Multivariate Contingent Claims, 1984.

2 Vgl. stellvertretend Nelken (Ed.), Exotic Options, 1996.

3 Dies gilt sowohl im Hinblick auf die modellmäßige Erfassung von Dividendenzahlungen bzw. zwischenzeitlichen Rückflussen als auch zur Frage der Existenz einer analytischen Lösung des Bewertungsmodells. Vgl. ebenfalls den ausführlichen Vergleich bei Perlitz/Peske/ Schrank, Real Options Valuation, 1999, S. 264.

4 „The two-state approach opens the door to the understanding of modern option pricing without the added complications associated with the solutions to stochastic differential equations ... One need not carry the two-state model to its continuous limit to derive many interesting insights into both the theoretical and practical applications of modern option pricing." Rendleman/Barter, Option Pricing, 1979, S. 1103. Vgl. ăhnlich Cox/Ross/Rubinstein, Option Pricing, 1979, S. 250. 


\section{Kapitel V}

\section{Berücksichtigung des Konkurrenzverhaltens bei der Bewertung von Investitionsmöglichkeiten}

\section{Ausgangsüberlegungen}

\subsection{Zwischenzeitlicher Konkurrenzeffekt}

Das in Kapitel IV vorgestellte Bewertungsmodell ist unter der Annahme entwickelt worden, daß die durch die strategische Akquisition zusätzlich geschaffene Investitionsmöglichkeit nicht durch zwischenzeitliche Investitionsentscheidungen bzw. Handlungen der Wettbewerber beeinträchtigt wird. Implizit wurde von einem Investor ausgegangen, dem die Investitionsmöglichkeit exklusiv zur Verfügung steht. Dies ist z.B. der Fall, wenn eine Produktidee durch ein Patent geschützt ist und die Konkurrenz über keine Substitute verfügt, mit denen es dem betrachteten Unternehmen den anvisierten Produktmarkt streitig machen kann.

Investitionsmöglichkeiten werden jedoch in der Regel mit Konkurrenten geteilt, so daß mehrere Unternehmen eine Realoption mit demselben Bezugsgut besitzen ${ }^{1}$. Genau genommen handelt es sich um ein gemeinsames Bezugsgut, innerhalb dessen die einzelnen Wettbewerber jeweils über eine Investitionsmöglichkeit in einer bestimmten unternehmensspezifischen Art und Höhe verfügen. Bei Investition erhalten sie einen bestimmten Anteil dieses gemeinsamen Bezugsguts. Dabei können Investitionen eines Unternehmens den für andere Wettbewerber relevanten Teil des Bezugsguts und somit auch den Wert deren Investitionsmöglichkeiten beeinflussen.

Um in einer solchen Konkurrenzsituation Realoptionen adäquat bewerten zu können, ist der Einfluß von Wettbewerbern in das Bewertungsmodell zu integrieren. Dazu kann in Anlehnung an die Erkenntnisse aus der Industrieökonomik zwischen dem direkten Effekt und dem Konkurrenzeffekt einer Investition unterschieden werden ${ }^{2}$. Der direkte Effekt drückt die durch die eigene Investition bedingte Veränderung des Unternehmenswerts unter Vernachlässigung eventueller Reaktionen der Wettbewerber aus. Hingegen verkörpert der Kon-

1 Zu ersten Gedanken dieser Art im Rahmen von Realoptionen vgl. Kester, Today's Options, 1984, S. $158 \mathrm{f}$.

2 Vgl. allgemein Tirole, Industrial Organization, 1988, S. 323 ff. 
kurrenzeffekt die Wertveränderung, die durch Reaktionen der Konkurrenz auf die geplante bzw. zu beobachtende Investition hervorgerufen werden.

In Abhängigkeit des Reife- und des Wettbewerbszustands von Produktmärkten stellen Investitionen eines einzelnen Unternehmens Vor- oder Nachteile für die anderen Wettbewerber dar ${ }^{1}$. Entsteht durch eine frühzeitige Investition ein exklusiver Vorteil, liegt für das investierende Unternehmen ein positiver Konkurrenzeffekt vor. Durch die Investition verringert sich das für die übrigen Wettbewerber vorhandene Bezugsgut bzw. steigen für ein gegebenes Bezugsgut die erforderlichen Investitionsauszahlungen, so daß der Wert ihrer Investitionsmöglichkeiten sinkt. Dies ist beispielsweise der Fall, wenn der Pionier aufgrund von Lernkurveneffekten Kostenvorteile gegenüber der Konkurrenz realisiert oder sich bereits frühzeitig Marktanteile sichern kann. Bei einem solchen first mover advantage kann es auch ohne sonstige zwischenzeitlich entgehende Rückflüsse sinnvoll sein, frühzeitig zu investieren.

Wird durch eine frühzeitige Investition ein Vorteil für alle Anbieter geschaffen, resultiert für den Investor ein negativer Konkurrenzeffekt. Durch die Investition vergrößert sich das für die anderen Wettbewerber zur Verfügung stehende Bezugsgut bzw. sinken die für ein bestimmtes Bezugsgut notwendigen Investitionsauszahlungen. Der Wert ihrer Investitionsmöglichkeiten steigt. Führt der Pionier beispielsweise Werbemaßnahmen durch, die den anvisierten Produktmarkt für Anbieter erst öffnet, profitieren Nachfolger hiervon, ohne dieselben Auszahlungen tätigen zu müssen. Das Gleiche gilt für Investitionen in kostensenkende Produktionstechnologien, die von der Konkurrenz leicht zu imitieren sind. In einem solchen Fall kann von einem first mover disadvantage gesprochen werden. Dies führt dazu, daß Investitionen tendenziell später durchgeführt werden.

Führen zwischenzeitliche Konkurrenzinvestitionen zu einer Erhöhung des Bezugsguts bzw. zu einer Senkung der Investitionsauszahlung des betrachteten Unternehmens, liegt ein konkurrenzbedingter Wertgewinn vor, der den Wert seiner Investitionsmöglichkeit erhöht. Ohne zwischenzeitliche Rückflüsse wird mit der Investitionsentscheidung möglichst lange gewartet. Die in Kapitel IV ermittelten Werte der Investitionsmöglichkeit können dann als Wertuntergrenzen angesehen werden. Führen demgegenüber zwischenzeitliche Konkurrenzinvestitionen hingegen zu einer Senkung des Bezugsguts bzw. zu einer Erhöhung der Investitionsauszahlung, verringert dies den Wert der Investitionsmög-

1 Für ähnliche Gedanken vgl. Cohen/Levinthal, Fortune Favors, 1994, S. 228 sowie S. 243. 
lichkeit des betrachteten Unternehmens. Für die Wahl des optimalen Investitionszeitpunkts ist nun der konkurrenzbedingte zwischenzeitliche Wertverlust gegen den reinen Wert des Abwartens abzuwägen.

Im folgenden werden ausschließlich Situationen betrachtet, in denen durch das Abwarten ein konkurrenzbedingter Wertverlust droht. Ohne zwischenzeitliche Rückflüsse stellen sie im Vergleich zu konkurrenzbedingten Wertgewinnen die interessanteren Entscheidungsfälle dar, da mit der Bewertung auch gleichzeitig der optimale Investitionszeitpunkt festgelegt wird. Darüber hinaus läßt sich die Mehrzahl der in diesem Kapitel angestellten Überlegungen problemlos auf Entscheidungssituationen mit konkurrenzbedingten Wertgewinnen übertragen.

An dieser Stelle sei darauf hingewiesen, daß die Richtung des Konkurrenzeffekts nicht nur - wie eben suggeriert - von Investitionsentscheidungen, sondern ebenfalls von nachgelagerten Preis- und Mengenentscheidungen abhängt ${ }^{1}$. Bei sogenannten strategischen Substituten ist z.B. die Entscheidung und das hierdurch angestrebte Ziel eines Wettbewerbers den Zielen der Konkurrenten entgegengesetzt. Die Erhöhung des Marktanteils eines Wettbewerbers führt c.p. zu geringeren Absatzmengen beim Konkurrenten. Bei sogenannten strategischen Komplementen entspricht das angestrebte Ziel eines Wettbewerbers dem Ziel des Konkurrenten. Von der Etablierung eines höheren Absatzpreises profitieren alle Anbieter. Im folgenden werden jedoch ausschließlich Investitionsentscheidungen in den Mittelpunkt der Betrachtung gerückt.

\subsection{Investitionsverhalten bei konkurrenzbedingten Wertverlusten}

Wenn zwischenzeitliche Investitionsentscheidungen der Konkurrenz keinen Einfluß auf die eigene Investitionsmöglichkeit haben und keine zwischenzeitlich entgehenden Rückflüsse existieren, wird die Investition zum spätest möglichen Zeitpunkt durchgeführt. Bei einer solchen exklusiven Investitionsmöglichkeit kann der reine Wert des Abwartens vollständig ausgeschöpft werden. Je mehr jedoch die Realoption mit Wettbewerbern geteilt wird, umso höher fällt der erwartete konkurrenzbedingte Wertverlust aus und umso früher wird investiert.

Im Rahmen einer - hier stets angenommenen - dynamischen Investitionsbetrachtung ist neben dem aktuellen Grad der Exklusivität dessen Veränderung

1 Vgl. stellvertretend Fudenberg/Tirole, Fat-Cat Effect, 1984, S. 361 ff.; Bulow/Geanakoplos/Klemperer, Multimarket Oligopoly, 1985, S. $491 \mathrm{ff}$. 
im Zeitablauf relevant ${ }^{1}$. Eine ursprünglich exklusive Investitionsmöglichkeit zur Einführung einer Produktinnovation wird zu einer mit Wettbewerbern geteilten Realoption, wenn es der Konkurrenz in der Zwischenzeit gelingt, ähnliche Produkte zu entwickeln. Zwischenzeitliche Veränderungen der Exklusivität sind somit stark von der im anvisierten Produktmarkt herrschenden Wettbewerbsintensität abhängig. Bei hoher Wettbewerbsintensität ist der erwartete konkurrenzbedingte Wertverlust größer als bei niedriger Wettbewerbsintensität, so daß eine frühzeitige Investition um so wahrscheinlicher wird ${ }^{2}$.

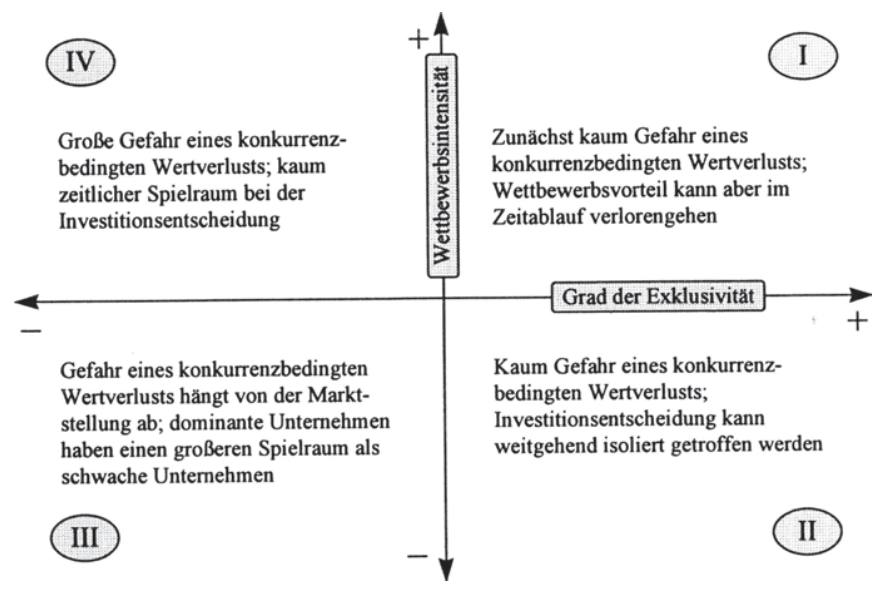

Abb. 30: Investitionsverhalten bei konkurrenzbedingten Wertverlusten

Quelle: In Anlehnung an Kester, Today's Options, 1984, S. 159.

In Abhängigkeit der eben erläuterten Bestimmungsfaktoren ,aktueller Grad der Exklusivitität“ und „Wettbewerbsintensität" läßt sich das Investitionsverhalten in vier Ausgangskonstellationen einteilen (vgl. Abb. 30). Bei hoher Wettbewerbsintensität und niedrigem Exklusivitätsgrad (Feld I) bzw. bei niedriger Wettbewerbsintensität und hohem Exklusivitätsgrad (Feld IV) ergeben sich eindeutige Handlungsempfehlungen. Feld IV entspricht der im vierten Kapitel implizit angenommenen Ausgangslage. Da das Unternehmen keine Konkurrenz zu fürchten braucht, erfolgt eine frühzeitige Investition nur bei hohen zwischenzeitlichen Rückflüssen. Ansonsten wird mit der Investition so lange wie

Vgl. Kester, Today's Options, 1984, S. 158.

2 Für ähnliche Überlegungen vgl. Kensinger, Value of Active Management, 1987, S. 38 sowie Wernerfelt/Karnani, Competitive Strategy, 1987, S. 189 ff., die eine frühzeitige Investition umso eher empfehlen, je größer der first mover advantage, je größer die Anzahl der Wettbewerber und je schwächer die eigene Wettbewerbsposition ist. 
möglich gewartet, um den reinen Wert des Abwartens vollständig auszuschöpfen. In Feld I existiert eine beachtliche Gefährdung der Investitionsmöglichkeit durch Wettbewerber. Es besteht kaum Flexibilität hinsichtlich des Investitionszeitpunkts. Im Extremfall kompensiert der Konkurrenzeffekt den Wert des Abwartens vollständig. Eine sofortige Investition ist die Folge. Der Kapitalwert wird zum alleinigen Entscheidungs- und Bewertungskriterium ${ }^{1}$.

Für die Felder II und III, in denen eine niedrige (hohe) Wettbewerbsintensität auf einen niedrigen (hohen) Exklusivitätsgrad trifft, lassen sich ad hoc keine eindeutigen Handlungsempfehlungen ableiten. In Feld III ist die Gefahr eines konkurrenzbedingten Wertverlusts vergleichsweise gering. Demgegenüber läßt sich die Investitionsmöglichkeit kaum gegenüber Handlungen der übrigen Wettbewerber schützen. Die umgekehrte Situation stellt sich in Feld II ein. Das betrachtete Unternehmen verfügt im Hinblick auf die Investitionsmöglichkeit über einen beachtlichen Wettbewerbsvorteil, der jedoch aufgrund der hohen Wettbewerbsintensität im Zeitablauf verloren gehen kann. In beiden Fällen muß der Wert des Abwartens einem möglichen konkurrenzbedingten Wertverlust gegenübergestellt werden.

\subsection{Beeinflußbarkeit des Konkurrenzverhaltens}

In den bisherigen Ausführungen ist implizit davon ausgegangen worden, daß sich durch eine frühzeitige Investition das Verhalten der Konkurrenz tatsächlich verändert. Lassen sich hingegen die Handlungen der Wettbewerber nicht durch eigene Entscheidungen beeinflussen, tritt der konkurrenzbedingte Wertverlust unabhängig davon ein, ob das betrachtete Unternehmen frühzeitig investiert oder noch abwartet. In einer solchen Situation nicht beeinflußbaren Konkurrenzverhaltens ist es stets optimal, die Investitionsentscheidung so lange wie möglich zu verschieben, um zumindest in den Genuß der verbesserten Informationsbasis über zukünftige Umweltentwicklungen zu gelangen ${ }^{2}$.

Demgegenüber setzt beeinflußbares Konkurrenzverhalten voraus, daß Unternehmen ihre eigene Handlungen aufgrund des Investitionsverhaltens der Wettbewerber tatsächlich überdenken. Durch eine frühzeitige Investition kann eventuell ein Konkurrent dazu veranlaßt werden, die seinerseits geplante Inve-

1 Vgl. auch Dixit, Investment and Hysteresis, 1992, S. 118.

2 Wenn sonstige, nicht konkurrenzbedingte zwischenzeitliche Rückflüsse existieren, sind für eine fruhzeitige Investition diese mit dem reinen Wert des Abwartens zu vergleichen. 
stition zu verschieben bzw. definitiv aufzugeben. Für die Wahl des optimalen Investitionszeitpunkts ist dann die konkrete Höhe des reinen Warte- und des Konkurrenzeffekts von Belang. Wieviel des konkurrenzbedingten Wertverlusts kann durch eine frühzeitige Investition abgewehrt werden? Welchen Wert haben die Informationen, auf die durch eine frühzeitige Investition verzichtet wird?

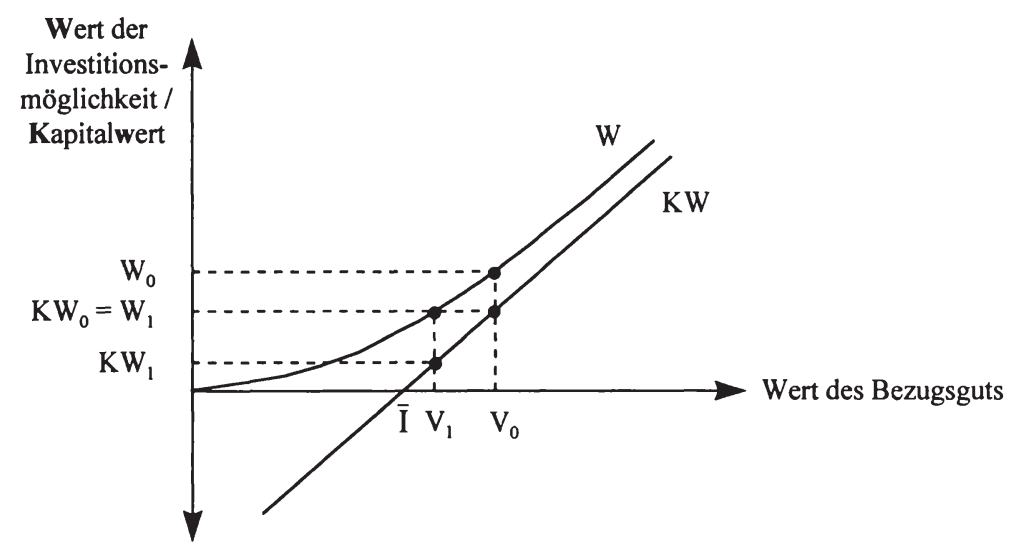

Abb. 31: Entscheidung über eine frühzeitige Investition bei beeinflußbarem Konkurrenzverhalten

Quelle: In Anlehnung an Kester, Growth Options, 1993, S. 194.

Der Abwägeprozeß zwischen dem reinen Wert des Abwartens und dem konkurrenzbedingten Wertverlust für den Fall, daß die anstehende und beeinflußbare Investition der Konkurrenz eine Verringerung des Bezugsguts zur Folge hat, ist in Abb. 31 graphisch dargestellt ${ }^{1}$. Die Kurve $W$ zeigt den (Realoptions-) Wert der Investitionsmöglichkeit in Abhängigkeit des Werts des Bezugsguts $V$ und der konstanten Investitionsauszahlung $\bar{I}$. Die Gerade $K W$ bildet entsprechend den traditionellen Kapitalwert ab. Bei einem Wert des Bezugsguts von $V_{0}$ beträgt der Wert der Investitionsmöglichkeit $W_{0}$. Dieser setzt sich aus dem traditionellen Kapitalwert $K W_{0}$ und dem reinen Wert des Abwartens $\left(W_{0}\right.$ $K W_{0}$ ) zusammen. Besteht nun die Gefahr, daß es aufgrund einer Konkurrenz-

1 Rein technisch bedingt ein sonstiger, nicht konkurrenzbedingter zwischenzeitlicher Rückfluß den gleichen Abwägeprozeß bzw. Werteffekt wie ein konkurrenzbedingter Wertverlust, der das Bezugsgut schmälert. Aufgrund des unterschiedlichen materiellen Gehalts wird jedoch im Verlauf dieser Arbeit streng zwischen diesen beiden Werteffekten unterschieden und gemäß der Zielsetzung dieses Kapitels letzterer in den Mittelpunkt der Betrachtung gerückt. 
investition zu einem Sinken des Bezugsguts von $V_{0}$ nach $V_{l}$ kommt, gilt es, den konkurrenzbedingten Wertverlust $\left(W_{0}-W_{l}\right)$ mit dem reinen Wert des Abwartens zu vergleichen ${ }^{1}$. Eine frühzeitige Investition ist erforderlich, falls der zuletzt genannte Wert kleiner ist als das durch das Konkurrenzverhalten bedingte Absinken des Werts der Investitionsmöglichkeit. In dem hier skizzierten Fall ist es unerheblich, ob frühzeitig investiert oder noch abgewartet wird, da sich der reine Warte- und der Konkurrenzeffekt exakt kompensieren.

Ähnliche Überlegungen lassen sich für eine konkurrenzbedingte Erhöhung der Investitionsauszahlung anstellen. Der Unterschied zu der eben dargestellten Entscheidungssituation liegt darin, daß die durch das Konkurrenzverhalten bedingte Senkung des Kapitalwerts nicht durch eine Verringerung der erwarteten diskontierten Rückflüsse $V$ bei konstanter Investitionsauszahlung $\bar{I}$, sondern durch eine gesteigerte Investitionsauszahlung $I$ bei nicht beeinflußtem Bezugsgut $\bar{V}$ zustande kommt. Graphisch bedeutet dies, daß sich jetzt sowohl die Kapitalwertgerade als auch die Kurve zur Abbildung des Realoptionswerts nach rechts verschieben. Die allgemeine Aussage, daß für eine frühzeitige Investition der Kapitalwert vor der Konkurrenzhandlung, die nun eine höhere Investitionsauszahlung zur Folge hat, größer sein sollte als der (Realoptions-) Wert der Investitionsmöglichkeit nach der Konkurrenzhandlung, gilt unverändert.

\subsection{Unsicherheit des Konkurrenzverhaltens}

Sind das Verhalten der Wettbewerber und die hieraus resultierenden Auswirkungen für den betrachteten Investor vollständig antizipierbar, kann der mögliche Konkurrenzeffekt als sicher eingestuft werden. Dies gilt auch für Fälle, in denen die Konkurrenzhandlung nur in Abhängigkeit des unsicheren Bezugsguts bekannt ist. In einem bestimmten Umweltzustand steht die konkurrenzbedingte absolute Verringerung des Bezugsguts bzw. Erhöhung der Investitionsauszahlung mit Sicherheit fest. Die einzige Unsicherheitsquelle resultiert aus der Wertentwicklung des Bezugsguts, die dazu führt, daß der konkurrenzbedingte Wertverlust zwar aus heutiger Sicht nicht für alle zukünftigen Zeitpunkte bekannt, jedoch in Abhängigkeit des Werts dieses Bezugsguts eindeutig festgelegt ist. Das Verhalten der Konkurrenz und der hiermit verbundene konkurrenzbedingte Wertverlust ist deterministischer Natur.

1 In der Realităt wird dieses Abwägen nicht exakt vor einer antizipierten Konkurrenzhandlung erfolgen, sondern eine bestimmte Zeit davor, um das Konkurrenzverhalten auch wirklich beeinflussen zu können. 
Ist das Verhalten der Konkurrenz selbst bei Kenntnis des Werts des Bezugsguts nicht vollständig absehbar, stellt der Konkurrenzeffekt selbst eine unsichere, von der Entwicklung des Bezugsguts unabhängige Größe dar ${ }^{1}$. Er unterliegt einem eigenständigen stochastischen Zufallsprozeß. In einer solchen Situation gehen zwei Unsicherheitsquellen in das Bewertungsmodell ein. Die Entscheidung über eine frühzeitige Investition wird auf Basis einer unsicheren Bezugsgutentwicklung und einer unsicheren Konkurrenzhandlung getroffen. Unsicherheit über die Konkurrenzhandlung kann zudem in zweierlei Hinsicht bestehen. Einerseits handelt es sich um die Frage, ob der Zeitpunkt der Konkurrenzhandlung, z.B. des Markteintritts bzw. der Marktbearbeitung, unsicher ist. Andererseits geht es darum, ob der durch die Konkurrenzhandlung hervorgerufene Wertverlust in seiner Höhe unsicher ist.

\subsection{Schlußfolgerungen für den weiteren Aufbau des Kapitels}

Der Wert einer mit Wettbewerbern geteilten Investitionsmöglichkeit setzt sich ebenfalls aus dem traditionellen Kapital- und dem Flexibilitätswert zusammen. Im Gegensatz zu einer exklusiven Investitionsmöglichkeit ist jedoch jeweils der Konkurrenzeffekt zu berücksichtigen (vgl. Abb. 32).

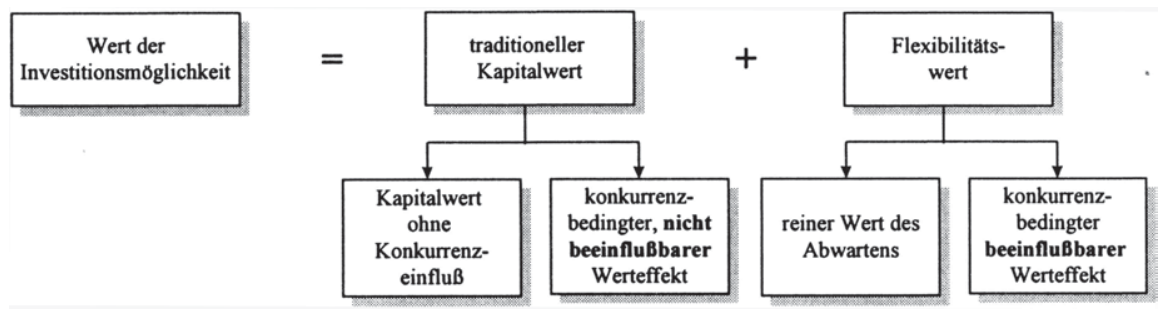

Abb. 32: Zusammensetzung des Werts einer Investitionsmöglichkeit unter Berücksichtigung des Konkurrenzeffekts

Einerseits ist vom Kapitalwert ohne Konkurrenzeinfluß, der sich bei passivem Wettbewerbsverhalten ergibt, der Werteffekt abzuziehen, der sich durch die nicht beeinflußbare Handlung der Konkurrenten ergibt. Andererseits ist beim

1 Wird die Unsicherheit des Konkurrenzeffekts nicht - wie hier geschehen - in Abhăngigkeit des Bezugsguts definiert, liegt bereits stochastisches Wettbewerbsverhalten vor, wenn die Höhe und/oder der zeitliche Eintritt der Konkurrenzauswirkung heute nicht bekannt ist. Im folgenden wird jedoch die in diesem Abschnitt eingefuhrte Begriffsbildung verwandt. 
Flexibilitätswert neben dem reinen Wert des Abwartens der konkurrenzbedingte beeinflußbare Werteffekt zu beachten ${ }^{1}$. Der reine Warteeffekt entsteht durch die in der Zwischenzeit verbesserte Informationsbasis über nicht beeinflußbare Umweltentwicklungen, auf die flexibel reagiert werden kann. Der Konkurrenzeffekt verkörpert hier Wertveränderungen, die durch zwischenzeitliche Handlungen der Wettbewerber hervorgerufen werden und sich durch eine frühzeitige Investition beeinflussen lassen.

Zur Einbeziehung des Konkurrenzeinflusses in die Wertermittlung von Investitionsmöglichkeiten ist es sinnvoll, das Wettbewerbsverhalten in verschiedene Kategorien einzuteilen. In Abstimmung mit den Ausführungen der beiden vorangegangenen Abschnitte wird im folgenden zum einen zwischen beeinflußbaren und nicht beeinflußbaren und zum anderen zwischen deterministischen und stochastischen Handlungen der Konkurrenz unterschieden.

Bei deterministischem sowie nicht beeinflußbarem Verhalten der Konkurrenz läßt sich das in Kapitel IV entwickelte Bewertungsmodell heranziehen. Der Konkurrenzeinfluß wird über die Prognose der erwarteten Rückflüsse und damit über den originär ermittelten Wert des Bezugsguts im Bewertungskalkül erfaßt ${ }^{2}$. Dabei ist zu beachten, daß die risikoadjustierte Verzinsung des Bezugsguts unter Einbeziehung des Konkurrenzeinflusses nur zufällig mit jener übereinstimmt, die ohne Berücksichtigung der Konkurrenzhandlung gelten würde ${ }^{3}$. Die Technik der Realoptionsbewertung bleibt jedoch unverändert.

Werden hingegen deterministische sowie beeinflußbare Handlungen der Konkurrenz angenommen, sind die bisher präsentieren Bewertungsansätze zu modifizieren. Es ist ein Bewertungskalkül zu entwickeln, das zum einen das Verhalten der Konkurrenz explizit berücksichtigt und zum anderen Empfehlungen für das eigene Investitionsverhalten enthält, um daraus ableitend den Wert von

1 Vgl. ähnlich Trigeorgis, Options Framework, 1988, S. 158 f. Die hier vorgenommene Einteilung abstrahiert von sonstigen, nicht konkurrenzbedingten zwischenzeitlichen Rückflüssen, die gegebenenfalls als dritte Komponente des Flexibilitătswerts zu berücksichtigen sind.

2 Zu einer solchen Bewertung vgl. z.B. Ang/Dukas, Competition, 1993, S. $171 \mathrm{ff}$.

3 Auf einem vollständigen Kapitalmarkt verfugen zwei unsichere Zahlungsströme nur dann uber die gleiche Verzinsung, wenn der eine Zahlungsstrom ein Vielfaches des anderen repräsentiert. Vgl. hierzu auch Abschnitt 2.2 in Kapitel VI. 
Investitionsmöglichkeiten $\mathrm{zu}$ ermitteln ${ }^{1}$. Hinsichtlich der Modellbildung werden im folgenden zwei Varianten unterschieden.

Die erste Variante faßt das Konkurrenzverhalten als exogene Modellgröße auf, indem einzelne Bewertungsparameter der im vorigen Kapitel dargestellten Ansätze uminterpretiert werden. Diese Modellerweiterung wird auf Basis des zeitdiskreten Binomialmodells für den Fall sinkender Marktanteile bzw. Absatzpreise sowie steigender Markteintrittsbarrieren diskutiert. Die zweite Variante integriert das Konkurrenzverhalten als endogene Modellgröße im Bewertungskalkül. Dazu sind die im vierten Kapitel präsentierten Verfahren in einer konzeptionellen Modifikation um spieltheoretische Elemente zu erweitern. In den bislang verwendeten Binomialbaum wird ein Spielbaum eingefügt, der die Interaktionen zwischen den Investitionsentscheidungen der Wettbewerber abbildet. Die Spieltheorie liefert dabei das Ergebnis der in der jeweiligen Teilperiode sowie dem entsprechenden Umweltzustand vorzufindenden Spielsituation.

Bei stochastischem Konkurrenzverhalten besteht das Problem, daß gleichzeitig zwei Unsicherheitsquellen im Bewertungskalkül zu berücksichtigen sind. Wird bei beiden Unsicherheitsgrößen eine Binomialverteilung unterstellt, ergeben sich in einer Teilperiode vier statt bisher zwei Umweltzustände. Dies führt da$\mathrm{zu}, \mathrm{da} ß$ im zeitdiskreten Binomialmodell ein risikoloses Hedgeportfolio aus Bezugsgut und Realoption bzw. ein die Rückflüsse der Realoption duplizierendes Portfolio aus Bezugsgut und risikolosem Wertpapier nicht mehr gebildet werden können. Somit existieren mehr Umweltzustände als risikobehaftete „Marktpreise“, so daß kein eindeutiges Preissystem vorliegt.

Zur Lösung dieses Problems können grundsätzlich zwei Wege beschritten werden. Einerseits lassen sich im zeitdiskreten Bewertungsansatz zusätzliche risikobehaftete Wertpapiere mit bekannten Marktpreisen einführen, um über genügend neue Preisinformationen wieder ein eindeutiges Preissystem entstehen zu lassen. Von dieser Möglichkeit wird jedoch hier abgesehen, da die relative Bewertung der Investitionsmöglichkeit im Verhältnis zu ihrem Bezugsgut und nicht die absolute Bewertung der Investitionsmöglichkeit im Verhältnis zu son-

1 Besteht der Konkurrenzeffekt sowohl aus einer beeinflußbaren als auch aus einer nicht zu beeinflussenden Komponente, so ist letztere bei der Bestimmung des originären Bezugsguts zu erfassen, wăhrend für erstere die im folgenden erlăuterten Modellerweiterungen in Frage kommen. 
stigen am Kapitalmarkt existierenden Vermögenspositionen im Mittelpunkt der Ausführungen stehen soll ${ }^{1}$.

Andererseits kann auf zeitstetige Bewertungsmodelle zurückgegriffen werden, die selbst bei unsicherer Entwicklung des Bezugsguts und stochastischem Konkurrenzverhalten in der Lage sind, den Wert einer Investitionsmöglichkeit mittels eines risikolosen Hedgeportfolios zu bestimmen. Dies geschieht, indem entweder der Zufallsprozeß des Bezugsguts durch einen anderen Verteilungstyp abgebildet oder ein weiterer Zufallsprozeß - z.B. für die unsichere Investitionsauszahlung oder den unsicheren zwischenzeitlichen Rückfluß - in das Bewertungsmodell integriert wird.

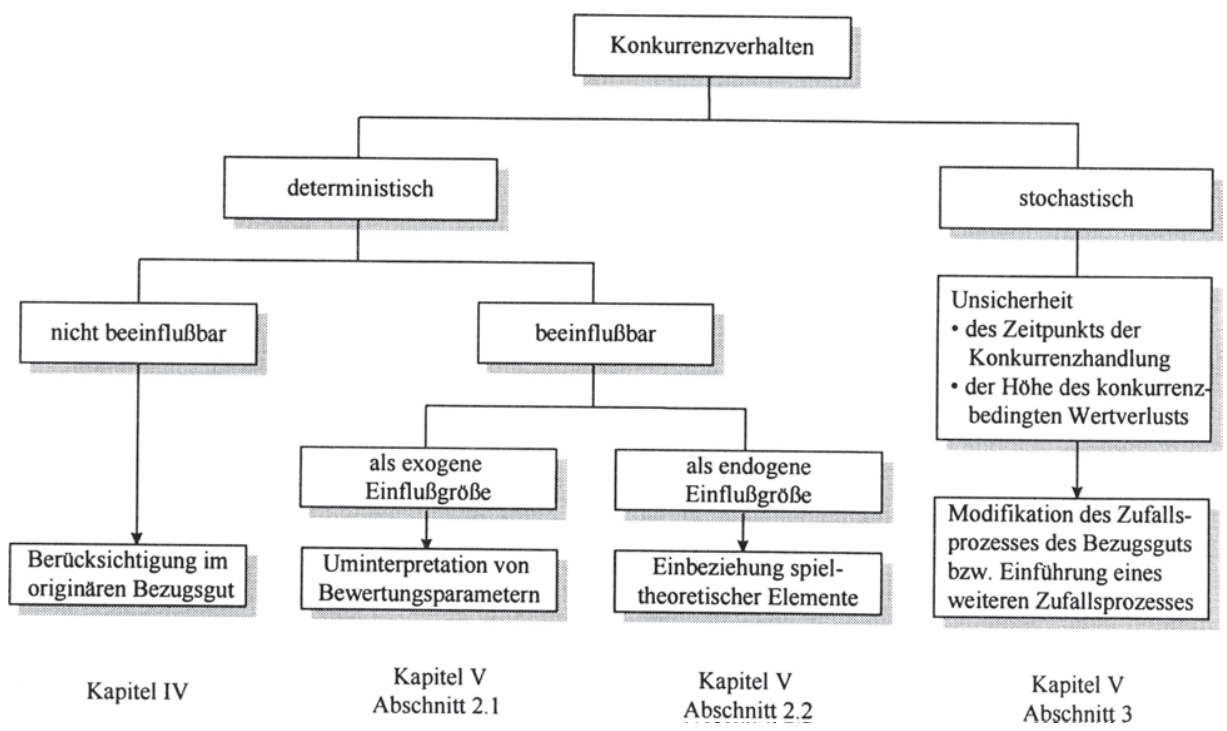

Abb. 33: Möglichkeiten zur Einbeziehung des Konkurrenzverhaltens im Realoptionsmodell

Bei stochastischen Wettbewerbshandlungen läßt sich der konkurrenzbedingte Wertverlust selbst bei Kenntnis der Umweltentwicklung nicht eindeutig abschätzen. Vor dem Hintergrund der zuvor getroffenen Einschränkung, keine zusätzlichen - über die relative Bewertung hinausgehenden ${ }^{2}$ - Preisinformationen zuzulassen, sind i.d.R. keine Aussagen zum optimalen Investitionszeit-

1 Vgl. hierzu auch die Ausführungen in Abschnitt 2.2 sowie 2.3 in Kapitel VI.

2 Es werden ausschließlich Preisinformationen zum risikolosen Zinsfuß sowie zu den im Optionspreismodell als unsicher unterstellten Bewertungsparameter berücksichtigt. 
punkt bei beeinflußbarer Konkurrenz möglich. Die in diesem Kapitel in Abschnitt 3 dargestellten Modelle erfassen somit lediglich Situationen, in denen die Konkurrenten von Investitionsentscheidungen des betrachteten Unternehmens unbeeinflußt sind. Ihre Bewertungsergebnisse stellen jedoch gleichzeitig eine Wertuntergrenze für den Fall beeinflußbarer Wettbewerber dar. Abb. 33 faßt die einzelnen Möglichkeiten zur Einbeziehung des Konkurrenzverhaltens zusammen. 


\section{Deterministischer Wettbewerbseffekt bei beeinflußbarem Konkurrenzverhalten}

\subsection{Konkurrenzverhalten als exogener Modellparameter}

\subsubsection{Sinkende Marktanteile und Absatzpreise}

Entscheidet sich das betrachtete Unternehmen für eine Verschiebung des Investitionszeitpunkts, gibt es Wettbewerbern die Gelegenheit, ihrerseits frühzeitig zu investieren. Dies kann bedeuten, daß ein bestimmter angestrebter Marktanteil bzw. Absatzpreis nicht mehr realisierbar ist, weil es der Konkurrenz zwischenzeitlich gelungen ist, sich in dem anvisierten Produktmarkt zu etablieren. Der durch die Verschiebung hinzu gewonnenen Flexibilität steht dann ein Marktanteilsverlust bzw. ein niedrigerer Absatzpreis gegenüber.

Wird wie im folgenden angenommen, daß sich dieser Wettbewerbseffekt durch eine eigene frühzeitige Investition vermeiden läßt, weist eine solche Konkurrenzsituation große Parallelen zum Bewertungsfall mit zwischenzeitlich entgehenden Rückflüssen auf' ${ }^{\prime}$. Bei einer frühzeitigen Investition geht der Vorteil verloren, in Zukunft auf Umweltentwicklungen flexibel zu reagieren. Dafür kann dem konkurrenzbedingten Wertverlust entgegengewirkt werden. Ähnlich wie im Fall der zwischenzeitlichen Rückflüsse ist deswegen in jedem Umweltzustand und Zeitpunkt der konkurrenzbedingte Wertverlust mit dem Wert des reinen Abwartens zu vergleichen².

Zur Illustration werden in Erweiterung des Beispiels aus Abschnitt 2.2 in Kapitel IV zwei zukünftige Konkurrenzinvestitionen angenommen, die zu einer Senkung des ursprünglich angenommenen Bezugsguts führen. Dabei ist es im folgenden unerheblich, ob diese absolute $K V_{t, s}$ bzw. prozentuale Wertverringerung $k v_{t, s}$ durch einen Marktanteilsverlust, einen niedrigeren Absatzpreis oder eine Kombination von beidem bedingt ist.

$$
\begin{aligned}
& V_{t, s_{u}}^{e x}=u \cdot V_{t-1, s}^{e x}-K V_{t, s_{w}} \\
& V_{t, s_{d}}^{e x}=d \cdot V_{t-1, s}^{e x}-K V_{t, s_{d}}
\end{aligned}
$$

1 Vgl. Abschnitt 2.4 in Kapitel IV.

2 Für eine Anwendung in einem zeitstetigen Bewertungsansatz vgl. Trigeorgis, Anticipated Competitive Entry, 1991, S. 150 f. 


$$
\text { mit } K V_{t, s}=V_{t, s} \cdot k v_{t, s}
$$

Es sei angenommen, daß die erste Konkurrenzinvestition nach der ersten Teilperiode erfolgt und eine Verringerung des Gegenwartswerts des Bezugsguts von $20 \%$ bewirkt, während die zweite nach der zweiten Teilperiode einen Wertverlust von $40 \%$ mit sich bringt ${ }^{1}$. Investiert das betrachtete Unternehmen eine Periode vor der erwarteten Konkurrenzhandlung, kann es den drohenden Konkurrenzeffekt erfolgreich abwehren. Die Bewertung der Investitionsmöglichkeit wird anhand der risikoneutralisierten Methode unter Berücksichtigung des zwischenzeitlichen Abwägens durchgeführt. Ist der konkurrenzbedingte Wertverlust in dem betreffenden Umweltzustand und Zeitpunkt größer als der reine Wert des Abwartens, wird sofort investiert. In Abb. 34 stellt $K V_{t, s}$ den absoluten konkurrenzbedingten Wertverlust in Umweltzustand $s$ der Teilperiode $t$ und $V_{t, s}{ }^{e x}$ den entsprechenden Wert des Bezugsguts nach Abzug des in diesem Umweltzustand und Zeitpunkt auftretenden Konkurrenzeffekts dar.

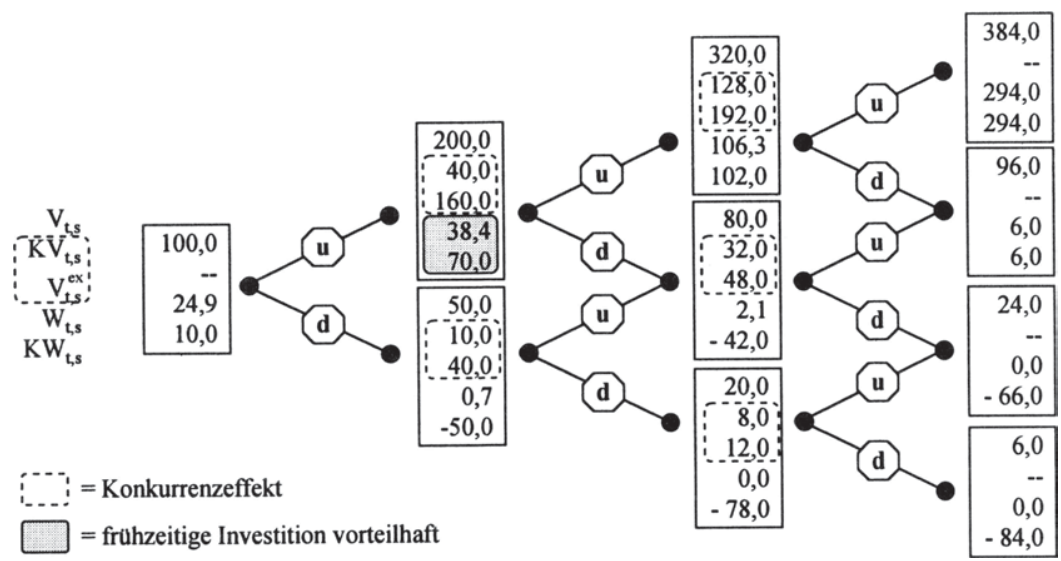

Abb. 34: Bewertung einer Investitionsmöglichkeit bei sinkendem Marktanteil bzw. Absatzpreis und zustandsunabhăngiger Konkurrenzhandlung

Im Vergleich zur Situation ohne Konkurrenzeinfluß fällt der Wert der Investitionsmöglichkeit von 54,5 Mio. € auf 24,9 Mio. €. Die Differenz von 29,6 Mio. $€$ setzt sich aus zwei Effekten zusammen. Zunächst verringert sich der Gegen-

1 Unter einer modellierten Konkurrenzhandlung können auch mehrere Wettbewerber verstanden werden, die kumuliert den besagten Konkurrenzeffekt verkörpern. Umgekehrt kann mit mehreren modellierten Konkurrenzhandlungen ein Wettbewerber dargestellt werden, der zu unterschiedlichen Zeitpunkten investiert. 
wartswert des Bezugsguts in der ersten und in der zweiten Teilperiode in jedem Umweltzustand, was c.p. zu einer Verringerung der Investitionsmöglichkeit von 40,7 Mio. $€$ auf 13,8 Mio. $€$ führt. Ein Teil dieses Verlusts kann durch eine frühzeitige Investition aufgefangen werden, bei der der Kapitalwert bei sofortiger Investition den Wert der Investitionsmöglichkeit bei weiterem Abwarten übersteigt. Dies ist nach einer Teilperiode in Umweltzustand $s=1$ der Fall, in dem der Kapitalwert 70,0 Mio. $€$ und der Wert der Investitionsmöglichkeit 38,4 Mio. $€$ beträgt ${ }^{1}$. Die frühzeitige Investition bringt einen Wertgewinn von rund $11[0,367 \cdot(70,0-38,4) / 1,05]$ Mio. $€$.

In dem eben erläuterten Beispiel reagieren die anderen Wettbewerber unabhängig von dem zum jeweiligen Zeitpunkt herrschenden Umweltzustand. Dies ist jedoch wenig realistisch. Vielmehr ist anzunehmen, daß sie sich in Abhängigkeit des Umweltzustands und damit des Werts ihres Bezugsguts für oder gegen eine Investition entscheiden. Wird aus diesem Grund nun vereinfachend davon ausgegangen, daß die Konkurrenz nur investiert, wenn der Wert des Bezugsguts des hier betrachteten Unternehmens 90 Mio. $€$ überschreitet ${ }^{2}$, droht der konkurrenzbedingte Wertverlust nicht in sämtlichen Umweltzuständen ${ }^{3}$. Nach der ersten Teilperiode erfolgt die Konkurrenzhandlung lediglich in Umweltzustand $s=1$ und nach der zweiten Teilperiode lediglich in den Umweltzuständen $s=1$ und $s=3^{4}$.

Durch die zustandsabhängige Modellierung des Konkurrenzverhaltens kommt es nach der zweiten Teilperiode zu vier verschiedenen Umweltzuständen (im Gegensatz zu den drei im vorigen Beispiel), da sich die Äste in den mittleren Szenarien nicht wieder vereinigen. Aus Platzgründen wird in Abb. 35 auf die Darstellung der dritten Teilperiode verzichtet. Der Wert der Investitionsmög-

1 Ohne den Konkurrenzeintritt in $t=2$ ergibt sich ein Wert der Investitionsmöglichkeit für $t=1$ und $s=1$ von 81,8 Mio. $€$. Der konkurrenzbedingte Wertverlust in $t=1$ beträgt folglich 43,4 $(81,8-38,4)$ Mio. $€$ und ist größer als der aufzugebende reine Wert des Abwartens von 11,8 (81,8 - 70) Mio. $€$. Das betrachtete Unternehmen wird deshalb investieren und den konkurrenzbedingten Wertverlust nicht hinnehmen.

2 Eine solche Vorgehensweise setzt voraus, daß das Bezugsgut der Wettbewerber den gleichen bzw. zumindest ähnlichen Unsicherheitseinflüssen im Hinblick auf die Umweltentwicklung unterliegt wie das Bezugsgut des betrachteten Unternehmens.

3 Zu Modellierungsalternativen bei umwelt- und zeitabhängigen zwischenzeitlichen Wertverlusten vgl. Abschnitt 2.4 in Kapitel IV sowie Meise, Realoptionen, 1998, S. 163 ff., der explizit die Anzahl der Wettbewerber, den durch eine frühzeitige Investition maximal zusätzlich zu erzielenden Marktanteil sowie dessen Abnahme im Zeitablauf betrachtet.

4 In den anderen Umweltzuständen der ersten und zweiten Teilperiode gilt: $K V_{t, s}=0$. 
lichkeit wird jedoch wie zuvor mit Hilfe des risikoneutralen Bewertungsansatzes ermittelt und beträgt nun 26,7 statt zuvor 24,9 Mio. $€$. Es ergibt sich rein zufällig die gleiche Investitionsstrategie wie bei zustandsunabhängiger Konkurrenzhandlung. Der Wertzuwachs in Höhe von 1,8 Mio. $€$ ist auf die Tatsache zurückzuführen, daß in dem unteren Ast des Binomialbaums bis auf eine Ausnahme kein wettbewerbsbedingter Wertverlust zu verzeichnen ist. Dadurch erhöht sich nach der ersten Teilperiode der Wert der Investitionsmöglichkeit in Umweltzustand $s=2$ um $3(3,7-0,7)$ Mio. $€$, wodurch besagte Differenz $(0,633 \cdot 3 / 1,05)$ resultiert.

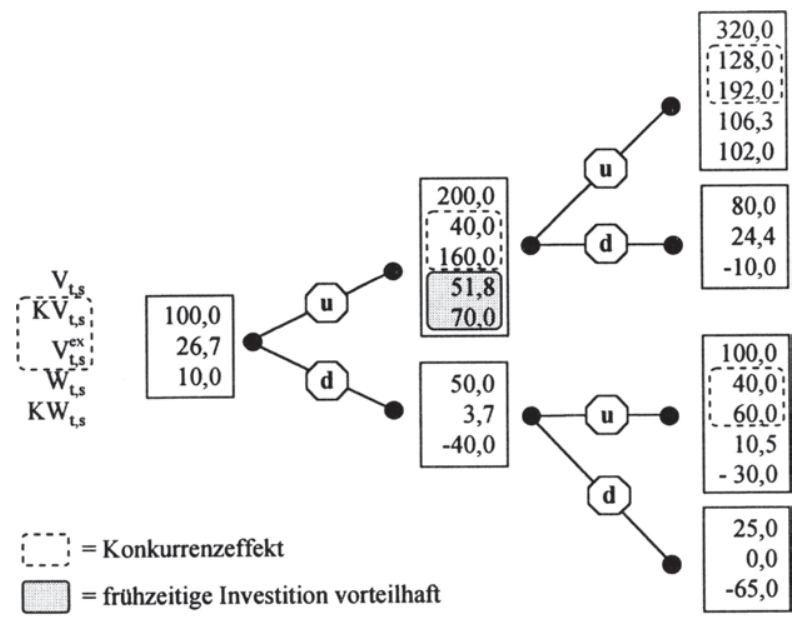

Abb. 35: Bewertung einer Investitionsmöglichkeit bei sinkendem Marktanteil bzw. Absatzpreis und zustandsabhängiger Konkurrenzhandlung

\subsubsection{Steigende Markteintrittsbarrieren}

Neben einem sinkenden Marktanteil bzw. Absatzpreis ist es auch vorstellbar, daß die Wahl eines späteren Investitionszeitpunkts höhere Markteintrittsbarrieren bedingt. Die bereits heute in den anvisierten Produktmarkt investierenden Unternehmen sind in der Zwischenzeit in der Lage, den Marktzutritt für andere Wettbewerber zu erschweren. Die Beschaffung zusätzlicher Informationen über die weitere Umweltentwicklung ist dann mit dem Nachteil verbunden, daß im Vergleich zur aktuellen Situation der zukünftige Marktzutritt nur durch größere Anstrengungen möglich ist.

Um eine solche Konkurrenzsituation zu modellieren, sei im folgenden angenommen, daß bei einer späteren Investition eine höhere Investitionssumme 
aufgebracht werden muß, um die zusätzlichen Eintrittshemmnisse zu überwinden und den Wert des Bezugsguts in der ursprünglich geplanten Höhe vollständig zu erhalten. Der „Basispreis“ der Investitionsmöglichkeit steigt im Zeitablauf an ${ }^{1}$, wobei $k v_{t, s}$ den prozentualen Anstieg der Investitionsauszahlung verkörpert.

$$
I_{t, s}=I_{t-1, s} \cdot\left(1+k v_{t, s}\right)
$$

In der jeweiligen Teilperiode läßt sich der traditionelle Kapitalwert somit folgendermaßen darstellen.

$$
K W_{t, s}=V_{t, s}-I_{t-1, s} \cdot\left(1+k v_{t, s}\right)
$$

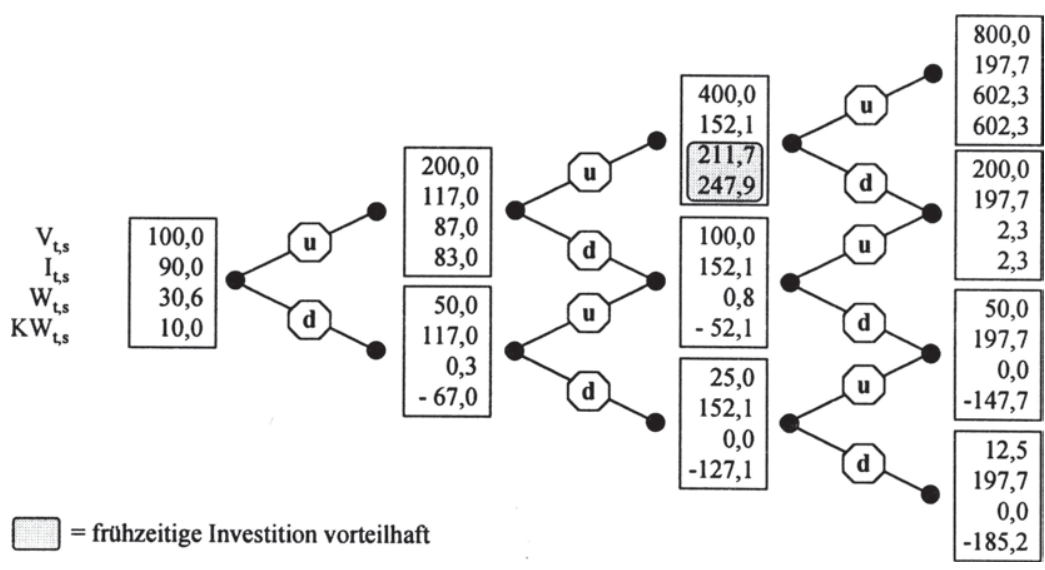

Abb. 36: Bewertung einer Investitionsmöglichkeit bei steigenden Markteintrittsbarrieren

Für die bisher betrachtete Investitionsmöglichkeit läßt sich anhand der risikoneutralisierten Bewertungsmethode ein Wert von 30,6 Mio. € ermitteln, falls bei konstanten Rückflüssen eine steigende Investitionsauszahlung von $30 \%$ pro Teilperiode unterstellt wird. In Abb. 36 stellt $I_{t, s}$ die erforderliche Investitionsauszahlung zum Zeitpunkt $t$ im Umweltzustand $s$ dar. In Umweltzustand $s=1$

1 Vgl. Kester, Growth Options, 1993, S. 202. Für eine Anwendung in einem zeitstetigen Bewertungsansatz vgl. Lee, Optimal Timing, 1988, S. 158. 
nach der zweiten Teilperiode ist es vorteilhaft, frühzeitig zu investieren und einem weiteren Anstieg der Markteintrittsbarrieren zuvorzukommen ${ }^{1}$.

In dem soeben skizzierten Beispiel ist unterstellt worden, daß die Wahl eines späteren Investitionszeitpunkts zwar eine erhöhte Ausgangsinvestition fordert, jedoch der Wert des Bezugsguts bei erfolgreichem Marktzutritt vollständig erhalten bleibt. Durch das Aufschieben der Investition sind die geplanten Marktanteile und Absatzpreise nicht gefährdet. Wird durch ein späteres Engagement zusätzlich eine konkurrenzbedingte Verringerung des Bezugsguts erwartet, lassen sich die beiden Wettbewerbseinflüsse auch kombiniert erfassen. Bei einer gleichzeitigen Verringerung des Gegenwartswerts der Rückflüsse sowie einer Erhöhung der Investitionsauszahlung sinkt ceteris paribus der Wert der Investitionsmöglichkeit. Die Tendenz zu einer frühzeitigen Investition wird verstärkt.

\subsection{Endogenisierung des Konkurrenzverhaltens im Bewertungsmodell}

\subsubsection{Begriffe und Grundlagen der Spieltheorie}

Untersuchungsgegenstand der Spieltheorie sind Entscheidungssituationen (im folgenden auch Spiele genannt), in denen das Ergebnis von den individuellen Entscheidungen mehrerer Entscheidungsträger (im folgenden auch Spieler genannt) abhängt, so daß der einzelne das Ergebnis nicht alleine bestimmen kann. Jeder Spieler ist sich dieser Interdependenz bewußt und weiß, daß sich alle anderen ebenfalls dieser Interdependenz bewußt sind und unter Berücksichtigung dieser Tatsache rational handeln ${ }^{2}$.

Das in der Literatur wohl bekannteste Einführungsbeispiel ist das Gefangenendilemma ${ }^{3}$ : Zwei Verdächtige, die zusammen ein schweres Verbrechen begangen haben und mangels Beweise nicht überführt werden können, kommen in Einzelhaft und werden getrennt zu dem Vorfall befragt. Wenn einer der beiden

1 Ohne den erwarteten weiteren Anstieg der Markteintrittsbarrieren ergibt sich ein Wert der Investitionsmöglichkeit für $t=2$ und $s=1$ von 255,2 Mio. $€$. Der konkurrenzbedingte Wertverlust beträgt folglich 43,5 (255,2 - 211,7) Mio. $€$ und ist größer als der aufzugebende reine Wert des Abwartens von 7,3 (255,2 - 247,9) Mio. $€$.

2 Zur Spieltheorie vgl. stellvertretend Fudenberg/Tirole, Game Theory, 1991; Eichberger, Game Theory, 1993; Rieck, Spieltheorie, 1993; Illing, Spieltheorie, 1995, S. 509 ff.; Holler/Illing, Spieltheorie, 1996; Güth, Spieltheorie, 1999.

3 Vgl. zu folgendem ausführlich Holler/llling, Spieltheorie, 1996, S. 2 ff. 
gesteht, der andere jedoch nicht, wird der gestehende Verbrecher aufgrund einer Kronzeugenregelung freigesprochen und der andere zur Höchststrafe von zehn Jahren verurteilt. Wenn beide gestehen, müssen beide für acht Jahre ins Gefängnis. Wenn beide nicht gestehen, werden sie aufgrund kleinerer Delikte lediglich zu einer einjährigen Gefängnisstrafe verurteilt. Der einzelne Verbrecher steht nun vor der Wahl, das Verbrechen zu gestehen bzw. nicht zu gestehen.

Die beiden Verdächtigen treffen ihre Entscheidung, ohne diejenige des anderen zu kennen. Da keine Kommunikation zwischen den Gefangenen gegeben ist, läßt sich das Einzelverhalten nicht aufeinander abstimmen. Es handelt sich um ein nicht-kooperatives Spiel, das in Abb. 37 in der sogenannten Matrixform dargestellt ist.

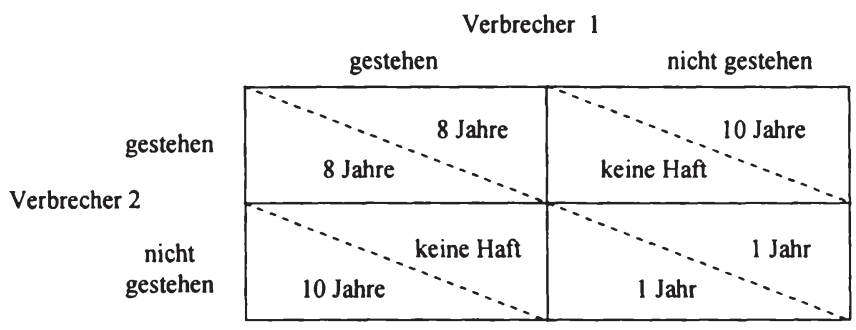

Abb. 37: Maxtrixform des Spiels „Gefangenendilemma“

Quelle: In Anlehnung an Holler/Illing, Spieltheorie, 1996, S. 3.

Beide Verdächtige werden in dieser Entscheidungssituation gestehen und damit eine achtjährige Strafe absitzen. Wenn Verbrecher 2 nicht gesteht, stellt sich Verbrecher 1 besser, wenn er gesteht $(0<1 \mathrm{Jahr})$. Auch wenn Verbrecher 2 gesteht, ist es für Verbrecher 1 ratsam, zu gestehen $(8<10 \mathrm{Jahre})$. Unabhängig von der Entscheidung des Verbrechers 2 ist für Verbrecher 1 das Ablegen eines Geständnisses die dominante Strategie. Verbrecher 2 steht vor der gleichen Situation und damit der gleichen Entscheidung. Somit kommt es zu einer Lösung, die bei rationalem Verhalten für den einzelnen Gefangenen die einzig richtige Entscheidung darstellt, obwohl die Kombination, daß beide nicht gestehen, effizienter ist als die eben präsentierte Lösung. Diese Ergebniskonstellation wird generell als Gefangenendilemma bezeichnet.

Selbst wenn sich die Verdächtigen vorher austauschen und eine Vereinbarung treffen, verändert sich das Ergebnis des Spiels nicht. Es sei denn, diese Abmachung kann bindend festgelegt werden. Dann handelt es sich im Gegensatz zu 
dem bisher betrachteten nicht-kooperativen Fall um ein kooperatives Spiel. Läßt sich hingegen keine bindende Vereinbarung treffen, liegt trotz einer möglichen Abmachung ein nicht-kooperatives Spiel vor, da u.U. für den Einzelnen ein Anreiz besteht, die vereinbarte Lösung nicht einzuhalten. Dies zeigt, daß in einer - im folgenden Verlauf der Arbeit unterstellten - nicht-kooperativen Ausgangssituation die Lösung des Spiels derart beschaffen sein muß, daß der einzelne Spieler kein Eigeninteresse hat, von dieser abzuweichen.

Die Lösung eines Spiels hängt zum einen von den Spielregeln und zum anderen von den Informationen, die den einzelnen Spielern zum Zeitpunkt ihrer Entscheidung zur Verfügung stehen, $a b^{1}$. Je nachdem, in welcher Reihenfolge die Spieler zum Zuge kommen, und je nachdem, ob der einzelne Spieler die Entscheidung des anderen bzw. die Auswirkungen der verschiedenen Entscheidungskombinationen beim anderen kennt, ergibt sich gegebenenfalls eine andere Lösung des betrachteten Spiels. Im Rahmen einer spieltheoretischen Analyse ist somit auf die exakte Festlegung der Spielregeln und Informationsstände zu achten $^{2}$.

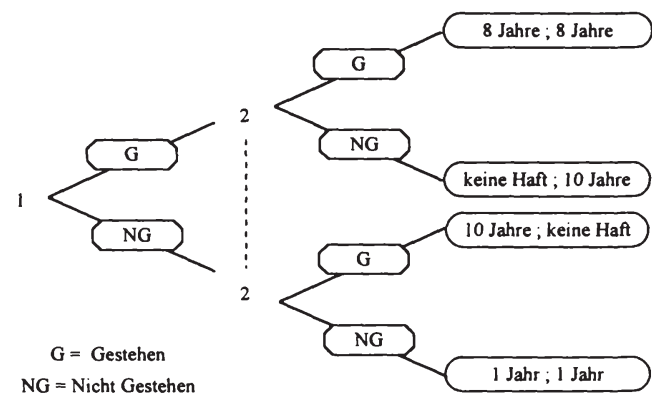

Abb. 38: Spielbaum des Spiels „Gefangenendilemma“

Zur Darstellung von Spielen existiert neben der Matrixform auch der Spielbaum $^{3}$. Der Zug des einzelnen Spielers wird hier als Entscheidungsknoten dargestellt, von dem verschiedene Zweige bzw. Handlungsalternativen abgehen.

2 Im folgenden werden lediglich ,einfache“ Spiele mit dominanten Strategien präsentiert. Im Vordergrund steht die konzeptionelle Einbeziehung spieltheoretischer Elemente in den realoptionsorientierten Bewertungsansatz und nicht die Analyse von (strategischen) Investitionsmöglichkeiten anhand spieltheoretischer Erkenntnisse.

3 Vgl. Rieck, Spieltheorie, 1993, S. 84 ff.; Güth, Spieltheorie, 1999, S. 36 ff. 
Hintereinander geschaltete Knoten geben dabei an, welcher Spieler wann zum Zuge kommt und über welche Informationen er zu diesem Zeitpunkt verfügt. Hat der einzelne Spieler kein Wissen darüber, welche Entscheidung der Gegenspieler trifft, kann er nicht unterscheiden an welchem Knoten er sich genau befindet. Dies wird durch eine gestrichelte Linie zwischen den von den einzelnen Spielern nicht zu unterscheidenden Knoten gekennzeichnet. Abb. 38 gibt den Spielbaum für das Beispiel des Gefangenendilemmas wieder. Die Darstellung eines Spiels als Spielbaum ist insofern interessant, als sie eine problemlose Integration spieltheoretischer Elemente in den bisher betrachteten Binomialbaum erlaubt.

\subsubsection{Spieltheoretische Interpretation von Investitionsmöglichkeiten}

Über die Einführung spieltheoretischer Elemente läßt sich das Investitionsverhalten von Wettbewerbern im Bewertungsmodell endogenisieren ${ }^{1}$. Die hiermit verbundene konzeptionelle Modifikation des Bewertungskalküls wird zunächst anhand der in Kapitel IV dargestellten exklusiven Investitionsmöglichkeit aufgezeigt, indem das dort skizzierte Beispiel mit dem Instrumentarium der Spieltheorie interpretiert wird. Darauf aufbauend erfolgt in einem zweiten Schritt die Integration des Konkurrenzverhaltens.

Der Inhaber einer exklusiven Investitionsmöglichkeit spielt ein Spiel gegen die Natur ${ }^{2}$. Dabei gilt es, auf Spielzüge eines fiktiven und nicht beeinflußbaren Gegners zu reagieren. Nach jeder Entwicklung der Natur (= Umwelt) trifft das betrachtete Unternehmen die Entscheidung, frühzeitig zu investieren oder noch eine weitere Teilperiode abzuwarten. Im Gegensatz zu dem Spiel des vorherigen Abschnitts besitzt hier der eine Spieler die Möglichkeit, den Spielzug des anderen abzuwarten. Im jeweiligen Teilspiel (jeweils ein Zug der beiden Spieler) kennt der Inhaber der exklusiven Investitionsmöglichkeit zum Entscheidungszeitpunkt die „Entscheidung“ der Natur sowie die Auswirkung dieser Entscheidung auf den Wert seiner Investitionsmöglichkeit.

Abb. 39 stellt die Investitionsmöglichkeit aus Abschnitt 2.2 in Kapitel IV als Spiel gegen die Natur dar. Dabei sind nur die Werte der ersten zwei Teilperioden abgebildet. Die fett gedruckte Linie kennzeichnet die Investitionsstrategie

1 Vgl. Trigeorgis, Real Options, 1996, S. 258.

2 Vgl. ähnlich Holler/Illing, Spieltheorie, 1996, S. 35, die allgemein auf Entscheidungen unter Unsicherheit eingehen. 
in Abhängigkeit der Umweltentwicklung. Es kann nicht überraschen, daß bei der hier aufgezeigten Investitionsmöglichkeit ohne zwischenzeitliche Rückflüsse der optimale Investitionszeitpunkt am Ende der Laufzeit liegt.

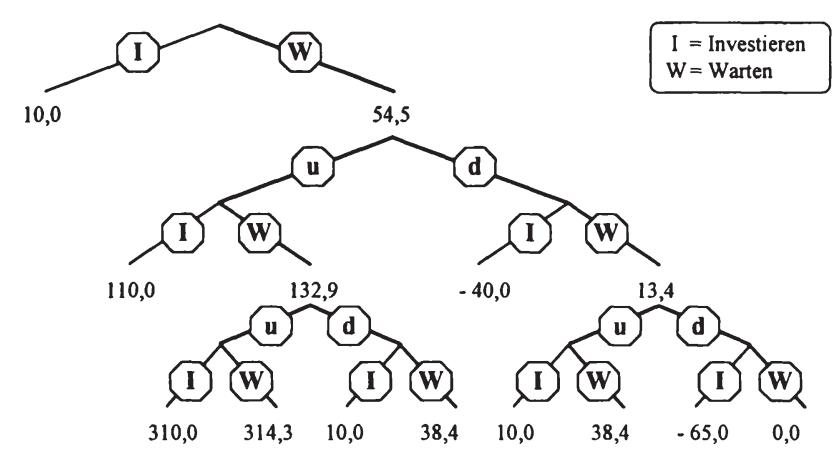

Abb. 39: Spieltheoretische Darstellung zur Bewertung einer exklusiven Investitionsmöglichkeit

Ist der Wert der Investitionsmöglichkeit hingegen durch beeinflußbare Wettbewerber bedroht, spielt der Inhaber der Investitionsmöglichkeit neben dem Spiel gegen die Natur gleichzeitig ein Spiel gegen die Konkurrenz ${ }^{1}$. In den einzelnen Teilperioden und Umweltzuständen kommt es jeweils zu einem Teilspiel, in dem jedes Unternehmen seine Entscheidung über eine frühzeitige Investition in Abhängigkeit der erwarteten Entscheidung der übrigen Wettbewerber trifft. Wie in Abschnitt 2.1 dieses Kapitels ist jeweils der reine Wert des Abwartens gegen einen möglichen konkurrenzbedingten Wertverlust abzuwägen. Im Gegensatz zu voriger Modellierung werden die Handlungen der Wettbewerber jedoch nicht exogen vorgegeben, sondern im Bewertungskalkül in Abhängigkeit der Umweltentwicklung und der Entscheidungen sämtlicher Wettbewerber endogen erfaßt.

Zur Illustration seien zwei Wettbewerber mit identischen Investitionsmöglichkeiten angenommen, die innerhalb des folgenden Teilspiels gleichzeitig entscheiden (vgl. Abb. 40) ${ }^{2}$. Wenn beide frühzeitig investieren, erzielen die Unternehmen den aktuellen Kapitalwert des Investitionsprojekts $\left(V_{0}-I\right)$. Entscheiden sich beide dafür, erst abzuwarten, bleibt für beide der Wert des Abwartens vollständig erhalten. Der Wert der Investitionsmöglichkeit beträgt $W_{0}$.

1 Vgl. Trigeorgis, Real Options, 1996, S. 58.

2 Vgl. Smit/Ankum, Game-Theoretic Approach, 1993, S. 244 f. 
Der Vorteil einer alleinigen frühzeitigen Investition wird dadurch berücksichtigt, daß für den Pionier der Wert des Bezugsguts größer ist als für den Fall, in dem beide gemeinsam frühzeitig investieren (d.h. $V_{0}{ }^{\text {Pionier }}>V_{0}$ ). Der Pionier realisiert somit einen höheren Kapitalwert $\left(V_{0}^{\text {Pionier }}-I\right)$ als bei gemeinsamer Investition. Analog dazu gilt, daß bei einem gemeinsamen Abwarten der Wert der Investitionsmöglichkeit größer ist als wenn der Konkurrent bereits investiert hat (d.h. $\left.W_{0}>W_{0}{ }^{\text {Nachfolger }}\right)$.

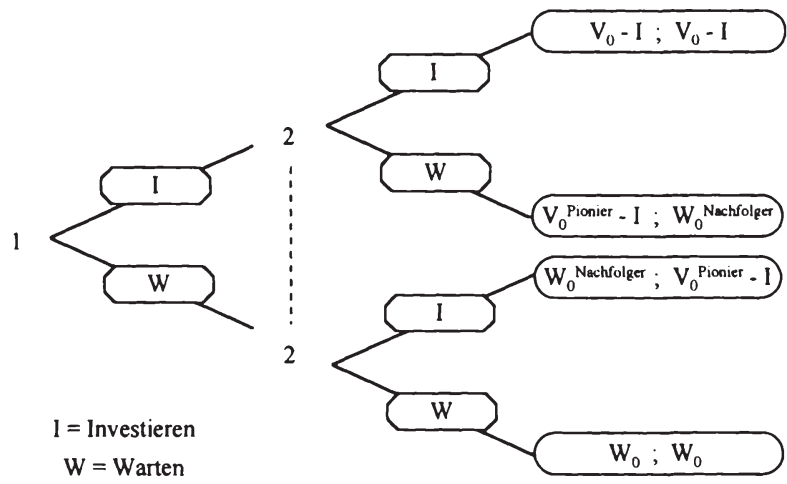

Abb. 40: Investitionsspiel mit zwei Wettbewerbern

Quelle: In Anlehnung an Smit/Ankum, Game-Theoretic Approach, 1993, S. 244.

In einem kooperativen Spiel vergleichen beide Wettbewerber lediglich den Kapitalwert $\left(V_{0}-I\right)$ mit dem Wert der Investitionsmöglichkeit $\left(W_{0}\right)$. Ohne zwischenzeitliche Rückflüsse entscheiden sich beide Unternehmen für ein weiteres Abwarten. In einem nicht-kooperativen Spiel kann es zu anderen Lösungen kommen. Ist der Kapitalwert für den Pionier $\left(V_{0}{ }^{\text {Pionier }}-I\right)$ größer als der Wert der Investitionsmöglichkeit bei gemeinsamen Abwarten $\left(W_{0}\right)$ und gleichzeitig der Wert der Investitionsmöglichkeit für den Nachfolger $\left(W_{0}{ }^{\text {Nachfolger }}\right)$ kleiner als der Kapitalwert bei gemeinsamer Investition $\left(V_{0}-I\right)$, entscheiden sich beide Wettbewerber für eine sofortige Investition. Wenn gleichzeitig der Wert der Investitionsmöglichkeit bei gemeinsamen Abwarten den Kapitalwert bei gemeinsamer Investition übersteigt, befinden sich die Unternehmen in dem zuvor geschilderten Gefangenendilemma. Es kommt zu einer im Vergleich mit dem Ergebnis des kooperativen Spiels suboptimalen Lösung. 


\subsubsection{Spieltheoretische Darstellung von Investitionsmöglichkeiten bei statischer Produktmarktstruktur}

\subsubsection{Ausgangssituation}

Zur weiteren Veranschaulichung der spieltheoretischen Darstellung von Investitionsmöglichkeiten wird das aus Abschnitt 2.2 in Kapitel IV bekannte Beispiel aufgegriffen und erweitert. Die dort geschilderte Investitionsmöglichkeit steht nun auch explizit einem Konkurrenten offen. Wie bisher haben die Investitions- sowie nachfolgenden Preis- und Mengenentscheidungen keine Auswirkungen auf das Marktvolumen des anvisierten Produktmarkts. Die einzelnen Wettbewerber sind folglich nicht in der Lage, über ihre Handlungen den Wert des gemeinsamen Bezugsguts zu beeinflussen. Dieser unterliegt weiterhin zu einhundert Prozent einer modellexogenen Unsicherheitsgröße.

In den beiden folgenden numerischen Beispielen werden zudem folgende, z.T. vereinfachende, Annahmen getroffen ${ }^{1}$ :

- Die beiden Wettbewerber sind nicht in der Lage, bindende Abmachungen zu treffen (nicht-kooperatives Spiel). Desweiteren entscheiden die Unternehmen in den einzelnen Teilspielen gleichzeitig.

- Der einzelne Wettbewerber erinnert sich an alle zuvor erfolgten Spielzüge bzw. nachträglich zu beobachtende Umweltentwicklungen (perfektes Erinnerungsvermögen). Er ist zudem vollständig über die Auswirkungen seiner Entscheidungen auf den Wert seiner Investitionsmöglichkeit informiert.

- Über die Wahl des Investitionszeitpunkts kann das einzelne Unternehmen in Abhängigkeit der Entscheidung des Konkurrenten den ihm anfangs zugedachten Marktanteil des vorgegeben Marktvolumens beeinflussen.

Unter diesen Bedingungen ergibt sich der Wert des Bezugsguts des betrachteten Unternehmens in der jeweiligen Teilperiode allgemein durch:

$$
\begin{aligned}
& V_{t, s_{u}}=u \cdot V_{t-1, s}^{G} \cdot\left(x_{M A}-k v_{t, s}\right) \\
& V_{t, s_{d}}=d \cdot V_{t-1, s}^{G} \cdot\left(x_{M A}-k v_{t, s}\right)
\end{aligned}
$$

1 Die Beispiele sind so konstruiert, daß sie mit den in Kapitel IV präsentierten Ergebnissen vergleichbar sind. 
wobei $V_{t, S}{ }^{G}$ den Wert des gemeinsamen Bezugsguts zum jeweiligen Zeitpunkt und Umweltzustand, $x_{M A}$ den dem betrachteten Unternehmen ursprünglich zugewiesenen Marktanteil und $k v_{t, s}$ den bei einer frühzeitigen Investition des Konkurrenten entstehenden prozentualen Marktanteilsverlust darstellt. Für den traditionellen Kapitalwert gilt dementsprechend ${ }^{1}$ :

$$
K W_{t, s}=V_{t, s}^{G} \cdot\left(x_{M A}-k v_{t, s}\right)-I .
$$

\subsubsection{Duopolbeispiel ohne dominante Marktstellung eines Wettbewerbers}

Zunächst wird von identischen Investitionsmöglichkeiten der beiden Wettbewerber ausgegangen. Letztere verfügen folglich über die gleiche Marktstellung und die gleiche Ausgangslage. Der Barwert der Rückflüsse aus der jeweiligen Investitionsmöglichkeit soll 100 Mio. $€$ und die jeweilige Investitionsauszahlung 90 Mio. $€$ betragen. Als gemeinsames Bezugsgut dient der Gegenwartswert der beiden Investitionsprojekte, der zum aktuellen Zeitpunkt 200 Mio. $€$ beträgt. Bei gemeinsamer Investition erhalten die Unternehmen jeweils 50\% dieses Gegenwartswerts, während bei einer alleinigen frühzeitigen Investition dem Pionier $60 \%$ und dem Nachfolger $40 \%$ zufallen $^{2}$. Falls sich die Konkurrenten gleichzeitig für eine frühzeitige Investition entscheiden, geht der Wert des Abwartens verloren, ohne daß daraus für ein Unternehmen ein Nutzen im Sinne eines Marktanteilszuwachses entsteht.

Der Wert des gemeinsamen Bezugsguts entwickelt sich entsprechend der Umweltdynamik aus dem Beispiel in Kapitel IV $(u=2$ und $d=0,5)$. Der risikolose Zinsfuß betrage weiterhin 5\% pro Teilperiode. Zur Bestimmung der Pseudowahrscheinlichkeiten werden die Steigungs- und Senkungsfaktoren des gemeinsamen Bezugsguts verwandt. Die risikoneutralisierten Wahrscheinlichkeiten belaufen sich auf $36,7 \%$ bzw. $63,3 \%$.

Abb. 41 enthält die für die jeweilige Investitionsmöglichkeit ermittelten Werte in Abhängigkeit der Teilperiode, des Umweltzustandes sowie der Investitionsentscheidung des Konkurrenten. Der erste Wert steht für das betrachtete Unternehmen (Spieler 1), wohingegen der zweite Wert für den Konkurrenten (Spie-

1 Wenn beide Unternehmen abwarten bzw. gleichzeitig investieren, gilt: $k v_{t, s}=0$. Bei einer fruhzeitigen Investition des betrachteten Unternehmens folgt ein Anteilsgewinn: $k v_{l, s}<0$.

$2 \mathrm{Zu}$ einem ähnlichen Zahlenbeispiel vgl. Smit/Ankum, Game-Theoretic Approach, 1993, S. $246 \mathrm{f}$. 
ler 2) gilt. Die Natur bzw. Umweltentwicklung wird durch den Spieler $N$ dargestellt. In den einzelnen - grau unterlegten - Teilspielen ist das jeweilige Spielergebnis fett gedruckt. Die optimale Entscheidung zwischen einer frühzeitigen Investition oder einem weiteren Abwarten ist in den einzelnen Teilperioden hervorgehoben. Aus Platzgründen wurde auf die Darstellung der Werte in der dritten Teilperiode verzichtet.

Die Berechnung des aktuellen Werts der jeweiligen Investitionsmöglichkeit erfolgt rekursiv. Hat das einzelne Unternehmen nicht schon in einer vorherigen Teilperiode sich für die Durchführung der Investition entschieden, kann es am Ende der Optionslaufzeit entweder investieren oder die Investitionsmöglichkeit endgültig aufgeben. Es wird dann investieren, falls der ihm zufallende Anteil am Gegenwartswert des Bezugsguts die zu entrichtende Investitionsauszahlung von 90 Mio. $€$ übersteigt.

In den vor dem Laufzeitende liegenden Teilperioden ist zu berücksichtigen, daß es gegebenenfalls zu einem Teilspiel kommen kann. Dies ist dann der Fall, wenn zu dem jeweiligen Zeitpunkt noch keiner der Wettbewerber investiert hat. Dies ist z.B. nach einer Teilperiode und einer Aufwärtsbewegung des gemeinsamen Bezugsguts der Fall. Wenn der Konkurrent investiert, ist der eigene Projektwert bei sofortiger Investition größer als wenn weiter abgewartet wird $(110>96$ Mio. $€)$. Im entgegengesetzten Fall, in dem der Konkurrent nicht investiert, ist ebenfalls eine frühzeitige Investition vorteilhaft $(150>131$ Mio. $€)$. Das betrachtete Unternehmen wird folglich in $t=1$ investieren. Da der Konkurrent vor der exakt gleichen Entscheidungssituation steht, investiert auch dieser in $t=1$. Die Lösung dieses Teilspiels bringt für beide Unternehmen ein Projektwert von 110 Mio. $€$ hervor, der zusammen mit dem Ergebnis aus dem Teilspiel bei einer Abwärtsbewegung des gemeinsamen Bezugsguts in die rekursive Bewertung der Investitionsmöglichkeit zum Zeitpunkt $t=0$ eingeht.

Die Lösung des zum Zeitpunkt $t=0$ stattfindenden Teilspiels ergibt den aktuellen Wert der Investitionsmöglichkeit. Da die Wettbewerber identischen Ausgangssituationen gegenüber stehen, ergibt sich für beide Konkurrenten der gleiche Wert der Investitionsmöglichkeit (46,5 Mio. €) sowie die gleiche, im folgenden erläuterte, Investitionsstrategie.

Zum heutigen Zeitpunkt wird nicht investiert, sondern eine weitere Teilperiode abgewartet. Bei einer Abwärtsbewegung des gemeinsamen Bezugsguts werden beide Unternehmen das Ende der Optionslaufzeit abwarten. Trotz Existenz von Teilspielen kommt es in diesen Umweltzuständen zu keinem Wertverlust der Investitionsmöglichkeit im Vergleich zu der Situation ohne Konkurrenzeinfluß. 


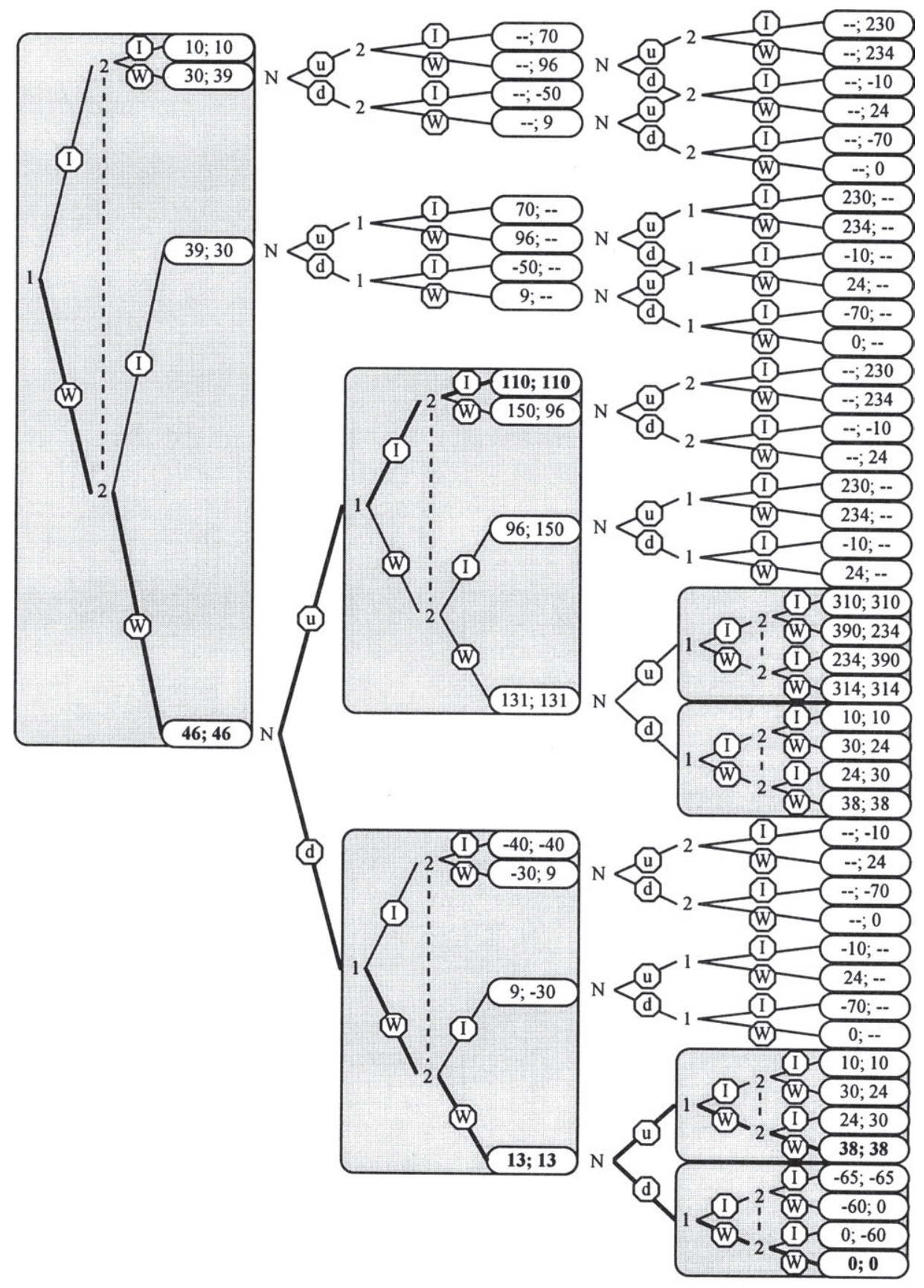

Abb. 41: Spieltheoretische Darstellung zur Bewertung einer Investitionsmöglichkeit im Duopol ohne dominante Marktstellung eines Wettbewerbers 
Bei einer Aufwärtsbewegung des gemeinsamen Bezugsguts nach einer Teilperiode entscheiden sich beide hingegen dafür, frühzeitig zu investieren, obwohl bei einem abgestimmten Investitionsverhalten ein weiteres Abwarten sinnvoll wäre. Es liegt der in den Abschnitten zuvor theoretisch erläuterte Fall des Gefangenendilemmas vor. Dies ist auch der Grund, wieso der Wert der Investitionsmöglichkeit hier um 8 Mio. $€$ niedriger ausfällt als derjenige ohne Konkurrenzeinfluß von 54,5 Mio. $€$. Eine Kooperation der Wettbewerber würde diesen Wertverlust verhindern.

\subsubsection{Duopolbeispiel mit dominanter Marktstellung eines Wettbewerbers}

In Abänderung des vorigen Beispiels wird nun angenommen, daß das betrachtete Unternehmen im Vergleich zu seinem Konkurrenten einen größeren Nutzen aus der geteilten Investition erzielen kann. Das betrachtete Unternehmen erhält bei gleichzeitiger Investition einen Marktanteil von 66,7\%, während dem Wettbewerber die restlichen 33,3\% zufallen. Das betrachtete Unternehmen verfügt somit über eine Investitionsmöglichkeit, dessen Rückflußpotential doppelt so groß ist wie dasjenige des Konkurrenten.

Der Barwert des Bezugsguts des betrachteten Unternehmens soll weiterhin 100 Mio. $€$ betragen, wodurch derjenige des Wettbewerbers 50 Mio. $€$ ausmacht. Der Barwert des gemeinsamen Bezugsguts beläuft sich somit auf 150 Mio. $€$. Beide benötigen weiterhin eine Investitionsauszahlung von 90 Mio. $€$, um in den Markt einzutreten. Bei einer frühzeitigen Investition kann der Pionier einen zusätzlichen Marktanteil von $10 \%$ zu Lasten des Nachfolgers erreichen. Dies würde für das betrachtete Unternehmen einen Marktanteil von $76,7 \%$ bedeuten, womit für den Konkurrenten nur noch 23,3\% übrig bleiben. Umgekehrt kann der Wettbewerber als frühzeitiger Pionier 43,3\% Marktanteil erlangen ${ }^{1}$.

Die Annahmen zur Wertentwicklung des gemeinsamen Bezugsguts $(u=2$ und $d=0,5$ ) sowie zur risikolosen Verzinsung ( $5 \%$ pro Teilperiode) bleiben bestehen. Die einzelnen Werte der Investitionsmöglichkeit in Abhängigkeit von der Teilperiode, vom Umweltzustand sowie von der Investitionsentscheidung des Konkurrenten können Abb. 42 entnommen werden. Der aktuelle Wert der Investitionsmöglichkeit beträgt für das betrachtete Unternehmen nun 57,0 Mio. $€$ und für den Wettbewerber 0,7 Mio. $€$.

1 Zu einem ăhnlichen Zahlenbeispiel vgl. Smit/Ankum, Game-Theoretic Approach, 1993, S. $247 \mathrm{f}$. 


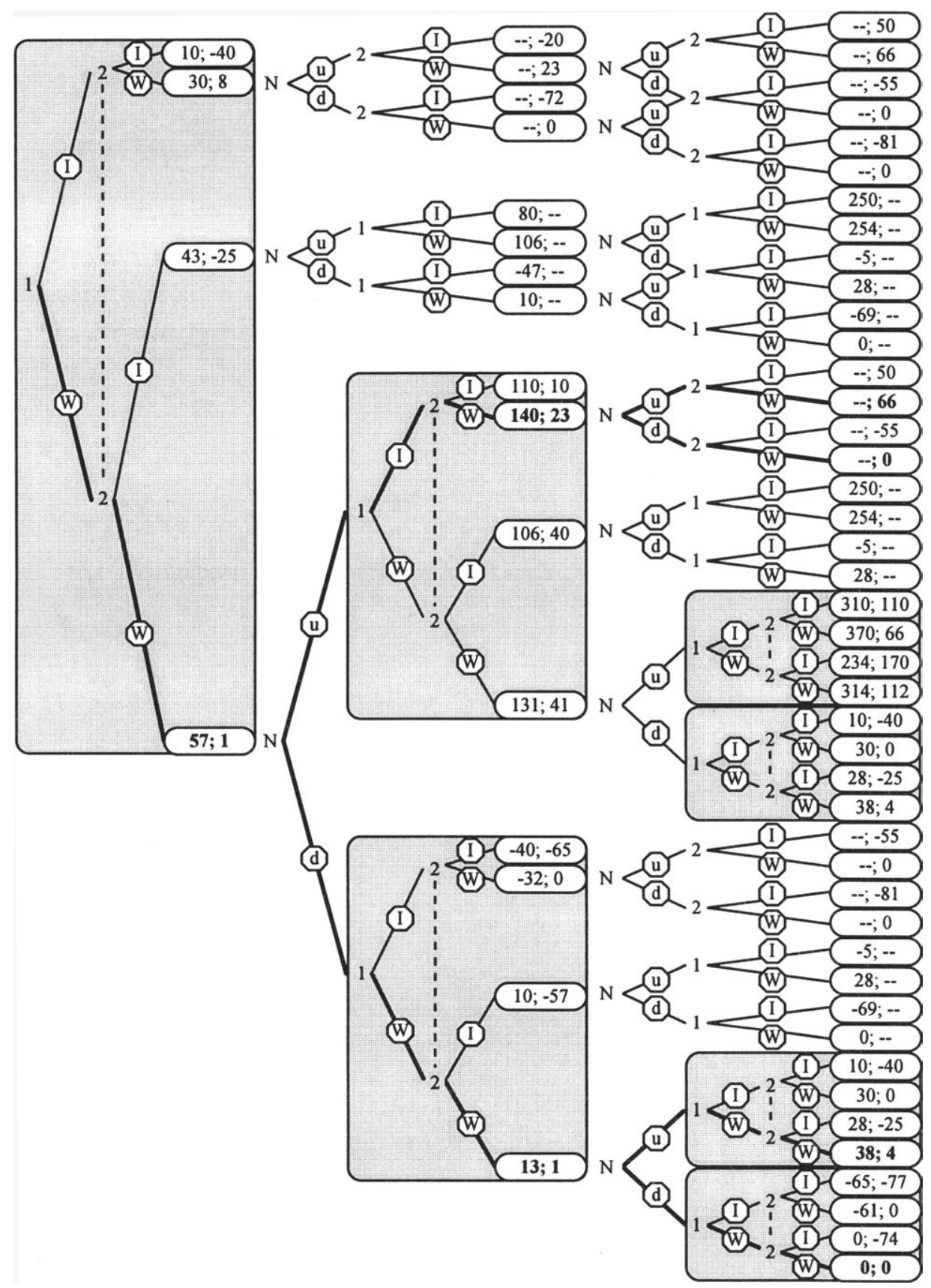

Abb. 42: Spieltheoretische Darstellung zur Bewertung einer Investitionsmöglichkeit im Duopol mit dominanter Marktstellung eines Wettbewerbers 
Während sich die Investitionsstrategie des betrachteten Unternehmens im Vergleich zum vorigen Beispiel nicht verändert hat, empfiehlt sich für den Konkurrenten eine andere Investitionspolitik. Zunächst warten die beiden Unternehmen die weitere Entwicklung ab. Das betrachtete Unternehmen investiert nach einer Teilperiode frühzeitig, wenn das gemeinsame Bezugsgut eine Aufwärtsbewegung vollzieht. Da sich der Konkurrent an dieser Stelle aufgrund der vorzufindenden Konstellation anders als zuvor für ein weiteres Abwarten entscheidet, kann das betrachtete Unternehmen einen zusätzlichen Marktanteil von $10 \%$ realisieren. Hierin liegt der Grund für den Anstieg des Werts der Investitionsmöglichkeit um 2,5 Mio. $€$ im Vergleich zur Situation ohne Konkurrenzeinfluß (54,5 Mio. €). Der Nutzen der Marktanteilserhöhung übersteigt knapp den aufgegebenen reinen Wert des Abwartens.

Wenn das betrachtete Unternehmen wie eben geschildert als Pionier investiert, gibt der Konkurrent die Investitionsmöglichkeit vollständig auf, falls in der Folgezeit das gemeinsame Bezugsgut nicht zwei weitere positive Entwicklungen aufweist. In diesen Umweltzuständen verdrängt das dominante Unternehmen den Wettbewerber vom Markt.

Bei einer Abwärtsbewegung des gemeinsamen Bezugsguts nach einer Teilperiode warten beide Unternehmen das Ende der Optionslaufzeit ab, um über eine endgültige Investition $\mathrm{zu}$ entscheiden. Für das betrachtete Unternehmen ist folglich in diesen Umweltzuständen weder eine investitionsstrategische noch eine wertmäßige Veränderung gegenüber der Situation ohne Konkurrenzeinfluß auszumachen.

\subsubsection{Spieltheoretische Darstellung der Modellansätze mit ursprünglich exogenem Konkurrenzverhalten}

In den Ansätzen, in denen das Konkurrenzverhalten als exogene Modellgröße aufgefaßt wird (vgl. Abschnitt 2.1 in Kapitel V), ist explizit unterstellt worden, daß die Investitionsstrategie des betrachteten Unternehmens von den Handlungen der Konkurrenz beeinflußt wird. Implizit wurde jedoch auch davon ausgegangen, daß die Konkurrenten ihrerseits ihre Investitionspolitik in Abhängigkeit von derjenigen des betrachteten Unternehmens festlegen. Folglich läßt sich die dort vorgenommene Bewertung von Investitionsmöglichkeiten mit Hilfe von wenigen zusätzlichen Annahmen ebenso spieltheoretisch darstellen.

Zur Illustration sei auf das Beispiel aus Abschnitt 2.1.1 dieses Kapitels zurückgegriffen, in dem zwei Konkurrenten zustandsabhängig handeln, falls das betrachteten Unternehmen nicht spätestens in der Teilperiode zuvor frühzeitig 
investiert und damit den ursprünglich geplanten Wert des Bezugsguts sichert. Das ohne Konkurrenzeinfluß vorhandene Bezugsgut des betrachteten Unternehmens wird nun als gemeinsames Bezugsgut definiert. Neben dem betrachteten Unternehmen werden zwei weitere Wettbewerber berücksichtigt.

Der erste Konkurrent, dessen Investition nach einer Teilperiode geplant ist, kann 20\% des nach einer Teilperiode gemeinsam zur Verfügung stehenden Bezugsguts erzielen. Bei einem Markteintritt dieses Konkurrenten verbleiben für das betrachtete Unternehmen $80 \%$ des Marktvolumens. Investiert der Wettbewerber nicht bzw. investiert das betrachtete Unternehmen sofort in $t=0$, gewinnt es durch den nicht vorhandenen Konkurrenzeinfluß bzw. die frühzeitige Investition $20 \%$ Marktanteil hinzu und beansprucht somit den gesamten Markt.

Zwischen dem betrachteten Unternehmen und dem zweiten Konkurrenten, dessen Investitionsmöglichkeit nach zwei Teilperioden besteht, existiert ein Aufteilungsverhältnis von $60 \% \mathrm{zu} 40 \%$. Dieses bezieht sich auf das zu diesem Zeitpunkt mit dem zweiten Konkurrenten gemeinsam geteilte Bezugsgut. Eine frühzeitige Investition sichert dem betrachteten Unternehmen den vom zweiten Konkurrenten beanspruchten 40\%-igen Marktanteil des nach der ersten Teilperiode noch nicht aufgeteilten Marktvolumens. Die Investitionsmöglichkeit des zweiten Konkurrenten ist somit abhängig von den zuvor vom ersten Konkurrenten und dem betrachteten Unternehmen getroffenen Entscheidungen ${ }^{1}$.

Für eine spieltheoretische Analyse der in Abschnitt 2.1.1 dieses Kapitels erfolgten Bewertung werden folgende zusätzliche Annahmen getroffen:

- Für die beiden Konkurrenten stellt die Investitionsmöglichkeit eine Jetzt oder Nie-Entscheidung dar. Ist zu dem geplanten Investitionszeitpunkt der Wert ihres Bezugsguts größer als ihre Investitionsauszahlung, werden sie investieren, ansonsten die Investition für immer aufgeben.

- Die Investitionsauszahlungen betragen 20 Mio. $€$ für den ersten und 35 Mio. $€$ für den zweiten Konkurrenten.

1 Bei einer Investition des ersten Konkurrenten und einem Abwarten des betrachteten Unternehmens in der ersten Teilperiode, stehen für den zweiten Konkurrenten und das betrachtete Unternehmen in der zweiten Teilperiode noch $80 \%$ des ursprünglichen Marktvolumens zur Verfugung. Der vom zweiten Konkurrenten zu diesem Zeitpunkt eventuell beanspruchte 40\% Marktanteil stellt somit 32\% des gesamten Marktvolumens dar. 
- Durch eine alleinige Investition können die beiden Konkurrenten keine zusätzlichen Marktanteile hinzugewinnen.

Abb. 43 stellt die beschriebene Investitionsmöglichkeit spieltheoretisch dar. Das betrachtete Unternehmen wird durch Spieler 1 verkörpert, während Spieler 2 den ersten Mitbewerber und Spieler 3 den zweiten Mitbewerber repräsentiert.

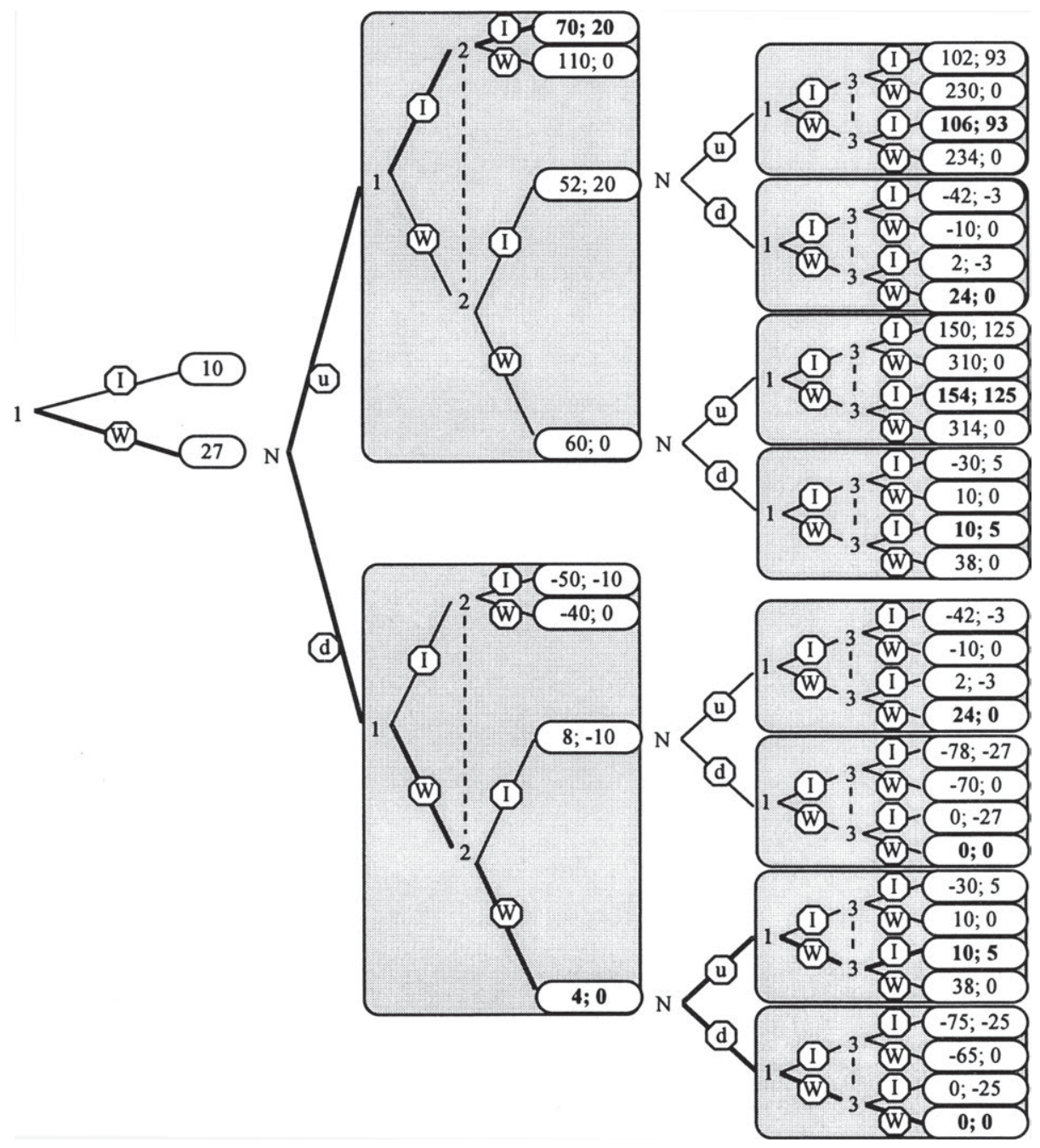

Abb. 43: Spieltheoretische Darstellung zur Bewertung einer Investitionsmöglichkeit mit ursprünglich exogener Konkurrenzhandlung 
Für das betrachtete Unternehmen resultiert der zuvor ermittelte Wert der Investitionsmöglichkeit von 26,7 Mio. $€$ sowie die bereits bekannte Investitionsstrategie. Zuerst wird die weitere Umweltentwicklung abgewartet, da der durch den ersten Konkurrenten bedingte Wertverlust niedriger ist als der reine Wert des Abwartens. Bei einer Aufwärtsbewegung des Bezugsguts nach einer Teilperiode wird investiert, um dem Markteintritt des zweiten Konkurrenten zuvorzukommen. Gleichzeitig investiert der erste Konkurrent. Bei einer Abwärtsbewegung des Bezugsguts nach einer Teilperiode investieren weder der erste Konkurrent noch das betrachtete Unternehmen, wodurch nach zwei Teilperioden der zweite Konkurrent eine Markteintrittschance erhält. Letztere wird von diesem wahrgenommen, falls sich das Bezugsgut in der zweiten Teilperiode erhöht.

\subsubsection{Spieltheoretische Darstellung von Investitionsmöglichkeiten bei dynamischer Produktmarktstruktur}

Bisher ist unterstellt worden, daß die Unsicherheit im Hinblick auf das gemeinsame Bezugsgut nicht durch die einzelnen Wettbewerber beeinflußt werden kann. Es wurde lediglich angenommen, daß sich zum Zeitpunkt einer frühzeitigen Investition das individuelle Bezugsgut des Pioniers vergrößert bzw. dasjenige des Nachfolgenden verkleinert. Dies ist als Marktanteilsgewinn bzw. -verlust bei zustandsabhängigem, jedoch für den einzelnen Umweltzustand vorgegebenem Marktvolumen interpretiert worden.

Der Vorteil einer derartigen statischen Struktur des Produktmarkts liegt darin, daß die am Kapitalmarkt geforderten Risikoprämien von den Entscheidungen der einzelnen Wettbewerber unberührt bleiben. Konstantes Risiko des gemeinsamen Bezugsguts im Zeitablauf vorausgesetzt, können die einmal ermittelten Pseudowahrscheinlichkeiten in allen Teilperioden verwendet werden. Wird jedoch das gemeinsame Bezugsgut auch durch die Entscheidungen der einzelnen Wettbewerber beeinflußt, kann diese Vereinfachung nicht mehr aufrecht erhalten werden. Die unsichere Wertentwicklung des gemeinsamen Bezugsguts wird dann durch die einzelnen Handlungen der Wettbewerber und die danach noch verbleibende modellexogene Unsicherheitsgröße bestimmt. Je nachdem, welche Individualentscheidungen getroffen werden und welche Produktmarktstruktur im Zeitablauf resultiert, ergeben sich andere Risikoprämien bzw. Pseudowahrscheinlichkeiten.

Zur Abbildung eines dynamischen Marktaufbaus bedarf es eines Modellrahmens, der in der Lage ist, den Einfluß individueller Entscheidungen auf die Struktur des Produktmarkts abzubilden. Einen solchen Modellrahmen in Ver- 
bindung mit Realoptionen präsentieren z.B. Smit/Trigeorgis, indem sie auf mikroökonomische Grundlagen eines Mengen- bzw. Preiswettbewerbs zurückgreifen ${ }^{1}$. In Abhängigkeit des Zeitpunkts der jeweiligen Investitionsentscheidungen entstehen unterschiedliche Marktformen, innerhalb derer die einzelnen Wettbewerber unter Berücksichtigung der jeweiligen Kostenstrukturen ihre Preis- bzw. Mengenentscheidungen optimieren.

Der Wert der Investitionsmöglichkeit wird wie auch in den Beispielen zuvor durch die optimale Investitionsstrategie bestimmt, die sich bei gleichzeitiger Berücksichtigung der unsicheren Umweltentwicklung und der unternehmerischen Flexibilität ergibt. Im Unterschied zu Abschnitt 2.2.3 hängt der optimale Investitionszeitpunkt nun explizit von der sich im Zeitablauf eventuell verändernden Marktform, der vorgegebenen Kostenstruktur sowie den in der jeweiligen Marktform optimal zu treffenden Preis/Mengen-Entscheidungen ab, die letztendlich die Rückflüsse aus der Investitionsmöglichkeit determinieren. Die in der jeweiligen Teilperiode durchzufürende Bewertungstechnik bleibt jedoch unverändert, weshalb im folgenden nicht näher auf diese Modellerweiterung eingegangen wird ${ }^{2}$.

Vgl. ausfuhrlich Smit/Trigeorgis, Strategic Investment, 1995, S. $11 \mathrm{ff}$.

$2 \mathrm{Zu}$ einem umfassenden numerischen Beispiel vgl. Smit/Trigeorgis, Strategic Investment, 1995, S. 21 ff. Für sonstige Bewertungsmodelle zur spieltheoretischen Darstellung und Analyse von Investitionsmöglichkeiten unter Unsicherheit, z.T. ohne Anwendung der Optionspreistheorie vgl. Appelbaum/Lim, Uncertainty, 1985; Mamer/McCardle, Competition, 1987; Vives, Competition, 1989; Smets, Direct Investment, 1991; Spencer/Brander, Flexibility, 1992; Grenadier, Strategic Exercise, 1996; Maggi, Endogenous Leadership, 1996; Nippel, Strategische Investitionsplanung, 1997, S. 83 ff.; Kulatilaka/Perotti, Growth Options, 1998. 


\section{Stochastischer Wettbewerbseffekt}

\subsection{Unsicherheit des Zeitpunkts der Konkurrenzhandlung}

In Abschnitt 2 dieses Kapitels wurde von deterministischen Handlungen der Konkurrenz ausgegangen. Der Zeitpunkt sowie die Höhe des konkurrenzbedingten Wertverlusts waren zwar ex ante nicht bekannt, jedoch in Abhängigkeit der stochastischen Entwicklung des Bezugsguts eindeutig festgelegt. Nun wird diese Annahme aufgehoben, so daß der Konkurrenzeffekt ebenfalls einem stochastischen unabhängigen Zufallsprozeß gehorcht. Zuerst wird auf Situationen eingegangen, in denen Unsicherheit über den Zeitpunkt des Konkurrenzeintritts herrscht, um anschließend Erweiterungen des Bewertungskalküls darzustellen, die eine unsichere Investitionsauszahlung (z.B. Markteintrittsbarrieren) oder eine unsichere zwischenzeitliche Verringerung des Bezugsguts (z.B. Marktanteilsverluste bzw. Preissenkungen) modellieren.

Zur Abbildung eines zeitlich unsicheren Konkurrenzeintritts kann u.a. das Bewertungsmodell von Cox und Ross herangezogen werden ${ }^{1}$. Hierbei wird das Bezugsgut neben der erwarteten momentanen Wachstumsrate $\mu$ durch einen Poissonprozeß beeinflußt. Über den Poissonprozeß läßt sich der zufällige Konkurrenzeintritt beschreiben, indem die diesen Prozeß bestimmenden Bewertungsparameter uminterpretiert werden ${ }^{2}$. Die Sprungkomponente $k$ verkörpert nun den konkurrenzbedingten Wertverlust für den Fall, daß ein Konkurrent in den Markt eintritt. Der Wert des Bezugsguts sinkt um den deterministischen Betrag $(1-k) \cdot V$, so daß bei Investition nach dem Konkurrenzeintritt nur noch ein Wert des Bezugsguts in Höhe von $k \cdot V$ erzielt werden kann ${ }^{3}$. Die Wahrscheinlichkeit, daß im nächsten Moment ein Konkurrent in den Markt eintritt und den eben erläuterten Wertverlust verursacht, wird mit $\lambda \cdot d t$ ausgedrückt.

$$
d V=[\mu-\lambda \cdot(k-1)] \cdot V \cdot d t+(k-1) \cdot V \cdot d \varphi
$$

wobei $\quad d \varphi=1$ mit Eintrittswahrscheinlichkeit $\lambda d t$

$d \varphi=0$ mit Eintrittswahrscheinlichkeit $l-\lambda d t$

mit $\quad 0<k<1$

1 Vgl. auch Abschnitt 4 in Kapitel IV.

2 Vgl. zu folgendem ausführlich Trigeorgis, Uncertain Competitive Arrivals, 1995, S. 5 ff.

3 Für ein ähnliches Bewertungsmodell mit stochastischem Sprungausmaß vgl. Pennings, Real Options, 1998, S. $20 \mathrm{ff}$. 
Je größer der Bewertungsparameter $\lambda$ ist, um so eher ist mit einem Konkurrenzeintritt zu rechnen. Ein hohes $\lambda$ stellt folglich Konkurrenzsituationen mit starker Wettbewerbsintensität und umgekehrt ein niedriges $\lambda$ Konkurrenzsituationen mit gemäßigter Wettbewerbsintensität dar. Für den Fall eines tatsächlichen Konkurrenzeintritts bewirkt eine relativ kleine Sprungkomponente $k$ eine beträchtliche Verringerung des Werts des Bezugsguts. Dieser Bewertungsparameter eignet sich somit, um die Wettbewerbsposition des betrachteten Unternehmens abzubilden. Bei einem schwachen Unternehmen wird ein niedriges $k$ gewählt, während bei einem dominanten Unternehmen ein hohes $k$ gilt.

Die Lösung des aufgestellten Bewertungsmodells ähnelt konzeptionell derjenigen aus dem Black/Scholes-Modell ${ }^{1}$. Der Wert der Kaufoption bzw. Investitionsmöglichkeit wird als Differenz zwischen dem gewichteten aktuellen Wert des Bezugsguts $V$ und der gewichteten, auf den heutigen Zeitpunkt abdiskontierten, zukünftigen Investitionsauszahlung $I$ bestimmt. Die Gewichte werden jedoch nicht wie im Black/Scholes-Modell auf Basis der Normalverteilung, sondern mit Hilfe der Gammaverteilung ermittelt.

$$
\begin{aligned}
& W=V \cdot \Gamma(k \cdot t, n)-I \cdot e^{-r_{f} \cdot t} \cdot \Gamma(t, n) \\
& \text { mit } n=\left\|\frac{\ln (V / I)+\left[r_{f}+\lambda \cdot(1-k)\right] \cdot t}{\ln (1 / k)}\right\|
\end{aligned}
$$

wobei $\Gamma(.)=$. Wert der Gammaverteilungsfunktion mit den Parametern $\alpha_{G}=n+1$ sowie $\beta_{G}=1$

Der soeben dargestellte Ansatz behandelt den eher unrealistischen Fall, in dem mit Ausnahme des modellierten Konkurrenzeintritts die sonstige Umweltentwicklung als sicher gilt. Ist neben dem stochastischen Konkurrenzeintritt auch die sonstige Umweltentwicklung unsicher, kann in Anlehnung an Merton der Zufallsproze $ß$ des Bezugsguts um eine Streuungskomponente $\sigma \cdot d z$ erweitert werden ${ }^{2}$.

$$
d V=[\mu-\lambda \cdot \mathrm{E}(k-1)] \cdot V \cdot d t+\sigma \cdot V \cdot d z+(k-1) \cdot V \cdot d \varphi
$$

1 Vgl. Trigeorgis, Real Options, 1996, S. 285 f.

2 Vgl. auch Abschnitt 4 in Kapitel IV. 
Diese Formulierung impliziert, daß der Wert des Bezugsguts größtenteils gemäß des im Black/Scholes-Modell unterstellten generellen Wiener Prozesses schwankt und zusätzlich an voneinander unabhängigen Zeitpunkten zufällig um einen deterministischen konkurrenzbedingten Betrag sinkt. Die Lösung dieses Bewertungsmodells läßt sich als gewichtete Summe von Black/Scholes-Ergebnissen $W_{B S}$ interpretieren, wobei letztere unter Berücksichtigung eines konkurrenzbedingten Wertverlusts ermittelt werden ${ }^{1}$. Dieser zwischenzeitlich entgehende Rückfluß wird mit Hilfe der Wahrscheinlichkeit eines Konkurrenzeintritts bzw. der Wettbewerbsintensität $\lambda$ und dem Wertverlust bei Konkurrenzeintritt $(1-k) \cdot V$ bestimmt ${ }^{2}$.

$$
\begin{aligned}
& W=\sum_{\varphi=0}^{\infty} \frac{\mathrm{e}^{-\lambda \cdot t} \cdot(\lambda \cdot t)^{\varphi}}{\varphi !} \cdot W_{B S} \\
& \text { mit } W_{B S}=V \cdot \mathrm{e}^{-\delta \cdot t} \cdot N\left(\hat{d}_{1}\right)-I \cdot \mathrm{e}^{-r_{f} \cdot t} \cdot N\left(\hat{d}_{2}\right) \\
& \text { sowie } \\
& \hat{d}_{1}=\frac{\ln \frac{V}{I}+\left(r_{f}-\delta+0,5 \cdot \sigma^{2}\right) \cdot t}{\sigma \cdot \sqrt{t}}, \hat{d}_{2}=\hat{d}_{1}-\sigma \cdot \sqrt{t} \\
& \text { und } \delta=\lambda \cdot(k-1)-(\varphi / t) \cdot \ln k
\end{aligned}
$$

Mit steigender Wahrscheinlichkeit eines Konkurrenzeintritts $\lambda$ und abnehmender Wettbewerbsposition $k$ steigt c.p. der Wert der Investitionsmöglichkeit ${ }^{3}$. Dieses Ergebnis, daß eine Investitionsmöglichkeit bei hoher Wettbewerbsintensität bzw. schwacher Wettbewerbsposition einen größeren Wert besitzt als bei niedriger Wettbewerbsintensität bzw. dominanter Wettbewerbsposition überrascht. Bei deterministischem Konkurrenzeffekt führte ein größerer konkurrenzbedingter Wertverlust zu einer Verringerung der Investitionsmöglichkeit. Hier ist jedoch nun der Konkurrenzeffekt nicht sicher, so daß der Aufschub der endgültigen Investitionsentscheidung - im Gegensatz zu einer sofortigen Investition - zu einer Absicherung gegen diesen konkurrenzbedingten Wertverlust

Vgl. Trigeorgis, Real Options, 1996, S. 287.

2 Wird der stochastische Konkurrenzeintritt aus dem Kalkül eleminiert ( $k=1$ bzw. $\lambda=0$ ), geht die Bewertungsgleichung in die Black/Scholes-Formel uber.

3 Vgl. Trigeorgis, Uncertain Competitive Arrivals, 1995, S. 14 f. 
führt. Diese Absicherung ist umso wertvoller, je größer die Gefahr eines Konkurrenzeintritts und der dann zu verlierende Betrag ist.

Das soeben erläuterte Ergebnis gilt jedoch nur, wenn sich der Barwert des Bezugsguts trotz des unterstellten stochastischen Konkurrenzeintritts nicht verändert. Bedingt die erhöhte Unsicherheit hingegen eine größere Risikoprämie des Bezugsguts, sinkt hierdurch der Barwert desselben und damit auch der Wert der Investitionsmöglichkeit. Letztgenannter Effekt steht dann dem werterhöhenden Versicherungseffekt gegenüber, so daß der Gesamteffekt der erhöhten Unsicherheit theoretisch nicht eindeutig feststeht ${ }^{1}$.

Eine interessante Variante des Bewertungsmodells stellt der Ansatz von Rei $\beta$ dar, in dem die auch bei Trigeorgis vorzufindende Jump to Ruin-Situation ${ }^{2}$ unter expliziter Berücksichtigung von Patentkosten untersucht wird ${ }^{3}$. Dieser Spezialfall unterstellt, daß ein Konkurrenzeintritt die eigene Investitionsmöglichkeit vollständig zunichte macht $(k=0)$. Mit Hilfe ihres Bewertungsmodells leitet die Autorin Investitionsstrategien ab, die einerseits den Zeitpunkt der Investitionsentscheidung und andererseits das Anmelden bzw. Nichtanmelden des Patents bestimmen.

\subsection{Unsicherheit der Höhe des konkurrenzbedingten Wertverlusts}

\subsubsection{Stochastische Markteintrittsbarrieren}

Wie in Abschnitt 2.1.2 dieses Kapitels erläutert, lassen sich unterschiedliche Markteintrittsbarrieren über verschiedene Höhen der Investitionsauszahlung darstellen. Sind die zum Zeitpunkt der endgültigen Investitionsentscheidung vorzufindenden Markteintrittsbarrieren selbst bei Kenntnis des Umweltzustands unsicher, bedeutet dies, daß die Höhe der Investitionsauszahlung als stochastischer Bewertungsparameter abgebildet werden muß.

Hierfür bieten sich die ursprünglich für Finanzoptionen entwickelten Bewertungskalküle von Fischer bzw. Margrabe $\mathrm{an}^{4}$. In diesen Modellen folgt neben dem Wert des Bezugsguts $V$ auch die Investitionsauszahlung $I$ einem generel-

Vgl. hierzu auch die Überlegungen in Abschnitt 3.1.1.2 in Kapitel VI.

2 Vgl. Trigeorgis, Uncertain Competitive Arrivals, 1995, S. $13 \mathrm{ff}$.

3 Vgl. Reiß, Investment and Competition, 1998, S. $640 \mathrm{ff}$.

4 Vgl. Fischer, Exercise Price is Uncertain, 1978, S. 170 ff.; Margrabe, Exchange, 1978, S. $177 \mathrm{ff}$. 
len Wiener Prozeß. Die Parameter $\mu_{V}$ bzw. $\mu_{I}$ stellen die erwarteten momentanen Wachstumsraten des Bezugsguts bzw. der Investitionsauszahlung dar, während $\sigma_{V}$ und $\sigma_{I}$ die entsprechenden Abweichungsraten verkörpern.

$$
\begin{gathered}
d V=\mu_{V} \cdot V \cdot d t+\sigma_{V} \cdot V \cdot d z \\
d I=\mu_{I} \cdot I \cdot d t+\sigma_{I} \cdot I \cdot d z
\end{gathered}
$$

Da Bezugsgut und Investitionsauszahlung Wertschwankungen unterliegen, die voneinander unabhängig sind, bedarf es zusätzlich eines Maßstabs, der den Gleichlauf der beiden stochastischen Zufallsprozesse beschreibt. Dies wird über die Angabe der Korrelation kor $_{V, I}$ zwischen dem Wert des Bezugsguts und der Höhe der Investitionsauszahlung bewerkstelligt.

In dem Lösungsansatz von Fischer entspricht die momentane Wachstumsrate der Investitionsauszahlung nicht der am Kapitalmarkt vorzufindenden Gleichgewichtsrendite. Aus diesem Grund wird auf ein am Kapitalmarkt existierendes Wertpapier $\hat{I}$ zurückgegriffen, dessen momentane Wachstumsrate per se die Bedingung einer Gleichgewichtsrendite $\hat{\mu}_{I}$ erfüllt und dessen Abweichungsrate mit derjenigen der Investitionsauszahlung übereinstimmt.

$$
d \hat{I}=\hat{\mu}_{I} \cdot \hat{I} \cdot d t+\sigma_{I} \cdot \hat{I} \cdot d z
$$

Fischer löst das aufgestellte Bewertungsproblem über die Bildung eines risikolosen Hedgeportfolios, in dem zu bestimmten Anteilen die Option, das Bezugsgut und das gehandelte Wertpapier enthalten sind. Während das Bezugsgut die Schwankungen des Hedgeportfolios absorbiert, die durch eine Veränderung des unsicheren Bezugsguts bedingt sind, wird das Wertpapier $\hat{I}$ zur Absicherung der unsicheren Investitionsauszahlung benötigt. Für den Wert der Investitionsmöglichkeit $W$ ergibt sich folgende Bewertungsformel ${ }^{1}$.

$$
W=V \cdot N\left(d_{l}\right)-I \cdot e^{-\left(\hat{\mu}_{l}-\mu_{l}\right) \cdot t} \cdot N\left(d_{2}\right)
$$

Bei einer im Zeitablauf konstanten und damit sicheren Investitionsauszahlung $\left(\mu_{I}=\sigma_{l}=0\right)$ und einer Interpretation der momentanen Wachstumsrate des am Kapitalmarkt gehandelten Wertpapiers als risikoloser Zinsfuß geht die Bewertungsgleichung in die Black/ScholesFormel über. 


$$
\begin{gathered}
\text { mit } \quad d_{l}=\frac{\ln \frac{V}{I}+\left(\hat{\mu}_{I}-\mu_{I}+0,5 \cdot \hat{\sigma}^{2}\right) \cdot t}{\hat{\sigma} \cdot \sqrt{t}} \\
\text { und } d_{2}=d_{I}-\hat{\sigma} \cdot \sqrt{t} \\
\text { sowie } \hat{\sigma}={\sigma_{V}}^{2}+\sigma_{I}{ }^{2}-2 \cdot \text { kor }_{V, I} \cdot \sigma_{V} \cdot \sigma_{I}
\end{gathered}
$$

Ein ähnliches Bewertungsmodell stammt von Margrabe, der die Option bewertet, einen risikobehafteten Vermögensgegenstand gegen einen anderen unsicheren Vermögensgegenstand einzutauschen. Wird die hier in den Blickpunkt gerückte Investitionsauszahlung als dieser zweite Vermögensgegenstand angesehen, ergibt sich für den Wert der Investitionsmöglichkeit die unten aufgeführte Bewertungsgleichung. Im Gegensatz zu Fischer geht Margrabe von einer momentanen Wachstumsrate der Investitionsauszahlung aus, die sich im Kapitalmarktgleichgewicht befindet. Dadurch entfällt zum einen die zuvor vorgenommene Abschlagskorrektur $\left(\hat{\mu}_{I}-\mu_{I}\right)$ bei der Bestimmung des Barwerts der Investitionsauszahlung $I$ und zum anderen die Berücksichtigung dieser Differenzrendite bei der Ermittlung des Parameters $d_{l}$.

$$
\begin{gathered}
W=V \cdot N\left(d_{1}\right)-I \cdot N\left(d_{2}\right) \\
\text { mit } d_{1}=\frac{\ln \frac{V}{I}+0,5 \cdot \hat{\sigma}^{2} \cdot t}{\hat{\sigma} \cdot \sqrt{t}} \\
\text { und } d_{2}=d_{1}-\hat{\sigma} \cdot \sqrt{t}
\end{gathered}
$$

Durch die Annahme einer stochastischen Investitionsauszahlung und damit unsicheren Markteintrittsbarrieren vergrößert sich die Bandbreite der am Laufzeitende erwarteten Optionswerte. Im Vergleich zum Fall mit deterministischem Auszahlungsbetrag erhöht sich somit das Gewinnpotential der zu beurteilenden Investitionsmöglichkeit, wohingegen das Verlustpotential aufgrund ihres asymmetrischen Risikocharakters unverändert bleibt, so daß c.p. der Wert der Investitionsmöglichkeit steigt. Dieses auf den ersten Blick merkwürdig anmutende Ergebnis wird klarer, wenn man sich den Versicherungscharakter einer Kaufoption vergegenwärtigt. Je mehr Unsicherheit über die Vorteilhaftigkeit des zu beurteilenden Investitionsprojekts besteht, um so wertvoller wird die Möglichkeit, die endgültige Entscheidung in die Zukunft zu verschieben. 


\subsubsection{Stochastische Marktanteilsverluste und Preissenkungen}

Wie in Abschnitt 2.1.1 dieses Kapitels angeführt, läßt sich bei einer Verschiebung des Investitionszeitpunkts der ursprünglich geplante Marktanteil bzw. Absatzpreis ggf. nicht mehr realisieren. Durch zwischenzeitliche Konkurrenzhandlungen sinkt der Wert des Bezugsguts. Diese konkurrenzbedingte Verringerung des Bezugsguts ist konzeptionell mit der Auszahlung einer Aktiendividende vergleichbar. Folglich kann bei Fällen, in denen die Höhe eines solchen konkurrenzbedingten Wertverlusts als unsicher gilt, auf Optionspreismodelle zurückgegriffen werden, die stochastische Dividenden oder stochastische zwischenzeitliche Rückflüsse abbilden. Die Bewertung von Investitionsmöglichkeiten erfolgt durch eine Uminterpretation der Bewertungsparameter.

Den beiden im folgenden - nur in ihren Grundgedanken - erläuterten Bewertungskalkülen ist gemeinsam, daß der Wert des Bezugsguts als genereller Wiener Prozeß modelliert wird. Im Modell von Geske zur Bewertung von Aktienoptionen wird zudem eine logarithmisch normalverteilte Dividendenrate unterstellt ${ }^{1}$. Der Autor nimmt an, daß die absolute Höhe der Dividendenrate vom Wert des Bezugsguts abhängt, so daß eine risikolose Hedgebewertung nicht möglich ist. Die Bewertung der Option erfolgt über die explizite Annahme bestimmter Risikopräferenzen. Geske wendet hierbei die Diskontierungstechnik von Rubinstein an, innerhalb derer unsichere zukünftige Zahlungsströme unter der Annahme konstant proportionaler Risikoaversion bewertet werden ${ }^{2}$. Wird im Bewertungskalkül von Geske die Höhe der Dividendenrate als deterministisch angesehen, resultiert die von Merton aufgestellte Bewertungsformel für europäische Kaufoptionen mit im Zeitablauf konstanter Dividendenrate ${ }^{3}$.

Im Rahmen des von Gibson/Schwartz vorgestellten Ansatzes zur Bewertung von Ölbohrrechten folgt die zwischenzeitlich entgehende "convenience yield“ einem Ornstein-Uhlenbeck-Proze $\beta^{4}$. Diese unsichere zwischenzeitlich entgehende „Rückflußrate“ $r_{D}$ weist folglich eine Tendenz auf, sich um ein bestimmtes vorgegebenes Niveau $\overline{r_{D}}$ zu bewegen (Mean Reverting-Eigenschaft). Die Geschwindigkeit, mit der diese zwischenzeitliche Rückflußrate auf dieses

1 Vgl. ausfuhrlich Geske, Stochastic Dividend Yield, 1978, S. 617 ff.

2 Vgl. Rubinstein, Uncertain Income Streams, 1976, S. 412 ff. Zur resultierenden Bewertungsformel vgl. Geske, Stochastic Dividend Yield, 1978, S. 621.

3 Vgl. Abschnitt 3.3 in Kapitel IV.

4 Vgl. ausfuhrlich Gibson/Schwartz, Stochastic Convenience Yield, 1990, S. 959 ff. 
Normalniveau zusteuert, wird durch $\eta$ abgebildet. Ihre Streuung ist durch $\sigma_{D}$ charakterisiert.

$$
d r_{D}=\eta \cdot\left(\overline{r_{D}}-r_{D}\right) \cdot d t+\sigma_{D} \cdot d z
$$

Zur Lösung eines solchen Bewertungsansatzes muß neben der Korrelation zwischen den beiden modellierten Zufallsprozessen ein „Marktpreis“ für das Risiko der unsicheren Rückflußrate angegeben werden. Die Bewertung dieses Risikos ist wie die Bewertung des Bezugsguts vor dem eigentlichen Optionskalkül durchzuführen. Gibson/Schwartz lösen die aufgestellte partielle Differentialgleichung mit Hilfe numerischer Methoden, da eine analytische Lösung nicht existiert ${ }^{1}$.

Durch die Einführung einer stochastischen Dividendenrate bzw. „convenience yield" $r_{D}$ schwankt der Wert des Bezugsguts mehr als bei deterministischen Vergleichsdaten. Hierdurch vergrößert sich die Bandbreite der asymmetrisch verteilten Optionswerte am Ende der Optionsfrist, so daß unter c.p.-Bedingungen - bei unverändertem Barwert des Bezugsguts - der Wert der Investitionsmöglichkeit steigt ${ }^{2}$.

In dem zurückliegenden Kapitel ist ausführlich auf die Berücksichtigung des Konkurrenzverhaltens bei der Bewertung von Investitionsmöglichkeiten eingegangen worden. Dabei wurde festgestellt, daß bei nicht beeinflußbarem (deterministischem) Konkurrenzverhalten der bereits in Kapitel IV entwickelte Bewertungskalkül herangezogen werden kann, während bei beeinflußbaren (deterministischen) Konkurrenzhandlungen das Bewertungsmodell $\mathrm{zu}$ modifizieren ist. Dazu wird das Konkurrenzverhalten entweder als exogene Modellgröße aufgefaßt und einzelne bereits bekannte Bewertungsparameter uminterpretiert oder ein modellendogenes Konkurrenzverhalten über die Integration spieltheoretischer Elemente erfaßt. Demgegenüber müssen bei stochastischem Konkurrenzverhalten zwei Unsicherheitsquellen gleichzeitig berücksichtigt werden, so daß entweder der Zufallsprozeß des Bezugsguts modifiziert oder ein weiterer Zufallsprozeß eingeführt wird.

Nach der in Kapitel IV und V erfolgten umfassenden Präsentation der Bewertungstechnik stehen nun Umsetzungsmöglichkeiten und -grenzen einer solchen Bewertung im Mittelpunkt des Interesses.

I Vgl. Gibson/Schwartz, Stochastic Convenience Yield, 1990, S. 962.

2 Vgl. stellvertretend Geske, Stochastic Dividend Yield, 1978, S. 622 f. 


\section{Kapitel VI}

\section{Umsetzungsmöglichkeiten und -grenzen des Realoptionskonzepts bei der Bewertung von Investitionsmöglichkeiten}

\section{1 Überblick}

Im Rahmen dieses Kapitels werden Fragestellungen diskutiert, die alle in direktem Zusammenhang mit einer praktischen Umsetzung der Realoptionsbewertung stehen. Wann und auf welche Weise läßt sich der Realoptionsansatz zur Bewertung der in den Kapiteln zuvor betrachteten, und aufgrund der strategischen Akquisition bedingten, zusätzlichen Investitionsmöglichkeit nutzen? Die im folgenden kurz erläuterten vier Aspekte sind dabei weitestgehend voneinander unabhängig.

Im zweiten Abschnitt werden die den Realoptionsbewertungsmodellen zugrunde liegenden und an verschiedenen Stellen dieser Arbeit bereits angeklungenen Kapitalmarktannahmen ausführlich diskutiert. Dies geschieht aus drei Beweggründen. Erstens sollen die in diesen Bewertungskalkülen z.T. implizit vorhandenen Marktannahmen aufgedeckt werden. Zweitens erfolgt ein Vergleich zu den in traditionellen Bewertungsmodellen unterstellten Marktprämissen mit dem Ziel, diejenigen der Realoptionspreistheorie entsprechend zu würdigen. Dazu wird letztere in die moderne Bewertungslehre der Investitions- und Finanzierungstheorie eingeordnet. Drittens läßt sich auf Basis einer solchen Diskussion der Blick für eine Anwendbarkeit der Realoptionsbewertung schärfen.

In den Beispielen des Kapitel IV und $\mathrm{V}$ ist vereinfachend davon ausgegangen worden, daß der Aufschub der zusätzlichen Investitionsmöglichkeit der einzige $\mathrm{zu}$ bewertende Handlungsspielraum darstellt. Wie muß hingegen vorgegangen werden, wenn, wie in realistischen Entscheidungssituationen anzunehmen, mehrere unternehmerische Handlungsspielräume gleichzeitig bestehen? In einem solchen Fall ist eine sorgfältige Analyse der jeweiligen Bezugsgüter zu fordern. Sind letztgenannte voneinander abhängig, so daß die einzelnen Handlungsspielräume sich gegenseitig bedingen, wird von verbundenen Realoptionen gesprochen. Die Bewertung solcher verbundenen Realoptionen ist Gegenstand des dritten Abschnitts.

Außerdem wurde in den bisherigen Ausführungen stets ein einwertiges Ergebnis für den Wert der zusätzlichen Investitionsmöglichkeit errechnet, wobei die hierzu erforderlichen Bewertungsparameter vorgegeben waren. Wie verändert sich jedoch der Wert der Investitionsmöglichkeit, wenn die ihm zugrundelie- 
genden Bewertungsparameter variiert werden? Wie lassen sich überhaupt solche Bewertungsparameter ermitteln? Und welchen Stellenwert könnte die Realoptionsbewertung im Kontext der wertorientierten Unternehmensführung einnehmen? Im vierten Abschnitt werden Instrumente, die u.U. eine Antwort auf diese Fragen ermöglichen, dargestellt und ihr paralleler Einsatz im Rahmen der Realoptionsbewertung diskutiert.

Der fünfte Abschnitt faßt die bisher zum Thema „Realoptionen“ durchgeführten empirischen Studien zusammen. Dabei geht es z.B. um die Fragestellung, ob Realoptionsbewertungsmodelle die beim Verkauf von Öl- und Gasförderungsrechten bzw. Grundstücken beobachtbaren Preise besser erklären können als traditionelle DCF-Verfahren. Zweitens wird anhand eines Vergleichs zwischen diskontierten Earnings Per Share-Prognosen und Börsenkursen belegt, daß in letztgenannten ein beträchtlicher, den Wachstumsoptionen der betreffenden Unternehmen zuzuschreibender Wertbestandteil vermutet werden kann. Schließlich wird auf Ergebnisse zum Verständnis und Einsatz der Realoptionstheorie eingegangen, die u.a. über Befragungen englischer und deutscher Finanz- und Rechnungswesenleiter erzielt worden sind.

\section{Ausführliche Diskussion der Kapitalmarktannahmen}

\subsection{Arbitragefreiheit}

In sämtlichen finanz- sowie realwirtschaftlichen Optionspreismodellen wird von einem arbitragefreien Kapitalmarkt ausgegangen. Auf einem nicht arbitragefreien Markt wäre es möglich, eine Vermögensposition aufzubauen, die einerseits einen nicht-negativen zukünftigen Rückfluß verspricht und andererseits heute eine Einzahlung ermöglicht. Vorausgesetzt die Marktteilnehmer ziehen stets ein höheres Einkommen einem niedrigeren vor, bestünde eine unbeschränkte Nachfrage nach dieser Vermögensposition, da sich mit ihrer Hilfe risikolose Arbitragegewinne realisieren ließen. Auf einem solchen Markt könnten sich keine stabilen Preise einstellen ${ }^{1}$.

Dies zeigt, daß die Annahme eines arbitragefreien Kapitalmarkts unabdingbar mit dem Wunsch eines Vergleichs zwischen zwei gehandelten Vermögenspositionen verknüpft ist. Zumindest für eine infinitesimal kleine Zeiteinheit müssen die ermittelten Preise am Kapitalmarkt stabil sein, ansonsten ist eine soge-

I Vgl. stellvertretend Nietert/Wilhelm, Arbitrage, 1998, S. 5. 
nannte marktorientierte Bewertung nicht möglich. Folglich gehen alle marktorientierten Bewertungstheorien von einem arbitragefreien Kapitalmarkt aus.

Formal setzt die Arbitragefreiheit voraus, daß zwischen dem Preis $P_{M, i}$ einer gehandelten Vermögensposition und dem zukünftig aufgrund dieser Vermögensposition zu erzielenden Rückfluß $C F_{i}$ ein linearer Zusammenhang besteht ${ }^{1}$. Für jedes einzelne auf dem Kapitalmarkt gehandelte Wertpapier $i$ existiert ein Preisfunktional, das im Fall endlicher Umweltzustände $s$ durch folgende Form gekennzeichnet ist.

$$
P_{M, i}=\sum_{s=1}^{S} \pi_{s} \cdot C F_{i, s} \text { für alle } i
$$

Die abgebildeten Diskontfaktoren $\pi_{s}$ entsprechen den Preisen von reinen Wertpapieren, wie sie von Arrow bzw. Debreu eingeführt wurden ${ }^{2}$. Der zustandsabhängige Preis eines reinen Wertpapiers stellt jenen Preis dar, der für ein Wertpapier zu zahlen ist, falls dieses in dem betreffenden Umweltzustand einen Rückfluß von einer Geldeinheit und in allen anderen Umweltzuständen keinen Rückfluß verspricht. Er ist einzig durch den Umweltzustand bedingt und hängt nicht von der Höhe des in diesem Umweltzustand zu erzielenden Rückflusses, der generell beurteilten Vermögensposition oder dessen Risikogehalt ab.

Die Prämisse eines arbitragefreien Kapitalmarkts sollte im Rahmen einer Realitätsüberprüfung nicht zu eng ausgelegt werden. Liegt z.B. ein kompetitiver Kapitalmarkt vor, auf dem sich die einzelnen Marktteilnehmer als Mengenanpasser verhalten ${ }^{3}$, ist davon auszugehen, daß sich risikolose Arbitragegewinne nur zufällig ergeben, in kürzester Zeit ausgenutzt werden ${ }^{4}$ und folglich ein nahezu arbitragefreier Kapitalmarkt existiert.

1 Vgl. zu folgendem Varian, Arbitrage, 1987, S. 59 f.; Nietert/Wilhelm, Arbitrage, 1998, S. 6 f.; Kruschwitz, Finanzierung und Investition, 1999, S. 147 f.

2 Vgl. allgemein Debreu, Value, 1959; Arrow, Optimal Allocation, 1964.

3 Zu einem kompetitiven Kapitalmarkt vgl. Kruschwitz, Finanzierung und Investition, 1999, S. $140 \mathrm{f}$.

4 Zur Ausnutzung risikoloser Arbitragegewinne unter realen Gegebenheiten vgl. kritisch Schleifer/Vishny, Limits of Arbitrage, 1997, S. $36 \mathrm{ff}$. 


\subsection{Vollständigkeit}

Die Existenz eines arbitragefreien Kapitalmarkts führt nicht zwangsläufig dazu, $\mathrm{da} ß$ eine zu beurteilende Vermögensposition gemäß des im vorigen Abschnitts aufgezeigten Preisfunktionals bewertet werden kann. Hierzu müssen die Preise der reinen Wertpapiere nicht nur existieren, sondern zudem eindeutig festgelegt $\operatorname{sein}^{1}$. Werden z.B. weniger linear unabhängige Wertpapiere $i$ gehandelt als zukünftige denkbare Umweltzustände $s$, wird von einem unvollständigen Kapitalmarkt gesprochen. Obwohl die zustandsabhängigen Rückflüsse aller gehandelten Vermögenspositionen sowie deren aktuelle Preise vorliegen, kann nicht auf ein eindeutiges Preissystem geschlossen werden, da weniger Gleichungen $i$ als Unbekannte $s$ vorliegen $(i<s)$. Im Gegenzug sind die Preise der reinen Wertpapiere eindeutig bestimmt, falls die Anzahl der zukünftigen Umweltzustände mit der Menge gehandelter linear unabhängiger Wertpapiere übereinstimmt $(i=s)$. Es liegt dann ein vollständiger Kapitalmarkt vor ${ }^{2}$.

In dem in dieser Arbeit schwerpunktmäßig verwendeten zeitdiskreten Binomialmodell wird implizit von einem vollständigen Kapitalmarkt ausgegangen. Die Arrow/Debreu-Preise sind in dem Zwei-Zeitpunkt-Zwei-Zustands-Modell durch die Existenz des Bezugsguts bzw. der zum Bezugsgut perfekt korrelierten und gehandelten Vermögensposition, des risikolosen Zinsfußes sowie dessen Marktpreisen eindeutig festgelegt. Zur Illustration sei auf das in Kapitel IV in Abschnitt 2.1.2 verwendete Einführungsbeispiel zurückgegriffen. Über die angenommene Wertentwicklung des risikobehafteten Bezugsguts sowie der risikolosen Vermögensposition lassen sich die Preise der reinen Wertpapiere errechnen.

$$
\begin{aligned}
& \pi_{1} \cdot 200+\pi_{2} \cdot 50=100 \\
& \pi_{1} \cdot 1,05+\pi_{2} \cdot 1,05=1
\end{aligned} \quad \Rightarrow \pi_{1}=0,349, \pi_{2}=0,603
$$

Mit Hilfe dieser Arrow/Debreu-Preise läßt sich der Wert der Investitionsmöglichkeit $W_{0}$ bestimmen ${ }^{3}$. Bei einer Investitionsauszahlung von 90 Mio. $€$ be-

I Vgl. u.a. Zimmermann, State-Preference Theorie, 1998, S. 13.

2 Vgl. Varian, Arbitrage, 1987, S. 60; Kruschwitz, Finanzierung und Investition, 1999, S. $150 \mathrm{ff}$.

3 Vgl. z.B. Banz/Miller, State-Contingent Claims, 1978, S. 653 ff. bzw. ähnlich Rao/Martin, Real Asset Investment Opportunities, 1981, S. 425 ff. 
läuft er sich auf 38,4 Mio. $€{ }^{1}$. Dieser Wert entspricht dem bereits in Kapitel IV ermittelten Ergebnis. Dies kann nicht überraschen, wenn man bedenkt, daß die im Rahmen der risikoneutralisierten Bewertungsmethode verwendeten Pseudowahrscheinlichkeiten $p_{s}$ und Arrow/Debreu-Preise $\pi_{s}$ die gleichen Informationen verarbeiten ${ }^{2}$. Werden diese beiden direkt in Beziehung gesetzt, wird die Analogie zwischen der eben durchgeführten Bewertung und derjenigen aus Kapitel IV und V besonders offensichtlich.

$$
\pi_{s}=\frac{p_{s}}{\left(1+r_{f}\right)}
$$

Wie bereits in Abschnitt 2.1.1.3 in Kapitel IV erwähnt, sind in den Pseudowahrscheinlichkeiten sowohl die für die einzelnen Umweltzustände angenommenen Eintrittswahrscheinlichkeiten $q_{s}$ als auch die Risikopräferenz des repräsentativen Marktteilnehmers bzw. des Marktes $m_{s}$ verarbeitet. Somit läßt sich der Arrow/Debreu-Preis auch wie folgt schreiben ${ }^{3}$.

$$
\pi_{s}=\frac{q_{s} \cdot m_{s}}{1+r_{f}}
$$

Durch die Einführung des inhaltlich an dieser Stelle nicht näher bestimmten zustandsabhängigen Risikoparameters ${ }^{4} m_{s}$ läßt sich abermals der Grundgedanke der in dieser Arbeit verfolgten risikoneutralisierten Bewertungsmethode verdeutlichen. Die Kovarianz zwischen diesem Risikoparameter und der zu beurteilenden unsicheren Zahlung führt zu einem Risikoabschlag beziehungsweise -zuschlag, mit dessen Hilfe ein Sicherheitsäquivalent gebildet werden

$$
W_{0}=0,349 \cdot 110+0,603 \cdot 0=38,4 .
$$

2 Die Pseudowahrscheinlichkeiten $p_{l}$ und $p_{2}$ sind mit Hilfe des risikolosen Zinsfußes $r_{f}$ sowie dem Steigungs- $u$ und dem Senkungsfaktor $d$ (angenommene Wertentwicklung des Bezugsguts) wie folgt bestimmt worden.

$$
p_{1}=\frac{\left(1+r_{f}\right)-d}{u-d} \text { und } p_{2}=1-p_{1}
$$

3 Vgl. zu ăhnlichen Darstellungen Copeland/Weston, Theory and Policy, 1988, S. 116; Franke/Hax, Finanzwirtschaft, 1994, S. 342; Nippel, Sichtweisen der Marktbewertung, 1996, S. 110; Nietert/Wilhelm, Arbitrage, 1998, S. $10 \mathrm{ff}$.

$4 \mathrm{Zu}$ dessen Bestimmung vgl. die Ausführungen im nächsten Abschnitt. 
kann. Demnach ist der Preis eines unsicheren Rückflusses $C F$ wie folgt definiert ${ }^{1}$.

$$
P_{M}=\frac{E(C F)+\operatorname{Cov}(C F, m)}{1+r_{f}}
$$

Da es sich bei der Kovarianz zwischen der zu bewertenden Zahlung und dem Risikofaktor um einen vom Umweltzustand unabhängigen Term handelt, läßt sich dieser in den Erwartungswert integrieren.

$$
P_{M}=\frac{E[C F+\operatorname{Cov}(C F, m)]}{1+r_{f}}=\frac{E^{*}(C F)}{1+r_{f}}
$$

Innerhalb der risikoneutralisierten Bewertungsmethode wird implizit von einer Zufallsvariablen $C F$ zu einer Zufallsvariablen $C F+\operatorname{Cov}(C F, m)$ übergegangen. Für eine Investitionsmöglichkeit und einen risikoaversen Marktteilnehmer bedeutet dies, daß die unsichere Zahlung in jedem Umweltzustand um einen Risikoabschlag gekürzt wird ${ }^{2}$ oder anders ausgedrückt: Die Wahrscheinlichkeitsverteilung wird um einen konstanten Risikoabschlag nach links verschoben (vgl. Abb. 44) ${ }^{3}$.

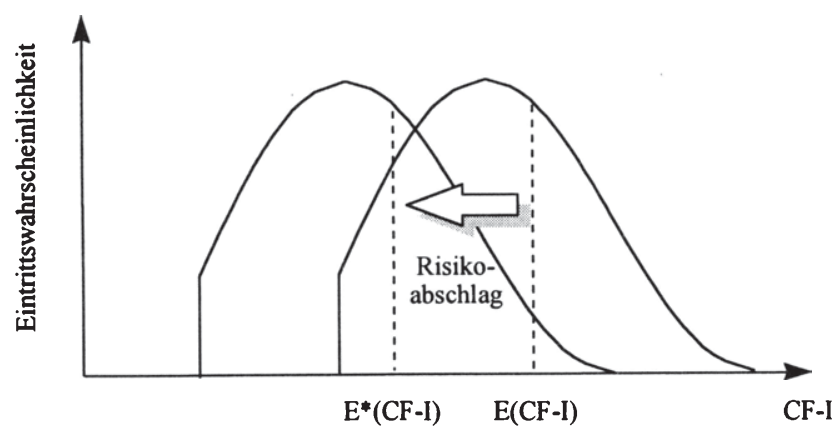

Abb. 44: Verschiebung der Wahrscheinlichkeitsverteilung einer Investitionsmöglichkeit innerhalb der risikoneutralisierten Bewertungsmethode

1 Vgl. zu folgendem Wilhelm, Arbitragefreiheit, 1981, S. 904; Nietert/Wilhelm, Arbitrage, 1998, S. 11.

2 In diesem Fall gilt: $\operatorname{Cov}(C F, m)<0$.

3 Vgl. ăhnlich bereits Harrison/Kreps, Martingales and Arbitrage, 1979, S. 383. 
Die neue Zufallsvariable weist die gleiche Standardabweichung auf wie die ursprüngliche Zufallsvariable. Lediglich der Erwartungswert der Zufallsvariablen hat sich in der Höhe geändert ${ }^{1}$. Der neue Erwartungswert $E^{*}$ berücksichtigt bereits das Marktrisiko und kann daher mit dem risikolosen Zinsfuß diskontiert werden, ohne daß eine solche Bewertung die tatsächlichen Risikopräferenzen der Marktteilnehmer unbeachtet läßt ${ }^{2}$. Dies ist auch der Grund, wieso von einem risikoneutralisierten Erwartungswert gesprochen wird.

Die in dieser Arbeit ausführlich dargestellte Bewertung von Investitionsmöglichkeiten kommt ohne die explizite Festlegung der eben aufgezeigten Marktbewertungsfaktoren (Arrow/Debreu-Preise $\pi_{s}$ bzw. zustandsabhängige Risikofaktoren $m_{s}$ ) aus. Es wird lediglich der risikolose Zinsfuß, der aktuelle Preis und die zukünftige Wertentwicklung des Bezugsguts vorausgesetzt, um bei einer bestimmten vorgegebenen Investitionsauszahlung den Preis (bzw. Wert) der Investitionsmöglichkeit im Verhältnis zu den beiden bestehenden Preisen zu bestimmen. Diese relative Bewertung, die keine Aussage über die „Richtigkeit“ zur Höhe des Barwerts des Bezugsguts sowie des risikolosen Zinsfußes trifft, geht durch die Akzeptanz der zuletzt genannten jedoch implizit von bestimmten Marktbewertungsfaktoren aus. Über die Preise der am Kapitalmarkt gehandelten Vermögenspositionen lassen sich auf einem arbitragfreien und vollständigen Kapitalmarkt stets Marktbewertungsfaktoren errechnen ${ }^{3}$.

In den zeitstetigen Realoptionsbewertungsmodellen ist i.d.R. eine unendliche Anzahl an zukünftigen Umweltzuständen unterstellt. Der Kapitalmarkt kann folglich nicht vollständig im eben erläuterten Sinn sein, da nicht anzunehmen ist, daß unendlich viele Wertpapiere gehandelt werden. Dies scheint auf den ersten Blick dazu zu führen, daß der Wert der Investitionsmöglichkeit nicht ermittelbar ist. Durch die in diesen Modellen ebenfalls angenommenen kontinuierlichen Handelsmöglichkeiten im Hinblick auf das Bezugsgut und die risikolose Vermögensposition ist es jedoch möglich, sich zu jedem erdenklichen Zeitpunkt gegen eine zukünftige Schwankung des Bezugsguts abzusichern. Dies wiederum führt dazu, daß der Kapitalmarkt für jede infinitesimal kleine

1 Die Eintrittswahrscheinlichkeit des neuen Erwartungswerts stimmt mit der Eintrittswahrscheinlichkeit des ursprünglichen Erwartungswerts überein.

$2 \mathrm{Zu}$ den theoretischen Grundlagen einer solchen Vorgehensweise vgl. Harrison/Kreps, Martingales and Arbitrage, 1979, S. $381 \mathrm{ff}$.

3 Vgl. ăhnlich Nippel, Stellungnahme zu Eble und Völker, 1994, S. 150 f.; Nietert/Wilhelm, Arbitrage, 1998, S. 13 und S. 16 f. 
Zeiteinheit vollständig ist ${ }^{1}$. Ein eindeutiger Wert der Investitionsmöglichkeit ist folglich ermittelbar, wenn von Marktteilnehmern ausgegangen wird, die ihr Hedge- oder Arbitrageportfolio nach jeder infinitesimal kleinen Wertänderung des Bezugsguts umschichten ${ }^{2}$.

\subsection{Handelbarkeit des Bezugsguts}

Im Rahmen der finanzwirtschaftlichen Optionspreistheorie wird von einem am Kapitalmarkt gehandelten Bezugsgut ausgegangen. Der aktuelle Preis dieses Bezugsguts enthält dabei sämtliche Informationen über unterstellte Eintrittswahrscheinlichkeiten der zukünftigen Umweltzustände sowie Risikopräferenzen der Marktteilnehmer, so daß die Aufgabe der Bewertung des Bezugsguts dem Kapitalmarkt überlassen wird. Unter Arbitragefreiheitsüberlegungen wird lediglich auf den theoretischen Wert der Option geschlossen.

Die Übertragung der finanzwirtschaftlichen Optionspreistheorie auf reale Investitionsprojekte wird durch die Tatsache erschwert, daß für die Mehrzahl der Bewertungssituationen das Bezugsgut nicht gehandelt wird ${ }^{3}$. Für diese Fälle muß ein gehandelter Werttitel bzw. ein Portfolio existieren, dessen unsichere

1 Die Vollständigkeit des Kapitalmarkts wird durch diejenige der Zeit ersetzt. Vgl. Nippel, Strategische Investitionsplanung, 1997, S. 19. Zur Realoptionsbewertung auf einem unvollständigen Kapitalmarkt vgl. Smith/Nau, Valuing Risky Projects, 1995, S. 804 ff.; Hoff, Investment, 1997, S. $14 \mathrm{ff}$.

2 Es sei daran erinnert, daß die Ergebnisse des zeitstetigen Black/Scholes-Modells auch bei zeitdiskreten Portfolioanpassungen gelten, wenn eine konstante relative Risikoaversion der Marktteilnehmer unterstellt wird. Vgl. Abschnitt 4 in Kapitel IV sowie die dort zitierte Literatur.

3 Wird fur das nicht gehandelte Bezugsgut eine Rendite erwartet, die unter der Gleichgewichtsrendite $\mu$ des Vergleichstitels liegt - z.B. aufgrund einer geringeren Liquididät - , kann der Vorschlag von McDonald und Siegel aufgegriffen werden. Der in die Optionspreisformel eingehende Wert des Vergleichstitels $V^{*}$ wird um die beschriebene Differenz $l$ verringert. Vgl. McDonald/Siegel, Below-Equilibrium Rate of Return, 1984, S. $262 \mathrm{ff}$.

$$
V=V^{*} \cdot e^{-1 \cdot t}
$$

Konzeptionell entspricht diese Korrektur derjenigen einer Dividendenrate. Vgl. Abschnitt 3.3 in Kapitel IV. 
Renditen mit den angenommenen unsicheren Renditen des Bezugsguts perfekt korreliert sind ${ }^{1}$.

Ist der Preis des gehandelten Vergleichspapiers direkt beobachtbar, kann dieser zur Bewertung der Investitionsmöglichkeit herangezogen werden. Im Preis sind dann bereits Eintrittswahrscheinlichkeiten und Risikopräferenzen der Marktteilnehmer verarbeitet. Ist hingegen der Preis des gehandelten Vergleichspapiers nicht direkt am Markt ablesbar, muß zuerst die absolute Bewertung des Bezugsguts durchgeführt werden, bevor sich in einem zweiten Schritt die relative Bewertung der Investitionsmöglichkeit anschließen läßt $\mathrm{t}^{2}$. Die Bewertung des Bezugsguts kann dabei mit Hilfe traditioneller Bewertungsmethoden angegangen werden, die auf die absolute Bewertung einer unsicheren Vermögensposition ausgerichtet sind.

Wird die absolute Bewertung des Bezugsguts z.B. auf Basis des Time State Preference Model durchgeführt ${ }^{3}$, wird ein repräsentativer Marktteilnehmer unterstellt, der seine Investitions-/Konsumentscheidung vor dem Hintergrund der Maximierung seines Erwartungsnutzens trifft. Hieraus resultiert ein zustandsabhängiger Risikofaktor $m_{s}$, der als Verhältnis zwischen dem Grenznutzen $U^{\prime}$ (.) der Zahlung in dem betreffenden Umweltzustand und dem über alle Umweltzustände erwarteten Grenznutzen $E\left[U^{\prime}().\right]$ beschrieben werden kann ${ }^{4}$.

$$
m_{s}=\frac{U^{\prime}\left(C F_{s}\right)}{E\left[U^{\prime}(C F)\right]}
$$

1 Vgl. die Ausfuhrungen in Abschnitt 2.1.2.2 in Kapitel IV. Für den Fall, daß die Renditen des Vergleichstitels und diejenigen des Bezugsguts nicht perfekt korrelieren vgl. Merton, Applications, 1998, S. $328 \mathrm{ff}$.

2 Zur relativen und absoluten Bewertung von Vermögenspositionen vgl. insbesondere Schöbel, Bewertung, 1995, S. 42 f. und S. 109 ff., der zur Unterscheidung die Begriffe ,arbitrageorientierte" und ,gleichgewichtsorientierte" Bewertung verwendet, sowie Nietert/Wilhelm, Arbitrage, 1998, S. 20 i.V.m. S. 23.

3 Vgl. allgemein zum TSPM Copeland/Weston, Theory and Policy, 1988, S. 109 ff.; Wosnitza, State Preference Ansatz, 1995, S. 593 ff.; Kruschwitz, Finanzierung und Investition, 1999, S. $209 \mathrm{ff}$.

4 Zur Ableitung sowie zur Unterscheidung in ein Individualkalkül und eine Gleichgewichtsbetrachtung vgl. Kruschwitz, Finanzierung und Investition, 1999, S. 211 ff. Für eine Anwendung im Rahmen der Optionsbewertung vgl. Nietert/Wilhelm, Arbitrage, 1998, S. 21 f. 
Auf Basis solcher zustandsabhängigen Risikofaktoren, dem risikolosen Zinsfuß und den individuell angenommenen Eintrittswahrscheinlichkeiten ließen sich anschließend Arrow/Debreu-Preise bilden, mit denen der theoretische Wert des Bezugsguts bestimmt werden könnte. Auf einem in Abschnitt 2.1 und 2.2 dieses Kapitels unterstellten arbitragefreien und vollständigen Kapitalmarkt sind sich alle Marktteilnehmer darüber einig, welche Umweltzustände in der $\mathrm{Zu}$ kunft eintreten können und welche Zahlungen in den betreffenden Umweltzuständen resultieren. Dies führt dazu, daß die Preise der reinen Wertpapiere für alle Marktteilnehmer identisch sind. Für ihre konkrete Bestimmung ist es folglich unter diesen Bedingungen sowohl möglich, sie aus dem aktuellen Preissystem gehandelter Wertpapiere „heraus" zu rechnen als auch auf die angenommenen Eintrittswahrscheinlichkeiten, den risikolosen Zinsfuß und die individuell aufgestellte Nutzenfunktion zu vertrauen ${ }^{1}$.

Wird hingegen die absolute Bewertung des Bezugsguts z.B. auf Basis des Capital Asset Pricing Model durchgeführt, läßt sich der Risikofaktor $m_{s}$ vollständig durch Gesamtmarktdaten ausdrücken. In der folgenden Gleichung zur Ermittlung des zustandsabhängigen Risikofaktors stellt $\lambda_{M}$ die Marktrisikoprämie, $E\left(r_{M}\right)$ die erwartete Marktrendite und $r_{M, s}$ die Marktrendite in dem betreffenden Umweltzustand $s$ dar $^{2}$.

$$
m_{s}=1+\lambda_{M} \cdot\left[E\left(r_{M}\right)-r_{M, s}\right]
$$

Für eine Bewertung auf Basis des CAPM werden im Vergleich zur Bewertung im TSPM jedoch zwei weitere Annahmen benötigt ${ }^{3}$. Die Marktteilnehmer haben homogene Erwartungen über die Eintrittswahrscheinlichkeiten der Umweltzustände und beurteilen Investitionen gemäß dem $\mu$ - $\sigma$-Prinzip. Durch letzteres ist die unterstellte Nutzenfunktion im Vergleich zum TSPM näher spezifiziert. Im Gegenzug bedarf es im CAPM nicht notwendigerweise eines vollständigen Kapitalmarkts ${ }^{4}$, da es sich um ein Gleichgewichtsmodell handelt,

Vgl. ähnlich Kruschwitz, Finanzierung und Investition, 1999, S. 212 und S. 216.

2 Zur Ableitung vgl. Nippel, Sichtweisen der Marktbewertung, 1996, S. 108 f.; Nietert/Willhelm, Arbitrage, 1998, S. 23 f.; Kruschwitz, Finanzierung und Investition, 1999, S. 256 f. Das Einsetzen dieses Risikofaktors in die im vorigen Abschnitt aufgezeigte Formel zur Bestimmung des Preises einer unsicheren Zahlung führt nach wenigen Umformungen zur „sicherheitsäquivalenten" Bewertungsformel des CAPM. Zu letzterer vgl. Abschnitt 2.1.2.1 in Kapitel III.

3 Vgl. Wosnitza, Praxisrelevanz, 1995, S. 701; Kruschwitz, Finanzierung und Investition, 1999, S. 161 f. i.V.m. S. 210.

4 Vgl. Nippel, Sichtweisen der Marktbewertung, 1996, S. 110. 
in dem die Marktteilnehmer aufgrund der unterstellten Prämissen zwangsläufig zum gleichen (Preis-) Ergebnis kommen.

Zur Bewertung des Bezugsguts werden sowohl im TSPM als auch im CAPM tatsächlich erwartete Eintrittswahrscheinlichkeiten und Risikopräferenzen explizit festgelegt, was auf den ersten Blick als Rückschritt der - in dieser Arbeit propagierten - zweistufigen Vorgehensweise zur Bewertung von Investitionsmöglichkeiten erscheinen mag ${ }^{1}$. Eine „direkte“ (absolute) Bewertung erfordert jedoch die gleichen Informationen, so daß dies zumindest kein Nachteil gegenüber bestehenden Verfahren darstellt. Worin aber besteht der Vorteil einer relativen Bewertung von Investitionsmöglichkeiten?

Durch die relative Bewertung braucht das in der Investitionsmöglichkeit vorhandene Risiko nicht explizit quantifiziert werden. Über das im Basisprojekt quantifizierte Risiko wird mit Hilfe von Arbitragefreiheitsüberlegungen quasi direkt auf den Wert der Investitionsmöglichkeit geschlossen, indem die Preise des Bezugsguts und der Investitionsmöglichkeit zueinander überprüft werden. Der „Umweg“ über die Beziehung der Investitionsmöglichkeit zu anderen im Markt gehandelten Vermögenspositionen entfällt.

Durch diese Eigenschaft besitzt das Realoptionskonzept gegenüber konkurrierenden Bewertungsansätzen erhebliche Vorteile im Rahmen der Implementierung. Durch die Abhängigkeit der Investitionsmöglichkeit vom Bezugsgut und dessen Abhängigkeit von der allgemeinen Umweltentwicklung unterliegt die Investitionsmöglichkeit zwei Unsicherheitsquellen. Wird angenommen, daß sich das für das Bezugsgut relevante Risiko im Zeitablauf nicht gravierend ändert, läßt sich bei der absoluten Bewertung des Bezugsguts von einem im Zeitablauf konstanten risikoadjustierten Zinsfuß ausgehen ${ }^{2}$. Ein solcher ist für die Investitionsmöglichkeit nicht mehr gegeben, da letztere neben der Unsicherheit über die allgemeine Umweltentwicklung zusätzlich vom spezifischen

1 Vgl. hierzu auch Fischer/Hahnenstein/Heitzer, Unternehmensbewertung, 1999, S. 1226, die die zweistufige Vorgehensweise gar als ,finanzierungstheoretisch ... inkonsistent bzw. unnötig" bezeichnen, da die Bewertung der Investitionsmöglichkeit grundsätzlich sowohl auf Basis des TSPM, des CAPM bzw. der OPT möglich sei und auf einem vollkommenen und vollständigen Kapitalmarkt auch zum gleichen Ergebnis fuhre. Sie leugnen jedoch nicht das „heuristische Potential“ eines solchen Verfahrens. Zu letzterem vgl. insbesondere Breuer/Gürtler/Schumacher, Realoptionen, 1999, S. 214, die der zweistufigen Vorgehensweise „eine eigenståndige Bedeutung für die Lösung praktischer Entscheidungsprobleme“ zusprechen.

2 Vgl. Abschnitt 2.2.2 in Kapitel III. 
Risiko des Bezugsguts abhängt. Jede Veränderung des Bezugsguts modifiziert das Risiko der Investitionsmöglichkeit, so daß sich dieses und damit auch der adäquate risikoadjustierte Zinsfuß kontinuierlich verändert ${ }^{1}$. Zur absoluten Bewertung der Investitionsmöglichkeit müßten konsequenterweise für jeden Umweltzustand und jeden Zeitpunkt der geeignete Zinsfuß ermittelt bzw. dynamische Programmierungsverfahren eingesetzt werden ${ }^{2}$.

\subsection{Zusammenfassung der Diskussion}

Ein marktorientierter Vergleich zwischen Vermögenspositionen setzt die in allen Bewertungsmodellen der modernen Investitions- und Finanzierungstheorie implizit oder explizit unterstellte Prämisse eines arbitragefreien Kapitalmarkts voraus. Sollen zusätzlich eindeutige Werte für die zu beurteilenden Vermögenspositionen ermittelt werden, muß der Kapitalmarkt zudem vollständig sein oder die Bedingungen des CAPM-Gleichgewichts erfüllen. Für die in dieser Arbeit vorgestellte relative Bewertung von Investitionsmöglichkeiten brauchen die allgemeinen Marktbewertungsfaktoren (z.B. Arrow/Debreu-Preise bzw. CAPM-Risikofaktoren) nicht explizit vorliegen, falls das Bezugsgut der Investitionsmöglichkeit am Kapitalmarkt gehandelt wird bzw. ein hierzu perfekt korrelierter Vermögenstitel beobachtbar ist.

Ist dies hingegen nicht der Fall, muß zuerst das Bezugsgut einer absoluten Bewertung unterzogen werden, bevor auf diesem Ergebnis aufbauend der Wert der Investitionsmöglichkeit ermittelt werden kann. Eine solche Vorgehensweise benötigt keine Informationen, die nicht auch bei traditionellen investitionstheoretischen Bewertungsverfahren vorausgesetzt werden. Zudem bietet sie den Vorteil, die sonst notwendige Bestimmung von zustands- und zeitabhängigen Zinsfüßen bzw. Anwendung von dynamischen Programmierungsverfahren zu umgehen. Gegenüber diesen Verfahren stellt das Realoptionskonzept für die im Rahmen dieser Arbeit aufgeworfene Problemstellung eine Komplexitätsreduktion dar.

Marktorientierte Bewertung setzt immer ein perfekt korreliertes Vergleichsgut voraus. Dies gilt unabhängig davon, ob die zum Bezugsgut perfekt korrelierte Vermögensposition explizit am Markt beobachtet werden kann oder ob jene im

1 Eine ăhnliche Argumentation läßt sich auch für Sicherheitsäquivalente führen.

2 Vgl. Pindyck, Irreversibility and Investment, 1988, S. 1122; Laux, Handlungsspielräume, 1993, S. 940. Zu dynamischen Programmierungsverfahren vgl. allgemein Dixit/Pindyck, Investment Under Uncertainty, 1994, S. 95 ff.; Sick, Real Options, 1995, S. 669 ff. 
Rahmen der absoluten Bewertung mit Hilfe von traditionellen Bewertungsverfahren implizit unterstellt wird ${ }^{1}$. Wenn ein solcher Vergleichstitel tatsächlich nicht existiert, ist grundsätzlich kein Bewertungsansatz für eine marktorientierte Wertermittlung geeignet ${ }^{2}$. Das nicht gehandelte Bezugsgut erweitert dann die Menge der in einem Markt vorhandenen (linear unabhängigen) Investitionsalternativen, wodurch die implizit oder explizit angenommenen Marktbewertungsfaktoren nicht mehr gelten ${ }^{3}$.

Stellt sich zum Schluß die Frage, welchen Informationsgehalt der optionspreistheoretisch ermittelte Wert einer Investitionsmöglichkeit besitzt, wenn das Bezugsgut auf Basis einer nicht marktorientierten Vorgehensweise - wie z.B. bei individuellen entscheidungstheoretischen Bewertungskalkülen - beurteilt wird. Zur Beantwortung kann auf die relative Gültigkeit eines Optionswerts zurückgegriffen werden. Das optionspreistheoretische Ergebnis stellt jenen theoretischen Wert dar, der sich auf einem arbitragefreien Kapitalmarkt einstellt, wenn die überwiegende Anzahl der Marktteilnehmer den aktuellen Preis des Bezugsguts sowie die in ihm enthaltenen Informationen akzeptiert ${ }^{4}$. Folglich ist der aus der Optionspreistheorie resultierende Wert der Investitionsmöglichkeit für den individuellen Bewerter relevant, wenn er glaubt, daß das Bezugsgut heute zu demjenigen Preis gehandelt würde, den er im Rahmen seines Individualkalküls ermittelt hat ${ }^{5}$.

1 Modellansätze zur absoluten Bewertung ermitteln den Preis eines unsicheren Zahlungsstroms, den dieser unter der Voraussetzung der Handelbarkeit besäße.

2 Bewerten heißt immer auch vergleichen. Eine marktorientierte Bewertung setzt damit zwingend die Existenz des Vergleichsmaßstabs am Kapitalmarkt voraus. Vgl. auch Ritchken/Rabinowitz, Contingent Claims Analysis, 1988, S. 142; Laux, Handlungsspielräume, 1993, S. 940; Copeland/Koller/Murrin, Valuation, 1994, S. 452; Sick, Real Options, 1995, S. 642; Kilka, Realoptionen, 1995, S. 77 f.; Mostowfi, Flexibilität, 1997, S. 590. Nach Breuer/Gürtler/Schuhmacher, Realoptionen, 1999, S. 214, besteht die Kunst jeder denkbaren Bewertung ,letztendlich darin, das als relevant zu erachtende Preissystem in möglichst sachgerechter Weise zu ermitteln".

3 Die Bewertung der Investitionsmöglichkeit ist dann nur noch über die explizite Formulierung einer entscheidungstheoretischen Nutzenfunktion möglich. Vgl. hierzu auch Pindyck, Irreversibility and Investment, 1991, S. 1122.

4 Vgl. ähnlich Varian, Arbitrage, 1987, S. 69.

$5 \mathrm{Zu}$ einer hierüber hinausgehenden, modellmäßigen Verknüpfung von entscheidungs- mit optionspreistheoretischen Erkenntnissen für den Fall des partiell vollständigen Kapitalmarkts vgl. Smith/Nau, Valuing Risky Projects, 1995, S. 806 ff.; Smith/McCardle, Integrating, 1998, S. $198 \mathrm{ff}$. 


\section{Verbundene Realoptionen}

\subsection{Art und Richtung der gegenseitigen Wertbeeinflussung}

In den Beispielen der vorherigen Kapitel stellt die strategische Akquisition einen Jetzt oder Nie-Beschluß dar, bei dem von der Möglichkeit einer späteren Desinvestition abgesehen wird. Zudem wird der Wert der zusätzlichen Investitionsmöglichkeit unter den Prämissen ermittelt, daß die Akquisition tatsächlich realisiert und neben dem Aufschub der endgültigen Entscheidung über die zusätzliche Investition keine weiteren unternehmerischen Handlungsspielräume bestehen. Das in diesem Abschnitt zu erläuternde Interdependenzproblem bei verbundenen Realoptionen tritt dann nicht auf. Der Unternehmenswert ergibt sich durch Addition des Werts des Akquisitionsprojekts ohne zusätzliche Investitionsmöglichkeit und dem (Realoptions-) Wert der zusätzlichen Investitionsmöglichkeit.

Existieren hingegen in einer Entscheidungssituation mehrere unternehmerische Handlungsspielräume gleichzeitig, ist eine präzise Analyse der einzelnen Bezugsgüter durchzuführen ${ }^{1}$. Bei voneinander unabhängigen Bezugsgütern lassen sich die für die jeweiligen Realoptionen isoliert ermittelten Ergebnisse zu einem Gesamtwert dieser Realoptionen addieren. Anders verhält es sich hingegen, wenn das Bezugsgut einer Realoption vom Bezugsgut eines anderen unternehmerischen Handlungsspielraums abhängt bzw. mehrere Realoptionen auf dasselbe Bezugsgut zurückgreifen. Dies ist z.B. der Fall, wenn bei einem Investitionsprojekt einerseits die Möglichkeit besteht, den Start zu verschieben und andererseits dasselbe Projekt $\mathrm{zu}$ einem darauffolgenden Zeitpunkt erweitert werden $\mathrm{kann}^{2}$.

1 Interaktionen, die durch die gleichzeitige Existenz von Optionen im real- und finanzwirtschaftlichen Bereich entstehen, werden an dieser Stelle nicht betrachtet. Letztgenannte Optionen sind z.B. dann gegeben, wenn bei einem eigenständigen und zum Teil fremdfinanzierten Investitionsprojekt die Möglichkeit besteht, das Fremdkapital nicht zurückzuzahlen, falls dieses das Gesamtkapital ubersteigt. Vgl. hierzu Trigeorgis, Interactions with Financial Flexibility, 1993, S. 216 ff. Daneben analysieren Childs/Ott/Triantis, Interrelated Projects, 1998, S. 305 ff. in einem Realoptionsansatz die Interaktion zwischen zwei Investitionsprojekten, die sich entweder nacheinander oder parallel entwickeln lassen, von denen jedoch nur eines implementiert werden soll. Auch solche Interaktionen stehen im folgenden nicht im Vordergrund.

2 Das Bezugsgut der Aufschubsoption entspricht dem Wert der Rückflüsse ohne Erweiterung zzgl. dem Wert der Erweiterungsoption. Darüber hinaus existiert das Bezugsgut der Erweiterungsoption nur dann, falls zuvor investiert wird. 
In solchen Situationen sind allgemein folgende Abhängigkeiten zu beachten ${ }^{1}$. Einerseits verfügen vorhergehende Realoptionen über ein Bezugsgut, dessen Wert sich durch den Wert der Projektrückflüsse und den Wert der nachfolgenden Realoptionen zusammensetzt. Anderseits verändert sich der Wert des Bezugsguts von nachfolgenden Realoptionen durch eine (Nicht-) Inanspruchnahme vorheriger Realoptionen. Durch diese gegenseitige Wertbeeinflussung entspricht der (Grenz-) Wert der jeweiligen Realoption nicht dem isoliert ermittelten Ergebnis und der Gesamtwert der verbundenen Realoptionen nicht deren Summe. Die Bewertung muß simultan erfolgen².

Um die Richtung der gegenseitigen Wertbeeinflussung zu untersuchen, wird im folgenden zum einen in vorhergehende und nachfolgende Realoptionen sowie zum anderen in verschiedene $\mathrm{Arten}^{3}$ unterschieden. Zuerst werden die Wertveränderungen bei vorhergehenden Realoptionen betrachtet. Bei Existenz einer nachfolgenden Realoption steigt der Wert des Bezugsguts einer vorhergehenden Realoption. Dadurch erhöht sich der Wert einer vorhergehenden Aufschubsoption, da im Vergleich zum isolierten Fall eine Inanspruchnahme wahrscheinlicher wird. Das gleiche gilt für eine vorhergehende Erweiterungsoption. Demgegenüber sinkt der Wert einer vorhergehenden Einschränkungs- oder Aufgabeoption, weil durch die Erhöhung des Werts des Bezugsguts die Realoption nun vergleichsweise in weniger Fällen in Anspruch genommen wird (vgl. Tab. 6). Wird die Aufschubsoption - wie hier angenommen - auf den erstmaligen Start eines Investitionsprojekts bezogen, kommen Situationen mit nachfolgenden Aufschubsoptionen nicht vor.

\begin{tabular}{|l|c|c|c|}
\hline $\begin{array}{l}\text { Wertveränderung von } \\
\text { vorhergehender Realoption }\end{array}$ & $\begin{array}{c}\text { Erweiterungs- } \\
\text { option }\end{array}$ & $\begin{array}{c}\text { Einschränkungs- } \\
\text { option }\end{array}$ & $\begin{array}{c}\text { Aufgabe- } \\
\text { option }\end{array}$ \\
\hline Aufschubsoption & $\uparrow$ & $\uparrow$ & $\uparrow$ \\
\hline Erweiterungsoption & $\uparrow$ & $\uparrow$ & $\uparrow$ \\
\hline Einschränkungsoption & $\downarrow$ & $\downarrow$ & $\downarrow$ \\
\hline Aufgabeoption & $\downarrow$ & $\downarrow$ & $\downarrow$ \\
\hline
\end{tabular}

Tab. 6: Richtung der Wertbeeinflussung von nachfolgenden Realoptionen auf den Wert von vorhergehenden Realoptionen mit demselben Bezugsgut

1 Vgl. Trigeorgis, Complex Options, 1996, S. 322.

2 Vgl. auch Dixit, Entry and Exit Decisions, 1989, S. 621.

3 Dazu werden explizit die Aufschubs-, Erweiterungs-, Einschränkungs- sowie Aufgabeoption betrachtet. 
Bei nachfolgenden Realoptionen ergeben sich folgende Wertveränderungen. Durch eine vorhergehende Aufschubsoption sinkt der Wert einer nachfolgenden Realoption, da im Extremfall des Nichtinvestierens das Bezugsgut für die nachfolgende Realoption vernichtet wird. Das gleiche Ergebnis gilt bei einer vorhergehenden Aufgabeoption, die bei Inanspruchnahme eine nachfolgende Realoption wertlos macht (vgl. Tab. 7). Eine vorhergehende Erweiterungsoption hingegen erhöht den Wert des Bezugsguts einer nachfolgenden Realoption, wodurch sich c.p. der Wert einer nachfolgenden Erweiterungsoption erhöht bzw. derjenige einer nachfolgenden Einschränkungs- oder Aufgabeoption sinkt. Umgekehrt verringert sich bei einer vorhergehenden Einschränkungsoption der Wert des Bezugsguts der nachfolgenden Realoption. Hierdurch sinkt c.p. der Wert einer nachfolgenden Erweiterungsoption, während sich der Wert einer nachfolgenden Einschränkungs- oder Aufgabeoption erhöht.

\begin{tabular}{|l|c|c|c|c|}
\hline $\begin{array}{l}\text { Wertveränderung } \\
\text { von nachfolgender Realoption }\end{array}$ & $\begin{array}{c}\text { Rufschubs- } \\
\text { option }\end{array}$ & $\begin{array}{c}\text { Erweite- } \\
\text { rungsoption }\end{array}$ & $\begin{array}{c}\text { Einschrän- } \\
\text { kungsoption }\end{array}$ & $\begin{array}{c}\text { Aufgabe- } \\
\text { option }\end{array}$ \\
\hline Erweiterungsoption & $\downarrow^{*}$ & $\uparrow$ & $\downarrow$ & $\downarrow^{* * * *}$ \\
\hline Einschränkungsoption & $\downarrow^{*}$ & $\downarrow, \uparrow^{* *}$ & $\uparrow, \downarrow^{* * *}$ & $\downarrow^{* * * *}$ \\
\hline Aufgabeoption & $\downarrow^{*}$ & $\downarrow, \uparrow^{* *}$ & $\uparrow, \downarrow^{* * *}$ & $\downarrow^{* * * *}$ \\
\hline
\end{tabular}

- Wird die vorhergehende Aufschubsoption nicht ausgeübt, sind die nachfolgenden Realoptionen wertlos.

** Geht die Inanspruchnahme der vorhergehenden Erweiterungsoption mit einer proportionalen Erhöhung des Desinvestitionserlöses einher, steigt der Wert der nachfolgenden Einschränkungs- oder Aufgabeoption.

*** Geht die Inanspruchnahme der vorhergehenden Einschränkungsoption mit einer proportionalen Verringerung des Desinvestitionserlöses einher, sinkt der Wert der nachfolgenden Einschränkungs- oder Aufgabeoption.

**** Wird die vorhergehende Aufgabeoption ausgeübt, sind die nachfolgenden Realoptionen wertlos.

Tab. 7: Richtung der Wertbeeinflussung von vorhergehenden Realoptionen auf den Wert von nachfolgenden Realoptionen mit demselben Bezugsgut

Im Rahmen einer nachfolgenden Einschränkungs- oder Aufgabeoption gilt es zu beachten, daß die zuletzt getätigte Aussage nur gilt, falls die vorhergehende Einschränkungsoption den erzielbaren Desinvestitionserlös unberührt läßt. Geht die Inanspruchnahme der vorhergehenden Einschränkungsoption mit einer proportionalen Verringerung des Desinvestitionserlöses einher, ändert sich das zuvor festgestellte Ergebnis. Der Wert der nachfolgenden Einschränkungs- 
oder Aufgabeoption sinkt ${ }^{1}$. Ähnliches gilt für Fälle, in denen sich durch eine vorhergehende in Anspruch genommene Erweiterungsoption der Desinvestitionserlös proportional erhöht. Hier steigt nun der Wert der späten Einschränkungs- oder Aufgabeoption.

\subsection{Ausmaß der gegenseitigen Wertbeeinflussung}

Nachdem im vorherigen Abschnitt diskutiert wurde, ob ein vorhergehender (nachfolgender) unternehmerischer Handlungsspielraum den Wert einer nachfolgenden (vorhergehenden) Realoption erhöht bzw. senkt, stehen nun Aussagen über die Größenordnung dieser gegenseitigen Wertbeeinflussung im Vordergrund. Als genereller Einflußfaktor läßt sich hierfür die Wahrscheinlichkeit einer gemeinsamen Inanspruchnahme der verbundenen Realoptionen nennen ${ }^{2}$.

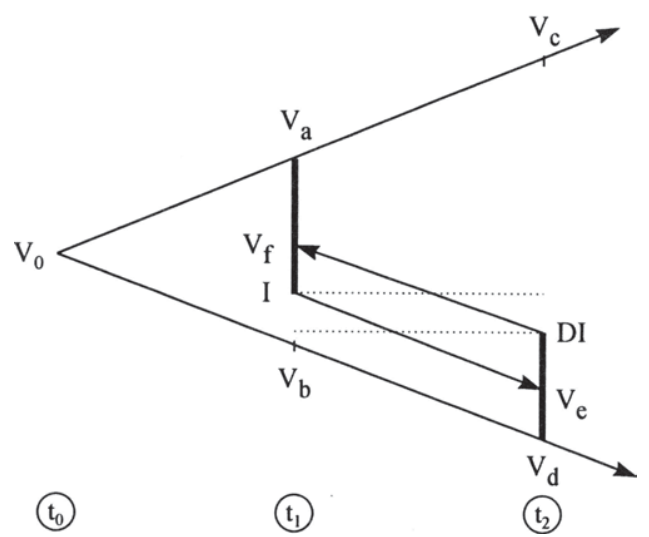

Abb. 45: Ausmaß der gegenseitigen Wertbeeinflussung zwischen Realoptionen mit demselben Bezugsgut

Quelle: In Anlehnung an Trigeorgis, Option Interactions, 1993, S. 6 f.

Abb. 45 verdeutlicht diesen Sachverhalt graphisch am Beispiel einer Erweiterungs- und einer Aufgabeoption. Bei einem heute gestarteten Investitionsprojekt besteht bis zum Zeitpunkt $t=1$ die Möglichkeit, das Projekt gegen eine zu

1 Vgl. Trigeorgis, Option Interactions, 1993, S. 8 f.

2 Zu diesem Ergebnis vgl. Trigeorgis, Option Interactions, 1993, S. 11. Diese Aussage gilt jedoch nicht für Extremfälle, in denen die Entscheidung über eine vorhergehende Realoption das Bezugsgut der nachfolgenden Realoption vollständig vernichten kann, wie z.B. bei einer vorhergehenden Aufschubs- oder Aufgabeoption. 
entrichtende Investitionsauszahlung in Höhe von $I$ zu vergrößern. Gleichzeitig wird angenommen, daß das Projekt in $t=2 \mathrm{zu}$ einem Desinvestitionserlös in Höhe von $D I$ (mit $D I<I)$ liquidiert werden kann ${ }^{1}$.

Der mögliche Verlauf des Bezugsguts $V$ wird durch die äußeren Begrenzungslinien gekennzeichnet. In $t=1$ liegt der Wert des Bezugsguts zwischen $V_{a}$ und $V_{b}$. Überschreitet der tatsächliche Wert des Bezugsguts in $t=1$ die zu entrichtende Investitionsauszahlung, wird das Projekt erweitert. Die Aufgabeoption hingegen wird wahrgenommen, falls in $t=2$ der Wert des Bezugsguts den Desinvestitionserlös unterschreitet, wobei ersterer aus Sicht von $t=0$ Werte zwischen $V_{c}$ und $V_{d}$ annimmt. Für eine gemeinsame Inanspruchnahme der beiden Realoptionen muß sich die Wertentwicklung des Bezugsguts in $t=1 \mathrm{zwi}$ schen $V_{a}$ und $I$ und in $t=2$ zwischen $V_{d}$ und $D I$ bewegen. Hierfür gibt es eine positive Wahrscheinlichkeit. Für den Fall, daß in $t=1$ investiert wird, kann das Bezugsgut in $t=2$ einen Wert zwischen $V_{c}$ und $V_{e}$ annehmen und überschneidet somit den Ausübungsbereich der Aufgabeoption. Eine Addition der für die Erweiterungs- und Aufgabeoption isoliert ermittelten Ergebnisse ist dann nicht gerechtfertigt.

Allgemein lassen sich folgende Tendenzaussagen ableiten ${ }^{2}$. Erstens ist die Addition der isoliert ermittelten Ergebnisse eher möglich, wenn es sich - wie in Abb. 45 dargestellt - um Realoptionen mit entgegengesetztem Optionscharakter handelt. Liegt in $t=2$ statt einer Aufgabe- z.B. eine Erweiterungsoption mit einer notwendigen Investitionsauszahlung in Höhe von $D I$ vor, fällt der gesamte Ausübungsbereich der nachfolgenden Realoption - und nicht wie zuvor nur ein Teil - in die nach Inanspruchnahme der vorhergehenden Realoption denkbare Wertentwicklung des Bezugsguts. Die Wahrscheinlichkeit für eine gemeinsame Inanspruchnahme steigt.

Zweitens ist die Addition der isoliert ermittelten Ergebnisse umso eher gegeben, je weniger die gegebenen Ausübungsbereiche übereinstimmen. Je mehr sich z.B. die zu entrichtende Investitionsauszahlung für die vorhergehende Erweiterungssoption dem Wert $V_{f}$ oder der Desinvestitionserlös für die nachfolgende Aufgabeoption dem Wert $V_{e}$ nähert, umso unwahrscheinlicher wird eine gemeinsame Inanspruchnahme. Sinkt die Wahrscheinlichkeit für eine gemein-

1 Implizit wird in dieser Darstellung davon ausgegangen, daß sich bei Inanspruchnahme der Erweiterungsoption der Desinvestitionserlös proportional zum Bezugsgut erhöht.

2 Vgl. Trigeorgis, Option Interactions, 1993, S. 8; Trigeorgis, Complex Options, 1996, S. 323. 
same Inanspruchnahme auf null $\left(I>V_{f}\right.$ oder $\left.D I<V_{e}\right)$ sind die isoliert ermittelten Ergebnisse addierbar.

Drittens ist die gegenseitige Wertbeeinflussung umso geringer, je rascher die möglichen Ausübungszeitpunkte aufeinander folgen. Bei einer unmittelbar nach $t=1 \mathrm{zu}$ treffenden Entscheidung über die Aufgabeoption, kann eine gemeinsame Inanspruchnahme nahezu ausgeschlossen werden. Wenn der Wert des Bezugsguts in $t=1$ zwischen $V_{a}$ und $I$ liegt, kann derselbe sich nicht kurz darauf zwischen $D I$ und $V_{d}$ bewegen.

Wie Trigeorgis und Kulatilaka an umfassenden Zahlenbeispielen zeigen ${ }^{1}$, ist der Wert, der durch einen zusätzlichen Handlungsspielraum bei Existenz anderer entsteht, i.d.R. geringer als der isoliert berechnete Wert ${ }^{2}$. Je größer die Zahl der verbundenen Realoptionen, umso geringer ist der zusätzliche Wert, den die einzelne Realoption zum Gesamtwert aller Realoptionen beiträgt. Folglich reicht es aus, im Rahmen der Bewertung eines Investitionsprojekts die „wesentlichen" Realoptionen zu berücksichtigen.

Die explizite Berücksichtigung der zuvor beschriebenen Interdependenzen führt dazu, daß in zeitstetigen Bewertungsansätzen nur noch selten eine analytische Lösung für die Wertermittlung von verbundenen Realoptionen existiert. Eine Ausnahme bildet das finanzwirtschaftliche Modell von Geske, in dem das Eigenkapital eines zum Teil fremdfinanzierten Unternehmens als Option auf den Wert des Gesamtkapitals verstanden wird ${ }^{3}$. Für den Fall, daß das Fremdkapital das Gesamtkapital übersteigt, begleichen die Eigentümer ihre Schulden nicht mehr. Die Eigenkapitaloption wird nicht ausgeübt. Bei einer derartigen Interpretation des Eigenkapitals stellt die in diesem Modell zu bewertende Aktienoption eine Option auf eine (Eigenkapital-) Option dar.

Investitionen, die einen ersten Meilenstein in einer ganzen Investitionsreihe verkörpern, unterliegen ähnlichen Eigenschaften wie die eben beschriebene Aktienoption. Durch Uminterpretation der Bewertungsparameter läßt sich folglich das Modell von Geske auf Markteintritts- oder Forschungs- und Entwicklungsprojekte übertragen, die erst nach mehreren Investitionszahlungen

1 Vgl. Trigeorgis, Option Interactions, 1993, S. 12 ff.; Kulatilaka, Substitutability and Complementarity, 1995, S. $126 \mathrm{ff}$.

2 Eine Ausnahme besteht u.a. fur den Fall, in dem eine vorhergehende Erweiterungsoption die Voraussetzung fur eine nachfolgende Erweiterungsoption bildet.

3 Vgl. Geske, Compound Options, 1979, S. 65 ff. 
erste Projektrückflüsse erwarten lassen. I.d.R. erfolgt jedoch die Bewertung von verbundenen Realoptionen nicht ohne Rückgriff auf numerische Methoden $^{1}$. Im folgenden werden anhand des zeitdiskreten Binomialmodells zwei Beispiele präsentiert, in denen der Gesamtwert der verbundenen Realoptionen einerseits unter und andererseits über der Summe der isoliert ermittelten Ergebnisse liegt.

\subsection{Beispiel für sich kompensierende Realoptionen}

Es sei angenommen, daß der Start der durch die strategische Akquisition zusätzlich geschaffenen Investitionsmöglichkeit lediglich um ein Jahr verschoben wird. Die Wertentwicklung des Bezugsguts soll dabei derjenigen aus dem Beispiel in Abschnitt 2.2 in Kapitel IV entsprechen. So beläuft sich der Wert des Bezugsguts heute auf 100 Mio. $€$, um nach einem Jahr entweder auf 200 Mio. $€(u=2)$ zu steigen oder auf 50 Mio. $€(d=0,5)$ zu fallen. Die Investitionsauszahlung beträgt weiterhin 90 Mio. $€$ und der risikolose Zinsfuß $5 \%$ p.a. Zusätzlich zu dieser in $t=1$ bestehenden Aufschubsoption besteht in $t=2$ die Möglichkeit, das Bezugsgut der zu beurteilenden Investitionsmöglichkeit um $50 \% \mathrm{zu}$ erweitern. Für eine weitere Investitionssumme von 30 Mio. $€$ wird in den einzelnen Umweltzuständen jeweils das 1,5-fache der zuvor geplanten Rückflüsse erwartet. Gemäß der angenommenen Wertentwicklung des Bezugsguts beträgt der in $t=2$ geltende Gegenwartswert dieser ursprünglich prognostizierten Rückflüsse 400 Mio. $€, 100$ Mio. $€$ bzw. 25 Mio. $€$.

Werden die unternehmerischen Handlungsspielräume isoliert bewertet, beläuft sich der Wert der Aufschubsoption (inklusive des traditionellen Kapitalwerts von 10 Mio. $€$ ) auf 38,4 Mio. $€$ und derjenige der Erweiterungsoption auf 29,2 Mio. $€^{2}$. Um den Interdependenzen Rechnung zu tragen, daß erstens das Projekt nur dann erweitert werden kann, wenn zuvor investiert wird sowie zweitens sich das Bezugsgut der Aufschubsoption aufgrund der nachfolgenden Erweiterungsoption erhöht, wird ein Roll Back-Verfahren angewandt. Zuerst wird die optimale Inanspruchnahme sowie der Wert der nachfolgenden Erweiterungsoption sowohl für den Fall einer vorherigen Investition als auch für den

1 Vgl. stellvertretend Trigeorgis, Multi-Option Investments, 1991, S. 311 ff. sowie Cortazar/Schwartz, Compound Option, 1993, S. $521 \mathrm{ff}$.

2 Für die Berechnung der isoliert ermittelten Ergebnisse muß jeweils unterstellt werden, daß die andere Realoption nicht existiert. Für die nachfolgende Erweiterungsoption bedeutet dies, daß die Erstinvestition von 90 Mio. DM in $t=0$ erfolgt, was aufgrund des positiven traditionellen Kapitalwerts von 10 Mio. DM nicht unrealistisch ist. 
Fall einer vorherigen Nichtinvestition erfaßt. Anschließend wird über die vorhergehende Aufschubsoption unter Berücksichtigung der bedingten Werte für die Erweiterungsoption entschieden. Die Diskontierung der einzelnen Werte erfolgt anhand des risikoneutralisierten Bewertungsansatzes mit den bekannten Pseudowahrscheinlichkeiten $p=36,7 \%$ und $1-p=63,3 \%$.

Für den Gesamtwert der beiden Realoptionen resultiert ein Ergebnis von 63,4 Mio. $€$ (vgl. Abb. 46). Dieser ist um 4,2 Mio. $€$ geringer als die Summe der isoliert berechneten Ergebnisse von 67,6 Mio. $€$. Die betrachteten unternehmerischen Handlungsspielräume stellen sich kompensierende Realoptionen dar. Der geringere Gesamtwert ist darauf zurückzuführen, daß in $t=2$ in Umweltzustand $s=3$ die isoliert betrachtet optimale Strategie der Erweiterung nicht besteht, weil in $t=1$ nicht investiert wird ${ }^{1}$. In Abb. 46 zeigt $V_{t, s}$ den Wert des Bezugsguts der jeweiligen Realoption im Zeitpunkt $t$ in Umweltzustand $s, I_{t, s}$ die bei Inanspruchnahme der jeweiligen Realoption fällige Investitionsauszahlung sowie $G W_{t, s}$ den entsprechenden Gesamtwert der verbundenen Handlungsspielräume.

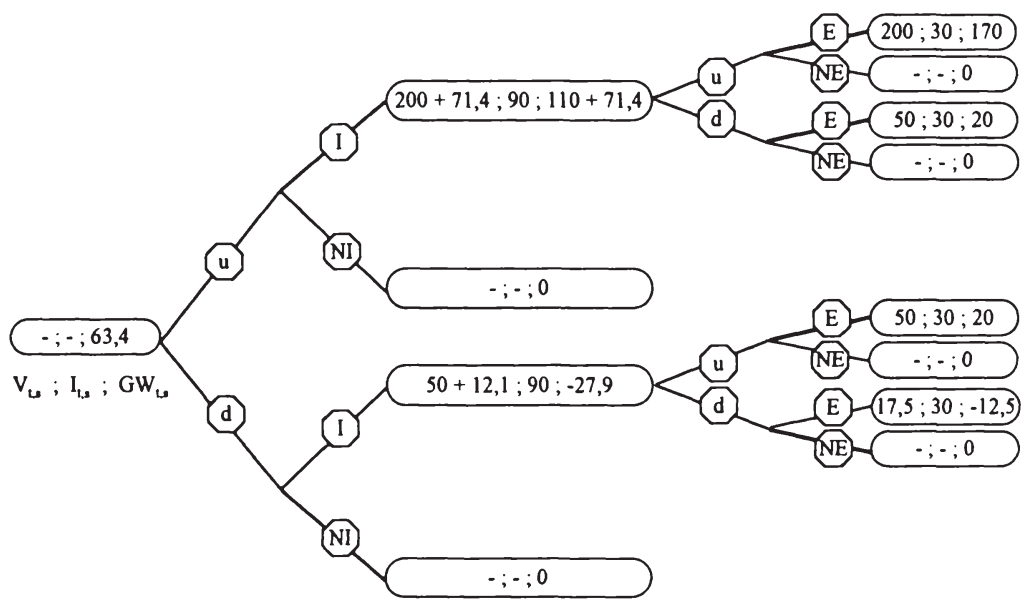

$\mathrm{I}=$ Investieren $\quad \mathrm{NI}=$ Nicht investieren $\quad \mathrm{E}=$ Erweitern $\quad \mathrm{NE}=$ Nicht erweitern

Abb. 46: Vorhergehende Aufschubs- und nachfolgende Erweiterungsoption als Beispiel für sich kompensierende Realoptionen 


\subsection{Beispiel für komplementäre Realoptionen}

Um aufzuzeigen, daß sich verbundene Handlungsspielräume im Hinblick auf ihren Gesamtwert auch positiv beeinflussen können, wird das eben eingeführte Beispiel leicht modifiziert. Der Start der zusätzlichen Investitionsmöglichkeit wird nun nicht in Frage gestellt und bereits heute durchgeführt. In $t=1$ besteht nun die Möglichkeit, für eine Investitionssumme von 45 Mio. $€$ den Wert des Bezugsguts des zu beurteilenden Investitionsprojekts auf das 1,5-fache zu erhöhen. Zusätzlich soll in einer nachfolgenden Erweiterungsoption in $t=2$ ebenfalls eine Erhöhung des Bezugsguts um 50\% denkbar sein und dies unabhängig davon, ob das Projekt bereits in $t=1$ erweitert worden ist oder nicht. Hierfür wäre eine Investitionsauszahlung von 70 Mio. $€$ fällig.

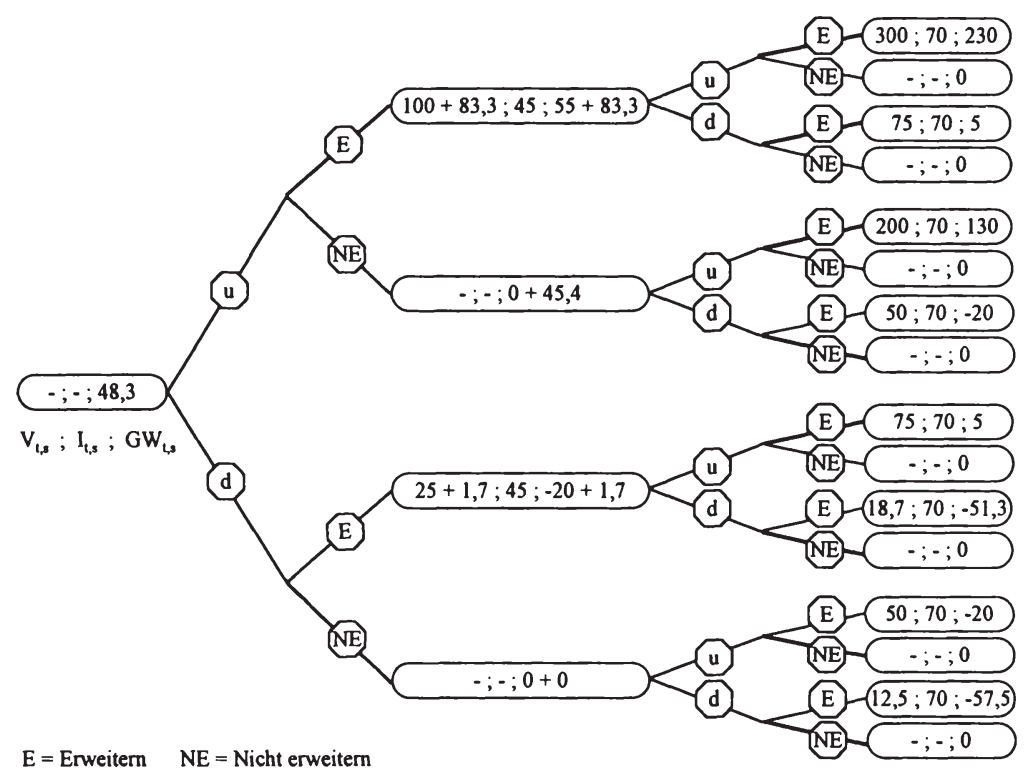

Abb. 47: Vorhergehende und nachfolgende Erweiterungsoption als Beispiel für komplementäre Realoptionen

Eine isolierte Berechnung der beiden Realoptionen ergibt Einzelwerte von 19,2 Mio. $€$ bzw. 15,9 Mio. $€^{1}$. Wird die Abhängigkeit zwischen den beiden Erweiterungsoptionen berücksichtigt, beläuft sich der Gesamtwert der Realoptio-

1 Für die Berechnung der isoliert ermittelten Ergebnisse muß unterstellt werden, daß die andere Realoption nicht existiert. 
nen auf 48,3 Mio. $€($ vgl. Abb. 47) 1 . Letzterer überragt um 13,2 Mio. $€$ die Summe der isoliert ermittelten Ergebnisse von 35,1 Mio. $€$. Bei den betrachteten Realoptionen handelt es sich um komplementäre Realoptionen. Der größere Gesamtwert ist auf den Umstand zurückzuführen, daß die nachfolgende Erweiterungsoption in den Umweltzuständen $s=1$ und $s=2$, in denen das Projekt bereits in $t=1$ vergrößert wird, wertvoller ist ( 230 Mio. $€$ bzw. 5 Mio. $€$ ) als in den Umweltsituationen $s=3$ und $s=4$ (130 Mio. $€$ bzw. 0 Mio. $€$ ), in denen die vorhergehende Erweiterungsoption nicht in Anspruch genommen wird $^{2}$. In Abb. 47 stellt $V_{t, s}$ wiederum den Wert des Bezugsguts der jeweiligen Realoption im Zeitpunkt $t$ in Umweltzustand $s, I_{t, s}$ die bei Inanspruchnahme der einzelnen Realoption fällige Investitionsauszahlung sowie $G W_{t, s}$ den Gesamtwert der verbundenen Realoptionen dar.

1 Zur Berechnung wird das bereits im vorigen Abschnitt erlăuterte Roll Back-Verfahren angewandt, das bei der Ausubungsentscheidung der jeweiligen Realoption die Existenz der anderen Realoption explizit berucksichtigt.

$2\left[0,367^{2} \cdot(230-130)+0,367 \cdot 0,633 \cdot(5-0)\right] / 1,05^{2}=13,2$. 


\section{4 Überlegungen zur praktischen Umsetzung der Realoptionsbewertung}

\subsection{Einsatz von Sensitivitätsanalysen}

\subsubsection{Isolierte Variation der Bewertungsparameter}

Wie bereits in Abschnitt 2.1.1.3 in Kapitel IV aufgezeigt, stellt die optionspreistheoretische Bewertung im Vergleich zu den Korrekturverfahren und den Sensitivitäts- bzw. Risikoanalysen keine weitere Technik im Umgang mit Unsicherheit dar, sondern kann als spezielles Korrekturverfahren für den Fall eines kontinuierlich wechselnden Risikos angesehen werden. Die im Rahmen der traditionellen Kapital- bzw. Barwertberechnung vorgeschlagenen Sensitivitätsanalysen lassen sich somit ebenfalls in der Realoptionsbewertung verwenden, indem das mit Hilfe eines bestimmten Optionsbewertungsmodells ermittelte Ergebnis auf seine Stabilität hin überprüft wird.

Inwieweit verändern sich die in Kapitel IV und V ermittelten Werte der durch die strategische Akquisition zusätzlich geschaffenen Investitionsmöglichkeit, wenn die zugrundeliegenden Bewertungsparameter systematisch variiert werden? So werden im folgenden Veränderungen des Barwerts sowie der Standardabweichung des Bezugsguts, der Investitionsauszahlung, des risikolosen Zinsfußes, der Laufzeit der Investitionsmöglichkeit sowie des zwischenzeitlich entgehenden Rückflusses bzw. Wertverlusts betrachtet ${ }^{1}$. Als Referenzwert dient dabei das in Abschnitt 3.3 in Kapitel IV im Rahmen des zeitstetigen Bewertungsansatzes ermittelte Ergebnis von 41,4 Mio. $€^{2}$.

Steigt der Barwert des Bezugsguts V, vergrößert sich c.p. der Wert der Investitionsmöglichkeit $W$, da im Fall einer zukünftigen Investition vergleichsweise höhere Rückflüsse erwartet werden.

$$
\frac{d W}{d V}>0
$$

1 Zu Sensitivitätsanalysen im Rahmen der Realoptionsbewertung vgl. stellvertretend Trigeorgis, Real Options Application, 1990, S. 159 ff.; Pindyck, Irreversibility and Investment, 1991, S. $1123 \mathrm{ff}$.

2 Der zeitstetige Bewertungsansatz wurde ausgewählt, weil er in dem hier untersuchten Beispiel eine analytische Bewertungsformel aufweist, welche eine effiziente Umsetzung der Sensitivitătsberechnungen erlaubt. Im Referenzfall sind für die Bewertungsparameter folgende numerische Werte angenommen worden: Barwert des Bezugsguts $V_{0}=100$ Mio. $€$, Investitionsauszahlung $I=90$ Mio. $€$, risikoloser Zinsfuß $r_{f}=5 \%$ p.a., Optionslaufzeit $t=3$ Jahre, Standardabweichung des Bezugsguts $\sigma=69,31 \%$, zwischenzeitlicher Rückfluß bzw. Wertverlust $r_{D}=5 \%$ p.a. 
Ein um 50\% erhöhter Barwert läßt den Wert der Investitionsmöglichkeit auf 76,0 Mio. $€(+84 \%)$ steigen. Demgegenüber bewirkt ein um $50 \%$ geringerer Barwert, daß der Wert der Investitionsmöglichkeit auf 12,7 Mio. $€(-69 \%)$ sinkt. Das Beispiel zeigt, daß das Ergebnis auf einen veränderten Barwert des Bezugsguts sehr sensibel reagiert (vgl. den Verlauf in Abb. 48). Dies kann nicht verwundern, wenn die in Abschnitt 2.1.3 in Kapitel IV vorgestellte $\mathrm{Zu}$ sammensetzung des Werts der Investitionsmöglichkeit bedacht wird. Neben dem Flexibilitätswert verkörpert dieser den traditionellen Kapitalwert und damit das erwartete Rückflußpotential des Bezugsguts ${ }^{1}$. Ein um $50 \%$ gestiegener Barwert des Bezugsguts bedingt z.B. eine Erhöhung des traditionellen Kapitalwerts von zuvor 10 Mio. $€$ auf nun 60 Mio. $€$. Der Flexibilitätswert ist dann von 31,4 Mio. $€$ auf 16,0 Mio. $€$ gesunken. Demgegenüber führt ein um 50\% gesunkenes Bezugsgut zu einem traditionellen Kapitalwert von -40 Mio. $€$. Der Flexibilitätswert ist nun auf 52,7 Mio. $€$ gestiegen.

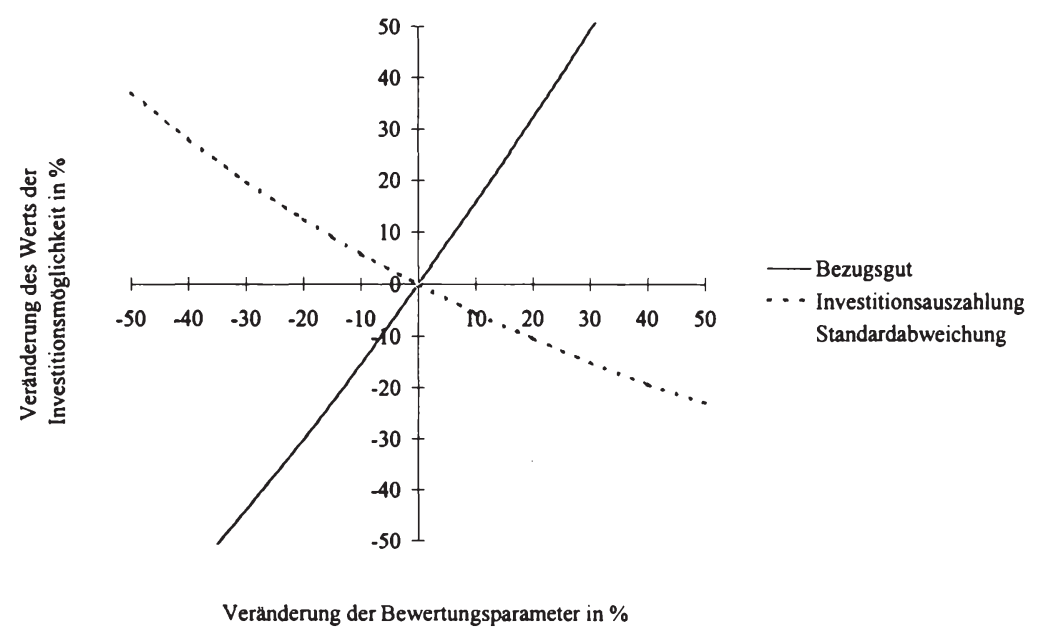

Abb. 48: Sensitivität des Werts der Investitionsmöglichkeit in Bezug auf den Barwert des Bezugsguts, die Investitionsauszahlung sowie die Standardabweichung des Bezugsguts

Steigt die zukünftig zu leistende Investitionsauszahlung $I$, sinkt c.p. der Wert der Investitionsmöglichkeit $W$.

$$
\frac{d W}{d I}<0
$$

1 Vgl. Abschnitt 2.1.3 in Kapitel IV. 
Auf eine solche Veränderung reagiert das Referenzergebnis jedoch weniger sensibel als bei modifiziertem Barwert des Bezugsguts. Eine erhöhte Investitionsauszahlung um $50 \%$ führt zu einem Wert der Investitionsmöglichkeit von 31,9 Mio. $€(-23 \%)$. Hingegen resultiert aus einer um $50 \%$ gesunkenen Investitionsauszahlung ein Wert der Investitionsmöglichkeit von 56,8 Mio. $€(+37 \%)$.

Wenn sich die Standardabweichung des Bezugsguts ${ }^{1} \sigma$ erhöht, steigt c.p. der Wert der Investitionsmöglichkeit $W$.

$$
\frac{d W}{d \sigma}>0
$$

Eine modifizierte Standardabweichung um $50 \%$ hat zur Folge, daß bei erhöhter Standardabweichung ein Wert der Investitionsmöglichkeit von 56,0 Mio. $€$ $(+35 \%)$ und bei geringerer Standardabweichung ein entsprechender Wert von 23,9 Mio. $€(-42 \%)$ resultiert.

Die Tatsache, daß eine erhöhte Standardabweichung des Bezugsguts zu einem höheren (Bar-) Wert der Investitionsmöglichkeit führt, steht auf den ersten Blick dem üblicherweise in der Investitions- und Finanzierungstheorie postulierten Rendite/Risiko-Zusammenhang entgegen. Gemäß letzterem bedingt eine erhöhte Standardabweichung (genauer ein erhöhtes bewertungsrelevantes Risiko) eine größere Rendite und damit bei gleichem Erwartungswert des unsicheren Rückflusses einen niedrigeren Barwert.

Dieser nur augenscheinlich vorhandene Widerspruch ist dadurch zu erklären, daß sich durch die höhere Standardabweichung des Bezugsguts c.p. ein höherer Erwartungswert des Rückflusses aus der Investitionsmöglichkeit ergibt ${ }^{2}$. Gleiches bewertungsrelevantes Risiko und damit eine unveränderte erwartete Rendite vorausgesetzt, führt ein höherer Erwartungswert zu einem höheren (Bar-) Wert der Investitionsmöglichkeit. Die höhere Unsicherheit über den zukünftigen Wert des Rückflusses erhöht das Chancenpotential, dem kein vergleichbares symmetrisches Verlustpotential gegenübersteht. Der Inhaber der Investitionsmöglichkeit ist nicht verpflichtet, die Investition durchzuführen, so daß schlimmstenfalls ein „Verlust“ von null resultiert.

1 Streng genommen handelt es sich um die Standardabweichung der Rendite des Bezugsguts.

2 Der Grund hierfur liegt in der asymmetrischen Risikostruktur der Investitionsmöglichkeit, welche durch die Existenz des unternehmerischen Handlungsspielraums bedingt ist. Vgl. Abschnitt 4.1 in Kapitel III. 
Aufgrund der c.p.-Bedingung bleibt der Barwert des Bezugsguts trotz modifizierter Standardabweichung bisher unverändert. Falls die Standardabweichung jedoch bewertungsrelevantes Risiko darstellt, müßte die c.p.-Bedingung aufgehoben und von einem geringeren Barwert des Bezugsguts ausgegangen werden. Der daraus resultierende Gesamteffekt auf den Wert der Investitionsmöglichkeit wird später im Rahmen der gleichzeitigen Variation von mehreren Bewertungsparametern diskutiert.

Steigt der risikolose Zinsfuß $r_{f}$, steigt c.p. der Wert der Investitionsmöglichkeit $W$, denn ein erhöhter risikoloser Zinsfuß läßt den Zinsertrag wachsen, der durch die Stundung der Investitionsauszahlung entsteht.

$$
\frac{d W}{d r_{f}}>0
$$

Auf etwaige Veränderungen reagiert das Referenzergebnis aber kaum (vgl. Abb. 49). Während ein erhöhter Zinsfuß um $50 \%$ in einem Wert der Investitionsmöglichkeit von 43,1 Mio. $€(+4 \%)$ resultiert, errechnet sich bei einem um $50 \%$ gesunkenen Zinsfuß ein Ergebnis von 39,6 Mio. $€(-4 \%)$. Im Rahmen einer solchen Veränderungsrechnung ist zusätzlich zu beachten, daß durch einen modifizierten risikolosen Zinsfuß sich auch gleichzeitig der Barwert des Bezugsguts verändert und somit die c.p.-Bedingung aufzuheben ist. Hiervon geht ein gegenläufiger Effekt auf den Wert der Investitionsmöglichkeit aus, der in Abschnitt 4.1.2 dieses Kapitels näher erläutert wird.

Durch eine erhöhte Laufzeit $t$ steigt c.p. der Wert der Investitionsmöglichkeit $W$, da mit zunehmender zeitlicher Entfernung gleichzeitig das Risiko des unsicheren Rückflusses des Bezugsguts steigt. Hierdurch wird die Bandbreite der am Laufzeitende denkbaren Werte dieses Rückflusses vergrößert, was durch die asymmetrische Risikostruktur des unternehmerischen Handlungsspielraums einen positiven Effekt auf den Wert der Investitionsmöglichkeit hat.

$$
\frac{d W}{d t}>0
$$

Eine erhöhte Laufzeit um 50\% führt zu einem neuen Ergebnis von 44,9 Mio. $€$ $(+8 \%)$. Demgegenüber bewirkt eine um 50\% geringere Laufzeit einen Wert der Investitionsmöglichkeit von 33,8 Mio. $€(-18 \%)$. Diese Beispieldaten veranschaulichen zudem das bereits aus der finanzwirtschaftlichen Optionspreistheo- 
rie bekannte Ergebnis ${ }^{1}$, daß mit abnehmender Restlaufzeit die Investitionsmöglichkeit überproportional an Wert verliert ${ }^{2}$.

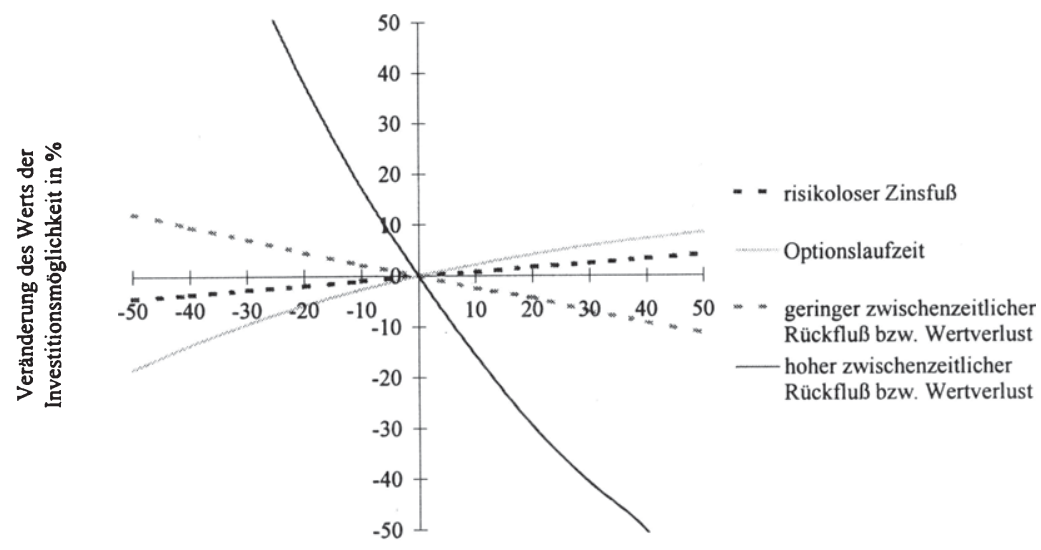

Verănderung der Bewertungsparameter in \%

Abb. 49: Sensitivität des Werts der Investitionsmöglichkeit in Bezug auf den risikolosen Zinsfuß, die Optionslaufzeit und einen geringen oder einen hohen zwischenzeitlichen Rückfluß bzw. Wertverlust ${ }^{3}$

Steigt der zwischenzeitliche Rückfluß bzw. Wertverlust $r_{D}$, sinkt c.p. der Wert der Investitionsmöglichkeit $W$. Ein wachsender zwischenzeitlicher Rückfluß bedeutet, daß ein höherer Betrag verloren geht, falls nicht sofort investiert wird.

$$
\frac{d W}{d r_{D}}<0
$$

Betrug bei einem zwischenzeitlichen Wertverlust von 5\% p.a. der Referenzwert 41,4 Mio. $€$, bewirkt eine Erhöhung um 50\% nun ein Ergebnis von 36,7 Mio. $€$ $(-11 \%)$ und eine Senkung um 50\% einen Wert der Investitionsmöglichkeit von 46,5 Mio. $€(+12 \%)$.

1 Vgl. stellvertretend Perridon/Steiner, Finanzwirtschaft, 1997, S. 319 f.

2 Bei einer Sensitivitätsanalyse der Laufzeit ist ähnlich wie beim risikolosen Zinsfuß auch ein gegenläufiger Effekt zu beobachten, falls die zuvor angenommene c.p.-Bedingung eines unveränderten Barwerts des Bezugsguts aufgegeben wird.

3 Beim hohen zwischenzeitlichen Rückfluß bzw. Wertverlust liegt systembedingt ein anderer Referenzwert zugrunde. 
Diese vergleichsweise geringe Sensitivität erhöht sich stark, wenn ein höherer Ausgangswert des zwischenzeitlichen Wertverlusts (z.B. durch Konkurrenzeinfluß) angenommen wird. Bei einem zwischenzeitlichen Wertverlust von $30 \%$ p.a. resultiert ein Referenzwert der Investitionsmöglichkeit von 11,5 Mio. $€$. Eine 50\%ige Erhöhung des zwischenzeitlichen Wertverlusts führt jetzt zu einem Wert der Investitionsmöglichkeit von 3,9 Mio. $€(-66 \%)$, während im entgegengesetzten Fall einer 50\%igen Verringerung ein Ergebnis von 25,4 Mio. $€$ $(+121 \%)$ resultiert ${ }^{1}$.

Zusammenfassend läßt sich festhalten, daß insbesondere der Barwert und die Standardabweichung des Bezugsguts sowie ein hoher zwischenzeitlicher Rückfluß bzw. Wertverlust als kritische Bewertungsparameter für die optionspreistheoretische Bestimmung des Werts der Investitionsmöglichkeit anzusehen sind. Auch bei der Veränderung der Investitionsauszahlung zeigt sich eine nicht zu unterschätzende Sensitivität, wobei jedoch in realistischen Bewertungs- und Entscheidungssituationen die Bestimmung zukünftig notwendiger Investitionssummen eventuell mit einer geringeren Unsicherheit einhergehen könnte als die zuvor genannten Bewertungsparameter.

\subsubsection{Parallele Variation der Bewertungsparameter}

Neben der isolierten Variation einzelner Bewertungsparameter lassen sich auch Auswirkungen bei gleichzeitiger Veränderung mehrerer Bewertungsparameter aufzeigen. Die parallele Variation bietet sich zum einen an, um Umweltsituationen ausfindig zu machen, in denen der ermittelte Wert der Investitionsmöglichkeit lediglich bei gleichzeitiger Variation von Parametern bedeutend vom Referenzergebnis abweicht. Zum anderen lassen sich hiermit Ergebnisveränderungen erkennen, bei denen die Variation eines Bewertungsparameters i.d.R. die Modifikation eines weiteren nach sich zieht.

Im folgenden sei exemplarisch auf die bereits im vorigen Abschnitt erwähnte Interdependenz eingegangen, daß durch eine erhöhte Standardabweichung des Bezugsguts der Wert der Investitionsmöglichkeit sowohl unmittelbar als auch i.d.R. mittelbar über einen veränderten Barwert des Bezugsguts beeinflußt wird. Eine erhöhte Standardabweichung führt einerseits unmittelbar zu einem erhöhten Wert der Investitionsmöglichkeit. Andererseits sinkt i.d.R. der Bar-

1 Für den risikolosen Zinsfuß ließe sich ebenfalls eine größere als die zuvor dargestellte Sensitivität aufzeigen, falls von einem wesentlich höheren Ausgangswert ausgegangen würde. Letzterer ist jedoch nicht realistisch. 
wert des Bezugsguts, wodurch mittelbar ein gegenläufiger Werteffekt resultiert $^{1}$. Da die Richtung des Gesamteffekts nicht allgemeingültig vorhergesagt werden kann, werden die Konsequenzen einer erhöhten Standardabweichung anhand eines konkreten Zahlenbeispiels verdeutlicht.

Dazu sei vereinfachend angenommen, daß zwischen der Standardabweichung und der Risikoprämie, die zur Bestimmung des Barwerts des Bezugsguts benötigt wird, eine lineare Beziehung besteht ${ }^{2}$. Eine erhöhte Standardabweichung um 50\% führt dann zu einer erhöhten Risikoprämie um 50\%. Zur Ermittlung des Barwerts des Bezugsguts wird die zeitstetige Diskontierung verwendet ${ }^{3}$, da die bisherigen Parametervariationen auf Basis des zeitstetigen Bewertungsansatzes analysiert worden sind.

Der im Referenzergebnis von 41,4 Mio. $€^{4}$ angenommene Barwert des Bezugsguts beträgt 100 Mio. $€$. Wird z.B. von einem Erwartungswert des unsicheren Gegenwartswerts des Bezugsguts von 125 Mio., 156 Mio. bzw. 195 Mio. $€$ nach einem, zwei bzw. drei Jahr(en) ausgegangen ${ }^{5}$, ist dies gleichbedeutend mit einer risikoadjustierten (zeitstetigen) Rendite des Bezugsguts von 22,3\% p.a. Bei einer risikolosen Verzinsung von 5\% p.a. resultiert somit eine Risikoprämie von 17,3\% p.a. Eine erhöhte Standardabweichung um 30\% (von 69,31 auf $90,10 \%$ ) bedingt aufgrund der getroffenen Annahme der linearen Transformation eine Risikoprämie von $22,5 \%$ p.a. und damit eine risikoadjustierte Rendite von $27,5 \%$ p.a.

Wird der nach drei Jahren erwartete Gegenwartswert des Bezugsguts von 195 Mio. $€$ mit dieser neuen Rendite abgezinst, führt dies zu einem Barwert von 85,6 Mio. $€$. Bei der nun angenommenen Standardabweichung von $90,10 \%$ errechnet sich ein Wert der Investitionsmöglichkeit von 40,8 Mio. $€$, der damit knapp niedriger als das Referenzergebnis ist. Während durch eine erhöhte

1 Vgl. auch McDonald/Siegel, Investment and Option, 1985, S. 333; Trigeorgis, Options Framework, 1988, S. 162; Laux, Handlungsspielräume, 1993, S. 954.

2 Im kapitalmarkttheoretischen Bewertungsmodell setzt dies voraus, daß sich trotz Variation der Standardabweichung die Korrelation zwischen zu bewertender Vermögensposition und Gesamtmarkt nicht verändert.

$3 V_{0}=E(C F) \cdot e^{-r_{A} \cdot t}$

4 Vgl. Fn. 2 auf S. 208.

$5 \quad 0,5 \cdot 200+0,5 \cdot 50=125$ Mio. $€ ; 0,25 \cdot 400+0,5 \cdot 100+0,25 \cdot 25=156,25$ Mio. $€$ usw. Vgl. das Beispiel aus Abschnitt 2.2 in Kapitel IV. 
Standardabweichung der Flexibilitätswert steigt, sinkt dementsprechend der traditionelle Kapitalwert, wodurch sich letztendlich der Wert der Investitionsmöglichkeit vergleichsweise wenig verändert. Im konkreten Zahlenbeispiel bedingt eine erhöhte Standardabweichung leicht sinkende Werte für die Investitionsmöglichkeit (vgl. Abb. 50) ${ }^{1}$.

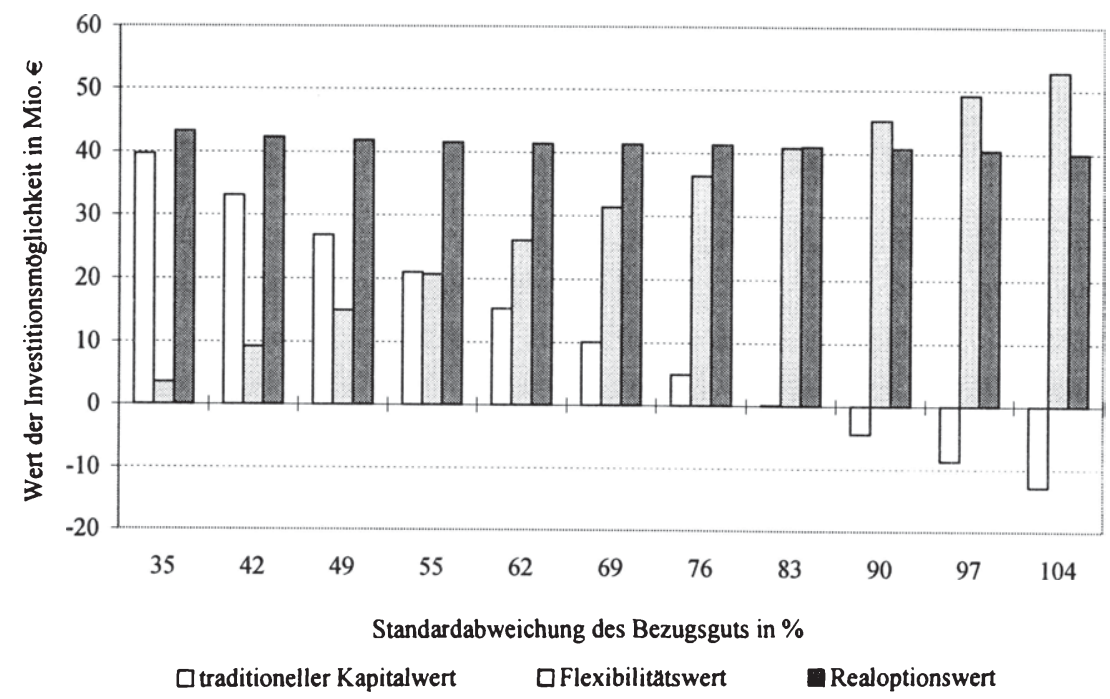

Abb. 50: Entwicklung des traditionellen Kapital- sowie des Flexibilitätswerts bei paralleler Variation des Barwerts sowie der Standardabweichung des Bezugsguts

Wie das folgende Beispiel zeigt, kann jedoch bei einer erhöhten Standardabweichung des Bezugsguts nicht generell von einer Senkung des Werts der Investitionsmöglichkeit ausgegangen werden. Wird im Gegensatz zu der eben analysierten dreijährigen Investitionsmöglichkeit nun eine einjährige Wachstumsoption ohne zwischenzeitlichen Rückfluß angenommen ${ }^{2}$, führt eine erhöhte Standardabweichung um $30 \%$ zu einer Erhöhung des ermittelten Ergebnisses von zuvor 32,9 Mio. $€$ auf nun 36,2 Mio. $€$. Die Steigung des Flexibilitätswerts ist größer als die Verringerung des traditionellen Kapitalwerts. Bei der gleichen

1 Diese Senkungstendenz wird hier durch den proportional zum Bezugsgut definierten zwischenzeitlichen Rückfluß abgeschwächt. Ein solcher bedingt, daß sich bei sinkendem Barwert auch die „Opportunitätskosten” verringern, wodurch c.p. die relative Bedeutung des Flexibilitătswerts steigt. Die Verringerung des Werts der Investitionsmöglichkeit ist folglich grőßer, wenn kein bzw. ein absoluter zwischenzeitlicher Rückfluß unterstellt wird.

2 Ansonsten gelten weiterhin folgende Ausgangswerte: Barwert des Bezugsguts $=100$ Mio. $€$, Investitionsauszahlung $=90$ Mio. $€$, risikoloser Zinsfuß $=5 \%$ p.a., Optionslaufzeit $=3$ Jahre, Standardabweichung des Bezugsguts $=69,31 \%$. 
dreijährigen Wachstumsoption führt eine um 30\% erhöhte Standardabweichung hingegen zu einem Wert von 50,2 Mio. $€$ bei zuvor 52,1 Mio. $€$. Die längere Laufzeit bedingt, daß der unmittelbare flexibilitätswerterhöhende Effekt schwächer ist als der mittelbare kapitalwertsenkende Effekt. Wie Tab. 8 aufzeigt, entsprechen sich die beiden Effekte bei einer Laufzeit von rund 2,5 Jahren.

\begin{tabular}{|c||c|c|c|c|c|}
\hline $\begin{array}{c}\text { Laufzeit in } \\
\text { Jahren }\end{array}$ & $t=1$ & $t=1,5$ & $t=2$ & $t=2,5$ & $t=3$ \\
\hline $\begin{array}{c}W_{0} \text { bei } \\
\sigma=69,31 \%\end{array}$ & 32,9 & 39,0 & 44,1 & 48,4 & 52,1 \\
\hline $\begin{array}{c}W_{0} \text { bei } \\
\sigma=90,10 \%\end{array}$ & 36,2 & 41,5 & 45,3 & 48,1 & 50,2 \\
\hline$\Delta$ & $+3,3$ & $+2,5$ & $+1,2$ & $-0,3$ & $-1,9$ \\
\hline
\end{tabular}

Tab. 8: Veränderung $\Delta$ des Werts der Investitionsmöglichkeit $W_{0}$ bei Variation der Standardabweichung des Bezugsguts $\sigma$, des Barwerts des Bezugsguts $V_{0}$ und der Laufzeit $t$

Wie bei einer modifizierten Standardabweichung sind auch bei einer Veränderung des risikolosen Zinsfußes sowie der Laufzeit grundsätzlich zwei Effekte zu berücksichtigen ${ }^{1}$. Bei einem erhöhten risikolosen Zinsfuß ergibt sich einerseits ein unmittelbarer Zinsertrag durch die Stundung der Investitionsauszahlung und andererseits sinkt durch die erhöhte Zeitpräferenz der Barwert des Bezugsguts. Demgegenüber steigt bei einer erhöhten Laufzeit das Chancenpotential der Investitionsmöglichkeit, während gleichzeitig der Barwert des Bezugsguts durch die „längere“ Diskontierung des unsicheren Rückflusses sinkt. Konzeptionell entstehen für den Wert der Investitionsmöglichkeit die gleichen Wirkungseffekte wie diejenigen die soeben im Rahmen einer veränderten Standardabweichung aufgezeigt wurden. Lediglich der Grund für die Barwertveränderung des Bezugsguts ist ein anderer.

\subsection{Nutzen der Risikosimulation}

In Abschnitt 3 in Kapitel III ist ausführlich dargelegt worden, warum sich die Risikosimulation nicht zur Ermittlung eines in die Entscheidungsfindung eingehenden Bar- bzw. Kapitalwerts eignet. Vor der Anwendung einer solchen Simulation muß einerseits der dem eingegangenen Risiko entsprechende Zinsfuß bekannt sein. Anderseits soll gerade mit Hilfe der Simulation dieses Risiko transparent gemacht werden, so daß für eine entscheidungsorientierte Risiko-

1 Vgl. Trigeorgis, Options Framework, 1988, S. 162. 
simulation ein Zirkelschluß besteht. Da das Problem des zu verwendenden Zinsfußes bereits innerhalb eines Projekts mit konstantem Investitionsrisiko auftritt, liegt es nahe, daß die Risikosimulation zur Bestimmung eines Werts der Investitionsmöglichkeit, dessen Risiko sich über die Laufzeit kontinuierlich ändert, gänzlich ungeeignet erscheint.

Die zuletzt getätigte Aussage darf nicht mit der Anwendung von Simulationstechniken verwechselt werden, bei denen ein Optionswert vor dem Hintergrund eines bereits bekannten Barwerts des Bezugsguts bestimmt wird. Mit Hilfe dieses Barwerts sowie dem risikolosen Zinsfuß wird eine risikoneutralisierte Wahrscheinlichkeitsverteilung für den Wert des Bezugsguts am Ende der Optionslaufzeit simuliert ${ }^{1}$. Auf diese Weise wird in zeitstetigen Bewertungsansätzen das analytische Lösen der partiellen Differentialgleichung umgangen. Ziel ist hierbei nicht, das Risiko des Bezugsguts bzw. der Option transparenter zu gestalten. Diese Information ist bereits in dem vorausgesetzten Barwert des Bezugsguts enthalten.

Unbeschadet der Tatsache, daß sich die Risikosimulation nicht als Bewertungsund Entscheidungsinstrument eignet, leistet diese wertvolle Dienste bei der Bestimmung von Eingangs- und Bewertungsparametern. So schlußfolgern Hertz/Thomas, daß viele Leser der Originalfachartikel wohl „wrongly interpreted risk analysis as an argument in methodology about investment decisionmaking. Unfortunately, by concentrating on this aspect, some people may have missed the broader perspective of risk analysis: ... a vehicle for examining the data surrounding a decision problem (...) in the light of all the pervasive uncertainties of the world“'2. Der wahre Nutzen der Risikosimulation wird nicht auf der Bewertungs- und Entscheidungsebene, sondern auf einer, wenngleich in ihrer Bedeutung nicht minder wichtigen Ebene vor der eigentlichen Wertermittlung gesehen ${ }^{3}$.

Im folgenden wird kurz aufgezeigt, auf welche Weise die Risikosimulation im Rahmen der Realoptionsbewertung eingesetzt werden kann. Dazu sind in einem ersten Schritt die ins Realoptionsbewertungsmodell eingehenden Bewertungsparameter zu identifizieren (vgl. auch Abb. 51). Die an dieser Stelle diskutierte Simulation bietet sich insbesondere für Bewertungsparameter an, die in

1 Vgl. stellvetretend Galitz, Financial Engineering, 1994, S. 214 ff.

2 Hertz/Thomas, Risk Analysis, 1983, S. 2.

3 Vgl. hierzu auch Sautter, Strategische Analyse, 1989, S. 320 ff. 
der Sensitivitätsanalyse als kritisch eingestuft wurden ${ }^{1}$, wie z.B. das Rückflußpotential des Bezugsguts oder der konkurrenzbedingte Wertverlust. Für die diesen Bewertungsparametern zugrundeliegenden Faktoren, wie z.B. das Marktvolumen, der Marktanteil, der Absatzpreis oder die variable Kosten, werden dann in einem zweiten Schritt der Verteilungstyp sowie die für den einzelnen Verteilungstyp benötigten Verteilungsparameter festgelegt.

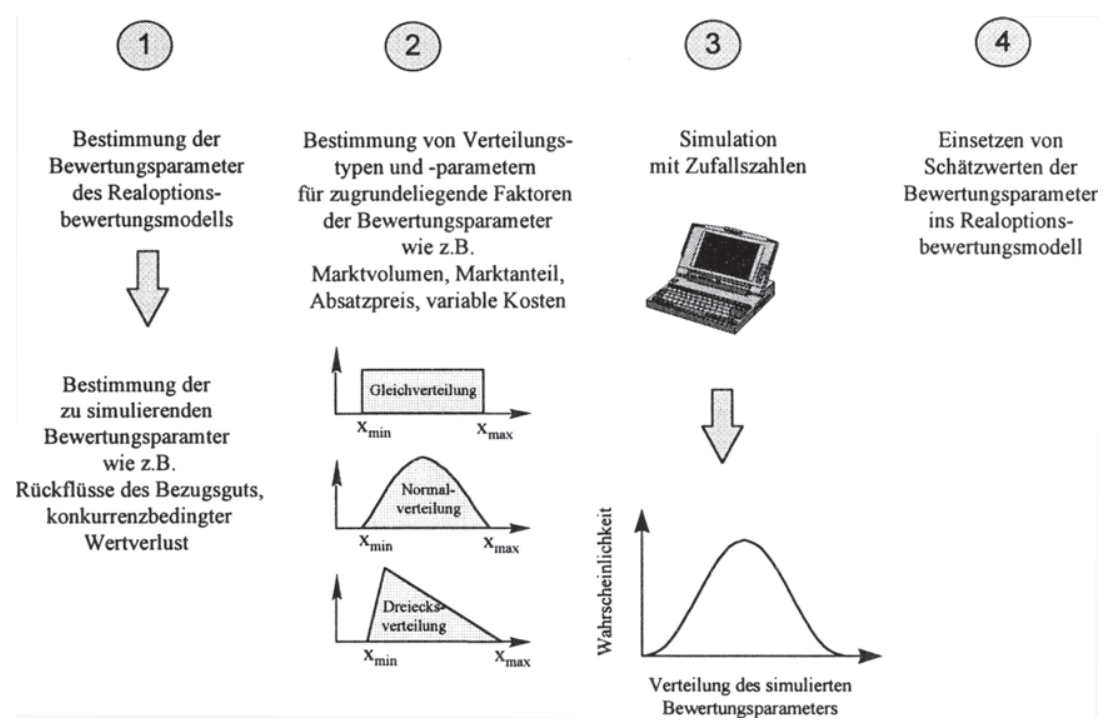

Abb. 51: Nutzen der Risikosimulation im Rahmen des Realoptionskonzepts

Anschließend lassen sich in einem dritten Schritt auf Basis eines Zufallszahlenkonzepts Verteilungen für die simulierten Bewertungsparameter aufstellen. Auf Basis dieser simulierten Verteilungen können abschließend wertvolle Informationen für die konkret ins Realoptionsbewertungsmodell einzusetzenden numerischen Schätzwerte gewonnen werden ${ }^{2}$. Beispielsweise läßt sich aus der Verteilung des Rückflußpotentials ein guter Schätzwert für die im Rahmen des Realoptionsbewertungsmodells zu verwendende Standardabweichung ablei$\operatorname{ten}^{3}$. Zudem bietet sie Hinweise zum Risikogehalt des Projekts und damit zur

1 Vgl. ähnlich Beyer von Morgenstern/Richter, Geschäftssteuerung, 1998, S. $124 \mathrm{f}$.

2 Zur Ermittlung einer Standardabweichung des Bezugsguts auf Basis der Standardabweichung des Absatzpreises vgl. z.B. Davis, Estimating Volatility, 1998, S. $728 \mathrm{ff}$.

3 Vgl. z.B. Luehrman, Getting Started, 1998, S. 58 sowie jüngst auch Hommel/Pritsch, Realoptionsansatz, 1999, S. 35 ff. 
Bestimmung des risikoadäquaten Zinsfußes, der zur Ermittlung des Barwerts des Bezugsguts gebraucht wird.

Inwieweit letztendlich die vorgeschaltete Risikosimulation $\mathrm{zu}$ einer fundierteren Realoptionsbewertung beitragen kann, ist auch davon abhängig, wie umfangreich das Struktur- und Planungsmodell der anstehenden Entscheidung modelliert wird. Je mehr Faktoren zur Beschreibung der Ausgangssituation verwendet werden, um so wertvoller dürften die aus der Simulation zu erzielenden Informationen sein.

\subsection{Integration strategischer Analyse- und Planungsinstrumente}

Im vorigen Abschnitt wurde aufgezeigt, welchen Beitrag die Risikosimulation u.a. bei der Prognose des erwarteten Rückflußpotentials leisten kann. Dabei ist von gegebenen Bandbreiten der in die Simulation eingehenden, den optionspreistheoretischen Bewertungsparametern zugrundeliegenden, Faktoren ausgegangen worden. Es wurde jedoch noch nicht diskutiert, wie solche Bandbreiten entstehen können.

Zur notwendigen Informationsbeschaffung bieten sich nahezu sämtliche strategischen Analyse- und Planungsinstrumente an, die im Rahmen der empirischen Planungsforschung (z.B. Erfahrungskurvenkonzept, Lebenszyklusanalyse, PIMS-Konzept), der Wettbewerbsanalyse (z.B. Branchenstrukturanalyse, Konkurrenzanalyse, Markt- und Portfolioanalyse) sowie der Unternehmensanalyse (z.B. Wertschöpfungskette, Stärken-/Schwächenanalyse) empfohlen werden' Mit ihrer Hilfe lassen sich einerseits Wettbewerbsfaktoren, wie z.B. die Exklusivität der Investitionsmöglichkeit, die Anzahl der Wettbewerber, die Wettbewerbsintensität oder die generelle Beeinflußbarkeit des Konkurrenzverhaltens erheben und andererseits bestehende Produktions- und Kostenstrukturen analysieren. Von besonderer Relevanz für die anschließende Umsetzung in optionspreistheoretische Bewertungsparameter können hierbei das Marktvolumen bzw. der Marktanteil, der erwartete Markteintritt der Konkurrenz sowie Vor- und Nachteile einer frühzeitigen Investition angesehen werden.

Während die Schätzung der zu leistenden Investitionsauszahlung und des risikolosen Zinsfußes als vergleichsweise unproblematisch gelten können, ist dies

$1 \mathrm{Zu}$ den Instrumenten im einzelnen vgl. Kreilkamp, Strategisches Management, 1987, S. 69 ff.; Aeberhard, Strategische Analyse, 1996, S. 115 ff.; Kreikebaum, Strategische Unternehmensplanung, 1997, S. 98 ff.; Welge/Al-Laham, Management, 1999, S. $183 \mathrm{ff}$. 
für die anderen Bewertungsparameter der Realoptionspreistheorie i.d.R. nicht der Fall. Ausgehend von der analysierten Unternehmens- und Wettbewerbssituation und den hieraus resultierenden qualitativen Strukturvariablen lassen sich jedoch eventuell quantifizierte Bandbreiten für die diesen Bewertungsparametern zugrundeliegenden Faktoren, wie z.B. Marktanteil und -volumen, gewinnen. Mit Hilfe dieser Bandbreiten und der Simulationstechnik können anschließend gemäß der im vorigen Abschnitt geschilderten Vorgehensweise Schätzwerte für die im Realoptionsbewertungsmodell benötigten Ausgangsdaten gebildet werden.

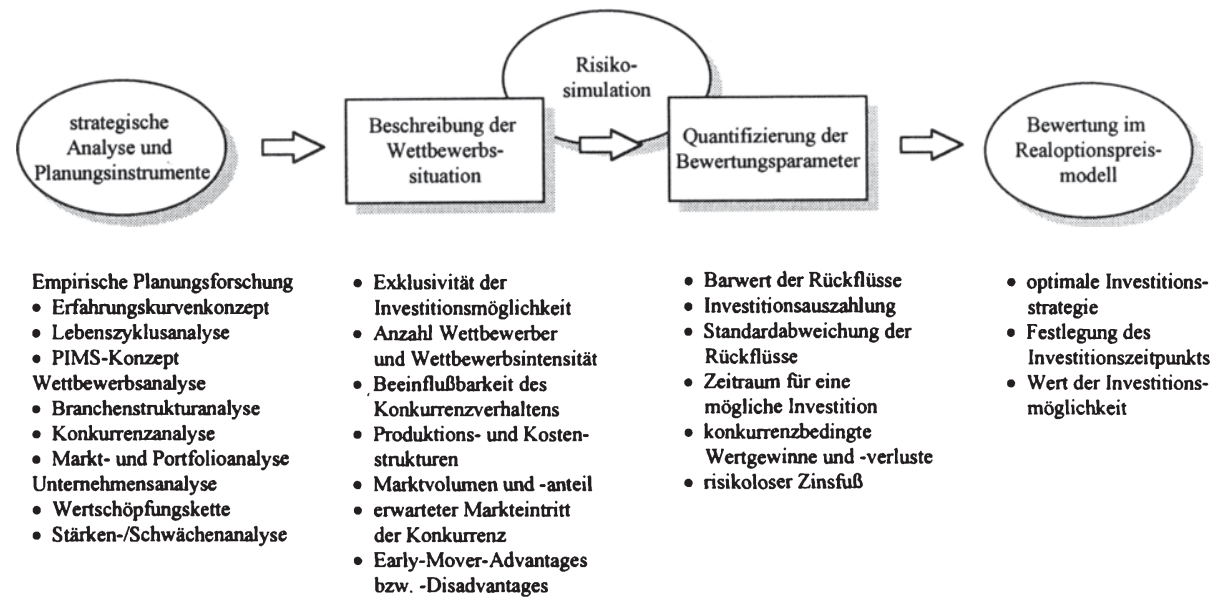

Abb. 52: Verbindung von strategischer Analyse bzw. Planung und optionspreistheoretischen Bewertungsansätzen

Gelingt die konzeptionelle Verbindung zwischen den spezifischen Wettbewerbsfaktoren einerseits und den Bewertungsparametern des verwendeten Realoptionsbewertungsmodells andererseits, lassen sich zudem, über die aus der Sensitivitätsanalyse bekannten c.p.-Wirkungen, konkrete Handlungsempfehlungen für eine mögliche Werterhöhung der Investitionsmöglichkeit ableiten ${ }^{1}$. Den an dieser Stelle nur skizzierten Grundgedanken der Einbeziehung strategischer Analyse- und Planungsinstrumente im Realoptionskalkül faßt Abb. 52 zusammen ${ }^{2}$.

1 Vgl. Leslie/Michaels, Real Options, 1997, S. 12 ff.

2 Vgl. ähnlich Meise, Realoptionen, 1998, S. 167. 


\subsection{Einbettung des Realoptionskonzepts in die wertorientierte Unter- nehmensführung}

In der Literatur zur wertorientierten Unternehmensführung werden eine Vielzahl von unterschiedlichen Steuerungsinstrumenten propagiert. Einerseits stehen einperiodige Erfolgskennzahlen ${ }^{1}$, wie z.B. EVA, EP, SHVA, CFROI, CVA, und andererseits die in Abschnitt 1.2.4 in Kapitel II dargestellten mehrperiodigen DCF-Verfahren zur Diskussion. Vor diesem Hintergrund stellt sich die Frage, in welchem Verhältnis der Realoptionsansatz zu diesen Steuerungsinstrumenten steht und ob es sich hierbei um konkurrierende oder ergänzende Ansätze zur Bewertung und Beurteilung von Geschäftsbereichen bzw. Akquisitions- und Investitionsprojekten handelt.

In den einperiodigen Erfolgskennzahlen spielen Zeitpräferenzen keine Rolle. Ziel dieser Konzepte ist es, den Wertbeitrag zu bestimmen, den das Beurteilungsobjekt innerhalb der vergangenen Periode erreicht hat bzw. in der Folgeperiode erreichen soll. Die Gemeinsamkeit dieser Instrumente liegt darin, daß sich die meisten auf die Bestimmung eines Residualeinkommens zurückführen lassen.

Wertbeitrag $=($ Kapitalrendite - Kapitalkostensatz $) \cdot$ investiertes Kapital

Bei Beurteilungsobjekten, die mit Hilfe dieser Kennzahlen gesteuert werden, sind ein Großteil der Investitionen bereits in der Vergangenheit getätigt worden. Im Rahmen der Überprüfung der letzten sowie der Planung der nächsten Periode geht es darum, die Realisierung des Erfolgs sicher zu stellen bzw. zu kontrollieren.

Demgegenüber zeichnen sich DCF-Verfahren dadurch aus, daß sie die mehrperiodige Rückflußstruktur von Beurteilungsobjekten explizit ins Kalkül einbeziehen. Aus diesem Grund sollten sie insbesondere bei aktuell zur Disposition stehenden Investitionsentscheidungen eingesetzt werden. Die in einer solchen Entscheidungssituation zwangsläufig vorhandene Unsicherheit bzgl. der Rück-

1 Zu den Konzepten im einzelnen sowie kritischen Anmerkungen vgl. Stewart, Value, 1990, S. 118 ff.; Lewis, Value Management, 1995, S. 40 ff.; Hesse, Periodischer Unternehmenserfolg, 1996, S. 121 ff.; Hachmeister, Erfolgsgröße, 1997, S. 556 ff.; Hostettler, EVA, 1997, S. 19 ff.; Dirrigl, Kollektive Investitionsrechnung, 1998, S. 18 ff.; Dirrigl, Wertorientierung und Konvergenz, 1998, S. 565 ff.; Pellens/Crasselt/Tomaszewski, Controlling, 1998, S. 891 ff.; Rappaport, Shareholder Value, 1998, S. 119 ff.; Crasselt/Pellens/Schremper, Konvergenz, 2000. 
flüsse der Investition wird über die Bildung von verschiedenen Umweltzuständen erfaßt.

Ein anderes Steuerungsinstrument wiederum erfordern Start Up-Investitionen, bei denen lediglich ein erster Meilenstein in einer Folge von Investitionserfordernissen erreicht wird. Neben der Unsicherheit der tatsächlichen Rückflüsse muß die Möglichkeit der Unternehmensleitung, flexibel auf zukünftige Umweltentwicklungen reagieren zu können, explizit ins Bewertungskalkül einbezogen werden. Dazu bedarf es eines über die traditionellen DCF-Ansätze hinausgehenden Instruments, in dem simultan zur Bewertung über den optimalen Investitionszeitpunkt entschieden wird. Ein solches Steuerungsinstrument stellt das Realoptionskonzept dar.

Während es sich innerhalb der Klasse der einperiodigen Erfolgskennzahlen bzw. derjenigen der DCF-Methoden vor allem um konkurrierende Konzepte handelt, ist der parallele Einsatz einer einperiodigen Erfolgskennzahl, eines DCF-Verfahrens und des Realoptionskonzepts in der wertorientierten Unternehmensführung nicht nur möglich, sondern wird jüngst explizit propagiert ${ }^{1}$. In Abhängigkeit der anstehenden Planungs- und Kontrollentscheidung und des Reifegrads des Beurteilungsobjekts, sollten verschiedene Steuerungsinstrumente zum Einsatz gelangen, die kombiniert dem Postulat der Wertorientierung gerecht werden ${ }^{2}$. Tab. 9 gibt die Eignung der einzelnen Steuerungsinstrumente im Hinblick auf die Berücksichtigung der Zahlungsstromorientierung, der Mehrperiodigkeit sowie der unternehmerischen Flexibilität wieder.

\begin{tabular}{|l|c|c|c|}
\hline Steueruingsinstrument & $\begin{array}{c}\text { Zahlungsstrom- } \\
\text { orientierung }\end{array}$ & Mehrperiodigkeit & $\begin{array}{c}\text { unternehmerische } \\
\text { Flexibilität }\end{array}$ \\
\hline $\begin{array}{l}\text { einperiodige wertorien- } \\
\text { tierte Erfolgskennzahl }\end{array}$ & $\checkmark$ & & \\
\hline DCF-Methode & $\checkmark$ & $\checkmark$ & \\
\hline Realoptionskonzept & $\checkmark$ & $\checkmark$ & $\checkmark$ \\
\hline
\end{tabular}

Tab. 9: Wertorientierte Steuerungsinstrumente im Vergleich

Quelle: In Anlehnung an Copeland/Keenan, Flexibility, 1998, S. 45.

1 Vgl. Beyer von Morgenstern/Richter, Geschäftssteuerung, 1998, S. 115 f. bzw. ähnlich Siegert/Böhme/Pfingsten/Picot, Lebenszyklus, 1997, S. 476 ff.; Copeland/Keenan, Flexibility, 1998, S. 45 f.

2 Zu konkreten Überlegungen einer Integration wertorientierter Planungs- und Kontrollrechnungen vgl. Dirrigl, Wertorientierung und Konvergenz, 1998, S. 553 ff.; Richter, Konzeption, 1999, S. $188 \mathrm{ff}$. 
Die Anwendung von verschiedenen Steuerungsinstrumenten führt dazu, daß ein spezifisches Investitionsprojekt bzw. ein Geschäftsbereich sowohl auf Basis von DCF- oder Realoptionsbewertungsmodellen als auch mit einperiodigen Erfolgskennzahlen beurteilt wird. Zu Beginn des Lebenszyklusses des Beurteilungsobjekts ist der Anteil des Flexibilitätswerts am Gesamtwert besonders hoch bzw. übersteigt diesen sogar (vgl. Abb. 53). Zur Steuerung sollten in diesen frühen Planungsphasen Realoptionsbewertungsmodelle eingesetzt werden ${ }^{1}$, die anschließend von mehrperiodigen DCF-Verfahren und später in der kurzfristigen Planungs- bzw. Kontrollphase von einperiodigen Erfolgskennzahlen ersetzt werden ${ }^{2}$.

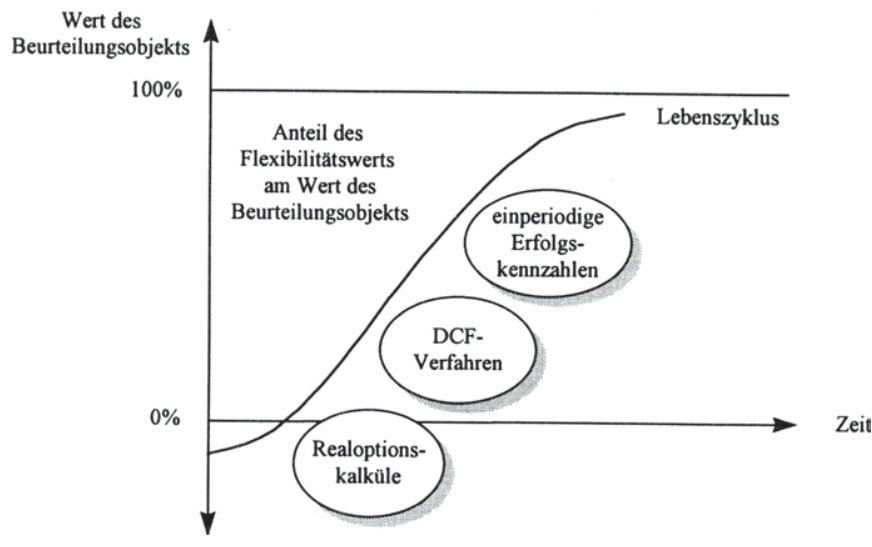

Abb. 53: Wertorientierte Steuerungsinstrumente in Abhängigkeit des Reifegrads eines Beurteilungsobjekts

Die Frage nach den einzusetzenden Steuerungsinstrumenten erfährt durch die Forderung nach einem ausgewogenen Beteiligungsportfolio, die im Rahmen der wertorientierten Unternehmensführung laut wird ${ }^{3}$, zusätzliche Brisanz. Wieviel Kapital soll in den Ausbau der bestehenden Geschäftsbereiche, den

1 Vgl. so auch Pfingsten, Lebenszyklus, 1998, S. 139.

2 Grundsătzlich kommt der Einsatz von Realoptionsbewertungsmodellen auch während und gegen Ende des Lebenszyklusses in Betracht, wenn es z.B. um die Bewertung von Erweiterungs-, Umstellungs- oder Desinvestitionsoptionen geht. Vergleichsweise dürften diese jedoch tendenziell einen geringeren Wert des Gesamtwerts ausmachen.

3 Vgl. stellvertretend Trigeorgis/Kasanen, Planning and Control Model, 1991, S. 17; Beyer von Morgenstern/Richter, Geschäftssteuerung, 1998, S. $126 \mathrm{f}$. 
Aufbau neuer Geschäftsbereiche bzw. zur Förderung von Geschäftsoptionen investiert werden ${ }^{1}$ ?

Das Streben nach einem solchen Beteiligungsportfolio bedingt neben neuen Planungsinstrumenten auch Veränderungen für die laufende Beurteilung bereits durchgeführter Investitionen ${ }^{2}$. Da es einerseits verständlich ist, daß Maßnahmen, die den persönlichen Interessen des untergeordneten Entscheidungsträgers entgegenstehen, von diesem nicht initiiert werden und andererseits die im Rahmen der Planung verwendeten Steuerungsinstrumente „Kapital- bzw. Realoptionswert" für Kontrollzwecke ungeeignet erscheinen, muß ein zu der modifizierten Planung im Einklang stehendes Kontroll- und Anreizsystem geschaffen werden ${ }^{3}$.

Im Hinblick auf die Umsetzung der Realoptionsbewertung in der Unternehmenspraxis bedeutet dies konkret, daß der im Rahmen des Realoptionskalküls optimal ermittelte Investitionszeitpunkt überwacht werden muß und in der Kontrollrechnung danach zu differenzieren ist, welche Geschäfts- bzw. Investitionsoptionen tatsächlich wahrgenommen wurden ${ }^{4}$. Eine ähnliche Idee entwickelt Agmon, der den tatsächlich realisierten Rückflüssen die gemäß dem bestimmten Umweltzustand geplanten - und nicht die über alle Umweltzustände hinweg erwarteten - Rückflüsse gegenüberstellt. Dies würde zu einem Steuerungsansatz bedingter Kennzahlen führen. Der Autor merkt jedoch selbst an, daß die Umsetzung und insbesondere der Steuerungserfolg eines solchen Konzepts davon abhängt, inwieweit der eingetretene Umweltzustand identifiziert werden $\mathrm{kann}^{5}$. An dieser Stelle zeigt sich ein erheblicher Forschungsbedarf, geeignete Kontroll- und Anreizsysteme zu schaffen, die den Planungsansatz der Realoptionsbewertung „begleiten“.

1 Für den realistischen Fall, daß heutige Investitionen einen Einfluß auf die Verfügbarkeit und den Wert zukünftiger Investitionsmöglichkeiten haben, vgl. Kasanen, Investment Opportunities, 1993, S. 252 ff. Zur Beantwortung dieser Fragen auf Basis qualitativer Aussagen des Realoptionskonzepts vgl. Luehrmann, Portfolio of Real Options, 1998, S. $89 \mathrm{ff}$.

2 Vgl. Kasanen/Trigeorgis, Flexibility and Control, 1993, S. $221 \mathrm{ff}$.

3 Bei Interessenskonflikten kann die Existenz von unternehmerischen Handlungsspielräumen auch einen - im Gegensatz zu den Annahmen dieser Arbeit - negativen Einfluß auf den Unternehmenswert ausüben. Vgl. hierzu auch Krahnen/Schmidt/Terberger, Flexibilität, 1985, S. 279.

4 Vgl. hierzu Trigeorgis/Kasanen, Planning and Control Model, 1991, S. 23.

5 Vgl. Agmon, Performance Evaluation and Real Options, 1993, S. 236 und S. 244. 


\section{Empirische Studien}

Nachdem in Abschnitt 4 dieses Kapitels auf Instrumente eingegangen worden ist, die eine praktische Umsetzung des Realoptionskalküls unterstützen, gewährt der nun folgende Abschnitt einen Einblick in bislang vorliegende empirische Ergebnisse. Hierbei wird ausschließlich auf Studien zurückgegriffen, die dem betriebswirtschaftlich ${ }^{1}$ orientierten Forschungsbereich der "real options“ zuzurechnen sind.

\subsection{Erklärungsgehalt von Realoptionsbewertungsmodellen}

\subsubsection{Untersuchung von Paddock/Siegel/Smith}

Die erste umfassendere Untersuchung zum Erklärungsgehalt von Realoptionsbewertungsmodellen stammt von Paddock/Siegel/Smith ${ }^{2}$. Die Autoren analysieren 21 von der US-amerikanischen Regierung im Jahre 1980 an die Industrie verkaufte Erschließungs- und Förderungsrechte. Beim Kauf eines dieser Rechte erhält das einzelne Unternehmen die exklusive Möglichkeit, innerhalb der nächsten fünf Jahre ein bestimmtes Petroleumfeld zu erschließen sowie die vermuteten Öl- und Gasreserven zu fördern. Wird das Unternehmen innerhalb dieser fünf Jahre nicht aktiv, erlischt das Erschließungs- und Förderungsrecht. Die Verfügungsgewalt liegt wieder auf Staatsebene.

Welchen Preis soll die Regierung für den Verkauf eines solchen Rechts verlangen bzw. welchen Preis sind die Industrieunternehmen bereit zu zahlen? Welchen Beitrag liefern Optionspreismodelle zur Bestimmung des Werts dieser Investitionsmöglichkeit? Die Autoren gehen diesen Fragen nach, indem sie die vom U.S. Geological Survey auf Basis von DCF-Methoden errechneten Werte mit den eigenen auf Basis von Optionspreismodellen ermittelten Ergebnissen vergleichen. Dabei greifen sie auf die gleiche, bereits von der US-Behörde verwendete, Datenbasis zurück ${ }^{3}$.

1 Zu empirischen Ergebnissen des volkswirtschaftlich orientierten Forschungsbereichs vgl. den Überblick bei Dixit/Pindyck, Investment under Uncertainty, 1994, S. 423 ff. sowie Sakellaris, Uncertain Market Conditions, 1995, S. 460 ff.; Caballero/Pindyck, Uncertainty, 1996, S. 65 ff.; Seppelfricke, Investitionstheorien unter Unsicherheit, 1996, S. 149 ff.

2 Vgl. Paddock/Siegel/Smith, Option Valuation, 1988, S. 494 ff.

3 Im Detail sind dies die subjektive Wahrscheinlichkeit, daß das betrachtete Feld nicht „trocken“ ist, Mittelwert und Varianz der vermuteten Öl- und Gasreserven sowie erwartete Erschließungs- und Förderungskosten. 
Anschließend stellen sie diesen beiden Wertansätzen die von den Unternehmen abgegebenen Preisangebote gegenüber. Bei diesem Vergleich ist zu beachten, daß sich die Prognosen bezüglich der Öl- und Gasmengen sowie der ErschlieBungs- und Förderungskosten erheblich unterscheiden können, wie auch der Korrelationskoeffizient zwischen den gegenübergestellten Ergebnissen nahelegt ${ }^{1}$. Von großem Interesse wäre ein Vergleich zwischen den von den Unternehmen vorgenommenen Bewertungen und solchen auf Basis der Optionspreistheorie, die auf den gleichen Prognosen basieren. Die Autoren bemängeln selbst, daß sie diesen Vergleich aufgrund fehlender Unternehmensdaten nicht durchführen können. Zudem darf nicht unberücksichtigt bleiben, daß es sich bei den Unternehmensangeboten nicht um Preisobergrenzen, sondern um Angebotspreise und somit um konservative Schätzwerte für den von den Unternehmen eingeschätzten (Maximal-) Wert dieser Rechte handelt.

\begin{tabular}{|l|c|c|}
\hline $\begin{array}{l}\text { Bewertungsmethode bzw. } \\
\text { Unternehmensangebote }\end{array}$ & $\begin{array}{c}\text { Mittelwert des } \\
\text { Untersuchungssamples } \\
\text { (in Mio. \$) }\end{array}$ & $\begin{array}{c}\text { Standardabweichung } \\
\text { des Untersuchungssamples }\end{array}$ \\
\hline $\begin{array}{l}\text { Optionsbewertung mit 12 \$ pro Öl- } \\
\text { einheit sowie 3 \$ pro Gaseinheit }\end{array}$ & 8,20 & 9,42 \\
\hline $\begin{array}{l}\text { Optionsbewertung mit 12 \$ pro Öl- } \\
\text { einheit sowie 2 \$ pro Gaseinheit }\end{array}$ & 4,13 & 5,56 \\
\hline $\begin{array}{l}\text { Discounted Cash Flow- } \\
\text { Methode }\end{array}$ & 4,93 & 6,32 \\
\hline $\begin{array}{l}\text { Höchstpreis der } \\
\text { Unternehmensangebote }\end{array}$ & 18,95 & 16,07 \\
\hline $\begin{array}{l}\text { Durchschnittspreis der } \\
\text { Unternehmensangebote }\end{array}$ & 6,03 & 3,58 \\
\hline
\end{tabular}

Tab. 10: Vergleich von Bewertungsmethoden und Unternehmensangeboten im Rahmen des Verkaufs von Rechten zur Erschließung und Förderung eines Petroleumfelds

Quelle: Paddock/Siegel/Smith, Option Valuation, 1988, S. 502

Die Ergebnisse von Paddock/Siegel/Smith hängen vom angenommenen Preis für eine Einheit gefördertes Gas ab. Gehen die Autoren von einem in Gesprächen mit Investmentbankern festgestellten Preis von 3 \$ aus, liegt der Mittelwert des Untersuchungssamples auf Basis von Optionspreismodellen bei 8 Mio. \$ und damit rund 3 Mio. \$ über demjenigen auf Basis von DCF-Verfahren von 5 Mio. \$. Wird hingegen eine strikte 1:6 (Gleichgewichts-) Relation zwi-

1 Während zwischen den Ergebnissen der US-Behörde und den OPT-Werten ein Korrelationskoeffizient von $\mathbf{9 8 \%}$ besteht, beträgt dieser zwischen den Unternehmensangeboten und den Werten der US-Behörde bzw. den OPT-Ergebnissen lediglich 39\%. Vgl. Paddock/Siegel/Smith, Option Valuation, 1988, S. $501 \mathrm{ff}$. 
schen dem Öl- (12\$) und dem Gaspreis (2\$) vorausgesetzt, unterschreitet der Optionswert knapp den DCF-Wert (vgl. Tab. 10) ${ }^{1}$.

Unabhängig hiervon beläuft sich der Mittelwert des Untersuchungssamples der von der Industrie abgegebenen Durchschnittspreise auf 6 Mio. \$, während der Mittelwert der Höchstpreise fast 19 Mio. \$ beträgt. Dieses Ergebnis könnte als Indiz gewertet werden, daß Unternehmen für den „eingekauften“ unternehmerischen Handlungsspielraum eine Sonderprämie bezahlen. $\mathrm{Ob}$ in der vorliegenden Untersuchung Realoptionsbewertungsmodelle diese eher erfassen als DCFVerfahren hängt - wie soeben dargestellt - vom eingesetzten Gaspreis ab.

\subsubsection{Untersuchung von Quigg}

Quigg analysiert 2.734 Grundstückskäufe, die zwischen Mitte 1976 und Ende 1979 in Seattle zu Investitionszwecken stattfanden ${ }^{2}$. Das erworbene Grundstück wird dabei als Option zum Bau eines Gebäudes aufgefaßt. Als Bezugsgut fungiert der Wert des auf dem Grundstück eventuell errichteten Bauwerks. Auf Basis eines zeitstetigen Bewertungsansatzes mit unendlicher Laufzeit ermittelt die Autorin sowohl den inneren als auch den Optionswert des Grundstücks ${ }^{3}$. Die Eingangsgrößen des Bewertungsmodells werden aus den Charakteristika von 3.200 bereits bebauten Flächen gewonnen.

Das untersuchte Sample wird von Quigg in drei Perioden (1976/77, 1978 bzw. 1979) sowie fünf Investitionszwecke (Business, Commercial, Industrial, LowDensity Residential und High-Density Residential) eingeteilt. Wie Tab. 11 zeigt, bewegen sich die Flexibilitätsprämien zwischen $1 \%$ und $30 \%$, wobei die höchsten bei Grundstückskäufen zu Industriezwecken auftreten. Die Autorin argumentiert, daß die von ihr ermittelten Werte eher als untere Grenze anzusehen sei, da der untersuchte Zeitraum als Expansionszeit gelte. Bei moderaterem

1 Vgl. Paddock/Siegel/Smith, Option Valuation, 1988, S. 496 i.V.m. S. 503. Vgl. auch Davis, Option Premiums, 1996, S. 179 f., der anhand von sieben veröffentlichten Fallstudien zur Bewertung von Ölfeldern schlußfolgert, daß der Optionswert den DCF-Wert um $41 \%$ übersteigt und damit rund die Hälfte der Differenz zwischen DCF- und Marktwert erklärt. Jedoch beläuft sich der ermittelte Optionswert auf lediglich acht Prozent der Investitionskosten und auf nur zwei bis drei Prozent des Bezugsguts.

2 Vgl. Quigg, Empirical Testing, 1993, S. $621 \mathrm{ff}$.

3 Der innere Wert des Grundstücks ist jener Wert, der sich bei sofortiger Bebauung ergibt. 
Wachstum mit im Durchschnitt weniger baulichen Vorhaben wären höhere Flexibilitätsprämien zu vermuten ${ }^{1}$.

\begin{tabular}{|l|c|c|c|c|}
\hline Subsample & Anzahl & $\begin{array}{c}\text { Mittelwert der } \\
\text { Optionswerte } \\
\text { (in Tsd. \$) }\end{array}$ & $\begin{array}{c}\text { Mittelwert der } \\
\text { inneren Werte } \\
\text { (in Tsd. \$) }\end{array}$ & $\begin{array}{c}\text { Mittelwert der } \\
\text { Flexibilitäts- } \\
\text { prämien in \% }\end{array}$ \\
\hline Business & 76 & 30,5 & 29,1 & 3,8 \\
$1976 / 77$ & 64 & 115,1 & 112,6 & 2,2 \\
1978 & 48 & 84,8 & 75,0 & 4,4 \\
1979 & 102 & 144,2 & 136,8 & 5,2 \\
\hline Commercial & 90 & 180,2 & 177,7 & 0,9 \\
$1976 / 77$ & 73 & 184,5 & 171,8 & 2,6 \\
1978 & & & & \\
1979 & 62 & 146,7 & 122,1 & 29,8 \\
\hline Industrial & 43 & 337,2 & 291,9 & 17,6 \\
$1976 / 77$ & 25 & 147,8 & 140,7 & 2,2 \\
1978 & & & & 4,9 \\
1979 & 490 & 90,1 & 84,6 & 11,2 \\
\hline Low-Density Residential & 401 & 47,2 & 44,0 & 1,2 \\
$1976 / 77$ & 340 & 51,1 & 49,2 & 10,4 \\
1978 & & & & 5,9 \\
1979 & 224 & 58,1 & 54,1 & 1,9 \\
\hline High-Density Residential & 336 & 41,0 & 37,5 & \\
$1976 / 77$ & 360 & 51,2 & 48,4 & \\
1978 & & & & \\
1979 & & & & \\
\hline
\end{tabular}

Tab. 11: Flexibilitätsprämien bei Grundstückskäufen

Quelle: Quigg, Empirical Testing, 1993, S. 636.

Um den Erklärungsgehalt von Bewertungsmodellen für tatsächlich gezahlte Kaufpreise zu messen, werden letztere in einer Regressionsanalyse als abhängige Variable eingesetzt. In einer ersten Regression wird der errechnete Optionswert und in einer zweiten Regression der ermittelte innere Wert als unabhängige Variable verwendet ${ }^{3}$. Der Erklärungsgehalt der ersten Regression ist zum Teil höher, zum Teil aber auch niedriger als derjenige der zweiten Regression, so daß hier kein zusätzlicher Erklärungsgehalt des Optionspreismodells feststellbar ist (vgl. Tab. 12). In einer dritten Variante setzt Quigg den inneren

1 Vgl. Quigg, Empirical Testing, 1993, S. 635.

2 Die Flexibilitätsprämie in \% ist wie folgt definiert: [(Optionswert - innerer Wert) / Optionswert] $\cdot 100$.

3 Auf eine Diskussion zur Zweckmäßigkeit einer solchen Vorgehensweise wird an dieser Stelle nicht eingegangen. 
Wert und die Differenz zwischen Options- und innerem Wert als unabhängige Variablen in die Regression ein. Der Erklärungsgehalt dieser dritten Regression übersteigt denjenigen der zweiten Regression in allen Subsamples, so daß hier ein - wenn auch geringer - zusätzlicher, über den inneren Wert hinausgehender Erklärungsgehalt des Realoptionswerts vermutet werden kann.

\begin{tabular}{|l|c|c|c|}
\hline unabhängige Variablen & Optionswert & innerer Wert & $\begin{array}{c}\text { innerer Wert sowie } \\
\text { (Optionswert - innerer Wert) }\end{array}$ \\
\hline Business & 96,3 & 97,5 & 98,5 \\
1977 & 93,2 & 94,5 & 95,6 \\
1978 & 96,0 & 97,4 & 97,9 \\
1979 & 96,1 & 96,0 & 96,9 \\
\hline Commercial & 94,5 & 93,5 & 94,2 \\
1977 & 90,0 & 87,8 & 89,8 \\
1978 & & & \\
1979 & 93,6 & 96,0 & 98,7 \\
\hline Industrial & 98,7 & 97,4 & 99,4 \\
1977 & 96,0 & 92,8 & 96,0 \\
1978 & & & 96,8 \\
1979 & 86,0 & 96,4 & 97,2 \\
\hline Low-density residential & 94,5 & 93,8 & 98,8 \\
1977 & 98,1 & 98,2 & 97,1 \\
1978 & & & 45,8 \\
1979 & 96,3 & 94,1 & 95,4 \\
\hline High-density residential & 44,9 & 41,2 & 92,1 \\
1977 & 94,4 & & \\
1978 & & & \\
1979 & & & \\
\hline
\end{tabular}

Tab. 12: Bestimmtheitsmaß $R^{2}$ von Regressionsanalysen zur Überprüfung der Relevanz von Realoptionsbewertungsmodellen bei Grundstückskäufen (in \%)

Quelle: Quigg, Empirical Testing, 1993, S. 637 und S. 638.

\subsection{Existenz von Realoptionswerten: Untersuchung von Kester}

Wie bereits in Abschnitt 4.3 in Kapitel II erwähnt, kann der Unternehmenswert als Summe von zwei verschiedenen Vermögensarten aufgefaßt werden. Einerseits besitzt ein Unternehmen Vermögenspositionen, dessen Rückflüsse nicht von zukünftigen Investitionen abhängen und anderseits solche, dessen Rückflüsse erst in Abhängigkeit zukünftiger Investitionsauszahlungen realisiert wer- 
den. Kester nutzt hierfür die Begriffe ,assets in place“ und „growth opportunities ${ }^{\alpha 1}$.

Über einen Vergleich zwischen dem aktuellen Börsenwert und erwarteten Gewinnen, die mit Hilfe der Kapitalkosten auf den gleichen Zeitpunkt abgezinst werden, versucht der Autor, einen Schätzwert für den Anteil der Wachstumsoptionen am Unternehmenswert zu erhalten ${ }^{2}$. Der für die nächste Periode erwartete Gewinn wird dabei als unendliche Rente aufgefaßt und auf Basis der Prognosen von Finanzanalysten ermittelt.

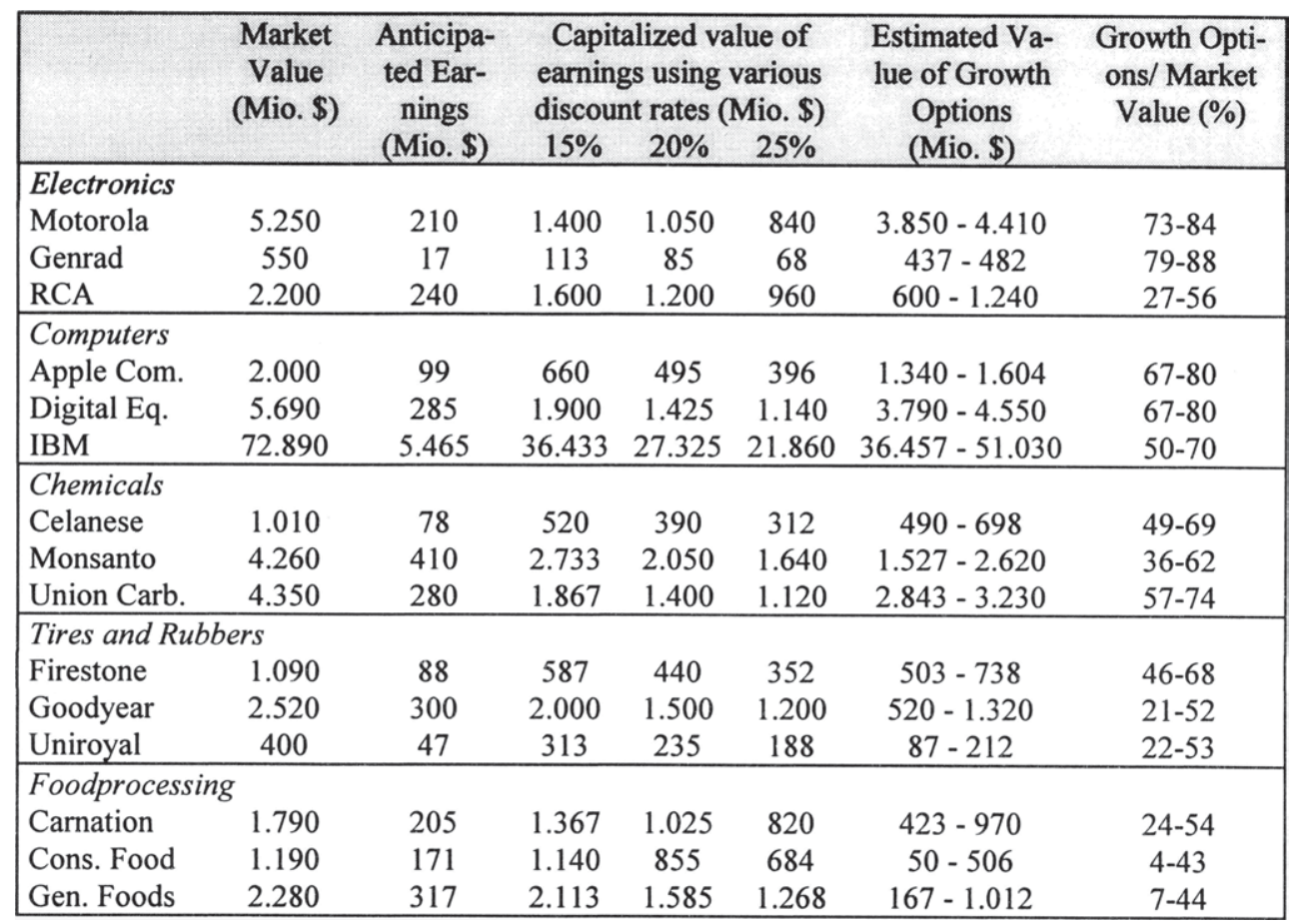

Tab. 13: Vermuteter Anteil der Wachstumsoptionen am Unternehmenswert

Quelle: Kester, Today's Options, 1984, S. 155.

In Tab. 13 fällt zum einen auf, daß der geschätzte Wertanteil der Wachstumsoptionen am Unternehmenswert in vielen Fällen nahezu die Hälfte des Bör-

1 Vgl. Kester, Today's Options, 1984, S. 154 f.

2 Eine solche Vorgehensweise setzt voraus, daß die Differenz zwischen kapitalisierten Gewinnen und dem Marktwert nicht auf sonstige börsenspezifische Vorgänge zurückzuführen ist. 
senwerts ausmacht. Zum anderen scheint dieser Anteil von der Zugehörigkeit des Unternehmens zu einer bestimmten Branche beeinflußt. Der intuitiv vermutete Zusammenhang eines höheren Anteils bei Unternehmen in der Computer- und Elektroindustrie kann bestätigt werden.

Kester wählt ausschließlich große Unternehmen aus, deren Werttitel am Kapitalmarkt stark gehandelt werden. Der Autor selbst vermutet, daß der untersuchte Wertanteil bei kleineren, äußerst innovativen und wachstumsträchtigen Firmen noch höher liegt. Werden bei einem solchen Unternehmen sogar heute und in naher Zukunft keine positiven Rückflüsse erwartet, läßt sich ein positiver Unternehmenswert lediglich über den Wert der vermuteten Wachstumsoptionen erklären.

\subsection{Zum Verständnis und Einsatz der Realoptionstheorie in der Unternehmenspraxis}

\subsubsection{Untersuchung von Busby/Pitts}

Busby/Pitts sendeten 1995 einen Fragebogen an alle hundert britische Unternehmen, die dem FT-SE 100 Index angehören ${ }^{1}$. Darin wurden die Finanzvorstände gebeten, zur Bedeutung unternehmerischer Flexibilität, zur Existenz von expliziten Analysetechniken bzw. organisatorischen Vorkehrungen bei der Beurteilung der unternehmerischen Flexibilität, zu den aus der Sensitivitätsanalyse bekannten optionspreistheoretischen ceteris paribus-Aussagen ${ }^{2}$ sowie zur Notwendigkeit eines expliziten Bewertungsinstruments Stellung zu nehmen.

Von den 100 versandten Fragebogen sind 44 beantwortet worden. Tab. 14 zeigt, daß die im Rahmen dieser Arbeit in den Mittelpunkt gerückten Aufschubs- bzw. Wachstumsoptionen besonders häufig vorkommen. Rund die Hälfte der Befragten ( $46 \%$ bzw. $54 \%$ ) berichteten, daß diese Realoptionen in über $60 \%$ der Investitionsfälle auftreten. Um so erstaunlicher ist es, daß hierfür i.d.R. (80\% bzw. $75 \%)$ keine expliziten Analysemethoden bzw. organisatorischen Vorkehrungen in den Unternehmen vorhanden sind.

In Bezug auf die Investitionsauszahlung und die Laufzeit einer Investitionsmöglichkeit standen die Antworten der Unternehmensvertreter im Einklang mit den in der Optionspreistheorie postulierten Zusammenhängen. Im Hinblick auf

Vgl. Busby/Pitts, Real Options in Practice, 1997, S. $173 \mathrm{ff}$.

2 Vgl. Abschnitt 4.1.1.1 dieses Kapitels. 
den risikolosen Zinsfuß und das Ausmaß der Unsicherheit waren die Meinungen eher heterogen. Insbesondere letzteres Ergebnis läßt sich eventuell durch den von einer Variation der Standardabweichung ausgehenden „Doppeleffekt“ erklären ${ }^{1}$.

\begin{tabular}{|c|c|c|c|c|c|}
\hline $\begin{array}{c}\text { Art der } \\
\text { Realoption }\end{array}$ & Aufschub & Aufgabe & $\begin{array}{l}\text { Erweiterung/ } \\
\text { Einschränkung }\end{array}$ & $\begin{array}{l}\text { technischer } \\
\text { Wandel }\end{array}$ & Wachstum \\
\hline \multicolumn{6}{|c|}{ Häufigkeit der Existenz unternehmerischer Flexibilität in Investitionsprojekten } \\
\hline über $80 \%$ & 30 & 5 & 14 & 7 & 26 \\
\hline über $60 \%$ & 46 & 14 & 30 & 17 & 54 \\
\hline über 40\% & 62 & 23 & 46 & 29 & 66 \\
\hline über $20 \%$ & 78 & 51 & 69 & 58 & 87 \\
\hline \multicolumn{6}{|c|}{$\begin{array}{l}\text { Existenz von expliziten Analysemethoden bzw. organisatorischen Vorkehrungen } \\
\text { zur Beurteilung der unternehmerischen Flexibilität }\end{array}$} \\
\hline $\mathrm{Ja}$ & 20 & 14 & 43 & - & 25 \\
\hline Nein & 80 & 86 & 57 & - & 75 \\
\hline
\end{tabular}

Tab. 14: Existenz sowie explizite Beurteilung unternehmerischer Flexibilität im Rahmen von Investitionsprojekten (in \% der Befragten)

Quelle: $\quad$ Busby/Pitts, Real Options in Practice, 1997, S. 174 und S. 176.

Rund die Hälfte der Unternehmensvertreter sprachen davon, daß systematische Methoden zur Beurteilung und Bewertung von unternehmerischer Flexibilität durchaus eine Notwendigkeit für ihr Unternehmen darstellen, jedoch hatten nur sehr wenige von der seit Mitte der 80'er Jahre stattfindenden Forschung zur optionspreistheoretischen Bewertung von Investitions- und Desinvestitionsmöglichkeiten gehört. Nur zwei Firmen prüften zum Befragungszeitpunkt die konkrete Anwendung des Realoptionskonzepts. Zwei weitere Befragte argumentierten, daß die vorhandenen Bewertungsmodelle für eine etwaige Anwendung in der Unternehmenspraxis benutzerfreundlicher konzipiert werden müssen.

\subsubsection{Untersuchung von HowellJägle}

Howell/Jägle führten zwischen Februar und Juni 1995 einen Feldversuch bei 82 Managern aus neun britischen Großunternehmen der Öl-, Flugzeug-, Telekommunikations-, Pharma- und Brauereiindustrie durch ${ }^{2}$. Neben einem umfangreichen Fragebogen zu qualitativen Aussagen und zur Häufigkeit von

Vgl. Abschnitt 4.1.1.2 dieses Kapitels.

2 Vgl. Howell/Jägle, Laboratory Evidence, 1997, S. 917 ff. 
Wachstumsoptionen sowie zum beruflichen Hintergrund der Befragten wurden den Entscheidungsträgern vierzehn Fallstudien präsentiert ${ }^{1}$, anhand derer sie hypothetische Investitionsentscheidungen treffen mußten.

Jede Fallstudie beschreibt ein Pilotprojekt, an das sich nach einem gewissen Zeitraum eventuell ein Folgeprojekt anschließen läßt. Das Pilotprojekt verursacht heute eine Investitionsauszahlung $I_{0}$, der noch keine Rückflüsse gegenüberstehen. Für die Folgeinvestitionsmöglichkeit ist der (aktuelle) Barwert $V_{0}$ der bei Investition $I_{l}$ entstehenden Rückflüsse angegeben. Die Volatilität $\sigma$ wird über die Spannbreite des zum möglichen Investitionszeitpunkt $t$ erwarteten Gegenwartswerts dieser Rückflüsse vermittelt.

Für die jeweiligen Fälle (vgl. Tab. 15), die durch einen unterschiedlichen Barwert des Bezugsguts (A1 - A7), eine unterschiedliche Volatilität (B1 - B5) oder eine unterschiedliche Laufzeit der Folgeinvestitionsmöglichkeit $(\mathrm{C} 1-\mathrm{C} 2)$ differieren, wurden die Befragten gebeten, den Gesamtwert von Pilot- und Folgeprojekt zu beziffern ${ }^{2}$. Die Ergebnisse aus $\mathrm{C} 1$ und $\mathrm{C} 2$ werden im folgenden nicht aufgeführt, da hier im Gegensatz zu den anderen Fallstudien gleichzeitig auch andere Bewertungsparameter modifiziert worden sind und eine entsprechende Interpretation somit umso schwieriger fällt. Der von den Entscheidungsträgern pro Fallstudie im Durchschnitt ermittelte Wert der Wachstumsoption wird im folgenden unter der Bezeichnung „empirisches Ergebnis“ wiedergegeben.

In zehn von zwölf Fällen übersteigt das empirische Ergebnis den traditionellen Kapitalwert. Dies zeigt, daß die Befragten ihre Investitionsentscheidung über das Folgeprojekt keineswegs ausschließlich anhand dieses Kriteriums begründen. Im Vergleich zu den theoretischen Optionswerten ist das empirische Ergebnis sogar in sieben Fällen signifikant größer und nur in drei Fällen signifikant kleiner. Werden zur Analyse der relativen Abweichung die Fälle A1, A2, $\mathrm{B} 1$ und $\mathrm{B} 2$ aufgrund der geringen absoluten Beträge und der damit verbundenen großen Hebelwirkung als Extremfälle ausgeschlossen, resultiert eine (ungewichtete) relative Überbewertung von $11 \%$. Dies könnte als Indiz gewertet werden, daß die Entscheidungsträger die Wachstumsoption annäherungs-

1 Aus Zeitgründen wurden dem einzelnen Teilnehmer lediglich drei der insgesamt vierzehn Fallstudien vorgelegt.

2 Durch Abzug der Investitionskosten des Pilotprojekts $I_{0}$ resultiert der vom Entscheidungsträger geschätzte Wert der Wachstumsoption. 
weise korrekt beurteilen ${ }^{1}$. Die Autoren selbst merken jedoch an, daß die Einzelergebnisse extrem weit streuen und damit dieses Ergebnis vorsichtig zu interpretieren sei $^{2}$.

\begin{tabular}{|c|c|c|c|c|c|c|c|}
\hline case study & A1 & $\mathrm{A} 2$ & A3 & A4 & A5 & A6 & A7 \\
\hline present value of CF's $V_{0}$ & 2,5 & 5 & 10 & 15 & 20 & 25 & 30 \\
\hline volatility $\sigma$ & 0,45 & 0,45 & 0,45 & 0,45 & 0,45 & 0,45 & 0,45 \\
\hline maturity $t$ & 3 & 3 & 3 & 3 & 3 & 3 & 3 \\
\hline investment cost $I_{l}$ & 12 & 12 & 12 & 12 & 12 & 12 & 12 \\
\hline theoretical option value * & 0,1 & 0,5 & 3,0 & 6,8 & 11,1 & 15,7 & 20,4 \\
\hline net present value $* *$ & $-7,5$ & -5 & 0 & 5 & 10 & 15 & 20 \\
\hline intrinsic value & 0 & 0 & 0 & 3 & 8 & 13 & 18 \\
\hline time value & 0,1 & 0,5 & 3 & 3,8 & 3,1 & 2,7 & 2,4 \\
\hline empirical valuation & 0 & 1,7 & 4 & 6,3 & 15,4 & 11,4 & 13,8 \\
\hline discrepancy & $-0,1$ & 1,2 & 1,0 & $-0,5$ & 4,3 & $-3,3$ & $-6,6$ \\
\hline $\begin{array}{l}\text { discrepancy/theoretical } \\
\text { option value (\%) }\end{array}$ & -133 & 240 & 33 & -7 & 39 & -21 & -32 \\
\hline significance $* * *$ & ns & $\mathrm{s}$ & $\mathrm{s}$ & ns & $\mathrm{s}$ & $\mathrm{s}$ & $\mathrm{s}$ \\
\hline sample size & 17 & 18 & 20 & 19 & 18 & 15 & 17 \\
\hline case study & B1 & B2 & $\overline{A 3}$ & B3 & B4 & B5 & \\
\hline present value of CF's $V_{0}$ & 10 & 10 & 10 & 10 & 10 & 10 & \\
\hline volatility $\sigma$ & 0,17 & 0,32 & 0,45 & 0,58 & 0,70 & 0,81 & \\
\hline maturity $t$ & 3 & 3 & 3 & 3 & 3 & 3 & \\
\hline initial investment cost $I_{I}$ & 12 & 12 & 12 & 12 & 12 & 12 & \\
\hline theoretical option value ${ }^{*}$ & 1,2 & 2,2 & 3,0 & 3,8 & 4,6 & 5,2 & \\
\hline net present value $* *$ & 0 & 0 & 0 & 0 & 0 & 0 & \\
\hline intrinsic value & 0 & 0 & 0 & 0 & 0 & 0 & \\
\hline time value & 1,2 & 2,2 & 3 & 3,8 & 4,6 & 5,2 & \\
\hline empirical valuation & 3,2 & 6,5 & 4,0 & 4,8 & 8,0 & 4,1 & \\
\hline discrepancy & 2 & 4,3 & 1 & 1 & 3,4 & $-1,1$ & \\
\hline $\begin{array}{l}\text { discrepancy/theoretical } \\
\text { option value }(\%)\end{array}$ & 167 & 195 & 33 & 26 & 74 & -21 & \\
\hline significance $* * *$ & $\mathrm{~s}$ & $\mathrm{~s}$ & $\mathrm{~s}$ & $\mathrm{~s}$ & $\mathrm{~s}$ & $\mathrm{~s}$ & \\
\hline sample size & 17 & 12 & 20 & 16 & 17 & 17 & \\
\hline
\end{tabular}

$* r_{f}=6 \% * * V_{0}-e^{-r_{f} \cdot t * *}(\mathrm{n}) \mathrm{s}=(\mathrm{not})$ significant at $5 \%$ level of significance

Tab. 15: Bewertungsparameter, theoretischer Optionswert sowie empirisches Ergebnis der einzelnen Fallstudien

Quelle: Howell/Jägle, Laboratory Evidence, 1997, S. 923.

1 In einem weiteren Schritt könnte gar vermutet werden, daß die Befragten die Wachstumsoption anhand der traditionellen flexiblen Investitionsplanung mit einem konstanten ,üblichen“ risikoadjustierten Zinsfuß bewertet haben. Dies würde zu der festgestellten Überbewertung fuhren.

2 Vgl. Howell/Jägle, Laboratory Evidence, 1997, S. 929. 
Durch eine Gegenüberstellung der einzelnen Fallstudienergebnisse lassen sich die in der Optionspreistheorie postulierten ceteris paribus-Beziehungen überprüfen. Mit Ausnahme des Falls B2 entsprechen die wiedergegebenen Durchschnittsergebnisse den theoretischen Zusammenhängen, es sei denn der Barwert des Bezugsguts (A6 und A7) bzw. die Volatilität (B5) ist extrem hoch. Dieses Ergebnis könnte als Indiz gewertet werden, daß die Entscheidungsträger $a b$ einer gewissen subjektiv empfundenen Unsicherheit das Projekt mit einem sehr hohen Risikoabschlag (bzw. den Zinsfuß mit einem sehr hohen Risikozuschlag) versehen, da sie es grundsätzlich als zu risikoreich einstufen ${ }^{1}$. Der unternehmerische Flexibilitätswert wird von den Managern insbesondere dann wahrgenommen, wenn der traditionelle Kapitalwert im Bereich der Investitionskosten liegt.

\subsubsection{Eigene Untersuchung im Rahmen von Akquisitionen}

Die im folgenden wiedergegebenen Ergebnisse beruhen auf einer im Frühjahr 1997 am Lehrstuhl für Internationale Unternehmensrechnung der Universität Münster durchgeführten umfangreichen Befragung zu methodischen Vorgehensweisen bei Akquisitions- und Steuerungsentscheidungen im Konzern ${ }^{2}$. Die Auswahl der Unternehmen erfolgte auf Basis folgender Kriterien: Zugehörigkeit zum DAX 100, Mutterunternehmen sowie mindestens 1 Mrd. DM Umsatz im Geschäftsjahr 1994 bzw. 1994/1995. Von den insgesamt 75 Mutterunternehmen erklärte sich das Management von 42 deutschen Konzernen zu persönlichen Interviews bereit.

Die überwiegende Mehrzahl der Konzerne verfolgt die Akquisitionsstrategien „Konzentration auf das Kerngeschäft“ (36 Unternehmen), „Eintritt in neue Märkte“ (35), „Know How-Transfer“ (32) und „Aufgabenzentralisierung“ (25). Hervorzuheben ist, daß diese Strategien vielfältige Interdependezen aufweisen und häufig parallel angestrebt werden. Als gängige Kombination wird von über $80 \%$ der befragten Konzerne die Stärkung der eigenen Kernkompetenzen durch Erschließung neuer Märkte erwähnt, sei es geographisch oder über den Zukauf

1 Die Autoren selbst bieten diese Erklărung lediglich für die Fallstudie B5 an. Wird bedacht, $\mathrm{da} ß$ das Risiko des Projekts im Fallstudientext über die absolute Spannbreite des Gegenwartswerts der Rückflüsse dargestellt wird und das empirische Ergebnis in den Fällen A6 und A7 unter dem traditionellen Kapitalwert liegt, unterstutzt dies die an dieser Stelle vorgenommene weitergehende Interpretation.

2 Vgl. auch Pellens/Rockholtz/Stienemann, Konzerncontrolling in Deutschland, 1997, S. $1933 \mathrm{ff}$. 
von ergänzenden Produkten und Technologien. Dieses Ergebnis läßt sich als Bestätigung der These deuten, daß die Mehrzahl der heute getätigten Akquisitionen strategisch bedingt sind und die Erschließung von neuen Investitionsgelegenheiten zum Ziel haben. Dabei ist zu vermuten, daß nicht alle dieser zusätzlichen Investitionsmöglichkeiten unmittelbar nach erfolgter Transaktion umgesetzt werden, so daß hier grundsätzlich ein Anwendungsgebiet des Realoptionskonzepts besteht.

Zur Beurteilung von Akquisitionsobjekten verwenden 31 Unternehmen das DCF- bzw. Ertragswertverfahren als zentrales Bewertungskalkül. Drei Unternehmen stützen ihre Bewertung hauptsächlich auf Multiplikatormethoden und sieben konnten sich nicht für ein zentrales Verfahren entscheiden. Letztere stehen im Einklang mit dem ansonsten belegten Ergebnis, daß in den meisten Fällen mehrere Verfahren gleichzeitig zum Einsatz kommen. Dabei dienen Multiplikatormethoden oft als erster Anhaltspunkt, bevor eine umfassende und detaillierte Bewertung anhand des DCF- oder Ertragswertkalküls durchgeführt wird. Substanzwertverfahren spielen nur in Ausnahmefällen zur Ermittlung eines Orientierungswerts eine Rolle, wenn z.B. der Goodwill vergleichsweise gering ist. Kein Unternehmen setzte zum Befragungszeitpunkt im Frühjahr 1997 ein Realoptionsbewertungsmodell ein ${ }^{1}$.

\begin{tabular}{|c|c|c|}
\hline Vorgehensweise & $\begin{array}{c}\text { Restrukturierungs- und } \\
\text { Synergieeffekte }\end{array}$ & Eintritt in neue Mărkte \\
\hline $\begin{array}{c}\text { quantitativ über } \\
\text { Investitionsrechnung }\end{array}$ & 33 & 11 \\
\hline $\begin{array}{c}\text { quantitativ über } \\
\text { Erfahrungswerte }\end{array}$ & 8 & 1 \\
\hline $\begin{array}{c}\text { qualitative } \\
\text { Beurteilung }\end{array}$ & - & 21 \\
\hline $\begin{array}{c}\text { keine } \\
\text { Berücksichtigung }\end{array}$ & 1 & 9 \\
\hline
\end{tabular}

Tab. 16: Berücksichtigung strategischer Faktoren bei Akquisitionen (Anzahl der Befragten)

Zur Frage ob und wie strategische Faktoren in die Bewertung einfließen, ergibt sich folgendes Bild. Restrukturierungs- und Synergieeffekte beziehen bis auf eine Ausnahme alle befragten Unternehmen in ihre quantitativen Überlegungen ein. Hiervon vertrauen acht auf eine grobe Abschätzung auf Basis von Erfah-

1 Rund drei Jahre später zeichnet sich in einer Neuauflage der Studie von 1997 ein leicht verändertes Bild ab. Von 50 Unternehmen gaben 5 Befragte an, den Einsatz von Optionspreismodellen zu testen bzw. zu diskutieren. 
rungswerten (vgl. Tab. 16). Beim Eintritt in neue Märkte berücksichtigen neun Firmen das ausgelöste zusätzliche Erfolgspotential nicht in ihrer Akquisitionsentscheidung und 21 gehen lediglich von einer qualitativen Beurteilung aus. Zwölf Konzerne versuchen eine Quantifizierung der zusätzlichen Investitionsmöglichkeiten, wobei ein Unternehmen auf Erfahrungswerte zurückgreift und elf Firmen diese Opportunitäten als sichere Investitionen in die der Bewertung zugrunde liegende Unternehmensplanung integriert. Durch eine solche Praxis bleiben die in diesen Investitionsprojekten i.d.R. vorhandenen Flexibilitätswerte unberücksichtigt.

\subsubsection{Untersuchung von Laamanen}

Laamanen untersucht den Optionscharakter von Akquisitionen, indem er die Verantwortlichen des Käufer- und Verkäuferunternehmens in persönlichen Interviews zu den Transaktionen befragt ${ }^{1}$. Als zu analysierende Untersuchungsgruppe wählt er Unternehmensübernahmen aus den Jahren 1987 - 1995, in denen zumindest ein finnisches und ein technologie-intensives Unternehmen beteiligt waren. Letzteres stellte zudem einen Existenzgründer als Eigentümer (u.U. auch zusammen mit einer Bank oder einem Risikokapitalgeber), verfügte über höchstens 500 Beschäftigte und eine Technologie, die für den Erwerber eine Neuheit darstellte.

\begin{tabular}{|c|c|c|c|c|}
\hline \multirow[b]{2}{*}{ hypothesized factor } & \multicolumn{2}{|c|}{ correlation analyses } & \multicolumn{2}{|c|}{ regression analyses } \\
\hline & $\begin{array}{l}\text { expected } \\
\text { sign }\end{array}$ & $\begin{array}{c}\text { Kendall } \\
\text { correlation } \\
\text { coefficient }\end{array}$ & $\begin{array}{c}\text { all } \\
\text { variables }\end{array}$ & $\begin{array}{l}\text { backward } \\
\text { elemination }\end{array}$ \\
\hline $\begin{array}{l}\text { age of the acq. Company } \\
\text { sales revenue of the acq. company } \\
\text { ln (personnel of the acq. company) } \\
\text { maturity of the acq. competencies } \\
\text { patentability of the acq. competencies } \\
\text { research and development intensity } \\
\text { industry trend at the time of acquisition }\end{array}$ & $\begin{array}{l}- \\
- \\
- \\
- \\
+ \\
+ \\
+\end{array}$ & $\begin{array}{l}-0,26 \mathrm{sf} \\
-0,04 \mathrm{~ns} \\
-0,07 \mathrm{~ns} \\
-0,52 \mathrm{sf} \\
+0,48 \mathrm{sf} \\
+0,45 \mathrm{sf} \\
+0,01 \mathrm{~ns}\end{array}$ & $\begin{array}{l}-1,47 \mathrm{sf} \\
+1,18 \mathrm{sf} \\
+0,90 \mathrm{sf}\end{array}$ & $\begin{array}{l}-1,32 \mathrm{sf} \\
+1,18 \mathrm{sf} \\
+0,97 \mathrm{sf}\end{array}$ \\
\hline
\end{tabular}

acq. $=$ acquired $\mathrm{ns}=$ not significant $\mathrm{sf}=$ significant

Tab. 17: Erklärungsgehalt hypothetischer Faktoren zum Optionscharakter von Akquisitionen

Quelle: Laamanen, Option Nature of Acquisitions, 1999, S. 159 und S. 160.

1 Vgl. Laamanen, Option Nature of Acquisitions, 1999, S. $154 \mathrm{ff}$. 
Laut Autor können von den 111 untersuchten Akquisitionen 58 (52\%) als „Einkauf" einer Wachstumsoption klassifiziert werden ${ }^{1}$. Des weiteren wird in einer Korrelationsanalyse festgestellt, daß das Umsatzvolumen, die Anzahl der Beschäftigten sowie der Industrietrend zum Zeitpunkt der Akquisition keinen signifikanten Gleichlauf mit dem analysierten Optionscharakter aufweisen, während das Alter des Akquisitionsobjekts, der Reifegrad sowie die Patentfähigkeit des erworbenen Wettbewerbsvorteils und die Forschungs- und Entwicklungsintensität als zu erklärende Variablen der Optionsausstattung verwendet werden können (vgl. Tab. 17). Auf Basis einer multiplen Regressionsanalyse, die mögliche Kollinearitäten der erklärenden Variablen in einem rückwärtsgerichteten Ausleseprozeß explizit berücksichtigt, zeigt sich anschließend, daß die drei zuletzt genannten Erklärungsfaktoren $77,5 \%$ des Optionscharakters vorhersagen. Hierbei übt der Reifegrad der erworbenen Technologie den größten Effekt aus.

\begin{tabular}{|c|c|c|c|c|}
\hline \multirow[b]{2}{*}{ hypothesized factor } & \multicolumn{2}{|c|}{ correlation analyses } & \multicolumn{2}{|c|}{ regression analyses } \\
\hline & $\begin{array}{l}\text { expected } \\
\text { sign }\end{array}$ & $\begin{array}{l}\text { Kendall } \\
\text { correlation } \\
\text { coefficient }\end{array}$ & $\begin{array}{c}\text { all } \\
\text { variables }\end{array}$ & $\begin{array}{l}\text { backward } \\
\text { elemination }\end{array}$ \\
\hline $\begin{array}{l}\text { relatedness of the companies } \\
\text { technological complementary } \\
\text { distribution complementary } \\
\text { financial complementary } \\
\text { proactive sales motive of the seller } \\
\text { industry trend after the acquisition }\end{array}$ & $\begin{array}{l}+ \\
+ \\
+ \\
+ \\
+ \\
+\end{array}$ & $\begin{array}{l}+0,45 \mathrm{sf} \\
+0,26 \mathrm{sf} \\
+0,34 \mathrm{sf} \\
-0,15 \mathrm{~ns} \\
+0,28 \mathrm{sf} \\
+0,41 \mathrm{sf}\end{array}$ & $\begin{array}{l}+5,73 \mathrm{sf} \\
+1,06 \mathrm{~ns} \\
-0,35 \mathrm{~ns} \\
+1,59 \mathrm{sf} \\
+2,07 \mathrm{sf}\end{array}$ & $\begin{array}{l}+5,25 \mathrm{sf} \\
\text { eleminated } \\
\text { eleminated } \\
+1,69 \mathrm{sf} \\
+1,90 \mathrm{sf} \\
\end{array}$ \\
\hline
\end{tabular}

$\mathrm{ns}=$ not significant $\mathbf{s f}=$ significant

Tab. 18: Erklärungsgehalt hypothetischer Faktoren zur Inanspruchnahme von Wachstumsoptionen bei Akquisitionen

Quelle: Laamanen, Option Nature of Acquisitions, 1999, S. 161 und S. 162.

In Erweiterung untersucht Laamanen die tatsächliche Inanspruchnahme der Wachstumsoption. In 28 (48\%) der zuvor festgestellten 58 Fälle ist die Option später auch ausgeübt worden. Hierzu weisen der Verwandschaftsgrad der Geschäftsfelder, das Verkaufsmotiv des ursprünglichen Eigentümers, der Industrietrend nach der Akquisition und die technologische sowie vertriebsmäßige Unterstützung des Erwerbers eine signifikante Korrelation auf (vgl. Tab. 18).

1 Laamanen berichtet zudem, daß bei 39 der 111 Akquisitionen bereits zuvor eine Kooperation bestand. Wăhrend von den vierzehn Eigenkapital-Kooperationen acht Optionscharakter aufwiesen, wurden in der Wahrnehmung der Befragten lediglich zwei der 25 Nichteigenkapital-Kooperationen als Wachstumsoption betrachtet. 
Für die finanzielle Unterstützung des Akquisiteurs läßt sich ein solcher $\mathrm{Zu}$ sammenhang nicht belegen. Auf Basis der bereits zuvor eingesetzten multiplen Regression folgt, daß die drei zuerst genannten Erklärungsfaktoren einen Prognosegrad von $84,3 \%$ erreichen.

Die soeben dargestellte Untersuchung zeigt, daß jede zweite analysierte Akquisition von den Beteiligten als Wachstumsoption interpretiert worden ist. Der Optionscharakter ist bei einer solchen Unternehmensübernahme umso eher gegeben, je intensiver das Akquisitionsobjekt forscht bzw. entwickelt, je innovativer die erworbene Technologie ist und je eher sich letztgenannte patentieren läßt.

Obwohl die empirische Realoptionsforschung noch sehr jung ist und umfassende Schlußfolgerungen erst nach weiteren Untersuchungen gezogen werden können, lassen sich auf Basis der in diesem Abschnitt präsentierten Studien folgende Tendenzen aufzeigen. Einerseits sind die Unternehmen bereit, bei Vorliegen eines unternehmerischen Handlungsspielraums eine "Sonderprämie“ zu bezahlen. Dies ließe sich dahingehend interpretieren, daß den Entscheidungsträgern das Wertpotential unternehmerischer Flexibilität durchaus bewußt ist. Andererseits sind jedoch bisher in den Unternehmen erst geringe bzw. kaum organisatorische Vorkehrungen getroffen worden, um dieses Wertpotential einer quantitativen Beurteilung zugänglich zu machen. Folglich gilt es, entsprechende Instrumente, wie z.B. den Realoptionsansatz, für eine praktische Umsetzung weiterzuentwickeln und in betrieblichen Entscheidungsprozessen $\mathrm{zu}$ installieren. 


\section{Kapitel VII}

\section{Zusammenfassung der Ergebnisse und Ausblick}

Im Mittelpunkt der vorliegenden Arbeit stand die optionspreistheoretische Bewertung unternehmerischer Flexibilität am Beispiel einer strategischen Akquisition. Dabei sind folgende Schwerpunkte gesetzt worden. Erstens wurden die bei Akquisitionen vielseitig denkbaren strategischen Faktoren systematisiert und der Aspekt der strategischen Flexibilität hiervon abgegrenzt. Zweitens ist auf Unterschiede zwischen Finanz- und Realoptionen eingegangen worden, da sich finanzwirtschaftliche Optionspreismodelle insbesondere bei einer mit Wettbewerbern geteilten Realoption nicht vollständig auf reale Investitionsbzw. Desinvestitionsmöglichkeiten übertragen lassen. Drittens wurde durch eine Auseinandersetzung mit den in den Realoptionsansätzen implizit unterstellten Kapitalmarktgegebenheiten die Anwendbarkeit dieser Bewertungsmodelle analysiert. Schließlich ist aufgezeigt worden, welche Stellung das Realoptionskonzept im Rahmen der wertorientierten Unternehmensführung einnehmen kann. Im einzelnen sind folgende Ergebnisse zu verzeichnen:

1. Die in der Literatur im Rahmen der strategischen Unternehmensbewertung angeführten strategischen Faktoren lassen sich grob in drei Kategorien einteilen: Restrukturierungspotentiale, Synergiepotentiale sowie Beitrag zur Umsetzung der geplanten Unternehmensstrategie. Da zur Bestimmung des Unternehmenswerts grundsätzlich sämtliche Faktoren zu berücksichtigen sind, ist eine solche Unterscheidung - wie auch diejenige zwischen strategischen und finanziellen Aspekten - nur insofern relevant, wie hiermit Transparenz über das Bewertungsobjekt bzw. die Bewertungssituation gewonnen werden kann.

2. Ein Vergleich der in der Literatur empfohlenen Konzepte zur strategischen Unternehmensbewertung zeigt, daß diese einerseits das Strategische auf unterschiedliche Art und Weise in die Bewertung einfließen lassen und andererseits spezielle strategische Aspekte in den Vordergrund rücken. Unabhängig von diesen spezifischen Konzepten können vier Weiterentwicklungen des aus der Investitionstheorie stammenden finanziellen Basiskalküls unterschieden werden: Integration strategischer Analyse- und Planungsinstrumente, Einbeziehung entscheidungstheoretischer Elemente, Integration finanzierungs- und kapitalmarkttheoretischer Erkenntnisse sowie pauschale Vorgehensweisen zur Wertermittlung einzelner Faktoren. 
3. Zur Berücksichtigung strategischer Aspekte in der Unternehmensbewertung sind zwei Grundhaltungen anzutreffen, die sich darin unterscheiden, ob die Auswirkungen strategischer Aspekte auf monetäre Größen quantifiziert werden sollen oder nicht. Bei Ablehnung lassen sich die strategischen Aspekte lediglich über einen entscheidungstheoretischen Zielnutzenvergleich oder über pauschale Vorgehensweisen berücksichtigen. Bei Befürwortung können einerseits Verknüpfungen zwischen bestimmten Werttreibern und den in das traditionelle Diskontierungsmodell eingehenden Bewertungsparametern erarbeitet oder andererseits Techniken zur Bewertung der in vielen Investitionsprojekten vorzufindenden Flexibilität des Entscheidungsaufschubs bzw. der Entscheidungsrevidierung analysiert werden.

4. Im Rahmen des realoptionsorientierten Ansatzes setzt sich der Wert des Unternehmens aus dem Barwert der Vermögenspositionen, deren Rückflüsse nicht durch zukünftige Investitionen beeinflußt sind, und dem Barwert solcher Vermögenspositionen, deren Rückflüsse sich erst in Abhängigkeit zukünftiger Investitionen bestimmen lassen, zusammen. Zur Ermittlung des zweiten Wertbestandteils werden Realoptionsbewertungsmodelle vorgeschlagen. Dies gilt unabhängig davon, ob es sich bei dem hier zu bewertenden strategischen Flexibilitätsaspekt um Restrukturierungspotentiale, Synergiepotentiale oder den Beitrag zur geplanten Strategieumsetzung handelt.

5. Im Rahmen der in der Unternehmenspraxis üblichen Risikozuschlagsmethode wird in traditionellen Bar- bzw. Kapitalwertmethoden ein im Zeitablauf einheitlicher risikoadjustierter Zinsfuß verwendet, um die Erwartungswerte von zukünftigen unsicheren Rückflüssen zu diskontieren. Eine solche Vorgehensweise ist jedoch nur gerechtfertigt, wenn das pro Periode auftretende Risiko des Akquisitions- bzw. Investitionsprojekts konstant ist und das Gesamtrisiko mit zunehmender Laufzeit in einem konstanten Verhältnis steigt. Die durch einen Entscheidungsaufschub von Investitionen gewonnene strategische Flexibilität führt dazu, daß sich das Risiko der Investitionsmöglichkeit (Basisprojekt zzgl. unternehmerischer Handlungsspielraum) gravierend von demjenigen des Basisprojekts unterscheidet.

6. Die Flexibilität des Entscheidungsaufschubs bedingt zudem, daß das Risiko der Investitionsmöglichkeit im Zeitablauf kontinuierlich wechselt. Zur Berücksichtigung dieses Umstands ist die traditionelle flexible Investitionsplanung kaum, die Sensitivitäts- oder die Risikoanalyse gar nicht geeignet. Erstere leistet zwar die notwendige Einbeziehung der strategischen 
Flexibilität ins Bewertungskalkül, scheitert jedoch an der Bestimmung perioden- und zustandsabhängiger Zinsfüße. Die anderen beiden Techniken fördern durch den Übergang auf mehrwertige Ergebnisse die Transparenz des tatsächlichen Risikogehalts des Akquisitions- bzw. Investitionsprojekts, sind aber nicht in der Lage, das durch die strategische Flexibilität entstehende Wertpotential abzubilden.

7. Zur Berücksichtigung eines im Zeitablauf kontinuierlich wechselnden Risikos sollten optionspreistheoretische Bewertungsverfahren eingesetzt werden. Sie stellen eine Lösung zur Bewertung von Investitionsmöglichkeiten dar, ohne die im Zeitablauf kontinuierlich modifizierte Risikoprämie explizit quantifizieren zu müssen. Das bereits im Barwert des Bezugsguts implizit verarbeitete Risiko wird unter der Annahme eines arbitragefreien Kapitalmarkts dazu verwendet, einen sogenannten risikoneutralisierten Erwartungswert aufzustellen, der dann entsprechend seinem Charakter mit dem risikolosen Zinsfuß abgezinst werden kann. In diesem Zusammenhang können Realoptionskalküle als „risikokorrigierte“ Version der traditionellen flexiblen Investitionsplanung angesehen werden.

8. Obwohl zwischen Finanzoptionen und Investitionsmöglichkeiten viele Parallelen bestehen, darf nicht übersehen werden, daß i.d.R. folgende Unterschiede bestehen:

- Das Bezugsgut der Investitionsmöglichkeit wird nicht auf einem organisierten Kapitalmarkt gehandelt. Dadurch muß vor der eigentlichen Bewertung der Investitionsmöglichkeit der Barwert des Bezugsguts geschätzt werden. Dies geschieht entweder mit Hilfe von Marktpreisen gehandelter Vergleichstitel oder auf Basis traditioneller Bar- bzw. Kapitalwertmethoden.

- Die Höhe der zukünftigen Investitionsauszahlung ist nicht vertraglich festgelegt. Bei Unsicherheit über die Investitionsauszahlung ist dieser Umstand im Bewertungskalkül zu berücksichtigen.

- Die Investitionsmöglichkeit steht dem Inhaber i.d.R. nicht exklusiv zur Verfügung, sondern er teilt diese mit Wettbewerbern. Für eine explizite Berücksichtigung des Konkurrenzverhaltens sind die finanzwirtschaftlichen Optionspreismodelle umzufunktionieren. Dies geschieht entweder durch Uminterpretation bereits bekannter Bewertungsparameter oder durch konzeptionelle Erweiterung dieser Modelle um spieltheoretische Elemente. 
- Die betrachtete Investitionsmöglichkeit schafft eventuell wiederum selbst neue Investitionsmöglichkeiten. In einem solchen Fall sind Folgeinvestitionsmöglichkeiten, die erst durch die Wahrnehmung einer ersten Investitionsmöglichkeit entstehen, bei der Bewertung zu berücksichtigen.

- Die Investitionsmöglichkeit ist nicht weiterveräußerbar, so daß ein drohender Wertverlust der Investitionsmöglichkeit sich nur durch eine frühzeitige Investition, jedoch nicht durch Verkauf an Dritte abwenden läßt.

9. Der Wert einer Investitionsmöglichkeit setzt sich aus dem traditionellen Kapitalwert bei frühzeitiger Investition und dem Flexibilitätswert zusammen. Letzterer verkörpert eine Schutzkomponente, die durch das Abwarten der zukünftigen Umweltentwicklung entsteht (reiner Wert des Abwartens). Gehen durch den Entscheidungsaufschub Rückflüsse definitiv verloren, enthält der Flexibilitätswert neben dem reinen Wert des Abwartens ebenfalls den sich negativ auswirkenden Wert der zwischenzeitlichen Rückflüsse. Ohne zwischenzeitliche Rückflüsse wird der Start der Investition so lange wie möglich hinausgezögert. Bei Existenz zwischenzeitlicher Rückflüsse muß hingegen der reine Wert des Abwartens gegen den Wert der zwischenzeitlichen Rückflüsse abgewogen werden. Parallel zur Bewertung wird die Wahl des optimalen Investitionszeitpunkts festgelegt. Eine frühzeitige Investition ist vorteilhaft, wenn der Wert der zwischenzeitlichen Rückflüsse den reinen Wert des Abwartens übersteigt.

10. In einer Konkurrenzsituation ist der zwischenzeitliche konkurrenzbedingte Werteffekt explizit in die in Punkt 9 angestellte Überlegung zu integrieren. Dabei sind die Konkurrenzhandlungen grundsätzlich danach zu unterscheiden, ob das betrachtete Unternehmen hiervon profitiert, und dies tendenziell zu einer Verschiebung der Investitionsentscheidung führt, oder ob das Verhalten der übrigen Wettbewerber einen Wertverlust nach sich zieht. Zur Bestimmung des optimalen Investitionszeitpunkts ist im zuletzt genannten Fall neben einem allgemeinen zwischenzeitlichen Rückfluß auch der konkurrenzbedingte beeinflußbare Wertverlust gegen den reinen Wert des Abwartens abzuwiegen. Im Rahmen einer spieltheoretischen Darstellung kann zudem gezeigt werden, daß die strategische Interaktion zwischen den Wettbewerbern u.U. eine frühzeitige Investition auslöst. In diesem Fall wird der Wert der Investitionsmöglichkeit auch dann geschmälert, wenn durch einen Entscheidungsaufschub keine zwischenzeitlichen Rückflüsse verloren gehen. 
11. Wird das Bezugsgut der Investitionsmöglichkeit am Kapitalmarkt gehandelt bzw. ist der Wert eines hierzu perfekt korrelierten Vermögenstitels beobachtbar, brauchen die allgemeinen Marktbewertungsfaktoren (z.B. Arrow/Debreu-Preise bzw. CAPM-Risikofaktoren) für eine relative Bewertung dieser Investitionsmöglichkeit nicht explizit vorzuliegen. Ist dies hingegen nicht der Fall, muß zuerst das Bezugsgut einer absoluten Bewertung unterzogen werden, bevor relativ zu diesem Ergebnis der Wert der Investitionsmöglichkeit ermittelt werden kann. Eine solche Vorgehensweise benötigt jedoch keine Informationen, die nicht auch bei traditionellen investitionstheoretischen Bewertungsverfahren vorausgesetzt werden. Zudem bietet sie den Vorteil, die sonst notwendige Bestimmung von zeit- und zustandsabhängigen Zinsfüßen bzw. Anwendung dynamischer Programmierungsverfahren zu umgehen, denen gegenüber der Realoptionsansatz eine Komplexitätsreduktion darstellt.

12. Mit Hilfe von Sensitivitätsanalysen läßt sich das auf Basis eines bestimmten Realoptionsbewertungsmodells ermittelte Ergebnis auf seine Stabilität hin überprüfen. Unter c.p.-Bedingungen steigt der Wert der Investitionsmöglichkeit, wenn sich der Barwert oder die Standardabweichung des Bezugsguts, der zeitliche Spielraum bis zur endgültigen Entscheidung oder der risikolose Zinsfuß erhöht bzw. die Investitionsauszahlung oder der zwischenzeitliche Rückfluß bzw. Wertverlust sinkt. Das auf den ersten Blick merkwürdig anmutende Ergebnis, daß eine erhöhte Unsicherheit in Form einer größeren Standardabweichung des Bezugsguts zu einer Steigerung des Werts der Investitionsmöglichkeit führt, ist durch das asymmetrische Chancen-/Risikopotential bei gleichzeitiger Annahme eines unveränderten Barwerts des Bezugsguts zu erklären. Sinkt hingegen durch die erhöhte Unsicherheit der Barwert des Bezugsguts, ergibt sich hierdurch für den Wert der Investitionsmöglichkeit ein negativer gegenläufiger Effekt. Der Gesamteffekt einer modifizierten Standardabweichung kann dann nicht mehr allgemeingültig vorhergesagt werden.

13. Während es sich innerhalb der Klasse der einperiodigen Erfolgskennzahlen (z.B. EVA, EP, CVA, SHVA, CFROI) bzw. derjenigen der DCFMethoden (z.B. FTE-, WACC-, APV-Ansatz) vor allem um konkurrierende Konzepte zur wertorientierten Unternehmensführung handelt, ist der parallele Einsatz einer einperiodigen Erfolgskennzahl, eines DCF-Verfahrens und des Realoptionskonzepts nicht nur möglich, sondern empfehlenswert. Immer dann, wenn in einer Planungsrechnung der Wert des unternehmerischen Handlungsspielraums explizit in die Beurteilung integriert werden 
soll, sollte über die Anwendung von Realoptionsbewertungsmodellen nachgedacht werden.

14. Vorliegende empirische Untersuchungen zeigen, daß Entscheidungsträger durchaus bereit sind, für den Erwerb unternehmerischer Flexibilität eine „Prämie“ zu bezahlen. Bisher werden die eingekauften Handlungsspielräume jedoch nur selten einer quantitativen Beurteilung zugänglich gemacht, da in den Unternehmen die dazu notwendigen organisatorischen Voraussetzungen fehlen. Folglich gilt es, entsprechende Instrumente, wie z.B. das Realoptionskonzept, für eine praktische Umsetzung weiterzuentwickeln und in betrieblichen Entscheidungsprozessen zu installieren.

Nach der in dieser Arbeit erfolgten Analyse der Bewertungsgrundlagen stellt sich für zukünftige Forschungsprojekte wie auch für Praktiker die Frage, welche Bedeutung der Realoptionsbewertung in Zukunft zukommen wird bzw. welche Voraussetzungen für einen entsprechenden Einsatz vor Ort geschaffen werden müssen. Bei welchen Investitionsprojekten läßt sich das Realoptionskonzept am einfachsten umsetzen? Wie schnell lassen sich für konkrete Investitionsprojekte die u.U. recht spezifischen zukünftigen Handlungsspielräume adäquat in einem Bewertungsmodell abbilden? Wie kann das mathematisch anspruchsvolle Bewertungsinstrumentarium auf Faustformeln reduziert werden, ohne hierbei die Qualität der Entscheidung zu beeinträchtigen?

Die Umsetzung des Realoptionskonzepts wird erstens davon abhängen, inwieweit es gelingt, die für die einzelnen Modelle benötigten Bewertungsparameter zu generieren. Existieren effiziente Kapitalmärkte, über die das entsprechende Datenmaterial zur Verfügung steht, läßt sich das Realoptionskonzept quasi direkt - ohne größere sonstige Voraussetzungen - einsetzen. Insofern ist es kein Zufall, daß bei der optionspreistheoretischen Bewertung von Investitionsmöglichkeiten zuerst an Anbieter von standardisierten Rohstoffen gedacht wurde. Aus den laufend notierten Preisen dieser Rohstoffe lassen sich gute Schätzwerte für den Barwert und die Standardabweichung des Bezugsguts gewinnen.

Eher zögerlich wird sich die Realoptionsbewertung zur Beurteilung von Investitionsmöglichkeiten durchsetzen, deren Risikoeinschätzung nicht durch eine permanente Marktnotierung der elementaren Bewertungsparameter vorgegeben ist. Dies ist z.B. für Forschungs- und Entwicklungs- bzw. Markteintrittsprojekte der Fall, bei denen u.U. noch nicht einmal Erfahrungswerte vorliegen. Hier ist ein Einsatz des Realoptionskonzepts nur denkbar, falls zuvor umfang- 
reiche Datenbanken aufgebaut werden, aus denen sich dann später die notwendigen Bewertungsparameter ableiten lassen ${ }^{1}$.

Zweitens wird der Einsatz der Realoptionsbewertung davon abhängen, inwieweit es gelingt, die in der Praxis vorzufindende Komplexität bzw. Spezifizität der mit der Investition verbundenen zukünftigen Handlungsspielräume zu modellieren. In diesem Kontext sind auch Investitionsmöglichkeiten zu erwähnen, bei denen der Wert des Bezugsguts von mehreren, voneinander unabhängigen, Unsicherheitsquellen beeinflußt wird. Die Realoptionsforschung profitiert jedoch hier glücklicherweise von der parallelen Modellentwicklung zur Bewertung von Finanzoptionen, so daß in vielen realistischen Entscheidungssituationen eventuell ein Rückgriff auf bzw. eine Anlehnung an finanzwirtschaftliche Optionspreismodelle erfolgen kann ${ }^{2}$.

Für eine Umsetzung in der Unternehmenspraxis ist drittens von Bedeutung, inwieweit es gelingt, das Realoptionskonzept bei den relevanten Entscheidungsträgern im Unternehmen zu kommunizieren. Wie weit läßt sich das mathematisch eher anspruchsvolle Bewertungsinstrumentarium auf Praktikabilitätsformeln reduzieren, ohne hierbei eine ausreichende theoretische Fundierung zu gefährden ${ }^{3}$ ? Hier gilt es, die Herausforderung anzunehmen, realoptionsorientierte Bewertungskonzepte mit zugrundeliegenden Spread Sheets zu entwikkeln, die von Praktikern akzeptiert werden ${ }^{4}$. In diesem Zusammenhang sind numerischen Methoden, wie z.B. dem Binomialmodell, gegenüber analytischen Lösungen, die in einem zeitstetigen Bewertungsansatz abgeleitet worden sind, eindeutig der Vorzug zu geben ${ }^{5}$.

1 Vgl. z.B. die Unternehmensbeispiele Merck und Philips bei Luehrman, Financial Engineering, 1994, S. 94 ff. sowie Lint/Pennings/Natter, Optionsmanagement, 1999, S. 922.

2 Von besonderem Interesse könnten zeit- und zustandsdiskrete Bewertungsmodelle sein, die mehrere Unsicherheitsquellen parallel abbilden. $\mathrm{Zu}$ solchen Ansătzen vgl. z.B. Kishomoto, Interest Rate, 1989; Schlag, Bewertung, 1995.

3 Für erste Versuche vgl. Stark, Irreversibility, 1990; Senn, Statistical Issues, 1996; Berk, Simple Approach, 1998; Luehrmann, Getting Started, 1998; McDonald, Rules of Thumb, 1998. Vgl. ebenfalls Lettau/Uhlig, Rules of Thumb, 1999, die für die „Überprüfung“ solcher Faustformeln eine Theorie anbieten.

4 Vgl. insbesondere Cortazar/Casassus, Implementing, 1998, S. 755 ff., die auch einen Einblick in ein entsprechendes Beispiel gewähren. Vgl. ebenfalls Pickles/Smith, Binomial Lattice Implementation, 1993, S. 1 ff.

5 Vgl. Ekern, Option Pricing Approach, 1988, S. 98. 
Zudem gilt es bei diesem Verhaltensaspekt auch zu beachten, daß die ermittelten Ergebnisse i.d.R. nur für die - zuvor in der Bewertung ermittelten - optimalen Investitionszeitpunkte gelten. Bei divergierenden Interessen zwischen untergeordneten Entscheidungsträgern und Unternehmensleitung macht dies den parallelen Einsatz eines Kontroll- und Anreizsystems erforderlich, das den Zeitpunkt der tatsächlichen Inanspruchnahme der einzelnen Investitionsmöglichkeiten steuert.

Schnell ließe sich gegen einen Einsatz des Realoptionskonzepts einwenden, daß aufgrund des u.U. weit in der Zukunft liegenden Prognosehorizonts das Erfolgspotential der Investitionsmöglichkeit nur schwer abzuschätzen sei. Der Realoptionsansatz verfügt jedoch exakt in einer solchen Situation über die entscheidenden Vorteile im Vergleich zu traditionellen Bewertungsverfahren'. Zudem ist die Durchführung von Sensitivitätsanalysen für alternative, die Flexibilität berücksichtigende Bewertungsverfahren weitaus schwieriger und mit einem geringeren Standardisierungsgrad ausgestattet ${ }^{2}$.

Selbst wenn eine quantitative Prognose des zukünftigen Erfolgspotentials in Frage gestellt wird, lassen sich über den Realoptionsansatz folgende Vorteile erzielen. Erstens wird explizit dargelegt, daß Unsicherheit nicht nur einen kapitalwertsenkenden, sondern auch einen flexibilitätswerterhöhenden Effekt auf die zu beurteilende Investitionsmöglichkeit hat ${ }^{3}$. Zweitens wird die Wahl des optimalen Investitionszeitpunkts explizit in die Überlegungen einbezogen, indem eine Investitionsmöglichkeit gedanklich in zwei sich gegenseitig ausschließende Projekte - Investition heute versus Investition morgen - eingeteilt wird ${ }^{4}$. Schließlich bleibt die im Realoptionsbewertungsmodell postulierte relative Bewertungsbeziehung zwischen Bezugsgut und Investitionsmöglichkeit

1 Vgl. ăhnlich Mason/Merton, Contingent Claims Analysis, 1985, S. 37.

2 Vgl. Stickel, Einsatz der Optionspreistheorie, 1997, S. 17.

3 Vgl. in diesem Kontext auch McGrath, Real Options Logic, 1997, S. 13 ff. sowie McGrath, Real Options Reasoning, S. 974 ff., die die Grundaussagen der Realoptionstheorie mit denjenigen aus der strategischen Management- bzw. Existenzgründungsliteratur verbindet.

4 Vgl. Dixit/Pindyck, Options Approach, 1995, S. 106; Mostowfi, Flexibilität, 1997, S. 591 sowie Slater/Reddy/Zwirlein, Strategic Investments, 1998, S. 455 f., die eine 9-FelderMatrix mit den Determinanten „Kapitalwertattraktivität“ und „Optionspotential“ vorschlagen. 
auch dann bestehen, wenn das zukünftige Erfolgspotential nur schwer abzuschätzen ist, so daß zumindest Vergleichsrechnungen möglich sind ${ }^{1}$.

Durch die Bewertung unternehmerischer Flexibilität mit dem Realoptionsansatz wird die Beurteilungsgrundlage für strategische Akquisitionen bzw. Investitionen entscheidend erweitert. Bemühungen, sämtliche Aspekte einer Investitionsentscheidung in einem Barwertkalkül zu verdichten, werden auch in Zukunft nicht von Erfolg gekrönt sein. Dies wird umso deutlicher, je mehr man Investitionen betrachtet, die in einem für alle Wettbewerber neuen Produktmarkt getätigt werden. Auch die optionspreistheoretische Bewertung wird das subjektive Gefühl und die Erfahrung des Entscheidungsträgers für den anvisierten Produktmarkt nicht ersetzen können ${ }^{2}$. Für die andere Seite der Entscheidungsfindungsmedaille ist jedoch davon auszugehen, daß das Realoptionskonzept „over the next one or two decades ${ }^{\text {“3 }} \mathrm{zu}$ einem Standardinstrument der Kapitalbudgetierung werden kann. Somit könnte in absehbarer Zeit das Motto gelten:

„Real options are not theoretical curiosities !‘4

1 Vgl. z.B. Kemna, Options in Real Markets, 1987, S. 166 f., die in einer praxisrelevanten Fallstudie den Barwert des Bezugsguts mit der leichter abzuschätzenden Investitionsauszahlung gleichsetzt, um anschließend für unterschiedliche Standardabweichungen und zwischenzeitliche Rückflüsse den Wert der unternehmerischen Flexibilität zu ermitteln.

2 Vgl. Laux, Handlungsspielräume, 1993, S. 956.

3 Sick, Real Options, 1995, S. 688.

4 Kulatilaka/Marcus, When Does DCF Fail?, 1992, S. 92. 


\section{Literaturverzeichnis}

Adam, D. (1996), Planung und Entscheidung: Modelle - Ziele - Methoden, 4. Aufl., Wiesbaden.

Aeberhard, K. (1996), Strategische Analyse - Empfehlungen zum Vorgehen und zu sinnvollen Methodenkombinationen, Bern u.a.

Agmon, T. (1993), Capital Budgeting and the Utilization of Full Information: Performance Evaluation and the Exercise of Real Options, in: Capital Budgeting under Uncertainty - New and Advanced Perspectives, ed. by Aggarwal, R., Engelwood Cliffs, S. 232 - 245.

Amram, M./Kulatilaka N. (1999), Disciplined Decisions - Aligning Strategy with the Financial Markets, in: Harvard Business Review, Vol. 77, S. 95 $-104$.

Amram, M./Kulatilaka, N. (1999), Real Options - Managing Strategic Investment in a Uncertain World, Boston.

Andres, P. (1998), Von der Black/Scholes-Optionspreisformel zum GARCHOptionsbewertungsmodell, Lohmar, Köln.

Ang, J. S./Dukas, S. P. (1993), Some Implications of Competition for Capital Investment, in: Capital Budgeting under Uncertainty, ed. by Aggarwal, R., Englewood Cliffs, S. 169 - 186.

Ansoff, I. (1965), Corporate Strategy - an Analytic Approach to Business Policy for Growth and Expansion, New York et al.

Appelbaum, E./Lim, C. (1985), Contestable Markets under Uncertainty, in: Rand Journal of Economics, Vol. 16, S. 28 - 40.

Arbeitskreis „Die Unternehmung im Markt“ der SG (1992), Synergie als Bestimmungsfaktor des Tätigkeitsbereichs (Geschäftsfelder und Funktionen) von Unternehmungen, in: Zeitschrift für betriebswirtschaftliche Forschung, 44. Jg., S. 963 - 973.

Arbeitskreis „Unternehmensbewertung im Rahmen der unternehmerischen Zielsetzung" der SG (1981), Unternehmensbewertung als Grundlage unternehmerischer Entscheidungen, hrsg. von Sieben, G./Zapf, B., Stuttgart.

Arrow, K. J. (1964), The Role of Securities in the Optimal Allocation of Risk Bearing, in: Review of Economic Studies, Vol. 31, S. 91 - 96.

Arrow, K. J./Fisher, A. C. (1974), Environmental Preservation, Uncertainty and Irreversibility, in: Quarterly Journal of Economics, Vol. 88, S. 312 319. 
Baetge, J./Krause, C. (1994), Die Berücksichtigung des Risikos in der Unternehmensbewertung - eine empirisch gestützte Betrachtung des Kalkulationszinsfußes, in: Betriebswirtschaftliche Forschung und Praxis, 46. Jg., S. $433-456$.

Bakshi, G./Cao, C./Chen, Z. (1997), Empirical Performance of Alternative Option Pricing Models, in: Journal of Finance, Vol. 52, S. 2003 - 2049.

Baldwin, C. Y. (1982), Optimal Sequential Investment When Capital is Not Readily Reversible, in: Journal of Finance, Vol. 37, S. 763 - 782.

Ballwieser, W. (1980), Möglichkeiten der Komplexitätsreduktion bei einer prognoseorientierten Unternehmensbewertung, in: Zeitschrift für betriebswirtschaftliche Forschung, 32. Jg., S. 50 - 73.

Ballwieser, W. (1981), Die Wahl des Kalkulationszinsfußes bei der Unternehmensbewertung unter Berücksichtigung von Risiko und Geldentwertung, in: Betriebswirtschaftliche Forschung und Praxis, 33. Jg., S. 97 - 114.

Ballwieser, W. (1990), Unternehmensbewertung und Komplexitätsreduktion, 3. Aufl., Wiesbaden.

Ballwieser, W. (1991), Unternehmensbewertung mit Hilfe von Multiplikatoren, in: Aktuelle Fragen der Finanzwirtschaft und der Unternehmensbesteuerung, hrsg. von Rückle, D., Wien, S. 47 - 66.

Ballwieser, W. (1993), Methoden der Unternehmensbewertung, in: Handbuch des Finanzmanagements, hrsg. von Gebhardt, G./Gerke, W./Steiner, M., München, S. 151 - 176.

Ballwieser, W. (1995), Aktuelle Aspekte der Unternehmensbewertung, in: Die Wirtschaftsprüfung, 48. Jg., S. 119 - 129.

Ballwieser, W. (1997), Eine neue Lehre der Unternehmensbewertung?, in: Der Betrieb, 50. Jg., S. 185 - 191.

Ballwieser, W. (1998), Unternehmensbewertung mit Discounted Cash FlowVerfahren, in: Die Wirtschaftsprüfung, 51 Jg., S. 81 - 92.

Ballwieser, W./Leuthier, R. (1986), Betriebswirtschaftliche Steuerberatung: Grundprinzipien, Verfahren und Probleme der Unternehmensbewertung, in: Deutsches Steuerrecht, 24. Jg., S. 545 - 551 und S. 604 - 610.

Bamberg, G./Coenenberg, A. G. (1996), Betriebswirtschaftliche Entscheidungslehre, 9. Aufl., München.

Banz, R. W./Miller, M. H. (1978), Prices for State-contingent Claims - Some Estimates and Applications, in: Journal of Business, Vol. 51, S. 653 672. 
Barone-Adesi G./Whaley, R. E. (1987), Efficient Analytic Approximation of American Option Values, in: Journal of Finance, Vol. 42, S. 301 - 320.

Barthel, C. W. (1996), Unternehmenswert: Die zuschlagsorientierten Bewertungsverfahren - Vom Buchwert-Zuschlagsverfahren zur strategischen Unternehmensbewertung, in: Der Betrieb, 49. Jg., S. 1349 - 1358.

Barthel, C. W. (1996), Unternehmenswert: Grundlagen und Varianten des Umsatzverfahrens, in: Deutsches Steuerrecht, 34. Jg., S. 1458 - 1464.

Barthel, C. W. (1996), Unternehmenswert: Theoretische Fundierung des Umsatzverfahrens, in: Deutsches Steuerrecht, 34. Jg., S. 1701 - 1707.

Beck, P. (1996), Unternehmensbewertung bei Akquisitionen - Methoden, Anwendungen, Probleme, Wiesbaden.

Beinert, M. (1997), Kurssprünge und der Wert deutscher Aktienoptionen Auswirkungen von Aktienkurssprüngen auf den Optionswert im Zeitraum 1983 - 1991, Wiesbaden.

Beißinger, T./Möller, J. (1994), Die Neue Investitionstheorie, in: Wirtschaftwissenschaftliches Studium, 23. Jg., S. 270 - 275.

Bell, A. (1993), Option Valuation in the Presence of Market Imperfections, Frankfurt.

Berk, J. (1998), A Simple Approach for Deciding when to Invest, Working Paper, University of Washington.

Berk, J./Green R./Naik V. (1999), Optimal Investment, Growth Options and Security Returns, in: Journal of Finance, Vol. 54, S. 1553 - 1607.

Bernanke, B. S. (1983), Irreversibility, Uncertainty and Cyclical Investment, in: Quarterly Journal of Economics, Vol. 98, S. 85 - 106.

Beyer von Morgenstern, I./Richter, F. (1998), Strategieentwicklung und Geschäftssteuerung trotz Unsicherheit, in: Rechnungswesen als Instrument für Führungsentscheidungen, hrsg. von Möller, H. P./Schmidt, F., Stuttgart, S. $111-131$.

Bischoff, J. (1994), Das Shareholder Value-Konzept - Darstellung, Probleme, Handhabungsmöglichkeiten, Wiesbaden.

Bitz, M. (1981), Entscheidungstheorie, München.

Black, F./Scholes, M. (1973), The Pricing of Options and Corporate Liabilities, in: Journal of Political Economy, Vol. 81, S. 637 - 654.

Blohm, H./Lüder, K. (1995), Investition, 8. Aufl., München. 
Blomeyer, E. C. (1986), An Analytic Approximation for the American Put Price for Options on Stocks with Dividends, in: Journal of Financial and Quantitative Analysis, Vol. 21, S. 229 - 233.

Böcking, H.-J./Nowak, K. (1999), Marktorientierte Unternehmensbewertung Darstellung und Würdigung der marktorientierten Vergleichsverfahren vor dem Hintergrund der deutschen Kapitalmarktverhältnisse, in: Finanz Betrieb, 1. Jg., S. 169 - 176.

Bös, M. (1991), Optionsbewertung und Kapitalmarkt, Bergisch Gladbach.

Bonini, C. (1977), Capital Investment Under Uncertainty with Abandonment Options, in: Journal of Financial and Quantitative Analysis, Vol. 12, S. 39 - 54.

Born, K. (1995), Überleitung von der Discounted Cash Flow-Methode (DCFMethode) zur Ertragswertmethode bei der Unternehmensbewertung, in: Der Betrieb, 49. Jg., S. 1885 - 1889.

Boyle, P. P. (1977), Options: A Monte Carlo Approach, in: Journal of Financial Economics, Vol. 4, S. 323 - 338.

Boyle, P. P./Lin, X. (1997), Valuation of Options on Several Risky Assets when There Are Transaction Costs, in: Advances in Futures and Options Research, Vol. 9, ed. by Boyle, P./Pemacchi, G./Ritchken, P., Greenwich, S. $111-127$.

Boyle, P. P./Vorst, T. (1992), Option Replication in Discrete Time with Transaction Costs, in: Journal of Finance, Vol. 47, S. 271 - 293.

Brealey, R. A./Myers, S. C. (1996), Principles of Corporate Finance, $5^{\text {th }}$ ed., New York et al.

Brennan, M. J. (1979), The Pricing of Contingent Claims in Discrete Time Models, in: Journal of Finance, Vol. 24, S. 53 - 68.

Brennan, M. J. /Schwartz, E. S. (1986), A New Approach to Evaluation Natural Resource Investments, in: The Revolution in Corporate Finance, ed. by Stern, J. M./Chew, D. H., New York, S. 78 - 87.

Brennan, M. J./Schwartz, E. S. (1977), The Valuation of American Put Options, in: Journal of Finance, Vol. 32, S. 449 - 462.

Brennan, M. J./Schwartz, E. S. (1985), Evaluating Natural Resource Investments, in: Journal of Business, Vol. 58, S. 135 - 157.

Brennan, M. J./Trigeorgis, L. (Ed.) (2000), Project Flexibility, Agency and Competition, Oxford et al.

Bretzke, W.-R. (1988), Risiken in der Unternehmensbewertung, in: Zeitschrift für betriebswirtschaftliche Forschung, 40. Jg., S. 813 - 823. 
Bretzke, W.-R. (1993), Unternehmungsbewertung in dynamischen Märkten, in: Betriebswirtschaftliche Forschung und Praxis, 45. Jg., S. 39 - 45.

Breuer, W. (1997), Die Marktwertmaximierung als finanzwirtschaftliche Entscheidungsregel, in: Wirtschaftswissenschaftliches Studium, 26. Jg., S. 222 - 226.

Breuer, W./Gürtler, M./Schuhmacher, J. (1999), Die Bewertung betrieblicher Realoptionen, in: Betriebswirtschaftliche Forschung und Praxis, 51. Jg., S. 213 - 232.

Broadie, M./Detemple, J. (1996), American Option Valuation - New Bounds, Approximations and A Comparison of Existing Methods, in: Review of Financial Studies, Vol. 9, S. 1213 - 1250.

Broyles, J. E./Cooper, I. A. (1981), Growth Opportunities and Real Investment Decisions, in: Risk, Capital Costs and Project Financing Decisions, ed. by von Derkinderen, F. G./Crum, R. L., Boston, S. 107 - 118.

Bühner, R./Spindler, H.-J. (1986), Synergieerwartungen bei Unternehmenszusammenschlüssen, in: Der Betrieb, 39. Jg., S. 601 - 606.

Bulow, J. I./Geanakolpos, J. D./Klemperer, P. D. (1985), Multimarket Oligopoly: Strategic Substitutes and Complements, in: Journal of Political Economy, Vol. 93, S. 488 - 511.

Busby, J. S./Pitts, C. G. (1997), Real Options in Practice: An Exploratory Survey of how Finance Officers Deal with Flexibility in Capital Appraisal, in: Management Accounting Research, Vol. 8, S. 169 - 186.

Busse von Colbe, W. (1957), Der Zukunftserfolg - Die Ermittlung des künftigen Unternehmenserfolges und seine Bedeutung für die Bewertung von Industrieunternehmen, Wiesbaden.

Busse von Colbe, W. (1970), Gesamtwert der Unternehmung, in: Handwörterbuch des Rechnungswesens, hrsg. von Kosiol, E. u.a., Stuttgart, Sp. 570 581.

Busse von Colbe, W./Laßmann, G. (1990), Betriebswirtschafttheorie, Band 3: Investitionstheorie, 3. Aufl., Berlin u.a.

Buzzell, R. D./Gale, B. T., (1989), Das PIMS-Programm - Strategien und Unternehmenserfolg, Wiesbaden.

Caballero, R. J./Pindyck, R. S. (1996), Uncertainty, Investment and Industry Evolution, in: International Economic Review, Vol. 37, S. 641 - 662.

Cheung, J. K. (1993), Managerial Flexibility in Capital Investment Decisions: Insights from the Real Options Literature, in: Journal of Accounting Literature, Vol. 12, S. 29 - 66. 
Childs, P. D./Ott, S. H./Triantis, A. J. (1998), Capital Budgeting for Interrelated Projects - A Real Options Approach, in: Journal of Financial and Quantitative Analysis, Vol. 33, S. 305 - 334.

Chung, K. H./Charoenwong C. (1991), Investment Options, Assets in Place, and the Risk of Stocks, in: Financial Management, Vol. 20, S. 21 - 33.

Coenenberg, A. G. (1970), Unternehmensbewertung mit Hilfe der MonteCarlo-Simulation, in: Zeitschrift für Betriebswirtschaft, 40. Jg., S. 793 804.

Coenenberg, A. G./Sautter, M. T. (1988), Strategische und finanzielle Bewertung von Unternehmensakquisitionen, in: Die Betriebswirtschaft, 48. Jg., S. $691-710$.

Cohen, W. M./Levinthal, D. A. (1994), Fortune Favors the Prepared Firm, in: Management Science, Vol. 40, S. 227 - 251.

Constantinides, G. (1980), Admissible Uncertainty in the Intertemporal Asset Pricing Model, in: Journal of Financial Economics, Vol. 8, S. 71 - 86.

Copeland, T./Keenan, P. T. (1998), How Much is Flexibility Worth?, in: McKinsey Quarterly, Vol. 34, S. 38 - 49.

Copeland, T./Keenan, P. T. (1998), Making Real Options Real, in: McKinsey Quarterly, Vol. 34, S. 128 - 141.

Copeland, T./Koller, T./Murrin, J. (1994), Valuation - Measuring and Managing the Value of Companies, $2^{\text {nd }}$ ed., New York et al.

Copeland, T./Weston, J. F. (1988), Financial Theory and Corporate Policy, $3^{\text {rd }}$ ed., Reading et al.

Cortazar, G./Casassus, J. (1998), Optimal Timing of a Mine Expansion: Implementing a Real Options Model, in: Quarterly Review of Economics and Finance, Special Issue, Vol. 38, Real Options: Developments and Applications, ed. by. Pinches, G. E., S. 755 - 769.

Cortazar, G./Schwartz, E. S. (1993), A Compound Option Model of Production and Intermediate Inventories, in: Journal of Business, Vol. 66, S. 517 - 540 .

Cox, J. C./Ross, S. A. (1976), The Valuation of Options for Alternative Stochastic Processes, in: Journal of Financial Economics, Vol. 3, S. 145 166.

Cox, J. C./Ross, S. A./Rubinstein, M. (1979), Option Pricing: A Simplified Approach, in: Journal of Financial Economics, Vol. 7, S. 229 - 263.

Cox, J. C./Rubinstein, M. (1985), Options Markets, Englewood Cliffs. 
Crasselt, N./Pellens, B./Schremper, R. (2000), Konvergenz wertorientierter Erfolgskennzahlen - Economic Value Added, Cash Flow Return on Investment und Cash Value Added, erscheint in: Das Wirtschaftsstudium, 29. Jg.

Crasselt, N./Tomaszewski C. (1999), Unternehmerische Flexibilität bei strategischen Akquisitionen - Einsatzmöglichkeiten von Optionspreismodellen, in: Controlling, 11. Jg., S. 517 - 524.

Crasselt, N./Tomaszewski, C. (1999), Realoptionen - eine neue Methode der Investitionsrechnung?, in: Wirtschaftswissenschaftliches Studium, 28. Jg., S. 556 - 559.

Cukierman, A. (1980), The Effects of Uncertainty on Investment under Risk Neutrality with Endogenous Information, in: Journal of Political Economy, Vol. 88, S. 462 - 475.

Damodaran, A. (1997), Corporate Finance - Theory and Practice, New York et al.

Davis, G. A. (1996), Option Premiums in Mineral Asset Pricing: Are They Important?, in: Land Economics, Vol. 72, S. 167 - 186.

Davis, G. A. (1998), Estimating Volatility and Dividend Yield When Valuing Real Options to Invest and Abandon, in: Quarterly Review of Economics and Finance, Special Issue, Vol. 38, Real Options: Developments and Applications, ed. by. Pinches, G. E., S. 725 - 754.

Debreu, G. (1959), Theory of Value: An Axiomatic Analysis of Economic Equilibrium, New York.

Delcker, M. (1992), Risiken beim Unternehmenskauf - Absicherung durch Besserungsoption, in: Der Betrieb, 45 Jg., S. 2453 - 2454.

Dennis, P./Rendleman, R. J. (1995), An LP Approach to Synthetic Option Replication with Transaction Costs and Multiple Security Selection, in: Advances in Futures and Options Research, Vol. 8, ed. by Boyle, P./Longstaff, F. A./Ritchken, P., Greenwich, S. 53 - 84.

Derman, E./Kani, I. (1994), Riding on a Smile, in: Risk, Vol. 7, S. 32 - 39.

Dirrigl, H. (1988), Die Bewertung von Beteiligungen an Kapitalgesellschaften: Betriebswirtschaftliche Methoden und steuerlicher Einfluß, Hamburg.

Dirrigl, H. (1994), Konzepte, Anwendungsgebiete und Grenzen einer strategischen Unternehmensbewertung, in: Betriebswirtschaftliche Forschung und Praxis, 46. Jg., S. 409 - 432. 
Dirrigl, H. (1998), Kollektive Investitionsrechnung und Unternehmensbewertung, in: Ergebnisse des Berliner Workshops „Unternehmensbewertung“ vom 7. Februar 1998, Diskussionsbeitrag des Fachbereichs Wirtschaftswissenschaft der Freien Universität Berlin, hrsg. von Kruschwitz, L./ Löffler, A., Berlin, S. 3 - 24.

Dirrigl, H. (1998), Wertorientierung und Konvergenz in der Unternehmensrechnung, in: Betriebswirtschaftliche Forschung und Praxis, 50. Jg., S. 540 - 579.

Dixit, A. K. (1989), Entry and Exit Decisions under Uncertainty, in: Journal of Political Economy, Vol. 97, S. 620 - 638.

Dixit, A. K. (1992), Investment and Hysteresis, in: Journal of Economic Perspectives, Vol. 6, S. 107 - 132.

Dixit, A. K./Pindyck, R. S. (1994), Investment Under Uncertainty, Princeton.

Dixit, A. K./Pindyck, R. S. (1995), The Options Approach to Capital Investment, in: Harvard Business Review, Vol. 73, S. 105 - 115.

Dörner, W. (1992), Die Unternehmensbewertung, in: Wirtschaftsprüferhandbuch, Band II, 10. Aufl., hrsg. vom IDW e.V., Düsseldorf, S. 1 - 136.

Drukarczyk, J. (1993), Theorie und Politik der Finanzierung, 2. Aufl., München.

Drukarczyk, J. (1995), DCF-Methoden und Ertragswertmethode - einige klärende Anmerkungen, in: Die Wirtschaftsprüfung, 48. Jg., S. 329 - 334.

Drukarczyk, J. (1998), Unternehmensbewertung, 2. Aufl., München.

Drukarczyk, J./Hunold, D. (1998), Unternehmensbewertung und periodische Unternehmenswertänderung - Zur Leistungsfähigkeit von Entity-, APVund Equity-Ansatz, Regensburger Diskussionsbeiträge zur Wirtschaftswissenschaft, Universität Regensburg.

Drukarczyk, J./Richter, F. (1995), Unternehmensgesamtwert, anteilseignerorientierte Finanzentscheidungen und APV-Ansatz, in: Die Betriebswirtschaft, 55. Jg., S. 559 - 580.

Dumas, B./Fleming, J./Whaley, R. E. (1998), Implied Volatility Functions Empirical Tests, in: Journal of Finance, Vol. 53, S. 2059 - 2106.

Dyl, E. A./Long, H. W. (1969), Abandonment Value and Capital Budgeting Comment, in: Journal of Finance, Vol. 24, S. 88 - 95.

Ebert, M. (1998), Evaluation von Synergien bei Unternehmenszusammenschlüssen, Hamburg. 
Eble, S./Völker, R (1993), Die Behandlung von Optionen in der betrieblichen Investitionsrechnung, in: Die Unternehmung, 47. Jg, S. 407 - 418.

Ediringshe, C./Naik, V./Uppal, R. (1993), Optimal Replication of Options with Transaction Costs and Trading Restrictions, in: Journal of Financial and Quantitative Analysis, Vol. 28, S. 117 - 138.

Egle, K./Trautmann, S. (1981), On Preference-Dependent Pricing of Contingent Claims, in: Geld, Banken und Versicherungen, hrsg. von Göppl, H./Henn, R., Königstein, S. 400 - 416.

Ehrensberger, S. (1993), Synergieorientierte Unternehmensintegration Grundlagen und Auswirkungen, Wiesbaden.

Ehrhardt, M. C. (1994), The Search for Value, Boston.

Eichberger, J. (1993), Game Theory for Economists, San Diego et al.

Ekern, S. (1988), An Option Pricing Approach to Evaluating Petroleum Projects, in: Energy Economics, Vol. 10, S. 91 - 99.

Emery, D./Parr, P./Mokkelbost, P./Gandhi, D./Saunders, A. (1978), An Investigation of Real Investment Decision Making with the Options Pricing Model, in: Journal of Business Finance \& Accounting, Vol. 5, S. 363 369.

Engeleiter, H.-J. (1970), Unternehmensbewertung, Stuttgart.

Englert, J. (1997), Die Bewertung freiberuflicher Praxen mit Hilfe branchentypischer Wertfindungsmethoden, in: Betriebs-Berater, 52. Jg., S. 142 149.

Fama, E. F. (1977), Risk-Adjusted Discount Rates and Capital Budgeting Under Uncertainty, in: Journal of Financial Economics, Vol. 5, S. 3 - 24.

Figlewski, S. (1989), Options Arbitrage in Imperfect Markets, in: Journal of Finance, Vol. 44, S. 1289 - 1311.

Finucane, T. J. (1994), Binomial Approximations of American Call Options Prices with Stochastic Volatilities, in: Advances in Futures and Options Research, Vol. 7, ed. by Chance, D./Trippi, R., Greenwich, S. 113 - 134.

Fischer, K. M. (1993), Die Aufstockung von Minderheitsbeteiligungen als Realoption beim Unternehmenskauf, in: Management von Unternehmensakquisitionen, hrsg. von Frank, G.-M./Stein, I., Stuttgart, S. 109 118.

Fischer, S. (1978), Call Option Pricing When the Exercise Price is Uncertain and the Valuation of Index Bonds, in: Journal of Finance, Vol. 33, S. 169 $-176$. 
Fischer, T. R./Hahnenstein, L./Heitzer, B. (1999), Kapitalmarkttheoretische Ansätze zur Berücksichtigung von Handlungsspielräumen in der Unternehmensbewertung, in: Zeitschrift für Betriebswirtschaft, 69. Jg., S. 1207 $-1232$.

Franke, G./Hax, H. (1994), Finanzwirtschaft des Unternehmens und Kapitalmarkt, 3. Aufl., Berlin, Heidelberg.

Fudenberg, D./Tirole, J. (1984), The Fat-Cat Effect, The Puppy-Dog Ploy, and The Lean und Hungry Look, in: American Economic Review, Vol. 74, S. 361 - 366.

Fudenberg, D./Tirole, J. (1991), Game Theory, Cambridge, London.

Galitz, L. (1994), Financial Engineering - Tools and Techniques to Manage Financial Risk, London.

Gälweiler, A. (1989), Synergiepotentiale, in: Handwörterbuch der Planung, hrsg. von Szyperski, N., Stuttgart, Sp. 1935 - 1943.

Gastineau, G. L. (1988), The Options Manual, $3^{\text {rd }}$ ed., New York.

Gerke, W./Bank, M. (1998), Finanzierung - Grundlagen für die Investitionsund Finanzierungsentscheidungen in Unternehmen, Stuttgart.

Geske, R. (1978), The Pricing of Options With Stochastic Dividend Yield, in: Journal of Finance, Vol. 33, S. 617 - 625.

Geske, R. (1979), A Note on an Analytical Valuation Formula for Unprotected American Call Options on Stocks with Known Dividends, in: Journal of Financial Economics, Vol. 7, S. 375 - 380.

Geske, R. (1979), The Valuation of Compound Options, in: Journal of Financial Economics, Vol. 7, S. 63 - 81.

Geske, R./Johnson, H. E. (1984), The American Put Option Valued Analytically, in: Journal of Finance, Vol. 39, S. 1511 - 1524.

Geske, R./Shastri, K. (1985), Valuation by Approximation: A Comparision of Alternative Option Valuation Techniques, in: Journal of Financial and Quantitative Analysis, Vol. 20, S. 45 - 71.

Geske, R./Trautmann, S. (1986), Option Valuation: Theory and Empirical Evidence, in: Capital Market Equilibria, hrsg. von Bamberg, G./Spremann, K., Berlin u.a., S. 79 - 133.

Gibson, R./Schwartz, E. S. (1990), Stochastic Convenience Yield and the Pricing of Oil Contingent Claims, in: Journal of Finance, Vol. 45, S. 959 976. 
Gilster, J./Lee, W. (1984), The Effects of Transaction Costs and Different Borrowing and Lending Rates on the Option Pricing Model - A Note, in: Journal of Finance, Vol. 39, S. 1215 - 1222.

Gintschel, A. (1999), Ein allgemeines Binomialmodell zur Bewertung von Realoptionen, in: Kredit und Kapital, 32. Jg., S. 60 - 84.

Göppl, H. (1980), Unternehmensbewertung und Capital-Asset-Pricing-Theorie, in: Die Wirtschaftsprüfung, 33. Jg., S. 237 - 245.

Goldenberg, D. H. (1991), A Unified Method for Pricing Options on Diffusion Processes, in: Journal of Financial Economics, Vol. 29, S. 3 - 34.

Grenadier, S. (1996), The Strategic Exercise of Options - Development Cascades and Overbuilding in Real Estate Markets, in: Journal of Finance, Vol. 51, S. 1653 - 1679.

Güth, W. (1999), Spieltheorie und ökonomische (Bei)Spiele, 2. Aufl., Berlin.

Hachmeister, D. (1996), Der Discounted Cash Flow als Unternehmenswert, in: Das Wirtschaftsstudium, 25. Jg., S. 357 - 366.

Hachmeister, D. (1996), Die Abbildung der Finanzierung im Rahmen verschiedener Discounted Cash Flow-Verfahren, in: Zeitschrift für betriebswirtschaftliche Forschung, 48. Jg., S. 251 - 277.

Hachmeister, D. (1997), Shareholder Value, in: Die Betriebswirtschaft, 57. Jg., S. 823 - 839.

Hachmeister, D. (1998), Der Discounted Cash Flow als Maß der Unternehmenswertsteigerung, 2. Aufl., Frankfurt.

Hachmeister, D. (1998), Diskontierung bei Unsicherheit, in: Ergebnisse des Berliner Work-shops „Unternehmensbewertung“ vom 7. Februar 1998, Diskussionsbeitrag des Fachbereichs Wirtschaftswissenschaft der Freien Universität Berlin, hrsg. von Kruschwitz, L./Löffler, A., Berlin, S. 25 33.

Hafner, R. (1988), Unternehmensbewertung bei mehrfacher Zielsetzung, in: Betriebswirtschaftliche Forschung und Praxis, 40. Jg., S. 485 - 504.

Hafner, R. (1989), Grenzpreisermittlung bei mehrfacher Zielsetzung - ein Beitrag zur Bewertung strategischer Unternehmensakquisitionen, Bergisch Gladbach.

Harrison, J. M./Kreps, D. M. (1979), Martingales and Arbitrage in Multiperiod Securities Markets, in: Journal of Economic Theory, Vol. 20, S. 381 -408 .

Hauck, W. (1991), Optionspreise: Märkte, Preisfaktoren, Kennzahlen, Wiesbaden. 
Haumer, H. (1983), Sequentielle stochastische Investitionsplanung, Wiesbaden.

Helbling, C. (1989), Unternehmenswertoptimierung durch Restrukturierungsmaßnahmen und durch Minimierung des betrieblichen Substanzwertes, in: Die Unternehmung, 43. Jg., S. 177 - 186.

Helbling, C. (1990), Wert und Preis eines Unternehmens, in: Index, o. Jg., S. 14 - 19.

Helling, N. (1994), Strategieorientierte Unternehmensbewertung - Instrumente und Techniken, Wiesbaden.

Henry, C. (1974), Investment Decisions Under Uncertainty: The Irreversibility Effect, in: American Economic Review, Vol. 64, S. 1006 - 1012.

Herter, R. N. (1992), Berücksichtigung von Optionen bei der Bewertung strategischer Investitionen, in: Controlling, 4. Jg., S. 320 - 327.

Hertz, D. (1964), Risk Analysis in Capital Investment, in: Harvard Business Review, Vol. 42, January-February, S. 95 - 106.

Hertz, D./Thomas, H. (1983), Risk Analysis and its Applications, New York et. al.

Hesse, T. (1996), Periodischer Unternehmenserfolg zwischen Realisationsund Antizipationsprinzip - Vergleich von Aktienrendite, Cash Flow und Economic Value Added, Stuttgart, Wien.

Hillier, F. (1963), The Derivation of Probabilistic Information for the Evaluation of Risky Investments, in: Management Science, Vol. 9, S. 443 - 457.

Hinterhuber, H. (1996), Strategische Unternehmensführung, I. Strategisches Denken: Vision, Unternehmenspolitik, Strategie, 6. Aufl., Berlin, New York.

Ho, T. S./Stapleton. R. C./Subrahmanyam, M. G. (1997), The Valuation of American Options with Stochastic Interest Rates: A Generalization of the Geske-Johnson Technique, in: Journal of Finance, Vol. 52, S. 827 - 840.

Ho, T. S./Lee, S.-B. (1986), Term Structure Movements and Pricing Interest Rate Contingent Claims, in: Journal of Finance, Vol. 41, S. 1011 - 1029.

Hoff, T. E. (1997), Investment under Uncertainty - State Prices in Incomplete Markets, Doctoral Dissertation, Stanford University.

Hoggard, T./Whalley, A. E./Wilmott, P. (1994), Hedging Option Portfolios in the Presence of Transaction Costs, in: Advances in Futures and Options Research, Vol. 7, ed. by Chance, D./Trippi, R., Greenwich, S. 21 35. 
Holler, M. J./Illing, G. (1996), Einführung in die Spieltheorie, 3. Aufl., Berlin.

Hommel, U./Müller, J. (1999), Realoptionsbasierte Investitionsbewertung, in: Finanz Betrieb, 1. Jg., S. 177 - 188.

Hommel, U./Pritsch, G. (1999), Investitionsbewertung und Unternehmensführung mit dem Realoptionsansatz, in: Handbuch Corporate Finance, hrsg. von Achleitner, A.-K./Thoma, G., Köln.

Hostettler, S. (1997), Economiv Value Added (EVA) - Darstellung und Anwendung auf Schweizer Aktiengesellschaften, 2. Aufl., Stuttgart, Wien.

Hsia, C.-C. (1983), On Binomial Option Pricing, in: Journal of Financial Research, Vol. 6, S. 41 - 46.

Hull, J. (1997), Options, Futures and Other Derivatives, $3^{\text {rd }}$ ed., London et al.

Hull, J./White, A. (1987), The Pricing of Options on Assets with Stochastic Volatilities, in: Journal of Finance, Vol. 42, S. 281 - 300.

IDW (1983), Stellungnahme HFA 2/1983: Grundsätze zur Durchführung von Unternehmensbewertungen, in: Die Wirtschaftsprüfung, 36. Jg., S. 468 480 .

IDW (1999), Entwurf IDW Standard: Grundsätze zur Durchführung von Unternehmensbewertungen, in: Die Wirtschaftsprüfung, 52. Jg., S. 200 216.

Illing, G. (1995), Spieltheorie in den Wirtschaftswissenschaften, in: Wirtschaftswissenschaftliches Studium, 24. Jg., S. 509 - 516.

Jackwerth, J./Rubinstein, M. (1996), Recovering Stochastic Processes from Option Prices, Working Paper, University of California at Berkeley.

Jaensch, G. (1966), Wert und Preis der ganzen Unternehmung, Köln u.a.

Jaensch, G. (1989), Unternehmensbewertung bei Akquisitionen in den USA, in: Zeitschrift für betriebswirtschaftliche Forschung, 41. Jg., S. 329 - 339.

Janschek, O. (1989), Unternehmensbewertung und Risiko, in: Jahrbuch für Controlling und Rechnungswesen '89, hrsg. von Seicht, G., Wien, S. 97 126.

Jarrow, R. A./Rudd, A. (1983), Option Pricing, Homewood.

Johnson, H. E. (1983), An Analytic Approximation for the American Put Price, in: Journal of Financial and Quantitative Analysis, Vol. 18, S. $141 \mathrm{ff}$.

Jonas, M. (1995), Unternehmensbewertung - Zur Anwendung der Discounted Cash Flow-Methode in Deutschland, in: Betriebswirtschaftliche Forschung und Praxis, 47. Jg., S. 83 - 98. 
Jurgeit, L. (1989), Bewertung von Optionen und bonitätsrisikobehafteten Finanztiteln - Anleihen, Kredite und Fremdfinanzierungsfazilitäten, Wiesbaden.

Kasanen, E. (1993), Creating Value by Spawning Investment Opportunities, in: Financial Management, Vol. 22, S. 251 - 258.

Kasanen, E./Trigeorgis, L. (1993), Flexibility, Synergy, and Control in Strategic Investment Planning, in: Capital Budgeting under Uncertainty New and Advanced Perspectives, ed. by Aggarwal, R., Englewood Cliffs, S. $208-231$.

Kasanen, E./Trigeorgis, L. (1994), A Market Utility Approach to Investment Valuation, in: European Journal of Operational Research, Vol. 74, S. 294 -309 .

Kasanen, E./Trigeorgis, L. (1995), Merging Finance Theory and Decision Analysis, in: Real Options in Capital Investment, ed. by Trigeorgis, L., Westport, S. 47 - 68.

Kemna, A. G. (1987), Options in Real and Financial Markets, Doctoral Dissertation, University of Rotterdam.

Kemna, A. G. (1993), Case Studies on Real Options, in: Financial Management, Vol. 22, S. 259 - 270.

Kensinger, J. W. (1987), Adding the Value of Active Management into the Capital Budgeting Equation, in: Midland Corporate Finance Journal, Vol. 5, S. $31-42$.

Kester, W. C. (1984), Today's Options for Tomorrow's Growth, in: Harvard Business Review, Vol. 62, S. 152 - 160.

Kester, W. C. (1986), An Options Approach to Corporate Finance, in: Handbook of Corporate Finance, ed. by Altman, E. I./McKinney, M. J., New York et al, Section 5.

Kester, W. C. (1993), Turning Growth Options Into Real Assets, in: Capital Budgeting under Uncertainty, ed. by Aggarwal, R., Englewood Cliffs, S. 187 - 207.

Kesting, H./Schulte-Mattler, H. (1992), Das binomiale Optionspreismodell, in: Wirtschaftswissenschaftliches Studium, 21. Jg., S. 211 - 215.

Kesting, H./Schulte-Mattler, H. (1992), Herleitung der Black/Scholes-Formel aus dem binomialen Optionspreismodell, in: Wirtschaftswissenschaftliches Studium, 21. Jg., S. 167 - 171.

Kilka, M. (1995), Realoptionen - Optionspreistheoretische Ansätze bei Investitionsentscheidungen unter Unsicherheit, Frankfurt. 
Kirsch, H.-J./Krause, C. (1996), Kritische Überlegungen zur Discounted Cash Flow-Methode, in: Zeitschrift für Betriebswirtschaft, 66. Jg., S. 793 $-812$.

Kishimoto, N. (1989), Pricing Contingent Claim under Interest Rate and Asset Price Risk, in: Journal of Finance, Vol. 44, S. 571 - 589.

Klump, R. (1985), Wiener-Prozesse und das Itô-Theorem, in: Wirtschaftswissenschaftliches Studium, 14. Jg., S. 183 - 185.

Koch, C. (1999), Optionsbasierte Unternehmensbewertung - Realoptionen im Rahmen von Akquisitionen, Wiesbaden.

Kogeler, R. (1992), Synergiemanagement im Akquisitions- und Integrationsprozeß von Unternehmungen, München.

Kogut, B. (1991), Joint Ventures and the Option to Expand and Acquire, in: Management Science, Vol. 37, S. 19 - 33.

Kogut, B./Kulatilaka, N. (1994), Options Thinking and Platform Investments: Investing in Opportunity, in: California Management Review, Vol. 36, S. $52-71$.

Krag, J. (1978), Die Berücksichtigung der Ungewißheit in der Unternehmungsbewertung mit Hilfe eines modifizierten Ertragswertkalküls, in: Zeitschrift für Betriebswirtschaft, 48. Jg., S. 439 - 451.

Krahnen, J. P./Schmidt, R. H./Terberger, E. (1985), Der ökonomische Wert von Flexibilität und Bindung, in: Information und Wirtschaftlichkeit, hrsg. von Ballwieser, W./Berger, K. H., Wiesbaden, S. 253 - 285.

Kreikebaum, H. (1997), Strategische Unternehmensplanung, 6. Aufl., Stuttgart, Berlin, Köln.

Kreilkamp, E. (1987), Strategisches Management und Marketing - Markt- und Wettbewerbsanalyse, Strategische Frühaufklärung, Portfoliomanagement, Berlin, New York.

Kruschwitz, L. (1998), Investitionsrechnung, 7. Aufl., Berlin, New York.

Kruschwitz, L. (1999), Finanzierung und Investition, 2. Aufl., München, Wien.

Kruschwitz, L./Löffler, A. (1998), Revisited, in: Ergebnisse des Berliner Workshops „Unternehmensbewertung“ vom 7. Februar 1998, Diskussionsbeitrag des Fachbereichs Wirtschaftswissenschaft der Freien Universität Berlin, hrsg. von Kruschwitz, L./Löffler, A., Berlin, S. 35 - 42.

Kruschwitz, L./Schöbel, R. (1984), Die Bewertung europäischer und amerikanischer Puts, in: Das Wirtschaftsstudium, 13. Jg., S. 378 - 386. 
Kruschwitz, L./Schöbel, R. (1984), Eine Einführung in die Optionspreistheorie, in: Das Wirtschaftsstudium, 13. Jg., S. 68 - 72, S. 116 - 121 und S. $171-176$.

Kruschwitz, L./Schöbel, R. (1987), Die Beurteilung riskanter Investitionen und das Capital Asset Pricing Model (CAPM), in: Wirtschaftswissenschaftliches Studium, 16. Jg., S. 67 - 72.

Künnemann, M. (1985), Objektivierte Unternehmensbewertung, Frankfurt.

Küting, K. (1981), Zur Bedeutung und Analyse von Verbundeffekten im Rahmen der Unternehmensbewertung, in: Betriebswirtschaftliche Forschung und Praxis, 33. Jg., S. 175 - 189.

Kulatilaka, N. (1995), Operating Flexibility in Capital Budgeting: Substitutability and Complementarity in Real Options, in: Real Options in Capital Investment, ed. by Trigeorgis, L., Westport, S. 121 - 132.

Kulatilaka, N./Marcus, A. J. (1988), General Formulation of Corporate Real Options, in: Research in Finance, Vol. 7, S. 183 - 199.

Kulatilaka, N./Marcus, A. J. (1992), Project Valuation under Uncertainty: When Does DCF Fail?, in: Journal of Applied Corporate Finance, Vol. 5, S. $92-100$.

Kulatilaka, N./Perotti, E. C. (1998), Strategic Growth Options, in: Management Science, Vol. 44, S. 1021 - 1031.

Kulatilaka, N./Trigeorgis, L. (1994), The General Flexiblitiy to Switch: Real Options Revisited, in: International Journal of Finance, Vol. 6, S. 778 798.

Laux, C. (1993), Handlungsspielräume im Leistungsbereich des Unternehmens: Eine Anwendung der Optionspreistheorie, in: Zeitschrift für betriebswirtschaftliche Forschung, 45. Jg., S. 933 - 958.

Laux, H. (1971), Flexible Investitionsplanung - Einführung in die Theorie der sequentiellen Entscheidungen bei Unsicherheit, Opladen.

Laux, H. (1971), Unternehmensbewertung bei Unsicherheit, in: Zeitschrift für Betriebswirtschaft, 41. Jg., S. 525 - 540.

Laux, H. (1995), Entscheidungstheorie, 3. Aufl., Berlin, Heidelberg, New York.

Lee, C. J. (1988), Capital Budgeting under Uncertainty: The Issue of Optimal Timing, in: Journal of Business Finance and Accounting, Vol. 15, S. 155 $-168$.

Leland, H. E. (1985), Option Pricing and Replication with Transaction Costs, in: Journal of Finance, Vol. 40, S. 1283 - 1301. 
Leslie, K. J./Michaels, M. P. (1997), The Real Power of Real Options, in: McKinsey Quarterly, Vol. 33, S. 4 - 22.

Lettau, M./Uhlig, H. (1999), Rules of Thumb versus Dynamic Programming, in: American Economic Review, Vol. 89, S. 148 - 174.

Levy, H./Levy, A. (1991), Option Valuation: An Extension of the Binomial Model, in: Advances in Futures and Options Research, Vol. 5, ed. by Fabozzi, F., S. 49 - 70.

Lewellen, W. G./Long, M. S. (1972), Simulation versus Single Value Estimates in Capital Expenditures Analysis, in: Decision Sciences, Vol. 3, S. 19 -35 .

Lewis, T. G. (1995), Steigerung des Unternehmenswerts - Total Value Management, 2. Aufl., Landsberg am Lech.

Liebler, H. (1996), Strategische Optionen - eine kapitalmarktorientierte Bewertung von Investitionen unter Unsicherheit, Konstanz.

Lint, O./Pennings, E./Natter, M. (1999), Optionsmanagement in F\&E - eine Fallstudie, in: Zeitschrift für betriebswirtschaftliche Forschung, 51. Jg., S. 990 - 1006.

Lintner, J. (1965), The Valuation of Risky Assets and the Selection of Risky Investments in Stock Portfolios and Capital Budgeting, in: Review of Economics and Statistics, Vol. 47, S. 13 - 37.

Lo, A. W./Wang, J. (1995), Implementing Option Pricing Models when Asset Returns are Predictable, in: Journal of Finance, Vol. 50, S. 87 - 129.

Loistl, O. (1992), Computergestütztes Wertpapiermanagement, 4. Aufl., München, Wien.

Loistl, O. (1994), Kapitalmarkttheorie, 3. Aufl., München.

Lorson, P. ( 1999), Shareholder Value-Ansätze - Zweck, Konzepte und Entwicklungstendenzen, in: Der Betrieb, 52. Jg., S. 1329 - 1339.

Luehrman, T. (1994), Financial Engeenering at Merck, in: Harvard Business Review, Vol. 72, S. 94 - 97.

Luehrman, T. (1998), Investment Opportunities as Real Options: Getting Started on the Numbers, in: Harvard Business Review, Vol. 76, S. 51 67.

Luehrman, T. (1998), Strategy as a Portfolio of Real Options, in: Harvard Business Review, Vol. 76, S. 89 - 99. 
Lund, D. (1991), Stochastic Models and Option Values: An Introduction, in: Stochastic Models and Option Values - Applications to Resources, Environment, and Investment Problems, ed. by Lund, D./ $\varnothing \mathrm{ksendahl,} \mathrm{B.,} \mathrm{Am-}$ sterdam et al, S. 3 - 18.

MacMillan, L. W. (1986), Analytic Approximation for the American Put Option, in: Advances in Futures and Options Research, Vol. 1, S. 119 - 139.

Maggi, G. (1996), Endogenous Leadership in a New Market, in: RAND Journal of Economics, Vol. 27, S. 641 - 659.

Majd, S./Pindyck, R. S. (1987), Time to Build, Option Value, and Investment Decisions, in: Journal of Financial Economics, Vol. 18, S. 7 - 27.

Mamer, J. W./McCardle, K. F. (1987), Uncertainty, Competition and the Adoption of New Technology, in: Management Science, Vol. 33, S. 161 177.

Mandl, G./Rabel, K. (1997), Unternehmensbewertung - eine praxisorientierte Einführung, Wien, Frankfurt.

Mann, T. (1994), Einführung in Wiener-Prozesse, in: Das Wirtschaftsstudium, 23. Jg., S. 674 - 676.

Mann, T. (1995), Wiener-Ito-Prozesse und Zeithorizonteffekte, in: Das Wirtschaftsstudium, 24. Jg., S. 299 - 304.

Margrabe, W. (1978), The Value of an Option to Exchange One Asset for Another, in: Journal of Finance, Vol. 33, S. 177 - 186.

Mason, S. P./Merton, R. C. (1985), The Role of Contingent Claims Analysis in Corporate Finance, in: Recent Advances in Corporate Finance, ed. by Altmann, E./ Subrahmanyam, M. G., Homewood, S. 7 - 54.

Matschke, M. (1975), Der Entscheidungswert der Unternehmung, Wiesbaden.

McDonald, R. L. (1998), Real Options and Rules of Thumb in Capital Budgeting, Working Paper, Northwestern University.

McDonald, R. L./Siegel, D. R. (1984), Option Pricing When the Underlying Asset Earns a Below-Equilibrium Rate of Return: A Note, in: Journal of Finance, Vol. 39, S. 261 - 265.

McDonald, R. L./Siegel, D. R. (1985), Investment and the Valuation of Firms when there is an Option to Shut Down, in: International Economic Review, Vol. 26, S. 331 - 349.

McDonald, R. L./Siegel, D. R. (1986), The Value of Waiting to Invest, in: Quarterly Journal of Economics, Vol. 101, S. 707 - 727. 
McGrath, R. G. (1997), A Real Options Logic for Initiating Technology Positioning Investments, in: Academy of Management Review, Vol. 22, S. 974 - 996.

McGrath, R. G. (1999), Falling Forward - Real Options Reasoning and Entrepreneurial Failure, in: Academy of Management Review, Vol. 24, S. 13 30.

Meise, F. (1998), Realoptionen als Investitionskalkül, München.

Merton, R. C. (1973), A Theory of Rational Option Pricing, in: Bell Journal of Economics and Management Sciences, Vol. 4, S. 141 - 183.

Merton, R. C. (1976), Option Pricing When Underlying Stock Returns are Discontinous, in: Journal of Financial Economics, Vol. 3, S. 125 - 144.

Merton, R. C. (1997), Applications of Option Pricing Theory - Twenty-Five Years Later, in: American Economic Review, Vol. 88, S. 323 - 349.

Mossin, J. (1966), Capital Asset Market, in: Econometrica, Vol. 34, S. 768 783.

Mostowfi, M. (1997), Bewertung von Investitionen unter Berücksichtigung zeitlicher Flexibilität, in: Betriebswirtschaftliche Forschung und Praxis, 49. Jg., S. 580 - 592.

Moxter, A. (1983), Grundsätze ordnungsmäßiger Unternehmensbewertung, 2. Aufl., 1993.

Münstermann, H. (1970), Wert und Bewertung der Unternehmung, Wiesbaden.

Myers, S. C. (1977), Determinants of Corporate Borrowing, in: Journal of Financial Economics, Vol. 5, S. 147 - 175.

Myers, S. C. (1984), Finance Theory and Financial Strategy, in: Interfaces, Vol. 14, S. 126 - 137.

Myers, S. C. (1986), The Valuation of an Acquisition Target, in: The Revolution in Corporate Finance, ed. by Stern, J. M./Chew, D. H., New York, S. 394 - 401.

Myers, S. C./Majd, S. (1990), Abandonment Value and Project Life, in: Advances in Futures and Options Research, Vol. 4, ed. by Fabozzi, Frank, Greenwich, S. 1 - 21.

Nelken, I. (Ed.) (1996), The Handbook of Exotic Options, Chicago.

Neumann, M. (1999), Optionsbewertung und Risikomessung mit impliziten Binomialbäumen, Wiesbaden. 
Neuss, W./Nippel, P. (1996), Was ist strategisch an strategischem Verhalten? Überlegungen zur Präzisierung eines inflationär benutzten Begriffs am Beispiel von Investitionsentscheidungen, in: Zeitschrift für betriebswirtschaftliche Forschung, 48. Jg., S. 423 - 441.

Nietert, B./Wilhelm, J. (1998), Arbitrage, Pseudowahrscheinlichkeiten und Martingale - ein didaktisch einfacher Zugang zur elementaren Bewertungstheorie für Derivate, Diskussionsbeitrag Nr. B-2-98 der wirtschaftswissenschaftlichen Fakultät der Universität Passau.

Nippel, P. (1994), Stellungnahme zu: Die Behandlung von Optionen in der betrieblichen Investitionsrechnung, von Eble und Völker, in: Die Unternehmung, 48. Jg., S. 149 - 152.

Nippel, P. (1996), Alternative Sichtweisen der Marktbewertung im CAPM, in: Wirtschaftswissenschaftliches Studium, 25. Jg., S. 106 - 111.

Nippel, P. (1997), Strategische Investitionsplanung und Finanzierung, Heidelberg.

Nippel, P. (1999), Zirkularitätsprobleme in der Unternehmensbewertung, in: Betriebswirtschaftliche Forschung und Praxis, 51. Jg., S. 333 - 347.

Nöldeke, G./Schmidt K. M. (1998), Sequential Investments and Options to Own, in: RAND Journal of Economics, Vol. 29, S. 633 - 653.

Oehler A. (1998), Abnehmende oder zunehmende relative Risikoaversion?, in: Zeitschrift für Bankrecht und Bankwirtschaft, 10. Jg., S. 230 - 236.

Ossadnik, W. (1995), Die Aufteilung von Synergieeffekten bei Fusionen, Stuttgart.

Paddock, J./Siegel, D./Smith, C. W. (1988), Option Valuation of Claims in Real Assets: The Case of Offshore Petroleum Leases, in: Quarterly Journal of Economics, Vol. 103, S. 479 - 508.

Paprottka, S. (1996), Unternehmenszusammenschlüsse - Synergiepotentiale und ihre Umsetzung durch Integration, Wiesbaden.

Parkinson, M. (1977), Option Pricing: The American Put, in: Journal of Business, Vol. 50, S. 21 - 36.

Peemöller, V. /Bömelburg, P./Denkmann, A. (1994), Unternehmensbewertung in Deutschland - eine empirische Erhebung, in: Die Wirtschaftsprüfung, 47. Jg., S. 741 - 749.

Peemöller, V./Keller, B./Rödl, M. (1996), Verfahren strategischer Unternehmensbewertung, in: Deutsches Steuerrecht, 34. Jg., S. 74 - 79.

Pellens, B. (Hrsg.) (1998), Unternehmenswertorientierte Entlohnungssysteme, Stuttgart. 
Pellens, B./Crasselt, N./Tomaszewski, C. (1998), Marktwertorientiertes Controlling in Dienstleistungsunternehmen, in: Handbuch Dienstleistungsunternehmen, hrsg. von Bruhn, M./Meffert, H., Wiesbaden, S. 891 - 912.

Pellens, B./Rockholtz, C./Stienemann, M. (1997), Marktwertorientiertes Konzerncontrolling in Deutschland, in: Der Betrieb, 50. Jg., S. 1933 - 1939.

Pennings, E. (1998), Real Options and Managerial Decision Making, Doctoral Dissertation, Erasmus University Rotterdam

Perin, S. (1996), Synergien bei Unternehmensakquisitionen - empirische Untersuchung von Finanz-, Markt- und Leistungssynergien, Wiesbaden.

Perlitz, M./Peske T./Schrank, R. (1999), Real Options Valuation - the New Frontier in R\&D Project Evaluation?, in: R \& D Management, Vol. 29, S. 255 - 269.

Perridon, L./Steiner, M. (1997), Finanzwirtschaft der Unternehmung, 9. Aufl., München.

Peschke, M. A. (1997), Wertorientierte Strategiebewertung - Modell, Konzeption und Umsetzung, Wiesbaden.

Petersen, M. C. (1994), Die finanzwirtschaftliche Bewertung strategischer Unternehmungskäufe - ein Konzept zur Evaluation von Akquisitionszielen im Rahmen der Unternehmensentwicklung, Bergisch Gladbach, Köln.

Pfingsten, F. (1998), Shareholder Value im Lebenszyklus - Methoden einer marktwertorientierten Unternehmensführung, Wiesbaden.

Pflüger, M./Ulrich, J. (1997), Amerikanische Optionen und Dividenden im Binomialmodell, in: Wirtschaftswissenschaftliches Studium, 26. Jg., S. 62 - 69 .

Pickles, E./Smith, J. L. (1993), Petroleum Property Valuation - A Binomial Lattice Implementation of Option Pricing Theory, in: Energy Journal, Vol. 14, S. 1 - 26.

Pinches, G. E. (Ed.) (1998), Real Options: Developments and Applications, in: Quarterly Review of Economics and Finance, Special Issue, Vol. 38, Stamford, London.

Pindyck, R. S. (1988), Irreversible Investment, Capacity Choice and the Value of the Firm, in: American Economic Review, Vol. 78, S. 969 - 985.

Pindyck, R. S. (1991), Irreversibility and the Explanation of Investment Behaviour, in: Stochastic Models and Option Values, ed. by Lund, D./Øksendal, B., Amsterdam et al. S. 129 - 141.

Pindyck, R. S. (1991), Irreversibility, Uncertainty and Investment, in: Journal of Economic Literature, Vol. 29, S. 1110 - 1148. 
Porter, M. E. (1986), Wettbewerbsvorteile: Spitzenleistungen erreichen und behaupten, Frankfurt.

Porter, M. E. (1995), Wettbewerbsstrategie: Methoden zur Analyse von Branchen und Konkurrenten, 8. Aufl., Frankfurt.

Porter, M. E. (1996), Wettbewerbsvorteile: Spitzenleistungen erreichen und behaupten, 4. Aufl., Frankfurt.

Prietze, O./Walker, A. (1995), Der Kapitalisierungszinsfuß im Rahmen der Unternehmensbewertung - eine empirische Analyse, in: Die Betriebswirtschaft, 55. Jg., S. 199 - 211.

Pümpin, C. (1990), Strategische Unternehmensbewertung, in: Der Schweizer Treuhänder, 64 Jg., S. 553 - 556.

Quigg, L. (1993), Empirical Testing of Real Option Pricing Models, in: Journal of Finance, Vol. 47, S. 621 - 640.

Rams, A. (1999), Realoptionsbasierte Unternehmensbewertung, in: Finanz Betrieb, 1. Jg., S. 349 - 364.

Rao, R./Martin, J. (1981), Another Look at the Use of Options Pricing Theory to Evaluate Real Asset Investment Opportunities, in: Journal of Business Finance and Accounting, Vol. 8, S. 421 - 429.

Rappaport, A. (1995), Shareholder Value - Wertsteigerung als Maßstab für die Unternehmensführung, Stuttgart.

Rappaport, A. (1998), Creating Shareholder Value - A Guide for Managers and Investors, $2^{\text {nd }}$ ed., New York et al.

Reinhardt, H. C. (1997), Kapitalmarktorientierte Bewertung industrieller F\&E-Projekte, Wiesbaden.

Reiß, A. (1998), Bewertung von Optionen unter Transaktionskosten, Heidelberg.

Reiß, A. (1998), Investment in Innovations and Competition: An Option Pricing Approach, in: Quarterly Review of Economics and Finance, Special Issue, Vol. 38, Real Options: Developments and Applications, ed. by. Pinches, G. E., S. 635 - 650.

Reißner, S. (1992), Synergiemanagement und Akquisitionserfolg, Wiesbaden.

Rendleman, R. J./Barter, B. J. (1979), Two-State Option Pricing, in: Journal of Finance, Vol. 34, S. 1093 - 1110. 
Richter, F. (1996), Die Finanzierungsprämissen des Entity-Ansatzes vor dem Hintergrund des APV-Ansatzes zur Bestimmung von Unternehmenswerten, in: Zeitschrift für betriebswirtschaftliche Forschung, 48. Jg., S. 1076 - 1097.

Richter, F. (1997), DCF-Methoden und Unternehmensbewertung: Analyse systematischer Abweichungen der Bewertungsergebnisse, in: Zeitschrift für Bankrecht und Bankwirtschaft, 9. Jg., S. $226-237$.

Richter, F. (1998), Unternehmensbewertung bei variablem Verschuldungsgrad, in: Zeitschrift für Bankrecht und Bankwirtschaft, 10. Jg., S. 379 389.

Richter, F. (1999), Konzeption eines marktwertorientierten Steuerungs- und Monitoringsystems, 2. Aufl., Frankfurt.

Rieck, C. (1993), Spieltheorie: Einführung für Wirtschafts- und Sozialwissenschaftler, Wiesbaden.

Ritchken, P./Rabinowitz, G. (1988), Capital Budgeting Using Contingent Claims Analysis: A Tutorial, in: Advances in Futures and Options Research, Vol. 3, ed. by Fabozzi, F. J., S. 119 - 143.

Ritchken, P./Trevor, R. (1999), Pricing Options under Generalized GARCH and Stochastic Volatility Processes, in: Journal of Finance, Vol. 54, S. 377 - 402.

Robichek, A. A./Myers, S. C. (1966), Conceptual Problems in the Use of Risk Adjusted Discount Rates, in: Journal of Finance, Vol. 21, S. 727 - 730.

Robichek, A. A./Van Horne, J. C. (1967), Abandonment Value and Capital Budgeting, in: Journal of Finance, Vol. 22, S. 577 - 589.

Robichek, A. A./Van Horne, J. C. (1969), Abandonment Value and Capital Budgeting (Reply), in: Journal of Finance, Vol. 24, S. 96 - 97.

Roll, R. (1977), An Analytic Valuation Formula for Unprotected American Call Option on Stock with Known Dividends, in: Journal of Financial Economics, Vol. 5, S. 251 - 258.

Ropella, W. (1989), Synergie als strategisches Ziel der Unternehmung, Berlin, New York.

Ross, S. A. (1978), A Simple Approach to the Valuation of Risky Streams, in: Journal of Business, Vol. 51, S. 453 - 475.

Ross, S. A./Westerfield, R. W./Jaffe J. F. (1996), Corporate Finance, $4^{\text {th }}$ ed., Boston.

Rubinstein, M. (1976), The Valuation of Uncertain Income Streams and the Pricing of Options, in: Bell Journal of Economics, Vol. 7, S. 407 - 425. 
Rubinstein, M. (1983), Displaced Diffusion Option Pricing, in: Journal of Finance, Vol. 38, S. 213 - 217.

Rubinstein, M. (1994), Implied Binomial Trees, in: Journal of Finance, Vol. 69, S. $771-818$.

Ruhnke, K. (1991), Unternehmensbewertung, Ermittlung der Preisobergrenze bei strategisch motivierten Akquisitionen, in: Der Betrieb, 44. Jg., S. 1889 - 1894.

Ruhnke, K. (1995), Ansätze zur Unternehmensbewertung und -preisfindung in Theorie und Praxis, in: Mergers \& Acquisitions-Review, 6. Jg., S. 6 - 15.

Sakellaris, P. (1995), Investment under Uncertain Market Conditions, in: Review of Economics and Statistics, Vol. 77, S. 455 - 469.

Sandler, G. (1991), Synergie: Konzept, Messung und Realisation - verdeutlicht am Beispiel der horizontalen Diversifikation durch Akquisition, Bamberg.

Sanfleber-Decher, M. (1992), Unternehmensbewertung in den USA, in: Die Wirtschaftsprüfung, 45. Jg., S. 597 - 603.

Sautter, M. T. (1989), Strategische Analyse von Unternehmensakquisitionen, Frankfurt.

Schäfer, K. (1994), Optionsbewertung mit Monte-Carlo-Methoden, Köln.

Scharlemann, U. (1996), Finanzwirtschaftliche Synergiepotentiale von Mergers und Acquisitions - Analyse nicht güterwirtschaftlicher Wertsteigerungseffekte von Unternehmenstransaktionen, Stuttgart, Wien.

Schlag, C. (1995), Bewertung derivativer Finanztitel in zeit- und zustandsdiskreten Modellen, Wiesbaden.

Schleifer, A./Vishny, R. W. (1997), In Limits of Arbitrage, in: Journal of Finance, Vol. 52, S. 35 - 55.

Schmidt, J. G. (1995), Die Discounted Cash Flow-Methode - nur eine kleine Abwandlung der Ertragswertmethode?, in: Zeitschrift für betriebswirtschaftliche Forschung, 47. Jg., S. 1088 - 1118.

Schmidt, J. G. (1996), Unternehmensbewertung mit Hilfe strategischer Erfolgsfaktoren, Frankfurt u.a.

Schmidt, R./Terberger, E. (1997), Grundzüge der Investitions- und Finanzierungstheorie, 4. Aufl., Wiesbaden.

Schnabel, H. J. (1998), Wertorientierte Vergütung von Führungskräften, Wiesbaden. 
Schneider, D. (1998), Marktwertorientierte Unternehmensrechnung: Pegasus mit Klumpfuß, in: Der Betrieb, 51. Jg., S. 1473 - 1478.

Schneider, J. (1988), Die Ermittlung strategischer Unternehmenswerte, in: Betriebswirtschaftliche Forschung und Praxis, 40. Jg., S. 522 - 531.

Schneider, J. (1991), Strategische Unternehmensbewertung als Teil der Akquisitionsplanung, in: Strategieentwicklung: Konzepte und Erfahrungen, hrsg. von Riekhof, H.-C., Stuttgart, S. 213 - 234.

Schöbel, R. (1995), Kapitalmarkt und zeitkontinuierliche Bewertung, Heidelberg.

Scholes, M. (1976), Taxes and the Pricing of Options, in: Journal of Finance, Vol. 21, S. 319 - 332.

Schroder, M. (1988), Adapting the Binomial Model to Value Options on Assets with Fixed Cash Payouts, in: Financial Analysts Journal, Vol. 44, S. 54 - 62 .

Schwetzler, B. (1998), Unternehmensbewertung unter Unsicherheit - Sicherheitsäquivalent- oder Risikozuschlagsmethode?, Arbeitspapier Nr. 21 der Handelshochschule Leipzig.

Schwetzler, B./Darijtschuk, N. (1999), Unternehmensbewertung mit Hilfe der DCF-Methode - eine Anmerkung zum „Zirkularitätsproblem", in: Zeitschrift für Betriebswirtschaft, 69. Jg., S. 295 - 318.

Selby, M. J./Hodges, S. D. (1987), On the Valuation of Compound Options, in: Management Science, Vol. 33, S. 347 - 355.

Senn, S. (1996), Some Statistical Issues in Project Priorization in the Pharmaceutical Industry, in: Statistics and Medicine, Vol. 15, S. 2689 - 2702.

Seppelfricke, P. (1996), Investitionstheorien unter Unsicherheit: eine theoretische und empirische Untersuchung für die Bundesrepublik Deutschland, Frankfurt.

Serfling, K./Pape, U. (1995), Theoretische Grundlagen und traditionelle Verfahren der Unternehmensbewertung, in: Das Wirtschaftsstudium, 24. Jg., S. 808 - 819 .

Serfling, K./Pape, U. (1996), Strategische Unternehmensbewertung und Discounted Cash Flow-Methode, in: Das Wirtschaftsstudium, 25. Jg., S. 57 64.

Sharpe, W. F. (1964), Capital Asset Prices - A Theory of Market Equilibrium under Conditions of Risk, in: Journal of Finance, Vol. 19, S. 425 - 442.

Sick, G. (1989), Capital Budgeting with Real Options, Monograph Series in Finance and Economics, Monograph 1989-3, New York. 
Sick, G. (1995), Real Options, in: Handbooks in Operations Research and Management Science, Vol. 9, Finance, ed. by Jarrow, R. A./Maksimovic, V./Ziemba, W. T., Amsterdam et al, S. 631 - 691.

Sieben, G. (1963), Der Substanzwert der Unternehmung, Wiesbaden.

Sieben, G. (1969), Die Bewertung von Unternehmen auf Grund von Erfolgsplänen bei heterogenen Zielen, in: Unternehmerische Planung und Entscheidung, hrsg. von Busse von Colbe, W./Meyer-Dohm, P., Bielefeld, S. $71-100$.

Sieben, G. (1983), Funktionen der Bewertung ganzer Unternehmen und von Unternehmensanteilen, in: Das Wirtschaftsstudium, 12. Jg., S. 539 - 542.

Sieben, G. (1988), Unternehmensstrategien und Kaufpreisbestimmung - ein Beitrag zur Bewertung ganzer Unternehmen, in: Festschrift 40 Jahre Der Betrieb, Stuttgart, S. 81 - 91.

Sieben, G. (1993), Unternehmensbewertung, in: Handwörterbuch der Betriebswirtschaft, hrsg. von Wittmann, W. u.a., 5. Aufl., Stuttgart, Sp. 4312 4331.

Sieben, G. (1995), Unternehmensbewertung: Discounted Cash Flow-Verfahren und Ertragswertverfahren - Zwei völlig unterschiedliche Ansätze?, in: Internationale Wirtschaftsprüfung, hrsg. von Lanfermann, J., Düsseldorf, S. 714 - 737.

Sieben, G./Diedrich, R. (1990), Aspekte der Wertfindung bei strategisch motivierten Unternehmensakquisitionen, in: Zeitschrift für betriebswirtschaftliche Forschung, 42. Jg., S. 794 - 809.

Sieben, G./Schildbach, T. (1979), Zum Stand der Entwicklung der Lehre von der Bewertung ganzer Unternehmungen, in: Deutsches Steuerrecht, 17. Jg. S. 455 - 461.

Sieben, G./Schildbach, T. (1994), Betriebswirtschaftliche Entscheidungstheorie, 4. Aufl., Düsseldorf.

Siegel, T. (1991), Das Risikoprofil als Alternative zur Berücksichtigung der Unsicherheit in der Unternehmensbewertung, in: Aktuelle Fragen der Finanzwirtschaft und der Unternehmensbesteuerung, hrsg. von Rückle, D., Wien, S. 619 - 638.

Siegel, T. (1994), Unternehmensbewertung, Unsicherheit und Komplexitätsreduktion, in: Betriebswirtschaftliche Forschung und Praxis, 46. Jg., S. 457 $-476$.

Siegert, T./Böhme, M./Pfingsten, F./Picot, A. (1997), Marktwertorientierte Unternehmensführung im Lebenszyklus, in: Zeitschrift für betriebswirtschaftliche Forschung, 49. Jg., S. 471 - 488. 
Slater, S. F./Reddy V. K./Zwirlein T. J. (1998), Evaluating Strategic Investments - Complementing Discounted Cash Flow Analysis with Options Analysis, in: Industrial Marketing Management, Vol. 27, S. 447 - 458.

Smets, F. (1991), Exporting versus Foreign Direct Investment - The Effect of Uncertainty, Irreversibility and Strategic Interactions, Working Paper, Yale University.

Smit, H. T. J./Ankum, L. A. (1993), A Real Options and Game-Theoretic Approach to Corporate Investment Strategy Under Competition, in: Financial Management, Vol. 22, S. 241 - 250.

Smit, H. T. J./Trigeorgis, L. (1995), Flexibility and Commitment in Strategic Investment, Working Paper 95.74, Tinbergen Institute Amsterdam.

Smith, C. W. (1976), Option Pricing: A Review, in: Journal of Financial Economics, Vol. 3, S. 3 - 51.

Smith, J. E./McCardle, K. F. (1998), Valuing Oil Properties - Integrating Option Pricing and Decision Analysis Approaches, in: Operations Research, Vol. 46, S. 198 - 217.

Smith, J. E./Nau, R. F. (1995), Valuing Risky Projects - Options Pricing Theory and Decision Analysis, in: Management Science, Vol. 41, S. 795 816.

Smith, K. W./Triantis, A. J. (1995), The Value of Options in Strategic Acquisitions, in: Real Options in Capital Investment, ed. by Trigeorgis, L., Westport, S. 135 - 149.

Spencer, B. J./Brander, J. A. (1992), Pre-Commitment and Flexibility - Applications to Oligopoly Theory, in: European Economic Review, Vol. 36, S. $1601-1626$.

Spremann, K. (1995), Flexibilität, in: Die Unternehmung, 48. Jg., S. 219 224.

Stapleton, R. C./Subrahmanyam, M. G. (1984), The Valuation of Multivariate Contingent Claims in Discrete Time Models, in: Journal of Finance, Vol. 39, S. 207 - 228.

Stapleton, R. C./Subrahmanyam, M. G. (1984), The Valuation of Options when Asset Returns Are Generated by a Binomial Process, in: Journal of Finance, Vol. 39, S. 1525 - 1539.

Stark, A. W. (1990), Irreversibility and the Capital Budgeting Process, in: Management Accounting Research, Vol. 1, S. 167 - 180.

Steidl, B. (1999), Synergiemanagement im Konzern - Organisatorische Grundlagen und Gestaltungsoptionen, Wiesbaden. 
Steiner, M./Bruns, C. (1998), Wertpapiermanagement, 6. Aufl., Stuttgart.

Steiner, M./Wallmeier, M. (1999), Unternehmensbewertung mit Discounted Cash Flow-Methoden und dem Economic Value Added-Konzept, in: Finanz Betrieb, 1. Jg., S. 1 - 10.

Stewart, B. G. (1990), The Quest for Value - The EVA Management Guide, New York.

Stickel, E. (1997), Der Einsatz der Optionspreistheorie zur Bewertung von Softwareentwicklungsprojekten, Working Paper, Universität Frankfurt/ Oder.

Stultz, R. M. (1982), Options on the Minimum or Maximum of Two Risky Assets: Analysis and Application, in: Journal of Financial Economics, Vol. 10, S. 161 - 185.

Teisberg, E. O. (1995), Methods for Evaluating Capital Investment under Uncertainty, in: Real Options in Capital Investment, ed. by Trigeorgis, L., Westport 1995, S. 31 - 46.

Terstege, U. (1995), Optionsbewertung: Möglichkeiten und Grenzen eines präferenz- und verteilungsfreien Ansatzes, Wiesbaden.

Tirole, J. (1988), The Theory of Industrial Organization, Cambridge, London.

Tourinho, O. (1979), The Valuation of Reserves of Natural Resources: An Option Pricing Approach, Doctoral Dissertation, University of California at Berkeley.

Trigeorgis, L. (1986), Valuing Real Investment Opportunities: An Options Approach to Strategic Capital Budgeting, Doctoral Dissertation, Harvard University.

Trigeorgis, L. (1988), A Conceptual Options Framework for Capital Budgeting, in: Advances in Futures and Options Research, Vol. 3, ed. by Fabozzi, F. J., Greenwich, S. 145 - 167.

Trigeorgis, L. (1990), A Real Options Application in Natural Resource Investments, in: Advances in Futures and Options Research, Vol. 4, ed. by Fabozzi, F. J., Greenwich, S. 153 - 164.

Trigeorgis, L. (1991), A Log-transformed Binomial Numerical Analysis Method for Valuing Complex Multi-Option Investments, in: Journal of Financial and Quantitative Analysis, Vol. 26, September, S. 309 - 326.

Trigeorgis, L. (1991), Anticipated Competitive Entry and Early Preemptive Investment in Deferrable Projects, in: Journal of Economics and Business, Vol. 43, S. 143 - 156. 
Trigeorgis, L. (1993), Real Options and Interactions with Financial Flexibility, in: Financial Management, Vol. 22, S. 202 - 224.

Trigeorgis, L. (1993), The Nature of Options Interactions and the Valuation of Investments with Multiple Real Options, in: Journal of Financial and Qualitative Analysis, Vol. 28, S. 1 - 20.

Trigeorgis, L. (1995), Real Options: An Overview, in: Real Options in Capital Investment, ed. by Trigeorgis, L., Westport, S. 1 - 28.

Trigeorgis, L. (1995), Valuing the Impact of Uncertain Competitive Arrivals on Deferrable Real Investment Opportunities, Working Paper, Boston University/University of Cyprus.

Trigeorgis, L. (1996), Evaluating Leases with Complex Operating Options, in: European Journal of Operational Research, Vol. 91, S. 315 - 329.

Trigeorgis, L. (1996), Real Options - Managerial Flexibility and Strategy in Resource Allocation, Cambridge.

Trigeorgis, L. (Ed.) (1995), Real Options in Capital Investment, Westport.

Trigeorgis, L./Kasanen, E. (1991), An Integrated Options-based Strategic Planning and Control Model, in: Managerial Finance, Vol. 17, S. 16 - 28.

Trigeorgis, L./Mason, S. P. (1987), Valuing Managerial Flexibility, in: Midland Corporate Finance Journal, Vol. 5, S. 14 - 21.

Valcarel, S. (1992), Ermittlung und Beurteilung des „strategischen Zuschlags“ als Brücke zwischen Unternehmenswert und Marktpreis, in: Der Betrieb, 45. Jg., S. 589 - 595.

Van Horne, J. C. (1998), Financial Management and Policy, $11^{\text {th }}$ ed., London.

Van Horne, J. C./Wachowicz, J. (1992), Fundamentals of Financial Management, $8^{\text {th }}$ ed., Englewood Cliffs.

Varian, H. R. (1987), The Arbitrage Principle in Financial Economics, in: Economic Perspectives, Vol. 1, S. 55 -72.

Vives, X. (1989), Technological Competition, Uncertainty and Oligopoly, in: Journal of Economic Theory, Vol. 48, S. 386 - 415.

Volpert, V. (1989), Kapitalwert und Ertragsteuern: Die Bedeutung der Finanzierungsprämisse für die Investitionsrechnung, Wiesbaden.

von Nitzsch, R. (1997), Investitionsbewertung und Risikofinanzierung, Stuttgart.

Weber, E. (1991), Berücksichtigung von Synergieeffekten bei der Unternehmensbewertung, in: Akquisition und Unternehmensbewertung, hrsg. von Baetge, J., Düsseldorf, S. 98 - 115. 
Welge, M./Al-Laham, A. (1999), Strategisches Management - Grundlagen, Prozess, Implementierung, 2. Aufl., Wiesbaden.

Wernerfelt, B./Karnani, A. (1987), Competitive Strategy under Uncertainty, in: Strategic Management Journal, Vol. 8, S. 187 - 194.

Whaley, R. E: (1981), On the Valuation of American Call Options on Stocks with Known Dividends, in: Journal of Financial Economics, Vol. 9, S. $207-211$.

Wilhelm, J. (1981), Zum Verhältnis von Capital Asset Pricing Model, Arbitrage Pricing Theory und Bedingungen der Arbitragefreiheit von Finanzmärkten, in: Zeitschrift für betriebswirtschaftliche Forschung, 33. Jg., S. $891-905$.

Wilhelm, J. (1983), Marktwertmaximierung - Ein didaktisch einfacher Zugang $\mathrm{zu}$ einem Grundlagenproblem der Investions- und Finanzierungstheorie, in: Zeitschrift für Betriebswirtschaft, 53. Jg., S. 516 - 534.

Wosnitza, M. (1995), Der State Preference Ansatz in der Finanzierungstheorie: Zur Praxisrelevanz des SPM, in: Das Wirtschaftsstudium, 24. Jg., S. 698 $-702$.

Wosnitza, M. (1995), Der State Preference Ansatz in der Finanzierungstheorie: Gleichgewichtstheoretische Grundlagen, in: Das Wirtschaftsstudium, 24. Jg., S. 593 - 597.

Ziegler, M. (1997), Synergieeffekte bei Unternehmenskäufen - Identifikation im Beschaffungs- und Produktionsbereich von Industriebetrieben, Wiesbaden.

Zimmermann, H. (1998), State-Preference Theorie und Asset Pricing - eine Einführung, Heidelberg.

Zimmermann, J. (1998), Investitionsbewertung mit Real Options - Ein mehrstufiges Verfahren für flexibel automatisierte Fertigungssysteme, Wien. 


\section{Bochumer Beiträge zur Unternehmungsführung und Unternehmensforschung \\ Herausgegeben vom Direktorium des Instituts für Unternehmungsführung und Unternehmensforschung der Ruhr-Universität Bochum}

Band 1 Busse von Colbe, Walther/Mattessich, Richard (Hrsg.): Der Computer im Dienste der Unternehmungsführung (1968)

Band 2 Busse von Colbe, Walther/Meyer-Dohm, Peter (Hrsg.): Unternehmerische Planung und Entscheidung (1969)

Band 3 Anthony, Robert N.: Harvard-Fälle aus der Praxis des betrieblichen Rechnungswesens. Herausgegeben von Richard V. Mattessich unter Mitarbeit von Klaus Herrnberger und Wolf Lange (1969)

Band 4 Mattessich, Richard: Die wissenschaftlichen Grundlagen des Rechnungswesens (1970)

Band 5 Schweim, Joachim: Integrierte Unternehmungsplanung (1969)

Band 6 Busse von Colbe, Walther (Hrsg.): Das Rechnungswesen als Instrument der Unternehmungsführung (1969)

Band 7 Domsch, Michel: Simultane Personal- und Investitionsplanung im Produktionsbereich (1970)

Band 8 Leunig, Manfred: Die Bilanzierung von Beteiligungen. Eine bilanztheoretische Untersuchung (1970)

Band 9 Franke, Reimund: Betriebsmodelle. Rechensystem für Zwecke der kurzfristigen Planung, Kontrolle und Kalkulation (1972)

Band 10 Wittenbrink, Hartwig: Kurzfristige Erfolgsplanung und Erfolgskontrolle mit Betriebsmodellen (1975)

Band 11 Lutter, Marcus (Hrsg.): Recht und Steuer der internationalen Unternehmensverbindungen (1972)

Band 12 Niebling, Helmut: Kurzfristige Finanzrechnung auf der Grundlage von Kosten- und Erlösmodellen (1973)

Band 13 Perlitz, Manfred: Die Prognose des Unternehmenswachstums aus Jahresabschlüssen deutscher Aktiengesellschaften (1973)

Band 14 Niggemann, Walter: Optimale Informationsprozesse in betriebswirtschaftlichen Entscheidungssituationen (1973)

Band 15 Reichardt, Harald: Der aktienrechtliche Abhängigkeitsbericht unter ökonomischen Aspekten (1974)

Band 16 Backhaus, Klaus: Direktvertrieb in der Investitionsgüterindustrie - Eine Marketing-Entscheiung (1974)

Band 17 Plinke, Wulff: Kapitalsteuerung in Filialbanken (1975)

Band 18 Steffen, Rainer: Produktionsplanung bei Fließbandfertigung (1977)

Band 19 Kolb, Jürgen: Industrielle Erlösrechnung - Grundlagen und Anwendungen (1978)

Band 20 Busse von Colbe, Walther/Lutter, Marcus (Hrsg.): Wirtschaftsprüfung heute: Entwicklung oder Reform? (1977)

Band 21 Uphues, Peter: Unternehmerische Anpassung in der Rezession (1979) 
Band 22 Gebhardt, Günther: Insolvenzprognosen aus aktienrechtlichen Jahresabschlüssen (1980)

Band 23 Domsch, Michel: Systemgestützte Personalarbeit (1980)

Band 24 Schmied, Volker: Alternativen der Arbeitsgestaltung und inre Bewertung (1982)

Band 25 Wäscher, Gerhard: Innerbetriebliche Standortplanung bei einfacher und mehrfacher Zielsetzung (1982)

Band 26 Weber, Martin: Entscheidungen bei Mehrfachzielen - Verfahren zur Unterstützung von Individual- und Gruppenentscheidungen (1983)

Band 27 Kroesen, Alfred: Instandhaltungsplanung und Betriebsplankostenrechnung (1983)

Band 28 Plinke, Wulf: Erlösplanung im industriellen Anlagengeschäft (1985)

Band 29 Chamoni, Peter: Simulation störanfälliger Systeme (1986)

Band 30 Arning, Andreas: Die wirtschaftliche Bewertung der Zentrenfertigung - Dargestellt am Beispiel einer Fertigungsinsel (1987)

Band 31 Gebhardt, Günther: Finanzielle Planung und Kontrolle bei internationaler Unternehmenstätigkeit

Band 32 Markiewicz, Michael: Ersatzteildisposition im Maschinenbau - Betriebswirtschaftliche Methoden der Planung und Überwachung (1988)

Band 33 Pellens, Bernd: Der Informationswert von Konzernabschlüssen - Eine empirische Untersuchung deutscher Börsengesellschaften (1989)

Band 34 Mrotzek, Rüdiger: Bewertung direkter Auslandsinvestitionen mit Hilfe betrieblicher Investitionskalküle (1989)

Band 35 Deppe, Joachim: Quality Circle und Lernstatt - Ein integrativer Ansatz (1989, 3. Auflage 1993)

Band 36 Rademacher, Michael: Arbeitszeitverkürzung und -flexibilisierung - Formen und betriebliche Auswirkungen (1990)

Band 37 Kaiser, Klaus: Kosten- und Leistungsrechung bei automatisierter Produktion (1991, 2. Auflage 1993)

Band 38 Müller, Hermann: Industrielle Abfallbewältigung - Entscheidungsprobleme aus betriebswirtschaftlicher Sicht (1991)

Band 39 Schörner, Peter: Gesetzliches Insiderhandelsverbot - Eine ordnungspolitische Analyse (1991)

Band 40 Bentler, Martin: Grundsätze ordnungsmäßiger Bilanzierung für die Equitymethode (1991)

Band 41 Brüggerhoff, Jürgen: Management von Desinvestitionen (1992)

Band 42 Bröker, Erich W.: Erfolgsrechnung im industriellen Anlagengeschäft - Ein dynamischer Ansatz auf Zahlungsbasis - (1993)

Band 43 Frankenberg, Peter: Transnationale Analyse US-amerikanischer und deutscher Jahresabschlüsse - Eine theoretische und empirische Untersuchung (1993)

Band 44 Kleinaltenkamp, Michael: Standardisierung und Marktprozeß - Entwicklungen und Auswirkungen im CIM-Bereich (1993)

Band 45 Pellens, Bernhard: Aktionärsschutz im Konzern - Empirische und theoretische Analyse der Reformvorschläge der Konzernverfassung (1994)

Band 46 Reckenfelderbäumer, Martin: Marketing-Accounting im Dienstleistungsbereich - Konzeption eines prozeßkostengestützten Instrumentariums (1995) 
Band 47 Knittel, Friedrich: Technikgestützte Kommunikation und Kooperation im Büro. Entwicklungshindernisse - Einsatzstrategien - Gestaltungskonzepte (1995)

Band 48 Riezler, Stephan: Lebenszyklusrechnung - Instrument des Controlling strategischer Projekte (1996)

Band 49 Schulte, Jörn: Rechnungslegung und Aktienkursentwicklung - Erklärung und Prognose von Aktienrenditen durch Einzel- und Konzernabschlußdaten (1996)

Band 50 Muhr, Martin: Zeitsparmodelle in der Industrie - Grundlagen und betriebswirtschaftliche Bedeutung mehrjähriger Arbeitszeitkonten (1996)

Band 51 Brotte, Jörg: US-amerikanische und deutsche Geschäftsberichte. Notwendigkeit, Regulierung und Praxis jahresabschlußergänzender Informationen (1997)

Band 52 Gersch, Martin: Vernetzte Geschäftsbeziehungen. Die Nutzung von EDI als Instrument des Geschäftsbeziehungsmanagement (1998)

Band 53 Währisch, Michael: Kostenrechnungspraxis in der deutschen Industrie. Eine empirische Studie (1998)

Band 54 Völkner, Peer: Modellbasierte Planung von Geschäftsprozeßabläufen (1998)

Band 55 Fülbier, Rolf Uwe: Regulierung der Ad-hoc-Publizität. Ein Beitrag zur ökonomischen Analyse des Rechts (1998)

\section{Band 1 - 55 erschienen beim Gabler Verlag Wiesbaden}

Band 56 Ane-Kristin Reif-Mosel: Computergestützte Kooperation im Büro. Gestaltung unter Berücksichtigung der Elemente Aufgabe, Struktur, Technik und Personal (2000)

Band 57 Claude Tomaszewski: Bewertung strategischer Flexibilität beim Unternehmenserwerb. Der Wertbeitrag von Realoptionen (2000) 


\section{Der Euro und die Folgen}

\section{Probleme und Perspektiven}

\section{Konzeption und Koordination: Alfons Lemper}

Frankfurt/M., Berlin, Bern, New York, Paris, Wien, 1998. 215 S.

ISBN 3-631-34021-4 · br. DM 38.-*

Die Einführung des Euro zum 1.1.1999 bringt nicht nur eine Fülle schwieriger und kostspieliger technischer Probleme mit sich, an deren Bewältigung allseits gearbeitet wird, sondern auch eine Reihe von Konsequenzen, die auf den ersten Blick nicht in Erscheinung treten, die gleichwohl für die Beurteilung des Gesamtprojektes „Europäische Währungsunion” (EWU) äußerst wichtig sind. Sie betreffen neben der prospektiven Stabilität der künftigen Währung und den Konsequenzen für die Kapitalanleger etwa Fragen nach den verbleibenden wirtschaftlichen Gestaltungsspielräumen für nationale Regierungen oder Tarifpartner, die Wahrscheinlichkeit eines innergemeinschaftlichen Finanzausgleichs oder Folgen für die Sozialstandards. Tiefgreifende Probleme ergeben sich auch für künftige Unternehmensmärkte. Die Lösung dieser - großenteils politischen - Probleme auf längere Sicht dürften für den Erfolg des Projektes EWU entscheidender werden als die bank- und bilanztechnischen Probleme, die derzeit meist im Vordergrund stehen.

Aus dem Inhalt: Prospektive Stabilität des Euro und seine internationale Position · Eingeschränkte Spielräume nationaler Wirtschaftspolitik · Finanzausgleich und Sozialstandards · Konsequenzen für Unternehmensmärkte

Frankfurt/M - Berlin - Bern - New York · Paris - Wien

Auslieferung: Verlag Peter Lang AG

Jupiterstr. 15, CH-3000 Bern 15

Telefax (004131) 9402131

«inklusive Mehrwertsteuer

Preisänderungen vorbehalten 